\title{
Access to Vinyl Ethers and Ketones with Hypervalent lodine Reagents as Oxy-Allyl Cation Synthetic Equivalents
}

\author{
Nina Declas ${ }^{[a]}$ and Jerome Waser*[a] \\ In the memory of Prof. Dr. Kilian Muñiz
}

\begin{abstract}
We report an Umpolung strategy of enol ethers to generate oxy-allyl cation equivalents based on the use of hypervalent iodine reagents. Under mild basic conditions, the addition of nucleophiles to aryloxy-substituted vinylbenziodoxolone (VBX) reagents, easily available in two steps from silyl alkynes, resulted in the stereoselective formation of substituted aryl enol ethers. The reaction was most efficient with phenols as nucleophiles, but preliminary results were also achieved for $\mathrm{C}$ - and $\mathrm{N}$ - nucleophiles. In absence of external nucleophiles, the 2-iodobenzoate group of the reagent was transferred. The obtained aryl enol ethers could then be transformed into $\alpha$-difunctionalized ketones by oxidation. The described "allyl cation"-like reactivity contrast with the well-established "vinyl-cation" behavior of alkenyl iodonium salts.
\end{abstract}

In standard organic reactions, new bonds are formed between atoms of opposite polarity. ${ }^{[1]}$ First introduced by Seebach, ${ }^{[2]}$ the Umpolung approach -inverting the reactivity of one of the partners- allowed chemical transformations impossible based on the inherent polarity of the reactants. In this context, hypervalent iodine compounds are broadly used in organic synthesis as efficient group transfer reagents via Umpolung of nucleophiles. ${ }^{[3]}$ Enolates, enol ethers and enamines are among the most important nucleophilic synthons in synthetic chemistry. ${ }^{[4]}$ The Umpolung of enolates with hypervalent iodine reagents is well established, ${ }^{[5]}$ but it is only recently that the involved enolonium species could be characterized by Szpilman and co-workers. ${ }^{[6]}$ Nevertheless, controlling transformations involving highly reactive intermediates formed in situ is challenging, and an access to stable reagents would be highly desirable.

Cyclic benziodoxol(on)es (BX) reagents are more stable and especially useful for group transfer reactions ${ }^{[7]}$ Recently, Miyake and co-workers ${ }^{[8]}$ and our group ${ }^{[9 a]}$ reported the first synthesis of enol ethers and enamides-based vinyl benziodoxol(on)es (VBX) reagents by the reaction of nucleophiles with ethynyl benziodoxol(on)es (EBX) ${ }^{[10]}$ (Scheme 1A, left). The enhanced reactivity of the hypervalent bond allowed the use of VBX as electrophiles in palladium-catalyzed Stille cross-couplings at room temperature for the formation of aryl, vinyl, alkynyl, and alkyl-substituted Z-enamides and enol ethers. ${ }^{[9 a]}$ Moreover, they reacted directly with thiol nucleophiles to form thio-enamides,

[a] N. Declas, Prof. Dr. J. Waser Laboratory of Catalysis and Organic Synthesis, Ecole Polytechnique Fédérale de Lausanne, EPFL, SB ISIC LCSO, BCH 4306 1015 Lausanne (Switzerland)

Email: jerome.waser@epfl.ch

Homepage : http://lcso.epfl.ch

Supporting information for this article is given via a link at the end of the document. Raw data for NMR, IR and MS is available at zenodo.org, DOI: 10.5281/zenodo.3894256 acting as vinyl cation equivalents, ${ }^{[9 a]}$ a well established polarityfor alkenyl iodonium salts. ${ }^{[11]}$

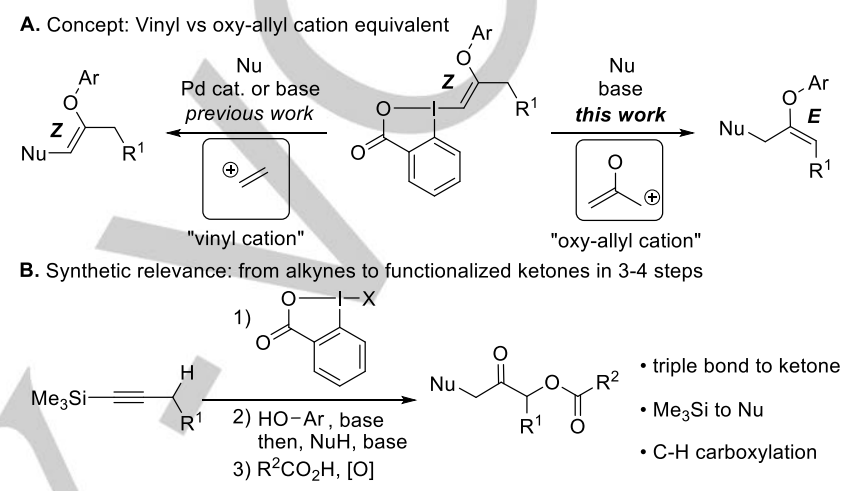

Scheme 1. O-Vinylbenziodoxolones (VBXs) as vinyl and oxy-allyl cation equivalents (A) and application to the synthesis of functionalized ketones (B).

Herein, we report a new mode of reactivity of O-VBX reagents, allowing the Umpolung of enol ethers to give formal oxy-allyl cations instead of vinyl cations (Scheme $1 \mathrm{~A}$, right). Oxy-allyl cations have been used as transient electrophilic specie for the reaction with nucleophiles ${ }^{[12]}$ or with dienes in $[4+3]$ cycloadditions. ${ }^{[13]}$ They are also formed in the versatile Nazarov cyclization. ${ }^{[14]}$ Enantioselective processes have been recently developed. ${ }^{[15]}$ Access to this type of reactive intermediates from hypervalent iodine reagents has not been reported to the best of our knowledge. Hypervalent iodoallyl intermediates have been proposed in sigmatropic [2,3] and [3,3] rearrangements. ${ }^{[16]}$ Reactive allylic cation intermediates were accessed recently using diazo-substituted hypervalent iodine reagents by Suero. ${ }^{[17]}$ In our work, we now disclose a different approach based on the treatment of O-VBX reagents with base and nucleophiles for the formation of C-O, C-N and C-C bonds in allylic position. The obtained aryl enol ethers were transformed into $\alpha$-difunctionalized ketones under oxidative conditions (Scheme 1B), resulting in a 34 steps synthesis from the corresponding silylated alkynes.

After having successfully used O-VBX reagents as vinyl cation equivalents with thiols, ${ }^{[9 a]}$ we attempted to extend this reactivity to phenols as nucleophiles. However, the reaction of $\mathrm{O}$ VBX (1a) and para-cresol (2a) under basic conditions led to the unexpected formation of allyl ether $\mathbf{3} \mathbf{a}$ as main product (Table 1). ${ }^{[18]}$ In 1,2-dimethoxyethane (DME) as solvent with 1.2 equivalents of potassium tert-butoxide as base, $\mathbf{3 a}$ could be obtained in $80 \%$ yield (entry 1 ). Other solvents gave a lower yield (See Supporting Information). When various bases were tested, cesium carbonate gave a similar NMR yield as $\mathrm{KO}$ tBu, but with higher reproducibility (entry 2), ${ }^{[19]}$ whereas organic bases, such 
as pyridine and triethylamine gave no reactivity (entries 3-4). OVBX 1a was also recovered when performing the reaction without base (entry 5). Incomplete conversion of 1a was observed when using a catalytic amount or one equivalent of base (entries 6 and 7 ). In the case of a larger excess of base (entry 8), formation of the allylic ester 4 a resulting from addition of 2-iodo benzoate was increased. In fact, small amounts of 4 a were always observed in this transformation. Allyl ester such as $\mathbf{4 a}$ are valuable building blocks in synthetic chemistry. Furthermore, all the parts of reagent 1a are incorporated in the product, resulting in high atom economy. ${ }^{[20]}$ Therefore, we optimized the formation of ester $\mathbf{4 a}$ in absence of external nucleophiles (see Supporting Information for details). In presence of $20 \mathrm{~mol} \%$ anisole as a non-participating nucleophilic additive, $\mathbf{4 a}$ could be obtained as the only product in $65 \%$ yield (entry 9 ).

Table 1. Optimisation of the addition of $p$-cresol (2a) to O-VBX 1a

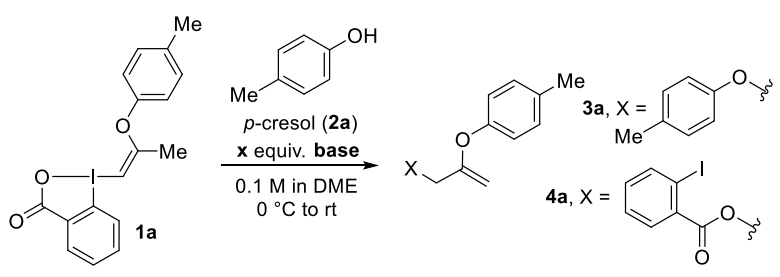

\begin{tabular}{ccccc}
\hline Entry & Base & $\begin{array}{c}\text { Base } \\
\text { equivalents }\end{array}$ & $\begin{array}{c}\text { Yield of 3a } \\
(\%)^{[a]}\end{array}$ & $\begin{array}{c}\text { Yield of 4a } \\
(\%)^{[a]}\end{array}$ \\
\hline 1 & $\mathrm{KOtBu}$ & 1.20 & 80 & $15 \%$ \\
2 & $\mathrm{Cs}_{2} \mathrm{CO}_{3}$ & 1.20 & 79 & $15 \%$ \\
3 & Pyridine & 1.20 & $\mathrm{NR}$ & $\mathrm{NR}$ \\
4 & $\mathrm{Et}{ }_{3} \mathrm{~N}$ & 1.20 & $\mathrm{NR}$ & $\mathrm{NR}$ \\
5 & none & - & $\mathrm{NR}$ & $\mathrm{NR}$ \\
6 & $\mathrm{Cs}_{2} \mathrm{CO}_{3}$ & 0.20 & 35 & $6 \%$ \\
7 & $\mathrm{Cs}_{2} \mathrm{CO}_{3}$ & 1.00 & 60 & $8 \%$ \\
8 & $\mathrm{Cs}_{2} \mathrm{CO}_{3}$ & 2.00 & 62 & $32 \%$ \\
\hline$\left[{ }^{[b]}\right.$ & $\mathrm{Cs}_{2} \mathrm{CO}_{3}$ & 1.20 & - & $65 \%$ \\
\hline
\end{tabular}

Reactions conditions: Substrate 1a $(0.100 \mathrm{mmol})$, para-cresol $(\mathbf{2 a})(0.100$ $\mathrm{mmol})$, base $(0.120 \mathrm{mmol})$ and $\operatorname{DME}(0.1 \mathrm{M})$ at $25^{\circ} \mathrm{C} . \mathrm{NR}=$ No reaction. ${ }^{\text {[a] NMR }}$ yield determined by addition of $0.1 \mathrm{mmol}$ of $\mathrm{CH}_{2} \mathrm{Br}_{2}$ as an internal standard after the reaction. When determined, the yield of $\mathbf{4 a}$ is given in parenthesis. ${ }^{[b]}$ Reaction performed without $\mathbf{2 a}$ in presence of 0.20 equiv. anisole as additive.

To explore the scope of formation of aryl enol ethers and esters, two conditions were used: conditions $\mathbf{A}$, starting from isolated O-VBX $\mathbf{1}$ and conditions $\mathbf{B}$, using an one-pot two-step procedure from the corresponding EBX reagent $\mathbf{5}$ without isolation of the intermediate O-VBX 1 (Scheme 2). For the addition of external nucleophiles to give allyl ethers $\mathbf{3}$, method $\mathbf{A}$ usually gave better yields. For allylic esters 4 , the yields were nearly identical for both methods, and the more practical method B was therefore preferred.

The scope of phenol nucleophiles was first investigated (Scheme 2A). On a $0.3 \mathrm{mmol}$ scale, using conditions A, substrate 3a was obtained in $83 \%$ yield. When conditions B where employed, only $63 \%$ yield was observed. The difference came from the larger amount of allyl ester $\mathbf{4 a}$ formed. ${ }^{[21]} 4$-Bromo- and 4-iodo-phenols afforded the corresponding products $\mathbf{3 b}$ and $\mathbf{3 c}$ in respectively $75 \%$ and $73 \%$ yields. 4-Methoxyphenol ether $\mathbf{3 d}$ was obtained in a reduced yield of $45 \%$, due to increased formation of ester $\mathbf{4 a}$. Boronic acid pinacol ester $\mathbf{3 e}$ was formed in $71 \%$ yield. A meta-methoxy group was also well tolerated (product 3f). When an unprotected amine-containing substrate was employed, 35\% of product $\mathbf{3 g}$ was still obtained, but significant decomposition was observed. Aryl enol ether $\mathbf{3 h}$ bearing an ortho ester group was obtained in $89 \%$ yield. A sterically highly hindered hydroxyacetophenone still afforded compound $3 \mathbf{i}$ in $43 \%$ yield. 2Naphthol was successfully converted into the corresponding aryl enol ether $\mathbf{3} \mathbf{j}$ in $72 \%$ yield.

The scope of the reaction could be extended to more complex phenols (Scheme 2B). Protected tyrosine gave the desired product 3k in $96 \%$ yield. Two natural products containing phenols -capsaicin and estradiol-could be used subsequently in the onepot protocol B to give highly functionalized product 3 I in $41 \%$ yield. Under the optimized conditions, the reaction worked best with phenols. Nevertheless, promising results were obtained with several C- and N-nucleophiles (Scheme 2B). Diketone and ketoester-derived products $\mathbf{3 m}$ and $\mathbf{3 n}$ were obtained in 31 and $65 \%$ yield respectively, whereas tosyl amide 30 was isolated in $33 \%$ yield.

Having explored the reactivity of various nucleophiles, we turned to the scope of O-VBX reagents with para-cresol (2a) as nucleophile (Scheme 2C). Propyl-substituted O-VBX 1b led to formation of allylic ether $3 p$ in $65 \%$ yield with a complete $E$ stereoselectivity. ${ }^{[22]}$ The formation of $3 p$ also confirmed that the new $\mathrm{C}-\mathrm{O}$ bond was formed at the position of the iodine atom. A longer alkyl chain was also well tolerated and product $\mathbf{3 q}$ was obtained in $57 \%$ yield. A hypervalent iodine reagent bearing a free alcohol could be converted into allylic ether $3 r$. Ethers $3 \mathrm{~s}$ and $3 \mathrm{t}$ containing a protected alkyne and a phthalimide group could also be accessed. We could also perform this transformation with $\mathrm{O}$ VBX bearing phenol with an iodide in ortho position to give products $3 u-w$ in $44-61 \%$ yield. An electron-donating group in para position was also well tolerated (products $\mathbf{3 x}$ and $\mathbf{3 y}$ ). When the reaction was examined for N-VBX reagent 11 bearing a sulfonamide group, the product $\mathbf{3 z}$ could be observed by NMR in about $30 \%$ yield. However, isolation of the product always led to partial decomposition and isomerization to the more stable internal alkene.

We then examined the synthesis of allyl esters using conditions B (Scheme 2D). Primary alkyl chains were well tolerated on the EBX reagents, and products $\mathbf{4 b - d}$ were obtained in good yield (Scheme 6A). EBXs bearing functional groups such as a protected alkyne, a phthalimide, an alkene or a chloride could be converted to the corresponding products $4 \mathrm{e}-\mathrm{h}$ in $60 \%$ to $82 \%$ yield. Halogen substituents were also tolerated. The ortho-iodo, metabromo and pentafluoro, derivates $4 \mathrm{i}-\mathrm{m}$ were obtained in $40 \%$ to $70 \%$ yield. In addition, phenols bearing an electron-donating methoxy group in para position or an electron-withdrawing aldehyde in ortho position could also be used to give products $4 \mathbf{n}$ p in 58-64\% yield. 


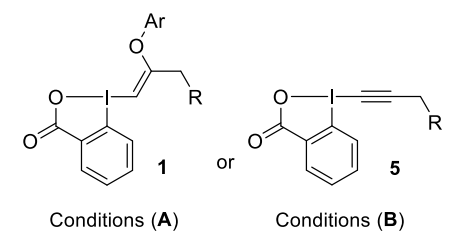

Conditions (A): 1.0 equiv. $\mathrm{Nu}-\mathrm{H}, 1.2$ equiv. $\mathrm{Cs}_{2} \mathrm{CO}_{3}$ DME $0.1 \mathrm{M}, 0^{\circ} \mathrm{C}$ to $\mathrm{rt}, 16 \mathrm{~h}$ DME $0.1 \mathrm{M}, \mathrm{rt}, 16 \mathrm{~h}$ then 0.2 equiv. anisole or 1.0 equiv. $\mathrm{Nu}-\mathrm{H}, 1.2$ equiv. $\mathrm{Cs}_{2} \mathrm{CO}_{3}$, DME $0.1 \mathrm{M}, \mathrm{rt}, 16 \mathrm{~h}$
Conditions (B): 1.0 equiv. $\mathrm{ArOH}, 10 \mathrm{~mol} \% \mathrm{Cs}_{2} \mathrm{CO}_{3}$
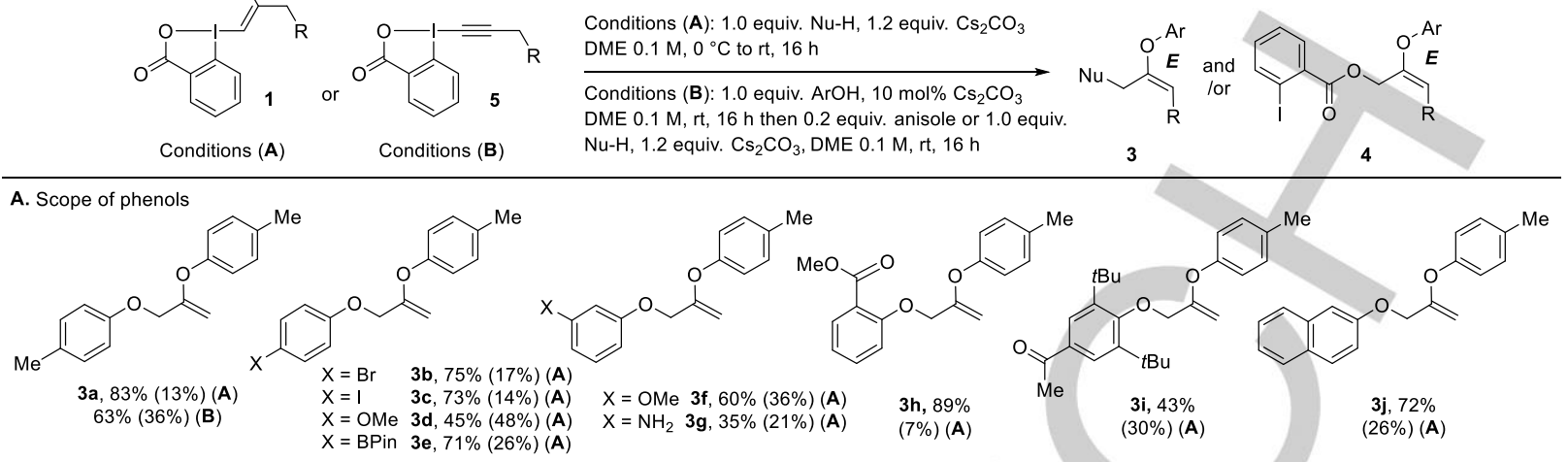

B. Scope of natural products and $\mathrm{C}$ - and $\mathrm{N}$-nucleophiles

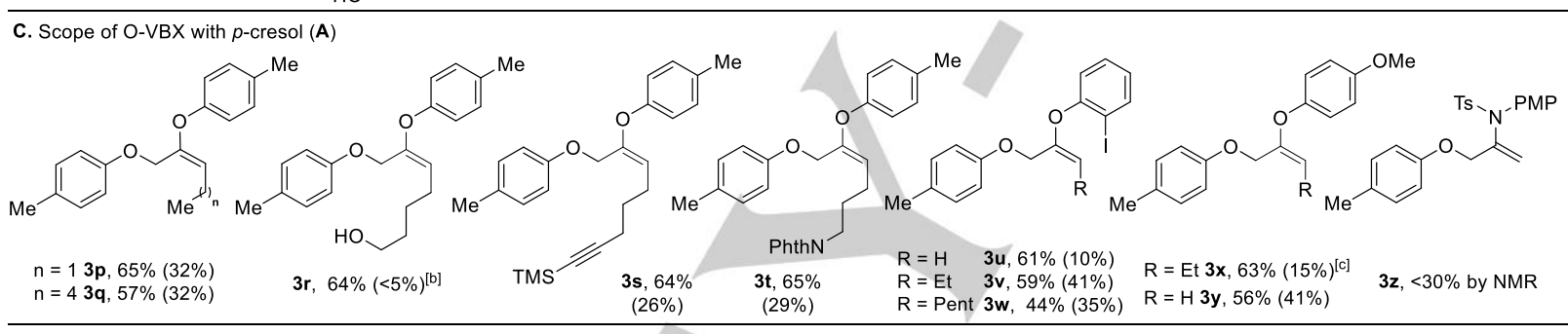

D. Scope without external nucleophiles (B)

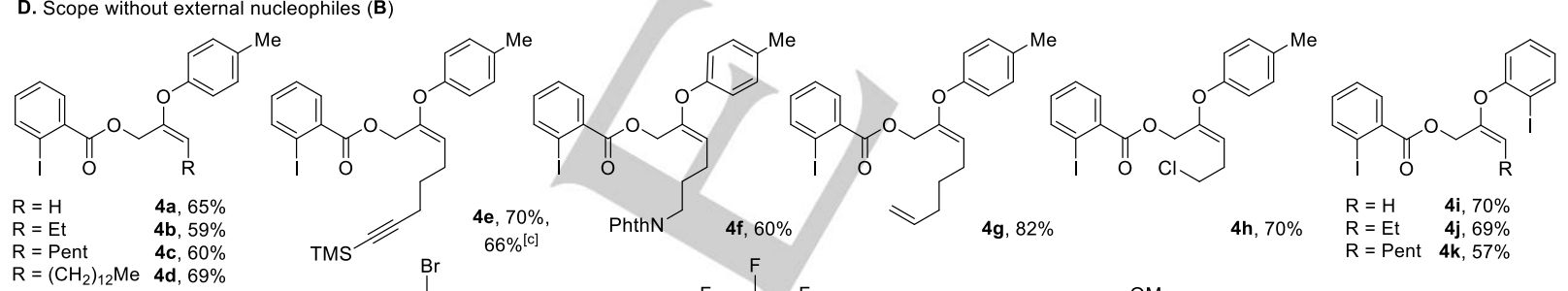
$60 \%$<smiles>C/C=C(\COC(=O)c1ccccc1I)Oc1c(F)c(F)c(F)c(F)c1F</smiles>

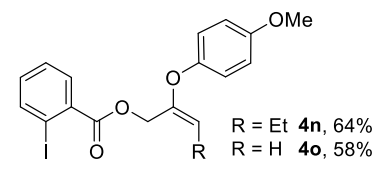

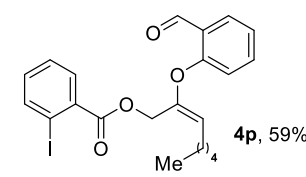

Scheme 2. Scope of the reaction. For A. B. and C. the yield of ester product $\mathbf{4}$ is given in parenthesis. ${ }^{[a]}$ Only partial conversion of $\mathbf{1 a}$ was observed. ${ }^{\left[{ }^{[b]}\right.}$ Reaction time was $24 \mathrm{~h}$. ${ }^{[c]}$ The reaction was performed on gram scale.

In some cases, formation of the O-VBX reagent $\mathbf{1}$ was successful, but the reaction stopped at this stage. In particular, this was the case for secondary alkyl groups, in absence of an allylic C-H bond, for sterically hindered O-VBX or for simple VBX lacking the ether group ${ }^{[23]}$ (see Scheme $\mathrm{S} 1$ in Supporting Information for more details). To highlight the efficiency of the transformation, gram scale syntheses were performed (Scheme $2 \mathrm{C}$ and $2 \mathrm{D}$ ). Allylic ether $3 \mathbf{x}$ was isolated in $63 \%$ yield using method A, while one-pot procedure $\mathbf{B}$ gave $66 \%$ of allylic ester 4 e. These yields are nearly identical to the one obtained on small scale, showing the robustness of the procedures.

We then examined further functionalization of the obtained aryl enol ethers (Scheme 3). The ortho-iodine substrate $3 \mathbf{u}$ could be used in a reported palladium oxidative cyclization ${ }^{[24]}$ to generate benzofuran 6 in high yield (Eq. 1). Under palladiumcatalyzed hydrogenation conditions, ketone 7 resulting from cleavage of the aryl-O bond and reduction of the aryl iodide was obtained in $96 \%$ yield (Eq. 2). Finally, the electron-rich nature of the enol ether make it well-suited for oxidative modification, with the potential for accessing more highly functionalized ketones. Indeed, when using 1.2 equivalents of meta-chloroperbenzoic acid ( $m$-CPBA) buffered with sodium bicarbonate, the epoxides $\mathbf{8 a}$ and $\mathbf{8 b}$ could be obtained in quantitative yield starting from the corresponding enol ethers (Eq. 3). ${ }^{[25]}$ Interestingly, the addition of the in situ generated meta-chlorobenzoic acid was observed in absence of buffer to give $\alpha$-benzoylated ketones such as $9 a$ and 
$9 \mathrm{~b}$ in moderate to good yields (Eq. 4). ${ }^{[26]}$ Treatment of the isolated epoxide $\mathbf{8 a}$ with acetic acid lead to the same regioselective formation of $\alpha$-acetoxy ketone $\mathbf{1 0}$ in $72 \%$ yield (Eq. 5). This straightforward oxidation method allows to make use of the formed enol ether resulting in an overall $\mathrm{C}-\mathrm{H}$ functionalization of the initial propargylic $\mathrm{C}-\mathrm{H}$ bond.

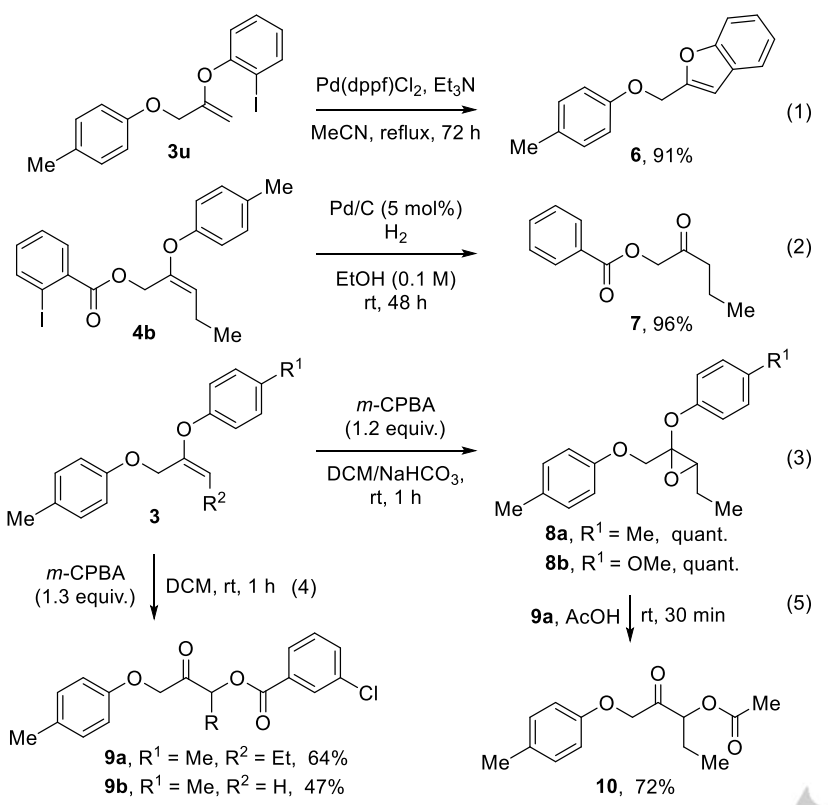

Scheme 3. Product modifications.

A speculative mechanism to rationalize the observed oxy-allyl cation-like reactivity would involve the isomerization of VBX 1 to the corresponding allyl iodane under basic conditions. The high leaving group ability of hypervalent iodine ${ }^{[27]}$ would then lead to the formation of an allyl cation. Indeed, ring-opening of an adjacent cyclopropane and a [4+3] reaction with furan in low yield were observed, supporting such an intermediate (See Scheme S5 and $\mathrm{S} 6$ in SI). However, the evidence does not allow to exclude a direct $\mathrm{SN}^{2}$ pathway and further experiments will be needed to understand the observed transformations.

In conclusion, hypervalent iodine reagents have been used as oxy-allyl cation surrogates for the stereoselective synthesis of aryl enol ethers by reaction with phenols. In absence of external nucleophiles, the in-situ generated benzoate group reacted, resulting in the formation of allylic esters. The reaction most probably proceeds via an electrophilic allylic intermediate and both $\mathrm{SN}^{1}$ or $\mathrm{SN}^{2}$ pathways appear feasible at this stage. The obtained enol ethers could be transformed into $\alpha$-difunctionalized ketones under oxidative conditions, demonstrating the synthetic utility of the transformation.

\section{Acknowledgements}

We thank the Swiss National Science Foundation (SNSF, grant nos. 200020_182798) and EPFL for financial support.
Keywords: Hypervalent lodine Reagents • Umpolung • Allyl Cation • Enol Ethers • Vinylbenziodoxolones

[1] E. J. Corey, X. M. Cheng, The logic of Chemical Synthesis; J. Wiley: New-York, 1989.

[2] D. Seebach, Angew. Chem. Int. Ed. 1979, 18, 239

[3] a) V. V. Zhdankin, Hypervalent lodine Chemistry: Preparation, Structure and Synthetic Applications of Polyvalent lodine Compounds, In Hypervalent lodine Chemistry; John Wiley \& Sons, Ltd, 2013, pp 1-20; b) A. Yoshimura, V. V. Zhdankin, Chem. Rev. 2016, 116, 3328.

[4] a) G. Stork, J. Szmuszkovicz, R. Terrell, A. Brizzolara, H. Landesman, J. Am. Chem. Soc. 1963, 85, 207; b) T. Mukaiyama, K. Banno, K. Narasaka, J. Am. Chem. Soc. 1974, 96, 7503; c) K. Gopalaiah, H. B. Kagan, Chem. Rev. 2011, 111, 4599.

[5] E. A. Merritt, B. Olofsson, Synthesis 2011, 517.

[6] a) S. Arava, J. N. Kumar, S. Maksymenko, M. A. Iron, K. N. Parida, P. Fristrup, A. M. Szpilman, Angew. Chem., Int. Ed. 2017, 56, 2599; b) S. Maksymenko, K. N. Parida, G. K. Pathe, A. A. More, Y. B. Lipisa, A. M. Szpilman, Org. Lett. 2017, 19, 6312; c) A. A. More, G. K. Pathe, K. N. Parida, S. Maksymenko, Y. B. Lipisa, A. M. Szpilman, J. Org. Chem. 2018, 83, 2442; d) K. N. Parida, G. K. Pathe, S. Maksymenko, A. M. Szpilman, Beilstein J. Org. Chem. 2018, 14, 992.

[7] a) Y. Li, D. P. Hari, M. V. Vita, J. Waser, Angew. Chem. Int. Ed. 2016, 55, 4436; b) D. P. Hari, P. Caramenti, J. Waser, J. Acc. Chem. Res. 2018, $51,3212$.

[8] B. Liu, C. -H. Lim, G. M. Miyake, J. Am. Chem. Soc. 2018, 140, 12829.

[9] a) P. Caramenti, N. Declas, R. Tessier, M. D. Wodrich, J. Waser, Chem. Sci. 2019, 10, 3223. Shortly thereafter Itoh and co-workers reported the addition of amides on unsubstituted EBX reagents: b) D. Shimbo, A Shibata, M. Yudasaka, T. Maruyama, N. Tada, B. Uno, A. Itoh, Org. Lett. 2019, 21, 9769.

[10] D. P. Hari, S. Nicolai, J. Waser, Alkynylations and Vinylations. PATAl'S Chemistry of Functional Groups 2018.

[11] T. Okuyama, T. Takino, T. Sueda, M. Ochiai, J. Am. Chem. Soc. 1995 $117,3360$.

[12] a) A. E. Favorskii, J. Russ. Phys.-Chem. Soc. 1894, 26, 559. b) R. B. Loftfield, J. Am. Chem. Soc. 1951, 73, 4707. Recent examples: c) M. Harmata, C. Huang, P. Rooshenas, P. R. Schreiner, Angew. Chem., Int. Ed. 2008, 47, 8696; d) Q. Tang, X. Chen, B. Tiwari, Y. R. Chi, Org. Lett. 2012, 14, 1922; e) B. Yang, X. Zhai, S. Feng, Z. Shao, Org. Chem. Front. 2018, 5, 2794; f) Y. Aota, Y. Doko, T. Kano, K. Maruoka, Eur. J. Org. Chem. 2020, 2020, 1907.

[13] Selected reviews: a) H. M. R. Hoffmann, Angew. Chem., Int. Ed. 1984 23, 1; b) I. V. Hartung, H. M. R. Hoffmann, Angew. Chem., Int. Ed. 2004 43, 1934; c) M. Harmata, Acc. Chem. Res. 2001, 34, 595; d) M. Harmata, P. Rashatasakhon, Tetrahedron 2003, 59, 2371; e) M. Harmata, Chem. Commun. 2010, 46, 8886; f) M. Harmata, Chem. Commun. 2010, 46, 8904; g) A. G. Lohse, R. P. Hsung, Chem. Eur. J. 2011, 17, 3812.

[14] Selected reviews: a) T. N. Grant, C. J. Rieder, F. G. West, Chem Commun. 2009, 5676; b) T. Vaidya, R. Eisenberg, A. J. Frontier ChemCatChem 2011, 3, 1531; c) N. Shimada, C. Stewart, M. A. Tius, Tetrahedron, 2011, 67, 5851

[15] a) M. N. Vander Wal, A. K. Dilger, D. W. C. MacMillan, Chem. Sci. 2013, 4, 3075; b) C. Liu, E. Z. Oblak, M. N. Vander Wal, A. K. Dilger, D. K Almstead, D. W. C. MacMillan, J. Am. Chem. Soc. 2016, 138, 2134; c) S. M. Banik, A. Levina, A. M. Hyde, E. N. Jacobsen, Science 2017, 358 761.

[16] Selected examples: a) K. Lee, D. Y. Kim, D. Y. Oh, Tetrahedron Lett 1988, 29, 667; b) H. R. Khatri, J. Zhu, Chem. Eur. J. 2012, 18, 12232; c) H. Nguyen, H. R. Khatri, J. Zhu, Tetrahedron Lett. 2013, 54, 5464; d) B. Xu, U. K. Tambar, J. Am. Chem. Soc. 2016, 138, 12073; e) B. Xu, U. K. Tambar, Angew. Chem., Int. Ed. 2017, 56, 9868; f) Y. C. Wu, S. Bouvet, S. Izquierdo, A. Shafir, Angew. Chem., Int. Ed. 2019, 58, 2617; Reviews: g) A. Shafir, Tetrahedron Lett. 2016, 57, 2673; h) I. F. D. Hyatt, L. Dave, N. David, K. Kaur, M. Medard, C. Mowdawalla, Org. Biomol. Chem. 2019, $17,7822$.

[17] Z. Wang, L. Jiang, P. Sarró, M. G. Suero, J. Am. Chem. Soc. 2019, 141, 15509. 
[18] The structure of 3a was confirmed by X-ray analysis. The data are available free of charge from the Cambridge Crystallographic Data Center (CCDC number 1989749).

[19] The reaction mixture was heterogenous, but no dependence on the batch of cesium carbonate was observed. Using finely grinded salt also did not change the result. See Supporting Information for details.

[20] Selected examples of atom-economical reactions with hypervalent iodine reagents: a) S. G. Modha, M. F. Greaney, J. Am. Chem. Soc. 2015, 137 1416; b) J. Buendia, B. Darses, P. Dauban, Angew. Chem. Int. Ed. 2015 54, 5697; c) D. P. Hari, J. Waser, J. Am. Chem. Soc. 2016, 138, 2190; d) M. Wang, J. Wei, Q. Fan, X. Jiang, Chem. Commun. 2017, 53, 2918 e) A. Boelke, P. Finkbeiner, B. J. Nachtsheim, Beilstein J. Org. Chem. 2018, 14, 1263; f) M. Wang, S. Chen, X. Jiang, Chem. Asian J. 2018, 13 2195; g) G. Pisella, A. Gagnebin, J. Waser, Org. Lett. 2020, accepted for publication, DOI: 10.1021/acs.orglett.0c01150.

[21] The reaction profile is usually very clean and lower yields were generally due to increase amount of ester $\mathbf{4 a}$. It is difficult to recognize clear trends in the formation of the ester products, although generally electron-rich phenols gave larger amounts of $\mathbf{4 a}$, in-line with the accelerating effect of nucleophilic additives. See Supporting Information for exact yields of isolated esters 4

[22] The structure of $3 p$ was confirmed by X-ray analysis. The data are available free of charge from the Cambridge Crystallographic Data Center (CCDC number 1989757). The structure and geometry of the other products is assumed to be the same based on the similar NMR spectra. The olefin geometry was further confirmed by NOE experiments on products $\mathbf{4 c}$ and $\mathbf{4 I}$.

[23] E. Stridfeldt, A. Seemann, M. J. Bouma, C. Dey, A. Ertan, B. Olofsson, Chem. Eur. J. 2016, 22, 16066

[24] L. Zhou, Y. Shi, X. Zhu, P. Zhang, Tetrahedron Lett. 2019, 60, 2005.

[25] No reaction was observed in presence of oxone and selectfluor led to a complex product mixture.

[26] a) C. L. Stevens, W. Malik, R. Pratt, J. Am. Chem. Soc. 1950, 72, 4758 b) C. L. Stevens, S. J. Dykstra, J. Am. Chem. Soc. 1953, 75, 5975 Review: c) A. Kirrmann, P. Duhamel, R. Nouri-Bimorghi, Justus Liebigs Ann. Chem. 1966, 691, 33.

[27] a) J. B. Dence, J. D. Roberts, J. Org. Chem. 1968, 33, 1251; b) G. A. Olah, J. R. DeMember, J. Am. Chem. Soc. 1969, 91, 2113; c) D. G. Morris, A. G. Shepherd, J. Chem. Soc. Chem. Commun. 1981, 1250; d) A. Flores, E. Cots, J. Bergès, K. Muñiz, Adv. Synth. Catal. 2019, 361, 2 f) A. E. Bosnidou, K. Muñiz, Chem. Eur. J. 2019, 25, 13654. 


\section{Entry for the Table of Contents}

Me $\mathrm{Si}=\mathrm{R}_{\mathrm{R}^{1}}$

Access to oxy-allyl cation equivalents from alkynes via hypervalent iodine reagents is described. The stereoselective transformation of Vinylbenziodoxolones (VBXs) gives aryl enol ethers bearing an allylic ether or ester group and corresponds to a Umpolung of the nucleophilic reactivity of enol ethers. The obtained products are easily transformed into $\alpha$-difunctionalized ketones under oxidative conditions.

@EPFL_CHEM_Tweet @LcsoLab 


\section{Supporting Information}

Table of Contents

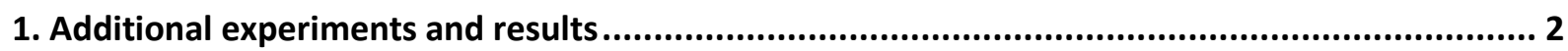

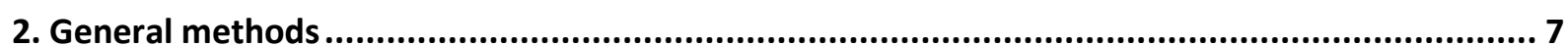

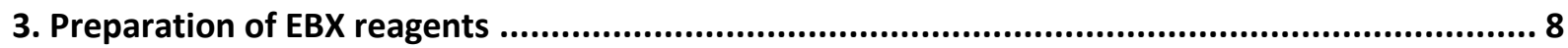

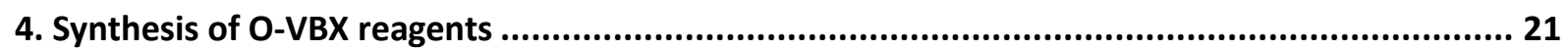

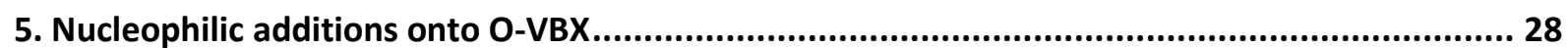

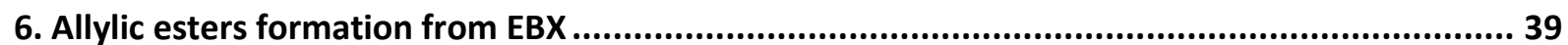

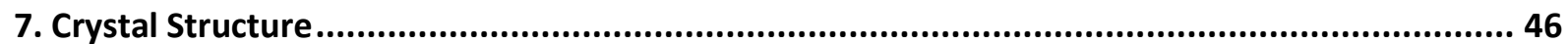

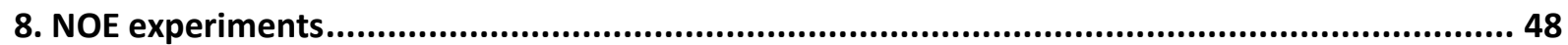

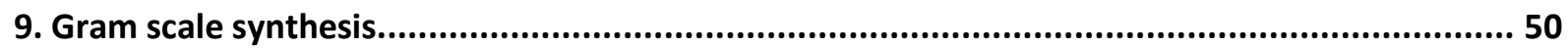

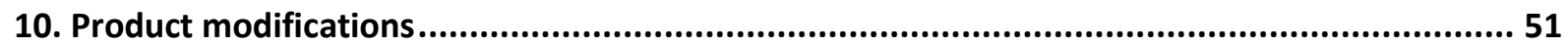

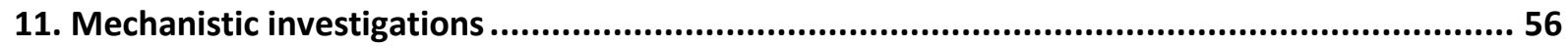

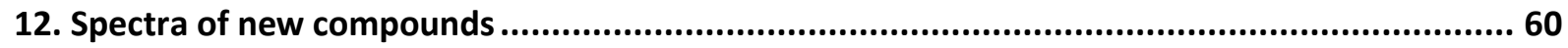

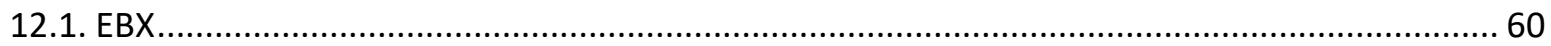

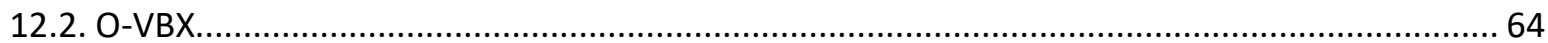

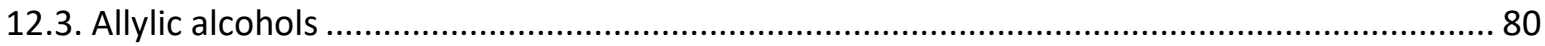

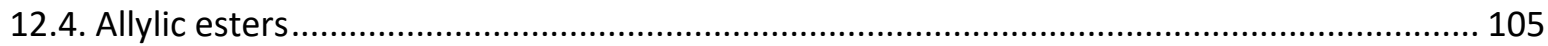

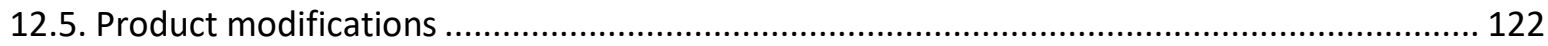

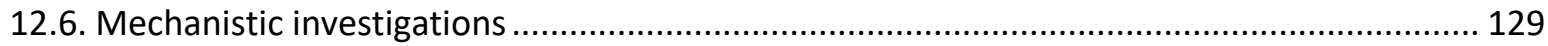




\section{Additional experiments and results}

\section{Limitations for the formation of allylic esters from EBX:}

In some cases, formation of the O-VBX reagent 1 was successful, but the reaction stopped at this stage (Scheme S1). In particular, this was the case for secondary alkyl groups. For cyclopropyl and cyclohexyl groups, the corresponding O-VBXs $1 \mathrm{~m}$ and $1 \mathrm{n}$ were recovered in $88 \%$ and $65 \%$ yield. Cyclopentylsubstituted $\mathrm{O}-\mathrm{vBX} 10$ decomposed under the reaction conditions. The reaction cannot proceed in absence of allylic C-H bond and $t$-Butyl-VBX $\mathbf{1 p}$ and phenyl-EBX 1q were isolated in excellent yield. In contrast, the reaction proceeded for all O-VBX reagents bearing a primary alkyl group, with the exception of sterically hindered ortho-dichlorobenzene-substituted O-VBX $\mathbf{1} r$, which could not be converted to the corresponding allylic ester. Finally, simple VBX $\mathbf{1 1}^{1}$ lacking the ether group was engaged in the procedure, but no reaction occurred and the starting material was recovered.<smiles></smiles>

$1 \mathrm{~m}, 88 \%$

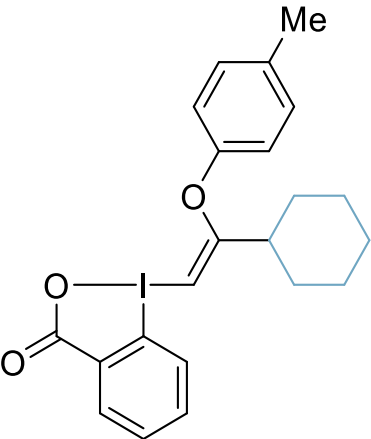

$1 \mathrm{n}, 65 \%$

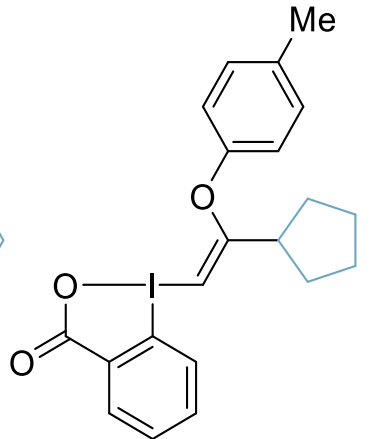

$10,--\%$<smiles>Cc1ccc(O/C(=C\I)C(C)(C)C)cc1</smiles>

$1 p, 80 \%$<smiles>Cc1ccc(O/C(=C\I2COC(=O)c3ccccc32)c2ccccc2)cc1</smiles>

1q, $99 \%$

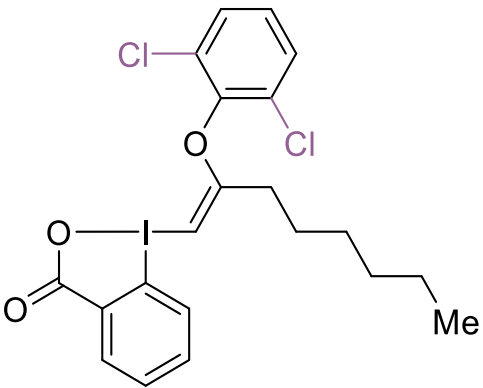

$1 \mathbf{r}, 83 \%$<smiles>CCC/C=C/I1COC(=O)c2ccccc21</smiles>

$11, N R$

Scheme S1: Scope of EBX 5 for the formation of allylic esters 4: reaction stopping at VBX stage.

The analysis data of coumpounds $1 \mathbf{m}-1 \mathrm{r}$ are described section 4.

\section{Mechanistic investigations:}

To gain insights into the reaction mechanism several control experiments were performed. We first wondered if allylic ester $\mathbf{4 a}$ was just an intermediate for the formation of allylic ether $\mathbf{3 a}$ via nucleophilic substitution (Scheme S2). When 4a was resubmitted to the reaction conditions, no substitution was observed, showing that $\mathbf{4 a}$ was not an intermediate in the synthesis of allylic ethers.

\footnotetext{
${ }^{1}$ E. Stridfeldt, A. Seemann, M. J. Bouma, C. Dey, A. Ertan, B. Olofsson, Chem. Eur. J. 2016, 22, 16066.
} 


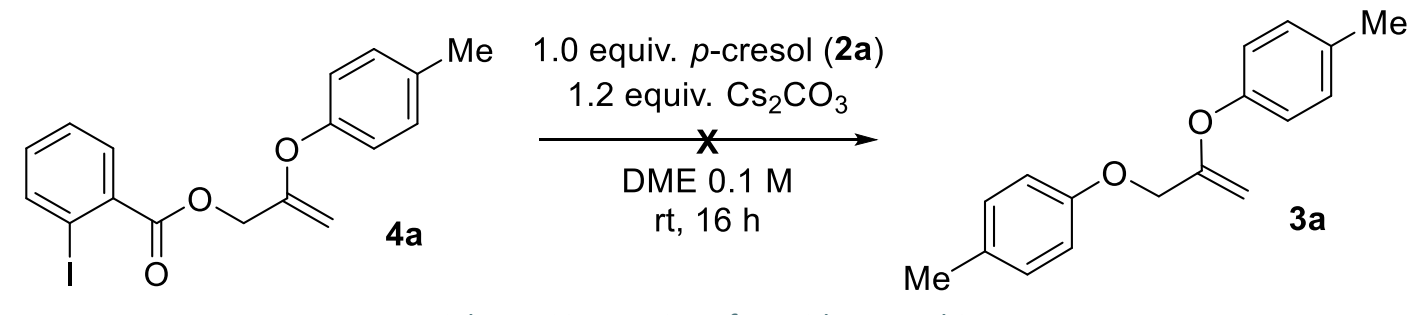

Scheme S2: Reaction of 4 a with $p$-cresol 2 a

We then wondered if the formation of 4 a was an intramolecular process, which would explain why it was difficult to supress completely. To check this hypothesis, a mixture of O-VBX reagents $1 \mathbf{a}$ and $\mathbf{1 t}$ bearing different groups on the benzene ring of the hypervalent iodine reagent and the one of the phenol was submitted to the reaction conditions. Four products $\mathbf{4 q} \mathbf{\mathbf { 4 }} \mathbf{4 \mathbf { q } ^ { \prime }}, \mathbf{4 r}$ and $\mathbf{4} \mathbf{r}^{\prime}$ were obtained in a similar proportion, showing that rapid cross-over was occurring and not a selective intramolecular process (Scheme S3).
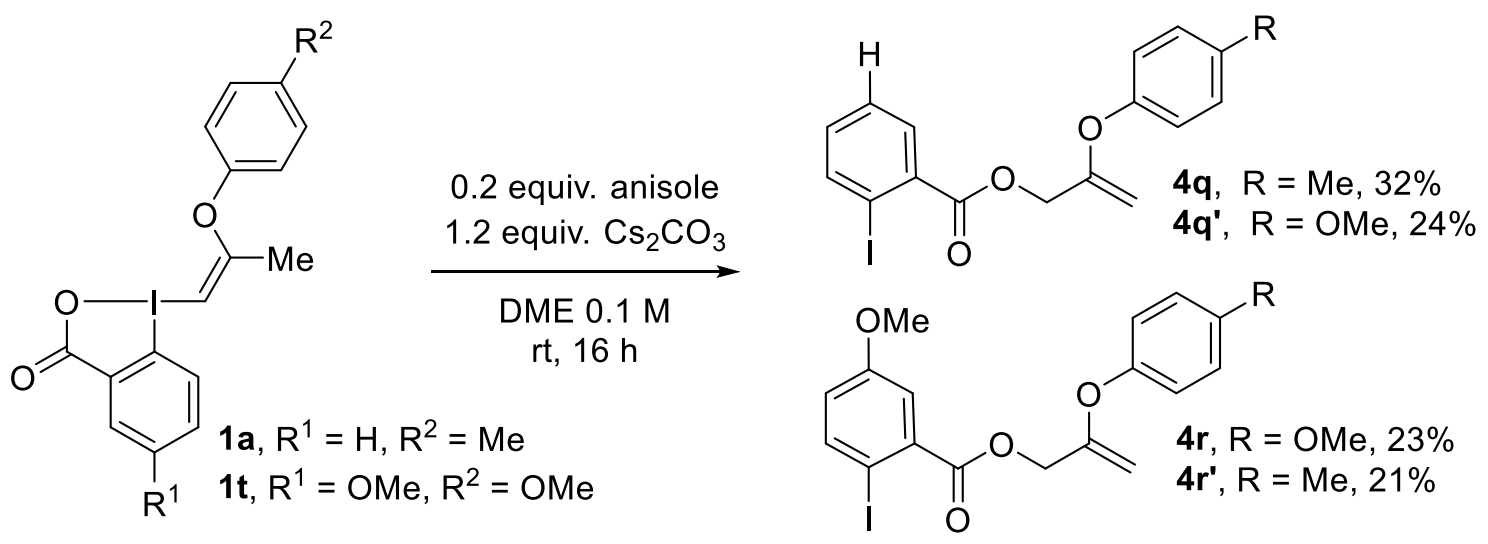

Scheme S3: Cross-over experiment.

We then investigated the nature of the reactive intermediate by attempting to trap it (Scheme S4). Addition of an equivalent of TEMPO or para-methoxystyrene to the reaction of O-VBX (1a) with paracresol (2a) did not affect the result. These indicated that radical or carbene intermediates, if at all present, would be short-lived and difficult to intercept.

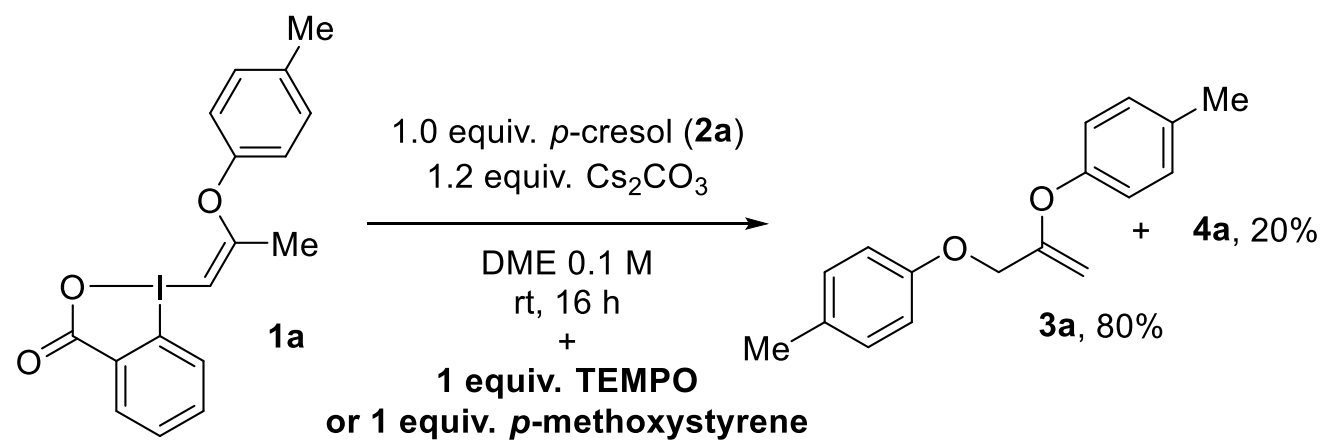

Scheme S4: Reaction of $1 \mathbf{a}$ with $p$-cresol $2 \mathbf{a}$ in presence of TEMPO or $p$-methoxystyrene.

We then wondered if the reaction could proceed through an oxy-allyl cation intermediate (Scheme S5). With furan as co-solvent starting from 1a, 4 a was still obtained in $50 \%$ yield. Formation of $(4+3)$ adduct 12 was observed by HRMS, but in too small amounts to be isolated. 


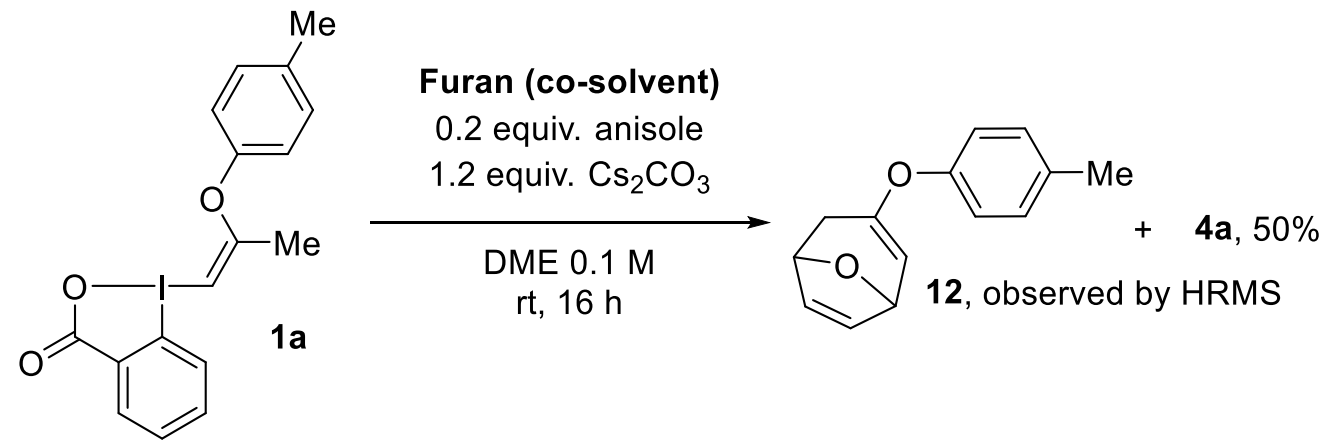

Scheme S5: Reaction of $\mathbf{1 a}$ with $p$-cresol $\mathbf{2 a}$ in presence of furan as co-solvent.

In order to gain further insight into the nature of the reactive intermediate, we then prepared the new EBX reagent 5q (Scheme S6). Under the one-pot reaction conditions, $\mathbf{5 q}$ was converted to diene $\mathbf{1 3}$ as main product, together with small amounts of $4 \mathrm{~s}$ and ketone $\mathbf{1 4}$. The formation of $\mathbf{1 3}$ could come from the regioselective nucleophilic attack of the in situ generated iodobenzoate on a putative oxy-allyl intermediate I, but a concerted mechanism with simultaneous cleavage of the C-I bond cannot be excluded. A control experiment showed that $\mathbf{4 s}$ did not react with benzoate to give $\mathbf{1 3}$.

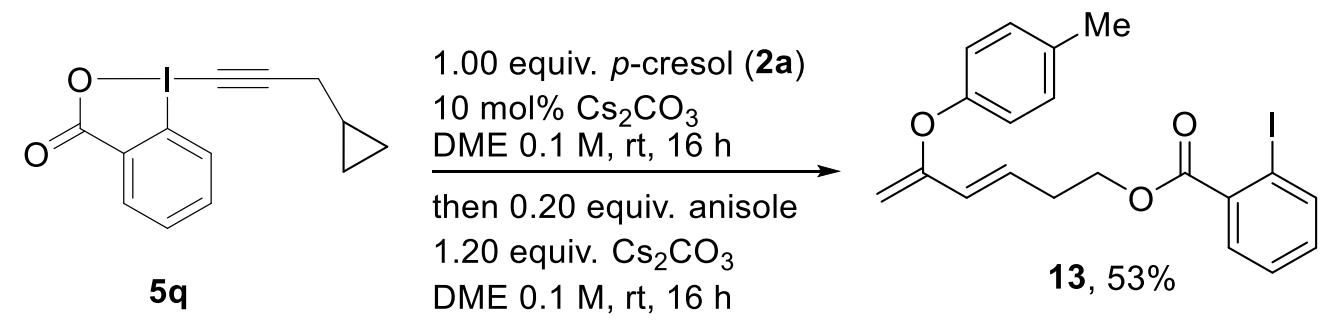<smiles>Cc1ccc(O/C(=C/C2CC2)COC(=O)c2ccccc2I)cc1</smiles>

4s, $18 \%$<smiles>O=C(COC(=O)c1ccccc1I)CC1CC1</smiles>

$14,4 \%$

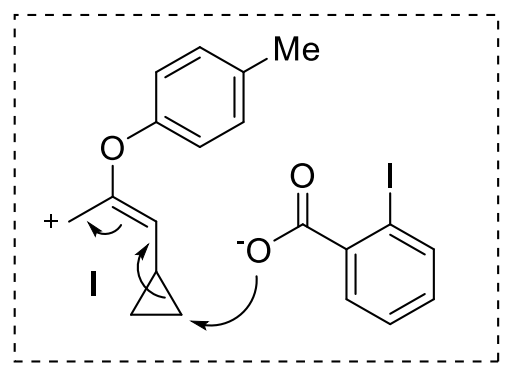

Scheme S6: Reaction of $\mathbf{5 a b}$ with $p$-cresol $\mathbf{2 a}$.

\section{Proposed mechanism:}

Based on the performed experiments, the following speculative mechanism can be proposed (Scheme S7). The formation of O-VBX 1 from EBX 5 was previously reported. ${ }^{2}$ Then, several pathways are available for O-VBX 1. Direct substitution, either via a vinyl cation intermediate or via a concerted/stepwise addition-elimination pathway as common for EBX reagents ${ }^{3}$ would lead to the vinylic product with thiol nucleophiles (Scheme S7 A). ${ }^{2}$ In presence of a catalytic amount of cesium carbonate, deprotonation of the vinyl position would lead to vinyl anion I, which can fragment to give back EBX 5 and the phenolate (Scheme S7 B). In our previous work, this equilibrium was shown to be feasible and strongly in the favour of VBX 1. In presence of an excess of base, a small amount of

2 P. Caramenti, N. Declas, R. Tessier, M. D. Wodrich, J. Waser, Chem. Sci. 2019, 10, 3223.

${ }^{3}$ a) R. Frei, M. D. Wodrich, D. P. Hari, P. A. Borin, C. Chauvier, J. Waser, J. Am. Chem. Soc. 2014, 136, 16563; b) M. D. Wodrich, P. Caramenti, J. Waser, Org. Lett. 2016, 18, 60. 
deprotonation at the allylic position could also occur to give allylic anion II, which can be drawn as its resonance structure II' and reprotonated $\alpha$ to the iodine atom to give allylic hypervalent iodine intermediate III (Scheme S7 C).

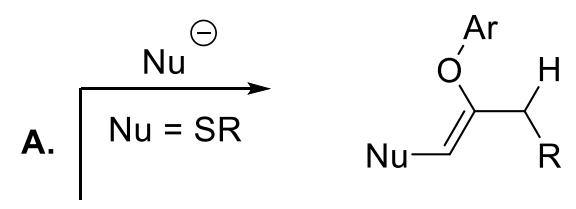

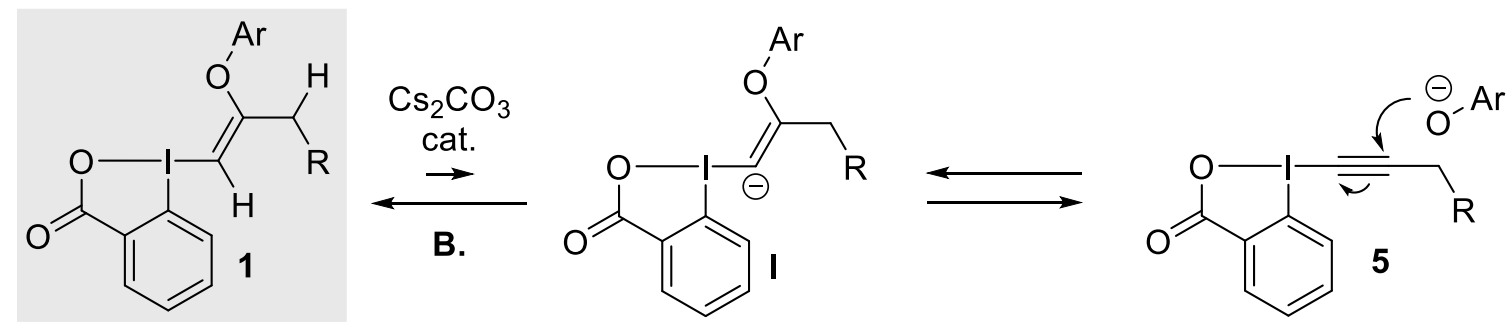

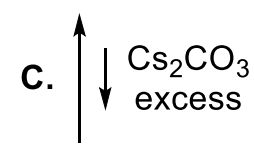

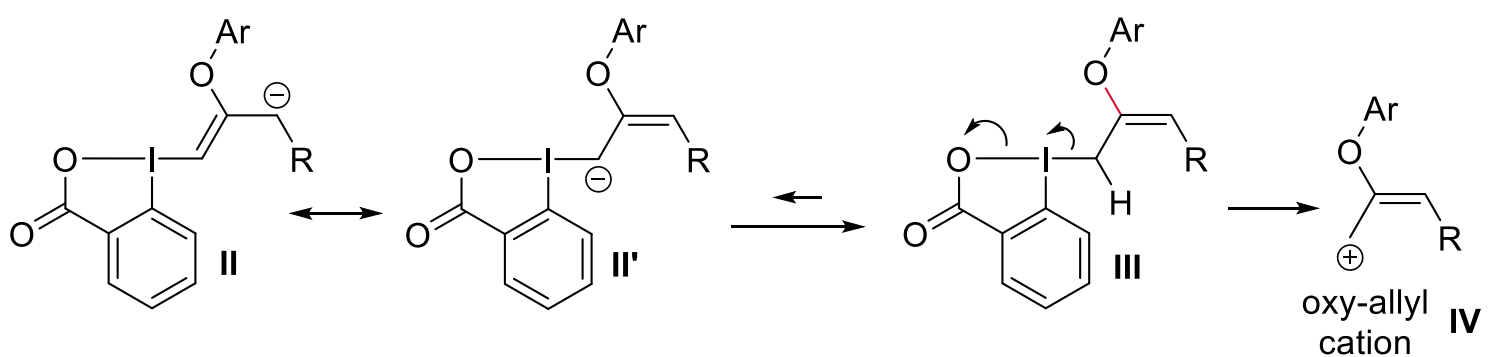

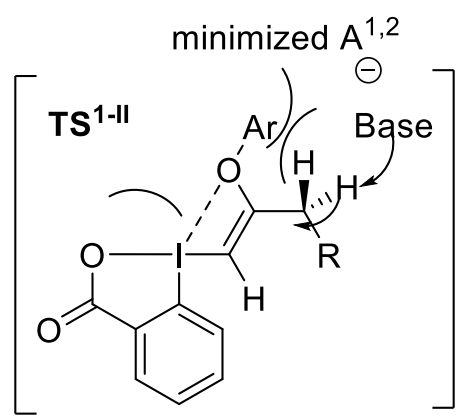<smiles>[R]/C=C(/OC)C([2H])[I-]([O-])([O-])c1ccccc1C(=O)O</smiles>

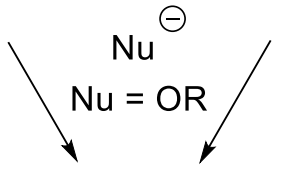

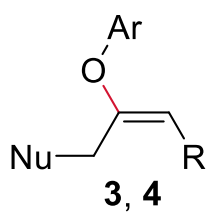

Scheme S7: Speculative reaction mechanism. A. Vinyl cation reactivity. B. Equilibrium with EBX. C. Oxo-allyl cation reactivity.

In the deprotonation event, it would be important to minimize the strong $A^{1,2}$ strain with the aryl group on the ether in $\mathbf{T S}^{1-1 \|}$. The aryl group is expected to point away from the iodine atom to limit steric interactions, and potentially also allow a favourable overlap of the oxygen lone-pair with the "sigmahole" of the hypervalent iodine. If reprotonation is significantly faster than bond rotation, the stereochemistry of the double-bond would be fixed. From allylic-I(III) intermediate III, two pathways could be envisaged. The well-known thermodynamic instability of alkyl-I(III) ${ }^{4}$ in general and allylic intermediates in particular ${ }^{5}$ would lead to an $\alpha$-elimination of the aryl iodide to deliver oxy-allyl cation

\footnotetext{
${ }^{4}$ a) J. B. Dence, J. D. Roberts, J. Org. Chem. 1968, 33, 1251; b) G. A. Olah, J. R. DeMember, J. Am. Chem. Soc. 1969, 91, 2113; c) D. G. Morris, A. G. Shepherd, J. Chem. Soc. Chem. Commun. 1981, 1250; d) A. Flores, E. Cots, J. Bergès, K. Muñiz, Adv. Synth. Catal. 2019, 361, 2; f) A. E. Bosnidou, K. Muñiz, Chem. Eur. J. 2019, 25, 13654.

${ }^{5}$ Selected examples: a) K. Lee, D. Y. Kim, D. Y. Oh, Tetrahedron Lett. 1988, 29, 667; b) H. R. Khatri, J. Zhu, Chem. Eur. J. 2012, 18, 12232; c) H. Nguyen, H. R. Khatri, J. Zhu, Tetrahedron Lett. 2013, 54, 5464; d) B. Xu, U. K. Tambar, J. Am. Chem. Soc. 2016, 138, 12073; e) B. Xu, U. K. Tambar, Angew. Chem., Int. Ed. 2017, 56, 9868; Reviews: f) A.
} 
species IV. Nucleophilic attack by the generated iodobenzoate, or by an external phenolate, would provide the desired allylic products $\mathbf{3}$ and $\mathbf{4}$ via an overall $\mathrm{SN}^{1}$ pathway. However, an alternative $\mathrm{SN}^{2}$ pathway by direct reaction of the nucleophile with allylic intermediate III cannot be excluded at this stage, especially when considering that no very strong support could be gathered for a free oxy-allyl cation intermediate IV. Finally, it is difficult to propose a rationalization for the anisole additive effect. One highly speculative explanation may be that a donor could coordinate to the iodine atom to give iodonium $\mathbf{V}$, resulting both in enhanced electrophilicity of the allylic system and enhanced nucleophilicity of the benzoate.

Shafir, Tetrahedron Lett. 2016, 57, 2673; g) I. F. D. Hyatt, L. Dave, N. David, K. Kaur, M. Medard, C. Mowdawalla, Org. Biomol. Chem. 2019, 17, 7822. 


\section{General methods}

All reactions were carried out in oven dried glassware under an atmosphere of nitrogen, unless stated otherwise. For quantitative flash chromatography, technical grade solvents were used. For flash chromatography for analysis, HPLC grade solvents from Sigma-Aldrich were used. THF, $\mathrm{Et}_{2} \mathrm{O}, \mathrm{CH}_{3} \mathrm{CN}$, toluene, hexane and $\mathrm{CH}_{2} \mathrm{Cl}_{2}$ were dried by passage over activated alumina under nitrogen atmosphere ( $\mathrm{H}_{2} \mathrm{O}$ content $<10 \mathrm{ppm}$, Karl-Fischer titration). The solvents were degassed through Freeze-Pump-Thaw method when mentioned. All chemicals were purchased from Acros, Aldrich, Fluka, VWR, Aplichem, or Merck and used as such unless otherwise stated. Chromatographic purification was performed as flash chromatography using Macherey-Nagel silica 40-63, $60 \AA$, with the solvents indicated as eluent under 0.1-0.5 bar pressure. TLC was performed on Merck silica gel 60 F254 TLC glass plates or aluminium plates and visualized with UV light, permanganate stain, CAN stain, or Anisaldehyde stain. Melting points were measured on a Büchi B-540 melting point apparatus using open glass capillaries, the data is uncorrected. ${ }^{1} \mathrm{H}$-NMR spectra were recorded on a Brucker DPX-400 $400 \mathrm{MHz}$ spectrometer in $\mathrm{CDCl}_{3}, \mathrm{DMSO}-\mathrm{d}_{6} \mathrm{CD}_{3} \mathrm{OD}, \mathrm{C}_{6} \mathrm{D}_{6}$ and $\mathrm{CD}_{2} \mathrm{Cl}_{2}$, all signals are reported in ppm with the internal chloroform signal at $7.26 \mathrm{ppm}$, the internal DMSO signal at $2.50 \mathrm{ppm}$ the internal methanol signal at $3.30 \mathrm{ppm}$, the internal dichloromethane signal at $5.30 \mathrm{ppm}$ as standard. The data is being reported as $(\mathrm{s}=$ singlet, $\mathrm{d}$ = doublet, $\mathrm{t}=$ triplet, $\mathrm{q}=$ quadruplet, $\mathrm{qi}=$ quintet, $\mathrm{m}=$ multiplet or unresolved, $\mathrm{br}=$ broad signal, app = apparent, coupling constant(s) in $\mathrm{Hz}$, integration, interpretation) ${ }^{13} \mathrm{C}-\mathrm{NMR}$ spectra were recorded with ${ }^{1} \mathrm{H}$-decoupling on a Brucker DPX-400 $100 \mathrm{MHz}$ spectrometer in $\mathrm{CDCl}_{3}, \mathrm{DMSO}-\mathrm{d}_{6}, \mathrm{CD}_{3} \mathrm{OD}$ or $\mathrm{CD}_{2} \mathrm{Cl}_{2}$, all signals are reported in ppm with the internal chloroform signal at $77.0 \mathrm{ppm}$, the internal DMSO signal at $39.5 \mathrm{ppm}$, the internal methanol signal at $49.0 \mathrm{ppm}$ and the internal dichloromethane signal at $54.0 \mathrm{ppm}$ as standard. Infrared spectra were recorded on a JASCO FT-IR B4100 spectrophotometer

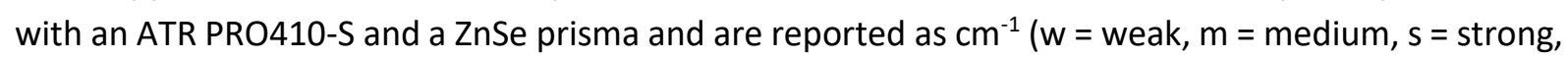
$\mathrm{br}=$ broad). High resolution mass spectrometric measurements were performed by the mass spectrometry service of ISIC at the EPFL on a MICROMASS (ESI) Q-TOF Ultima API. Optical rotations were measured on a Schmidt+Haensch (Polartronic M) polarimeter using a $10 \mathrm{~cm}$ cell with a $\mathrm{Na} 589$ $\mathrm{nm}$ filter. The specific solvents and concentrations (in $10 \mathrm{mg} / \mathrm{mL}$ ) are indicated. 


\section{Preparation of EBX reagents}

The synthesis of the precursors for EBX reagents (except $\mathbf{5 f}, \mathbf{5 0}, \mathbf{5 p}$ and $\mathbf{5 q}$ ) and their starting materials had been already described before in our group. ${ }^{6,7}$ The procedures here reported are taken from the cited publications to facilitate reproduction of the results by having all the data in the same file.

\section{General procedure for the synthesis of EBX from boronic acid:}<smiles>O=C(O)c1ccccc1I</smiles>

15
1. 1.10 equiv. mCPBA,<smiles>[R]C#CB1OC2(C)OC1(C)OC21OCCO1</smiles><smiles>[R]C#CI1COC(=O)c2ccccc21</smiles>

5

GP1: Following a slightly modified procedure, ${ }^{8}$ 2-iodobenzoic acid 15 (1.00 equiv.), paratoluenesulfonic acid monohydrate (1.10 equiv.) and meta-chloroperoxybenzoic acid (mCPBA-70\%, 1.10 equiv.) were dissolved in dichloromethane and 2,2,2-trifluoroethanol (1:1 mixture, $0.27 \mathrm{M})$. The mixture was stirred at room temperature under nitrogen for 1 hour, after which the correspondent alkyl-1-boronic acid pinacol ester (1.40 equiv.) was added in one portion. The reaction mixture was stirred for 2.5 hours at room temperature, filtered and concentrated in vacuo. The resulting oil was dissolved in dichloromethane $(30 \mathrm{~mL})$ and under vigorous stirring, saturated aq. $\mathrm{NaHCO}_{3}(30 \mathrm{~mL})$ was added. The mixture was stirred for 15 minutes, the two layers were separated and the aqueous phase was extracted with additional portions of dichloromethane $(3 \times 25 \mathrm{~mL})$. The combined organic layers were washed with brine $(25 \mathrm{~mL})$, dried over $\mathrm{MgSO}_{4}$, filtered and concentrated in vacuo. The crude product was purified by flash column chromatography (DCM:MeOH 9:1) to afford the desired compounds 5 .

\section{Propynyl-1,2-benziodoxol-3(1H)-one (5a)}<smiles>CC#CI1OC(=O)c2ccccc21</smiles>

Following GP1 on $4.30 \mathrm{mmol}$ scale and using propynyl-1-boronic acid pinacol ester ( $4.85 \mathrm{~g}, 21.2 \mathrm{mmol}, 1.40$ equiv.), propynyl-1,2-benziodoxol-3(1H)-one $\mathbf{5 a}$ (1.03 g, $3.60 \mathrm{mmol}, 84 \%)$ was obtained as a white solid. $\mathbf{R}_{f}: 0.10$ (EtOAc). $\mathbf{M p}$ 124-150 ${ }^{\circ} \mathrm{C}$ (decomposition). ${ }^{1} \mathrm{H}$ NMR (400 MHz, Chloroform-d) $\delta 8.41-8.35$ (m, $1 \mathrm{H}, \operatorname{ArH}), 8.22-8.14(\mathrm{~m}, 1 \mathrm{H}, \operatorname{ArH}), 7.79-7.68(\mathrm{~m}, 2 \mathrm{H}, \mathrm{ArH}), 2.27\left(\mathrm{~s}, 3 \mathrm{H}, \mathrm{CCCH}_{3}\right) .{ }^{13} \mathrm{C}$ NMR $(100 \mathrm{MHz}$, Chloroform-d) $\delta 166.7,134.8,132.5,131.6,126.4,115.6,105.1,39.0,5.7 .{ }^{9}$ IR $\vee 2183(\mathrm{w}), 1607$ (s), 1559 $(\mathrm{m}), 1350(\mathrm{~m}), 746(\mathrm{~m}), 730(\mathrm{~m}) . \mathrm{HRMS}(\mathrm{ESI}) \mathrm{C}_{10} \mathrm{H}_{8} \mathrm{IO}_{2}{ }^{+}[\mathrm{M}+\mathrm{H}]^{+}$286.9564; found 286.9561.

\section{(Pent-1-ynyl)-1,2-benziodoxol-3(1H)-one (5b)}

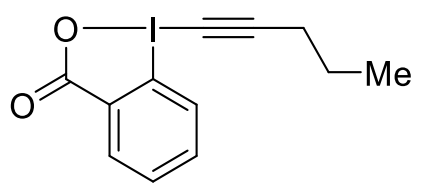

Following GP1 on $4.00 \mathrm{mmol}$ scale and using 1-pentynyl-1-boronic acid pinacol ester (1.09 g, $4.60 \mathrm{mmol}, 1.15$ equiv.) (pent-1-ynyl)-1,2benziodoxol-3(1H)-one $\mathbf{5 b}(0.754 \mathrm{~g}, 2.40 \mathrm{mmol}, 60 \%)$ was obtained as a white oil. ${ }^{10}{ }^{1} \mathrm{H}$ NMR (400 MHz, Chloroform-d) $\delta 8.40$ (ddd, $J=7.4,3.8,2.3$

$\mathrm{Hz}, 1 \mathrm{H}, \operatorname{ArH}), 8.26-8.09(\mathrm{~m}, 1 \mathrm{H}, \mathrm{ArH}), 7.75$ (dddd, $J=6.0,4.6,2.8,1.8 \mathrm{~Hz}, 2 \mathrm{H}, \operatorname{ArH}), 2.58$ (td, $J=7.1$,

\footnotetext{
${ }^{6}$ R. Frei, M. D. Wodrich, D. P. Hari, P.-A. Borin, C. Chauvier, J. Waser, J. Am. Chem. Soc. 2014, 136, 16563.

${ }^{7}$ D. P. Hari, J. Waser, J. Am. Chem. Soc. 2016, 138, 2190.

${ }^{8}$ M. J. Bouma, B. Olofsson, Chem. - A Eur. J. 2012, 18, 14242.

${ }^{9}$ One aromatic carbon signal was not resolved.

${ }^{10} \mathrm{NB}$ : the compound was isolated as an extremely viscous oil and retains organic solvent.
} 
$1.6 \mathrm{~Hz}, 2 \mathrm{H}, \mathrm{CH}_{2}$ ), $1.68\left(\mathrm{dtd}, J=14.7,7.2,2.1 \mathrm{~Hz}, 2 \mathrm{H}, \mathrm{CH}_{2}\right), 1.08\left(\mathrm{td}, J=7.6,2.1 \mathrm{~Hz}, 3 \mathrm{H}, \mathrm{CH}_{3}\right) .{ }^{13} \mathrm{C}$ NMR $\left(\mathrm{CDCl}_{3}, 100 \mathrm{MHz}\right) 166.8,134.6,132.1,131.3,126.2,115.5,109.5,50.4,38.8,22.3,21.6,13.4$. IR v 2960 (w), 2875 (w), $2172(w), 1732(\mathrm{~m}), 1654$ (s), 1465 (w), 1439 (w), 1342 (w), 1296 (m), 1252 (m), 1109 (w), $1016(w), 832(m), 743(s)$. HRMS (ESI) calcd for $\mathrm{C}_{12} \mathrm{H}_{12} \mathrm{IO}_{2}{ }^{+}[\mathrm{M}+\mathrm{H}]^{+}$314.9877; found 314.9882.

\section{(5-Chloropent-1-ynyl)-1,2-benziodoxol-3(1H)-one (5i)}<smiles>O=C1OI(C#CCCCCl)c2ccccc21</smiles>

Following GP1 on $15.2 \mathrm{mmol}$ scale and using 5-chloro-1-pentynyl-1boronic acid pinacol ester (4.85 g, $21.2 \mathrm{mmol}, 1.40$ equiv.), (5chloropent-1-ynyl)-1,2-benziodoxol-3(1H)-one 5i (3.76 g, $10.8 \mathrm{mmol}$, 71\%) was obtained as a white solid. Mp: $138.5-141.7^{\circ} \mathrm{C}$. $\mathbf{R}_{\mathbf{f}}: 0.15$ (EtOAC 100\%). ${ }^{1} \mathbf{H}$ NMR (400 MHz, Chloroform-d) $\delta$ 8.41-8.34 (m, $\left.1 \mathrm{H}, \mathrm{ArH}\right), 8.22-8.13(\mathrm{~m}, 1 \mathrm{H}, \mathrm{ArH}), 7.82-7.68$ $(\mathrm{m}, 2 \mathrm{H}, \mathrm{ArH}), 3.71\left(\mathrm{t}, J=6.1 \mathrm{~Hz}, 2 \mathrm{H}, \mathrm{ClCH}_{2} \mathrm{CH}_{2}\right), 2.82\left(\mathrm{t}, J=6.9 \mathrm{~Hz}, 2 \mathrm{H}, \mathrm{CCCH}_{2} \mathrm{CH}_{2}\right), 2.18-2.05(\mathrm{~m}, 2 \mathrm{H}$, $\left.\mathrm{ClCH}_{2} \mathrm{CH}_{2}\right) .{ }^{13} \mathrm{C}$ NMR $\left(\mathrm{CDCl}_{3}, 100 \mathrm{MHz}\right) \delta 166.8,134.9,132.5,131.6,131.6,126.4,115.8,107.1,43.4$, 41.2, 30.7, 18.0. IR v $2942(w), 2866(w), 2171(w), 2091(w), 1727(w), 1617(s), 1556(w), 1441(w)$, $1339(\mathrm{~m}), 1213(\mathrm{w}), 1023(\mathrm{w}), 846(\mathrm{w}), 742(\mathrm{~s})$. The characterization data corresponded to the reported values. $^{8}$

\section{2-Cyclopropylethynyl-1,2-benziodoxol-3(1H)-one (5j)}<smiles>O=C1OI(C#CC2CC2)c2ccccc21</smiles>

Following GP1 on $25.8 \mathrm{mmol}$ scale and using 5-chloro-1-pentynyl-1-boronic acid pinacol ester (4.85 g, $21.2 \mathrm{mmol}, 1.40$ equiv.), (cyclopropylethynyl)trimethylsilane $(5.00 \mathrm{~g}, 36.2 \mathrm{mmol}, 1.40$ equiv.) 2cyclopropylethynyl-1,2-benziodoxol-3(1H)-one 5j (2.11 g, $6.76 \mathrm{mmol}, 26 \%)$ was obtained as a white solid. $\mathbf{M p}: 174.2-177.6{ }^{\circ} \mathrm{C}$ (Dec.). $\mathbf{R}_{\mathbf{f}}: 0.46$ (EtOAc:MeOH, 9:1). ${ }^{1} \mathbf{H}$ NMR (400 $\mathrm{MHz}$, Chloroform-d) $\delta 8.34$ (dd, $J=7.0,2.1 \mathrm{~Hz}, 1 \mathrm{H}, \mathrm{ArH}), 8.18-8.09(\mathrm{~m}, 1 \mathrm{H}, \mathrm{ArH}), 7.81-7.63(\mathrm{~m}, 2 \mathrm{H}, \operatorname{ArH})$, $1.59(\mathrm{tt}, J=8.2,5.0 \mathrm{~Hz}, 1 \mathrm{H}, \mathrm{CH}), 1.07-0.85\left(\mathrm{~m}, 4 \mathrm{H}, \mathrm{CH}_{2} \mathrm{CH}_{2}\right) .{ }^{13} \mathrm{C}$ NMR $\left(\mathrm{CDCl}_{3}, 100 \mathrm{MHz}\right) \delta 166.7,134.7$, 132.3, 131.7, 131.4, 126.2, 115.9, 113.3, 35.0, 9.8, 1.1. IR v $3464(w), 3077(w), 3012(w), 2238(w)$, $2159(\mathrm{~m}), 1607(\mathrm{~s}), 1559(\mathrm{~m}), 1438(\mathrm{~m}), 1338(\mathrm{~m}), 1298(\mathrm{~m}), 833(\mathrm{~m}), 744(\mathrm{~s}), 691(\mathrm{~m})$. HRMS (ESI) calcd. for $\mathrm{C}_{12} \mathrm{H}_{10} \mathrm{IO}_{2}{ }^{+}[\mathrm{M}+\mathrm{H}]^{+} 312.9720$; found 312.9719. Data reported in literature. ${ }^{6}$

\section{2-Cyclohexylethynyl-1,2-benziodoxol-3(1H)-one (5k)}

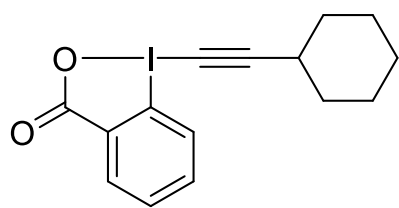

Following GP1 on $4.00 \mathrm{mmol}$ scale and using ethynylcyclohexane $(0.732 \mathrm{~g}$, $5.60 \mathrm{mmol}, 1.40$ equiv) 2-cyclohexylethynyl-1,2-benziodoxol-3(1H)-one $\mathbf{5 k}$ $(0.850 \mathrm{~g}, 2.40 \mathrm{mmol}, 60 \%)$ was obtained as a white amorphous solid. $\mathbf{R}_{\mathbf{f}}$ : 0.44 (DCM:MeOH 9:1). ${ }^{1} \mathrm{H}$ NMR (400 MHz, Chloroform- $d$ ) $\delta 8.28$ (t, $J=6.0$ $\mathrm{Hz}, 1 \mathrm{H}, \mathrm{ArH}), 8.10(\mathrm{t}, J=5.8 \mathrm{~Hz}, 1 \mathrm{H}, \mathrm{ArH}), 7.65(\mathrm{dp}, J=12.9,6.6 \mathrm{~Hz}, 2 \mathrm{H}, \mathrm{ArH}), 2.68(\mathrm{~h}, J=4.7,4.2 \mathrm{~Hz}, 1 \mathrm{H}$, $\mathrm{CH}), 1.82\left(\mathrm{~d}, J=12.5 \mathrm{~Hz}, 2 \mathrm{H}, \mathrm{CH}_{2}\right.$ ), $1.67\left(\mathrm{~d}, J=10.7 \mathrm{~Hz}, 2 \mathrm{H}, \mathrm{CH}_{2}\right), 1.46\left(\mathrm{t}, J=10.4 \mathrm{~Hz}, 3 \mathrm{H}, \mathrm{CH}_{2}\right), 1.29$ (d, J $\left.=10.2 \mathrm{~Hz}, 3 \mathrm{H}, \mathrm{CH}_{2}\right) .{ }^{13} \mathrm{C} \mathrm{NMR}\left(\mathrm{CDCl}_{3}, 100 \mathrm{MHz}\right) \delta$ 166.7, 134.3, 131.9, 131.4, 130.9, 126.1, 115.5, 113.4, 38.7, 31.9, 30.4, 25.3, 24.4. IR $v 2899(\mathrm{~m}), 2877(\mathrm{~m}), 1634(\mathrm{~s}), 1579(\mathrm{~s}), 1494(\mathrm{w}), 1307(\mathrm{~s}), 1241(\mathrm{w})$, $1049(w), 980(w), 876(w), 817$ (w). HRMS (ESI) calcd for $\mathrm{C}_{15} \mathrm{H}_{16} \mathrm{IO}_{2}{ }^{+}[\mathrm{M}+\mathrm{H}]^{+}$355.0190; found 355.0192.

\section{2-Cyclopentylethynyl-1,2-benziodoxol-3(1H)-one (5I)}<smiles>O=C1OI(C#CC2CCCC2)c2ccccc21</smiles>

Following GP1 on $4.00 \mathrm{mmol}$ scale and using ethynylcyclopentane $(0.649 \mathrm{~g}$, $5.60 \mathrm{mmol}, 1.40$ equiv.) at $50{ }^{\circ} \mathrm{C}, 2$-cyclopentylethynyl-1,2-benziodoxol$3(1 \mathrm{H})$-one $5 \mathrm{l}(0.950 \mathrm{~g}, 2.79 \mathrm{mmol}, 70 \%)$ was obtained as a white amorphous solid. $\mathbf{R}_{\mathrm{f}}: 0.40$ (DCM:MeOH 9:1). ${ }^{1} \mathrm{H}$ NMR (400 MHz, Chloroform-d) $\delta 8.28$ (t, 
$J=6.0 \mathrm{~Hz}, 1 \mathrm{H}, \operatorname{ArH}), 8.08(\mathrm{t}, J=6.4 \mathrm{~Hz}, 1 \mathrm{H}, \operatorname{ArH}), 7.66(\mathrm{tt}, J=13.4,7.0 \mathrm{~Hz}, 2 \mathrm{H}, \operatorname{ArH}), 2.91(\mathrm{q}, J=6.7 \mathrm{~Hz}$, $1 \mathrm{H}, \mathrm{CH}), 1.96\left(\mathrm{dd}, J=13.6,7.5 \mathrm{~Hz}, 2 \mathrm{H}, \mathrm{CH}_{2}\right), 1.68\left(\mathrm{~d}, J=13.9 \mathrm{~Hz}, 4 \mathrm{H}, \mathrm{CH}_{2}\right), 1.63-1.48\left(\mathrm{~m}, 2 \mathrm{H}, \mathrm{CH}_{2}\right) .{ }^{13} \mathrm{C}$ NMR $\left(\mathrm{CDCl}_{3}, 100 \mathrm{MHz}\right) \delta 166.7,134.4,131.9,131.5,131.0,126.1,115.5,113.7,38.3,33.5,31.3,24.9$. IR $\vee 2960$ (w), $2868(\mathrm{w}), 2165(\mathrm{w}), 1649(\mathrm{~s}), 1610(\mathrm{~s}), 1560(\mathrm{~m}), 1439(\mathrm{~m}), 1333(\mathrm{~m}), 1295(\mathrm{~m}), 1222(\mathrm{w})$, $1008(\mathrm{~m}), 833(\mathrm{w}), 752(\mathrm{~m})$. HRMS (ESI) calcd for $\mathrm{C}_{14} \mathrm{H}_{14} \mathrm{IO}_{2}{ }^{+}[\mathrm{M}+\mathrm{H}]^{+} 341.0033$; found 341.0036.

\section{Preparation of hydroxy-BX:}<smiles>O=C(O)c1ccccc1I</smiles>

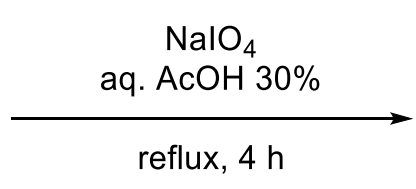<smiles>[R]c1ccc2c(c1)C(=O)OI2O</smiles>

GP2: Following a reported procedure, ${ }^{6} \mathrm{NaIO}_{4}$ (1.05 equiv.) and 2-iodobenzoic acid 15 (1.00 equiv.) were suspended in $30 \%(\mathrm{v} / \mathrm{v})$ aq. AcOH $(48 \mathrm{~mL}$ for $33.8 \mathrm{mmol})$. The mixture was vigorously stirred and refluxed for $4 \mathrm{~h}$. The reaction mixture was then diluted with cold water $(180 \mathrm{~mL})$ and allowed to cool to room temperature, protecting it from light. After $1 \mathrm{~h}$, the crude product was collected by filtration, washed on the filter with ice water $(3 \times 20 \mathrm{~mL})$ and acetone $(3 \times 20 \mathrm{~mL})$, and air-dried in the dark to give the pure product 16 .

\section{1-Hydroxy-5-methyl-1,2-benziodoxol-3-(1H)-one (16a)}<smiles>O=C1OI(O)c2ccccc21</smiles>

Following GP2 on $33.8 \mathrm{mmol}$ scale, 1-hydroxy-1,2-benziodoxol-3-(1H)-one 16a (8.3 g, $31 \mathrm{mmol}, 98 \%)$ was obtained as a white solid. ${ }^{1} \mathrm{H}$ NMR (400 MHz, $\left.\left(\mathrm{CD}_{3}\right)_{2} \mathrm{SO}\right): \delta 8.02$ (dd, J = 7.7, 1.4 Hz, 1H, ArH), $7.97(\mathrm{~m}, 1 \mathrm{H}, \mathrm{ArH}), 7.85$ (dd, J = 8.2, 0.7 Hz, 1H, ArH), 7.71 $(\mathrm{td}, J=7.6,1.2 \mathrm{~Hz}, 1 \mathrm{H}, \mathrm{ArH}) .{ }^{13} \mathrm{C}$ NMR $\left(100 \mathrm{MHz},\left(\mathrm{CD}_{3}\right)_{2} \mathrm{SO}\right): \delta 167.7,134.5,131.5$, 131.1, 130.4, 126.3, 120.4. IR $3083(w), 3060(w), 2867(w), 2402(w), 1601(m), 1585(m), 1564(m)$, $1440(\mathrm{~m}), 1338(\mathrm{~s}), 1302(\mathrm{~m}), 1148(\mathrm{~m}), 1018(\mathrm{w}), 834(\mathrm{~m}), 798(\mathrm{w}), 740(\mathrm{~s}), 694(\mathrm{~s}), 674(\mathrm{~m}), 649(\mathrm{~m})$. The values of the NMR spectra are in accordance with reported literature data. ${ }^{6}$

\section{1-Hydroxy-5-methyl-1,2-benziodoxol-3-(1H)-one (16b)}<smiles>Cc1ccc2c(c1)C(=O)OI2O</smiles>

Following GP2 on $1.91 \mathrm{mmol}$ scale, 1-hydroxy-5-methyl-1,2-benziodoxol-3-(1H)-one 16b $(0.402 \mathrm{~g}, 1.45 \mathrm{mmol}, 76 \%)$ was obtained as a white amorphous solid. ${ }^{1} \mathbf{H}$ NMR (400 MHz, Dimethyl Sulfoxide- $\left.d_{6}\right) \delta 7.96(\mathrm{~s}, 1 \mathrm{H}, \mathrm{OH}), 7.84(\mathrm{~s}, 1 \mathrm{H}, \mathrm{ArH}), 7.78(\mathrm{~d}, J=8.0$ $\mathrm{Hz}, 1 \mathrm{H}, \mathrm{ArH}), 7.69(\mathrm{~d}, J=8.0 \mathrm{~Hz}, 1 \mathrm{H}, \mathrm{ArH}), 2.47\left(\mathrm{~s}, 3 \mathrm{H}, \mathrm{ArCH}_{3}\right) . \delta 8.00(\mathrm{~s}, 1 \mathrm{H}, \mathrm{ArH}), 7.72$ - $7.61(\mathrm{~m}, 1 \mathrm{H}, \mathrm{Ar} H), 7.59-7.47(\mathrm{~m}, 1 \mathrm{H}, \mathrm{ArH}), 3.88\left(\mathrm{~s}, 3 \mathrm{H}, \mathrm{OCH}_{3}\right) .{ }^{13} \mathrm{C}$ NMR $13 \mathrm{C}$ NMR (100 MHz, Dimethyl Sulfoxide- $d_{6}$ ) $\delta 168.2,141.0,135.7,131.9,126.4,117.2,20.6$. The values of the NMR spectra are in accordance with reported literature data. ${ }^{11}$

\section{1-Hydroxy-5-methoxy-1,2-benziodoxol-3-(1H)-one (16c)}<smiles>COc1ccc2c(c1)C(=O)OI2O</smiles>

Following GP2 on $3.60 \mathrm{mmol}$ scale, 1-hydroxy-5-methoxy-1,2-benziodoxol-3-(1H)one $16 \mathrm{c}(0.838 \mathrm{~g}, 2.85 \mathrm{mmol}, 79 \%)$ was obtained as a white amorphous solid. ${ }^{1} \mathbf{H}$ NMR (400 MHz, Dimethyl Sulfoxide- $\left.d_{6}\right) \delta 8.00(\mathrm{~s}, 1 \mathrm{H}, \mathrm{ArH}), 7.72-7.61(\mathrm{~m}, 1 \mathrm{H}, \mathrm{ArH})$, $7.59-7.47(\mathrm{~m}, 1 \mathrm{H}, \mathrm{ArH}), 3.88\left(\mathrm{~s}, 3 \mathrm{H}, \mathrm{OCH}_{3}\right) .{ }^{13} \mathrm{C}$ NMR $13 \mathrm{C}$ NMR $(100 \mathrm{MHz}$, Dimethyl 
Sulfoxide- $\left.d_{6}\right) \delta 167.9,162.0,133.5,127.6,122.0,115.4,109.5,56.4$. The values of the NMR spectra are in accordance with reported literature data. ${ }^{11}$

\section{General procedure for the synthesis of EBX from hydroxy-BX:}

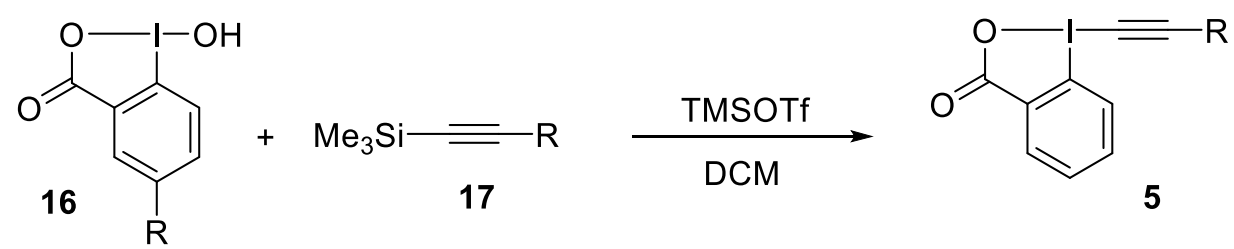

GP3: Following a reported procedure, ${ }^{12}$ to a solution of 2-iodosylbenzoic acid 16 (1.00 equiv.) in dry $\mathrm{DCM}(\mathrm{c}=0.2 \mathrm{M}$ ) was added trimethylsilyl trifluoromethanesulfonate (1.10 equiv.) dropwise at room temperature and the reaction mixture was stirred for $1 \mathrm{~h}$. After this time, the corresponding trimethylethynylsilane 17 (1.10 equiv.) was added and the mixture was stirred for $6 \mathrm{~h}$ at room temperature. The reaction mixture was then quenched with saturated aqueous $\mathrm{NaHCO}_{3}$ solution and extracted with dichloromethane ( 3 times). The combined organic extracts were dried over $\mathrm{MgSO}_{4}$, filtered and concentrated under reduced pressure. The residue was purified by column chromatography using DCM/methanol as eluent to give the corresponding EBX reagent 5.

\section{1-[Phenylethynyl]-1,2-benziodoxol-3(1H)-one (5n)}<smiles>O=C1OI(C#Cc2ccccc2)c2ccccc21</smiles>

Following GP2 on $41.5 \mathrm{mmol}$ scale, 1-[phenylethynyl]-1,2-benziodoxol-3(1H)one $5 \mathrm{n}\left(6.08 \mathrm{~g}, 17.4 \mathrm{mmol}, 46 \%\right.$ ) as a white solid. $\mathrm{Mp}$ (Dec.) $155.0-160.0^{\circ} \mathrm{C}$ (lit 153-155 $\mathrm{C}) .{ }^{1} \mathrm{H}$ NMR $\left(400 \mathrm{MHz}, \mathrm{CDCl}_{3}\right) \delta 8.46(\mathrm{~m}, 1 \mathrm{H}, \mathrm{ArH}), 8.28(\mathrm{~m}, 1 \mathrm{H}, \mathrm{ArH})$, $7.80(\mathrm{~m}, 2 \mathrm{H}, \operatorname{ArH}), 7.63(\mathrm{~m}, 2 \mathrm{H}, \operatorname{ArH}), 7.48(\mathrm{~m}, 3 \mathrm{H}, \operatorname{ArH}) .{ }^{13} \mathrm{C}$ NMR $(100 \mathrm{MHz}$, $\left.\mathrm{CDCl}_{3}\right) \delta 163.9,134.9,132.9,132.5,131.6,131.3 .130 .8,128.8,126.2,120.5,116.2,106.6,50.2$. Data reported in literature. ${ }^{12}$

\section{5-Methyl-propynyl-1,2-benziodoxol-3(1H)-one (5o)}<smiles>CC#CI1COC(=O)c2cc(C)ccc21</smiles>

Following GP3 on $1.44 \mathrm{mmol}$ scale, 5-methyl-propynyl-1,2-benziodoxol-3(1H)one 50 ( $0.235 \mathrm{~g}, 783 \mu \mathrm{mol}, 54 \%$ - about $85 \%$ purity) was obtained as a white solid Rf: 0.57 (DCM:MeOH 9:1). Mp: $137^{\circ} \mathrm{C} .{ }^{1} \mathrm{H}$ NMR (400 MHz, Chloroform-d) $\delta 8.15(\mathrm{~s}, 1 \mathrm{H}, \mathrm{ArH}), 7.98(\mathrm{~d}, J=8.4 \mathrm{~Hz}, 1 \mathrm{H}, \mathrm{ArH}), 7.52(\mathrm{dd}, J=8.5,2.2 \mathrm{~Hz}, 1 \mathrm{H}$, $\mathrm{ArH}), 2.46$ (s, 3H, $\left.\mathrm{ArCH}_{3}\right), 2.23\left(\mathrm{~s}, 3 \mathrm{H}, \mathrm{CH}_{3}\right) .{ }^{13} \mathrm{C} \mathrm{NMR}(100 \mathrm{MHz}$, Chloroform-d) $\delta 167.4,142.3,135.7$ (2 Carbon signals are overlapping), 132.9, 126.3, 111.7, 105.1, 37.7, 20.8, 5.6. IR v $2979(\mathrm{~m}), 2913(\mathrm{w}), 2245(\mathrm{w}), 2175(\mathrm{w}), 1625(\mathrm{~s}), 1574(\mathrm{~m}), 1456(\mathrm{~m}), 1404(\mathrm{~m}), 1316(\mathrm{~m}), 1253(\mathrm{~m})$, $1067(\mathrm{~m}), 1004(\mathrm{~m}), 909(\mathrm{~s}), 786(\mathrm{~m}), 730(\mathrm{~s}), 645(\mathrm{~m})$. HRMS (ESI/QTOF) m/z: [M + H] ${ }^{+}$Calcd for $\mathrm{C}_{11} \mathrm{H}_{10} \mathrm{IO}_{2}{ }^{+}$300.9720; Found 300.9724.

\section{5-Methoxy-propynyl-1,2-benziodoxol-3(1H)-one (5p)}<smiles>CC#CI1COC(=O)c2cc(OC)ccc21</smiles>

Following GP3 on $2.72 \mathrm{mmol}$ scale, 5-methoxy-propynyl-1,2-benziodoxol3(1H)-one 5 aa $(0.273 \mathrm{~g}, 864 \mu \mathrm{mol}, 32 \%)$ was obtained as a white solid Rf: 0.51 (DCM:MeOH 9:1). Mp: $176{ }^{\circ} \mathrm{C} .{ }^{1} \mathrm{H}$ NMR (400 MHz, Chloroform-d) $\delta 7.97$ (d, $J=$ $9.1 \mathrm{~Hz}, 1 \mathrm{H}, \operatorname{ArH}), 7.91(\mathrm{~d}, J=3.0 \mathrm{~Hz}, 1 \mathrm{H}, \operatorname{ArH}), 7.30(\mathrm{dd}, J=9.1,3.0 \mathrm{~Hz}, 1 \mathrm{H}, \operatorname{ArH})$,

\footnotetext{
${ }^{11}$ S. Bertho, R. Rey-Rodriguez, C. Colas, P. Retailleau, I. Gillaizeau, Chem. - Eur. J. 2017, 23, 17674-17677.

${ }^{12}$ S. Nicolai, C. Piemontesi, J. Waser, Angew. Chem. Int. Ed. 2011, 50, 4680.
} 
$3.93\left(\mathrm{~s}, 3 \mathrm{H}, \mathrm{OCH}_{3}\right), 2.26\left(\mathrm{~s}, 3 \mathrm{H}, \mathrm{CH}_{3}\right) .{ }^{13} \mathrm{C}$ NMR $(100 \mathrm{MHz}$, Chloroform-d) $\delta 166.6,163.3,133.2,127.0$, 123.1, 115.5, 104.7, 103.3, 56.4, 38.5, 5.7. IR v $2175(\mathrm{w}), 1631$ (s), $1577(\mathrm{~s}), 1464(\mathrm{~m}), 1418(\mathrm{~m}), 1317$ (s), $1231(\mathrm{~m}), 1031(\mathrm{~m}), 1000(\mathrm{w}), 907(\mathrm{~m}), 789$ (m), 734 (s). HRMS (ESI/QTOF) m/z: [M + H] ${ }^{+}$Calcd for $\mathrm{C}_{11} \mathrm{H}_{10} \mathrm{IO}_{3}{ }^{+}$316.9669; Found 316.9676.

\section{One pot procedure for the synthesis of EBX:}

\section{Octynyl-1,2-benziodoxol-3(1H)-one (5c)}

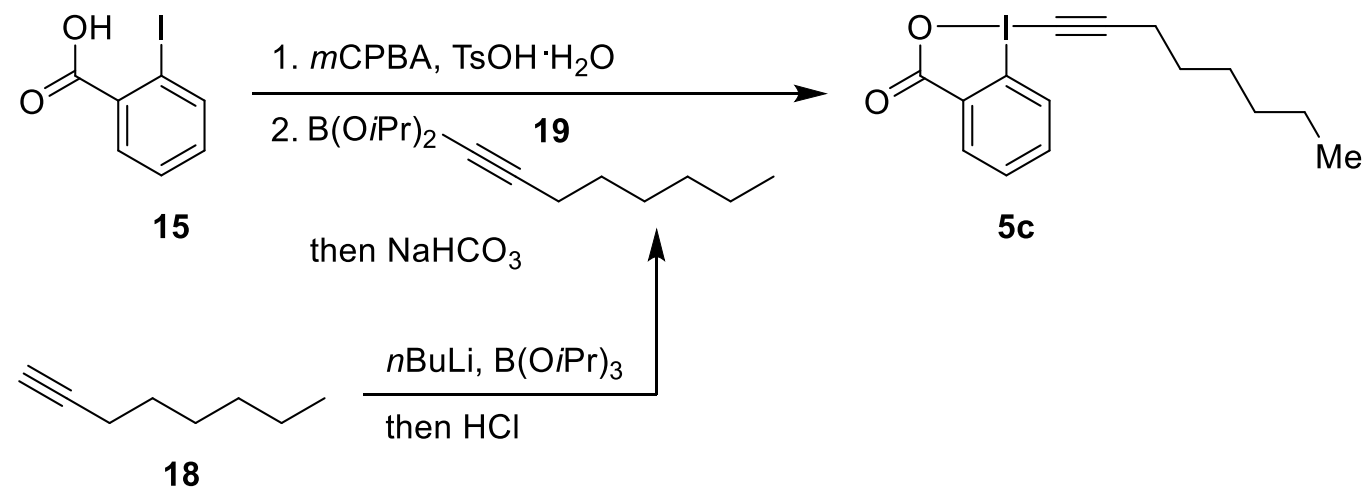

Following a slightly modified procedure, ${ }^{13}$ a solution of 1-octyne $18(747 \mathrm{mg}, 6.78 \mathrm{mmol}, 1.00$ eq.) and dry diethyl ether $(7.0 \mathrm{~mL})$ was cooled to $-78^{\circ} \mathrm{C}$, at which temperature $1.6 \mathrm{M} \mathrm{nBuLi}$ in hexanes $(4.24 \mathrm{~mL}$, $6.78 \mathrm{mmol}, 1.00$ eq.) was added dropwise. The mixture was stirred at $-78^{\circ} \mathrm{C}$ for 90 minutes and then canullated into a to $-78{ }^{\circ} \mathrm{C}$ pre-cooled solution consisting of triisopropyl borate $(1.56 \mathrm{~mL}, 6.78 \mathrm{mmol}$, 1.00 eq.) and dry diethyl ether $(7.0 \mathrm{~mL})$. The reaction mixture was stirred at $-78{ }^{\circ} \mathrm{C}$ for 2 hours, after which $2.0 \mathrm{M} \mathrm{HCl}$ in diethyl ether $(3.73 \mathrm{~mL}, 7.46 \mathrm{mmol}, 1.10$ eq.) was added. The cooling bath was removed and the mixture was stirred for an additional 60 minutes. After filtration and solvent removal, Kugelrohr distillation ( $75^{\circ} \mathrm{C}$ at $0.6 \mathrm{mbar}$ ) furnished pure diisopropyloct-1-ynylboronate $(\mathbf{1 9}, 940 \mathrm{mg}$, $3.95 \mathrm{mmol}, 58 \%$ yield) as a colorless liquid. ${ }^{1} \mathrm{H}$ NMR ( $\mathrm{CDCl}_{3}, 400 \mathrm{MHz}$ ): $\delta 4.55$ (sept, $2 \mathrm{H}, \mathrm{J}=6.2 \mathrm{~Hz}$, ${ }^{\mathrm{P}} \mathrm{Pr}$ $\mathrm{CH}), 2.27\left(\mathrm{t}, 2 \mathrm{H}, \mathrm{J}=7.0 \mathrm{~Hz}\right.$, propargyl $\left.\mathrm{CH}_{2}\right), 1.60-1.48\left(\mathrm{~m}, 2 \mathrm{H}, \mathrm{CH}_{2}\right), 1.45-1.24\left(\mathrm{~m}, 6 \mathrm{H}, \mathrm{CH}_{2}\right), 1.19(\mathrm{~d}, 12$ $\left.\mathrm{H}, J=6.2 \mathrm{~Hz},{ }^{\mathrm{i} P r}-\mathrm{CH}_{3}\right), 0.89\left(\mathrm{t}, 3 \mathrm{H}, J=6.9 \mathrm{~Hz}\right.$, alkyl $\left.\mathrm{CH}_{3}\right)$. The values of the ${ }^{1} \mathrm{H}$ NMR spectrum are in accordance with reported literature data. ${ }^{14}$

Following a slightly modified procedure, ${ }^{8}$ 2-iodobenzoic acid 15 (692 mg, 2.79 mmol, 1.00 eq.), paratoluenesulfonic acid monohydrate $\left(\mathrm{TsOH}^{\mathrm{H}} \mathrm{H}_{2} \mathrm{O}, 531 \mathrm{mg}, 2.79 \mathrm{mmol}, 1.00\right.$ eq.) and metachloroperoxybenzoic acid (mCPBA-70\%, $756 \mathrm{mg}, 3.07 \mathrm{mmol}, 1.10$ eq.) were dissolved in dichloromethane $(4.5 \mathrm{~mL})$ and 2,2,2-trifluoroethanol $(4.5 \mathrm{~mL})$. The mixture was stirred at room temperature under nitrogen for 1 hour, after which diisopropyloct-1-ynylboronate $(\mathbf{1 9}, 930 \mathrm{mg}, 3.90$ $\mathrm{mmol}, 1.40$ eq.) was added in one portion. The reaction mixture was stirred for 2 hours at room temperature, filtered and concentrated in vacuo. The resulting oil was dissolved in dichloromethane $(30 \mathrm{~mL})$ and under vigorous stirring, saturated aq. $\mathrm{NaHCO}_{3}(30 \mathrm{~mL})$ was added. The mixture was stirred for 15 minutes, the two layers were separated and the aqueous layer was extracted with additional portions of dichloromethane $(3 \times 10 \mathrm{~mL})$. The combined organic layers were dried over $\mathrm{MgSO}_{4}$, filtered and concentrated in vacuo. The crude product was purified by flash column chromatography (ethyl

\footnotetext{
${ }^{13}$ H. C. Brown, N. G. Bhat, M. Srebnik, Tetrahedron Lett. 1988, 29, 2631.

${ }^{14}$ R. Morita, E. Shirakawa, T. Tsuchimoto, Y. Kawakami, Y. Org. Biomol. Chem. 2005, 3, 1263.
} 


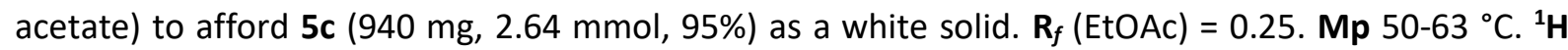
NMR $\left(\mathrm{CDCl}_{3}, 400 \mathrm{MHz}\right): 8$ 8.42-8.35 (m, $\left.1 \mathrm{H}, \mathrm{ArH}\right), 8.20-8.13(\mathrm{~m}, 1 \mathrm{H}, \mathrm{ArH}), 7.78-7.69(\mathrm{~m}, 2 \mathrm{H}, \mathrm{ArH}), 2.59$ $\left(\mathrm{t}, 2 \mathrm{H}, \mathrm{J}=7.1 \mathrm{~Hz}, \mathrm{CCCH}_{2}\right), 1.70-1.58(\mathrm{~m}, 2 \mathrm{H}), 1.51-1.39(\mathrm{~m}, 2 \mathrm{H}), 1.38-1.26(\mathrm{~m}, 4 \mathrm{H}), 0.94-0.86(\mathrm{~m}, 3 \mathrm{H}$, $\left.\mathrm{CH}_{2} \mathrm{CH}_{3}\right) .{ }^{13} \mathrm{C} \mathrm{NMR}\left(\mathrm{CDCl}_{3}, 100 \mathrm{MHz}\right): \delta$ 166.7, 134.7, 132.5, 131.7, 131.6, 126.3, 115.7, 109.9, 39.4, 31.3, 28.7, 28.3, 22.6, 20.6, 14.1. IR $2930(w), 2858(w), 2166(w), 1619(s), 1561(w), 1439(w), 1331(m)$, $1297(\mathrm{~m}), 832(\mathrm{w}), 748(\mathrm{~m}) . \mathrm{HRMS}(\mathrm{ESI}) \mathrm{C}_{15} \mathrm{H}_{18} \mathrm{OO}_{2}{ }^{+}[\mathrm{M}+\mathrm{H}]^{+}$calc. $=357.0346 ;[\mathrm{M}+\mathrm{H}]^{+}$obs. $=357.0339$.

\section{5-Pentanolethynyl-1,2-benziodoxol-3(1H)-one (5d)}

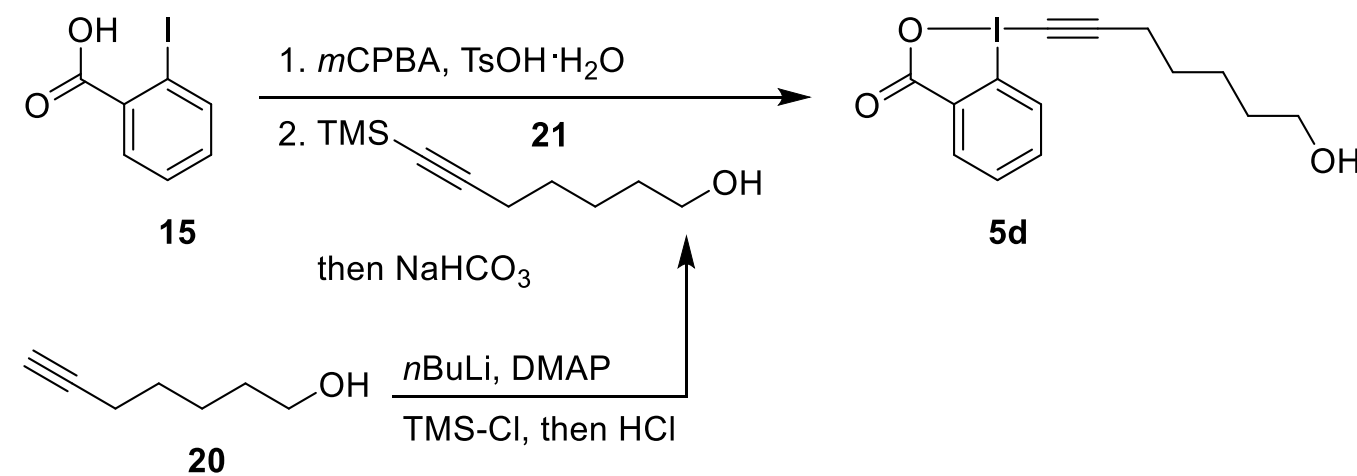

Following a slightly modified procedure, ${ }^{15} 2.5 \mathrm{M} \mathrm{nBuLi}$ in hexanes (39.2 mL, $98.0 \mathrm{mmol}, 2.20$ eq.) was added at $-78^{\circ} \mathrm{C}$ under nitrogen to a mixture of hept-6-yn-1-ol $20(5.00 \mathrm{~g}, 44.6 \mathrm{mmol}, 1.00 \mathrm{eq}$.) and dry THF (150 mL), followed by 4-dimethylaminopyridine (DMAP, $1.36 \mathrm{~g}, 11.1 \mathrm{mmol}, 0.25$ eq.). The mixture was stirred at $-78{ }^{\circ} \mathrm{C}$ for 60 minutes, after which trimethylsilyl chloride (TMS-Cl, $20.4 \mathrm{~mL}, 156 \mathrm{mmol}$, 3.50 eq.) was added dropwise. The cooling bath was removed and the reaction stirred for 2 hours. Next, $1.0 \mathrm{~N}$ aq. $\mathrm{HCl}(50 \mathrm{~mL})$ was added and the solution was stirred vigorously for 30 minutes at room temperature. The mixture was diluted with EtOAc $(200 \mathrm{~mL})$ and extracted. The aqueous layer was extracted with additional portions of EtOAc $(3 \times 50 \mathrm{~mL})$. The combined organic layers were washed with sat. aq. $\mathrm{NaHCO}_{3}(100 \mathrm{~mL})$, brine $(50 \mathrm{~mL})$, dried over $\mathrm{MgSO}_{4}$, filtered and concentrated in vacuo. The crude product was purified by flash column chromatography (pentane:EtOAc 4:1) to afford 7(trimethylsilyl)hept-6-yn-1-ol (21, $8.22 \mathrm{~g}, 43.5 \mathrm{mmol}, 97 \%)$ as a colorless oil. ${ }^{1} \mathrm{H} \mathbf{~ N M R}\left(\mathrm{CDCl}_{3}, 400 \mathrm{MHz}\right)$ : $\delta 3.61\left(\mathrm{t}, 2 \mathrm{H}, J=6.5 \mathrm{~Hz}, \mathrm{CH}_{2} \mathrm{OH}\right.$ ), 2.21 (t, $\left.2 \mathrm{H}, J=7.0 \mathrm{~Hz}, \mathrm{CCCH}_{2}\right), 1.73$ (bs, $\left.1 \mathrm{H}, \mathrm{CH}_{2} \mathrm{OH}\right), 1.61-1.48$ (m, 4 $\mathrm{H}), 1.48-1.38(\mathrm{~m}, 2 \mathrm{H}), 0.11$ (s, $9 \mathrm{H}, \mathrm{TMS}) .{ }^{13} \mathrm{C}$ NMR $\left(\mathrm{CDCl}_{3}, 100 \mathrm{MHz}\right): \delta 107.4,84.6,62.8,32.3,28.5$, $25.1,19.9,0.3$. The values of the ${ }^{1} \mathrm{H}$ NMR spectrum are in accordance with reported literature data. ${ }^{16}$

Following a slightly modified procedure, ${ }^{8}$ 2-iodobenzoic acid 15 (7.69 g, $31.0 \mathrm{mmol}, 1.00$ eq.), paratoluenesulfonic acid monohydrate $\left(\mathrm{TsOH} \cdot \mathrm{H}_{2} \mathrm{O}, 5.90 \mathrm{~g}, 31.0 \mathrm{mmol}, 1.00\right.$ eq.) and metachloroperoxybenzoic acid (mCPBA-70\%, $8.41 \mathrm{~g}, 34.1 \mathrm{mmol}, 1.10$ eq.) were dissolved in dichloromethane $(57 \mathrm{~mL}$ ) and 2,2,2-trifluoroethanol $(57 \mathrm{~mL})$. The mixture was stirred at room temperature under nitrogen for 1 hour, after which 7-(trimethylsilyl)hept-6-yn-1-ol (21, 8.00 g, 43.4 mmol, 1.40 eq.) was added in one portion. The reaction mixture was stirred for 18 hours at room temperature, filtered and concentrated in vacuo. The resulting oil was dissolved in dichloromethane $(500 \mathrm{~mL})$ and under vigorous stirring, saturated aq. $\mathrm{NaHCO}_{3}(500 \mathrm{~mL})$ was added. The mixture was stirred for 60 minutes, the two layers were separated and the aqueous layer was extracted with additional portions of dichloromethane $(3 \times 150 \mathrm{~mL})$. The combined organic layers were dried over

\footnotetext{
${ }^{15}$ P. A. Peixoto, J. A. Richard, R. Severin, D. Y. Chen, Org. Lett. 2011, 13, 5724.

${ }^{16}$ F. Rodier, M. Rajzmann, J. L. Parrain, G. Chouraqui, L. Commeiras, Chem. Eur. J. 2013, 19, 2467.
} 
$\mathrm{MgSO}_{4}$, filtered and concentrated in vacuo. The crude product was purified by flash column chromatography (EtOAc:MeOH 95:5) to afford 5d (3.56 g, $9.94 \mathrm{mmol}, 32 \%)$ as a white solid. $\mathbf{R}_{\boldsymbol{f}}$ (EtOAc:MeOH 9:1) = 0.24. Mp 115-120 ${ }^{\circ} \mathrm{C} .{ }^{1} \mathrm{H} \mathrm{NMR}\left(\mathrm{CDCl}_{3}, 400 \mathrm{MHz}\right): \delta 8.33$ (dd, $1 \mathrm{H}, J=7.2,2.0 \mathrm{~Hz}$, $\operatorname{ArH}), 8.15(\mathrm{~d}, 1 \mathrm{H}, J=8.0 \mathrm{~Hz}, \mathrm{ArH}), 7.79-7.64(\mathrm{~m}, 2 \mathrm{H}, \mathrm{ArH}), 3.66\left(\mathrm{t}, 2 \mathrm{H}, J=5.9 \mathrm{~Hz}, \mathrm{CH}_{2} \mathrm{OH}\right), 2.59(\mathrm{t}, 2 \mathrm{H}$, $\left.J=6.9 \mathrm{~Hz}, \mathrm{CCCH}_{2}\right), 1.73-1.49\left(\mathrm{~m}, 7 \mathrm{H}, \mathrm{CH}_{2}\right.$ and $\left.\mathrm{OH}\right) .{ }^{13} \mathrm{C} \mathrm{NMR}\left(\mathrm{CDCl}_{3}, 100 \mathrm{MHz}\right): \delta 167.0,134.8,132.3$, 131.6, 131.5, 126.5, 115.7, 109.7, 62.3, 39.2, 32.1, 28.0, 25.3, 20.6. IR $\vee 3351$ (w), $2934(w), 2170(w)$, $1623(\mathrm{~s}), 1585(\mathrm{~m}), 1561(\mathrm{w}), 1439(\mathrm{w}), 1333(\mathrm{~m}), 1300(\mathrm{w}), 1058(\mathrm{w}), 911(\mathrm{~m}), 832(\mathrm{w}), 732(\mathrm{~s}), 689$ (m). $\mathrm{HRMS}(\mathrm{ESI}) \mathrm{C}_{14} \mathrm{H}_{16} \mathrm{IO}_{3}{ }^{+}[\mathrm{M}+\mathrm{H}]^{+}$calc. $=359.0139 ;[\mathrm{M}+\mathrm{H}]^{+}$obs. $=359.0136$.

\section{8-(Trimethylsilyl)octa-1,7-diyn-1-yl-1,2-benziodoxol-3(1H)-one (5e)}

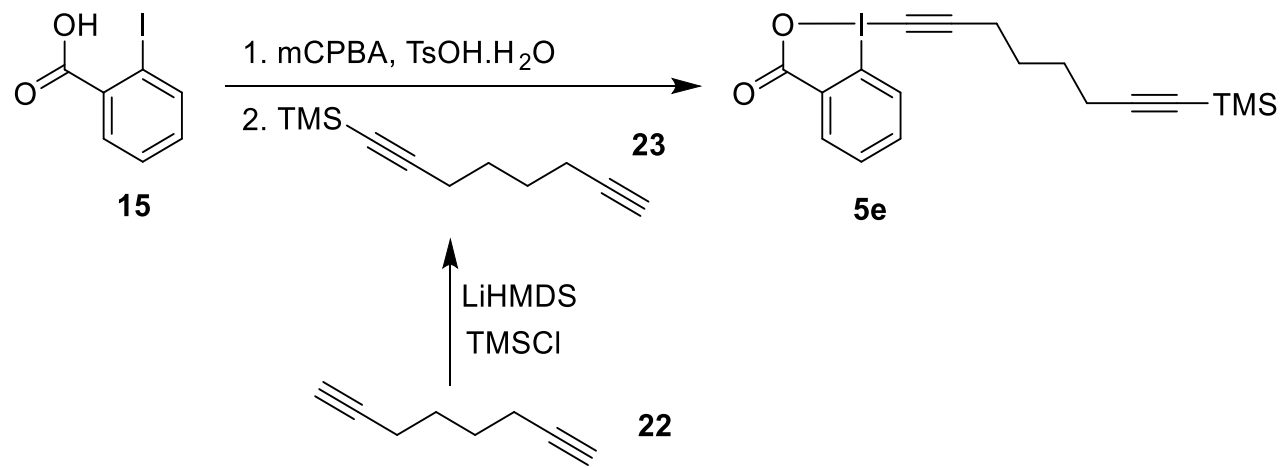

Following a slightly modified procedure, ${ }^{6}$ to a solution of 1,7-octadiyne $22(10.6 \mathrm{~g}, 100 \mathrm{mmol}, 1.00$ equiv) in dry THF (150 mL) was added at $-78^{\circ} \mathrm{C}$ under nitrogen $1 \mathrm{M}$ lithium bis(trimethylsilyl)amide in THF (LiHMDS, $100 \mathrm{~mL}, 100 \mathrm{mmol}, 1.00$ equiv.). The solution was stirred at $-78^{\circ} \mathrm{C}$ for 30 minutes, after which trimethylsilyl chloride ( $\mathrm{TMSCl}, 13.0 \mathrm{~mL}, 100 \mathrm{mmol}, 1.00$ equiv.) was added dropwise. The reaction was warmed to room temperature and stirred for $2 \mathrm{~h}$. The reaction was cooled to $0{ }^{\circ} \mathrm{C}$ and quenched by adding water $(10 \mathrm{~mL})$. The mixture was diluted with $1 \mathrm{M} \mathrm{HCl}(200 \mathrm{~mL})$ and extracted with diethyl ether $(100 \mathrm{~mL}$ and $2 \times 75 \mathrm{~mL})$. The combined organic layers were washed with brine $(200 \mathrm{~mL})$, dried over $\mathrm{MgSO}_{4}$, filtered and concentrated in vacuo. The crude product was purified by vacuum distillation using a $20 \mathrm{~cm}$ Vigreux column (oil bath set to $98{ }^{\circ} \mathrm{C}$ at $0.3 \mathrm{mbar}$ ) furnishing pure trimethyl(octa-1,7-diyn-1-yl)silane $\mathbf{2 3}$ (8.37 g, $46.9 \mathrm{mmol}, \mathbf{4 7 \%}$ ) as a colorless liquid. $\mathbf{R}_{\mathbf{f}}$ : 0.2 (Pentane). ${ }^{1} \mathrm{H}$ NMR $\left(\mathrm{CDCl}_{3}, 400 \mathrm{MHz}\right) \delta 2.28-2.17(\mathrm{~m}, 4 \mathrm{H}), 1.93(\mathrm{t}, J=2.7 \mathrm{~Hz}, 1 \mathrm{H}, \mathrm{CCH}), 1.68-1.57(\mathrm{~m}, 4 \mathrm{H}), 0.13(\mathrm{~s}$, 9H, TMS). ${ }^{13} \mathrm{C}$ NMR $\left(\mathrm{CDCl}_{3}, 100 \mathrm{MHz}\right) \delta 107.0,84.9,84.2,68.6,27.7,27.6,19.5,18.1,0.3$. IR v 3309 (w), $2951(w), 2175(w), 1250(m), 912(w), 841(s), 761(m), 734(m)$. Data reported in literature. ${ }^{6}$

Following a slightly modified procedure, ${ }^{8} 2$-iodobenzoic acid 15 (8.43 g, $33.3 \mathrm{mmol}, 1.00$ equiv.), paratoluenesulfonic acid monohydrate ( $\left(\mathrm{TsOH} . \mathrm{H}_{2} \mathrm{O}, 6.40 \mathrm{~g}, 33.3 \mathrm{mmol}, 1.00\right.$ equiv.) and metachloroperoxybenzoic acid (mCPBA-70\%, $9.04 \mathrm{~g}, 36.7 \mathrm{mmol}, 1.10$ equiv.) were dissolved in $\mathrm{CH}_{2} \mathrm{Cl}_{2}(60$ $\mathrm{mL}$ ) and 2,2,2-trifluoroethanol $(60 \mathrm{~mL})$. The mixture was stirred at room temperature under nitrogen for $1 \mathrm{~h}$, after which trimethyl(octa-1,7-diyn-1-yl)silane $\mathbf{2 3}$ (8.32 g, $46.7 \mathrm{mmol}, 1.40$ equiv.) was added. The reaction mixture was stirred for $15 \mathrm{~h}$ at room temperature and then filtered and concentrated in vacuo. The resulting light being solid was dissolved in $\mathrm{CH}_{2} \mathrm{Cl}_{2}(500 \mathrm{~mL})$ and under vigorous stirring, saturated solution of $\mathrm{NaHCO}_{3}(500 \mathrm{~mL})$ was added. The mixture was stirred for $1 \mathrm{~h}$, the two layers were separated and the aqueous layer was extracted with additional portions of $\mathrm{CH}_{2} \mathrm{Cl}_{2}(3 \times 150 \mathrm{~mL})$. The combined organic layers were dried over $\mathrm{MgSO}_{4}$, filtered and concentrated in vacuo. The crude product was purified by flash column chromatography using ethyl acetate to afford 5 e $(4.20 \mathrm{~g}, 9.90$ mmol, 30\%) as a white solid. $\mathbf{M p}: 152.3-155.6^{\circ} \mathrm{C} . \mathbf{R}_{\mathbf{f}}: 0.59$ (EtOAc:MeOH 9:1). ${ }^{1} \mathbf{H} \mathbf{~ N M R}\left(\mathrm{CDCl}_{3}, 400 \mathrm{MHz}\right.$ ) 
$\delta 8.37(\mathrm{dd}, J=6.7,2.3 \mathrm{~Hz}, 1 \mathrm{H}, \operatorname{ArH}), 8.17(\mathrm{dd}, J=7.8,1.5 \mathrm{~Hz}, 1 \mathrm{H}, \operatorname{ArH}), 7.82-7.66(\mathrm{~m}, 2 \mathrm{H}, \operatorname{ArH}), 2.63(\mathrm{t}, J$ $=6.8 \mathrm{~Hz}, 2 \mathrm{H},), 2.29(\mathrm{t}, J=6.7 \mathrm{~Hz}, 2 \mathrm{H}), 1.83-1.62(\mathrm{~m}, 4 \mathrm{H}), 0.13(\mathrm{~s}, 9 \mathrm{H}, \mathrm{TMS}) .{ }^{13} \mathrm{C} \mathrm{NMR}\left(\mathrm{CDCl}_{3}, 100 \mathrm{MHz}\right) \delta$ 166.7, 134.8, 132.4, 131.7, 131.5, 126.3, 115.7, 109.1, 106.4, 85.4, 40.0, 27.7, 27.3, 20.2, 19.4, 0.3. IR v 2955 (w), 2170 (w), 1647 (m), 1621 (s), 1439 (w), 1329 (m), 1296 (w), 1249 (m), 840 (s), 746 (s). HRMS (ESI) calcd. for $\mathrm{C}_{18} \mathrm{H}_{22} \mathrm{OO}_{2} \mathrm{Si}^{+}[\mathrm{M}+\mathrm{H}]^{+} 425.0428$; found 425.0433. Data reported in literature. ${ }^{6}$

2-(6-(3-Oxo-1,2-benziodoxol-3(1H)-yl)hex-5-yn-1-yl)isoindoline-1,3-dione (5f)

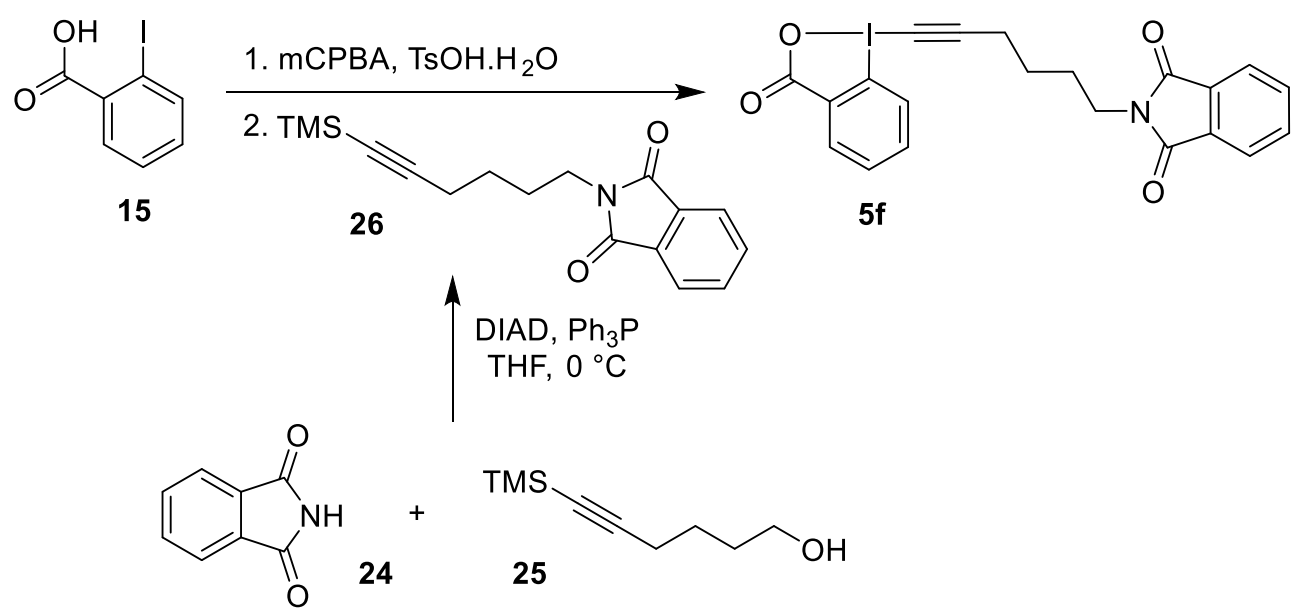

To a stirring solution of 6-(trimethylsilyl)hex-5-yn-1-ol 25 (852 mg, $5.00 \mathrm{mmol}, 1.00$ equiv.) in THF (16.7 $\mathrm{mL}$ ) was added triphenylphosphine $(1.44 \mathrm{~g}, 5.50 \mathrm{mmol}, 1.10$ equiv.) and DIAD (1.15 mL, $5.50 \mathrm{mmol}$, 1.10 equiv.) at $0{ }^{\circ} \mathrm{C}$. The reaction mixture was stirred at this temperature for $15 \mathrm{~min}$ and then, phthalimide (24) (750 mg, $5.10 \mathrm{mmol}, 1.02$ equiv.) was added. The reaction was continued at room temperature for $5 \mathrm{~h}$, then cold water $(20 \mathrm{~mL})$ was added and the product was extracted with EtOAc ( 3 $x 40 \mathrm{~mL}$ ). The combined organic layers were washed with brine $(50 \mathrm{~mL})$, dried over anhydrous $\mathrm{MgSO}_{4}$ and the solvent was removed under reduced pressure. The crude product was purified by column chromatography using $\mathrm{Et}_{2} \mathrm{O}$ /pentane $10: 90$ as eluent to furnish 2-(6-(trimethylsilyl)hex-5-yn-1yl)isoindoline-1,3-dione (26) as a white solid (1.37 g, $4.58 \mathrm{mmol}, 92 \%)$. M.p. $68-70{ }^{\circ} \mathrm{C} ; \mathbf{R}_{\mathbf{f}}=0.26$ (Et ${ }_{2} \mathrm{O} /$ pentane 10:90); ${ }^{1} \mathrm{H}$ NMR (400 MHz, $\left.\mathrm{CDCl}_{3}\right) \delta 7.83$ (dd, J=5.4, 3.1 Hz, 2H, ArH), 7.70 (dd, J = 5.4, $3.1 \mathrm{~Hz}, 2 \mathrm{H}, \mathrm{ArH}$ ), $3.70\left(\mathrm{t}, J=7.0 \mathrm{~Hz}, 2 \mathrm{H}, \mathrm{CH}_{2} \mathrm{NPhth}\right), 2.27\left(\mathrm{t}, J=7.1 \mathrm{~Hz}, 2 \mathrm{H}, \mathrm{C} \equiv \mathrm{CCH}_{2}\right), 1.85-1.74(\mathrm{~m}, 2 \mathrm{H}$, $\left.\mathrm{CH}_{2}\right), 1.61$ - $1.49\left(\mathrm{~m}, 2 \mathrm{H}, \mathrm{CH}_{2}\right), 0.12(\mathrm{~s}, 9 \mathrm{H}, \mathrm{TMS}) ;{ }^{13} \mathrm{C} \mathrm{NMR}\left(101 \mathrm{MHz}, \mathrm{CDCl}_{3}\right) \delta 168.5,134.0,132.3,123.3$, 106.7, 85.1, 37.6, 27.8, 25.9, 19.6, 0.3; IR $\left(v_{\max }, \mathrm{cm}^{-1}\right) 2955(\mathrm{w}), 2931(\mathrm{w}), 2170(\mathrm{w}), 1770(\mathrm{w}), 1705(\mathrm{~m})$, $1440(\mathrm{w}), 1390(\mathrm{~s}), 1352(\mathrm{~m}), 1324(\mathrm{~m}), 1247(\mathrm{~m}), 1035(\mathrm{~m}), 904(\mathrm{~m}), 841(\mathrm{~s}), 761(\mathrm{~s}), 718(\mathrm{~s}), 638(\mathrm{~m})$; HRMS (ESI/QTOF) m/z: [M+H] ${ }^{+}$Calcd. for $\mathrm{C}_{17} \mathrm{H}_{22} \mathrm{NO}_{2} \mathrm{Si}^{+} 300.1414$; Found 300.1413.

Following a slightly modified procedure, ${ }^{8} 2$-iodobenzoic acid 15 (496 mg, $2.00 \mathrm{mmol}, 1.00$ equiv.), paratoluenesulfonic acid monohydrate $\left(\mathrm{TsOH} \bullet \mathrm{H}_{2} \mathrm{O}, 380 \mathrm{mg}, 2.00 \mathrm{mmol}, 1.00\right.$ equiv.) and metachloroperoxybenzoic acid ( $m$ CPBA-70\%, $493 \mathrm{mg}, 2.20 \mathrm{mmol}, 1.10$ equiv.) were dissolved in $\mathrm{CH}_{2} \mathrm{Cl}_{2}$ (3 $\mathrm{mL}$ ) and 2,2,2-trifluoroethanol ( $3 \mathrm{~mL})$. The mixture was stirred at room temperature under nitrogen for $1 \mathrm{~h}$, after which 2-(6-(trimethylsilyl)hex-5-yn-1-yl)isoindoline-1,3-dione 26 (599 mg, $2.00 \mathrm{mmol}$, 1.00 equiv.) was added. The reaction mixture was stirred for $15 \mathrm{~h}$ at room temperature and then filtered and concentrated in vacuo. The resulting light beige solid was dissolved in $\mathrm{CH}_{2} \mathrm{Cl}_{2}(30 \mathrm{~mL})$ and under vigorous stirring, a saturated solution of $\mathrm{NaHCO}_{3}(30 \mathrm{~mL})$ was added. The mixture was stirred for $1 \mathrm{~h}$, the two layers were separated and the aqueous layer was extracted with additional portions of $\mathrm{CH}_{2} \mathrm{Cl}_{2}$ (3 $\times 20 \mathrm{~mL}$ ). The combined organic layers were dried over $\mathrm{MgSO}_{4}$, filtered and concentrated 
in vacuo. The crude product was purified by flash column chromatography using ethyl acetate to afford 2-(6-(3-oxo-1,2-benziodoxol-3(1H)-yl)hex-5-yn-1-yl)isoindoline-1,3-dione $5 f$ (300 mg, 634 umol, 32\%) as a white solid. Rf: 0.54 (DCM:MeOH 9:1). $\mathbf{M p :} 168-172{ }^{\circ} \mathrm{C} .{ }^{1} \mathrm{H}$ NMR (400 MHz, Chloroform-d) $\delta 8.40$ (dd, $J=7.3,1.9 \mathrm{~Hz}, 1 \mathrm{H}, \mathrm{ArH}), 8.16(\mathrm{dd}, J=8.1,1.1 \mathrm{~Hz}, 1 \mathrm{H}, \operatorname{ArH}), 7.85(\mathrm{dd}, J=5.4,3.0 \mathrm{~Hz}, 2 \mathrm{H}, \operatorname{ArH}), 7.82$ - $7.69(\mathrm{~m}, 4 \mathrm{H}, \mathrm{ArH}), 3.76\left(\mathrm{t}, J=7.0 \mathrm{~Hz}, 2 \mathrm{H}, \mathrm{CH}_{2}\right), 2.67\left(\mathrm{t}, J=7.0 \mathrm{~Hz}, 2 \mathrm{H}, \mathrm{CH}_{2}\right), 1.95-1.83\left(\mathrm{~m}, 2 \mathrm{H}, \mathrm{CH}_{2}\right)$, 1.78-1.67 (m, 2H, CH $)^{13}{ }^{13} \mathrm{C} \mathrm{NMR} \mathrm{(101} \mathrm{MHz,} \mathrm{Chloroform-d)} \mathrm{\delta} \mathrm{168.} \mathrm{6,} \mathrm{166.6,} \mathrm{135.0,} \mathrm{134.3,} \mathrm{132.5,} \mathrm{132.1,}$ 131.6, 131.6, 126.3, 123.5, 115.7, 108.6, 40.7, 37.3, 27.8, 25.4, 20.1. IR $2937(\mathrm{w}), 2164(\mathrm{w}), 1768(\mathrm{~m})$, 1710 (s), 1647 (s), 1619 (s), $1441(\mathrm{~m}), 1398(\mathrm{~m}), 1329(\mathrm{~m}), 1181(\mathrm{w}), 1031(\mathrm{w}), 914(\mathrm{~m}), 835$ (w), 727 (s), 687 (w). HRMS (ESI/QTOF) m/z: [M + H] ${ }^{+}$Calcd for $\mathrm{C}_{21} \mathrm{H}_{17} \mathrm{INO}_{4}{ }^{+}$474.0197; Found 474.0203.

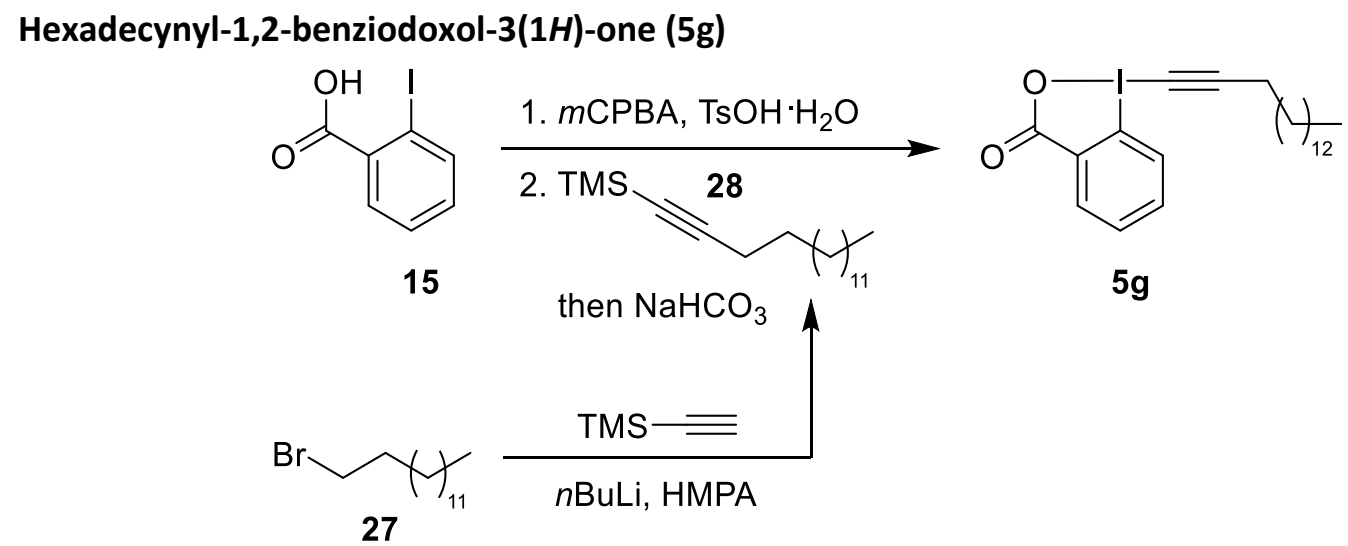

To a mixture of trimethylsilylacetylene $(8.33 \mathrm{~g}, 85.0 \mathrm{mmol}, 1.20 \mathrm{eq}$.) and dry THF ( $46 \mathrm{~mL})$ was added at $-78{ }^{\circ} \mathrm{C}$ under nitrogen $2.5 \mathrm{M} n \mathrm{BBLi}$ in hexanes $(33.9 \mathrm{~mL}, 85.0 \mathrm{mmol}, 1.20$ eq.) over a 10 minute time period. The resulting light yellow solution was stirred at $-78{ }^{\circ} \mathrm{C}$ for 60 minutes, after which a mixture consisting of 1-bromotetradecane 27 (19.6 g, $70.7 \mathrm{mmol}, 1.00 \mathrm{eq}$.), hexamethylphosphoramide (HMPA, $14.2 \mathrm{~mL}, 78.0 \mathrm{mmol}, 1.10$ eq.) and dry THF ( $23 \mathrm{~mL}$ ) was slowly added via cannula over a 20 minute time period. The reaction mixture was stirred for 60 minutes at $-78^{\circ} \mathrm{C}$, followed by 24 hours of stirring at room temperature. The reaction was quenched at $0{ }^{\circ} \mathrm{C}$ with saturated aq. $\mathrm{NH}_{4} \mathrm{Cl}(50 \mathrm{~mL})$ and diluted with water $(10 \mathrm{~mL})$ and EtOAc $(50 \mathrm{~mL})$. The two layers were separated and the aq. layer was extracted with additional portions of EtOAc $(3 \times 50 \mathrm{~mL})$. The combined organic layers were washed with water $(2 \times 100 \mathrm{~mL})$, brine $(100 \mathrm{~mL})$, dried over $\mathrm{MgSO}_{4}$, filtered and concentrated in vacuo. The light brown crude liquid was finally pushed through a small plug of silica gel with pentane as eluent to afford pure hexadec-1-yn-1-yltrimethylsilane $(\mathbf{2 8}, 19.3 \mathrm{~g}, 65.5 \mathrm{mmol}, 92.7 \%$ yield) as a colorless liquid. $\mathbf{R}_{f}$ (pentane) $=0.78 .{ }^{1} \mathrm{H}$ NMR $\left(\mathrm{CDCl}_{3}, 400 \mathrm{MHz}\right): \delta 2.19\left(\mathrm{t}, 2 \mathrm{H}, \mathrm{J}=7.1 \mathrm{~Hz}, \mathrm{CCCH}_{2}\right), 1.54-1.44\left(\mathrm{~m}, 2 \mathrm{H}, \mathrm{CH}_{2}\right)$,

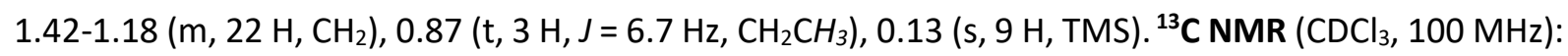
${ }^{17} \delta 107.7,84.3,32.2,29.9,29.8,29.7,29.6,29.3,29.0,28.9,22.9,20.0,14.3,0.3$. IR $\vee 2924(\mathrm{~m}), 2854$

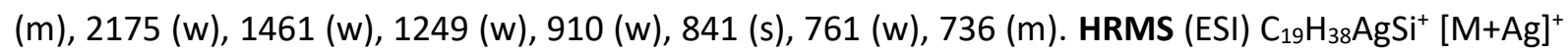
calc. $=401.1794 ;[\mathrm{M}+\mathrm{Ag}]^{+}$obs. $=401.1798$.

Following a slightly modified procedure, ${ }^{8}$ 2-iodobenzoic acid 15 (8.00 g, $32.2 \mathrm{mmol}, 1.00$ eq.), paratoluenesulfonic acid monohydrate $\left(\mathrm{TsOH} \cdot \mathrm{H}_{2} \mathrm{O}, 6.13 \mathrm{~g}, 32.2 \mathrm{mmol}, 1.00 \mathrm{eq}.\right)$ and metachloroperoxybenzoic acid (mCPBA-70\%, $8.74 \mathrm{~g}, 35.5 \mathrm{mmol}, 1.10$ eq.) were dissolved in dichloromethane $(60 \mathrm{~mL})$ and 2,2,2-trifluoroethanol $(60 \mathrm{~mL})$. The mixture was stirred at room temperature under nitrogen for 1 hour, after which hexadec-1-yn-1-yltrimethylsilane (28, $13.3 \mathrm{~g}, 45.1$

\footnotetext{
${ }^{17}$ Some signals were not resolved at $100 \mathrm{MHz}$.
} 
mmol, 1.40 eq.) was added in one portion. The reaction mixture was stirred for 14 hours at room temperature, filtered and concentrated in vacuo. The resulting oil was dissolved in dichloromethane $(400 \mathrm{~mL})$ and under vigorous stirring, saturated aq. $\mathrm{NaHCO}_{3}(400 \mathrm{~mL})$ was added. The mixture was stirred for 60 minutes, the two layers were separated and the aqueous layer was extracted with additional portions of dichloromethane $(3 \times 100 \mathrm{~mL})$. The combined organic layers were dried over $\mathrm{MgSO}_{4}$, filtered and concentrated in vacuo. The crude product was purified by flash column chromatography (ethyl acetate) to afford $\mathbf{5 g}(6.02 \mathrm{~g}, 12.9 \mathrm{mmol}, 40 \%)$ as a white solid. $\mathbf{R}_{\boldsymbol{f}}($ EtOAc) $=$ 0.36. Mp 102.6-105.3 ${ }^{\circ} \mathrm{C} .{ }^{1} \mathrm{H} ~ N M R ~\left(\mathrm{CDCl}_{3}, 400 \mathrm{MHz}\right): \delta 8.44-8.37(\mathrm{~m}, 1 \mathrm{H}, \mathrm{ArH}), 8.21-8.14(\mathrm{~m}, 1 \mathrm{H}, \mathrm{ArH})$, 7.80-7.70 (m, $2 \mathrm{H}, \mathrm{ArH}), 2.59\left(\mathrm{t}, 2 \mathrm{H}, J=7.1 \mathrm{~Hz}, \mathrm{CCCH}_{2}\right), 1.65\left(\mathrm{p}, 2 \mathrm{H}, J=7.1 \mathrm{~Hz}, \mathrm{CCCH}_{2} \mathrm{CH}_{2}\right), 1.52-1.40$ (m, $2 \mathrm{H}), 1.39-1.19\left(\mathrm{~m}, 20 \mathrm{H}, \mathrm{CH}_{2}\right), 0.86\left(\mathrm{t}, 3 \mathrm{H}, J=6.7 \mathrm{~Hz}, \mathrm{CH}_{2} \mathrm{CH}_{3}\right) .{ }^{13} \mathrm{C}$ NMR $\left(\mathrm{CDCl}_{3}, 100 \mathrm{MHz}\right):{ }^{5} \delta$ 166.6, 134.7, 132.5, 131.7, 131.6, 126.2, 115.7, 109.9, 39.5, 32.1, 29.8, 29.7, 29.6, 29.5, 29.2, 29.1, 28.3, 22.8, 20.6, 14.3. IR $\vee 2924(\mathrm{~s}), 2853(\mathrm{~m}), 2166(\mathrm{w}), 1649(\mathrm{~m}), 1623(\mathrm{~m}), 1439(\mathrm{w}), 908(\mathrm{~m}), 736(\mathrm{~s})$. HRMS (ESI) $\mathrm{C}_{23} \mathrm{H}_{34} \mathrm{IO}_{2}^{+}[\mathrm{M}+\mathrm{H}]^{+}$calc. $=469.1598 ;[\mathrm{M}+\mathrm{H}]^{+}$obs. $=469.1614$.

(Oct-6-en-1-ynyl)-1,2-benziodoxol-3(1H)-one (5h)

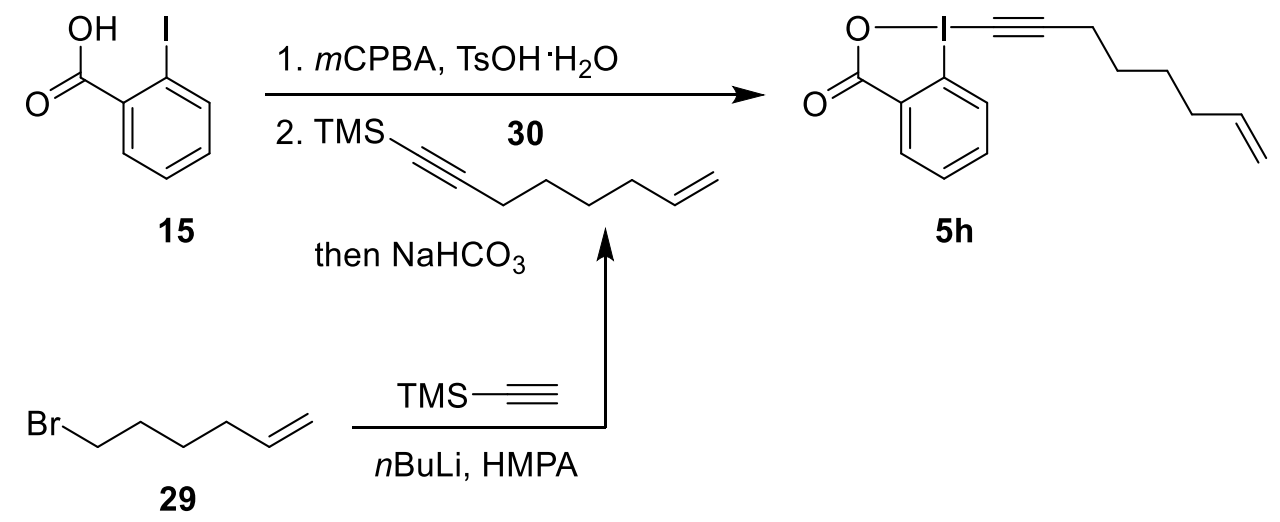

To a mixture of trimethylsilylacetylene $(7.23 \mathrm{~g}, 73.6 \mathrm{mmol}, 1.20 \mathrm{eq}$.) and dry THF $(40 \mathrm{~mL})$ was added at $-78{ }^{\circ} \mathrm{C}$ under nitrogen $2.5 \mathrm{M} \mathrm{nBuLi}$ in hexanes $(31.9 \mathrm{~mL}, 80.0 \mathrm{mmol}, 1.30$ eq.) over a 10 minute time period. The resulting light yellow solution was stirred at $-78{ }^{\circ} \mathrm{C}$ for 60 minutes, after which a mixture consisting of 6-bromohexene 29 (10.0 g, $61.3 \mathrm{mmol}, 1.00$ eq.), hexamethylphosphoramide (HMPA, $12.0 \mathrm{~mL}, 67.5 \mathrm{mmol}, 1.10$ eq.) and dry THF (20 mL) was slowly added via cannula over a 20 minute time period. The reaction mixture was stirred for 60 minutes at $-78^{\circ} \mathrm{C}$, followed by 24 hours of stirring at room temperature. The reaction was quenched at $0{ }^{\circ} \mathrm{C}$ with saturated aq. $\mathrm{NH}_{4} \mathrm{Cl}(50 \mathrm{~mL})$ and diluted with water $(5 \mathrm{~mL})$ and EtOAc $(50 \mathrm{~mL})$. The two layers were separated and the aq. layer was extracted with additional portions of EtOAc $(3 \times 50 \mathrm{~mL})$. The combined organic layers were washed with water $(2 \times 100 \mathrm{~mL})$, brine $(100 \mathrm{~mL})$, dried over $\mathrm{MgSO}_{4}$, filtered and concentrated in vacuo. The light brown crude liquid was finally pushed through a small plug of silica gel with pentane as eluent to afford pure trimethyl(oct-7-en-1-yn-1-yl)silane (30, $10.6 \mathrm{~g}, 58.8 \mathrm{mmol}, 95.9 \%$ yield) as a colorless liquid. ${ }^{1} \mathbf{H}$ NMR $\left(\mathrm{CDCl}_{3}, 400 \mathrm{MHz}\right.$ ): $\delta 5.79$ (ddt, $\left.1 \mathrm{H}, J=16.9,10.2,6.7 \mathrm{~Hz}, \mathrm{CH}_{2} \mathrm{CHCH}_{2}\right), 5.04-4.91\left(\mathrm{~m}, 2 \mathrm{H}, \mathrm{CH}_{2} \mathrm{CHCH}_{2}\right.$ ), $2.22\left(\mathrm{t}, 2 \mathrm{H}, \mathrm{J}=6.9 \mathrm{~Hz}, \mathrm{CH}_{2}\right), 2.11-2.01\left(\mathrm{~m}, 2 \mathrm{H}, \mathrm{CH}_{2}\right), 1.58-1.43\left(\mathrm{~m}, 4 \mathrm{H}, \mathrm{CH}_{2}\right), 0.14(\mathrm{~s}, 9 \mathrm{H}, \mathrm{TMS}) .{ }^{13} \mathrm{C} \mathrm{NMR}$ $\left(\mathrm{CDCl}_{3}, 100 \mathrm{MHz}\right): \delta 138.8,114.7,107.6,84.5,33.3,28.2,28.1,19.9,0.3$. The values of the NMR spectra are in accordance with reported literature data. ${ }^{18}$

\footnotetext{
${ }^{18}$ H. Urabe, F. Sato, J. Am. Chem. Soc. 1999, 121, 1245.
} 
Following a slightly modified procedure, ${ }^{8}$ 2-iodobenzoic acid 15 (9.82 g, $39.6 \mathrm{mmol}, 1.00$ eq.), paratoluenesulfonic acid monohydrate $\left(\mathrm{TsOH} \cdot \mathrm{H}_{2} \mathrm{O}, 7.53 \mathrm{~g}, 39.6 \mathrm{mmol}, 1.00 \mathrm{eq}.\right)$ and metachloroperoxybenzoic acid (mCPBA-70\%, $10.7 \mathrm{~g}, 43.6 \mathrm{mmol}, 1.10$ eq.) were dissolved in dichloromethane $(73 \mathrm{~mL})$ and 2,2,2-trifluoroethanol $(73 \mathrm{~mL})$. The mixture was stirred at room temperature under nitrogen for 1 hour, after which trimethyl(oct-7-en-1-yn-1-yl)silane (30, 10.0 g, 55.4 mmol, 1.40 eq.) was added in one portion. The reaction mixture was stirred for 14 hours at room temperature, filtered and concentrated in vacuo. The resulting oil was dissolved in dichloromethane $(700 \mathrm{~mL})$ and under vigorous stirring, saturated aq. $\mathrm{NaHCO}_{3}(700 \mathrm{~mL})$ was added. The mixture was stirred for 1 hour, the two layers were separated and the aqueous layer was extracted with additional portions of dichloromethane $(3 \times 200 \mathrm{~mL})$. The combined organic layers were dried over $\mathrm{MgSO}_{4}$, filtered and concentrated in vacuo. The crude product was purified by flash column chromatography (ethyl acetate) to afford $5 \mathrm{~h}(2.60 \mathrm{~g}, 7.34 \mathrm{mmol}, 19 \%)$ as a white solid. In addition, starting trimethyl(oct7-en-1-yn-1-yl)silane (77, $3.20 \mathrm{~g}, 17.7 \mathrm{mmol})$ was recovered and re-submitted to the above described conditions to afford additional $11 \mathrm{~m}(1.18 \mathrm{~g}, 3.33 \mathrm{mmol}, 28 \%)$ as a white solid, giving an overall yield of $27 \%$ brsm. $\mathbf{R}_{f}($ EtOAC $)=0.34 . \mathrm{Mp} 48-58{ }^{\circ} \mathrm{C} .{ }^{1} \mathbf{H} \mathbf{~ N M R}\left(\mathrm{CDCl}_{3}, 400 \mathrm{MHz}\right): \delta 8.43-8.36(\mathrm{~m}, 1 \mathrm{H}, \mathrm{ArH}), 8.21-$ $8.13(\mathrm{~m}, 1 \mathrm{H}, \mathrm{ArH}), 7.80-7.69(\mathrm{~m}, 2 \mathrm{H}, \mathrm{ArH}), 5.81$ (ddt, $1 \mathrm{H}, \mathrm{J}=17.0,10.2,6.7 \mathrm{~Hz}, \mathrm{CH}_{2} \mathrm{CHCH}_{2}$ ), 5.10-4.95 $\left(\mathrm{m}, 2 \mathrm{H}, \mathrm{CH}_{2} \mathrm{CHCH}_{2}\right), 2.61(\mathrm{t}, 2 \mathrm{H}, \mathrm{J}=7.0 \mathrm{~Hz}), 2.17-2.07(\mathrm{~m}, 2 \mathrm{H}), 1.73-1.51(\mathrm{~m}, 4 \mathrm{H}) .{ }^{13} \mathrm{C} \mathrm{NMR}\left(\mathrm{CDCl}_{3}, 100\right.$ $\mathrm{MHz}): \delta 166.7,138.1,134.8,132.5,131.6,131.6,126.2,115.7,115.2,109.5,39.7,33.2,28.1,27.7,20.4$. IR 3294 (w), 2912 (w), 2869 (w), 1731 (w), $1650(w), 1625$ (w), $1447(\mathrm{~m}), 1250(\mathrm{w}), 1101(\mathrm{~s}), 1018(\mathrm{~m})$, 747 (s). HRMS (ESI) $\mathrm{C}_{15} \mathrm{H}_{16} \mathrm{IO}_{2}{ }^{+}[\mathrm{M}+\mathrm{H}]^{+}$calc. $=355.0189 ;[\mathrm{M}+\mathrm{H}]^{+}$obs. $=355.0182$.

\section{3,3-Dimethylbutynyl-1,2-benziodoxol-3(1H)-one (5m)}<smiles>O=C(O)c1ccccc1I</smiles>

15

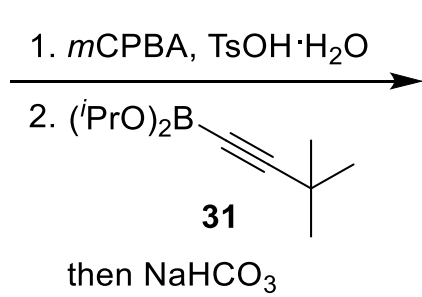

then $\mathrm{NaHCO}_{3}$

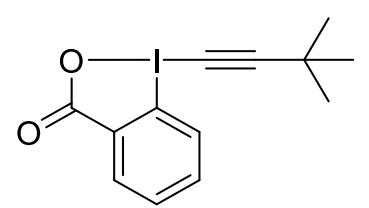

$5 \mathrm{~m}$

Following a slightly modified procedure, ${ }^{8}$ 2-iodobenzoic acid 15 (1.64 g, $6.59 \mathrm{mmol}, 1.00$ eq.), paratoluenesulfonic acid monohydrate $\left(\mathrm{TsOH} \cdot \mathrm{H}_{2} \mathrm{O}, 1.25 \mathrm{~g}, 6.59 \mathrm{mmol}, 1.00 \mathrm{eq}.\right)$ and metachloroperoxybenzoic acid (mCPBA-70\%, $1.79 \mathrm{~g}, 7.25 \mathrm{mmol}, 1.10$ eq.) were dissolved in dichloromethane $(12 \mathrm{~mL})$ and 2,2,2-trifluoroethanol $(12 \mathrm{~mL})$. The mixture was stirred at room temperature under nitrogen for 1 hour, after which diisopropyl (3,3-dimethylbut-1-yn-1-yl)boronate $(31,1.94 \mathrm{~g}, 9.23 \mathrm{mmol}, 1.40 \mathrm{eq}$.) was added in one portion. The reaction mixture was stirred for 1 hour at room temperature, filtered and concentrated in vacuo. The resulting oil was dissolved in dichloromethane $(120 \mathrm{~mL})$ and under vigorous stirring, saturated aq. $\mathrm{NaHCO}_{3}(120 \mathrm{~mL})$ was added. The mixture was stirred for 60 minutes, the two layers were separated and the aqueous layer was extracted with additional portions of dichloromethane $(3 \times 50 \mathrm{~mL})$. The combined organic layers were dried over $\mathrm{MgSO}_{4}$, filtered and concentrated in vacuo. The crude product was purified by flash column chromatography (ethyl acetate) to afford $5 \mathrm{~m}(2.06 \mathrm{~g}, 6.28 \mathrm{mmol}, 95 \%)$ as a white solid. $\mathbf{R}_{f}($ EtOAc) $=$ 0.36. $\mathrm{Mp} \mathrm{189-192}{ }^{\circ} \mathrm{C} .{ }^{1} \mathrm{H}$ NMR $\left(\mathrm{CDCl}_{3}, 400 \mathrm{MHz}\right): \delta 8.39-8.33(\mathrm{~m}, 1 \mathrm{H}, \mathrm{ArH}), 8.13-8.07(\mathrm{~m}, 1 \mathrm{H}, \operatorname{ArH})$, 7.78-7.66 (m, $2 \mathrm{H}, \mathrm{ArH}), 1.34(\mathrm{~s}, 9 \mathrm{H}, t \mathrm{Bu}) .{ }^{13} \mathrm{C}$ NMR $\left(\mathrm{CDCl}_{3}, 100 \mathrm{MHz}\right): \delta$ 166.7, 134.7, 132.4, 131.6, 131.5, 126.0, 117.5, 115.7, 38.2, 30.6, 29.7. IR v 3463 (w), $2971(w), 2171(w), 1646$ (s), 1622 (s), 1440 $(\mathrm{w}), 1332(\mathrm{~m}), 1248(\mathrm{~m}), 913(\mathrm{w}), 832(\mathrm{~m}), 745(\mathrm{~s})$. HRMS (ESI) $\mathrm{C}_{13} \mathrm{H}_{14} \mathrm{IO}_{2}{ }^{+}[\mathrm{M}+\mathrm{H}]^{+}$calc. = 329.0033; $[\mathrm{M}+\mathrm{H}]^{+}$obs. $=329.0023$. 
1-(3-Cyclopropylprop-1-yn-1-yl)-1,2-benziodoxol-3(1H)-one (5q)<smiles>O=C(O)c1ccccc1I</smiles>

15
1. $m \mathrm{CPBA}, \mathrm{TsOH} \cdot \mathrm{H}_{2} \mathrm{O}$

2. TMS

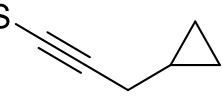

then $\mathrm{NaHCO}_{3}$
33<smiles>O=C1OI(C#CCC2CC2)c2ccccc21</smiles>

$5 q$

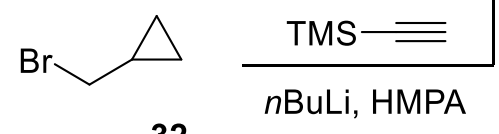

32

To a mixture of trimethylsilylacetylene $(2.36 \mathrm{~g}, 24.0 \mathrm{mmol}, 1.20 \mathrm{eq}$.) and dry THF ( $20 \mathrm{~mL})$ was added at $-78{ }^{\circ} \mathrm{C}$ under nitrogen $2.5 \mathrm{M} n \mathrm{BuLi}$ in hexanes $(10.4 \mathrm{~mL}, 26.0 \mathrm{mmol}, 1.30$ eq.) over a 10 minute time period. The resulting light yellow solution was stirred at $-78{ }^{\circ} \mathrm{C}$ for 60 minutes, after which a mixture consisting of (bromomethyl)cyclopropane $32(2.70 \mathrm{~g}, 20.0 \mathrm{mmol}, 1.00 \mathrm{eq}$.$) , hexamethylphosphoramide$ (HMPA, $3.83 \mathrm{~mL}, 22.0 \mathrm{mmol}, 1.10$ eq.) and dry THF ( $2 \mathrm{~mL}$ ) was slowly added via cannula over a 20 minute time period. The reaction mixture was stirred for 60 minutes at $-78^{\circ} \mathrm{C}$, followed by 24 hours of stirring at room temperature. The reaction was quenched at $0{ }^{\circ} \mathrm{C}$ with saturated aq. $\mathrm{NH}_{4} \mathrm{Cl}(20 \mathrm{~mL})$ and diluted with water $(5 \mathrm{~mL})$ and $\mathrm{Et}_{2} \mathrm{O}(20 \mathrm{~mL})$. The two layers were separated and the aq. layer was extracted with additional portions of $\mathrm{Et}_{2} \mathrm{O}(3 \times 20 \mathrm{~mL})$. The combined organic layers were washed with water $(2 \times 20 \mathrm{~mL})$, brine $(20 \mathrm{~mL})$, dried over $\mathrm{MgSO}_{4}$, filtered and concentrated in vacuo. The light brown crude liquid was finally pushed through a small plug of silica gel with pentane as eluent to afford the (3-cyclopropylprop-1-yn-1-yl)trimethylsilane 33 (2.72 g, 17.9 mmol, 89\%).

Following a slightly modified procedure, ${ }^{8}$ 2-iodobenzoic acid 15 (2.33 g, $9.38 \mathrm{mmol}, 1.00$ eq.), paratoluenesulfonic acid monohydrate $\left(\mathrm{TsOH} \cdot \mathrm{H}_{2} \mathrm{O}, 1.78 \mathrm{~g}, 9.38 \mathrm{mmol}, 1.00 \mathrm{eq}.\right)$ and metachloroperoxybenzoic acid (mCPBA-70\%, $2.31 \mathrm{~g}, 10.3 \mathrm{mmol}, 1.10$ eq.) were dissolved in dichloromethane $(16 \mathrm{~mL})$ and 2,2,2-trifluoroethanol $(16 \mathrm{~mL})$. The mixture was stirred at room temperature under nitrogen for 1 hour, after which (3-cyclopropylprop-1-yn-1-yl)trimethylsilane (33, $2.00 \mathrm{~g}, 13.1 \mathrm{mmol}, 1.40 \mathrm{eq}$.) was added in one portion. The reaction mixture was stirred for 14 hours at room temperature, filtered and concentrated in vacuo. The resulting oil was dissolved in dichloromethane $(30 \mathrm{~mL})$ and under vigorous stirring, saturated aq. $\mathrm{NaHCO}_{3}(30 \mathrm{~mL})$ was added. The mixture was stirred for 1 hour, the two layers were separated and the aqueous layer was extracted with additional portions of dichloromethane $(3 \times 20 \mathrm{~mL})$. The combined organic layers were dried over $\mathrm{MgSO}_{4}$, filtered and concentrated in vacuo. The crude product was purified by flash column chromatography (DCM/methanol) to afford $\mathbf{5 q}(1.08 \mathrm{~g}, 3.31 \mathrm{mmol}, 35 \%)$ as a white solid. $\mathbf{R f :} 0.61$ (DCM:MeOH 9:1). Mp: $132-137{ }^{\circ} \mathrm{C} .{ }^{1} \mathrm{H}$ NMR (400 MHz, Methylene Chloride- $\left.d_{2}\right) \delta 8.34-8.28(\mathrm{~m}, 1 \mathrm{H}$, $\operatorname{ArH}), 8.27-8.22(\mathrm{~m}, 1 \mathrm{H}, \mathrm{ArH}), 7.77(\mathrm{~m}, 2 \mathrm{H}, \mathrm{ArH}), 2.61\left(\mathrm{~d}, J=6.3 \mathrm{~Hz}, 2 \mathrm{H}, \mathrm{CH}_{2}\right), 1.08(\mathrm{ttt}, J=8.0,6.3,4.8$ $\mathrm{Hz}, 1 \mathrm{H}, \mathrm{CH}), 0.66-0.53\left(\mathrm{~m}, 2 \mathrm{H}, \mathrm{CH}_{2}\right), 0.35-0.27\left(\mathrm{~m}, 2 \mathrm{H}, \mathrm{CH}_{2}\right) .{ }^{13} \mathrm{C} \mathrm{NMR}(101 \mathrm{MHz}$, Methylene Chloride$\left.d_{2}\right) \delta 166.7,135.1,132.4,132.2,131.9,126.9,116.3,108.5,40.3,25.4,9.9,4.7$. IR $v 3425(\mathrm{~m}), 3073$ $(\mathrm{m}), 3005(\mathrm{~m}), 2921(\mathrm{~m}), 2167(\mathrm{~m}), 1616(\mathrm{~s}), 1560(\mathrm{~s}), 1514(\mathrm{~m}), 1443(\mathrm{~s}), 1328(\mathrm{~s}), 1296(\mathrm{~s}), 1243(\mathrm{~m})$, $1011(\mathrm{~s}), 950(\mathrm{~m}), 833(\mathrm{~s}), 751(\mathrm{~s}), 681(\mathrm{~m}), 643(\mathrm{~m})$. HRMS (ESI/QTOF) m/z: [M + H] Calcd for $\mathrm{C}_{13} \mathrm{H}_{12} \mathrm{IO}_{2}{ }^{+}$ 326.9877; Found 326.9879. 


\section{Preparation of $(E)-1-(P e n t-1-e n-1-y l)-1 \lambda^{3}$-benzo[d][1,2]iodaoxol-3(1H)-one 11:}

1-Acetoxy-1,2-benziodoxol-3-(1H)-one (81)<smiles>O=C1OI(O)c2ccccc21</smiles><smiles>CCOCCOCCOC</smiles><smiles>CC(=O)OI1C(=O)OC1=O</smiles>

Following a reported procedure, ${ }^{19}$ 2-iodosylbenzoic acid $16 \mathrm{a}(20.8 \mathrm{~g}, 79.0 \mathrm{mmol}, 1.00$ equiv.) was suspended in acetic anhydride $\left(75.0 \mathrm{~mL}, 788 \mathrm{mmol}, 10.0\right.$ equiv.) and heated to reflux $\left(140{ }^{\circ} \mathrm{C}\right)$ until complete dissolution (about $15 \mathrm{~min}$ ). The resulting clear solution was allowed to cool to room temperature and then cooled to $5^{\circ} \mathrm{C}$ overnight. The white crystals were filtered, washed with pentane $(3 \times 30 \mathrm{~mL})$ and dried under reduced pressure to afford 1-acetoxy-1,2-benziodoxol-3-(1H)-one 34 as a white solid (22.3 g, $73.0 \mathrm{mmol}, 92 \%) .{ }^{1} \mathrm{H}$ NMR (CDCl3, $\left.400 \mathrm{MHz}\right) \delta 8.24(\mathrm{dd}, J=7.6,1.6 \mathrm{~Hz}, 1 \mathrm{H}, \mathrm{ArH})$, $8.00(\mathrm{dd}, J=8.3,1.0 \mathrm{~Hz}, 1 \mathrm{H}, \mathrm{ArH}), 7.92(\mathrm{ddd}, J=8.4,7.2,1.6 \mathrm{~Hz}, 1 \mathrm{H}, \mathrm{ArH}), 7.71(\mathrm{td}, J=7.3,1.1 \mathrm{~Hz}, 1 \mathrm{H}$, $\mathrm{ArH}), 2.25$ (s, $\left.3 \mathrm{H}, \mathrm{COCH}_{3}\right) ;{ }^{13} \mathrm{C} \mathrm{NMR}(\mathrm{CDCl} 3,100 \mathrm{MHz}) \delta 176.5,168.2,136.2,133.3,131.4,129.4,129.1$, $118.4,20.4$. The values of the NMR spectra are in accordance with reported literature data. ${ }^{20}$

(E)-1-(Pent-1-en-1-yl)-1 $\lambda^{3}$-benzo[d][1,2]iodaoxol-3(1H)-one (11)
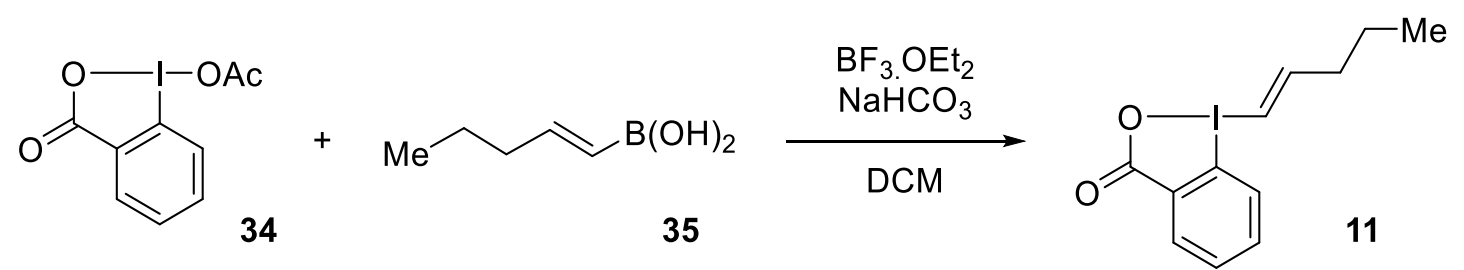

Following the reported procedure ${ }^{21}$, a solution of the corresponding trans-1-penten-1-yboronic acid 35 (1.30 mmol, 1.00 equiv.) in dry DCM (13 mL) was added BF3:OEt2 (0.198 mL, $1.56 \mathrm{mmol}, 1.20$ equiv.) dropwise at $0{ }^{\circ} \mathrm{C}$. After 15 minutes, 1 - acetoxy-1,2-benziodoxol-3-(1H)-one 34 (477 mg, 1.56 mmol, 1.20 equiv.) was added in one portion at $0{ }^{\circ} \mathrm{C}$. The reaction mixture was allowed to warm up to room temperature and stirred until the reaction was completed ( 4 to $24 \mathrm{~h}$, monitored by TLC using $\mathrm{MeOH} / \mathrm{DCM} 5: 95)$. The reaction was then quenched with a saturated solution of $\mathrm{NaHCO} 3(13 \mathrm{~mL})$ and stirred vigorously for $1 \mathrm{~h}$. The resulting suspension was filtered and the filtrate was extracted with DCM $(3 \times 20 \mathrm{~mL})$. The combined organic layers were washed with water $(3 \times 20 \mathrm{~mL})$, dried over MgSO4, filtered and the solvent was removed under reduced pressure. The resulting solid was dissolved in DCM (minimum amount until dissolution) and precipitated in Et2O (ca. $150 \mathrm{~mL}$ ). After precipitation at $4{ }^{\circ} \mathrm{C}$ for $2 \mathrm{~h}$, the solid was filtered and washed with Et2O to afford (E)-1-(pent-1-en1-yl)- $1 \lambda^{3}$ benzo[d][1,2]iodaoxol-3(1H)-one (11) as a white solid (115 mg, $0.364 \mathrm{mmol}, 28 \%$ ). M.p. (dec.) 154-160 ${ }^{\circ} \mathrm{C} ; \mathbf{R f}=0.15(\mathrm{MeOH} / \mathrm{DCM} 5: 95) ;{ }^{1} \mathbf{H}$ NMR $(400 \mathrm{MHz}, \mathrm{MeOD}) \delta 8.34-8.22(\mathrm{~m}, 1 \mathrm{H}, \mathrm{ArH}), 7.80-7.63(\mathrm{~m}$, $3 \mathrm{H}, \mathrm{ArH}$ ), 7.16 (dt, J=14.9, $7.0 \mathrm{~Hz}, 1 \mathrm{H}, \mathrm{ICHCHCH}$ ), 6.87 (dt, J=15.0, $1.4 \mathrm{~Hz}, 1 \mathrm{H}, \mathrm{ICHCHCH}$ ), 2.49 (qd, J $\left.=7.2,1.5 \mathrm{~Hz}, 2 \mathrm{H}, \mathrm{CHCH}_{2} \mathrm{CH}_{2}\right), 1.65\left(\mathrm{~h}, J=7.4 \mathrm{~Hz}, 2 \mathrm{H}, \mathrm{CH}_{2} \mathrm{CH}_{2} \mathrm{CH}_{3}\right), 1.05\left(\mathrm{t}, J=7.4 \mathrm{~Hz}, 3 \mathrm{H}, \mathrm{CH}_{2} \mathrm{CH}_{3}\right) ;{ }^{13} \mathrm{C}$ NMR (101 MHz, MeOD) $\delta$ 169.7, 160.4, 134.9, 133.1, 131.6, 128.7, 114.7, 100.1, 38.6, 22.2, 13.7; IR (vmax, cm-1) $2987(\mathrm{~s}), 2973(\mathrm{~s}), 2905$ (s), $1748(\mathrm{~m}), 1737(\mathrm{~m}), 1649(\mathrm{~m}), 1559(\mathrm{~m}), 1540(\mathrm{~m}), 1512(\mathrm{~m})$, $1395(\mathrm{~m}), 1255(\mathrm{~m}), 1079(\mathrm{~s}), 1054(\mathrm{~s}), 863(\mathrm{~m})$; HRMS (ESI) calcd for $\mathrm{C}_{12} \mathrm{H}_{14} \mathrm{IO}_{2}{ }^{+}[\mathrm{M}+\mathrm{H}]^{+} 317.0033$; found 317.0033 .

\footnotetext{
${ }^{19}$ P. Caramenti, S. Nicolai, J. Waser, Chem. Eur. J. 2017, 23, 14702-14706.

${ }^{20}$ P. Eisenberger, S. Gischig, A. Togni, Chem. Eur. J. 2006, 12, 2579.

${ }^{21}$ G. Pisella, A. Gagnebin, J. Waser, ChemRxiv. Preprint, 2019, https://doi.org/10.26434/chemrxiv.7892513.v1
} 


\section{Synthesis of O-VBX reagents}

\section{General Procedure for the Synthesis O-VBX.}<smiles>[R]C#CI1COC(=O)c2ccccc21</smiles>

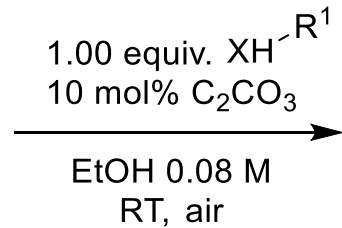<smiles>[R]C([R])=CI1COC(=O)c2ccccc21</smiles>

In a glass vial, the correspondent sulfonamide or phenol (1.00 equiv.) was dissolved in EtOH (0.08 M). $\mathrm{Cs}_{2} \mathrm{CO}_{3}(10 \mathrm{~mol} \%)$ was added and the mixture stirred vigorously for 5 minutes. Then the corresponding EBX 5 was added in one portion (1.00 equiv.) and the reaction was left stirring for 12 hours if not specifically specified otherwise. The reaction was stopped, the $\mathrm{EtOH}$ removed under reduced pressure and the crude purified via column chromatography using DCM:MeOH (20:1) as eluent to provide 1 .

\section{(Z)-(1-Prop-1-en-2-yl-2-oxy)-4-methylbenzene-1,2-benziodoxol-3-(1H)-one (1a)}

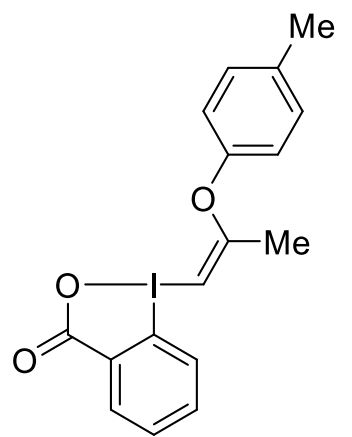

Starting from EBX 5a (286 mg, $1.00 \mathrm{mmol}),(Z)-(1-p r o p-1-e n-2-y l-2-o x y)-4-$ methylbenzene-1,2-benziodoxol-3-(1H)-one 1a $(226 \mathrm{mg}, 0.572 \mathrm{mmol}, 57 \%$ yield) was obtained, as a white amorphous solid. Rf: 0.48 (DCM:MeOH 9:1). ${ }^{1} \mathbf{H}$ NMR (400 MHz, Chloroform-d) $\delta 8.40-8.34(\mathrm{~m}, 1 \mathrm{H}, \mathrm{ArH}), 7.64-7.52(\mathrm{~m}, 3 \mathrm{H}$, $\operatorname{ArH}), 7.11-7.04(\mathrm{~m}, 2 \mathrm{H}, \operatorname{ArH}), 6.77(\mathrm{~d}, J=8.5 \mathrm{~Hz}, 2 \mathrm{H}, \operatorname{ArH}), 5.80(\mathrm{~d}, J=1.1 \mathrm{~Hz}$, $1 \mathrm{H}$, vinyl $H$ ), $2.27\left(\mathrm{~s}, 3 \mathrm{H}, \mathrm{CH}_{3}\right), 2.18\left(\mathrm{~d}, \mathrm{~J}=0.9 \mathrm{~Hz}, 3 \mathrm{H}, \mathrm{CH}_{3}\right) .{ }^{13} \mathrm{C}$ NMR $(101 \mathrm{MHz}$, Chloroform-d) $\delta 167.1,166.8,151.2,135.4,133.7,133.1,132.7,130.4,130.3$, 125.3, 120.0, 113.7, 77.3 (1 Carbon signal overlaps with Chloroform-d), 20.7, 19.2. IR v 1603 (s), 1559 (w), 1505 (s), 1437 (w), 1357 (w), 1275 (w), $1211(\mathrm{~m})$, 837 (w). HRMS (ESI) calcd for $\mathrm{C}_{17} \mathrm{H}_{16} \mathrm{IO}_{3}{ }^{+}[\mathrm{M}+\mathrm{H}]^{+}$395.0139; found 395.0148. The olefin geometry was assigned by analogy to NMR data of compound 11. The values of the NMR spectra are in accordance with reported literature data. ${ }^{2}$

\section{(Z)-(1-Pent-1-en-2-yl-2-oxy)-4-methylbenzene-1,2-benziodoxol-3-(1H)-one (1b)}

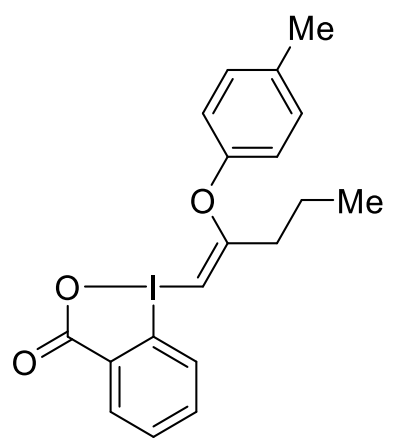

Starting from EBX 5b (314 mg, $1.00 \mathrm{mmol}),(Z)$-(1-pent-1-en-2-yl-2-oxy)-4methylbenzene-1,2-benziodoxol-3-(1H)-one 1 b (172 mg, $0.407 \mathrm{mmol}, 41 \%$ yield) was obtained as a white amorphous solid. Rf: 0.50 (DCM:MeOH 9:1). ${ }^{1} \mathrm{H}$ NMR (400 MHz, Chloroform-d) $\delta 8.49-8.31(\mathrm{~m}, 1 \mathrm{H}, \mathrm{ArH}), 7.60(\mathrm{~m}, 3 \mathrm{H}$, $\operatorname{ArH}), 7.09(\mathrm{~d}, J=7.9 \mathrm{~Hz}, 2 \mathrm{H}, \operatorname{ArH}), 6.78(\mathrm{~d}, J=7.9 \mathrm{~Hz}, 2 \mathrm{H}, \operatorname{ArH}), 5.85(\mathrm{~s}, 1 \mathrm{H}$, vinylH), $2.48\left(\mathrm{t}, J=7.6 \mathrm{~Hz}, 2 \mathrm{H}, \mathrm{CH}_{2}\right), 2.29\left(\mathrm{~s}, 3 \mathrm{H}, \mathrm{CH}_{3}\right), 1.60(\mathrm{q}, J=7.5 \mathrm{~Hz}, 2 \mathrm{H}$, $\left.\mathrm{CH}_{2}\right), 0.96\left(\mathrm{t}, J=7.4 \mathrm{~Hz}, 3 \mathrm{H}, \mathrm{CH}_{3}\right) .{ }^{13} \mathrm{C}$ NMR $(101 \mathrm{MHz}$, Chloroform-d) $\delta 170.4$, $166.5,151.5,134.9,133.8,133.1,132.9,130.6,130.5,125.1,119.1,113.9$, 80.1, 34.4, 20.7, 20.4, 13.5. IR $\vee 1601$ (w), 1505 (w), $1430(w), 1266(\mathrm{~m})$, $1205(\mathrm{~m}), 1143(\mathrm{w}), 740(\mathrm{~s}), 703(\mathrm{~m}), 660(\mathrm{~m})$. HRMS (ESI) calcd for $\mathrm{C}_{19} \mathrm{H}_{20} \mathrm{IO}_{3}{ }^{+}[\mathrm{M}+\mathrm{H}]^{+}$423.0452; found 423.0452. The olefin geometry was assigned by analogy to NMR data of compound 11 . The values of the NMR spectra are in accordance with reported literature data. ${ }^{2}$ 
(Z)-1-(2-(p-tolyloxy)oct-1-en-1-yl)-1,2-benziodoxol-3-(1H)-one (1c)

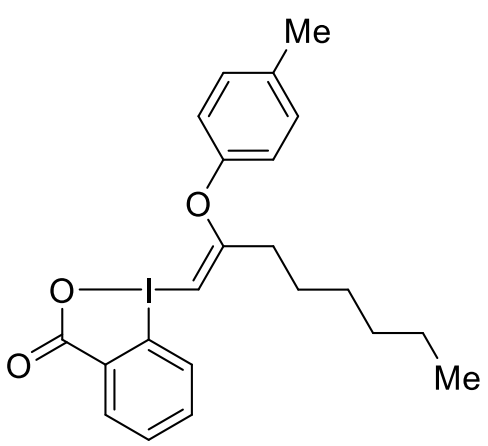

Starting from EBX 5c $(0.107 \mathrm{~g}, 0.300 \mathrm{mmol}),(Z)-1$-(2-(p-tolyloxy)oct1-en-1-yl)-1,3-benziodoxol-3(1H)-one 1c ( $80.0 \mathrm{mg}, 0.172 \mathrm{mmol}, 57 \%$ yield) was obtained, as a white amorphous solid. Rf: 0.50 (DCM:MeOH 9:1). ${ }^{1} \mathrm{H}$ NMR (400 MHz, Chloroform-d) $\delta 8.48-8.42$ $(\mathrm{m}, 1 \mathrm{H}, \mathrm{ArH}), 7.67-7.60(\mathrm{~m}, 3 \mathrm{H}, \mathrm{ArH}), 7.14-7.07(\mathrm{~m}, 2 \mathrm{H}, \mathrm{ArH}), 6.86$ - $6.79(\mathrm{~m}, 2 \mathrm{H}, \mathrm{ArH}), 5.90(\mathrm{~s}, 1 \mathrm{H}$, vinylH), $2.51(\mathrm{t}, J=7.6 \mathrm{~Hz}, 2 \mathrm{H}$, $\mathrm{CCH}_{2} \mathrm{CH}_{2} \mathrm{CH}_{2} \mathrm{CH}_{2} \mathrm{CH}_{2} \mathrm{CH}_{3}$ ), 2.31 (s, 3H, $\left.\mathrm{ArCH}_{3}\right), 1.58(\mathrm{p}, J=7.5 \mathrm{~Hz}, 2 \mathrm{H}$, $\left.\mathrm{CCH}_{2} \mathrm{CH}_{2} \mathrm{CH}_{2} \mathrm{CH}_{2} \mathrm{CH}_{2} \mathrm{CH}_{3}\right), \quad 1.39-1.19$ (m, $6 \mathrm{H}$, $\left.\mathrm{CCH}_{2} \mathrm{CH}_{2} \mathrm{CH}_{2} \mathrm{CH}_{2} \mathrm{CH}_{2} \mathrm{CH}_{3}\right), 0.88\left(\mathrm{t}, J=6.8 \mathrm{~Hz}, 3 \mathrm{H}, \mathrm{CH}_{3}\right) .{ }^{13} \mathrm{C}$ NMR (101 $\mathrm{MHz}$, Chloroform-d) $\delta 170.8,167.2,151.7,135.2,133.5,133.5,133.2,130.8,130.6,125.7,119.5,114.5$, 79.8, 32.6, 31.5, 28.8, 27.1, 22.6, 20.9, 14.1. IR $3056(\mathrm{~m}), 2929(\mathrm{~m}), 2858(\mathrm{~m}), 1605(\mathrm{~s}), 1505(\mathrm{~s}), 1340$ $(\mathrm{m}), 1272(\mathrm{~s}), 1206(\mathrm{~s}), 1117(\mathrm{~m}), 1008(\mathrm{~m}), 927(\mathrm{~m}), 832(\mathrm{~s}), 748(\mathrm{~s}), 693(\mathrm{~m})$. HRMS (ESI/QTOF) m/z: $[\mathrm{M}+\mathrm{H}]^{+}$Calcd for $\mathrm{C}_{22} \mathrm{H}_{26} \mathrm{IO}_{3}{ }^{+} 465.0921 ;$ Found 465.0921 . The olefin geometry was assigned by analogy to NMR data of compound 11 .

\section{(Z)-1-(7-hydroxy-2-(p-tolyloxy)hept-1-en-1-yl)-1,3-benziodoxol-3-(1H)-one (1d)}

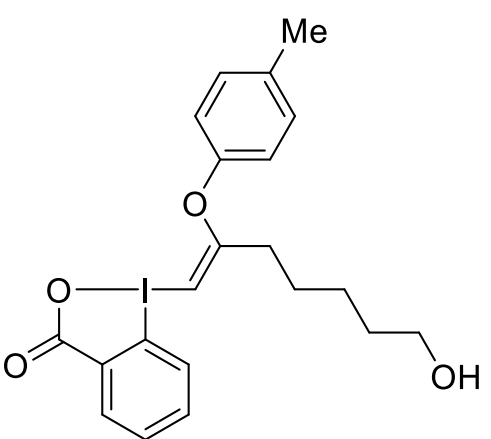

Starting from EBX 5d $(0.107 \mathrm{~g}, 0.300 \mathrm{mmol}),(Z)-1-(7-h y d r o x y-2-(p-$ tolyloxy)hept-1-en-1-yl)-1,3-benziodoxol-3-(1H)-one 1d (0.110 g, $0.237 \mathrm{mmol}, 79 \%$ yield) was obtained, as a white solid. Rf: 0.27 (DCM:MeOH 9:1). Mp: $151-172{ }^{\circ} \mathrm{C} .{ }^{1} \mathbf{H}$ NMR $\left(400 \mathrm{MHz}\right.$, Methanol- $\left.d_{4}\right)$ $\delta 8.29$ (dd, $J=7.5,1.9 \mathrm{~Hz}, 1 \mathrm{H}, \mathrm{ArH}), 7.89(\mathrm{dd}, J=8.0,1.2 \mathrm{~Hz}, 1 \mathrm{H}, \mathrm{ArH})$, $7.78(\mathrm{td}, J=8.1,7.6,1.9 \mathrm{~Hz}, 1 \mathrm{H}, \mathrm{ArH}), 7.75-7.69(\mathrm{~m}, 1 \mathrm{H}, \mathrm{ArH}), 7.18$ $(\mathrm{d}, J=8.2 \mathrm{~Hz}, 2 \mathrm{H}, \mathrm{ArH}), 6.97-6.90(\mathrm{~m}, 2 \mathrm{H}, \operatorname{ArH}), 6.28(\mathrm{~s}, 1 \mathrm{H}$, vinyl $\mathrm{H})$, 3.55 (t, J = 6.2 Hz, 2H, $\left.\mathrm{CH}_{2} \mathrm{CH}_{2} \mathrm{CH}_{2} \mathrm{CH}_{2} \mathrm{CH}_{2} \mathrm{OH}\right), 2.65$ (t, $J=7.6 \mathrm{~Hz}, 2 \mathrm{H}$, $\mathrm{CH}_{2} \mathrm{CH}_{2} \mathrm{CH}_{2} \mathrm{CH}_{2} \mathrm{CH}_{2} \mathrm{OH}$ ), 2.32 (s, $\left.3 \mathrm{H}, \mathrm{CH}_{3}\right), 1.66$ (p, J = $7.5 \mathrm{~Hz}, 2 \mathrm{H}$, $\mathrm{CH}_{2} \mathrm{CH}_{2} \mathrm{CH}_{2} \mathrm{CH}_{2} \mathrm{CH}_{2} \mathrm{OH}$ ), 1.49 (m, $4 \mathrm{H}, \mathrm{CH}_{2} \mathrm{CH}_{2} \mathrm{CH}_{2} \mathrm{CH}_{2} \mathrm{CH}_{2} \mathrm{OH}$ ). ${ }^{13} \mathrm{C}$ NMR (101 MHz, Methanol-d $d_{4}$ ) $\delta$ 172.3, 170.2, 153.0, 136.4, 135.2, 134.7, 133.4, 131.8, 131.5, 128.5, 120.8, 114.3, 79.7, 62.6, 33.1, 33.0, 27.9, 26.3, 20.7. IR 3334 (m), 2946 (m), 2833 (w), 2518 (w), $2224(w), 2042$ (w), 1667 (w), $1454(w), 1029$ (s), 740 (m). HRMS HRMS (ESI/QTOF) m/z: $[\mathrm{M}+\mathrm{H}]^{+}$Calcd for $\mathrm{C}_{21} \mathrm{H}_{24} \mathrm{IO}_{4}{ }^{+} 467.0714$; Found 467.0718. The olefin geometry was assigned by analogy to NMR data of compound $\mathbf{1}$.

\section{(Z)-1-(2-(p-tolyloxy)-8-(trimethylsilyl)oct-1-en-7-yn-1-yl)-1,3-benziodoxol-3-(1H)-one (1e)}

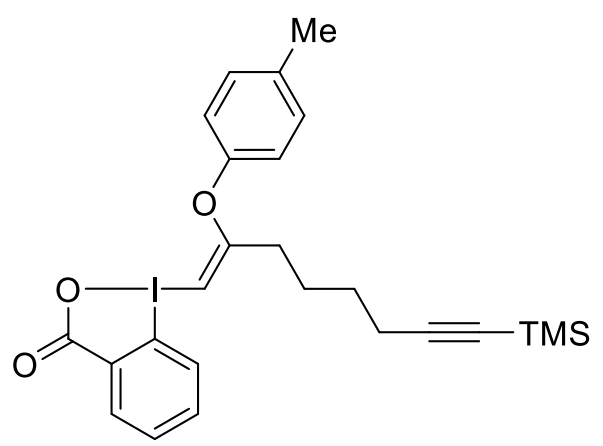
Starting from EBX 5e $(0.127 \mathrm{~g}, 0.300 \mathrm{mmol}),(Z)-1-(2-(p-$ tolyloxy)-8-(trimethylsilyl)oct-1-en-7-yn-1-yl)-1,3benziodoxol-3-(1H)-one $1 \mathrm{e}$ (78.0 $\mathrm{mg}, 0.146 \mathrm{mmol}, 49 \%$ yield) was obtained, as a white solid. Rf: 0.42 (DCM:MeOH 9:1). Mp: 116-119 ${ }^{\circ} \mathrm{C} .{ }^{1} \mathrm{H}$ NMR (400 MHz, Methylene Chloride- $\left.d_{2}\right) \delta 8.35$ (dq, $J=5.6,2.3,1.8 \mathrm{~Hz}, 1 \mathrm{H}, \operatorname{ArH}), 7.66(\mathrm{~d}, J=3.1 \mathrm{~Hz}, 3 \mathrm{H}, \operatorname{ArH})$, $7.14(\mathrm{~d}, J=8.1 \mathrm{~Hz}, 2 \mathrm{H}, \mathrm{ArH}), 6.83(\mathrm{~d}, J=8.1 \mathrm{~Hz}, 2 \mathrm{H}, \mathrm{ArH}), 5.89$ $\left(\mathrm{s}, 1 \mathrm{H}\right.$, vinylH), $2.54\left(\mathrm{t}, J=7.5 \mathrm{~Hz}, 2 \mathrm{H}, \mathrm{CH}_{2}\right), 2.31\left(\mathrm{~s}, 3 \mathrm{H}, \mathrm{ArCH}_{3}\right)$, $2.23\left(\mathrm{t}, J=6.9 \mathrm{~Hz}, 2 \mathrm{H}, \mathrm{CH}_{2}\right), 1.70\left(\mathrm{~d}, J=8.0 \mathrm{~Hz}, 2 \mathrm{H}, \mathrm{CH}_{2}\right), 1.54$ $\left(\mathrm{p}, J=7.1 \mathrm{~Hz}, 2 \mathrm{H}, \mathrm{CH}_{2}\right), 0.13\left(\mathrm{~d}, J=1.1 \mathrm{~Hz}, 9 \mathrm{H}, \mathrm{Si}\left(\mathrm{CH}_{3}\right)_{3}\right) .{ }^{13} \mathrm{C}$ NMR $\left(101 \mathrm{MHz}\right.$, Methylene Chloride- $\left.d_{2}\right) \delta$ $170.9,166.6,152.1,135.7,134.5,133.7,133.0,131.1,131.0,125.9,119.8,114.6,106.9,85.5,80.9$, 32.5, 28.3, 26.6, 21.0, 19.9, 0.4. IR $v 3001$ (w), 2817 (w), $2170(w), 1598(\mathrm{~s}), 1503(\mathrm{~m}), 1356(\mathrm{~m}), 1248$ 
(m), $1205(\mathrm{~m}), 1136(\mathrm{~m}), 984(\mathrm{~m}), 840(\mathrm{~s}), 746$ (s), $687(\mathrm{~m})$. HRMS (ESI/QTOF) m/z: [M + H] Calcd for $\mathrm{C}_{25} \mathrm{H}_{30} \mathrm{O}_{3} \mathrm{Si}^{+}$533.1003; Found 533.1008. The olefin geometry was assigned by analogy to NMR data of compound 11 .

(Z)-2-(6-(3-Oxo-1,2-benziodoxol-3-(1H)-yl)-5-( $p$-tolyloxy)hex-5-en-1-yl)isoindoline-1,3-dione (1f)

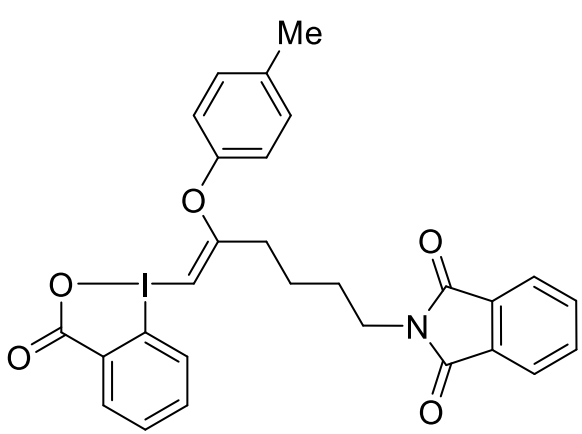

Starting from EBX $\mathbf{5 f}(300 \mathrm{mg}, 634 \mu \mathrm{mol}),(Z)-2-(6-(3-0 \times 0-1,2-$ benziodoxol-3-(1H)-yl)-5-(p-tolyloxy)hex-5-en-1yl)isoindoline-1,3-dione 1f (180 mg, $310 \mu \mathrm{mol}, 49 \%$ yield) was obtained, as an white solid. Rf: 0.68 (DCM:MeOH 9:1). Mp: 87$92{ }^{\circ} \mathrm{C} .{ }^{1} \mathrm{H}$ NMR $(400 \mathrm{MHz}$, Chloroform-d) $\delta 8.45-8.37(\mathrm{~m}, 1 \mathrm{H}$, $\operatorname{ArH}), 7.88-7.77(m, 2 \mathrm{H}, \mathrm{ArH}), 7.77-7.68(\mathrm{~m}, 2 \mathrm{H}, \mathrm{ArH}), 7.66-$ $7.53(\mathrm{~m}, 3 \mathrm{H}, \mathrm{ArH}), 7.04(\mathrm{~d}, J=8.2 \mathrm{~Hz}, 2 \mathrm{H}, \operatorname{ArH}), 6.75(\mathrm{~d}, J=8.5$ $\mathrm{Hz}, 2 \mathrm{H}, \mathrm{ArH}), 5.91\left(\mathrm{~s}, 1 \mathrm{H}\right.$, vinylH), $3.67\left(\mathrm{t}, J=6.9 \mathrm{~Hz}, 2 \mathrm{H}, \mathrm{CH}_{2}\right)$, $2.57\left(\mathrm{t}, J=7.5 \mathrm{~Hz}, 2 \mathrm{H}, \mathrm{CH}_{2}\right), 2.26\left(\mathrm{~s}, 3 \mathrm{H}, \mathrm{CH}_{3}\right), 1.72(\mathrm{tt}, J=13.0$, $7.1 \mathrm{~Hz}, 2 \mathrm{H}, \mathrm{CH}_{2}$ ), 1.59 (dq, J = 9.7, 7.3 Hz, 2H, CHz). ${ }^{13} \mathrm{C}$ NMR (101 MHz, Chloroform-d) $\delta 169.8,168.5$, $166.7,151.5,135.3,134.3,133.9,133.4,133.2,132.1,130.8,130.7,125.3,123.5,119.2,114.1,80.8$, 37.2, 32.0, 27.9, 24.3, 20.9. IR v $3067(\mathrm{w}), 2939(\mathrm{~m}), 1768(\mathrm{~m}), 1710(\mathrm{~s}), 1607(\mathrm{~s}), 1506(\mathrm{~m}), 1442(\mathrm{~m})$, $1397(\mathrm{~m}), 1347(\mathrm{~m}), 1208(\mathrm{~m}), 1178(\mathrm{~m}), 1119(\mathrm{w}), 996(\mathrm{w}), 917(\mathrm{~m}), 825(\mathrm{~m}), 728(\mathrm{~s}), 687(\mathrm{~m})$. HRMS (ESI/QTOF) $\mathrm{m} / \mathrm{z}:[\mathrm{M}+\mathrm{H}]^{+}$Calcd for $\mathrm{C}_{28} \mathrm{H}_{25} \mathrm{INO}_{5}{ }^{+}$582.0772; Found 582.0778. The olefin geometry was assigned by analogy to NMR data of compound 1 I.

\section{(Z)-1-(2-(2-iodophenoxy)prop-1-en-1-yl)-1,2-benziodoxol-3-(1H)-one (1g)}

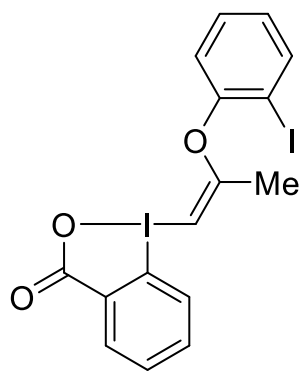

Starting from EBX $5 a(86.0 \mathrm{mg}, 0.300 \mathrm{mmol}),(Z)-1-(2-(2$-iodophenoxy)prop-1en-1-yl)-1,2-benziodoxol-3-(1H)-one $1 \mathrm{~g}$ (50.5 $\mathrm{mg}, 0.100 \mathrm{mmol}, 33 \%$ yield) was obtained, as an off-white sticky solid. Rf: 0.45 (DCM:MeOH 9:1). ${ }^{1} \mathbf{H}$ NMR (400 $\mathrm{MHz}$, Chloroform-d) $\delta 8.45-8.38(\mathrm{~m}, 1 \mathrm{H}, \mathrm{ArH}), 7.78(\mathrm{dd}, J=7.9,1.6 \mathrm{~Hz}, 1 \mathrm{H}, \mathrm{ArH})$, $7.75-7.67(\mathrm{~m}, 1 \mathrm{H}, \mathrm{ArH}), 7.64-7.55(\mathrm{~m}, 2 \mathrm{H}, \mathrm{ArH}), 7.33(\mathrm{td}, J=7.7,1.6 \mathrm{~Hz}, 1 \mathrm{H}$, $\operatorname{ArH}), 7.04-6.90(\mathrm{~m}, 2 \mathrm{H}, \operatorname{ArH}), 5.86(\mathrm{~d}, J=1.1 \mathrm{~Hz}, 1 \mathrm{H}, \operatorname{vinylH}), 2.16(\mathrm{~d}, J=0.9 \mathrm{~Hz}$, $\left.3 \mathrm{H}, \mathrm{CH}_{3}\right) .{ }^{13} \mathrm{C}$ NMR (101 MHz, Chloroform-d) $\delta 167.1,166.3,153.4,140.1,133.8$, 133.4, 133.0, 130.8, 130.1, 128.0, 126.1, 122.1, 114.1, 90.3, 77.7, 19.9. IR v 3060 (m), 2778 (m), 1604 (s), $1348(\mathrm{~m}), 1261(\mathrm{~m}), 1218(\mathrm{~m}), 1125(\mathrm{~m}), 1069(\mathrm{~m}), 1011(\mathrm{~s}), 912$ (s), 832 (s), 739 (s). HRMS (ESI/QTOF) m/z: [M + H] $]^{+}$Calcd for $\mathrm{C}_{16} \mathrm{H}_{13} \mathrm{I}_{2} \mathrm{O}_{3}{ }^{+}$506.8949; Found 506.8956. The olefin geometry was assigned by analogy to NMR data of compound 11 .

\section{(Z)-1-(2-(2-iodophenoxy)pent-1-en-1-yl)-1,2-benziodoxol-3-(1H)-one (1h)}

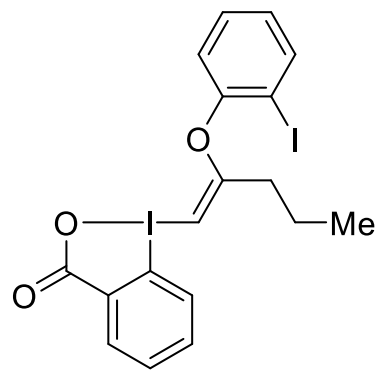

Starting from EBX $5 \mathbf{b}(500 \mathrm{mg}, 1.60 \mathrm{mmol}),(Z)$-1-(2-(2-iodophenoxy)pent-1en-1-yl)-1,2-benziodoxol-3-(1H)-one $1 \mathrm{~h}$ (0.450 $\mathrm{mg}, 0.842 \mathrm{mmol}, 53 \%$ yield) was obtained, as a white solid. Rf: 0.53 (DCM:MeOH 9:1). Mp: $176-177^{\circ} \mathrm{C}$. ${ }^{1} \mathrm{H}$ NMR (400 MHz, Chloroform- $d$ ) $\delta 8.48-8.42(\mathrm{~m}, 1 \mathrm{H}, \mathrm{ArH}), 7.80$ (dd, $J=$ 7.9, $1.5 \mathrm{~Hz}, 1 \mathrm{H}, \mathrm{ArH}), 7.70-7.59(\mathrm{~m}, 3 \mathrm{H}, \mathrm{ArH}), 7.31$ (ddd, $J=8.1,7.5,1.6 \mathrm{~Hz}$, $1 \mathrm{H}, \mathrm{ArH}), 6.99-6.88(\mathrm{~m}, 2 \mathrm{H}, \mathrm{ArH}), 5.90(\mathrm{~d}, J=1.0 \mathrm{~Hz}, 1 \mathrm{H}$, vinylH), $2.44-2.35$ $\left(\mathrm{m}, 2 \mathrm{H}, \mathrm{CH}_{2}\right), 1.71-1.58\left(\mathrm{~m}, 2 \mathrm{H}, \mathrm{CH}_{2}\right), 1.00\left(\mathrm{t}, \mathrm{J}=7.4 \mathrm{~Hz}, 3 \mathrm{H}, \mathrm{CH}_{3}\right) .{ }^{13} \mathrm{C}$ NMR

(101 MHz, Chloroform-d) $\delta$ 169.6, 166.7, 153.3, 140.3, 133.9, 133.4, 133.1, 130.8, 129.9, 127.5, 125.7, 120.5, 114.1, 89.5, 80.0, 34.7, 20.4, 13.8. IR v 3062 (w), 2966 (m), $2872(w), 1722(w), 1604(s), 1578$ $(\mathrm{m}), 1559(\mathrm{~m}), 1506(\mathrm{w}), 1465(\mathrm{~s}), 1438(\mathrm{~m}), 1342(\mathrm{~m}), 1300(\mathrm{~m}), 1258(\mathrm{~m}), 1221(\mathrm{~s}), 1124(\mathrm{~m}), 1043$ 
(m), $1019(\mathrm{~m}), 908(\mathrm{~m}), 829(\mathrm{~m}), 741$ (s), $687(\mathrm{~m}), 644$ (w). HRMS (ESI/QTOF) m/z: [M + H] ${ }^{+}$Calcd for $\mathrm{C}_{18} \mathrm{H}_{17} \mathrm{l}_{2} \mathrm{O}_{3}{ }^{+} 534.9262$; Found 534.9266. The olefin geometry was assigned by analogy to NMR data of compound 11 .

(Z)-1-(2-(2-lodophenoxy)oct-1-en-1-yl)-1,2-benziodoxol-3-(1H)-one (1i)

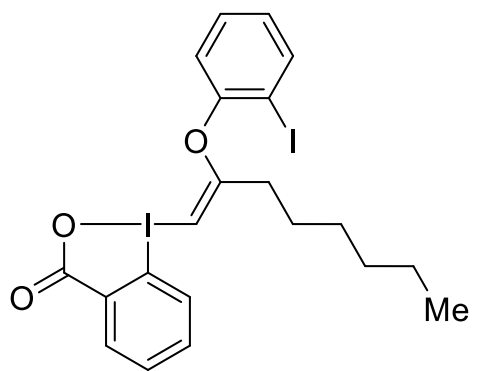

Starting from EBX $5 c \quad(0.142 \mathrm{~g}, 0.400 \mathrm{mmol}), \quad(Z)-1-(2-(2-$ lodophenoxy)oct-1-en-1-yl)-1,2-benziodoxol-3-(1H)-one 1i (0.190 g, $0.330 \mathrm{mmol}, 82 \%$ yield) was obtained, as a white solid. Rf: 0.51 (DCM:MeOH 9:1). Mp: $130-133.5^{\circ} \mathrm{C} .{ }^{1} \mathrm{H}$ NMR $(400 \mathrm{MHz}$, Chloroformd) $\delta 8.48-8.41(\mathrm{~m}, 1 \mathrm{H}, \mathrm{ArH}), 7.79(\mathrm{dd}, J=7.8,1.6 \mathrm{~Hz}, 1 \mathrm{H}, \mathrm{ArH}), 7.70$ - $7.66(\mathrm{~m}, 1 \mathrm{H}, \mathrm{ArH}), 7.62$ (hept, $J=5.1 \mathrm{~Hz}, 2 \mathrm{H}, \mathrm{ArH}), 7.36-7.27(\mathrm{~m}$, $1 \mathrm{H}, \mathrm{ArH}), 6.94(\mathrm{td}, J=8.5,2.9 \mathrm{~Hz}, 2 \mathrm{H}, \mathrm{ArH}), 5.94(\mathrm{~s}, 1 \mathrm{H}$, vinylH), 2.43 $\left(\mathrm{t}, J=7.6 \mathrm{~Hz}, 2 \mathrm{H}, \mathrm{CH}_{2}\right), 1.59\left(\mathrm{p}, J=7.6 \mathrm{~Hz}, 2 \mathrm{H}, \mathrm{CH}_{2}\right), 1.41-1.22\left(\mathrm{~m}, 6 \mathrm{H}, \mathrm{CH}_{2}\right), 0.88\left(\mathrm{t}, J=6.7 \mathrm{~Hz}, 3 \mathrm{H}, \mathrm{CH}_{3}\right)$. ${ }^{13} \mathrm{C}$ NMR $(101 \mathrm{MHz}$, Chloroform-d) $\delta 169.9,167.1,153.3,140.3,133.6,133.5,133.1,130.8,129.9$, 127.4, 126.0, 120.6, 114.3, 89.5, 80.0, 32.7, 31.5, 28.8, 27.0, 22.6, 14.1. IR $\vee 3061$ (w), 2925 (m), 2859 $(\mathrm{m}), 1601(\mathrm{~s}), 1460(\mathrm{~m}), 1341(\mathrm{~s}), 1219(\mathrm{~s}), 1119(\mathrm{~m}), 1011(\mathrm{~m}), 920(\mathrm{w}), 825(\mathrm{~m}), 745(\mathrm{~s}), 681(\mathrm{~m}), 641$ (m). HRMS (ESI/QTOF) m/z: $[\mathrm{M}+\mathrm{H}]^{+}$Calcd for $\mathrm{C}_{21} \mathrm{H}_{23} \mathrm{I}_{2} \mathrm{O}_{3}{ }^{+}$576.9731; Found 576.9742. The olefin geometry was assigned by analogy to NMR data of compound 11 .

(Z)-1-(2-(4-Methoxyphenoxy)pent-1-en-1-yl)-1,2-benziodoxol-3-(1H)-one (1j)

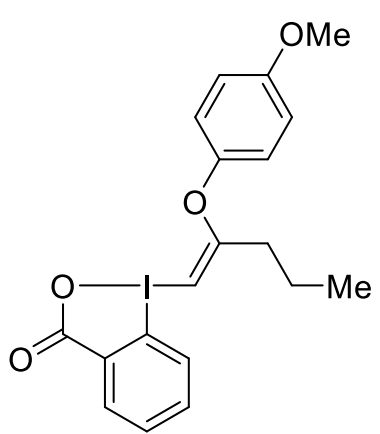

Starting from EBX 5b $(2.00 \mathrm{~g}, 6.40 \mathrm{mmol}), \quad(Z)-1-(2-(4-$ methoxyphenoxy)pent-1-en-1-yl)-1,2-benziodoxol-3-(1H)-one 1j (2.10 g, $4.80 \mathrm{mmol}, 75 \%$ yield) was obtained, as a white solid. Rf: 0.53 (DCM:MeOH 9:1). Mp: $175.8-180.9^{\circ} \mathrm{C} .{ }^{1} \mathbf{H}$ NMR $(400 \mathrm{MHz}$, Chloroform-d) $\delta 8.49-8.41$ (m, 1H, ArH), $7.66-7.57(\mathrm{~m}, 3 \mathrm{H}, \mathrm{ArH}), 6.83(\mathrm{~s}, 4 \mathrm{H}, \mathrm{ArH}), 5.79(\mathrm{~d}, J=0.9 \mathrm{~Hz}$, $1 \mathrm{H}$, vinylH), $3.77\left(\mathrm{~s}, 3 \mathrm{H}, \mathrm{OCH}_{3}\right), 2.46\left(\mathrm{t}, J=7.6 \mathrm{~Hz}, 2 \mathrm{H}, \mathrm{CH}_{2}\right), 1.61(\mathrm{~h}, J=7.4$ $\left.\mathrm{Hz}, 2 \mathrm{H}, \mathrm{CH}_{2}\right), 0.97\left(\mathrm{t}, J=7.4 \mathrm{~Hz}, 3 \mathrm{H}, \mathrm{CH}_{3}\right) .{ }^{13} \mathrm{C}$ NMR (101 MHz, Chloroform-d) $\delta 170.9,166.7,157.2,147.2,134.0,133.3,133.1,130.8,125.1,120.8,115.1$, 114.0, 79.0, 55.8, 34.5, 20.6, 13.7. IR $\vee 3667(\mathrm{~m}), 2975(\mathrm{~s}), 2904$ (s), $2355(\mathrm{w})$, 2233 (w), 1607 (s), 1503 (s), 1445 (m), 1348 (s), 1294 (m), 1245 (s), 1207 (s), 1055 (s), 909 (s), 836 (m), 736 (s), 648 (m). HRMS (ESI/QTOF) m/z: [M + H] ${ }^{+}$Calcd for $\mathrm{C}_{19} \mathrm{H}_{20} \mathrm{OO}{ }_{4}{ }^{+}$439.0401; Found 439.0403. The olefin geometry was assigned by analogy to NMR data of compound 11 .

(Z)-1-(2-(4-Methoxyphenoxy)prop-1-en-1-yl)-1,2-benziodoxol-3-(1H)-one (1k)

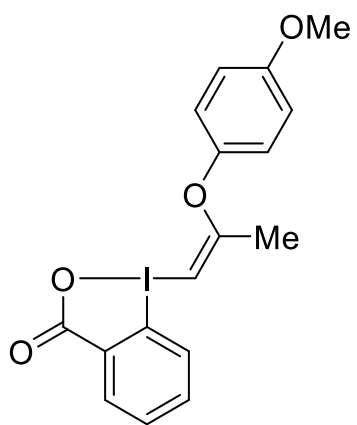

Starting from EBX 5a $(86.0 \mathrm{mg}, \quad 0.300 \mathrm{mmol})$, (Z)-1-(2-(4methoxyphenoxy)prop-1-en-1-yl)-1,2-benziodoxol-3-(1H)-one 1k $(63.0$ mg, $0.154 \mathrm{mmol}, 51 \%$ yield) was obtained, as an off-white sticky solid. Rf: 0.46 (DCM:MeOH 9:1). ${ }^{1} \mathrm{H}$ NMR (400 MHz, Chloroform-d) $\delta 8.47-8.39(\mathrm{~m}, 1 \mathrm{H}$, $\operatorname{ArH}), 7.74-7.66(\mathrm{~m}, 1 \mathrm{H}, \mathrm{ArH}), 7.67-7.60(\mathrm{~m}, 2 \mathrm{H}, \mathrm{ArH}), 6.96-6.89(\mathrm{~m}, 2 \mathrm{H}$, $\operatorname{ArH}), 6.89-6.74(\mathrm{~m}, 2 \mathrm{H}, \operatorname{ArH}), 5.86(\mathrm{~d}, J=1.2 \mathrm{~Hz}, 1 \mathrm{H}$, vinylH), $3.77(\mathrm{~s}, 3 \mathrm{H}$, $\mathrm{OCH}_{3}$ ), 2.21 (s, 3H, $\left.\mathrm{CH}_{3}\right) .{ }^{13} \mathrm{C}$ NMR (101 MHz, Chloroform-d) $\delta$ 167.6, 167.4, 157.5, 147.1, 133.6, 133.5, 133.2, 130.7, 125.7, 121.7, 115.0, 114.3, 76.4, 55.8, 19.5. IR v 3620 (s), 3041 (s), 2778 (s), 1610 (s), 1507 (s), 1208 (s), 1127 (s), 1070 (s), 977 (s), 908 (s), 844 (s), 736 (s). HRMS (ESI/QTOF) m/z: [M + H] Calcd for $\mathrm{C}_{17} \mathrm{H}_{16} \mathrm{IO}_{4}{ }^{+}$ 
411.0088; Found 411.0092. The olefin geometry was assigned by analogy to NMR data of compound 11.

(Z)-N-(1-Prop-1-en-2-yl)-N-(4-methoxyphenyl)-4-methylbenzenesulfonamide-1,2-benziodoxol-3-

(1H)-one (1l) Starting from EBX 5a $(286 \mathrm{mg}, 1.00 \mathrm{mmol})$, (Z)- $N$-(1-prop-1-en-2-yl)- $N$-(4-<smiles>C/C(=C/I(C)c1ccccc1C(=O)O)N([12F])P</smilesmethoxyphenyl)-4-methylbenzenesulfonamide-1,2-benziodoxol-3-(1H)-one $\mathbf{1 l}$ (383 $\mathrm{mg}, 0.680 \mathrm{mmol}, 68 \%$ yield) was obtained, as a white solid. Rf: 0.30 (DCM:MeOH 9:1). Mp: $92.4^{\circ} \mathrm{C}-96.3{ }^{\circ} \mathrm{C} .{ }^{1} \mathrm{H}$ NMR (400 MHz, Chloroform-d) $\delta 8.39$ $(\mathrm{dd}, J=7.4,1.8 \mathrm{~Hz}, 1 \mathrm{H}, \mathrm{ArH}), 7.62-7.55(\mathrm{~m}, 3 \mathrm{H}, \mathrm{ArH}), 7.51$ (ddd, J = 9.0, 7.2, 1.9 $\mathrm{Hz}, 1 \mathrm{H}, \operatorname{ArH}), 7.35(\mathrm{dd}, J=8.1,1.1 \mathrm{~Hz}, 1 \mathrm{H}, \operatorname{ArH}), 7.31-7.27(\mathrm{~m}, 2 \mathrm{H}, \operatorname{ArH}), 6.99-$ $6.93(\mathrm{~m}, 2 \mathrm{H}, \operatorname{Ar} H), 6.80(\mathrm{~d}, J=1.4 \mathrm{~Hz}, 1 \mathrm{H}$, vinyl $H), 6.77-6.71(\mathrm{~m}, 2 \mathrm{H}, \mathrm{ArH}), 3.73$ (s, 3H, OMe), $2.43\left(\mathrm{~s}, 3 \mathrm{H}, \mathrm{CH}_{3}\right), 2.21\left(\mathrm{~s}, 3 \mathrm{H}, \mathrm{CH}_{3}\right) .{ }^{13} \mathrm{C}$ NMR (101 MHz, Chloroformd) $\delta 166.9,160.0,152.6,145.2,135.4,133.8,133.3,132.78,130.6,130.3,129.9,129.8,128.0,126.1$, 114.8, 114.6, 105.4, 55.5, 22.9, 21.6. IR $\vee 2970(\mathrm{~m}), 1757(\mathrm{w}), 1654(\mathrm{~s}), 1575(\mathrm{~s}), 1481(\mathrm{~s}), 1230(\mathrm{~m})$, $1195(w), 1170(w), 1081(w)$. HRMS (ESI) calcd for $\mathrm{C}_{24} \mathrm{H}_{23} \mathrm{INO}_{5} \mathrm{~S}^{+}[\mathrm{M}+\mathrm{H}]^{+} 564.0336$; found 564.0339. The structure of the obtained regioisomer was confirmed by crystal structure. The values of the NMR spectra are in accordance with reported literature data.Error! Bookmark not defined.

\section{(Z)-1-(2-Cyclopropyl-2-(p-tolyloxy)vinyl)-1,2-benziodoxol-3-(1H)-one (1m)}

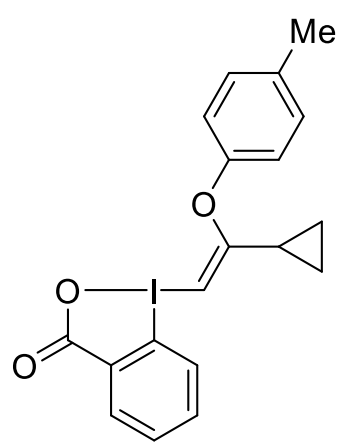

Starting from EBX $5 \mathbf{j}(31.0 \mathrm{mg}, 0.100 \mathrm{mmol}),(Z)-1-(2-c y c l o p r o p y l-2-(p-$ tolyloxy)vinyl)-1/3-benziodoxol-3(1H)-one $1 \mathrm{~m}$ ( $37.0 \mathrm{mg}, 88.0 \mu \mathrm{mol}, 88 \%$ yield) was obtained, as a white amorphous solid. Rf: 0.43 (DCM:MeOH 9:1). ${ }^{1} \mathbf{H}$ NMR (400 MHz, Chloroform-d) $\delta 8.51-8.35(\mathrm{~m}, 1 \mathrm{H}, \mathrm{ArH}), 7.64-7.58(\mathrm{~m}, 2 \mathrm{H}, \mathrm{ArH})$, $7.56-7.51(\mathrm{~m}, 1 \mathrm{H}, \operatorname{Ar} H), 7.16-7.01(\mathrm{~m}, 2 \mathrm{H}, \operatorname{Ar} H), 6.92-6.77(\mathrm{~m}, 2 \mathrm{H}, \operatorname{Ar} H)$, $5.72(\mathrm{~s}, 1 \mathrm{H}$, vinyl $H), 2.29\left(\mathrm{~s}, 3 \mathrm{H}, \mathrm{CH}_{3}\right), 1.67(\mathrm{tt}, J=8.2,5.3 \mathrm{~Hz}, 1 \mathrm{H}, \mathrm{CH}), 1.02-$ $0.88\left(\mathrm{~m}, 4 \mathrm{H}, \mathrm{CH}_{2}\right) .{ }^{13} \mathrm{C}$ NMR (101 MHz, Chloroform-d) $\delta 172.7,166.8,152.5$, 135.0, 133.9, 133.3, 133.0, 130.7, 130.6, 125.2, 119.2, 114.4, 75.5, 20.8, 14.3, 9.7. IR $v 3672(\mathrm{~m}), 3059(\mathrm{~m}), 2805(\mathrm{~m}), 1603(\mathrm{~s}), 1504(\mathrm{~s}), 1344(\mathrm{~m}), 1203(\mathrm{~s})$, 1141 (s), 1065 (s), 1012 (s), 932 (s), 824 (s), 737 (s). HRMS (ESI/QTOF) m/z: [M + H] Calcd for $\mathrm{C}_{19} \mathrm{H}_{18} \mathrm{IO}_{3}{ }^{+}$ 421.0295; Found 421.0296. The olefin geometry was assigned by analogy to NMR data of compound 11.

\section{(Z)-1-(2-Cyclohexyl-2-(p-tolyloxy)vinyl)-1,2-benziodoxol-3-(1H)-one (1n)}

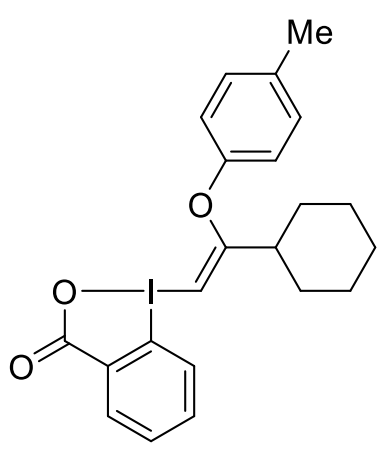

Starting from EBX $5 \mathbf{k}(35.0 \mathrm{mg}, 0.100 \mathrm{mmol}),(Z)-1-(2-c y c l o h e x y l-2-(p-$ tolyloxy)vinyl)-1,2-benziodoxol-3-(1H)-one 1n $(30.0 \mathrm{mg}, 65.0 \mu \mathrm{mol}, 65 \%$ yield) was obtained, as a white amorphous solid. Rf: 0.34 (DCM:MeOH 9:1). ${ }^{1} \mathrm{H}$ NMR $(400 \mathrm{MHz}$, Chloroform-d) $\delta 8.48-8.32(\mathrm{~m}, 1 \mathrm{H}, \mathrm{ArH}), 7.64-7.58(\mathrm{~m}$, $2 \mathrm{H}, \mathrm{ArH}), 7.58-7.52(\mathrm{~m}, 1 \mathrm{H}, \mathrm{ArH}), 7.14-7.01(\mathrm{~m}, 2 \mathrm{H}, \mathrm{ArH}), 6.88-6.69(\mathrm{~m}$, $2 \mathrm{H}, \operatorname{ArH}), 6.04(\mathrm{~s}, 1 \mathrm{H}$, vinylH), $2.42(\mathrm{tt}, J=11.7,3.3 \mathrm{~Hz}, 1 \mathrm{H}, \mathrm{CH}), 2.29(\mathrm{~s}, 3 \mathrm{H}$, $\left.\mathrm{CH}_{3}\right), 2.11-2.00\left(\mathrm{~m}, 2 \mathrm{H}, \mathrm{CH}_{2}\right), 1.85-1.75\left(\mathrm{~m}, 2 \mathrm{H}, \mathrm{CH}_{2}\right), 1.74-1.65(\mathrm{~m}, 1 \mathrm{H}$, $\mathrm{CH}_{2}$ ), 1.39 (qd, $\left.J=12.0,3.3 \mathrm{~Hz}, 2 \mathrm{H}, \mathrm{CH}_{2}\right), 1.28-1.11\left(\mathrm{~m}, 3 \mathrm{H}, \mathrm{CH}_{2}, \mathrm{CH}_{2}\right) .{ }^{13} \mathrm{C}$ NMR (101 MHz, Chloroform-d) $\delta$ 174.9, 167.2, 152.4, 134.5, 133.6, 133.4, 133.1, 130.7, 130.7, 125.6, 118.4, 114.5, 81.7, 41.2, 31.8, 26.0, 25.9, 20.8. IR v 3052 (w), 2931 (m), 2857 (w), $1604(\mathrm{~m}), 1504(\mathrm{~m}), 1444(\mathrm{w}), 1342(\mathrm{~m}), 1202(\mathrm{~m}), 1173(\mathrm{w}), 1116(\mathrm{w}), 1010(\mathrm{w}), 906(\mathrm{~s}), 826(\mathrm{~m})$, 
728 (s), 687 (w), 644 (m). HRMS (ESI/QTOF) m/z: [M + H] ${ }^{+}$Calcd for $\mathrm{C}_{22} \mathrm{H}_{24} \mathrm{IO}_{3}{ }^{+}$463.0765; Found 463.0768. The olefin geometry was assigned by analogy to NMR data of compound 11 .

(Z)-1-(3,3-dimethyl-2-(p-tolyloxy)but-1-en-1-yl)-1,2-benziodoxol-3-(1H)-one (1p)

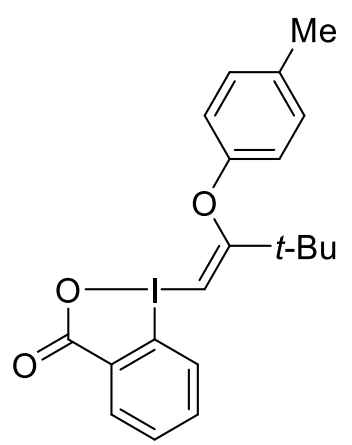

Starting from EBX $5 \mathrm{~m}(33.0 \mathrm{mg}, 0.100 \mathrm{mmol}),(Z)-1-(3,3-$ dimethyl-2-(ptolyloxy)but-1-en-1-yl)-1,2-benziodoxol-3-(1H)-one 1p (35.0 mg, $80.0 \mu \mathrm{mol}$, $80 \%$ yield) was obtained, as a white amorphous solid. Rf: 0.43 (DCM:MeOH 9:1). ${ }^{1} \mathrm{H}$ NMR (400 MHz, Chloroform-d) $\delta 8.40(\mathrm{dd}, J=5.9,3.4 \mathrm{~Hz}, 1 \mathrm{H}, \operatorname{ArH})$, $7.67-7.58(\mathrm{~m}, 2 \mathrm{H}, \mathrm{ArH}), 7.53-7.42(\mathrm{~m}, 1 \mathrm{H}, \mathrm{ArH}), 7.12-7.01(\mathrm{~m}, 2 \mathrm{H}, \mathrm{ArH})$, $6.80(\mathrm{~d}, J=8.6 \mathrm{~Hz}, 2 \mathrm{H}, \mathrm{ArH}), 6.13(\mathrm{~s}, 1 \mathrm{H}$, vinyl $\mathrm{H}), 2.27\left(\mathrm{~s}, 3 \mathrm{H}, \mathrm{CH}_{3}\right), 1.37(\mathrm{~s}, 9 \mathrm{H}$, $\left.\mathrm{C}\left(\mathrm{CH}_{3}\right)_{3}\right) .{ }^{13} \mathrm{C}$ NMR $(101 \mathrm{MHz}$, Chloroform-d) $\delta 177.3,166.8,155.2,134.2$, 133.6, 133.4, 133.2, 131.3, 130.8, 125.4, 116.8, 115.2, 80.9, 40.4, 28.8, 20.8. IR $\vee 3246(\mathrm{~m}), 2994(\mathrm{~m}), 1739(\mathrm{~s}), 1658(\mathrm{~s}), 1631$ (s), $1583(\mathrm{~s}), 1552(\mathrm{~s}), 1508$ (s), $1394(\mathrm{~s}), 1363(\mathrm{~s}), 1320(\mathrm{~s}), 1263(\mathrm{~s}), 1214(\mathrm{~s}), 1173(\mathrm{~s}), 1072(\mathrm{~m}), 1021(\mathrm{~s}), 972(\mathrm{~m}), 848(\mathrm{~s}), 829(\mathrm{~s})$, 752 (s). HRMS (ESI/QTOF) m/z: $[\mathrm{M}+\mathrm{H}]^{+}$Calcd for $\mathrm{C}_{20} \mathrm{H}_{22} \mathrm{OO}_{3}{ }^{+}$437.0608; Found 437.0595. The olefin geometry was assigned by analogy to NMR data of compound 1 .

\section{(Z)-1-(2-phenyl-2-(p-tolyloxy)vinyl)-1,2-benziodoxol-3-(1H)-one (1q)}

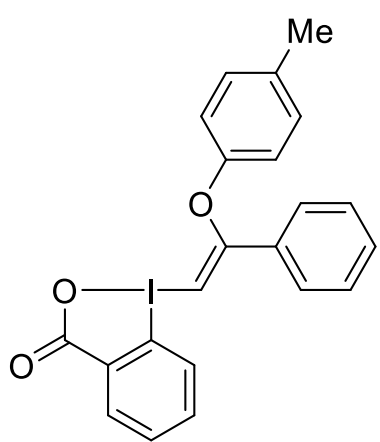

Starting from EBX $5 \mathrm{n}(35.0 \mathrm{mg}, 0.100 \mathrm{mmol}),(Z)-1-(2-$ phenyl-2-( $p$ tolyloxy)vinyl)-1,2-benziodoxol-3-(1H)-one 1q (45.3 mg, $99.0 \mu \mathrm{mol}, 99 \%$ yield) was obtained, as a white amorphous solid. Rf: 0.31 (DCM:MeOH 9:1). ${ }^{1} \mathrm{H}$ NMR (400 MHz, Chloroform-d) $\delta 8.51-8.41(\mathrm{~m}, 1 \mathrm{H}, \mathrm{ArH}), 7.69-7.57$ $(\mathrm{m}, 5 \mathrm{H}, \mathrm{ArH}), 7.42(\mathrm{dq}, J=8.9,7.1,6.6 \mathrm{~Hz}, 3 \mathrm{H}, \mathrm{ArH}), 6.98(\mathrm{~d}, J=8.4 \mathrm{~Hz}, 2 \mathrm{H}$, $\mathrm{ArH}), 6.80-6.72(\mathrm{~m}, 2 \mathrm{H}, \mathrm{ArH}), 6.66\left(\mathrm{~s}, 1 \mathrm{H}\right.$, vinylH), $2.21\left(\mathrm{~s}, 3 \mathrm{H}, \mathrm{CH}_{3}\right) .{ }^{13} \mathrm{C} \mathrm{NMR}$ (101 MHz, Chloroform-d) $\delta 167.2,165.6,153.6,133.9,133.7,133.3,133.2$, 131.7, 131.5, 130.9, 130.6, 129.3, 128.1, 126.1, 117.2, 115.0, 86.4, 20.7. IR v $3066(\mathrm{w}), 1604(\mathrm{~s}), 1505(\mathrm{~m}), 1442(\mathrm{w}), 1347(\mathrm{~m}), 1283(\mathrm{~m}), 1201(\mathrm{~m}), 1033$ (w), $1016(\mathrm{w}), 911(\mathrm{~m}), 824(\mathrm{~m}), 737$ (s), $697(\mathrm{~m}), 649$ (w). HRMS (ESI/QTOF) m/z: [M + H] ${ }^{+}$Calcd for $\mathrm{C}_{22} \mathrm{H}_{18} \mathrm{IO}_{3}{ }^{+} 457.0295 ;$ Found 457.0287. The olefin geometry was assigned by analogy to NMR data of compound 11 .

(Z)-1-(2-(2,6-dichlorophenoxy)oct-1-en-1-yl)-1,3-benziodoxol-3(1H)-one (1r)

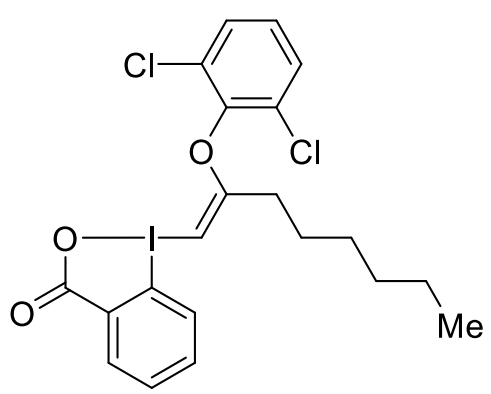

Starting from EBX 5c $(36.0 \mathrm{mg}, 0.100 \mathrm{mmol}),(Z)-1-(2-(2,6-$ dichlorophenoxy)oct-1-en-1-yl)-1,3-benziodoxol-3(1H)-one $\quad \mathbf{1 r}$ (43.0 mg, $83.0 \mu \mathrm{mol}, 83 \%$ yield) was obtained, as a white solid. $\mathbf{R f :}$ 0.46 (DCM:MeOH 9:1). ${ }^{1} \mathbf{H}$ NMR (400 MHz, Chloroform-d) $\delta 8.45$ (dd, $J=7.2,2.0 \mathrm{~Hz}, 1 \mathrm{H}, \operatorname{ArH}), 7.69$ (dd, $J=7.7,1.5 \mathrm{~Hz}, 1 \mathrm{H}, \operatorname{ArH}), 7.67$ - $7.56(\mathrm{~m}, 2 \mathrm{H}, \operatorname{ArH}), 7.34(\mathrm{~d}, J=8.1 \mathrm{~Hz}, 2 \mathrm{H}, \operatorname{ArH}), 7.15(\mathrm{dd}, J=8.6$, $7.7 \mathrm{~Hz}, 1 \mathrm{H}, \mathrm{ArH}), 5.81(\mathrm{t}, J=1.1 \mathrm{~Hz}, 1 \mathrm{H}$, vinyl $\mathrm{H}), 2.38-2.25(\mathrm{~m}, 2 \mathrm{H}$, $\left.\mathrm{CH}_{2} \mathrm{CH}_{2} \mathrm{CH}_{2} \mathrm{CH}_{2} \mathrm{CH}_{2} \mathrm{CH}_{3}\right), \quad 1.62$ (t, J $=7.8 \mathrm{~Hz}, 2 \mathrm{H}$, $\left.\mathrm{CH}_{2} \mathrm{CH}_{2} \mathrm{CH}_{2} \mathrm{CH}_{2} \mathrm{CH}_{2} \mathrm{CH}_{3}\right), 1.45-1.19\left(\mathrm{~m}, 6 \mathrm{H}, \mathrm{CH}_{2} \mathrm{CH}_{2} \mathrm{CH}_{2} \mathrm{CH}_{2} \mathrm{CH}_{2} \mathrm{CH}_{3}\right), 0.88\left(\mathrm{t}, \mathrm{J}=6.9 \mathrm{~Hz}, 3 \mathrm{H}, \mathrm{CH}_{3}\right) .{ }^{13} \mathrm{C}$ NMR (101 MHz, Chloroform-d) $\delta$ 170.1, 167.1, 145.7, 133.6, 133.4, 133.1, 130.8, 129.4, 129.3, 127.6, 126.1, 114.0, 76.1, 32.1, 31.4, 28.8, 26.7, 22.6, 14.1. IR $v 3067(\mathrm{~m}), 2935(\mathrm{~m}), 2864(\mathrm{~m}), 1738(\mathrm{~m}), 1665(\mathrm{~m})$, $1597(\mathrm{~s}), 1562(\mathrm{~m}), 1442(\mathrm{~s}), 1336(\mathrm{~m}), 1246(\mathrm{~s}), 1124(\mathrm{~m}), 1006(\mathrm{~m}), 916(\mathrm{~m}), 830(\mathrm{~m}), 734(\mathrm{~s}), 725(\mathrm{~s})$, 
$678(\mathrm{~m}), 643(\mathrm{~m})$. HRMS (ESI/QTOF) m/z: [M + H] Calcd for $\mathrm{C}_{21} \mathrm{H}_{22} \mathrm{Cl}_{2} \mathrm{IO}_{3}{ }^{+}$518.9985; Found 518.9993. The olefin geometry was assigned by analogy to NMR data of compound 1 .

(Z)-5-Methyl-1-(2-(4-methoxyphenoxy)prop-1-en-1-yl)-1,2-benziodoxol-3-(1H)-one (1s)<smiles>COc1ccc(O/C(C)=C/I2OC(=O)c3cc(C)ccc32)cc1</smiles>

Starting from EBX 50 (90.0 mg, $0.300 \mathrm{mmol})$, (Z)-5-methyl-1-(2-(4methoxyphenoxy)prop-1-en-1-yl)-1,2-benziodoxol-3-(1H)-one 1s (83.0 mg, $0.196 \mathrm{mmol}, 65 \%$ yield) was obtained, as an off-white solid. Rf: 0.53 (DCM:MeOH 9:1). Mp: $168{ }^{\circ} \mathrm{C} .{ }^{1} \mathrm{H}$ NMR (400 MHz, Methylene chloride- $d_{2}$ ) $\delta$ $8.19(\mathrm{~d}, J=2.2 \mathrm{~Hz}, 1 \mathrm{H}, \operatorname{ArH}), 7.55-7.44(\mathrm{~m}, 2 \mathrm{H}, \operatorname{ArH}), 6.92-6.81(\mathrm{~m}, 4 \mathrm{H}, \operatorname{Ar} H)$, $5.68\left(\mathrm{~d}, J=1.1 \mathrm{~Hz}, 1 \mathrm{H}\right.$, vinylH), $3.77\left(\mathrm{~s}, 3 \mathrm{H}, \mathrm{OCH}_{3}\right), 2.48\left(\mathrm{~s}, 3 \mathrm{H}, \mathrm{CH}_{3}\right), 2.17(\mathrm{~d}, J$ $\left.=1.0 \mathrm{~Hz}, 3 \mathrm{H}, \mathrm{CH}_{3}\right) .{ }^{13} \mathrm{C}$ NMR $\left(101 \mathrm{MHz}\right.$, Methylene chloride- $\left.d_{2}\right) \delta 168.0,166.9$, $158.0,147.7,141.9,134.5,134.3,133.6,125.6,122.1,115.3,110.7,77.0$, 56.2, 21.0, 19.7. IR $\vee 3667(\mathrm{~m}), 2979(\mathrm{~s}), 2903(\mathrm{~s}), 1615(\mathrm{~m}), 1499(\mathrm{~m}), 1400$ (m), $1241(\mathrm{~m}), 1212(\mathrm{~m}), 1059(\mathrm{~s}), 901(\mathrm{w}), 790(\mathrm{w}), 712(\mathrm{w}), 677(\mathrm{w})$. HRMS

(ESI/QTOF) m/z: $[\mathrm{M}+\mathrm{H}]^{+}$Calcd for $\mathrm{C}_{18} \mathrm{H}_{181} \mathrm{O}_{4}{ }^{+}$425.0244; Found 425.0244. The olefin geometry was assigned by analogy to NMR data of compound $\mathbf{1 1}$.

\section{(Z)-5-Methoxy-1-(2-(4-methoxyphenoxy)prop-1-en-1-yl)-1,2-benziodoxol-3-(1H)-one (1t)}<smiles>COc1ccc(O/C(C)=C/I2OC(=O)c3cc(OC)ccc32)cc1</smiles>

Starting from EBX $5 p(158 \mathrm{mg}, 0.500 \mathrm{mmol}),(Z)-5-M e t h o x y-1-(2-(4-$ methoxyphenoxy)prop-1-en-1-yl)-1,2-benziodoxol-3-(1H)-one 1t (133 mg, $0.302 \mathrm{mmol}, 60 \%$ yield) was obtained, as a white solid. Rf: 0.50 (DCM:MeOH 9:1). Mp: $180{ }^{\circ} \mathrm{C} .{ }^{1} \mathrm{H}$ NMR (400 MHz, Methylene chloride- $\left.d_{2}\right) \delta 7.91(\mathrm{~d}, J=3.0$ $\mathrm{Hz}, 1 \mathrm{H}, \operatorname{ArH}), 7.50(\mathrm{~d}, J=9.0 \mathrm{~Hz}, 1 \mathrm{H}, \operatorname{ArH}), 7.21(\mathrm{dd}, J=8.9,3.1 \mathrm{~Hz}, 1 \mathrm{H}, \operatorname{Ar} H)$, $6.93-6.81(\mathrm{~m}, 4 \mathrm{H}, \operatorname{Ar} H), 5.68\left(\mathrm{~d}, J=1.1 \mathrm{~Hz}, 1 \mathrm{H}\right.$, vinylH), $3.92\left(\mathrm{~s}, 3 \mathrm{H}, \mathrm{OCH}_{3}\right)$, $3.77\left(\mathrm{~s}, 3 \mathrm{H}, \mathrm{OCH}_{3}\right), 2.17\left(\mathrm{~d}, J=1.0 \mathrm{~Hz}, 3 \mathrm{H}, \mathrm{CH}_{3}\right) .{ }^{13} \mathrm{C}$ NMR (101 MHz, Methylene chloride- $\left.d_{2}\right) \delta 168.1,166.6,162.9,158.0,147.6,136.2,126.5,122.1,121.3$, 116.4, 115.4, 102.5, 76.7, 56.6, 56.2, 19.7. IR $\vee 3676(\mathrm{w}), 2979(\mathrm{~s}), 2903(\mathrm{~s})$, $1618(\mathrm{~m}), 1571(\mathrm{~m}), 1498(\mathrm{~m}), 1461(\mathrm{~m}), 1403(\mathrm{~m}), 1342(\mathrm{~m}), 1214(\mathrm{~m}), 1058$

(s), $953(w), 902(w), 712(m)$. HRMS (ESI/QTOF) m/z: [M + H] ${ }^{+}$Calcd for $\mathrm{C}_{18} \mathrm{H}_{18} \mathrm{IO}_{5}{ }^{+}$441.0193; Found 441.0191. The olefin geometry was assigned by analogy to NMR data of compound 11 . 


\section{Nucleophilic additions onto O-VBX}

\section{Optimization procedure and table for the synthesis of 3a:}

In a round-bottom flask were added $p$-cresol $(10.8 \mathrm{mg}, 0.100 \mathrm{mmol}, 1.00$ equiv.) and cesium carbonate (39.0 mg, $0.120 \mathrm{mmol}, 1.20$ equiv.). Anhydrous DME (1.00 mL, $0.1 \mathrm{M}$ ) was introduced at $0{ }^{\circ} \mathrm{C}$ and the solution was stirred at room temperature for $10 \mathrm{~min}$. O-VBX $1 \mathrm{a}$ ( $39.0 \mathrm{mg}, 0.100 \mathrm{mmol}, 1.00$ equiv.) was added to the reaction mixture under open air and the reaction mixture was stirred at room temperature for $16 \mathrm{~h}$.

Table S1: Optimisation studies of the addition of $p$-cresol (2a) to O-VBX 1a.

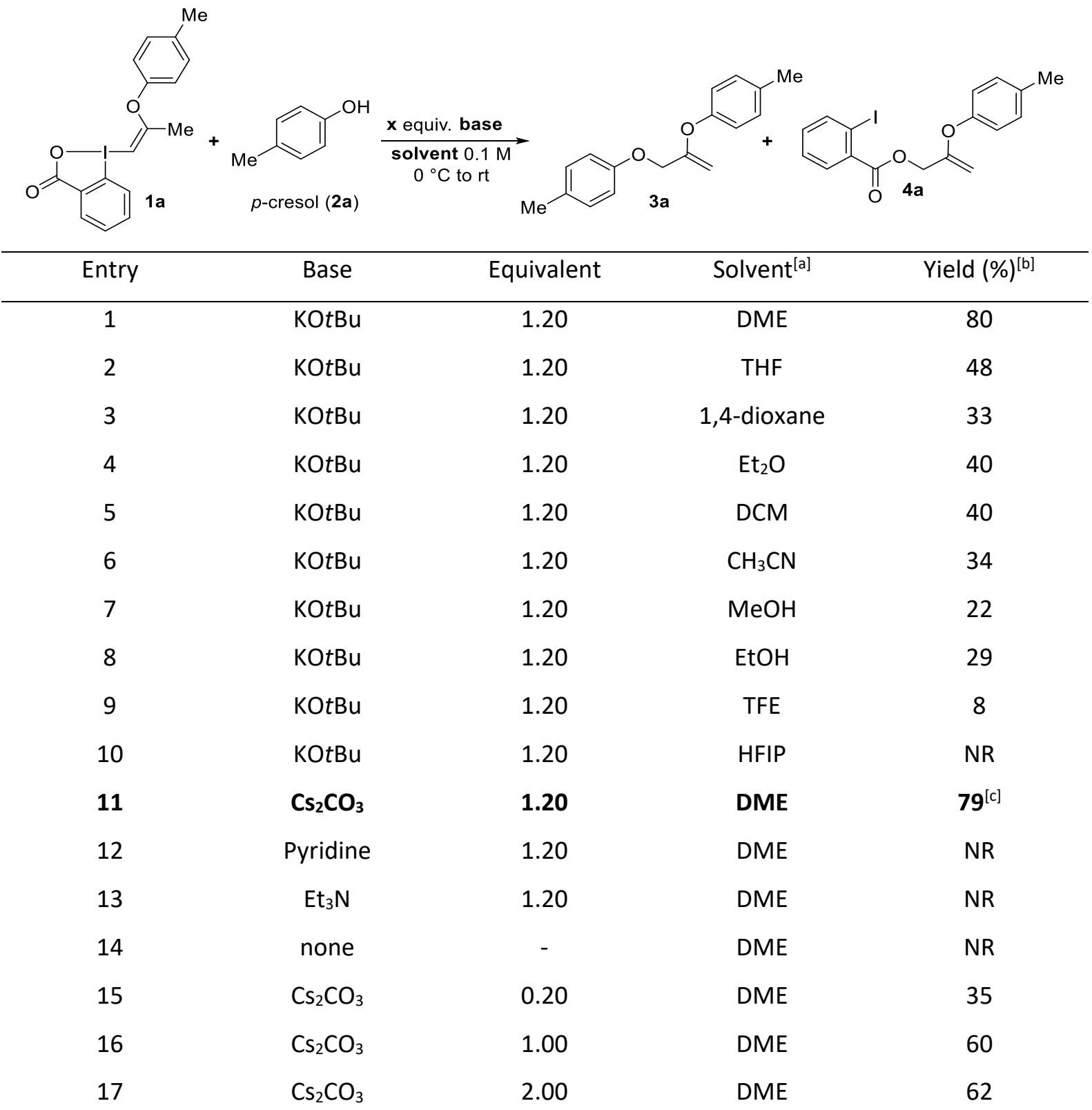

Reactions were performed on $0.1 \mathrm{mmol}$ scale: Substrate 1a $(0.100 \mathrm{mmol})$, para-cresol (2a) $(0.100 \mathrm{mmol})$, base $(0.120 \mathrm{mmol})$ and solvent $(0.1 \mathrm{M})$ at $25^{\circ} \mathrm{C}$. NR = No reaction. ${ }^{[\mathrm{a}]}$ Concentration based on $1 \mathrm{a}: 0.1 \mathrm{M}$; ${ }^{[\mathrm{b}]} \mathrm{NMR}$ yield determined by addition of $0.1 \mathrm{mmol}$ of $\mathrm{CH}_{2} \mathrm{Br}_{2}$ as an internal standard after the reaction. ${ }^{[\mathrm{c}]} 3 \mathrm{a}$ was obtained with $10 \%$ (NMR yield) of $4 a$. 
Cesium carbonate effect: The reaction is heterogeneous. The size of the particles is not influencing the reaction. When the reaction was performed with finely grinded salt, same isolated yield was obtained for $3 p$. Also, two batches of $\mathrm{Cs}_{2} \mathrm{CO}_{3}$ were tested for the synthesis of $3 p$ and the same yield was obtained.

\section{One-pot two-step procedure for the synthesis of 3a:}<smiles>CC#CI1COC(=O)c2ccccc21</smiles>

1.00 equiv. $p$-cresol (2a)

$10 \mathrm{~mol} \% \mathrm{Cs}_{2} \mathrm{CO}_{3}$

DME $0.1 \mathrm{M}, \mathrm{rt}, 16 \mathrm{~h}$

then 1.00 equiv. $p$-cresol (2a)

1.20 equiv. $\mathrm{Cs}_{2} \mathrm{CO}_{3}$

DME $0.1 \mathrm{M}, \mathrm{rt}, 16 \mathrm{~h}$<smiles>C=C(COC(=O)c1ccccc1I)Oc1ccc(C)cc1</smiles>

To increase the efficiency of the new aryl allyl ether synthesis, a one-pot two-step procedure was examined, starting from EBX $5 \mathrm{a}$. Using the optimized conditions previously developed for the synthesis of O-VBXs, but replacing EtOH with DME, Me-EBX (5a) was efficiently converted into O-VBX 1a. Then another equivalent of cresol $\mathbf{2 a}$ and an excess of base were added. After 16 hours, allyl ether $\mathbf{3 a}$ was obtained in $63 \%$ yield, together with $36 \%$ allyl ester $4 a$. Therefore, the one-pot procedure is efficient, but leads to the formation of a larger amount of allyl ester $4 a$.

\section{General procedure for nucleophilic additions onto O-vBX:}<smiles>[R]C/C(=C\I(C)c1ccccc1C(=O)OC)Oc1ccc(C)cc1</smiles>

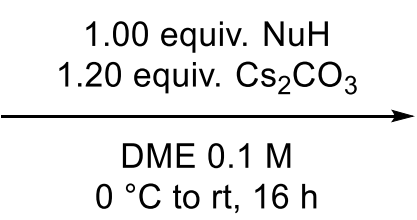

DME $0.1 \mathrm{M}$<smiles>[R]C=C(C[NH])Oc1ccc(C)cc1</smiles>

3

Nucleophile ( $0.300 \mathrm{mmol}, 1$ equiv.) and cesium carbonate $(0.117 \mathrm{~g}, 0.360 \mathrm{mmol}, 1.20$ equiv.) were added to a round-bottom flask. Anhydrous DME (3.00 mL, $0.1 \mathrm{M})$ was introduced at $0{ }^{\circ} \mathrm{C}$ and the solution was stirred at room temperature for $10 \mathrm{~min}$. O-VBX reagent $1(0.300 \mathrm{mmol}$, 1equiv.) was added to the reaction mixture under open air and the reaction mixture was stirred at room temperature for $16 \mathrm{~h}$. The reaction mixture was filtrated, solvent was removed under reduced pressure and the crude material was purified by column chromatography (pentane:ethyl acetate 9:1) to provide $\mathbf{3}$ as a sticky solid. 
<smiles>C=C(COc1ccc(C)cc1)Oc1ccc(C)cc1</smiles>

Starting from O-VBX 1a $(0.118 \mathrm{~g}, 0.300 \mathrm{mmol})$ and $p$-cresol 2a (32.4 $\mathrm{mg}, \quad 0.300 \mathrm{mmol}), \quad 4,4$ '-(prop-2-ene-1,2diylbis(oxy))bis(methylbenzene) 3a $(63.0 \mathrm{mg}, 0.248 \mathrm{mmol}, 83 \%$ yield) and $4 \mathrm{a}(15.3 \mathrm{mg}, 38.8 \mu \mathrm{mol}, 13 \%)$ were obtained, as a colorless amorphous solids. Rf: 0.70 (Pentane:EtOAc 9:1). ${ }^{1} \mathbf{H}$ NMR (400 MHz, Chloroform-d) $\delta 7.14$ (d, J = 8.2 Hz, 2H, ArH), $7.12-7.07$ $(\mathrm{m}, 2 \mathrm{H}, \mathrm{ArH}), 7.00-6.95(\mathrm{~m}, 2 \mathrm{H}, \mathrm{ArH}), 6.92-6.87(\mathrm{~m}, 2 \mathrm{H}, \mathrm{ArH})$, $4.61\left(\mathrm{~s}, 2 \mathrm{H}, \mathrm{CCH}_{2} \mathrm{O}\right), 4.57-4.50\left(\mathrm{~m}, 1 \mathrm{H}, \mathrm{CH}_{2} \mathrm{CO}\right), 4.19\left(\mathrm{~d}, \mathrm{~J}=2.2 \mathrm{~Hz}, 1 \mathrm{H}, \mathrm{CH}_{2} \mathrm{CO}\right), 2.33\left(\mathrm{~s}, 3 \mathrm{H}, \mathrm{CH}_{3}\right), 2.30$ $\left(\mathrm{s}, 3 \mathrm{H}, \mathrm{CH}_{3}\right) .{ }^{13} \mathrm{C}$ NMR (101 MHz, Chloroform-d) $\delta 158.8,156.5,152.8,134.1,130.6,130.3,130.0,120.8$, 115.0, 90.4, 67.9, 20.9, 20.7. IR v 3027 (w), 2925 (m), 2849 (w), $1650(w), 1612(w), 1586(w), 1510(s)$, $1459(w), 1370(w), 1294(m), 1224(s), 1180(w), 1072(w), 1040(w), 958(w), 907(w), 818(m), 729$ (m), 660 (w). HRMS (ESI) calcd for $\mathrm{C}_{17} \mathrm{H}_{18} \mathrm{NaO}_{2}^{+}[\mathrm{M}+\mathrm{Na}]^{+} 277.1199$; found 277.1189.

\section{1-Bromo-4-((2-(p-tolyloxy)allyl)oxy)benzene (3b)}<smiles>C=C(COc1ccc(Br)cc1)Oc1ccc(C)cc1</smiles>
Starting from O-VBX 1a $(0.118 \mathrm{~g}, 0.300 \mathrm{mmol})$ and 4-bromophenol 2b $\quad(51.9 \quad \mathrm{mg}, \quad 0.300 \quad \mathrm{mmol}), \quad$ 1-bromo-4- $((2-(p-$ tolyloxy)allyl)oxy)benzene $\mathbf{3 b}$ ( $71.3 \mathrm{mg}, 0.223 \mathrm{mmol}, 75 \%$ yield) and 4a $(20.1 \mathrm{mg}, 51.0 \mu \mathrm{mol}, 17 \%)$ were obtained, as a colorless amorphous solids. Rf: 0.67 (Pentane:EtOAc 9:1). ${ }^{1} \mathrm{H}$ NMR (400 MHz, Chloroform-d) $\delta 7.46-7.33(\mathrm{~m}, 2 \mathrm{H}, \mathrm{ArH}), 7.18-7.09(\mathrm{~m}, 2 \mathrm{H}, \mathrm{ArH})$, $7.00-6.92(\mathrm{~m}, 2 \mathrm{H}, \mathrm{ArH}), 6.92-6.83(\mathrm{~m}, 2 \mathrm{H}, \mathrm{ArH}), 4.61\left(\mathrm{~s}, 2 \mathrm{H}, \mathrm{CCH}_{2} \mathrm{O}\right), 4.51(\mathrm{dd}, J=2.3,1.1 \mathrm{~Hz}, 1 \mathrm{H}$, $\left.\mathrm{CH}_{2} \mathrm{CO}\right), 4.20\left(\mathrm{~d}, \mathrm{~J}=2.3 \mathrm{~Hz}, 1 \mathrm{H}, \mathrm{CH}_{2} \mathrm{CO}\right), 2.34(\mathrm{~s}, 3 \mathrm{H}, \mathrm{ArCH}) .{ }^{13} \mathrm{C}$ NMR $(101 \mathrm{MHz}$, Chloroform-d) $\delta 158.3$, 157.7, 152.6, 134.3, 132.4, 130.3, 120.8, 117.0, 113.6, 90.7, 68.0, 20.9. IR $v 3033$ (w), 2929 (w), 1650 (w), $1587(w), 1507(m), 1488(s), 1455(w), 1388(w), 1288(m), 1227(s), 1167(w), 1064(w), 1011(w)$, $963(w), 908(m), 828(m), 734(m)$. HRMS (APPI/LTQ-Orbitrap) m/z: [M] ${ }^{+}$Calcd for $\mathrm{C}_{16} \mathrm{H}_{15}{ }^{79} \mathrm{BrO}_{2}{ }^{+}$ 318.0250; Found 318.0240.

\section{1-lodo-4-((2-(p-tolyloxy)allyl)oxy)benzene (3c)}

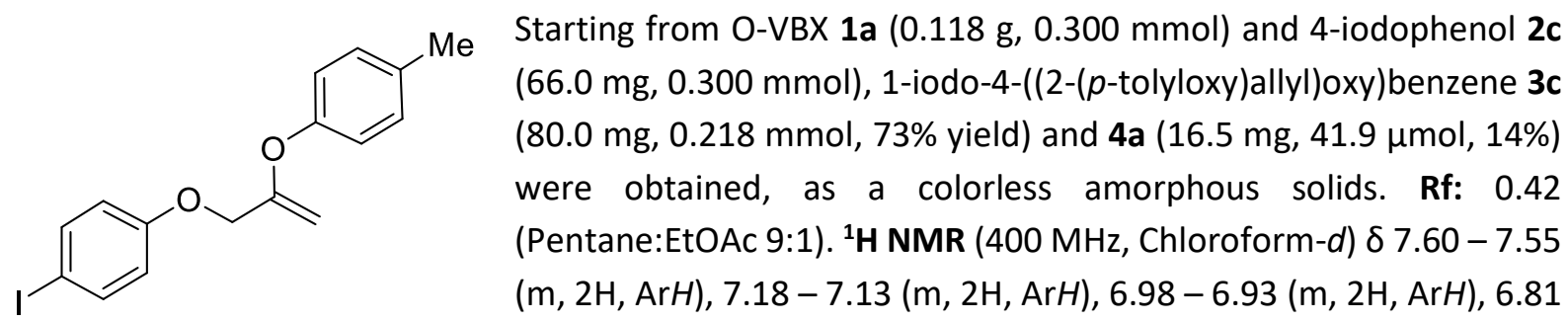

- $6.75(\mathrm{~m}, 2 \mathrm{H}, \mathrm{ArH}), 4.60\left(\mathrm{~d}, J=0.8 \mathrm{~Hz}, 2 \mathrm{H}, \mathrm{CCH}_{2} \mathrm{O}\right), 4.51$ (dd, J = 2.2, $1.1 \mathrm{~Hz}, 1 \mathrm{H}, \mathrm{CH}_{2} \mathrm{CO}$ ), 4.20 (d, J= 2.4 $\mathrm{Hz}, 1 \mathrm{H}, \mathrm{CH}_{2} \mathrm{CO}$ ), 2.34 (s, 3H, $\mathrm{ArCH}_{3}$ ). ${ }^{13} \mathrm{C}$ NMR (101 MHz, Chloroform-d) $\delta$ 158.5, 158.2, 152.6, 138.4, 134.3, 130.3, 120.8, 117.6, 90.7, 83.5, 67.9, 20.9. IR $v 3051$ (w), 2958 (w), 2928 (w), 1739 (w), $1494(w)$, 1265 (m), 1231 (w), 827 (w), 735 (s), 702 (s) HRMS (APPI/LTQ-Orbitrap) m/z: [M] ${ }^{+}$Calcd for $\mathrm{C}_{16} \mathrm{H}_{15} \mathrm{IO}_{2}{ }^{+}$ 366.0111; Found 366.0108. 
<smiles>C=C(COc1ccc(OC)cc1)Oc1ccc(C)cc1</smiles>

Starting from O-VBX 1a $(0.118 \mathrm{~g}, 0.300 \mathrm{mmol})$ and 4methoxyphenol $2 \mathrm{~d}(37.2 \mathrm{mg}, 0.300 \mathrm{mmol}), 1-\mathrm{methoxy}-4-((2-(p-$ tolyloxy)allyl)oxy)benzene $\mathbf{3 d}$ (36.2 $\mathrm{mg}, 0.134 \mathrm{mmol}, 45 \%$ yield) and $4 \mathrm{a}$ ( $56.8 \mathrm{mg}, 0.144 \mathrm{mmol}, 48 \%$ ) were obtained, as a colorless amorphous solids. Rf: 0.61 (Pentane:EtOAc 9:1). ${ }^{1} \mathbf{H}$ NMR (400 $\mathrm{MHz}$, Chloroform-d) $\delta 7.17-7.11(\mathrm{~m}, 2 \mathrm{H}, \mathrm{ArH}), 7.00-6.91(\mathrm{~m}$, $4 \mathrm{H}, \mathrm{ArH}), 6.88-6.82(\mathrm{~m}, 2 \mathrm{H}, \mathrm{ArH}), 4.59\left(\mathrm{~d}, J=0.9 \mathrm{~Hz}, 2 \mathrm{H}, \mathrm{CCH}_{2} \mathrm{O}\right), 4.52\left(\mathrm{dd}, J=2.1,1.0 \mathrm{~Hz}, 1 \mathrm{H}, \mathrm{CH}_{2} \mathrm{CO}\right)$, $4.18\left(\mathrm{~d}, J=2.2 \mathrm{~Hz}, 1 \mathrm{H}, \mathrm{CH}_{2} \mathrm{CO}\right), 3.78\left(\mathrm{~s}, 3 \mathrm{H}, \mathrm{OCH}_{3}\right), 2.33\left(\mathrm{~s}, 3 \mathrm{H}, \mathrm{CH}_{3}\right) .{ }^{13} \mathrm{C}$ NMR (101 MHz, Chloroform-d) $\delta$ 158.9, 154.3, 152.7 (2 Carbon signals are overlapping), 134.1, 130.3, 120.8, 116.3, 114.7, 90.5, 68.6, 55.9, 20.9. IR $\vee 3056$ (w), 2927 (w), 2842 (w), 1589 (w), 1507 (s), 1461 (w), 1276 (m), 1226 (s), 1159 (w), $1111(w), 1026(w), 964(w), 929(w), 831(m), 747(m), 714(m)$. HRMS (APPI/LTQ-Orbitrap) m/z: $[\mathrm{M}]^{+}$Calcd for $\mathrm{C}_{17} \mathrm{H}_{18} \mathrm{O}_{3}{ }^{+}$270.1250; Found 270.1252 .

\section{4,4,5,5-Tetramethyl-2-(4-((2-(p-tolyloxy)allyl)oxy)phenyl)-1,3,2-dioxaborolane (3e)}

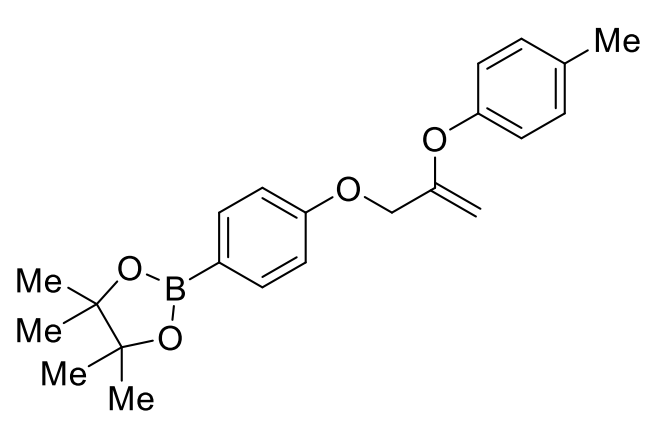

Starting from O-VBX 1a $(0.118 \mathrm{~g}, 0.300 \mathrm{mmol})$ and $4-$ (4,4,5,5-tetramethyl-1,3,2-dioxaborolan-2-yl)phenol $\quad \mathbf{2 e}$ (66.0 mg, $0.300 \mathrm{mmol}), 4,4,5,5$-tetramethyl-2-(4-((2-(ptolyloxy)allyl)oxy)phenyl)-1,3,2-dioxaborolane 3e (78.3 $\mathrm{mg}, 0.214 \mathrm{mmol}, 71 \%$ yield) and $4 \mathrm{a}$ ( $30.7 \mathrm{mg}, 77.9 \mu \mathrm{mol}$, 26\%) were obtained, as a colorless amorphous solids. Rf: 0.51 (Pentane:EtOAc 9:1). ${ }^{1}$ H NMR (400 MHz, Methylene Chloride- $\left.d_{2}\right) \delta 7.74-7.69(\mathrm{~m}, 2 \mathrm{H}, \mathrm{ArH}), 7.21-7.14(\mathrm{~m}, 2 \mathrm{H}$, $\mathrm{ArH}), 7.02-6.94(\mathrm{~m}, 4 \mathrm{H}, \mathrm{ArH}), 4.66\left(\mathrm{~s}, 2 \mathrm{H}, \mathrm{CCH}_{2} \mathrm{O}\right), 4.53\left(\mathrm{dt}, J=2.0,0.9 \mathrm{~Hz}, 1 \mathrm{H}, \mathrm{CH}_{2} \mathrm{CO}\right), 4.18$ (d, J= 2.3 $\left.\mathrm{Hz}, 1 \mathrm{H}, \mathrm{CH}_{2} \mathrm{CO}\right), 2.33\left(\mathrm{~s}, 3 \mathrm{H}, \mathrm{ArCH}_{3}\right), 1.32\left(\mathrm{~s}, 12 \mathrm{H}, \mathrm{CH}_{3}\right) .{ }^{13} \mathrm{C}$ NMR (101 MHz, Chloroform-d) $\delta$ 161.1, 158.5, 152.7, 136.6, 134.1, 130.3 (2 Carbon signals are overlapping), 120.8, 114.4, 90.6, 83.7, 67.4, 25.0, 20.9. IR $\vee 3034(w), 2987(w), 2253(w), 1722(w), 1604(m), 1505(m), 1360(s), 1323(w), 1217$ (m), $1159(\mathrm{~m}), 1143(\mathrm{~m}), 1087(\mathrm{~m}), 905$ (s), 829 (w), 734 (s), 652 (m). HRMS (APPI/LTQ-Orbitrap) m/z: $[\mathrm{M}]^{+}$Calcd for $\mathrm{C}_{22} \mathrm{H}_{27} \mathrm{BO}_{4}{ }^{+}$366.1997; Found 366.2000.

\section{1-Methoxy-3-((2-(p-tolyloxy)allyl)oxy)benzene (3f)}

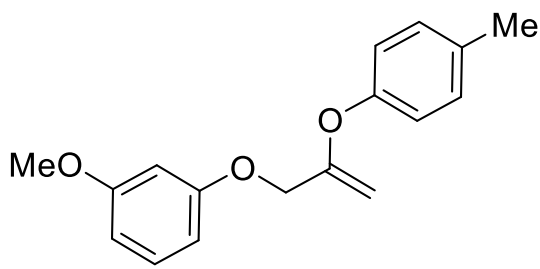

Starting from O-VBX 1a $(0.118 \mathrm{mg}, 0.300 \mathrm{mmol})$ and $3-$ methoxyphenol $2 \mathrm{f}(37.2 \mathrm{mg}, 0.300 \mathrm{mmol}), 1$-methoxy-3-((2-(ptolyloxy)allyl)oxy)benzene $\mathbf{3 f}$ ( $49.0 \mathrm{mg}, 0.181 \mathrm{mmol}, 60 \%$ yield) and $4 \mathrm{a}$ ( $42.5 \mathrm{mg}, 0.108 \mathrm{mmol}, 36 \%$ ) were obtained, as a colorless amorphous solids. Rf: 0.58 (Pentane:EtOAc 9:1). ${ }^{1} \mathbf{H}$ NMR (400 $\mathrm{MHz}$, Acetonitrile- $\left.d_{3}\right) \delta 7.24-7.15(\mathrm{~m}, 3 \mathrm{H}, \mathrm{ArH}), 6.99-6.93(\mathrm{~m}$, $2 \mathrm{H}, \mathrm{ArH}), 6.62$ - $6.53(\mathrm{~m}, 3 \mathrm{H}, \mathrm{ArH}), 4.63\left(\mathrm{~d}, J=0.8 \mathrm{~Hz}, 2 \mathrm{H}, \mathrm{CH}_{2} \mathrm{CO}\right), 4.55\left(\mathrm{dd}, J=2.1,1.0 \mathrm{~Hz}, 1 \mathrm{H}, \mathrm{CCH}_{2} \mathrm{O}\right.$ ), $4.14\left(\mathrm{~d}, J=2.1 \mathrm{~Hz}, 1 \mathrm{H}, \mathrm{CCH}_{2} \mathrm{O}\right), 3.77\left(\mathrm{~s}, 3 \mathrm{H}, \mathrm{OCH}_{3}\right), 2.32\left(\mathrm{~s}, 3 \mathrm{H}, \mathrm{CH}_{3}\right) .{ }^{13} \mathrm{C}$ NMR $(101 \mathrm{MHz}$, Acetonitrile$\left.d_{3}\right) \delta 161.9,160.6,159.8,153.6,135.2,131.2,131.0,121.4,107.9,107.7,102.1,91.6,68.4,55.9,20.7$. IR v $3665(\mathrm{~m}), 3091(\mathrm{~m}), 2989(\mathrm{~m}), 2906(\mathrm{~m}), 2601(\mathrm{~m}), 2262(\mathrm{~s}), 1886(\mathrm{w}), 1799(\mathrm{w}), 1639(\mathrm{w}), 1581$ (w), 1489 (s), 1404 (w), $1334(w), 1192(\mathrm{~m}), 1149(\mathrm{~m}), 1040$ (s), 841 (s), 777 (s), 689 (s). HRMS (APCI/QTOF) m/z: [M + H] $]^{+}$Calcd for $\mathrm{C}_{17} \mathrm{H}_{19} \mathrm{O}_{3}{ }^{+}$271.1329; found 271.1329 . 
<smiles>C=C(COc1cccc(N)c1)Oc1ccc(C)cc1</smiles>

Starting from O-VBX 1a $(0.118 \mathrm{~g}, 0.300 \mathrm{mmol})$ and 3-aminophenol $2 \mathrm{~g}$ (33.0 mg, $0.300 \mathrm{mmol}), 3-((2-(p$-tolyloxy)allyl)oxy)aniline $\mathbf{3 g}$ $(27.0 \mathrm{mg}, 0.106 \mathrm{mmol}, 35 \%$ yield) was obtained, as a orange amorphous solid and $4 \mathrm{a}(24.7 \mathrm{mg}, 62.6 \mu \mathrm{mol}, 21 \%)$ was obtained as a colorless amorphous solid. Rf: 0.62 (Pentane:EtOAc 7:3). ${ }^{1} \mathbf{H}$ NMR (400 MHz, Chloroform-d) $\delta 7.14$ (d, J = 8.2 Hz, 2H, ArH), 7.13 - $7.07(\mathrm{~m}, 1 \mathrm{H}, \mathrm{ArH}), 7.00$ - $6.94(\mathrm{~m}, 2 \mathrm{H}, \mathrm{ArH}), 6.48$ (ddd, J = 8.2, 2.3, $0.9 \mathrm{~Hz}, 1 \mathrm{H}, \mathrm{ArH}), 6.44-6.39(\mathrm{~m}$, $2 \mathrm{H}, \mathrm{ArH}), 4.61-4.58\left(\mathrm{~m}, 2 \mathrm{H}, \mathrm{CCH}_{2} \mathrm{O}\right), 4.54\left(\mathrm{dt}, J=2.0,1.0 \mathrm{~Hz}, 1 \mathrm{H}, \mathrm{CH}_{2} \mathrm{CO}\right), 4.19\left(\mathrm{~d}, J=2.3 \mathrm{~Hz}, 1 \mathrm{H}, \mathrm{CH}_{2} \mathrm{CO}\right.$ ), 2.33 (s, 3H, ArCH A $^{13}{ }^{13} \mathrm{C}$ NMR (101 MHz, Chloroform-d) $\delta$ 159.8, 158.7, 152.8, 145.9, 134.1, 130.3, 130.3, 120.8, 109.4, 106.3, 103.2, 90.5, 67.6, 20.9. IR v $3464(\mathrm{w}), 3378(\mathrm{~m}), 3221(\mathrm{w}), 2929(\mathrm{~m}), 1823(\mathrm{~m})$, 1607 (s), 1501 (s), $1459(\mathrm{~m}), 1294(\mathrm{~m}), 1219(\mathrm{~m}), 1188(\mathrm{~s}), 1166$ (s), $1158(\mathrm{~s}), 1071(\mathrm{w}), 1029(\mathrm{w}), 966$ (w), $910(\mathrm{~m}), 840(\mathrm{~m}), 735(\mathrm{~m})$. HRMS (ESI/QTOF) m/z: [M + H] ${ }^{+}$Calcd for $\mathrm{C}_{16} \mathrm{H}_{18} \mathrm{NO}_{2}{ }^{+}$256.1332; Found 256.1325 .

\section{Methyl 2-((2-(p-tolyloxy)allyl)oxy)benzoate (3h)}

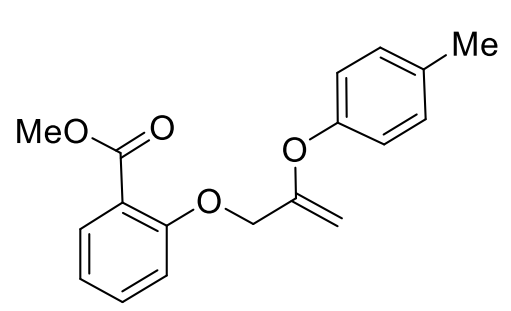

Starting from O-VBX 1a $(0.118 \mathrm{~g}, 0.300 \mathrm{mmol})$ and methyl 2hydroxybenzoate $2 \mathrm{~h}(46.1 \mathrm{mg}, 0.300 \mathrm{mmol})$, methyl 2-((2-(ptolyloxy)allyl)oxy)benzoate $3 \mathrm{~h}(80.1 \mathrm{mg}, 0.268 \mathrm{mmol}, 89 \%$ yield) and $4 \mathrm{a}(8.00 \mathrm{mg}, 20.3 \mu \mathrm{mol}, 7 \%)$ were obtained, as a colorless amorphous solids. Rf: 0.41 (Pentane:EtOAc 9:1). ${ }^{1} \mathbf{H}$ NMR $(400 \mathrm{MHz}$, Chloroform-d) $\delta 7.84$ (dd, J = 7.7, 1.9 Hz, 1H, ArH), 7.47 (ddd, $J=8.4$, 7.3, $1.8 \mathrm{~Hz}, 1 \mathrm{H}, \mathrm{ArH}), 7.18-7.12(\mathrm{~m}, 2 \mathrm{H}, \mathrm{ArH}), 7.06-7.00(\mathrm{~m}, 2 \mathrm{H}, \mathrm{ArH}), 7.00-6.96(\mathrm{~m}, 2 \mathrm{H}, \mathrm{ArH}), 4.72$ (dt, J = 2.3, $1.1 \mathrm{~Hz}, 1 \mathrm{H}, \mathrm{CH}_{2} \mathrm{CO}$ ), $4.70\left(\mathrm{~s}, 2 \mathrm{H}, \mathrm{CCH}_{2} \mathrm{O}\right), 4.23\left(\mathrm{~d}, J=2.2 \mathrm{~Hz}, 1 \mathrm{H}, \mathrm{CH}_{2} \mathrm{CO}\right), 3.90\left(\mathrm{~s}, 3 \mathrm{H}, \mathrm{OCH}_{3}\right)$, 2.34 (s, 3H, ArCH A $^{13}{ }^{13} \mathrm{C} \mathrm{NMR}(101 \mathrm{MHz}$, Chloroform-d) $\delta$ 166.9, 158.1, 158.0, 152.7, 134.1, 133.6, 132.0, 130.3, 121.0, 120.9, 120.7, 114.0, 90.2, 68.2, 52.2, 20.9. IR $v 2952(\mathrm{~m}), 1726(\mathrm{~s}), 1654(\mathrm{~m}), 1602(\mathrm{~m})$, $1499(\mathrm{~m}), 1456(\mathrm{~m}), 1389(\mathrm{~m}), 1301$ (s), $1244(\mathrm{~s}), 1134(\mathrm{~m}), 1085$ (s), $1054(\mathrm{~m}), 964(\mathrm{~m}), 909(\mathrm{~m}), 840$ (m), 750 (s). HRMS (ESI/QTOF) m/z: [M + Na] $]^{+}$Calcd for $\mathrm{C}_{18} \mathrm{H}_{18} \mathrm{NaO}_{4}{ }^{+} 321.1097$; Found 321.1100.

\section{1-(3,5-Di-tert-butyl-4-((2-(p-tolyloxy)allyl)oxy)phenyl)ethan-1-one (3i)}<smiles>C=C(COc1c(C(C)(C)C)cc(C(C)=O)cc1C(C)(C)C)Oc1ccc(C)cc1</smiles>

Starting from O-VBX 1a (39.4 mg, $0.100 \mathrm{mmol})$ and 1-(3,5-di-tertbutyl-4-hydroxyphenyl)ethanone $2 \mathbf{i}(24.8 \mathrm{mg}, 0.100 \mathrm{mmol}), 1-$ (3,5-di-tert-butyl-4-((2-( -tolyloxy)allyl)oxy)phenyl)ethan-1-one $3 i$ (17.0 mg, $43.0 \mu \mathrm{mol}, 43 \%$ yield) and 4 a ( $35.4 \mathrm{mg}, 89.8 \mu \mathrm{mol}$, $30 \%)$ were obtained, as a colorless amorphous solids. Rf: 0.54 (Pentane:EtOAc 9:1). ${ }^{1} \mathrm{H}$ NMR (400 MHz, Chloroform-d) $\delta 7.27$ (s, $2 \mathrm{H}, \mathrm{ArH}), 7.15-7.09(\mathrm{~m}, 2 \mathrm{H}, \mathrm{ArH}), 6.92-6.87(\mathrm{~m}, 2 \mathrm{H}, \mathrm{ArH}), 4.16$ (d, $\left.J=1.6 \mathrm{~Hz}, 1 \mathrm{H}, \mathrm{CH}_{2} \mathrm{CO}\right), 4.01\left(\mathrm{~d}, J=1.8 \mathrm{~Hz}, 1 \mathrm{H}, \mathrm{CH}_{2} \mathrm{CO}\right), 3.56-3.51\left(\mathrm{~m}, 2 \mathrm{H}, \mathrm{CCH}_{2} \mathrm{O}\right), 2.34(\mathrm{~s}, 3 \mathrm{H}$, $\left.\mathrm{COCH}_{3}\right), 2.32\left(\mathrm{~s}, 3 \mathrm{H}, \mathrm{ArCH}_{3}\right), 1.35\left(\mathrm{~s}, 18 \mathrm{H}, \mathrm{C}\left(\mathrm{CH}_{3}\right)_{3}\right) .{ }^{13} \mathrm{C}$ NMR $(101 \mathrm{MHz}$, Chloroform-d) $\delta$ 171.3, 162.8, 153.2, 146.6, 142.2, 134.8, 133.7, 130.1, 127.0, 120.9, 89.9, 40.7, 35.5, 31.6, 22.9, 20.9. IR $\vee 2962$ (s), $2921(\mathrm{~m}), 2877(\mathrm{~m}), 1761(\mathrm{~s}), 1640(\mathrm{~m}), 1604(\mathrm{~m}), 1506(\mathrm{~s}), 1476(\mathrm{~m}), 1428(\mathrm{~m}), 1365(\mathrm{~s}), 1268(\mathrm{~s}), 1222$ (s), $1195(\mathrm{~s}), 1185(\mathrm{~s}), 1109(\mathrm{~s}), 1011(\mathrm{~m}), 961(\mathrm{~m}), 905(\mathrm{~m}), 822(\mathrm{~s}), 742(\mathrm{~m}), 700(\mathrm{~m}), 635(\mathrm{~m})$. HRMS (ESI/OTOF) $\mathrm{m} / \mathrm{z}:[\mathrm{M}+\mathrm{Na}]^{+}$Calcd for $\mathrm{C}_{26} \mathrm{H}_{34} \mathrm{NaO}_{3}{ }^{+}$417.2400; Found 417.2405. 


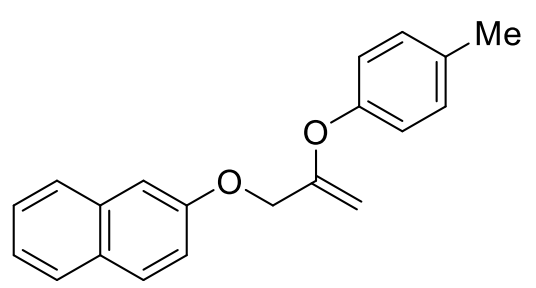

Starting from O-VBX 1a $(0.118 \mathrm{~g}, 0.300 \mathrm{mmol})$ and naphthalene-2ol $\quad 2 \mathbf{j} \quad(43.3 \quad \mathrm{mg}, \quad 0.300 \quad \mathrm{mmol}), \quad 2-((2-(p-$ tolyloxy)allyl)oxy)naphthalene $3 \mathbf{j}(62.4 \mathrm{mg}, 0.215 \mathrm{mmol}, 72 \%$ yield) and 4 a $(30.6 \mathrm{mg}, 77.6 \mu \mathrm{mol}, 26 \%)$ were obtained, as a colorless amorphous solids. Rf: 0.66 (Pentane:EtOAc 9:1). ${ }^{1} \mathbf{H}$ NMR (400 MHz, Chloroform-d) $\delta 7.78(\mathrm{td}, J=8.7,6.0 \mathrm{~Hz}, 3 \mathrm{H}, \mathrm{ArH}), 7.47$ (ddd, $J=8.2,6.8,1.3 \mathrm{~Hz}, 1 \mathrm{H}, \operatorname{ArH}), 7.37$ (ddd, $J=8.2,6.8,1.3 \mathrm{~Hz}, 1 \mathrm{H}, \operatorname{ArH}), 7.31-7.26(\mathrm{~m}, 2 \mathrm{H}, \operatorname{Ar} H$ ), $7.21-7.15(\mathrm{~m}, 2 \mathrm{H}, \mathrm{ArH}), 7.05-7.00(\mathrm{~m}, 2 \mathrm{H}, \mathrm{ArH}), 4.78\left(\mathrm{~s}, 2 \mathrm{H}, \mathrm{CCH}_{2} \mathrm{O}\right), 4.64(\mathrm{dd}, J=2.2,1.1 \mathrm{~Hz}, 1 \mathrm{H}$, $\mathrm{CH}_{2} \mathrm{CO}$ ), $4.27\left(\mathrm{~d}, J=2.3 \mathrm{~Hz}, 1 \mathrm{H}, \mathrm{CH}_{2} \mathrm{CO}\right), 2.36\left(\mathrm{~s}, 3 \mathrm{H}, \mathrm{CH}_{3}\right) .{ }^{13} \mathrm{C}$ NMR $(101 \mathrm{MHz}$, Chloroform-d) $\delta 158.5$, 156.5, 152.7, 134.6, 134.2, 130.3, 129.6, 129.3, 127.8, 127.0, 126.5, 124.0, 120.8, 119.1, 107.6, 90.8, 67.8, 20.9. IR v $3051(w), 2923(w), 1830(w), 1637(m), 1602(m), 1506(s), 1463(w), 1393(w), 1259$ (s), 1216 (s), $1182(\mathrm{~m}), 1127$ (w), 1024 (w), 963 (w), 839 (m), 741 (s), 714 (m). HRMS (APPI/LTQOrbitrap) m/z: [M] Calcd for $\mathrm{C}_{20} \mathrm{H}_{18} \mathrm{O}_{2}{ }^{+}$290.1301; Found 290.1295 .

Ethyl (S)-2-acetamido-3-(4-((2-(p-tolyloxy)allyl)oxy)phenyl)propanoate (3k)

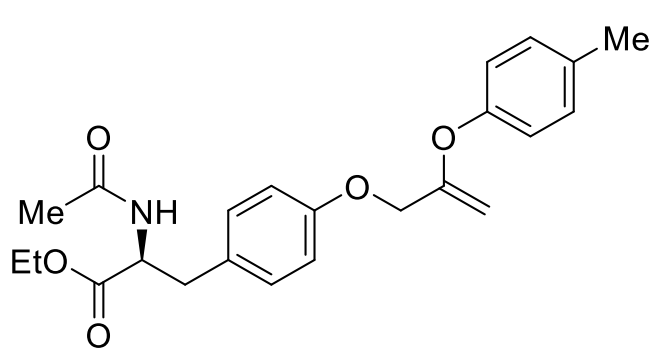

Starting from O-VBX 1a $(0.118 \mathrm{mg}, 0.300 \mathrm{mmol})$ and $(S)$ ethyl 2-acetamido-3-(4-hydroxyphenyl)propanoate hydrate $\mathbf{2 k}(81.0 \mathrm{mg}, 0.300 \mathrm{mmol})$, ethyl (S)-2-acetamido3-(4-((2-(p-tolyloxy)allyl)oxy)phenyl)propanoate $\quad 3 \mathbf{k}$ (0.115 g, $0.289 \mathrm{mmol}, 96 \%$ yield) was obtained, as a colorless amorphous solid. Rf: 0.45 (DCM:MeOH 9:1). Mixture of rotamers not identified yet. ${ }^{1} \mathbf{H}$ NMR $(400 \mathrm{MHz}$,

Acetonitrile- $\left.d_{3}\right) \delta 7.23-7.17(\mathrm{~m}, 2 \mathrm{H}, \mathrm{ArH}), 7.17-7.11(\mathrm{~m}, 2 \mathrm{H}, \mathrm{ArH}), 6.99-6.89(\mathrm{~m}, 4 \mathrm{H}, \mathrm{ArH}), 6.66(\mathrm{~d}$, $J=7.8 \mathrm{~Hz}, 1 \mathrm{H}, \mathrm{NH}), 4.63\left(\mathrm{~s}, 2 \mathrm{H}, \mathrm{OCH}_{2} \mathrm{CO}\right), 4.58-4.50\left(\mathrm{~m}, 2 \mathrm{H}\right.$, vinylH, $\left.\operatorname{ArCH}_{2} \mathrm{CHNH}\right), 4.13(\mathrm{~d}, J=2.1 \mathrm{~Hz}$, $1 \mathrm{H}$, vinylH), 4.09 (q, $J=7.1 \mathrm{~Hz}, 2 \mathrm{H}, \mathrm{OCH}_{2} \mathrm{CH}_{3}$ ), 3.02 (dd, $J=13.9,6.0 \mathrm{~Hz}, 1 \mathrm{H}, \mathrm{ArCH}_{2} \mathrm{CHNH}$ ), $2.95-2.85$ (m, $1 \mathrm{H}, \mathrm{ArCH} \mathrm{CHNH}_{2} \mathrm{CH}, 2.31$ (s, 3H, $\left.\mathrm{ArCH}_{3}\right), 1.85\left(\mathrm{~s}, 3 \mathrm{H}, \mathrm{COCH}_{3}\right), 1.17\left(\mathrm{t}, J=7.1 \mathrm{~Hz}, 3 \mathrm{H}, \mathrm{OCH}_{2} \mathrm{CH}_{3}\right) .{ }^{13} \mathrm{C} \mathrm{NMR}$ (101 MHz, Acetonitrile- $d_{3}$ ) $\delta$ 172.6, 170.6, 159.8, 158.3, 153.6, 135.1, 131.3, 131.2, 130.5, 121.4, 115.7, 91.6, 68.3, 61.8, 54.9, 37.4, 22.7, 20.7, 14.4. IR v3332 (w), 2991 (w), 2931 (w), 2866 (w), 2254 (w), 1737 $(\mathrm{m}), 1664(\mathrm{~m}), 1614(\mathrm{w}), 1505(\mathrm{~s}), 1442(\mathrm{w}), 1377(\mathrm{w}), 1221(\mathrm{~s}), 1127(\mathrm{~m}), 1024(\mathrm{w}), 910(\mathrm{~m}), 822(\mathrm{~m})$, 736 (s), 656 (w), 612 (w). HRMS (ESI/QTOF) m/z: [M + Na] ${ }^{+}$Calcd for $\mathrm{C}_{23} \mathrm{H}_{27} \mathrm{NNaO}_{5}{ }^{+}$420.1781; found 420.1781. $[\alpha]_{D}^{20}=+46.8\left(\mathrm{c}=1.00, \mathrm{CHCl}_{3}\right)$.

(E)-N-(4-((3-)((8R,9S,13S,14S,17S)-17-Hydroxy-13-methyl-7,8,9,11,12,13,14,15,16,17-decahydro-6Hcyclopenta[a]phenanthren-3-yl)oxy)prop-1-en-2-yl)oxy)-3-methoxybenzyl)-8-methylnon-6enamide (3I)

One pot procedure: In a glass vial, Capsaicin 21 (31.0 mg, $0.100 \mathrm{mmol}, 1.00$ equiv.) was dissolved in DME (0.1 M). Cesium carbonate ( $3.26 \mathrm{mg}, 10.0 \mu \mathrm{mol}, 0.10$ equiv.) was added and the mixture stirred vigorously for $5 \mathrm{~min}$. Then EBX 5a was added in one portion $(29.0 \mathrm{mg}, 0.100 \mathrm{mmol}, 1.00$ equiv.) and the reaction was left stirring for 16 hours at room temperature. Then cesium carbonate $(39.0 \mathrm{mg}, 0.120$ mmol, 1.20 equiv.) and Estradiol 2l' $(27.0 \mathrm{mg}, 0.100 \mathrm{mmol}, 1.00$ equiv.) were added and the solution was stirred at room temperature for 16 hours. The reaction mixture was filtrated, solvent was removed under reduced pressure and the crude material was purified by column chromatography 
(pentane:ethyl acetate 9:1) to provide compound 31 ( $25.0 \mathrm{mg}, 41.0 \mu \mathrm{mol}, 41 \%$ yield), as a white amorphous solid. Rf: 0.57 (DCM:MeOH 9:1). ${ }^{1} \mathrm{H}$ NMR (400 MHz, Acetonitrile- $\left.d_{3}\right) \delta 7.21(\mathrm{~d}, J=8.6 \mathrm{~Hz}$, $1 \mathrm{H}, \operatorname{Ar} H), 6.97(\mathrm{~d}, J=8.2 \mathrm{~Hz}, 2 \mathrm{H}, \operatorname{ArH}), 6.83(\mathrm{dd}, J=8.1,1.9 \mathrm{~Hz}, 1 \mathrm{H}, \operatorname{ArH}), 6.76(\mathrm{dd}, J=8.4,2.9 \mathrm{~Hz}, 1 \mathrm{H}$,

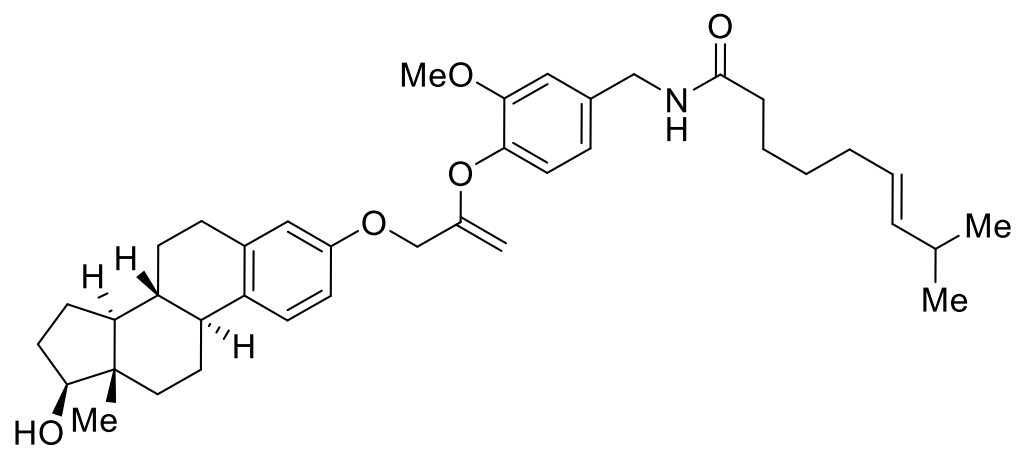
$\operatorname{ArH}), 6.71(\mathrm{~d}, J=2.9 \mathrm{~Hz}, 1 \mathrm{H}, \operatorname{ArH})$, $5.46-5.31(\mathrm{~m}, 2 \mathrm{H}, \mathrm{vinyl} H), 4.62(\mathrm{~s}$, $\left.2 \mathrm{H}, \mathrm{OCH}_{2} \mathrm{CO}\right), 4.39(\mathrm{~d}, \mathrm{~J}=2.2 \mathrm{~Hz}$, $\left.1 \mathrm{H}, \mathrm{OCCH}_{2}\right), 4.30(\mathrm{~d}, J=6.1 \mathrm{~Hz}, 2 \mathrm{H}$, $\mathrm{ArCH}_{2} \mathrm{NH}$ ), $3.92(\mathrm{~d}, J=2.2 \mathrm{~Hz}, 1 \mathrm{H}$, $\left.\mathrm{OCCH}_{2}\right), 3.81(\mathrm{~d}, J=4.8 \mathrm{~Hz}, 1 \mathrm{H}$, $\mathrm{OH}), 3.77\left(\mathrm{~s}, 3 \mathrm{H}, \mathrm{OCH}_{3}\right), 3.61(\mathrm{td}, \mathrm{J}$ $=8.5,4.2 \mathrm{~Hz}, 1 \mathrm{H}, \mathrm{CH}), 2.91-2.74$ $\left(\mathrm{m}, 2 \mathrm{H}, \mathrm{CH}_{2}\right), 2.64(\mathrm{~d}, J=6.4 \mathrm{~Hz}$, $1 \mathrm{H}, \mathrm{NH}), 2.31\left(\mathrm{dq}, J=12.3,3.7 \mathrm{~Hz}, 1 \mathrm{H}, \mathrm{CH}_{2}\right), 2.26-2.16(\mathrm{~m}, 2 \mathrm{H}, \mathrm{CH}+\mathrm{CH}), 2.15\left(\mathrm{~s}, 2 \mathrm{H}, \mathrm{CH}_{2}\right), 2.03-1.95$ $\left(\mathrm{m}, 1 \mathrm{H}, \mathrm{CH}_{2}\right.$ ), 1.87 (ddt, $\left.J=13.3,8.3,3.0 \mathrm{~Hz}, 2 \mathrm{H}, \mathrm{CH}_{2}\right), 1.67$ (ddt, $\left.J=12.2,5.1,2.6 \mathrm{~Hz}, 1 \mathrm{H}, \mathrm{CH}_{2}\right), 1.61-$ $1.55\left(\mathrm{~m}, 2 \mathrm{H}, \mathrm{CH}_{2}\right), 1.55-1.46\left(\mathrm{~m}, 1 \mathrm{H}, \mathrm{CH}_{2}\right), 1.45-1.40\left(\mathrm{~m}, 1 \mathrm{H}, \mathrm{CH}_{2}\right), 1.40-1.22\left(\mathrm{~m}, 6 \mathrm{H}, \mathrm{CH}_{2}+\mathrm{CH}_{2}+\right.$ $\mathrm{CH}_{2}+\mathrm{CH}_{2}+\mathrm{CH}_{2}$ ) , 1.17 (ddd, J=14.5, 11.3, $5.5 \mathrm{~Hz}, 2 \mathrm{H}, \mathrm{CH}_{2}+\mathrm{CH}_{2}$ ), 0.94 (d, J = 6.8 Hz, 4H, $\mathrm{CH}+\mathrm{CHCH}_{3}$ ), $0.86\left(\mathrm{~d}, J=6.6 \mathrm{~Hz}, 3 \mathrm{H}, \mathrm{CHCH}_{3}\right), 0.73\left(\mathrm{~s}, 3 \mathrm{H}, \mathrm{CCH}_{3}\right) .{ }^{13} \mathrm{C} \mathrm{NMR}\left(101 \mathrm{MHz}\right.$, Acetonitrile-d $\left.d_{3}\right) \delta$ 173.7, 159.7, 157.2, 152.5, 142.9, 139.1, 138.8, 138.7, 134.3, 127.8, 127.4, 123.3, 120.5, 115.8, 113.3, 113.2, 89.2, 82.0, 68.1, 56.5, 50.9, 44.9, 44.1, 43.1, 39.9, 37.7, 36.7, 32.9, 31.8, 30.9, 30.5, 30.0, 28.0, 27.3 26.1, 23.8, 23.0, 22.9, 11.7. IR v $3062(\mathrm{~m}), 2924(\mathrm{~s}), 2851(\mathrm{~m}), 1648(\mathrm{~s}), 1542(\mathrm{~s}), 1507(\mathrm{~s}), 1460(\mathrm{~m}), 1355$ (m), $1274(\mathrm{~s}), 1221(\mathrm{~s}), 1156(\mathrm{~s}), 1068(\mathrm{w}), 1025(\mathrm{~s}), 966(\mathrm{~m}), 826(\mathrm{~m}), 735$ (m). HRMS (ESI/QTOF) m/z:

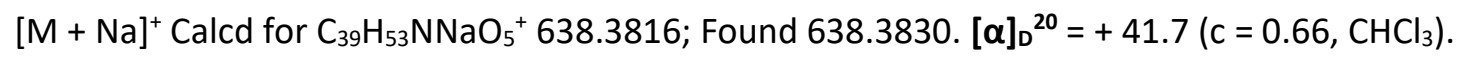

\section{2-Methyl-2-(2-(p-tolyloxy)allyl)cyclopentane-1,3-dione (3m)}

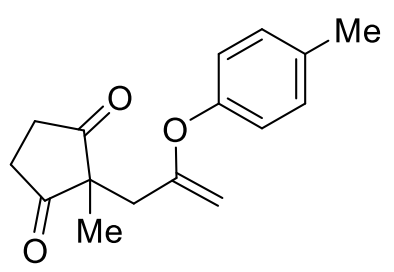

Starting from O-VBX 1a $(39.4 \mathrm{mg}, 0.100 \mathrm{mmol})$ and 2-methylcyclopentane1,3-dione $\quad 2 \mathrm{~m} \quad(11.2 \quad \mathrm{mg}, \quad 0.100 \quad \mathrm{mmol}), \quad 2$-methyl-2-(2-(ptolyloxy)allyl)cyclopentane-1,3-dione $3 \mathrm{~m}(8.00 \mathrm{mg}, 31.0 \mu \mathrm{mol}, 31 \%$ yield) was obtained, as a colorless amorphous solid. The allyl ester 4a was formed in 15\% NMR yield, but not isolated. Rf: 0.20 (Pentane:EtOAc 9:1). ${ }^{1} \mathbf{H}$ NMR (400 MHz, Chloroform-d) $\delta 7.10$ (d, $J=8.1 \mathrm{~Hz}, 2 \mathrm{H}, \mathrm{ArH}), 6.81-6.75$ (d, $J=$

$8.1 \mathrm{~Hz}, 2 \mathrm{H}, \mathrm{ArH}), 4.11\left(\mathrm{dd}, J=2.3,1.1 \mathrm{~Hz}, 1 \mathrm{H}, \mathrm{CH}_{2} \mathrm{CO}\right), 3.81\left(\mathrm{~d}, \mathrm{~J}=2.4 \mathrm{~Hz}, 1 \mathrm{H}, \mathrm{CH}_{2} \mathrm{CO}\right), 2.83\left(\mathrm{~s}, 2 \mathrm{H}, \mathrm{CCH}_{2} \mathrm{C}\right.$ ), $2.81-2.69\left(\mathrm{~m}, 4 \mathrm{H}, \mathrm{OCCH}_{2} \mathrm{CH}_{2} \mathrm{CO}\right), 2.31\left(\mathrm{~s}, 3 \mathrm{H}, \mathrm{ArCH}_{3}\right), 1.16\left(\mathrm{~s}, 3 \mathrm{H}, \mathrm{CCH}_{3}\right) .{ }^{13} \mathrm{C} \mathbf{N M R}(101 \mathrm{MHz}$, Chloroform-d) $\delta 216.0,158.1,151.8,134.6,130.4,120.8,89.0,54.1,39.9,34.9,20.9,20.9$. IR $v 3465$ $(\mathrm{m}), 3038(\mathrm{~m}), 2925(\mathrm{~m}), 2867(\mathrm{~m}), 1724(\mathrm{~s}), 1644(\mathrm{~m}), 1506(\mathrm{~s}), 1451(\mathrm{~m}), 1422(\mathrm{~m}), 1378(\mathrm{~m}), 1277$ (m), 1215 (s), $1159(\mathrm{~m}), 1067(\mathrm{~m}), 958(\mathrm{~m}), 894(\mathrm{~m}), 838(\mathrm{~m}), 739(\mathrm{~m}), 617(\mathrm{~m})$. HRMS (ESI/QTOF) m/z: $[\mathrm{M}+\mathrm{Na}]^{+}$Calcd for $\mathrm{C}_{16} \mathrm{H}_{18} \mathrm{NaO}_{3}{ }^{+}$281.1148; Found 281.1148.

\section{Ethyl 2-acetyl-2-methyl-4-( $p$-tolyloxy)pent-4-enoate (3n)}

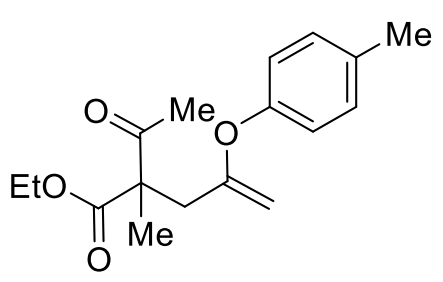

Starting from O-VBX 1a $(39.4 \mathrm{mg}, 0.100 \mathrm{mmol})$ and ethyl 2-methyl-3oxobutanoate $2 \mathrm{n}(15.2 \mathrm{mg}, 0.100 \mathrm{mmol})$, ethyl 2-acetyl-2-methyl-4-( $p$ tolyloxy)pent-4-enoate $3 \mathrm{n}$ (19.0 mg, $65.0 \mu \mathrm{mol}, 65 \%$ yield) was obtained, as a colorless amorphous solid. The allyl ester 4 a was formed in 15\% NMR yield, but not isolated. Rf: 0.42 (Pentane:EtOAc 9:1). ${ }^{1} \mathbf{H}$ NMR (400 MHz, Chloroform-d) $\delta 7.15-7.08(\mathrm{~m}, 2 \mathrm{H}, \mathrm{ArH}), 6.89-6.84$ (m, $2 \mathrm{H}, \mathrm{ArH}), 4.18\left(\mathrm{q}, J=7.1 \mathrm{~Hz}, 2 \mathrm{H}, \mathrm{OCH}_{2} \mathrm{CH}_{3}\right), 4.07$ (d, J = $\left.1.9 \mathrm{~Hz}, 1 \mathrm{H}, \mathrm{CH}_{2} \mathrm{CO}\right), 3.87(\mathrm{~d}, J=2.0 \mathrm{~Hz}, 1 \mathrm{H}$, 
$\left.\mathrm{CH}_{2} \mathrm{CO}\right), 2.95-2.78\left(\mathrm{~m}, 2 \mathrm{H}, \mathrm{CCH}_{2} \mathrm{C}\right), 2.31\left(\mathrm{~s}, 3 \mathrm{H}, \mathrm{ArCH}_{3}\right), 2.20\left(\mathrm{~s}, 3 \mathrm{H}, \mathrm{COCH}_{3}\right), 1.46\left(\mathrm{~s}, 3 \mathrm{H}, \mathrm{CCH}_{3}\right), 1.23$ (t, $\left.J=7.1 \mathrm{~Hz}, 3 \mathrm{H}, \mathrm{OCH}_{2} \mathrm{CH}_{3}\right) .{ }^{13} \mathrm{C}$ NMR $(101 \mathrm{MHz}$, Chloroform-d) $\delta 204.5,172.5,159.5,152.2,134.1,130.1$, 121.1, 90.2, 61.5, 58.5, 39.4, 26.1, 20.8, 18.7, 14.0. IR v 3039 (m), $2990(\mathrm{~m}), 2935$ (w), 1714 (s), 1640 $(\mathrm{m}), 1507(\mathrm{~m}), 1452(\mathrm{~m}), 1359(\mathrm{~m}), 1293(\mathrm{~m}), 1252(\mathrm{~s}), 1220(\mathrm{~s}), 1182(\mathrm{~s}), 1107(\mathrm{~m}), 1020(\mathrm{~m}), 971(\mathrm{~m})$, $932(\mathrm{~m}), 836(\mathrm{~m}), 740(\mathrm{~m}), 655(\mathrm{~m})$. HRMS (ESI/QTOF) m/z: $[\mathrm{M}+\mathrm{Na}]^{+}$Calcd for $\mathrm{C}_{17} \mathrm{H}_{22} \mathrm{NaO}_{4}{ }^{+} 313.1410$; Found 313.1414.

\section{$N$-(4-Methoxyphenyl)-4-nitro- $N$-(2-( $p$-tolyloxy)allyl)benzenesulfonamide (30)}<smiles>C=C(CN(c1ccc(OC)cc1)S(=O)(=O)c1ccc([N+](=O)[O-])cc1)Oc1ccc(C)cc1</smiles>

Starting from O-VBX 1a $(39.4 \mathrm{mg}, 0.100 \mathrm{mmol})$ and $\mathrm{N}$-(4methoxyphenyl)-4-nitrobenzenesulfonamide $20(31.0 \mathrm{mg}$, $0.100 \quad \mathrm{mmol}), \quad \mathrm{N}$-(4-methoxyphenyl)-4-nitro- $N$ - $(2-(p-$ tolyloxy)allyl) benzene sulfonamide $30(15.0 \mathrm{mg}, 33.0 \mu \mathrm{mol}$, $33 \%$ yield) was obtained, as a colorless amorphous solid. The allyl ester 4a was formed in 15\% NMR yield, but not isolated. Rf: 0.33 (Pentane:EtOAc 9:1). ${ }^{1} \mathbf{H}$ NMR (400 MHz, Methylene Chloride- $\left.d_{2}\right) \delta 8.30-8.21(\mathrm{~m}, 2 \mathrm{H}, \mathrm{ArH}), 7.89-7.79(\mathrm{~m}, 2 \mathrm{H}, \mathrm{ArH}), 7.14-7.07(\mathrm{~m}, 2 \mathrm{H}, \mathrm{ArH}), 7.06-6.98$ $(\mathrm{m}, 2 \mathrm{H}, \mathrm{ArH}), 6.92-6.79(\mathrm{~m}, 2 \mathrm{H}, \mathrm{ArH}), 6.76-6.66(\mathrm{~m}, 2 \mathrm{H}, \mathrm{ArH}), 4.37\left(\mathrm{~s}, 2 \mathrm{H}, \mathrm{CCH}_{2} \mathrm{~N}\right), 4.16(\mathrm{~d}, J=2.3 \mathrm{~Hz}$, $\left.1 \mathrm{H}, \mathrm{CH}_{2} \mathrm{CO}\right), 3.91\left(\mathrm{~d}, J=2.3 \mathrm{~Hz}, 1 \mathrm{H}, \mathrm{CH}_{2} \mathrm{CO}\right), 3.81\left(\mathrm{~s}, 3 \mathrm{H}, \mathrm{OCH}_{3}\right), 2.30\left(\mathrm{~s}, 3 \mathrm{H}, \mathrm{ArCH}_{3}\right) .{ }^{13} \mathrm{C} \mathrm{NMR}(101 \mathrm{MHz}$, Methylene Chloride- $\left.d_{2}\right) \delta 160.2,157.9,152.6,150.6,145.4,135.0,131.1,131.0,130.7,129.6,124.5$, 121.1, 114.9, 92.1, 56.0, 54.7, 21.0. IR $\vee 3340(\mathrm{~m}), 2970(\mathrm{~m}), 2880(\mathrm{~m}), 1435(\mathrm{~s}), 1307(\mathrm{~m}), 1091(\mathrm{~s})$, 1046 (s), 880 (s), 777 (s). HRMS (ESI/QTOF) m/z: [M + Na] $]^{+}$Calcd for $\mathrm{C}_{23} \mathrm{H}_{22} \mathrm{~N}_{2} \mathrm{NaO}_{6} \mathrm{~S}^{+} 477.1091$; Found 477.1091.

\section{(E)-4,4'-(Pent-1-ene-2,3-diylbis(oxy))bis(methylbenzene) (3p)}

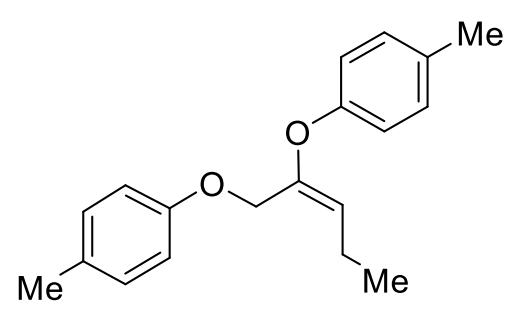

Starting from O-VBX 1b $(0.127 \mathrm{~g}, 0.300 \mathrm{mmol})$ and $p$-cresol $2 \mathrm{a}(32.4$ $\mathrm{mg}, \quad 0.300 \quad \mathrm{mmol}), \quad(E)-4,4$ '-(pent-1-ene-2,3diylbis(oxy))bis(methylbenzene) $3 p$ (55.0 mg, $0.195 \mathrm{mmol}, 65 \%$ yield) and $\mathbf{4 b}(40.5 \mathrm{mg}, 95.6 \mu \mathrm{mol}, 32 \%)$ were obtained, as a colorless amorphous solids. Rf: 0.65 (Pentane:EtOAc 9:1). ${ }^{1} \mathbf{H}$ NMR (400 MHz, Acetonitrile- $d_{3}$ ) $\delta 7.13$ (dd, $J=7.4,1.4 \mathrm{~Hz}, 2 \mathrm{H}, \mathrm{ArH}$ ), 7.11 - $7.06(\mathrm{~m}, 2 \mathrm{H}, \mathrm{ArH}), 6.90-6.86(\mathrm{~m}, 2 \mathrm{H}, \mathrm{ArH}), 6.86-6.81(\mathrm{~m}, 2 \mathrm{H}, \mathrm{ArH}), 5.05(\mathrm{t}, J=7.9 \mathrm{~Hz}, 1 \mathrm{H}, \mathrm{CCHO})$, $4.61\left(\mathrm{~s}, 2 \mathrm{H}, \mathrm{CH}_{2} \mathrm{CO}\right), 2.28\left(\mathrm{~s}, 3 \mathrm{H}, \mathrm{ArCH}_{3}\right), 2.25$ (s, 3H, $\left.\mathrm{ArCH}_{3}\right), 2.13$ (p, J = 7.5 Hz, 2H, $\mathrm{CHCH}_{2} \mathrm{CH}_{3}$ ), 0.95 (t, $\left.J=7.5 \mathrm{~Hz}, 3 \mathrm{H}, \mathrm{CHCH}_{2} \mathrm{CH}_{3}\right) .{ }^{13} \mathrm{C} \mathrm{NMR}(101 \mathrm{MHz}$, Chloroform-d) $\delta 156.6,154.0,149.6,132.5,130.3,130.0$, 129.8, 119.1, 116.8, 115.0, 64.4, 20.7, 20.5, 20.0, 14.8. IR $3665(\mathrm{~m}), 3408(\mathrm{~m}), 2968(\mathrm{~s}), 2929(\mathrm{~s}), 1722$ $(\mathrm{m}), 1611(\mathrm{~m}), 1512(\mathrm{~s}), 1441(\mathrm{~m}), 1385(\mathrm{~m}), 1239(\mathrm{~s}), 1170(\mathrm{~m}), 1061(\mathrm{~s}), 890(\mathrm{~m}), 820(\mathrm{~s}), 738(\mathrm{~m})$. HRMS (ESI/QTOF) m/z: [M + Na] ${ }^{+}$Calcd for $\mathrm{C}_{19} \mathrm{H}_{22} \mathrm{NaO}_{2}{ }^{+}$305.1512; Found 305.1514.

\section{(E)-4,4'-(Oct-2-ene-1,2-diylbis(oxy))bis(methylbenzene) (3q)}<smiles>CCCCC/C=C(/COc1ccc(C)cc1)Oc1ccc(C)cc1</smiles>
Starting from O-VBX 1c $(0.139 \mathrm{~g}, 0.300 \mathrm{mmol})$ and $p$-cresol 2a $(32.4$ $\mathrm{mg}, \quad 0.300 \quad \mathrm{mmol}), \quad(E)-4,4$ '-(oct-2-ene-1,2diylbis(oxy))bis(methylbenzene) 3q (55.0 mg, $0.170 \mathrm{mmol}, 57 \%$ yield) and $4 \mathrm{c}(44.6 \mathrm{mg}, 95.3 \mu \mathrm{mol}, 32 \%)$ were obtained, as a colorless amorphous solids. Rf: 0.8 (Pentane:EtOAc 9:1). ${ }^{\mathbf{1}} \mathbf{H}$ NMR $\left(400 \mathrm{MHz}\right.$, Acetonitrile- $\left.d_{3}\right) \delta 7.16-7.11(\mathrm{~m}, 2 \mathrm{H}, \mathrm{ArH}), 7.11-7.06$ $(\mathrm{m}, 2 \mathrm{H}, \mathrm{ArH}), 6.90-6.85(\mathrm{~m}, 2 \mathrm{H}, \mathrm{ArH}), 6.85-6.81(\mathrm{~m}, 2 \mathrm{H}, \mathrm{ArH}), 5.05\left(\mathrm{t}, J=8.0 \mathrm{~Hz}, 1 \mathrm{H}, \mathrm{OCCHCH}_{2}\right), 4.61$ 
(s, 2H, OCH $\mathrm{OCO}_{2}, 2.28\left(\mathrm{~s}, 3 \mathrm{H}, \mathrm{ArCH}_{3}\right), 2.25\left(\mathrm{~s}, 3 \mathrm{H}, \mathrm{ArCH}_{3}\right), 2.10$ (q, J = 7.5 Hz, 2H, CH $\left.\mathrm{CH}_{2}\right), 1.40-1.30(\mathrm{~m}$, $\left.2 \mathrm{H}, \mathrm{CH}_{2}\right), 1.30-1.23\left(\mathrm{~m}, 4 \mathrm{H}, \mathrm{CH}_{2} \mathrm{CH}_{2}\right), 0.87\left(\mathrm{t}, J=6.8 \mathrm{~Hz}, 3 \mathrm{H}, \mathrm{CH}_{3}\right) .{ }^{13} \mathrm{C}$ NMR (101 MHz, Acetonitrile- $d_{3}$ ) $\delta 157.5,155.1,150.7,133.4,131.3,131.0,130.8,119.6,116.9,115.8,64.7,32.0,30.3,26.9,23.1,20.6$, 20.4, 14.3. IR $v 3669$ (m), 2976 (s), 2912 (s), 1506 (s), 1388 (s), 1220 (s), 1057 (s), 890 (m), 815 (m), 723 (m). HRMS (ESI/QTOF) m/z: [M + Na $]^{+}$Calcd for $\mathrm{C}_{22} \mathrm{H}_{28} \mathrm{NaO}_{2}{ }^{+}$347.1982; Found 347.1972.

\section{(E)-6,7-bis(p-Tolyloxy)hept-5-en-1-ol (3r)}<smiles>Cc1ccc(OC/C(=C/CCCCO)Oc2ccc(C)cc2)cc1</smiles>

Starting from O-VBX 1d $(0.140 \mathrm{~g}, 0.300 \mathrm{mmol})$ and $p$-cresol $2 \mathrm{a}(32.4$ $\mathrm{mg}, 0.300 \mathrm{mmol})$, and following the general procedure with $24 \mathrm{~h}$ reaction time, $(E)$-6,7-bis( $p$-tolyloxy)hept-5-en-1-ol $3 r(63.0 \mathrm{mg}$, $0.193 \mathrm{mmol}, 64 \%$ yield) was obtained, as a colorless amorphous solid. Rf: 0.8 (DCM:MeOH 9:1). ${ }^{1} \mathbf{H}$ NMR (400 MHz, Acetonitrile- $d_{3}$ ) $\delta 7.16-7.09(\mathrm{~m}, 2 \mathrm{H}, \mathrm{ArH}), 7.11-7.06(\mathrm{~m}, 2 \mathrm{H}, \mathrm{ArH}), 6.90-6.86(\mathrm{~m}$, $2 \mathrm{H}, \mathrm{ArH}), 6.86-6.81(\mathrm{~m}, 2 \mathrm{H}, \mathrm{ArH}), 5.05\left(\mathrm{t}, J=8.0 \mathrm{~Hz}, 1 \mathrm{H}, \mathrm{OCCHCH}_{2}\right), 4.61\left(\mathrm{~s}, 2 \mathrm{H}, \mathrm{OCH}_{2} \mathrm{CO}\right), 3.46(\mathrm{t}, J=$ $6.4 \mathrm{~Hz}, 2 \mathrm{H}, \mathrm{CH}_{2} \mathrm{OH}$ ), $2.28\left(\mathrm{~s}, 3 \mathrm{H}, \mathrm{ArCH}_{3}\right), 2.26\left(\mathrm{~s}, 3 \mathrm{H}, \mathrm{ArCH}_{3}\right), 2.13$ (q, J = 7.5 Hz, 2H, CH2), $1.51-1.42(\mathrm{~m}$, $\left.2 \mathrm{H}, \mathrm{CH}_{2}\right), 1.41-1.34\left(\mathrm{~m}, 2 \mathrm{H}, \mathrm{CH}_{2}\right) .{ }^{13} \mathrm{C}$ NMR $\left(101 \mathrm{MHz}\right.$, Acetonitrile- $\left.d_{3}\right) \delta$ 157.5, 155.1, 150.9, 133.5, 131.3, 131.0, 130. 8, 119.6, 116.6, 115.8, 64.7, 62.3, 32.9, 27.0, 26.8, 20.6, 20.4. IR v 3001 (m), 2944 (m), $1608(\mathrm{~m}), 1507(\mathrm{~s}), 1224(\mathrm{~s}), 1063(\mathrm{~m}), 1013$ (s), $916(\mathrm{~m}), 821(\mathrm{~s}), 741(\mathrm{~m})$. HRMS (ESI/QTOF) m/z: $[\mathrm{M}+\mathrm{Na}]^{+}$Calcd for $\mathrm{C}_{21} \mathrm{H}_{26} \mathrm{NaO}_{3}{ }^{+} 349.1774$; Found 349.1771.

\section{(E)-(7,8-bis(p-Tolyloxy)oct-6-en-1-yn-1-yl)trimethylsilane (3s)}

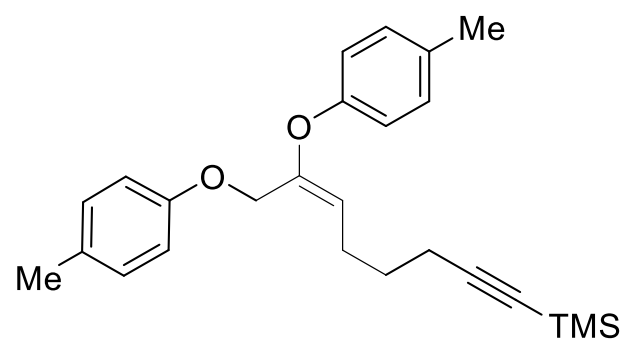

Starting from O-VBX $1 \mathrm{e}(0.160 \mathrm{~g}, 0.300 \mathrm{mmol})$ and $p$-cresol 2a (32.4 mg, $0.300 \mathrm{mmol}),(E)-(7,8$-bis $(p$-tolyloxy)oct-6-en1-yn-1-yl)trimethylsilane $3 s$ ( $75.0 \mathrm{mg}, 0.191 \mathrm{mmol}, 64 \%$ yield) and $4 \mathrm{e}(41.5 \mathrm{mg}, 77.9 \mu \mathrm{mol}, 26 \%)$ were obtained, as a colorless amorphous solids. Rf: 0.8 (Pentane:EtOAc 9:1). ${ }^{1} \mathbf{H}$ NMR (400 MHz, Acetonitrile- $\left.d_{3}\right) \delta 7.16-7.11(\mathrm{~m}, 2 \mathrm{H}, \mathrm{ArH})$, $7.11-7.07(\mathrm{~m}, 2 \mathrm{H}, \mathrm{ArH}), 6.91-6.86(\mathrm{~m}, 2 \mathrm{H}, \mathrm{ArH}), 6.86-$ $6.82(\mathrm{~m}, 2 \mathrm{H}, \mathrm{ArH}), 5.00\left(\mathrm{t}, J=8.0 \mathrm{~Hz}, 1 \mathrm{H}, \mathrm{OCCHCH}_{2}\right), 4.63\left(\mathrm{~s}, 2 \mathrm{H}, \mathrm{OCH}_{2} \mathrm{CO}\right), 2.28\left(\mathrm{~s}, 3 \mathrm{H}, \mathrm{ArCH}_{3}\right), 2.25(\mathrm{~s}$, $\left.3 \mathrm{H}, \mathrm{ArCH}_{3}\right), 2.20\left(\mathrm{td}, J=7.3,3.3 \mathrm{~Hz}, 4 \mathrm{H}, \mathrm{CH}_{2} \mathrm{CH}_{2}\right), 1.50\left(\mathrm{p}, J=7.1 \mathrm{~Hz}, 2 \mathrm{H}, \mathrm{CH}_{2}\right), 0.08\left(\mathrm{~s}, 9 \mathrm{H}, \mathrm{Si}\left(\mathrm{CH}_{3}\right)_{3}\right) .{ }^{13} \mathrm{C}$ NMR (101 MHz, Acetonitrile- $\left.d_{3}\right) \delta$ 157. 5, 154.9, 151.7, 133.6, 131.3, 131.1, 130.8, 119.8, 115.9, 115.1, 108.1, 85. 5, 64.8, 29.5, 25.9, 20.6, 20.5, 19.5, 0.1. IR v 3621 (w), $3096(w), 2964$ (w), $2602(w), 2262$ (s), $1633(\mathrm{~m}), 1514(\mathrm{~m}), 1227(\mathrm{~m}), 1187(\mathrm{~m}), 1038(\mathrm{~s}), 841$ (s), $681(\mathrm{w})$. HRMS (ESI/QTOF) m/z: [M + Na] Calcd for $\mathrm{C}_{25} \mathrm{H}_{32} \mathrm{NaO}_{2} \mathrm{Si}^{+}$415.2064; Found 415.2070.

\section{(E)-2-(5,6-bis(p-Tolyloxy)hex-4-en-1-yl)isoindoline-1,3-dione (3t)}

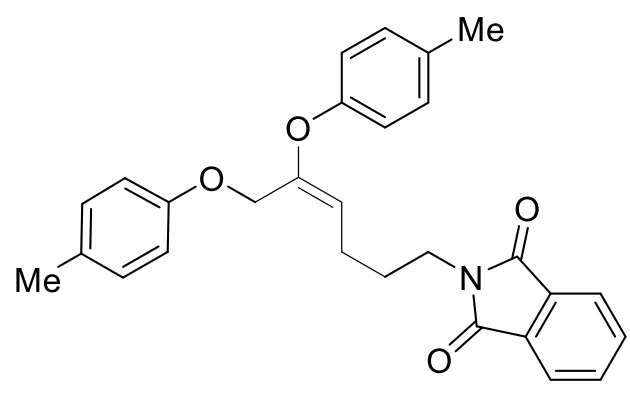
Starting from O-VBX 1f (174 $\mathrm{mg}, 0.300 \mathrm{mmol}$ ) and $p$-cresol 2a (32.4 mg, $0.300 \mathrm{mmol}),(E)-2-(5,6$-bis( $p$-tolyloxy)hex-4en-1-yl)isoindoline-1,3-dione $3 t$ ( $86.3 \mathrm{mg}, 0.195 \mathrm{mmol}, 65 \%$ yield with $10 \%$ impurities) and $\mathbf{4 f}$ ( $50.6 \mathrm{mg}, 86.7 \mu \mathrm{mol}, 29 \%)$ were obtained, as a colorless amorphous solids. Rf: 0.40 (Pentane:EtOAc 9:1). ${ }^{1} \mathbf{H}$ NMR (400 MHz, Acetonitrile- $d_{3}$ ) $\delta$ $7.81-7.74(\mathrm{~m}, 4 \mathrm{H}, \mathrm{ArH}), 7.11(\mathrm{dd}, J=7.4,1.4 \mathrm{~Hz}, 2 \mathrm{H}, \mathrm{ArH})$, $7.07-7.03(\mathrm{~m}, 2 \mathrm{H}, \mathrm{ArH}), 6.90-6.84(\mathrm{~m}, 2 \mathrm{H}, \mathrm{ArH}), 6.82-$ $6.76(\mathrm{~m}, 2 \mathrm{H}, \mathrm{ArH}), 5.03(\mathrm{t}, J=8.0 \mathrm{~Hz}, 1 \mathrm{H}, \mathrm{OCCH}), 4.59\left(\mathrm{~s}, 2 \mathrm{H}, \mathrm{OCH}_{2} \mathrm{CO}\right), 3.60\left(\mathrm{t}, J=7.0 \mathrm{~Hz}, 2 \mathrm{H}, \mathrm{CH}_{2}\right), 2.28$ 
(s, 3H, $\left.\mathrm{CH}_{3}\right), 2.24\left(\mathrm{~s}, 3 \mathrm{H}, \mathrm{CH}_{3}\right), 2.16\left(\mathrm{q}, J=7.7 \mathrm{~Hz}, 2 \mathrm{H}, \mathrm{CH}_{2}\right), 1.69\left(\mathrm{p}, J=7.2 \mathrm{~Hz}, 2 \mathrm{H}, \mathrm{CH}_{2}\right) .{ }^{13} \mathrm{C}$ NMR (101 $\mathrm{MHz}$, Acetonitrile- $\left.d_{3}\right) \delta 169.3,157.4,154.8,151.5,135.0,133.6,133.2,131.3,131.0,130.8,123.7$, 119.7, 115.8, 115.1, 64.9, 38.1, 29.4, 24.5, 20.6, 20.4. IR $v 2934(\mathrm{~m}), 1770(\mathrm{~m}), 1712(\mathrm{~s}), 1609(\mathrm{~m}), 1508$ $(\mathrm{s}), 1459(\mathrm{~m}), 1397(\mathrm{~m}), 1364(\mathrm{~m}), 1221(\mathrm{~s}), 1173(\mathrm{~m}), 1114(\mathrm{~m}), 1020(\mathrm{~m}), 899(\mathrm{~m}), 815(\mathrm{~m}), 720(\mathrm{~m})$. HRMS (ESI/QTOF) $\mathrm{m} / \mathrm{z}:[\mathrm{M}+\mathrm{Na}]^{+}$Calcd for $\mathrm{C}_{28} \mathrm{H}_{27} \mathrm{NNaO}_{4}{ }^{+}$464.1832; Found 464.1840.

\section{1-lodo-2-((3-(p-tolyloxy)prop-1-en-2-yl)oxy)benzene (3u)}

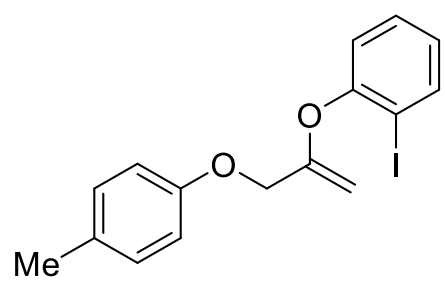

Starting from O-VBX $1 \mathrm{~g}(152 \mathrm{mg}, 0.300 \mathrm{mmol})$ and $p$-cresol 2a $(32.4 \mathrm{mg}$, $0.300 \mathrm{mmol}), 1$-iodo-2-((3-( $p$-tolyloxy)prop-1-en-2-yl)oxy)benzene $3 \mathbf{u}$ (66.5 $\mathrm{mg}, 0.182 \mathrm{mmol}, 61 \%$ yield) was obtained, as a yellow amorphous solid and $\mathbf{4 i}$ (11.8 mg, $29.9 \mu \mathrm{mol}, 10 \%)$ was obtained, as a colorless amorphous solid. Rf: 0.7 (Pentane:EtOAc 9:1). ${ }^{1} \mathbf{H}$ NMR $(400 \mathrm{MHz}$, Acetonitrile- $\left.d_{3}\right) \delta 7.89$ (dd, $\left.J=7.9,1.6 \mathrm{~Hz}, 1 \mathrm{H}, \mathrm{ArH}\right), 7.42$ (ddd, $J=8.0$, 7.4, $1.6 \mathrm{~Hz}, 1 \mathrm{H}, \mathrm{ArH}), 7.18-7.08(\mathrm{~m}, 3 \mathrm{H}, \mathrm{ArH}), 6.99(\mathrm{td}, J=7.6,1.5 \mathrm{~Hz}, 1 \mathrm{H}, \mathrm{ArH}), 6.95-6.89(\mathrm{~m}, 2 \mathrm{H}$, $\operatorname{ArH}), 4.68\left(\mathrm{~d}, J=0.9 \mathrm{~Hz}, 2 \mathrm{H}, \mathrm{OCH}_{2} \mathrm{CO}\right), 4.57\left(\mathrm{dt}, J=2.5,1.0 \mathrm{~Hz}, 1 \mathrm{H}, \mathrm{OCCH}_{2}\right), 4.00(\mathrm{~d}, J=2.5 \mathrm{~Hz}, 1 \mathrm{H}$, $\left.\mathrm{OCCH}_{2}\right), 2.27\left(\mathrm{~s}, 3 \mathrm{H}, \mathrm{CH}_{3}\right) .{ }^{13} \mathrm{C} \mathrm{NMR}\left(101 \mathrm{MHz}\right.$, Acetonitrile- $\left.d_{3}\right) \delta 158.7,157.2,155.3,140.8,131.5,131.0$, 130.8, 127.7, 123.0, 115.8, 91.3, 90.5, 68.1, 20.4. IR v $3667(\mathrm{~m}), 2991(\mathrm{~s}), 2908(\mathrm{~s}), 1512(\mathrm{~m}), 1401(\mathrm{~m})$, $1231(\mathrm{~s}), 1068(\mathrm{~s}), 1028(\mathrm{~s}), 905(\mathrm{~m}), 825(\mathrm{~m}), 742(\mathrm{~m})$. HRMS (APPI/QTOF) m/z: [M] ${ }^{+}$Calcd for $\mathrm{C}_{16} \mathrm{H}_{15} \mathrm{IO}_{2}{ }^{+}$ 366.0111; Found 366.0111.

\section{(E)-1-lodo-2-((1-(p-tolyloxy)pent-2-en-2-yl)oxy)benzene (3v)}

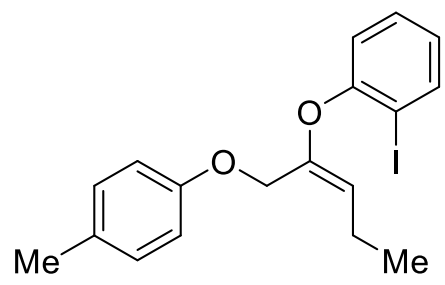

Starting from O-VBX $1 \mathrm{~h}(160 \mathrm{mg}, 0.300 \mathrm{mmol})$ and $p$-cresol 2a $(32.4 \mathrm{mg}$, $0.300 \mathrm{mmol}),(E)$-1-iodo-2-((1-( $p$-tolyloxy)pent-2-en-2-yl)oxy)benzene $3 v(70.0 \mathrm{mg}, 0.178 \mathrm{mmol}, 59 \%$ yield) and $4 \mathrm{j}$ ( $51.9 \mathrm{mg}, 0.121 \mathrm{mmol}, 41 \%)$ were obtained, as a colorless amorphous solids. Rf: 0.61 (Pentane:EtOAc 9:1). ${ }^{1} \mathrm{H}$ NMR $\left(400 \mathrm{MHz}\right.$, Acetonitrile- $\left.d_{3}\right) \delta 7.82(\mathrm{dd}, J=$ 7.9, $1.5 \mathrm{~Hz}, 1 \mathrm{H}, \mathrm{ArH}), 7.34$ (ddd, J = 8.2, 7.3, $1.6 \mathrm{~Hz}, 1 \mathrm{H}, \operatorname{ArH}), 7.13-7.06$ $(\mathrm{m}, 2 \mathrm{H}, \mathrm{ArH}), 7.04(\mathrm{dd}, J=8.2,1.4 \mathrm{~Hz}, 1 \mathrm{H}, \mathrm{ArH}), 6.93-6.79(\mathrm{~m}, 3 \mathrm{H}, \mathrm{ArH}), 5.04(\mathrm{t}, J=7.9 \mathrm{~Hz}, 1 \mathrm{H}, \mathrm{OCCH})$, $4.66\left(\mathrm{~s}, 2 \mathrm{H}, \mathrm{OCH}_{2} \mathrm{CO}\right.$ ), 2.25 (s, 3H, $\left.\mathrm{ArCH}_{3}\right), 2.14$ (q, J=7.8 Hz, 2H, $\left.\mathrm{CH}_{2}\right), 0.97$ (t, J=7.5 Hz, 3H, $\mathrm{CH}_{3}$ ). ${ }^{13} \mathrm{C}$ NMR $\left(101 \mathrm{MHz}\right.$, Acetonitrile- $\left.d_{3}\right) \delta 157.4,156.6,149.6,140.6,131.4,130.8,130.7,126.0,119.9,119.1$, 115.9, 88.9, 64.7, 20.5, 20. 5, 14.9. IR $v 3671(w), 2969(\mathrm{~m}), 2872(w), 2604(w), 2360(w), 1758(w)$, $1680(\mathrm{w}), 1612(\mathrm{~m}), 1580(\mathrm{~m}), 1510(\mathrm{~s}), 1465(\mathrm{~s}), 1439(\mathrm{~m}), 1393(\mathrm{w}), 1259(\mathrm{~m}), 1227(\mathrm{~s}), 1174(\mathrm{~m}), 1119$ $(\mathrm{m}), 1108(\mathrm{~m}), 1041(\mathrm{~m}), 1020(\mathrm{~s}), 939(\mathrm{~m}), 887(\mathrm{~m}), 817(\mathrm{~m}), 752(\mathrm{~m}), 718(\mathrm{w}), 687(\mathrm{w}), 645(\mathrm{w})$. HRMS (ESI/QTOF) $\mathrm{m} / \mathrm{z}:[\mathrm{M}+\mathrm{Na}]^{+}$Calcd for $\mathrm{C}_{18} \mathrm{H}_{19} \mathrm{INaO}_{2}{ }^{+}$417.0322; Found 417.0323 .

\section{(E)-1-lodo-2-((1-(p-tolyloxy)oct-2-en-2-yl)oxy)benzene (3w)}

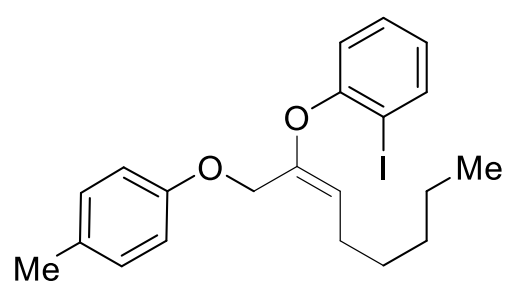

Starting from O-VBX 1i (173 mg, $0.300 \mathrm{mmol})$ and $p$-cresol 2a (32.4 $\mathrm{mg}, \quad 0.300 \quad \mathrm{mmol}), \quad(E)$-1-iodo-2-((1-( $p$-tolyloxy)oct-2-en-2yl)oxy)benzene $3 \mathbf{w}(57.0 \mathrm{mg}, 0.131 \mathrm{mmol}, 44 \%$ yield) and $\mathbf{4 k}$ (48.7 $\mathrm{mg}, 0.105 \mu \mathrm{mol}, 35 \%)$ were obtained, as a colorless amorphous solids. Rf: 0.86 (Pentane:EtOAc 9:1). ${ }^{1} \mathbf{H}$ NMR (400 MHz, Acetonitrile- $\left.d_{3}\right) \delta 7.83(\mathrm{dd}, J=7.9,1.5 \mathrm{~Hz}, 1 \mathrm{H}, \operatorname{ArH}), 7.35$ (ddd, $J=$ 8.1, 7.4, 1.6 Hz, 1H, ArH), 7.12-7.07 (m, 2H, ArH), 7.03 (dd, J = 8.2, 1.5 Hz, 1H, ArH), $6.90-6.82(\mathrm{~m}$, $3 \mathrm{H}, \mathrm{ArH}), 5.01(\mathrm{t}, J=8.0 \mathrm{~Hz}, 1 \mathrm{H}, \mathrm{OCCH}), 4.66\left(\mathrm{~s}, 2 \mathrm{H}, \mathrm{OCH}_{2} \mathrm{CO}\right), 2.26\left(\mathrm{~s}, 3 \mathrm{H}, \mathrm{ArCH}_{3}\right), 2.18-2.08(\mathrm{~m}, 2 \mathrm{H}$, $\left.\mathrm{CH}_{2}\right), 1.39-1.22\left(\mathrm{~m}, 6 \mathrm{H}, \mathrm{CH}_{2}\right), 0.91-0.83\left(\mathrm{~m}, 3 \mathrm{H}, \mathrm{CH}_{3}\right) .{ }^{13} \mathrm{C}$ NMR $\left(101 \mathrm{MHz}\right.$, Acetonitrile- $\left.d_{3}\right) \delta 157.4$, 
156.7, 150.0, 140.6, 131.4, 130.8, 130.7, 126.1, 120.0, 117.4, 116.0, 89.0, 64.8, 31.9, 30.2, 26.9, 23.1, 20.4, 14.3. IR $\vee 3666(\mathrm{~m}), 2979(\mathrm{~s}), 2902(\mathrm{~s}), 2322(\mathrm{w}), 2261(\mathrm{~m}), 2069(\mathrm{w}), 1643(\mathrm{w}), 1443(\mathrm{w}), 1401$ (m), $1238(\mathrm{~m}), 1056(\mathrm{~s}), 888(\mathrm{~m}), 840(\mathrm{~m}), 687(\mathrm{w})$. HRMS (ESI/QTOF) m/z: [M + Na] ${ }^{+}$Calcd for $\mathrm{C}_{21} \mathrm{H}_{25} \mathrm{INaO}_{2}{ }^{+}$459.0791; Found 459.0794 .

\section{(E)-1-Methoxy-4-((1-(p-tolyloxy)pent-2-en-2-yl)oxy)benzene (3x)}<smiles>CC/C=C(/COc1ccc(C)cc1)Oc1ccc(OC)cc1</smiles>

Starting from O-VBX $1 \mathrm{j}(1 \mathrm{~g}, 2.28 \mathrm{mmol})$ and $p$-cresol $2 \mathrm{a}(0.247 \mathrm{~g}$, $2.28 \mathrm{mmol}), \quad(E)$-1-methoxy-4-((1-(p-tolyloxy)pent-2-en-2yl)oxy)benzene $3 x(0.427 \mathrm{~g}, 1.43 \mathrm{mmol}, 63 \%$ yield) and $4 \mathrm{n}(0.164$ $\mathrm{g}, 0.340 \mathrm{mmol}, 15 \%)$ were obtained, as a colorless amorphous solids. Rf: 0.57 (Pentane:EtOAc 9:1). ${ }^{1} \mathbf{H}$ NMR (400 MHz, Acetonitrile- $\left.d_{3}\right) \delta 7.13-7.07(\mathrm{~m}, 2 \mathrm{H}, \mathrm{ArH}), 6.95-6.81(\mathrm{~m}, 6 \mathrm{H}$, $\operatorname{ArH}), 4.94(\mathrm{t}, J=7.9 \mathrm{~Hz}, 1 \mathrm{H}, \mathrm{OCCH}), 4.62\left(\mathrm{~s}, 2 \mathrm{H}, \mathrm{OCH}_{2} \mathrm{CO}\right), 3.75\left(\mathrm{~s}, 3 \mathrm{H}, \mathrm{OCH}_{3}\right), 2.26\left(\mathrm{~s}, 3 \mathrm{H}, \mathrm{CH}_{3}\right), 2.11(\mathrm{p}$, $\left.J=7.6 \mathrm{~Hz}, 2 \mathrm{H}, \mathrm{CH}_{2}\right), 0.93\left(\mathrm{t}, J=7.5 \mathrm{~Hz}, 3 \mathrm{H}, \mathrm{CH}_{3}\right) \cdot{ }^{13} \mathrm{C}$ NMR $\left(101 \mathrm{MHz}\right.$, Acetonitrile- $\left.d_{3}\right) \delta 157.5,156.7$, 151.4, 150.4, 131.3, 130.8, 121.4, 116.4, 115.8, 115.6, 64.8, 56.1, 20.4 (2C), 15.1. IR v 3666 (m), 2979 (s), $2902(\mathrm{~s}), 2333(\mathrm{~m}), 2260(\mathrm{~m}), 1938(\mathrm{w}), 1617(\mathrm{~m}), 1506(\mathrm{~m}), 1445(\mathrm{~m}), 1400(\mathrm{~m}), 1240(\mathrm{~m}), 1056(\mathrm{~s})$, $874(\mathrm{~m}), 839(\mathrm{~m}), 742(\mathrm{w}), 687(\mathrm{~m})$. HRMS (APPI/QTOF) m/z: [M] ${ }^{+}$Calcd for $\mathrm{C}_{19} \mathrm{H}_{22} \mathrm{O}_{3}{ }^{+}$298.1563; Found 298.1551.

\section{1-Methoxy-4-((3-(p-tolyloxy)prop-1-en-2-yl)oxy)benzene (3y)}<smiles>C=C(COc1ccc(C)cc1)Oc1ccc(OC)cc1</smiles>

Starting from O-VBX 1k (123 mg, $0.300 \mathrm{mmol})$ and $p$-cresol $2 \mathbf{a}$ (32.4 mg, $0.300 \mathrm{mmol}), 1$-methoxy-4-((3-(p-tolyloxy)prop-1-en2-yl)oxy)benzene 3y (45.1 mg, $0.167 \mathrm{mmol}, 56 \%$ yield) and 40 (48.3 $\mathrm{mg}, 0.122 \mathrm{mmol}, 41 \%)$ were obtained, as a colorless amorphous solids. Rf: 0.60 (Pentane:EtOAc 9:1). ${ }^{1} \mathbf{H}$ NMR (400 $\mathrm{MHz}$, Acetonitrile- $\left.d_{3}\right) \delta 7.14-7.09(\mathrm{~m}, 2 \mathrm{H}, \mathrm{ArH}), 7.02-6.96(\mathrm{~m}$, $2 \mathrm{H}, \mathrm{ArH}), 6.95-6.87(\mathrm{~m}, 4 \mathrm{H}, \mathrm{ArH}), 4.61\left(\mathrm{~d}, J=0.8 \mathrm{~Hz}, 2 \mathrm{H}, \mathrm{OCH}_{2} \mathrm{CO}\right), 4.47\left(\mathrm{dd}, J=2.0,1.0 \mathrm{~Hz}, 1 \mathrm{H}, \mathrm{OCCH}_{2}\right)$, $4.04\left(\mathrm{~d}, J=2.1 \mathrm{~Hz}, 1 \mathrm{H}, \mathrm{OCCH}_{2}\right), 3.77\left(\mathrm{~s}, 3 \mathrm{H}, \mathrm{OCH}_{3}\right), 2.27\left(\mathrm{~s}, 3 \mathrm{H}, \mathrm{CH}_{3}\right) .{ }^{13} \mathrm{C}$ NMR (101 MHz, Acetonitrile$\left.d_{3}\right) \delta 160.6,157.5,157.2,149.1,131.4,130.8,122.9,115.8,115.7,90.4,68.5,56.2,20.4$. IR $\vee 2962$ (s), $2921(\mathrm{~s}), 1647(\mathrm{~m}), 1615(\mathrm{~m}), 1508(\mathrm{~s}), 1456(\mathrm{~m}), 1394(\mathrm{~m}), 1289(\mathrm{~m}), 1217(\mathrm{~s}), 1039(\mathrm{~s}), 963(\mathrm{~m}), 916$ (m), 832 (s). HRMS (APPI/QTOF) m/z: [M] ${ }^{+}$Calcd for $\mathrm{C}_{17} \mathrm{H}_{18} \mathrm{O}_{3}{ }^{+} 270.1250$; Found 270.1239 . 


\section{Allylic esters formation from EBX}

\section{Optimization procedure and table for the synthesis of 4a:}

In a glass vial, phenol $(0.100 \mathrm{mmol}, 1.00$ equiv.) was dissolved in $1.00 \mathrm{~mL}$ of DME (0.1 M). Cesium carbonate $(10.0 \mu \mathrm{mol}, 0.10$ equiv.) was added and the mixture stirred vigorously for $5 \mathrm{~min}$. Then the corresponding EBX 5 was added in one portion $(0.100 \mathrm{mmol}, 1.00$ equiv.) and the reaction was left stirring for 16 hours at room temperature. Then cesium carbonate $(0.120 \mathrm{mmol}, 1.20$ equiv.) and anisole $(20.0 \mu \mathrm{mol}, 0.20$ equiv.) were added and the solution was stirred at room temperature for 16 hours. The reaction mixture was filtrated, solvent was removed under reduced pressure and the crude material was purified by column chromatography (pentane : ethyl acetate 9:1) to provide product 4 as a sticky solid.

Table S2: Optimisation studies for the transformation of 1a into $4 a$.

\begin{tabular}{|c|c|c|c|c|c|}
\hline Entry & Additive & Equiv. & Temperature $\left({ }^{\circ} \mathrm{C}\right)$ & Remaining 1a (\%) ${ }^{[a]}$ & Yield $(\%)^{[a]}$ \\
\hline 1 & - & - & $25^{\circ} \mathrm{C}$ & 36 & $0-30$ \\
\hline 2 & Anisole & 1.0 & $25^{\circ} \mathrm{C}$ & $<5$ & 45 \\
\hline 3 & Anisole & 1.0 & $0-25^{\circ} \mathrm{C}$ & $9-60$ & $34-87$ \\
\hline 4 & Anisole & 0.20 & $25^{\circ} \mathrm{C}$ & $<5$ & 65 \\
\hline 5 & Anisole & 0.20 & $0-25^{\circ} \mathrm{C}$ & 34 & 61 \\
\hline $6^{[\mathrm{b}]}$ & Anisole & 1.00 & $25^{\circ} \mathrm{C}$ & $>95$ & NR \\
\hline $7^{[b]}$ & DMAP & 2.00 & $25^{\circ} \mathrm{C}$ & $>95$ & NR \\
\hline
\end{tabular}

Reaction conditions: Substrate $1 \mathrm{a}(0.100 \mathrm{mmol}), \mathrm{Cs}_{2} \mathrm{CO}_{3}(0.120 \mathrm{mmol})$, additive (xx equiv.) and DME $(0.1 \mathrm{M})$. NR: no reaction. ${ }^{[\mathrm{a}]} \mathrm{NMR}$ yield given, calculated using $7.0 \mu \mathrm{L}$ of dibromomethane as internal standard. ${ }^{[b]}$ The reaction was performed without $\mathrm{Cs}_{2} \mathrm{CO}_{3}$.

In absence of external nucleophiles, formation of 4 a was sometimes observed, together with decomposition of 1a (entry 1). However, the yield was never higher than $30 \%$ and this result was not reproducible. During screening of nucleophiles, electron-rich phenols have led to increased amount of ester $4 \mathbf{a}$. Anisole was then tested as additive to promote the reaction as an electron-rich arene lacking the free $\mathrm{OH}$ group. Indeed, compounds 4 a was now obtained, but with low reproducibility in yield and conversion (entry 2), in moderate yield. At $0{ }^{\circ} \mathrm{C}$, the yield was improved in some instance, but the transformation still suffered of low reproducibility, with yields between 34 and $87 \%$ (entry 3 ). A better reproducibility was obtained using 0.20 equivalents of anisole at room temperature, giving $4 a$ in $65 \%$ yield (entry 4). Starting at $0{ }^{\circ} \mathrm{C}$, a similar yield was obtained but no full conversion was observed (entry 5). Furthermore, to confirm the role of $\mathrm{Cs}_{2} \mathrm{CO}_{3}$, the reaction was performed in absence of base and with 1.00 equivalent of anisole (entry 6 ). No product was formed and 1a was recovered. Thereby, for 
the reaction to proceed efficiently, a base and an additive seem to be required. We then wonder if DMAP could play both roles (entry 7), but no conversion was observed.

\section{One-pot two-step procedure for the synthesis of 4a:}

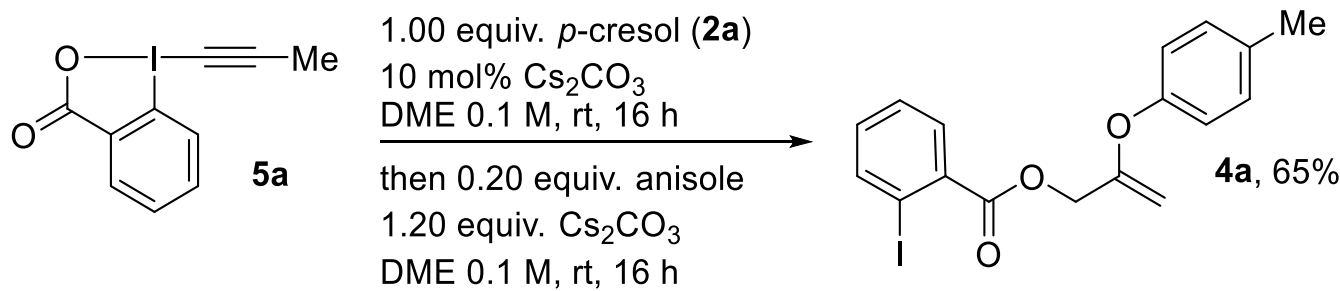

With the optimized conditions in hand, a one-pot two-step procedure was applied to the synthesis of 4a. After conversion of Me-EBX (5a) to O-VBX 1a, anisole and an excess of base were added to the reaction mixture. The product 4 a could be isolated in the same yield as when starting from isolated $1 \mathbf{a}$.

\section{General procedure for allylic esters formation from EBX:}

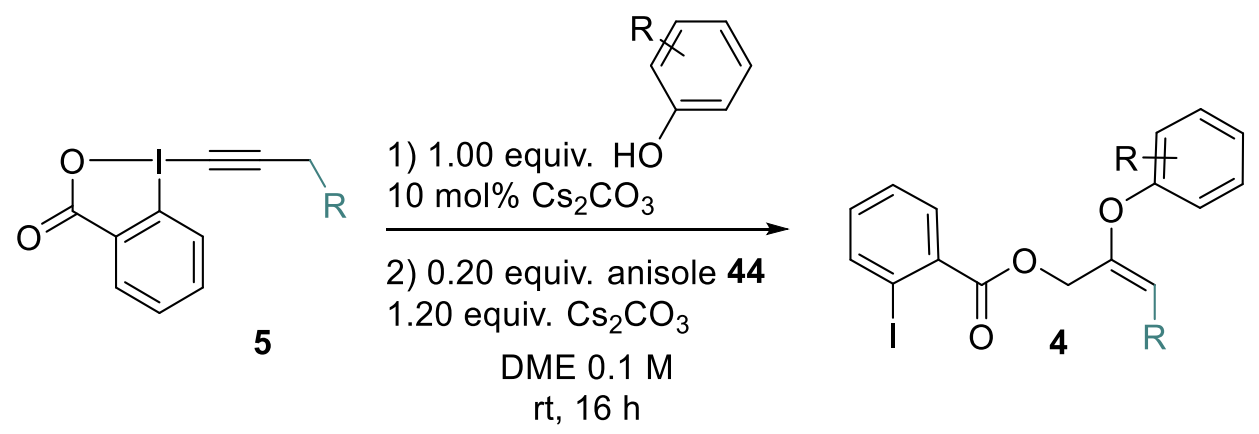

In a glass vial, phenol $(0.100 \mathrm{mmol}, 1.00$ equiv.) was dissolved in $1.00 \mathrm{~mL}$ of DME (0.1 M). Cesium carbonate $(10.0 \mu \mathrm{mol}, 0.10$ equiv.) was added and the mixture stirred vigorously for $5 \mathrm{~min}$. Then the corresponding EBX 5 was added in one portion $(0.100 \mathrm{mmol}, 1.00$ equiv.) and the reaction was left stirring for 16 hours at room temperature. Then cesium carbonate $(0.120 \mathrm{mmol}, 1.20$ equiv.) and anisole $(20.0 \mu \mathrm{mol}, 0.20$ equiv.) were added and the solution was stirred at room temperature for 16 hours. The reaction mixture was filtrated, solvent was removed under reduced pressure and the crude material was purified by column chromatography (pentane : ethyl acetate 9:1) to provide product 4 as a sticky solid.

\section{2-(p-Tolyloxy)allyl 2-iodobenzoate (4a)}<smiles>C=C(COC(=O)c1ccccc1I)Oc1ccc(C)cc1</smiles>

Starting from EBX 5a $(0.118 \mathrm{mg}, 0.300 \mathrm{mmol}), 2$-(p-tolyloxy)allyl 2iodobenzoate $4 \mathrm{a}$ ( $77.0 \mathrm{mg}, 0.195 \mathrm{mmol}, 65 \%$ yield) was obtained, as a colorless amorphous solid. Rf: 0.58 (Pentane:EtOAc 9:1). ${ }^{1} \mathbf{H}$ NMR (400 MHz, Chloroform-d) $\delta 8.01$ (dd, $J=7.9,1.2 \mathrm{~Hz}, 1 \mathrm{H}, \operatorname{ArH}$ ), 7.87 (dd, $J=7.8,1.7 \mathrm{~Hz}, 1 \mathrm{H}, \operatorname{ArH}), 7.41(\mathrm{td}, J=7.6,1.2 \mathrm{~Hz}, 1 \mathrm{H}, \operatorname{ArH}), 7.20-$ $7.12(\mathrm{~m}, 3 \mathrm{H}, \mathrm{ArH}), 7.02-6.96(\mathrm{~m}, 2 \mathrm{H}, \mathrm{ArH}), 4.94(\mathrm{~d}, J=0.6 \mathrm{~Hz}, 2 \mathrm{H}$, $\left.\mathrm{CCH}_{2} \mathrm{O}\right), 4.55\left(\mathrm{~d}, J=2.3 \mathrm{~Hz}, 1 \mathrm{H}, \mathrm{CH}_{2} \mathrm{CO}\right), 4.23\left(\mathrm{~d}, J=2.3 \mathrm{~Hz}, 1 \mathrm{H}, \mathrm{CH}_{2} \mathrm{CO}\right), 2.33\left(\mathrm{~s}, 3 \mathrm{H}, \mathrm{CH}_{3}\right) .{ }^{13} \mathrm{C}$ NMR (101 $\mathrm{MHz}$, Chloroform-d) $\delta 166.1,157.5,152.6,141.6,134.8,134.2,133.0,131.4,130.3,128.1,120.8,94.5$, 92.1, 65.0, 20.9. IR $v 3463(w), 3030(w), 2929(w), 1736(s), 1729(s), 1651(w), 1588(w), 1505(m)$, $1457(\mathrm{w}), 1430(\mathrm{~m}), 1373(\mathrm{w}), 1289$ (s), 1243 (s), 1134 (s), 1101 (s), 1045 (w), 1017 (m), 964 (w), 839 (m), $741(\mathrm{~s}), 697$ (w). HRMS (ESI/QTOF) $\mathrm{m} / \mathrm{z}:[\mathrm{M}+\mathrm{Na}]^{+}$Calcd for $\mathrm{C}_{17} \mathrm{H}_{15} \mathrm{INaO}_{3}{ }^{+}$416.9958; Found 416.9964 . 


\section{2-(p-Tolyloxy)pent-1-en-3-yl 2-iodobenzoate (4b)}

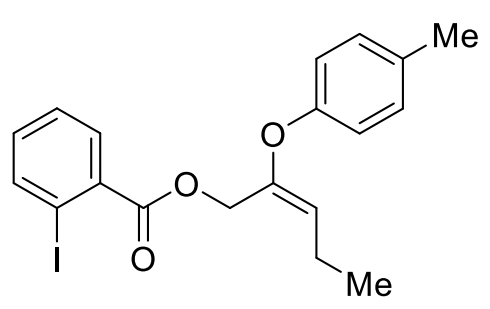

Starting from EBX $5 \mathbf{b}(42.2 \mathrm{mg}, 0.100 \mathrm{mmol}), 2$-(p-tolyloxy)pent-1en-3-yl 2-iodobenzoate 4 b $(25.0 \mathrm{mg}, 59.0 \mu \mathrm{mol}, 59 \%$ yield) was obtained, as a colorless amorphous solid. Rf: 0.60 (Pentane:EtOAc 9:1). ${ }^{1} \mathrm{H}$ NMR (400 MHz, Chloroform-d) $\delta 7.99$ (dd, $J=7.9,1.2 \mathrm{~Hz}, 1 \mathrm{H}$, $\operatorname{ArH}), 7.73(\mathrm{dd}, J=7.8,1.7 \mathrm{~Hz}, 1 \mathrm{H}, \operatorname{ArH}), 7.37(\mathrm{td}, J=7.6,1.2 \mathrm{~Hz}, 1 \mathrm{H}$, $\operatorname{ArH}), 7.14(\mathrm{td}, J=7.7,1.7 \mathrm{~Hz}, 1 \mathrm{H}, \mathrm{ArH}), 7.12-7.08(\mathrm{~m}, 2 \mathrm{H}, \mathrm{ArH}), 6.95$ - $6.90(\mathrm{~m}, 2 \mathrm{H}, \mathrm{ArH}), 5.12(\mathrm{t}, J=7.9 \mathrm{~Hz}, 1 \mathrm{H}, \mathrm{CCHO}), 4.99\left(\mathrm{~s}, 2 \mathrm{H}, \mathrm{CH}_{2} \mathrm{CO}\right), 2.31\left(\mathrm{~s}, 3 \mathrm{H}, \mathrm{ArCH}_{3}\right), 2.21(\mathrm{p}, J=$ $7.6 \mathrm{~Hz}, 2 \mathrm{H}, \mathrm{CH}_{2} \mathrm{CH}_{3}$ ), 1.01 (t, J = 7.5 Hz, 3H, $\mathrm{CH}_{2} \mathrm{CH}_{3}$ ). ${ }^{13} \mathrm{C} \mathrm{NMR}$ (101 MHz, Chloroform-d) $\delta$ 166.4, 154.1, $148.3,141.5,134.9,132.8,132.7,131.3,130.2,128.0,119.1,118.1,94.4,60.9,20.8,20.2,15.0$. IR v $3464(w), 3030(w), 2957(w), 2256(w), 1728(m), 1607(w), 1590(w), 1517(w), 1462(w), 1427(w)$, $1372(\mathrm{w}), 1286(\mathrm{~m}), 1254(\mathrm{~m}), 1123(\mathrm{~m}), 1017(\mathrm{~m}), 904(\mathrm{~m}), 821(\mathrm{w}), 741(\mathrm{~s}), 649(\mathrm{w})$. HRMS (ESI/QTOF) $\mathrm{m} / \mathrm{z}:[\mathrm{M}+\mathrm{H}]^{+}$Calcd for $\mathrm{C}_{19} \mathrm{H}_{20} \mathrm{IO}_{3}{ }^{+}$423.0452; Found 423.0449 .

\section{(E)-2-(p-Tolyloxy)oct-2-en-1-yl 2-iodobenzoate (4c)}<smiles>CCCCC/C=C(/COC(=O)c1ccccc1I)Oc1ccc(C)cc1</smiles>

Starting from EBX 5c (46.4 mg, $0.100 \mathrm{mmol}),(E)-2$-( $p$-tolyloxy)oct-2en-1-yl 2-iodobenzoate $4 \mathrm{c}(27.8 \mathrm{mg}, 60.0 \mu \mathrm{mol}, 60 \%$ yield $)$ was obtained, as a colorless amorphous solid. Rf: 0.69 (Pentane:EtOAc 9:1). ${ }^{1} \mathrm{H}$ NMR (400 MHz, Chloroform-d) $\delta 7.99$ (dd, $J=7.9,1.2 \mathrm{~Hz}, 1 \mathrm{H}$, $\operatorname{ArH}), 7.73(\mathrm{dd}, J=7.8,1.7 \mathrm{~Hz}, 1 \mathrm{H}, \operatorname{ArH}), 7.36(\mathrm{td}, J=7.6,1.2 \mathrm{~Hz}, 1 \mathrm{H}$, $\operatorname{ArH}), 7.17-7.07(\mathrm{~m}, 3 \mathrm{H}, \mathrm{ArH}), 7.00-6.87(\mathrm{~m}, 2 \mathrm{H}, \mathrm{ArH}), 5.14(\mathrm{t}, J=$ $\left.8.0 \mathrm{~Hz}, 1 \mathrm{H}, \mathrm{CCHCH}_{2}\right), 4.99\left(\mathrm{~s}, 2 \mathrm{H}, \mathrm{CCH}_{2} \mathrm{O}\right), 2.31\left(\mathrm{~s}, 3 \mathrm{H}, \mathrm{ArCH}_{3}\right), 2.18(\mathrm{q}, J=7.5 \mathrm{~Hz}, 2 \mathrm{H}$, $\left.\mathrm{CHCH}_{2} \mathrm{CH}_{2} \mathrm{CH}_{2} \mathrm{CH}_{2} \mathrm{CH}_{3}\right), 1.38\left(\mathrm{t}, J=7.1 \mathrm{~Hz}, 2 \mathrm{H}, \mathrm{CHCH}_{2} \mathrm{CH}_{2} \mathrm{CH}_{2} \mathrm{CH}_{2} \mathrm{CH}_{3}\right), 1.34-1.21(\mathrm{~m}, 4 \mathrm{H}$, $\mathrm{CHCH}_{2} \mathrm{CH}_{2} \mathrm{CH}_{2} \mathrm{CH}_{2} \mathrm{CH}_{3}$ ), $0.95-0.80\left(\mathrm{~m}, 3 \mathrm{H}, \mathrm{CH}_{3}\right) .{ }^{13} \mathrm{C}$ NMR (101 MHz, Chloroform-d) $\delta 166.3,154.2$, $148.5,141.5,134.9,132.8,132.7,131.3,130.2,128.0,119.0,117.0,94.4,60.9,31.5,29.8,26.7,22.6$, 20.8, 14.2. IR v $3458(w), 3038(w), 2929(m), 2861(w), 2254(w), 1731(m), 1610(w), 1505(s), 1456$ $(\mathrm{w}), 1369(\mathrm{w}), 1282(\mathrm{~m}), 1246(\mathrm{~m}), 1216(\mathrm{~s}), 1170(\mathrm{~m}), 1128(\mathrm{~m}), 1105(\mathrm{~m}), 1016(\mathrm{~m}), 958(\mathrm{w}), 904(\mathrm{~m})$, 820 (w), 741 (s), 648 (w). HRMS (ESI/QTOF) m/z: [M + H] ${ }^{+}$Calcd for $\mathrm{C}_{22} \mathrm{H}_{26} \mathrm{IO}_{3}{ }^{+}$465.0921; Found 465.0923.

(E)-2-( $p$-Tolyloxy)hexadec-2-en-1-yl 2-iodobenzoate (4d)

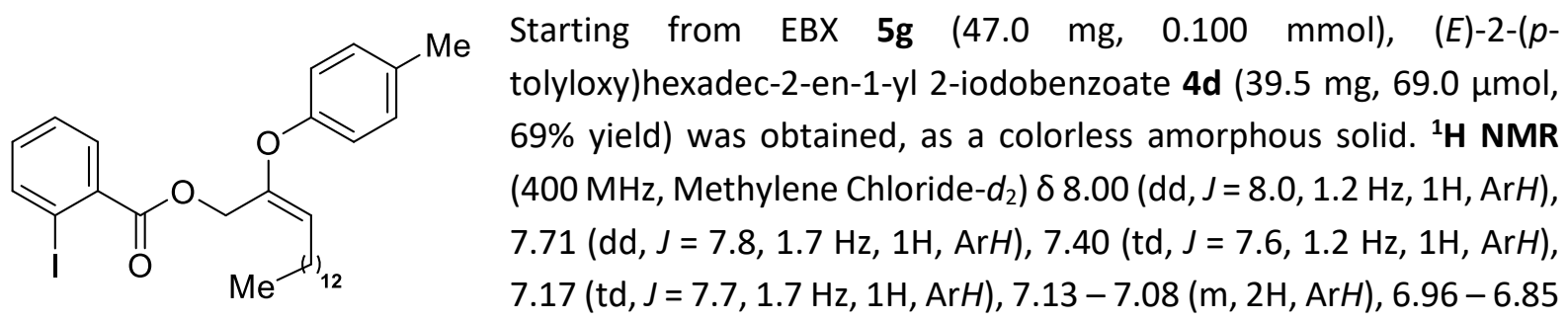

$(\mathrm{m}, 2 \mathrm{H}, \mathrm{ArH}), 5.14\left(\mathrm{t}, J=8.0 \mathrm{~Hz}, 1 \mathrm{H}, \mathrm{CCHCH}_{2}\right), 4.96\left(\mathrm{~s}, 2 \mathrm{H}, \mathrm{CCH}_{2} \mathrm{O}\right), 2.30\left(\mathrm{~s}, 3 \mathrm{H}, \mathrm{ArCH}_{3}\right), 2.18(\mathrm{q}, J=7.5$ $\left.\mathrm{Hz}, 2 \mathrm{H}, \mathrm{CH}_{2}\right), 1.43-1.33\left(\mathrm{~m}, 2 \mathrm{H}, \mathrm{CH}_{2}\right), 1.33-1.22\left(\mathrm{~m}, 2 \mathrm{H}, \mathrm{CH}_{2}\right), 0.92-0.83\left(\mathrm{~m}, 3 \mathrm{H}, \mathrm{CH}_{3}\right) .{ }^{13} \mathrm{C}$ NMR (101 $\mathrm{MHz}$, Methylene Chloride- $\left.d_{2}\right) \delta 166.6,154.6,148.9,141.8,135.6,133.2,133.2,131.5,130.6,128.5$, 119.2 , 117.7, 94.4, 61.3, 32.5, 30.6, 30.3, 30.3, 30.2, 30.2, 30.2, 30.0, 29.9, 29.7, 27.1, 23.3, 20.9, 14.5. IR $\vee 2959$ (m), 2923 (m), 1732 (s), 1503 (s), 1400 (s), 1256 (s), 1075 (s), 1069 (s), 741 (s). HRMS (ESI/QTOF) m/z: [M + Na] ${ }^{+}$Calcd for $\mathrm{C}_{30} \mathrm{H}_{41} \mathrm{INaO}_{3}{ }^{+}$599.1993; Found 599.2001. 
(E)-2-(p-Tolyloxy)-8-(trimethylsilyl)oct-2-en-7-yn-1-yl 2-iodobenzoate (4e)

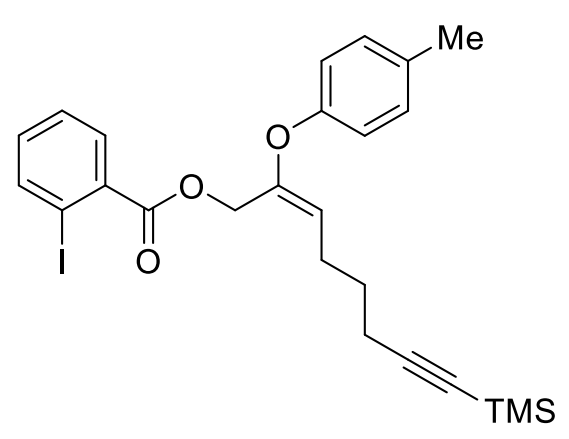

Starting from EBX 5e $(42.0 \mathrm{mg}, 0.100 \mathrm{mmol}),(E)-2$-( $p$-tolyloxy)8-(trimethylsilyl)oct-2-en-7-yn-1-yl 2-iodobenzoate 4e (37.3 mg, $70.0 \mu \mathrm{mol}, 70 \%$ yield) was obtained, as a colorless amorphous solid. Rf: 0.64 (Pentane:EtOAc 9:1). ${ }^{1} \mathbf{H}$ NMR $(400 \mathrm{MHz}$, Chloroform-d) $\delta 7.99$ (dd, $J=7.9,1.2 \mathrm{~Hz}, 1 \mathrm{H}, \operatorname{ArH}), 7.74$ (dd, $J=$ 7.8, 1.7 Hz, 1H, ArH), 7.37 (td, J = 7.6, $1.2 \mathrm{~Hz}, 1 \mathrm{H}, \mathrm{ArH}), 7.14$ (td, $J=7.8,1.9 \mathrm{~Hz}, 1 \mathrm{H}, \operatorname{ArH}), 7.10(\mathrm{~d}, J=8.3 \mathrm{~Hz}, 2 \mathrm{H}, \operatorname{ArH}), 6.95-6.89$ $(\mathrm{m}, 2 \mathrm{H}, \mathrm{ArH}), 5.04\left(\mathrm{t}, J=8.0 \mathrm{~Hz}, 1 \mathrm{H}, \mathrm{CCHCH}_{2}\right), 5.01\left(\mathrm{~s}, 2 \mathrm{H}, \mathrm{CCH}_{2} \mathrm{O}\right)$, $2.30\left(\mathrm{~d}, J=7.2 \mathrm{~Hz}, 5 \mathrm{H}, \mathrm{CHCH}_{2} \mathrm{CH}_{2} \mathrm{CH}_{2} \mathrm{C}, \mathrm{ArCH}_{3}\right), 2.25(\mathrm{t}, J=7.1 \mathrm{~Hz}$,

$2 \mathrm{H}, \mathrm{CHCH}_{2} \mathrm{CH}_{2} \mathrm{CH}_{2} \mathrm{C}$ ), 1.60 (p, J = 7.2 Hz, 2H, CHCH $\mathrm{CH}_{2} \mathrm{CH}_{2} \mathrm{C}$ ), 0.14 (s, 9H, Si( $\left.\left.\mathrm{CH}_{3}\right)_{3}\right) \cdot{ }^{13} \mathrm{C}$ NMR $(101 \mathrm{MHz}$, Chloroform-d) $\delta$ 166.3, 153.9, 149.8, 141.4, 135.0, 133.0, 132.8, 131.3, 130.3, 128.0, 119.3, 114.7, 106.8, 94.3, 85.3, 60.9, 28.9, 25.7, 20.8, 19.3, 0.3. IR 3666 (w), 2978 (s), $2902(\mathrm{~s}), 2172(\mathrm{w}), 1734(\mathrm{~m})$, $1676(\mathrm{w}), 1589(\mathrm{w}), 1506(\mathrm{~m}), 1399(\mathrm{~m}), 1249(\mathrm{~s}), 1232(\mathrm{~s}), 1221(\mathrm{~s}), 1054(\mathrm{~s}), 1028(\mathrm{~s}), 901(\mathrm{~m}), 842(\mathrm{~s})$, 741 (s), 642 (w). HRMS (ESI/QTOF) m/z: [M + Na] ${ }^{+}$Calcd for $\mathrm{C}_{25} \mathrm{H}_{29} \mathrm{INaO}_{3} \mathrm{Si}^{+} 555.0823$; Found 555.0834.

(E)-6-(1,3-Dioxoisoindolin-2-yl)-2-(p-tolyloxy)hex-2-en-1-yl 2-iodobenzoate (4f)

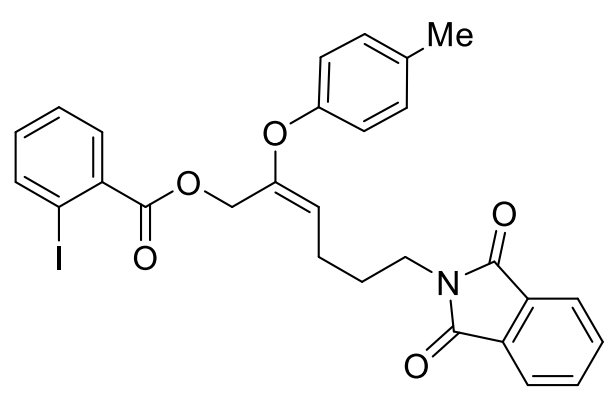

Starting from EBX $5 f(47.0 \mathrm{mg}, 0.100 \mathrm{mmol}),(E)-6-(1,3-$ dioxoisoindolin-2-yl)-2-( $p$-tolyloxy)hex-2-en-1-yl 2- $\mathbf{4 f}$ (35.0 $\mathrm{mg}, 60.0 \mathrm{\mu mol}, 60 \%$ yield) was obtained, as a colorless amorphous solid. Rf: 0.28 (Pentane:EtOAc 9:1). ${ }^{1} \mathbf{H}$ NMR (400 $\mathrm{MHz}$, Acetonitrile- $\left.d_{3}\right) \delta 7.99(\mathrm{dd}, J=7.9,1.1 \mathrm{~Hz}, 1 \mathrm{H}, \mathrm{ArH}), 7.77$ (tdd, $J=8.8,5.4,3.9 \mathrm{~Hz}, 4 \mathrm{H}, \mathrm{ArH}), 7.64$ (dd, $J=7.8,1.7 \mathrm{~Hz}, 1 \mathrm{H}$, $\operatorname{ArH}), 7.44(\mathrm{td}, J=7.6,1.1 \mathrm{~Hz}, 1 \mathrm{H}, \mathrm{ArH}), 7.21(\mathrm{td}, J=7.7,1.7$ $\mathrm{Hz}, 1 \mathrm{H}, \mathrm{ArH}), 7.14-7.09(\mathrm{~m}, 2 \mathrm{H}, \mathrm{ArH}), 6.91-6.86(\mathrm{~m}, 2 \mathrm{H}$, $\operatorname{ArH}), 5.11(\mathrm{t}, J=7.9 \mathrm{~Hz}, 1 \mathrm{H}, \mathrm{OCCH}), 4.94\left(\mathrm{~s}, 2 \mathrm{H}, \mathrm{OCH}_{2} \mathrm{CO}\right), 3.63\left(\mathrm{t}, J=7.0 \mathrm{~Hz}, 2 \mathrm{H}, \mathrm{CH}_{2}\right), 2.26(\mathrm{~d}, J=11.3$ $\left.\mathrm{Hz}, 5 \mathrm{H}, \mathrm{CH}_{2}, \mathrm{CH}_{3}\right), 1.74\left(\mathrm{p}, J=7.2 \mathrm{~Hz}, 2 \mathrm{H}, \mathrm{CH}_{2}\right) .{ }^{13} \mathrm{C} \mathrm{NMR}\left(101 \mathrm{MHz}\right.$, Acetonitrile- $\left.d_{3}\right) \delta$ 169.3, 167.2, 154.9, $150.0,142.0,136.5,135.0,133.8,133.7,133.2,131.5,131.1,129.2,123.7,119.6,116.5,94.0,61.5$, 38.1, 29.4, 24.7, 20.6. IR v 2942 (w), 1769 (w), 1705 (s), 1611 (w), $1583(w), 1503$ (m), 1465 (w), 1434 (w), $1394(\mathrm{~m}), 1369(\mathrm{~m}), 1283(\mathrm{~m}), 1244(\mathrm{~m}), 1219(\mathrm{~m}), 1169(\mathrm{~m}), 1129(\mathrm{~m}), 1099(\mathrm{~m}), 1041(\mathrm{~m}), 1015$ (m), $954(w), 919(w), 888(w), 823(w), 747(w), 718$ (s). HRMS (ESI/QTOF) m/z: [M + Na] ${ }^{+}$Calcd for $\mathrm{C}_{28} \mathrm{H}_{24} \mathrm{INNaO}_{5}{ }^{+}$604.0591; Found 604.0589.

\section{(E)-2-(p-Tolyloxy)octa-2,7-dien-1-yl 2-iodobenzoate (4g)}

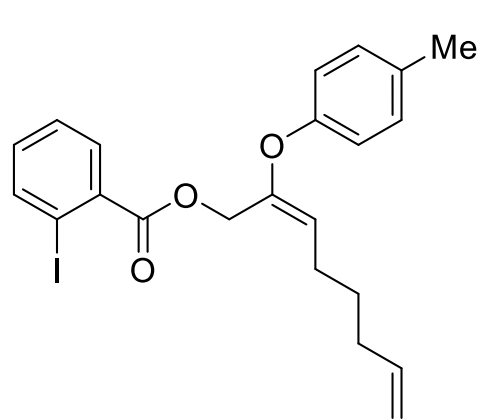

Starting from EBX $5 \mathrm{~h}(35.0 \mathrm{mg}, 0.100 \mathrm{mmol}),(E)-2-(p$-tolyloxy)octa2,7-dien-1-yl 2-iodobenzoate $\mathbf{4 g}$ (38.0 mg, $82.0 \mu \mathrm{mol}, 82 \%$ yield) was obtained, as a colorless amorphous solid. Rf: 0.66 (Pentane:EtOAc 9:1). ${ }^{1} \mathrm{H}$ NMR $(400 \mathrm{MHz}$, Chloroform-d) $\delta 7.99$ (dd, $J=7.9,1.2 \mathrm{~Hz}, 1 \mathrm{H}$, $\operatorname{ArH}), 7.73(\mathrm{dd}, J=7.8,1.7 \mathrm{~Hz}, 1 \mathrm{H}, \operatorname{ArH}), 7.37(\mathrm{td}, J=7.6,1.2 \mathrm{~Hz}, 1 \mathrm{H}$, $\mathrm{ArH}), 7.18-7.07(\mathrm{~m}, 3 \mathrm{H}, \mathrm{ArH}), 6.95-6.89(\mathrm{~m}, 2 \mathrm{H}, \mathrm{ArH}), 5.78$ (ddt, $J=$ $\left.16.9,10.2,6.7 \mathrm{~Hz}, 1 \mathrm{H}, \mathrm{CH}_{2} \mathrm{CHCH}_{2}\right), 5.10\left(\mathrm{t}, J=7.9 \mathrm{~Hz}, 1 \mathrm{H}, \mathrm{OCCHCH}_{2}\right)$, $5.04-4.93\left(\mathrm{~m}, 4 \mathrm{H}, \mathrm{CCH}_{2} \mathrm{O}, \mathrm{CH}_{2} \mathrm{CHCH}_{2}\right), 2.31\left(\mathrm{~s}, 3 \mathrm{H}, \mathrm{ArCH}_{3}\right), 2.20$ (q, J $\left.=7.6 \mathrm{~Hz}, 2 \mathrm{H}, \mathrm{CHCH}_{2} \mathrm{CH}_{2} \mathrm{CH}_{2} \mathrm{CH}\right), 2.14-2.03\left(\mathrm{~m}, 2 \mathrm{H}, \mathrm{CHCH}_{2} \mathrm{CH}_{2} \mathrm{CH}_{2} \mathrm{CH}\right), 1.49(\mathrm{p}, J=7.4 \mathrm{~Hz}, 2 \mathrm{H}$, $\left.\mathrm{CHCH}_{2} \mathrm{CH}_{2} \mathrm{CH}_{2} \mathrm{CH}\right) .{ }^{13} \mathrm{C}$ NMR $(101 \mathrm{MHz}$, Chloroform-d) $\delta 166.4,154.0,148.9,141.5,138.5,134.9,132.8$, 132.8, 131.3, 130.2, 128.0, 119.1, 116.1, 115.0, 94.4, 61.0, 33.3, 29.3, 26.1, 20.8. IR v 2927 (m), 1728 
(s), $1585(w), 1505$ (s), $1458(w), 1436(w), 1287$ (s), 1249 (s), 1219 (s), 1168 (w), $1127(\mathrm{~m}), 1096(\mathrm{~s})$, $1044(\mathrm{~m}), 1015$ (s), $949(\mathrm{w}), 914(\mathrm{~m}), 821(\mathrm{~m}), 741$ (s). HRMS (ESI/QTOF) m/z: [M + H] ${ }^{+}$Calcd for $\mathrm{C}_{22} \mathrm{H}_{24} \mathrm{IO}_{3}{ }^{+}$463.0765; Found 463.0768 .

(E)-5-Chloro-2-(p-tolyloxy)pent-2-en-1-yl 2-iodobenzoate (4h)<smiles>Cc1ccc(O/C(=C/CCCl)COC(=O)c2ccccc2I)cc1</smiles>

Starting from EBX 5i $(35.0 \mathrm{mg}, 0.100 \mathrm{mmol}),(E)-5$-chloro-2- $(p$ tolyloxy)pent-2-en-1-yl 2-iodobenzoate $4 \mathrm{~h}$ (32.0 mg, $70.0 \mu \mathrm{mol}, 70 \%$ yield) was obtained, as a colorless amorphous solid. Rf: 0.45 Pentane : EtOAc 9:1). ${ }^{1} \mathrm{H}$ NMR (400 MHz, Chloroform- $d$ ) $\delta 8.00$ (dd, $J=7.9,1.2$ $\mathrm{Hz}, 1 \mathrm{H}, \mathrm{ArH}), 7.77(\mathrm{dd}, J=7.8,1.7 \mathrm{~Hz}, 1 \mathrm{H}, \mathrm{ArH}), 7.39$ (td, $J=7.6,1.2$ $\mathrm{Hz}, 1 \mathrm{H}, \mathrm{ArH}), 7.20-7.05(\mathrm{~m}, 3 \mathrm{H}, \mathrm{ArH}), 7.00-6.88(\mathrm{~m}, 2 \mathrm{H}, \mathrm{ArH}), 5.02$ (d, $\left.J=2.5 \mathrm{~Hz}, 3 \mathrm{H}, \mathrm{CCH}_{2} \mathrm{O}, \mathrm{OCCHCH}_{2}\right), 3.53\left(\mathrm{t}, J=6.7 \mathrm{~Hz}, 2 \mathrm{H}, \mathrm{CH}_{2} \mathrm{CH}_{2} \mathrm{Cl}\right.$ ), $2.66(\mathrm{dt}, J=8.1,6.7 \mathrm{~Hz}, 2 \mathrm{H}$, $\mathrm{CH}_{2} \mathrm{CH}_{2} \mathrm{Cl}$ ), 2.32 (s, 3H, ArCH$)_{3} .{ }^{13} \mathrm{C}$ NMR (101 MHz, Chloroform-d) $\delta$ 166.2, 153.2, 151.7, 141.4, 134.7, 133.3, 132.8, 131.2, 130.2, 127.9, 119.6, 109.9, 94.3, 60.8, 44.3, 30.0, 20.7. IR $\vee 3666$ (w), 2979 (s), $2902(\mathrm{~s}), 1401(\mathrm{~m}), 1253(\mathrm{w}), 1064$ (s), 886 (w), 767 (w), 717 (w). HRMS (ESI/QTOF) m/z: [M + H] Calcd for $\mathrm{C}_{19} \mathrm{H}_{19} \mathrm{CllO}_{3}{ }^{+}$457.0062; Found 457.0062.

\section{2-(2-lodophenoxy)allyl 2-iodobenzoate (4i)}<smiles>C=C(COC(=O)c1ccccc1I)Oc1ccccc1I</smiles>

Starting from EBX 5a $(29.0 \mathrm{mg}, 0.100 \mathrm{mmol})$ and 2-iodophenol $(22.0 \mathrm{mg}$, $0.100 \mathrm{mmol}$ ), 2-(2-iodophenoxy)allyl 2-iodobenzoate $4 \mathbf{i}(35.6 \mathrm{mg}, 70.0$ $\mu \mathrm{mol}, 70 \%$ yield) was obtained, as a yellow amorphous solid. Rf: 0.62 (Pentane:EtOAc 9:1). ${ }^{1} \mathbf{H}$ NMR $\left(400 \mathrm{MHz}\right.$, Acetonitrile- $\left.d_{3}\right) \delta 8.05$ (dd, $J=$ 8.0, $1.2 \mathrm{~Hz}, 1 \mathrm{H}, \mathrm{ArH}), 7.89$ (ddd, $J=7.8,2.8,1.6 \mathrm{~Hz}, 2 \mathrm{H}, \operatorname{ArH}), 7.50$ (td, $J=$ 7.6, $1.2 \mathrm{~Hz}, 1 \mathrm{H}, \mathrm{ArH}$ ), 7.43 (ddd, $J=8.1,7.3,1.5 \mathrm{~Hz}, 1 \mathrm{H}, \operatorname{ArH}), 7.30-7.22$ $(\mathrm{m}, 1 \mathrm{H}, \operatorname{ArH}), 7.19$ (dd, J = 8.1, 1.5 Hz, 1H, ArH), 6.99 (ddd, J = 8.0, 7.3, 1.5 Hz, 1H, ArH), 4.99 (d, J = 0.6 $\left.\mathrm{Hz}, 2 \mathrm{H}, \mathrm{OCH}_{2} \mathrm{CO}\right), 4.66\left(\mathrm{dt}, J=2.7,0.7 \mathrm{~Hz}, 1 \mathrm{H}, \mathrm{OCCH}_{2}\right), 4.07$ (d, $\left.J=2.7 \mathrm{~Hz}, 1 \mathrm{H}, \mathrm{OCCH}_{2}\right) .{ }^{13} \mathrm{C}$ NMR (101 $\mathrm{MHz}$, Acetonitrile- $\left.d_{3}\right) \delta 167.0,157.3,155.2,142.2,140.9,136.0,134.0,131.9,131.1,129.3,127.8$, 122.9, 94.2, 93.4, 90.3, 65.2. IR $\vee 3666$ (s), $3091(\mathrm{~m}), 2995(\mathrm{~s}), 2905(\mathrm{~s}), 2261(\mathrm{~s}), 1625(\mathrm{~m}), 1401(\mathrm{~m})$, $1230(\mathrm{~m}), 1068(\mathrm{~s}), 1027(\mathrm{~s}), 909(\mathrm{~m}), 839(\mathrm{~s}), 748(\mathrm{~m})$. HRMS (ESI/QTOF) m/z: [M + H] ${ }^{+}$Calcd for $\mathrm{C}_{16} \mathrm{H}_{13} \mathrm{I}_{2} \mathrm{O}_{3}{ }^{+}$506.8949; Found 506.8949.

\section{(E)-2-(2-lodophenoxy)pent-2-en-1-yl 2-iodobenzoate (4j)}

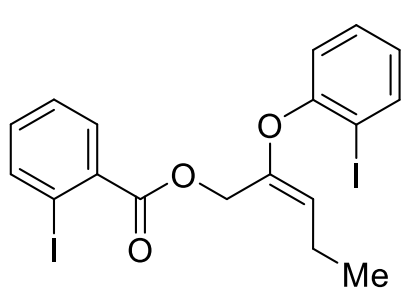

Starting from EBX $\mathbf{5 b}(31.0 \mathrm{mg}, 0.100 \mathrm{mmol}),(E)$-2-(2-iodophenoxy)pent2-en-1-yl 2-iodobenzoate $4 \mathbf{j}(37.0 \mathrm{mg}, 69.0 \mu \mathrm{mol}, 69 \%$ yield) was obtained, as a colorless amorphous solid. Rf: 0.43 (Pentane:EtOAc 9:1). ${ }^{1} \mathbf{H}$ NMR (400 $\mathrm{MHz}$, Acetonitrile- $\left.d_{3}\right) \delta 8.05-7.96(\mathrm{~m}, 1 \mathrm{H}, \mathrm{ArH}), 7.83(\mathrm{dd}, J=7.9,1.6 \mathrm{~Hz}$, $1 \mathrm{H}, \operatorname{ArH}), 7.73(\mathrm{dd}, J=7.8,1.7 \mathrm{~Hz}, 1 \mathrm{H}, \mathrm{ArH}), 7.45(\mathrm{td}, J=7.6,1.2 \mathrm{~Hz}, 1 \mathrm{H}$, $\operatorname{ArH}), 7.35$ (ddd, $J=8.0,7.3,1.6 \mathrm{~Hz}, 1 \mathrm{H}, \operatorname{ArH}), 7.23(\mathrm{td}, J=7.7,1.7 \mathrm{~Hz}, 1 \mathrm{H}$,

$\operatorname{ArH}), 7.07(\mathrm{dd}, J=8.2,1.5 \mathrm{~Hz}, 1 \mathrm{H}, \operatorname{ArH}), 6.87(\mathrm{td}, J=7.6,1.5 \mathrm{~Hz}, 1 \mathrm{H}, \operatorname{ArH}), 5.09(\mathrm{t}, J=7.9 \mathrm{~Hz}, 1 \mathrm{H}, \mathrm{OCCH})$, $5.02\left(\mathrm{~s}, 2 \mathrm{H}, \mathrm{OCH}_{2} \mathrm{CO}\right), 2.23\left(\mathrm{p}, J=7.5 \mathrm{~Hz}, 2 \mathrm{H}, \mathrm{CH}_{2}\right), 1.01\left(\mathrm{t}, J=7.5 \mathrm{~Hz}, 3 \mathrm{H}, \mathrm{CH}_{3}\right) .{ }^{13} \mathrm{C} \mathrm{NMR}(101 \mathrm{MHz}$, Acetonitrile- $\left.d_{3}\right) \delta 167.0,156.4,148.1,142.1,140.7,136.2,133.9,131.8,130.8,129.2,126.2,120.0$, 119.8, 94.2, 89.0, 61.2, 20.6, 14.9. IR v $3672(\mathrm{~m}), 3091$ (w), 3062 (w), $2969(\mathrm{~s}), 2872(\mathrm{~m}), 2604(\mathrm{w}), 1881$ $(w), 1873(w), 1730(s), 1680(w), 1581(m), 1511(w), 1465(s), 1437(m), 1391(m), 1345(w), 1307(w)$, $1281(\mathrm{~m}), 1246(\mathrm{~s}), 1228(\mathrm{~s}), 1181(\mathrm{~m}), 1124(\mathrm{~m}), 1095(\mathrm{~s}), 1066(\mathrm{~m}), 1042(\mathrm{~s}), 1017(\mathrm{~s}), 944(\mathrm{~m}), 884$ (w), $848(w), 833(\mathrm{~m}), 803(\mathrm{w}), 743(\mathrm{~s}), 717$ (w), $687(\mathrm{w}), 640$ (m). HRMS (ESI/OTOF) m/z: [M + H] ${ }^{+}$Calcd

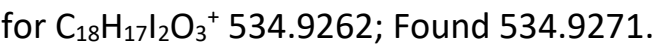




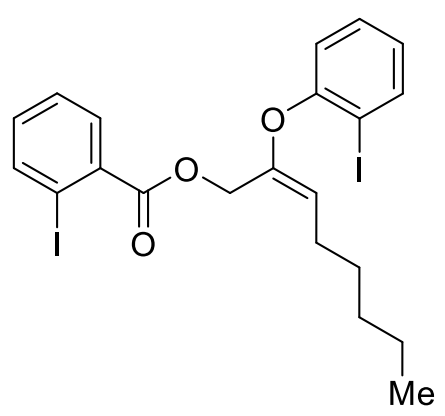

Starting from EBX 5c (36.0 mg, $0.100 \mathrm{mmol})$ and 2-iodophenol $(22.0 \mathrm{mg}$, $0.100 \mathrm{mmol}),(E)$-2-(2-iodophenoxy)oct-2-en-1-yl 2-iodobenzoate $\mathbf{4 k}$ (33.0 $\mathrm{mg}, 57.0 \mu \mathrm{mol}, 57 \%$ yield) was obtained, as a colorless amorphous solid. Rf: 0.80 (Pentane:EtOAc 9:1). ${ }^{1} \mathbf{H}$ NMR (400 MHz, Acetonitrile- $d_{3}$ ) $\delta 8.02(\mathrm{dd}, J=7.9,1.2 \mathrm{~Hz}, 1 \mathrm{H}, \mathrm{ArH}), 7.83(\mathrm{dd}, J=7.9,1.6 \mathrm{~Hz}, 1 \mathrm{H}, \mathrm{ArH})$, $7.74(\mathrm{dd}, J=7.8,1.7 \mathrm{~Hz}, 1 \mathrm{H}, \operatorname{ArH}), 7.46(\mathrm{td}, J=7.6,1.2 \mathrm{~Hz}, 1 \mathrm{H}, \operatorname{ArH}), 7.35$ (ddd, $J=8.2,7.3,1.5 \mathrm{~Hz}, 1 \mathrm{H}, \operatorname{ArH}), 7.23(\mathrm{td}, J=7.7,1.7 \mathrm{~Hz}, 1 \mathrm{H}, \mathrm{ArH}), 7.07$ (dd, $J=8.2,1.5 \mathrm{~Hz}, 1 \mathrm{H}, \mathrm{ArH}), 6.88(\mathrm{td}, J=7.6,1.5 \mathrm{~Hz}, 1 \mathrm{H}, \mathrm{ArH}), 5.05(\mathrm{t}, J$ $=8.0 \mathrm{~Hz}, 1 \mathrm{H}, \mathrm{OCCH}), 5.02\left(\mathrm{~s}, 2 \mathrm{H}, \mathrm{OCH}_{2} \mathrm{CO}\right), 2.20\left(\mathrm{q}, \mathrm{J}=7.3 \mathrm{~Hz}, 2 \mathrm{H}, \mathrm{CH}_{2}\right), 1.44-1.36\left(\mathrm{~m}, 2 \mathrm{H}, \mathrm{CH}_{2}\right), 1.32-$ $1.28\left(\mathrm{~m}, 4 \mathrm{H}, \mathrm{CH}_{2}\right), 0.91-0.83\left(\mathrm{~m}, 3 \mathrm{H}, \mathrm{CH}_{3}\right) .{ }^{13} \mathrm{C} \mathrm{NMR}\left(101 \mathrm{MHz}\right.$, Acetonitrile- $\left.d_{3}\right) \delta 167.0,156.4,148.6$, $142.1,140.7,136.2,133.9,131.8,130.8,129.2,126.3,120.0,118.3,94.2,89.1,61.2,32.0,30.2,27.0$, 23.1, 14.3. IR v $3666(\mathrm{~m}), 2979(\mathrm{~s}), 2903$ (s), 2351 (w), 2260 (m), $1613(\mathrm{w}), 1459(\mathrm{w}), 1400(\mathrm{~m}), 1250$ $(\mathrm{m}), 1050(\mathrm{~s}), 883(\mathrm{w}), 838(\mathrm{~m}), 751(\mathrm{w}), 686(\mathrm{w})$. HRMS (ESI/QTOF) m/z: [M + Na] ${ }^{+}$Calcd for $\mathrm{C}_{21} \mathrm{H}_{22} \mathrm{l}_{2} \mathrm{NaO}_{3}{ }^{+}$598.9551; Found 598.9560.

\section{(E)-2-(3-Bromophenoxy)-5-chloropent-2-en-1-yl 2-iodobenzoate (4I)}

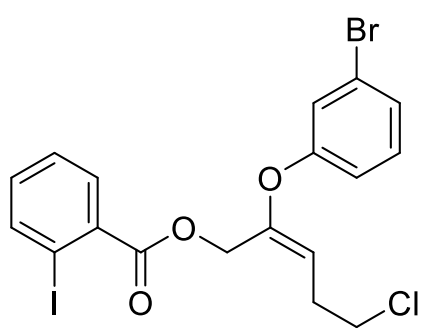

Starting from EBX 5i (52.2 mg, $0.100 \mathrm{mmol}),(E)$-2-(3-bromophenoxy)-5chloropent-2-en-1-yl 2-iodobenzoate $4 \mathbf{l}(27.0 \mathrm{mg}, 52.0 \mu \mathrm{mol}, 52 \%$ yield) was obtained, as a colorless amorphous solid. Rf: 0.55 (Pentane:EtOAc 9:1). ${ }^{1} \mathrm{H}$ NMR (400 MHz, Chloroform-d) $\delta 8.00$ (dd, $J=8.0,1.2 \mathrm{~Hz}, 1 \mathrm{H}$, $\operatorname{ArH}), 7.73(\mathrm{dd}, J=7.8,1.7 \mathrm{~Hz}, 1 \mathrm{H}, \mathrm{ArH}), 7.39(\mathrm{td}, J=7.6,1.2 \mathrm{~Hz}, 1 \mathrm{H}, \mathrm{ArH})$, $7.25-7.13(\mathrm{~m}, 4 \mathrm{H}, \mathrm{ArH}), 7.00$ (ddd, J = 7.5, 2.3, $1.5 \mathrm{~Hz}, 1 \mathrm{H}, \mathrm{ArH}), 5.22(\mathrm{t}$, $J=8.0 \mathrm{~Hz}, 1 \mathrm{H}$, vinylH), $5.00\left(\mathrm{~s}, 2 \mathrm{H}, \mathrm{OCH}_{2} \mathrm{C}\right), 3.59(\mathrm{t}, J=6.5 \mathrm{~Hz}, 2 \mathrm{H}$, $\mathrm{CH}_{2} \mathrm{CH}_{2} \mathrm{Cl}$ ), $2.72\left(\mathrm{dt}, J=8.0,6.5 \mathrm{~Hz}, 2 \mathrm{H}, \mathrm{CH}_{2} \mathrm{CH}_{2} \mathrm{Cl}\right.$ ). ${ }^{13} \mathrm{C}$ NMR (101 MHz, Chloroform-d) $\delta 166.2,157.0$, $150.5,141.6,134.5,133.1,131.4,131.0,128.1,126.6,123.0,122.3,117.7,113.9,94.4,60.6,44.2,30.0$. IR $\vee 3667(\mathrm{~m}), 3062(\mathrm{~m}), 3011(\mathrm{~m}), 2948(\mathrm{w}), 1731(\mathrm{~m}), 1583(\mathrm{~m}), 1469(\mathrm{~m}), 1432(\mathrm{w}), 1247(\mathrm{~s}), 1218$ $(\mathrm{m}), 1132(\mathrm{~m}), 1065(\mathrm{~s}), 1017(\mathrm{~s}), 912(\mathrm{~s}), 861(\mathrm{~m}), 822(\mathrm{w}), 781(\mathrm{~m}), 736$ (s). HRMS (ESI/QTOF) m/z: [M $+\mathrm{H}]^{+}$Calcd for $\mathrm{C}_{18} \mathrm{H}_{16}{ }^{79} \mathrm{Br}^{35} \mathrm{ClIO}_{3}{ }^{+}$520.9011; Found 520.9011 .

\section{2-(Perfluorophenoxy)pent-1-en-3-yl 2-iodobenzoate (4m)}<smiles>CC/C=C(/COC(=O)c1ccccc1I)Oc1c(F)c(F)c(F)c(F)c1F</smiles>

Starting from EBX 5b $(49.8 \quad \mathrm{mg}, \quad 0.100 \quad \mathrm{mmol}), \quad 2-$ (perfluorophenoxy)pent-1-en-3-yl 2-iodobenzoate $4 \mathrm{~m}$ (20.1 mg, 40.0 $\mu \mathrm{mol}, 40 \%$ yield) was obtained, as a colorless amorphous solid. $\mathbf{R f :} 0.65$ (Pentane:EtOAc 9:1). ${ }^{1} \mathrm{H}$ NMR (400 MHz, Chloroform- $d$ ) $\delta 8.02$ (dd, $J=$ 7.9, $1.2 \mathrm{~Hz}, 1 \mathrm{H}, \mathrm{ArH}), 7.87$ (dd, J = 7.8, $1.7 \mathrm{~Hz}, 1 \mathrm{H}, \operatorname{ArH}), 7.42$ (td, $J=7.6$, $1.2 \mathrm{~Hz}, 1 \mathrm{H}, \mathrm{ArH}), 7.17(\mathrm{td}, J=7.6,1.7 \mathrm{~Hz}, 1 \mathrm{H}, \mathrm{ArH}), 5.07\left(\mathrm{~s}, 2 \mathrm{H}, \mathrm{CH}_{2} \mathrm{CO}\right)$, $4.82(\mathrm{tt}, J=7.8,1.3 \mathrm{~Hz}, 1 \mathrm{H}, \mathrm{CCHO}), 2.25-2.14\left(\mathrm{~m}, 2 \mathrm{H}, \mathrm{CHCH}_{2} \mathrm{CH}_{3}\right), 0.99$ (t, $\left.J=7.5 \mathrm{~Hz}, 3 \mathrm{H}, \mathrm{CHCH}_{2} \mathrm{CH}_{3}\right) .{ }^{13} \mathrm{C}$ NMR (101 MHz, Chloroform-d) $\delta$ 166.1, 149.1, 141.6, 134.5, 133.1, $131.4,128.1,112.9,94.5,60.2,19.9,14.8$. The carbon signals of the pentafluorobenzene ring could not be resolved. ${ }^{19} \mathrm{~F}$ NMR (376 MHz, Chloroform-d) $\delta-153.70--154.37(\mathrm{~m}),-160.23(\mathrm{t}, J=21.9 \mathrm{~Hz}),-161.98$ - -162.63 (m). IR $v 3060(w), 2970(w), 2939(w), 2876(w), 1738(m), 1685(w), 1577(w), 1516(s), 1470$ (m), 1437 (w), $1386(w), 1245(\mathrm{~m}), 1183(\mathrm{~m}), 1094(\mathrm{~m}), 996$ (s), 741 (s). HRMS (ESI/QTOF) m/z: [M + $\mathrm{Na}]^{+}$Calcd for $\mathrm{C}_{18} \mathrm{H}_{12} \mathrm{~F}_{5} \mathrm{INaO}_{3}{ }^{+} 520.9644$; Found 520.9651 . 
(E)-2-(4-Methoxyphenoxy)pent-2-en-1-yl 2-iodobenzoateiodobenzoate (4n)

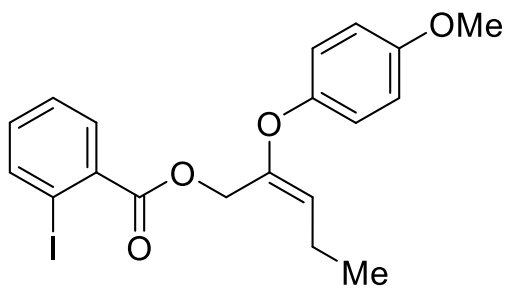

Starting from EBX $\mathbf{5 b}(31.0 \mathrm{mg}, 0.100 \mathrm{mmol})$ and 4-methoxyphenol (12.0 mg, $0.100 \mathrm{mmol}),(E)-2-(4-m e t h o x y p h e n o x y) p e n t-2-e n-1-y l$ 2iodobenzoateiodobenzoate $4 \mathrm{n}(28.0 \mathrm{mg}, 64.0 \mu \mathrm{mol}, 64 \%$ yield) was obtained, as a colorless amorphous solid. Rf: 0.52 (Pentane:EtOAc 9:1). ${ }^{1} \mathrm{H}$ NMR (400 MHz, Acetonitrile- $\left.d_{3}\right) \delta 8.02(\mathrm{dd}, J=7.9,1.1 \mathrm{~Hz}$, $1 \mathrm{H}, \mathrm{ArH}), 7.68(\mathrm{dd}, J=7.8,1.7 \mathrm{~Hz}, 1 \mathrm{H}, \mathrm{ArH}), 7.47(\mathrm{td}, J=7.6,1.2 \mathrm{~Hz}$, $1 \mathrm{H}, \operatorname{ArH}), 7.23(\mathrm{td}, J=7.7,1.7 \mathrm{~Hz}, 1 \mathrm{H}, \operatorname{ArH}), 6.98-6.93(\mathrm{~m}, 2 \mathrm{H}, \operatorname{ArH}), 6.90-6.85(\mathrm{~m}, 2 \mathrm{H}, \operatorname{ArH}), 5.03(\mathrm{t}, J$ $=7.9 \mathrm{~Hz}, 1 \mathrm{H}, \mathrm{OCCH}), 4.97\left(\mathrm{~s}, 2 \mathrm{H}, \mathrm{OCH}_{2} \mathrm{CO}\right), 3.74\left(\mathrm{~s}, 3 \mathrm{H}, \mathrm{OCH}_{3}\right), 2.19\left(\mathrm{p}, J=7.6 \mathrm{~Hz}, 2 \mathrm{H}, \mathrm{CH}_{2}\right), 0.98(\mathrm{t}, J=$ $\left.7.5 \mathrm{~Hz}, 3 \mathrm{H}, \mathrm{CH}_{3}\right) .{ }^{13} \mathrm{C}$ NMR $\left(101 \mathrm{MHz}\right.$, Acetonitrile- $\left.d_{3}\right) \delta 167.3,156.7,150.4,149.9,142.0,136.7,133.8$, 131.5, 129.2, 121.2, 117.8, 115.6, 94.0, 61.5, 56.2, 20.6, 15.1. IR v $2963(\mathrm{~m}), 1730(\mathrm{~s}), 1672(\mathrm{~m}), 1582$ $(\mathrm{m}), 1504(\mathrm{~s}), 1460(\mathrm{~m}), 1280(\mathrm{~s}), 1247(\mathrm{~s}), 1214(\mathrm{~s}), 1123(\mathrm{~m}), 1105(\mathrm{~m}), 1025(\mathrm{~m}), 946(\mathrm{~m}), 837(\mathrm{~m})$, 745 (m). HRMS (ESI/QTOF) m/z: [M + H] Calcd for $\mathrm{C}_{19} \mathrm{H}_{20} \mathrm{OO}_{4}{ }^{+} 439.0401$; Found 439.0404 .

\section{2-(4-Methoxyphenoxy)allyl 2-iodobenzoate (40)}<smiles>C=C(COC(=O)c1ccccc1I)Oc1ccc(OC)cc1</smiles>

Starting from EBX 5a (29.0 mg, $0.100 \mathrm{mmol})$ and 4-methoxyphenol (12.0 mg, $0.100 \mathrm{mmol}$ ), 2-(4-methoxyphenoxy)allyl 2-iodobenzoate 40 (23.8 mg, $58.0 \mu \mathrm{mol}, 58 \%$ yield) was obtained, as a colorless amorphous solid. Rf: 0.50 (Pentane:EtOAc 9:1). ${ }^{1} \mathbf{H}$ NMR (400 MHz, Chloroform-d) $\delta 8.01(\mathrm{dd}, J=7.9,1.2 \mathrm{~Hz}, 1 \mathrm{H}, \mathrm{ArH}), 7.88$ (dd, $J=7.8$, $1.7 \mathrm{~Hz}, 1 \mathrm{H}, \mathrm{ArH}), 7.42(\mathrm{td}, J=7.6,1.2 \mathrm{~Hz}, 1 \mathrm{H}, \mathrm{ArH}), 7.17(\mathrm{td}, J=7.6$, $1.7 \mathrm{~Hz}, 1 \mathrm{H}, \mathrm{ArH}), 7.06-6.99(\mathrm{~m}, 2 \mathrm{H}, \mathrm{ArH}), 6.91-6.85(\mathrm{~m}, 2 \mathrm{H}, \mathrm{ArH}), 4.94\left(\mathrm{~s}, 2 \mathrm{H}, \mathrm{OCH}_{2} \mathrm{CO}\right), 4.51(\mathrm{~d}, J=$ $\left.2.4 \mathrm{~Hz}, 1 \mathrm{H}, \mathrm{OCCH}_{2}\right), 4.16\left(\mathrm{~d}, \mathrm{~J}=2.4 \mathrm{~Hz}, 1 \mathrm{H}, \mathrm{OCCH}_{2}\right), 3.79\left(\mathrm{~s}, 3 \mathrm{H}, \mathrm{OCH}_{3}\right) .{ }^{13} \mathrm{C}$ NMR $(101 \mathrm{MHz}$, Chloroformd) $\delta 166.1,158.0,156.6,148.2,141.6,134.8,133.0,131.3,128.1,122.2,114.8,94.4,91.2,65.0,55.7$. IR v $3667(\mathrm{~m}), 2994(\mathrm{~m}), 2907(\mathrm{~m}), 1732(\mathrm{~s}), 1649(\mathrm{~m}), 1588(\mathrm{w}), 1505$ (s), $1436(\mathrm{w}), 1388(\mathrm{w}), 1290(\mathrm{~m})$, $1219(\mathrm{~s}), 1105(\mathrm{~m}), 1027$ (s), $963(\mathrm{~m}), 908$ (m), 841 (m), 745 (s). HRMS (ESI/QTOF) m/z: [M + H]+ Calcd for $\mathrm{C}_{17} \mathrm{H}_{16} \mathrm{IO}_{4}{ }^{+}$411.0088; Found 411.0094 .

\section{(E)-2-(2-Formylphenoxy)oct-2-en-1-yl 2-iodobenzoate (4p)}

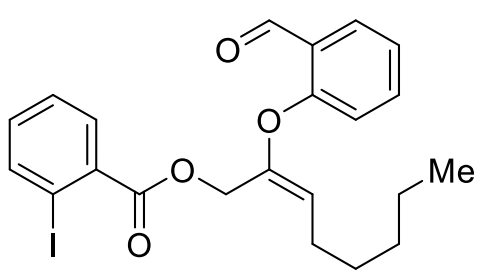

Starting from EBX $5 c \quad(36.0 \mathrm{mg}, 0.100 \mathrm{mmol})$ and 2hydroxybenzaldehyde $(10.7 \mu \mathrm{L}, \quad 0.100 \quad \mathrm{mmol}), \quad(E)-2-(2-$ formylphenoxy)oct-2-en-1-yl 2-iodobenzoate $4 \mathrm{p}$ (28.0 $\mathrm{mg}, 59.0$ $\mu \mathrm{mol}, 59 \%$ yield) was obtained, as a colorless amorphous solid. Rf: 0.55 (Pentane:EtOAc 9:1). ${ }^{1} \mathrm{H}$ NMR $\left(400 \mathrm{MHz}\right.$, Acetonitrile- $\left.d_{3}\right) \delta 10.35$ (d, J = 0.8 Hz, 1H), 8.01 (dd, J = 7.9, 1.2 Hz, 1H, ArH), 7.80 (dd, J = 8.0, $1.8 \mathrm{~Hz}, 1 \mathrm{H}, \mathrm{ArH}), 7.64$ (dd, $J=7.8,1.7 \mathrm{~Hz}, 1 \mathrm{H}, \mathrm{ArH}), 7.60$ (ddd, $J=8.3,7.3,1.8 \mathrm{~Hz}, 1 \mathrm{H}, \operatorname{ArH}), 7.44(\mathrm{td}, J=$ 7.6, 1.2 Hz, 1H, ArH), 7.26- $7.15(\mathrm{~m}, 3 \mathrm{H}, \mathrm{ArH}), 5.26(\mathrm{t}, \mathrm{J}=8.0 \mathrm{~Hz}, 1 \mathrm{H}, \mathrm{OCCH}), 5.06\left(\mathrm{~s}, 2 \mathrm{H}, \mathrm{OCH}_{2} \mathrm{CO}\right), 2.23$ (q, J = 7.4 Hz, 2H, CH 2$), 1.48-1.37\left(\mathrm{~m}, 2 \mathrm{H}, \mathrm{CH}_{2}\right), 1.30$ (ddd, J = 7.2, 4.4, 3.1 Hz, 4H, CH 2$), 0.93-0.80(\mathrm{~m}$, $\left.3 \mathrm{H}, \mathrm{CH}_{3}\right) .{ }^{13} \mathrm{C}$ NMR $\left(101 \mathrm{MHz}\right.$, Acetonitrile- $\left.d_{3}\right) \delta$ 190.0, 167.1, 159.9, 149.0, 142.1, 136.9, 136.3, 133.9, 131.5, 129.2, 128.9, 127.8, 124.4, 120.7, 119.7, 94.1, 61.3, 32.0, 30.1, 27.1, 23.1, 14.3. IR v 3646 (s), 2938 (s), 2260 (s), 1875 (s), 1733 (s), 1686 (s), 1593 (s), 1467 (s), 1282 (s), 1253 (s), 1038 (s), 903 (s), 840 (s), 755 (s). HRMS (ESI/QTOF) m/z: [M + Na] ${ }^{+}$Calcd for $\mathrm{C}_{22} \mathrm{H}_{23} \mathrm{INaO}_{4}{ }^{+}$501.0533; Found 501.0538. 


\section{Crystal Structure}

\section{Crystal structure of 3a:}

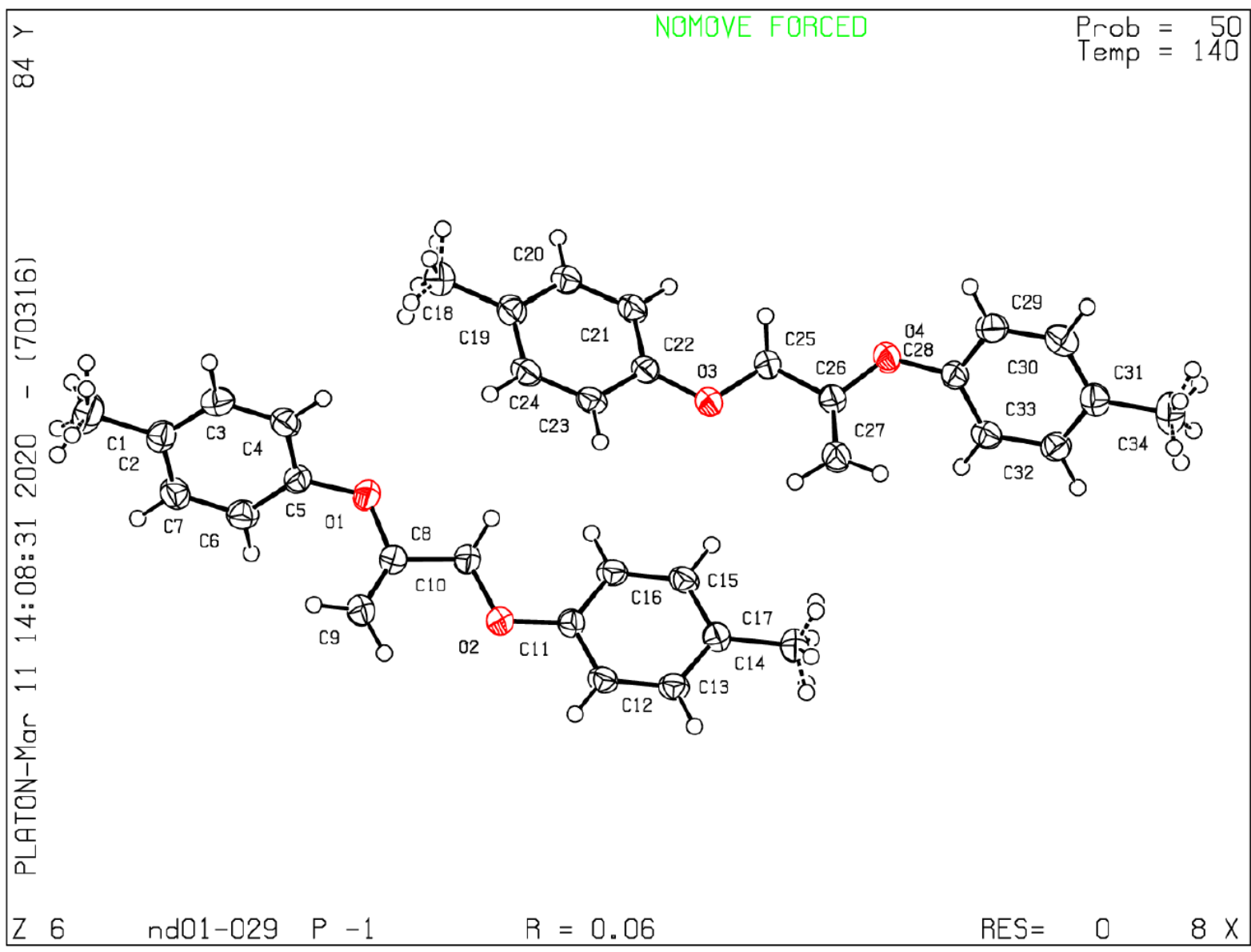

A single crystal was grown by slow diffusion of the solution of 3a in ethyl acetate and pentane mixture. Supplementary crystallographic data for this compound have been deposited at Cambridge Crystallographic Data Centre (1989749) and can be obtained free of charge via www.ccdc.cam.ac.uk/data request/cif. 


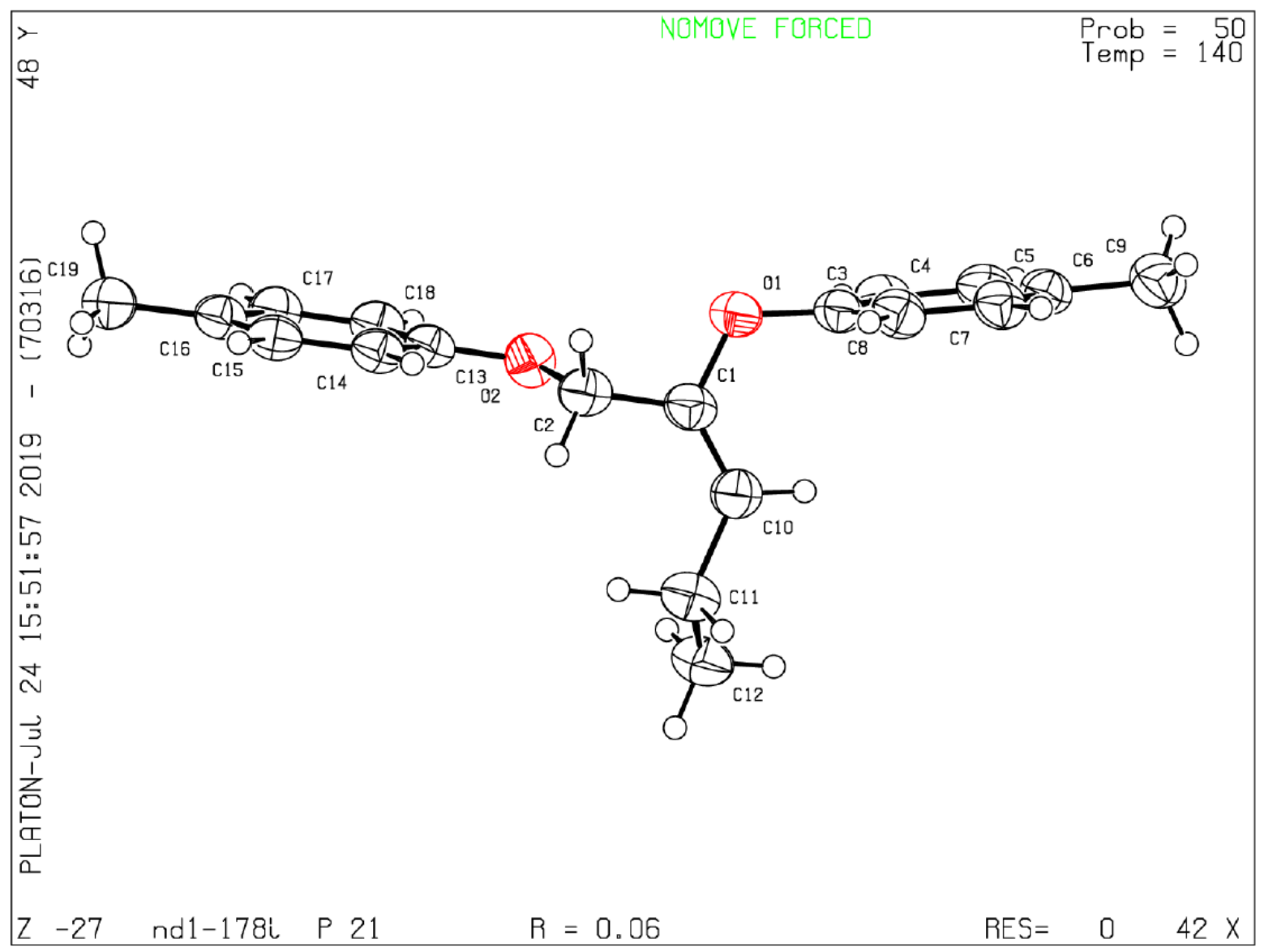

A single crystal was grown by slow diffusion of the solution of $3 p$ in ethyl acetate and pentane mixture. Supplementary crystallographic data for this compound have been deposited at Cambridge Crystallographic Data Centre (1989757) and can be obtained free of charge via www.ccdc.cam.ac.uk/data request/cif. 


\section{NOE experiments}

\section{NOE experiment on compound 4c:}
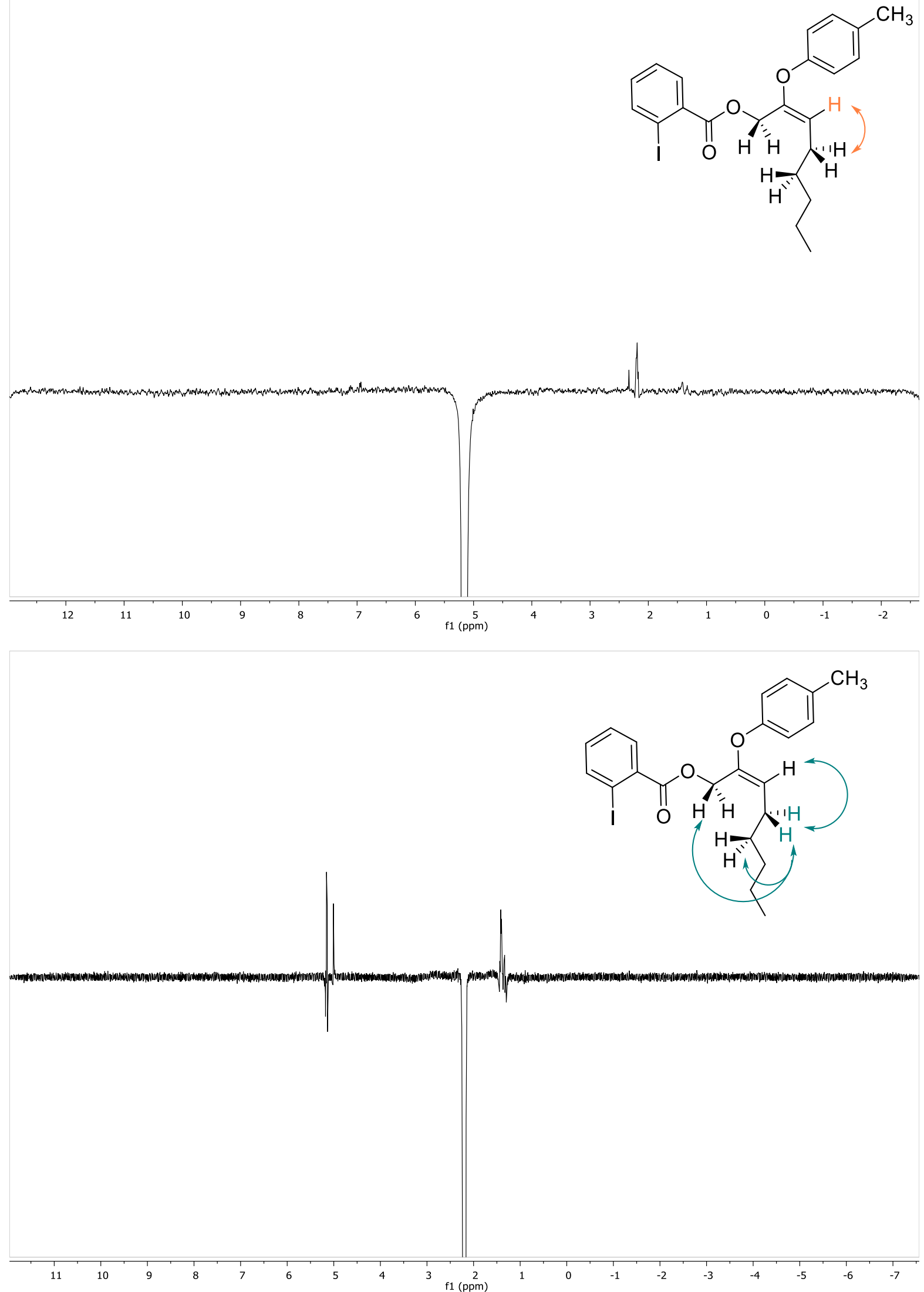

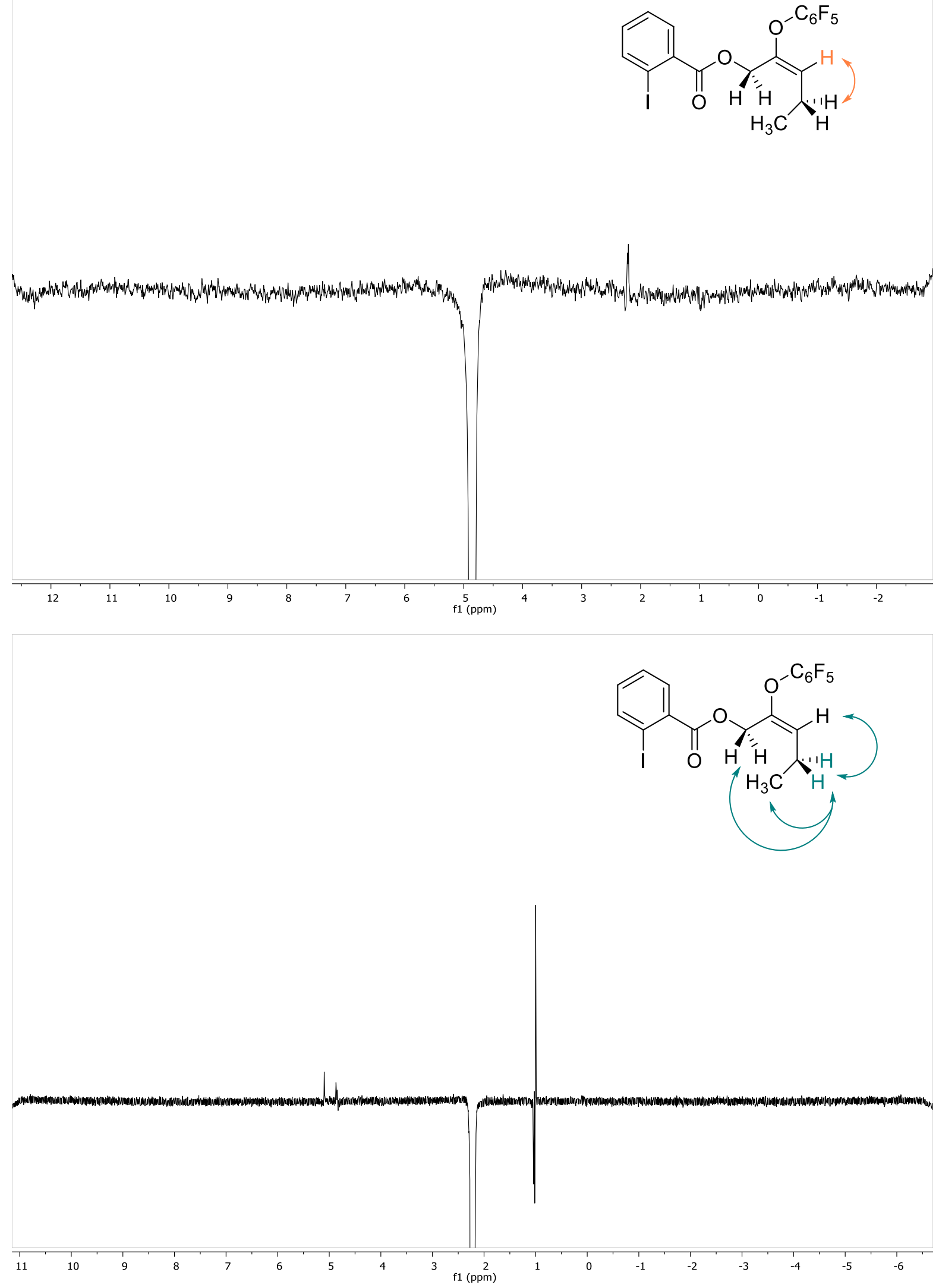


\section{Gram scale synthesis}

\section{(E)-2-(p-Tolyloxy)-8-(trimethylsilyl)oct-2-en-7-yn-1-yl 2-iodobenzoate (4e)}<smiles>CS(C)(=O)=CCCCCC#CI1COC(=O)c2ccccc21</smiles>

1) 1.00 equiv. $p$-cresol $10 \mathrm{~mol} \% \mathrm{Cs}_{2} \mathrm{CO}_{3}$

2) 0.20 equiv. anisole 1.20 equiv. $\mathrm{Cs}_{2} \mathrm{CO}_{3}$

DME $0.1 \mathrm{M}$ rt, $16 \mathrm{~h}$<smiles>Cc1ccc(O/C(=C\CCCC#CC(C)(C)C)COC(=O)c2ccccc2I)cc1</smiles>

In a round-bottom flask, $p$-cresol $(0.255 \mathrm{~g}, 2.36 \mathrm{mmol}, 1.00$ equiv.) was dissolved in $23.5 \mathrm{~mL}$ of DME $(0.1 \mathrm{M})$. Cesium carbonate $(77.0 \mathrm{mg}, 0.236 \mathrm{mmol})$ was added and the mixture stirred vigorously for 5 $\mathrm{min}$. Then the corresponding EBX $5 \mathrm{e}$ was added in one portion $(1.00 \mathrm{~g}, 2.36 \mathrm{mmol}, 1.00$ equiv. $)$ and the reaction was left stirring for 16 hours at room temperature. Then cesium carbonate $(0.921 \mathrm{~g}, 2.83$ $\mathrm{mmol}, 1.20$ equiv.) and anisole $(0.471 \mathrm{mmol}, 51.0 \mu \mathrm{L})$ were added and the solution was stirred at room temperature for 16 hours. The reaction mixture was filtrated, the solvent was removed under reduced pressure and the crude material was purified by column chromatography using phosphate buffered silica $\left(\mathrm{Na}_{2} \mathrm{HPO}_{4}\right)$ and pentane/ethyl acetate as eluent. $(E)$-2-( $p$-tolyloxy)-8-(trimethylsilyl)oct-2-en-7-yn1 -yl 2-iodobenzoate $4 \mathrm{e}(0.825 \mathrm{~g}, 1.55 \mathrm{mmol}, 66 \%$ yield) was obtained, as a colorless sticky solid.

\section{(E)-1-Methoxy-4-((1-(p-tolyloxy)pent-2-en-2-yl)oxy)benzene (3x)}<smiles>CCC/C(=C\I(C)c1ccccc1C(=O)O)Oc1ccc(OC)cc1</smiles><smiles>CC/C=C(/COc1ccc(C)cc1)Oc1ccc(OC)cc1</smiles>

In a round-bottom flask, $p$-cresol $(0.247 \mathrm{~g}, 2.28 \mathrm{mmol}, 1$ equiv.) and cesium carbonate $(0.892 \mathrm{~g}, 2.74$ mmol, 1.20 equiv.) were added. Anhydrous DME (23 mL, $0.1 \mathrm{M})$ was introduced at $0{ }^{\circ} \mathrm{C}$ and the solution was stirred at room temperature for $10 \mathrm{~min}$. O-VBX reagent $1 \mathrm{j}(1.00 \mathrm{~g}, 2.28 \mathrm{mmol}, 1$ equiv.) was added to the reaction mixture under open air and the reaction mixture was stirred at room temperature for $16 \mathrm{~h}$. The reaction mixture was filtrated, the solvent was removed under reduced pressure and the crude material was purified by column chromatography using Pentane:EtOAc (9:1). (E)-1-Methoxy-4((1-( $p$-tolyloxy)pent-2-en-2-yl)oxy)benzene $3 x(0.427 \mathrm{~g}, 1.43 \mathrm{mmol}, 63 \%$ yield) was obtained, as a colorless sticky solid. 


\section{Product modifications}

\section{Palladium oxidation cyclization:}

\section{2-((p-Tolyloxy)methyl)benzofuran (6)}

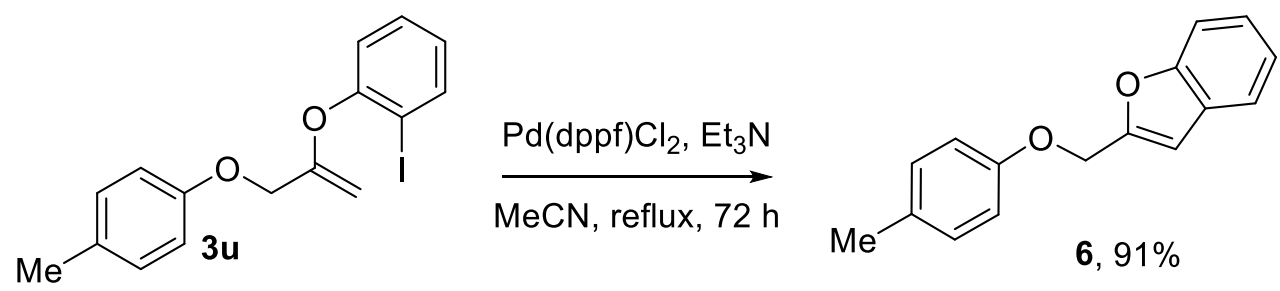

Following a reported procedure, ${ }^{22}$ to a microwave vial charged with 1-iodo-2-((3-(p-tolyloxy)prop-1en-2-yl)oxy)benzene $3 \mathbf{u}(36.6 \mathrm{mg}, 0.1 \mathrm{mmol}), \mathrm{Pd}(\mathrm{dppf}) \mathrm{Cl}_{2}(7.32 \mathrm{mg}, 10.0 \mu \mathrm{mol})$ and triethylamine $(0.028 \mathrm{~mL}, 0.20 \mathrm{mmol})$ was added dry $\operatorname{MeCN}(0.27 \mathrm{~mL})$ under $\mathrm{N}_{2}$. The reaction mixture was stirred at $82{ }^{\circ} \mathrm{C}$ for 72 hours to go to complete conversion. The solution was then filtrated and concentrated under reduced pressure. The crude was purified by preparative TLC (Pentane:EtOAc 80:20) to provide 2-((p-tolyloxy)methyl)benzofuran $6(21.6 \mathrm{mg}, 91.0 \mu \mathrm{mol}, 91 \%$ yield) as a white solid. Rf: 0.7 (Pentane:EtOAc 9:1). Mp: $78{ }^{\circ} \mathrm{C} .{ }^{1} \mathrm{H}$ NMR $\left(400 \mathrm{MHz}\right.$, Methylene Chloride- $\left.d_{2}\right) \delta 7.61-7.54(\mathrm{~m}, 1 \mathrm{H}, \mathrm{Ar} H)$, 7.49 (dq, $J=8.4,1.0 \mathrm{~Hz}, 1 \mathrm{H}, \mathrm{ArH}), 7.30$ (ddd, $J=8.3,7.2,1.4 \mathrm{~Hz}, 1 \mathrm{H}, \operatorname{ArH}), 7.24(\mathrm{td}, J=7.5,1.1 \mathrm{~Hz}, 1 \mathrm{H}$, $\operatorname{ArH}), 7.15-7.07(\mathrm{~m}, 2 \mathrm{H}, \mathrm{ArH}), 6.94-6.86(\mathrm{~m}, 2 \mathrm{H}, \mathrm{ArH}), 6.79(\mathrm{~d}, J=0.9 \mathrm{~Hz}, 1 \mathrm{H}, \mathrm{OCCH}), 5.13(\mathrm{~d}, J=0.7$ $\left.\mathrm{Hz}, 2 \mathrm{H}, \mathrm{OCH}_{2} \mathrm{C}\right), 2.29\left(\mathrm{~s}, 3 \mathrm{H}, \mathrm{CH}_{3}\right) .{ }^{13} \mathrm{C}$ NMR $\left(101 \mathrm{MHz}\right.$, Methylene Chloride- $\left.d_{2}\right) \delta$ 156.7, 155.7, 153.9, 131.4, 130.5, 128.6, 125.1, 123.4, 121.6, 115.3, 111.8, 106.5, 63.6, 20.7. IR v 3112 (m), 3045 (s), 2925 (s), 2861 (s), 1735 (s), 1612 (s), 1513 (s), 1458 (s), 1377 (s), 1287 (s), 1247 (s), 1182 (s), 1017 (s), 950 (s), 858 (s), 816 (s), 747 (s), 710 (s). HRMS (APPI/QTOF) m/z: [M] ${ }^{+}$Calcd for $\mathrm{C}_{16} \mathrm{H}_{13} \mathrm{O}_{2}{ }^{+}$237.0910; Found 237.0902 .

\section{Palladium reduction:}

\section{2-Oxopentyl benzoate (7)}<smiles>CC/C=C(/COC(=O)c1ccccc1I)Oc1ccc(C)cc1</smiles>

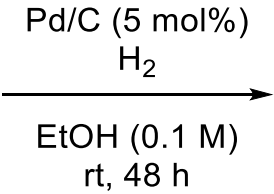

$\mathrm{rt}, 48 \mathrm{~h}$<smiles>CCCC(=O)COC(=O)c1ccccc1</smiles>

2-(p-Tolyloxy)pent-1-en-3-yl 2-iodobenzoate $4 \mathrm{~b}(42.2 \mathrm{mg}, 0.100 \mathrm{mmol}), \mathrm{Pd} / \mathrm{C}$ (loading $5 \mathrm{wt} . \%, 11.8$ $\mathrm{mg}, 5 \mathrm{~mol} \%)$ and $\mathrm{EtOH}(2.90 \mathrm{~mL}, 0.035 \mathrm{M}$ ) were added into a flame dried test tube equipped with a teflon coated stir bar. The tube was placed under hydrogen atmosphere using an autoclave (10 bar) and the solution was stirred at room temperature for $24 \mathrm{~h}$. 2-Oxopentyl benzoate 7 (19.9 mg, 96.0 $\mu \mathrm{mol}, 96 \%$ yield) was obtained, as a colorless sticky solid. Rf: 0.51 (Pentane:EtOAc 9:1). ${ }^{1} \mathbf{H}$ NMR (400 $\mathrm{MHz}$, Acetonitrile- $\left.d_{3}\right) \delta 8.09-8.02(\mathrm{~m}, 2 \mathrm{H}, \mathrm{ArH}), 7.70-7.62(\mathrm{~m}, 1 \mathrm{H}, \mathrm{ArH}), 7.57-7.48(\mathrm{~m}, 2 \mathrm{H}, \operatorname{Ar} H)$, $4.91\left(\mathrm{~s}, 2 \mathrm{H}, \mathrm{OCH}{ }_{2} \mathrm{CO}\right), 2.48\left(\mathrm{t}, J=7.3 \mathrm{~Hz}, 2 \mathrm{H}, \mathrm{COCH}_{2} \mathrm{CH}_{2} \mathrm{CH}_{3}\right), 1.61\left(\mathrm{~h}, J=7.3 \mathrm{~Hz}, 2 \mathrm{H}, \mathrm{COCH}_{2} \mathrm{CH}_{2} \mathrm{CH}_{3}\right), 0.93$ $\left(\mathrm{t}, J=7.4 \mathrm{~Hz}, 3 \mathrm{H}, \mathrm{COCH}_{2} \mathrm{CH}_{2} \mathrm{CH}_{3}\right) .{ }^{13} \mathrm{C}$ NMR $\left(101 \mathrm{MHz}\right.$, Acetonitrile- $\left.d_{3}\right) \delta$ 205.0, 166.5, 134.4, 130.5, 130.4, 129.7, 69.4, 41.0, 17.5, 13.8. IR $2963(\mathrm{w}), 2878(\mathrm{w}), 2261$ (m), $1726(\mathrm{~s}), 1719(\mathrm{~s}), 1458(\mathrm{w}), 1415$ $(\mathrm{m}), 1375(\mathrm{~m}), 1275(\mathrm{~s}), 1116(\mathrm{~m}), 1056(\mathrm{~m}), 1031(\mathrm{~m}), 984(\mathrm{w}), 840(\mathrm{~m}), 713(\mathrm{~s})$. HRMS (APCI/QTOF) $\mathrm{m} / \mathrm{z}:[\mathrm{M}+\mathrm{Na}]^{+}$Calcd for $\mathrm{C}_{12} \mathrm{H}_{14} \mathrm{NaO}_{3}{ }^{+} 229.0835$; Found 229.0833 .

\footnotetext{
${ }^{22}$ L. Zhou, Y. Shi, X. Zhu, P. Zhang, Tetrahedron Lett. 2019, 60, 2005-2008.
} 


\section{Epoxidation:}<smiles>[R]C=C(COc1ccc(C)cc1)Oc1ccc([R])cc1</smiles>
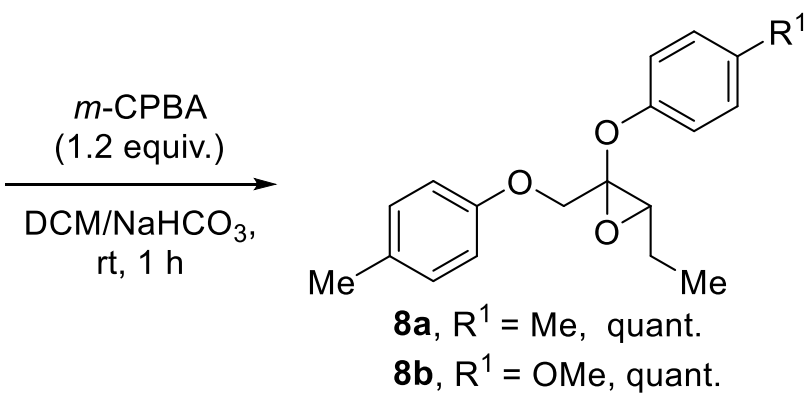

To a solution of $3\left(0.300 \mathrm{mmol}, 1.0\right.$ equiv.) in $\mathrm{DCM}(3.0 \mathrm{~mL})$ was added $\mathrm{NaHCO}_{3}(3.0 \mathrm{~mL})$ and then 3 chloroperbenzoic acid $(0.360 \mathrm{mmol}, 1.2$ equiv.). The resulting mixture was stirred at room temperature for $5 \mathrm{~h}$. A saturated aqueous solution of $\mathrm{NaHCO}_{3}(3 \mathrm{~mL})$ was added to the reaction mixture and stirred for $10 \mathrm{~min}$. The organic layers were extracted with dichloromethane three times. The combined extracts were washed with brine and dried over anhydrous sodium sulfate. The sulfate was removed by filtration and the filtrate was concentrated under reduced pressure to provide product 8 as a yellowish oil.

\section{3-Ethyl-2-(p-tolyloxy)-2-((p-tolyloxy)methyl)oxirane (8a)}<smiles>CCC1OC1(COc1ccc(C)cc1)Oc1ccc(C)cc1</smiles>

Starting from $3 p(85.0 \mathrm{mg}, 0.300 \mathrm{mmol})$, 3-ethyl-2-(p-tolyloxy)-2(( $p$-tolyloxy)methyl)oxirane 8a ( $90.0 \mathrm{mg}, 0.300 \mathrm{mmol}$, quant. yield) was obtained, as a yellowish oil. Rf: 0.81 (Pentane:EtOAc 9:1). ${ }^{1} \mathbf{H}$ NMR $\left(400 \mathrm{MHz}\right.$, Acetonitrile- $\left.d_{3}\right) \delta 7.15-7.04(\mathrm{~m}, 4 \mathrm{H}, \mathrm{ArH}), 7.00-$ $6.92(\mathrm{~m}, 2 \mathrm{H}, \mathrm{ArH}), 6.86-6.76(\mathrm{~m}, 2 \mathrm{H}, \mathrm{ArH}), 4.44(\mathrm{dd}, J=11.2,0.8$ $\left.\mathrm{Hz}, 1 \mathrm{H}, \mathrm{OCH}_{2} \mathrm{C}\right), 4.09\left(\mathrm{~d}, J=11.2 \mathrm{~Hz}, 1 \mathrm{H}, \mathrm{OCH}_{2} \mathrm{C}\right), 3.21-3.15(\mathrm{~m}$, $\left.1 \mathrm{H}, \mathrm{OCHCH}_{2}\right), 2.27\left(\mathrm{~s}, 3 \mathrm{H}, \mathrm{ArCH}_{3}\right), 2.25\left(\mathrm{~s}, 3 \mathrm{H}, \mathrm{ArCH}_{3}\right), 1.71-1.56\left(\mathrm{~m}, 2 \mathrm{H}, \mathrm{CH}_{2} \mathrm{CH}_{3}\right), 1.04(\mathrm{t}, J=7.5 \mathrm{~Hz}$, $\left.3 \mathrm{H}, \mathrm{CH}_{2} \mathrm{CH}_{3}\right) .{ }^{13} \mathrm{C}$ NMR $\left(101 \mathrm{MHz}\right.$, Acetonitrile- $\left.d_{3}\right) \delta 157.3,152.9,133.9,131.7,130.9,130.9,120.4$, 115.5, 86.7, 66.8, 64.0, 22.1, 20.6, 20.4, 10.4. IR $3032(\mathrm{~m}), 2972(\mathrm{~m}), 2928(\mathrm{~m}), 2878(\mathrm{~m}), 1612(\mathrm{~m})$, $1587(\mathrm{~m}), 1510(\mathrm{~s}), 1462(\mathrm{~m}), 1289(\mathrm{~m}), 1221(\mathrm{~s}), 1175(\mathrm{~m}), 1127(\mathrm{~m}), 1108(\mathrm{~m}), 1051(\mathrm{~m}), 1016(\mathrm{~m})$, $930(\mathrm{~m}), 843(\mathrm{~m}), 818(\mathrm{~s}), 768(\mathrm{~m}), 737(\mathrm{~m}), 701(\mathrm{~m})$. HRMS (ESI/QTOF) m/z: [M + Na] ${ }^{+}$Calcd for $\mathrm{C}_{19} \mathrm{H}_{22} \mathrm{NaO}_{3}{ }^{+}$321.1461; Found 321.1464.

\section{3-Ethyl-2-(4-methoxyphenoxy)-2-((p-tolyloxy)methyl)oxirane (8b)}

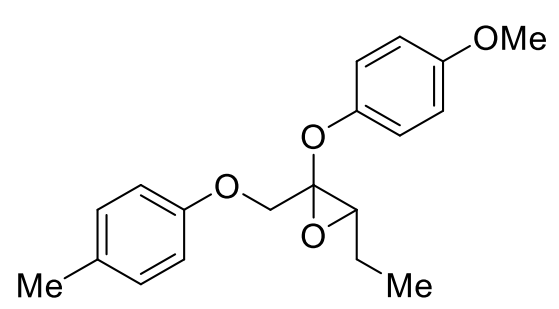

Starting from $3 \mathbf{x}(30.0 \mathrm{mg}, 0.100 \mathrm{mmol})$, 3-ethyl-2-(4methoxyphenoxy)-2-((p-tolyloxy)methyl)oxirane 8 b $(31.0 \mathrm{mg}$, $0.100 \mathrm{mmol}$, quant. yield) was obtained, as a yellowish oil. Rf: 0.55 (Pentane:EtOAc 9:1). ${ }^{1} \mathbf{H}$ NMR (400 MHz, Acetonitrile- $\left.d_{3}\right) \delta$ $7.12-7.06(\mathrm{~m}, 2 \mathrm{H}, \mathrm{ArH}), 7.03-6.98(\mathrm{~m}, 2 \mathrm{H}, \mathrm{ArH}), 6.87-6.81(\mathrm{~m}$, $4 \mathrm{H}, \mathrm{ArH}), 4.41\left(\mathrm{dd}, J=11.2,0.9 \mathrm{~Hz}, 1 \mathrm{H}, \mathrm{OCH}_{2} \mathrm{C}\right), 4.06(\mathrm{~d}, J=11.1$

$\mathrm{Hz}, 1 \mathrm{H}, \mathrm{OCH}_{2} \mathrm{C}$ ), $3.73\left(\mathrm{~s}, 3 \mathrm{H}, \mathrm{OCH}_{3}\right.$ ), 3.17 (td, J $\left.=6.6,0.8 \mathrm{~Hz}, 1 \mathrm{H}, \mathrm{OCHCH}_{2}\right), 2.25$ (d, J=0.8 Hz, 3H, ArCH $)_{3}$, 1.62 (qd, $J=7.5,6.4 \mathrm{~Hz}, 2 \mathrm{H}, \mathrm{CH}_{2} \mathrm{CH}_{2}$ ), 1.02 (t, $\left.J=7.5 \mathrm{~Hz}, 3 \mathrm{H}, \mathrm{CH}_{3}\right) .{ }^{13} \mathrm{C} \mathrm{NMR}\left(101 \mathrm{MHz}\right.$, Acetonitrile- $d_{3}$ ) $\delta$ 157.3, 157.0, 148.4, 131.7, 130.9, 122.2, 115.5, 115.4, 87.2, 66.9, 63.8, 56.1, 22.1, 20.4, 10.3. IR v 2979 (w), $2940(w), 2880(w), 1732(w), 1613(w), 1588(w), 1507(s), 1469(w), 1439(w), 1393(w), 1291(m)$, $1242(\mathrm{~s}), 1211(\mathrm{~s}), 1178(\mathrm{~m}), 1126(\mathrm{w}), 1106(\mathrm{~m}), 1038(\mathrm{~m}), 1012(\mathrm{w}), 930(\mathrm{~m}), 823(\mathrm{~m}), 736(\mathrm{~m}), 705$ (w). HRMS (APPI/LTQ-Orbitrap) m/z: [M] ${ }^{+}$Calcd for $\mathrm{C}_{19} \mathrm{H}_{22} \mathrm{O}_{4}{ }^{+}$314.1513; Found 314.1513. 
<smiles>[R]C=C(COc1ccc(C)cc1)Oc1ccc([R])cc1</smiles>

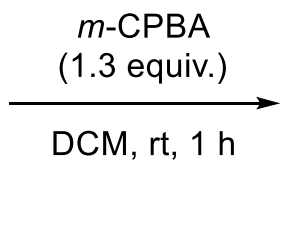

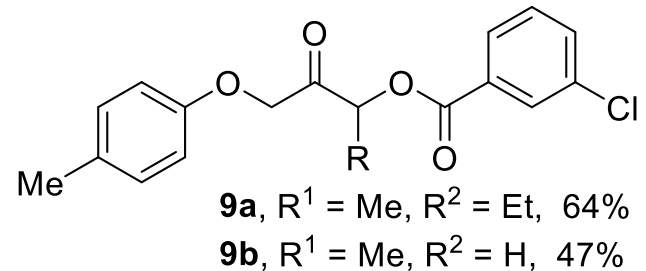

To a solution of $\mathbf{3}(0.300 \mathrm{mmol}, 1.0$ equiv.) in DCM $(3.0 \mathrm{~mL})$ was added 3 -chloroperbenzoic acid $(0.390$ mmol, 1.3 equiv.). The resulting mixture was stirred at room temperature for $17 \mathrm{~h}$. A saturated aqueous solution of $\mathrm{NaHCO}_{3}(3 \mathrm{~mL})$ was added to the reaction mixture and stirred for $10 \mathrm{~min}$. The organic layers were extracted with dichloromethane three times. The combined extracts were washed with brine and dried over anhydrous sodium sulfate. The sulfate was removed by filtration and the filtrate was concentrated under reduced pressure. The crude was purified by column chromatography (pentane:ethyl acetate 9:1) to provide product 9 as a colorless sticky solid.

\section{2-0xo-1-(p-tolyloxy)pentan-3-yl 3-chlorobenzoate (9a)}<smiles>CCC(OC(=O)c1cccc(Cl)c1)C(=O)COc1ccc(C)cc1</smiles>

Starting from $3 p(85.0 \mathrm{mg}, 0.300 \mathrm{mmol}), 2-0 x 0-1-(p-$ tolyloxy)pentan-3-yl 3-chlorobenzoate 9 a $(66.5 \mathrm{mg}, 0.19$ $\mathrm{mmol}, 64 \%$ yield) was obtained, as a colorless oil. $\mathbf{R f :} 0.43$ (Pentane:EtOAc 9:1). ${ }^{1} \mathbf{H}$ NMR (400 MHz, Acetonitrile- $\left.d_{3}\right) \delta$ $8.05(\mathrm{t}, J=1.9 \mathrm{~Hz}, 1 \mathrm{H}, \mathrm{ArH}), 8.01(\mathrm{dt}, J=7.8,1.4 \mathrm{~Hz}, 1 \mathrm{H}$, $\operatorname{ArH}), 7.68$ (ddd, $J=8.1,2.3,1.1 \mathrm{~Hz}, 1 \mathrm{H}, \mathrm{ArH}), 7.52(\mathrm{t}, J=7.9 \mathrm{~Hz}, 1 \mathrm{H}, \mathrm{ArH}), 7.14-7.07(\mathrm{~m}, 2 \mathrm{H}, \operatorname{ArH}), 6.85$ - $6.77(\mathrm{~m}, 2 \mathrm{H}, \mathrm{ArH}), 5.43(\mathrm{dd}, J=7.8,4.4 \mathrm{~Hz}, 1 \mathrm{H}, \mathrm{OCCHO}), 4.98-4.82\left(\mathrm{~m}, 2 \mathrm{H}, \mathrm{OCH}_{2} \mathrm{CO}\right), 2.26(\mathrm{~s}, 3 \mathrm{H}$, $\mathrm{ArCH}_{3}$ ), 2.08 (ddd, $J=14.6,7.3,3.8 \mathrm{~Hz}, 1 \mathrm{H}, \mathrm{CH}_{2}$ ), 1.99 (dd, $J=15.0,7.6 \mathrm{~Hz}, 1 \mathrm{H}, \mathrm{CH}_{2}$ ), 1.07 (t, $J=7.4 \mathrm{~Hz}$, $\left.3 \mathrm{H}, \mathrm{CH}_{3}\right) .{ }^{13} \mathrm{C}$ NMR $\left(101 \mathrm{MHz}\right.$, Acetonitrile- $\left.d_{3}\right) \delta 203.7,165.6,156.7,135.2,134.4,132.3,131.8,131.4$, 130.9, 130.2, 129.0, 115.3, 79.3, 71.6, 24.5, 20.4, 9.9. IR $3673(\mathrm{~m}), 3093(\mathrm{~m}), 2978(\mathrm{~s}), 2878(\mathrm{~m}), 2604$ $(w), 1934(w), 1746(m), 1720(m), 1592(w), 1512(m), 1472(w), 1427(w), 1392(m), 1302(m), 1244$ $(\mathrm{m}), 1181(\mathrm{w}), 1070(\mathrm{~s}), 1043(\mathrm{~s}), 957(\mathrm{w}), 897(\mathrm{w}), 870(\mathrm{w}), 847(\mathrm{~m}), 832(\mathrm{~m}), 751(\mathrm{~m}), 687(\mathrm{w}), 673(\mathrm{w})$, 632 (w). HRMS (ESI/QTOF) m/z: [M + Na] ${ }^{+}$Calcd for $\mathrm{C}_{19} \mathrm{H}_{19} \mathrm{ClNaO}_{4}{ }^{+} 369.0864$; Found 369.0863.

\section{2-0xo-3-(p-tolyloxy)propyl 3-chlorobenzoate (9b)}<smiles>Cc1ccc(OCC(=O)COC(=O)c2cccc(Cl)c2)cc1</smiles>

Starting from 3a $(25.0 \mathrm{mg}, 0.100 \mathrm{mmol}), 2-0 \times 0-3-(p-$ tolyloxy)propyl 3-chlorobenzoate $9 \mathrm{~b}(15.0 \mathrm{mg}, 47.0 \mu \mathrm{mol}$, $47 \%$ yield) was obtained, as a colorless oil. Rf: 0.29 (Pentane:EtOAc 9:1). ${ }^{1} \mathbf{H}$ NMR (400 MHz, Acetonitrile- $\left.d_{3}\right) \delta$ $8.04(\mathrm{t}, J=1.9 \mathrm{~Hz}, 1 \mathrm{H}, \mathrm{ArH}), 7.99(\mathrm{dt}, J=7.8,1.3 \mathrm{~Hz}, 1 \mathrm{H}$, $\operatorname{ArH}), 7.68$ (ddd, $J=8.1,2.2,1.1 \mathrm{~Hz}, 1 \mathrm{H}, \mathrm{ArH}), 7.52(\mathrm{t}, J=7.9 \mathrm{~Hz}, 1 \mathrm{H}, \operatorname{ArH}), 7.18-7.09(\mathrm{~m}, 2 \mathrm{H}, \operatorname{ArH}), 6.88$ - $6.80(\mathrm{~m}, 2 \mathrm{H}), 5.16\left(\mathrm{~s}, 2 \mathrm{H}, \mathrm{CH}_{2}\right), 4.82\left(\mathrm{~s}, 2 \mathrm{H}, \mathrm{CH}_{2}\right), 2.27\left(\mathrm{~s}, 3 \mathrm{H}, \mathrm{ArCH}_{3}\right) .{ }^{13} \mathrm{C} \mathrm{NMR}(101 \mathrm{MHz}$, Acetonitrile$\left.d_{3}\right) \delta 201.3,165.4,156.7,135.2,134.4,132.3,131.9,131.5,130.9,130.2,128.9,115.3,72.0,68.3,20.4$. IR $\vee 2975(\mathrm{~m}), 2926(\mathrm{~m}), 1732(\mathrm{~s}), 1613(\mathrm{~m}), 1576(\mathrm{~m}), 1511(\mathrm{~s}), 1457(\mathrm{w}), 1431(\mathrm{~m}), 1411(\mathrm{~m}), 1379$ $(\mathrm{m}), 1290(\mathrm{~s}), 1255(\mathrm{~s}), 1244(\mathrm{~s}), 1123(\mathrm{~m}), 1109(\mathrm{~m}), 1065(\mathrm{~m}), 1053(\mathrm{~m}), 985(\mathrm{w}), 898(\mathrm{w}), 818(\mathrm{~m}), 752$ (m), 719 (w), 676 (m), 629 (m). HRMS (nanochip-ESI/LTQ-Orbitrap) m/z: [M] Calcd for $\mathrm{C}_{17} \mathrm{H}_{15} \mathrm{ClNaO}_{4}{ }^{+}$ 341.0551; Found 341.0554. 
<smiles>CCC1OC1(COc1ccc(C)cc1)Oc1ccc(C)cc1</smiles><smiles>CCC(OC(C)=O)C(=O)COc1ccc(C)cc1</smiles>

$10,72 \%$

A solution of 3-ethyl-2-( $p$-tolyloxy)-2-(( $p$-tolyloxy)methyl)oxirane $8 a(0.200 \mathrm{mmol}, 1.0$ equiv. $)$ in acetic acid $(1.0 \mathrm{~mL})$ was stirred at room temperature for 30 minutes. The excess of acetic acid was removed by co-evaporation with toluene. The crude was purified by preparative TLC (pentane:ethyl acetate 9:1) to provide the 2-oxo-1-( $p$-tolyloxy)pentan-3-yl acetate $10(36.0 \mathrm{mg}, 0.144 \mathrm{mmol}, 72 \%$ yield) as a colorless oil. Rf: 0.32 (Pentane:EtOAc 9:1). ${ }^{1} \mathbf{H}$ NMR (400 MHz, Methylene Chloride- $d_{2}$ ) $\delta 7.14-7.06$ $(\mathrm{m}, 2 \mathrm{H}, \mathrm{ArH}), 6.81-6.76(\mathrm{~m}, 2 \mathrm{H}, \mathrm{ArH}), 5.20(\mathrm{dd}, J=8.0,4.3 \mathrm{~Hz}, 1 \mathrm{H}, \mathrm{OCCHO}), 4.79-4.65(\mathrm{~m}, 2 \mathrm{H}$, $\mathrm{OCH}_{2} \mathrm{CO}$ ), $2.28\left(\mathrm{~s}, 3 \mathrm{H}, \mathrm{ArCH}_{3}\right), 2.13\left(\mathrm{~s}, 3 \mathrm{H}, \mathrm{OCCH}_{3}\right), 2.00-1.87\left(\mathrm{~m}, 1 \mathrm{H}, \mathrm{CH}_{2} \mathrm{CH}_{3}\right), 1.81$ (ddt, J = 15.3, 14.5, $\left.7.4 \mathrm{~Hz}, 1 \mathrm{H}, \mathrm{CH}_{2} \mathrm{CH}_{3}\right), 1.00\left(\mathrm{t}, J=7.4 \mathrm{~Hz}, 3 \mathrm{H}, \mathrm{CH}_{3}\right) .{ }^{13} \mathrm{C}$ NMR $\left(101 \mathrm{MHz}\right.$, Methylene Chloride- $\left.d_{2}\right) \delta 203.7$, 171.1, 156.2, 131.7, 130.6, 114.9, 78.0, 71.7, 24.3, 20.9, 20.7, 10.0. IR 3469 (w), 2974 (w), 2926 (w), $1736(\mathrm{~s}), 1612(\mathrm{w}), 1588(\mathrm{w}), 1511(\mathrm{~s}), 1459(\mathrm{w}), 1433(\mathrm{w}), 1374(\mathrm{~m}), 1294(\mathrm{~m}), 1227(\mathrm{~s}), 1177(\mathrm{w}), 1096$ $(\mathrm{m}), 1057(\mathrm{~m}), 1019(\mathrm{~m}), 970(\mathrm{~m}), 902(\mathrm{w}), 816(\mathrm{~m}), 717(\mathrm{w}), 678(\mathrm{w})$. HRMS (nanochip-ESI/LTQOrbitrap) $\mathrm{m} / \mathrm{z}:[\mathrm{M}+\mathrm{Na}]^{+}$Calcd for $\mathrm{C}_{14} \mathrm{H}_{18} \mathrm{NaO}_{4}{ }^{+}$273.1097; Found 273.1085.

The regioselectivity of the addition can be explained by acid activation of the epoxide (I) to form oxonium II, followed by addition of the carboxylic acid to give III and finally acyl migration (Scheme S8). This mechanism is well-establish for structurally similar epoxides derived from alkyl vinyl ethers. ${ }^{23}$

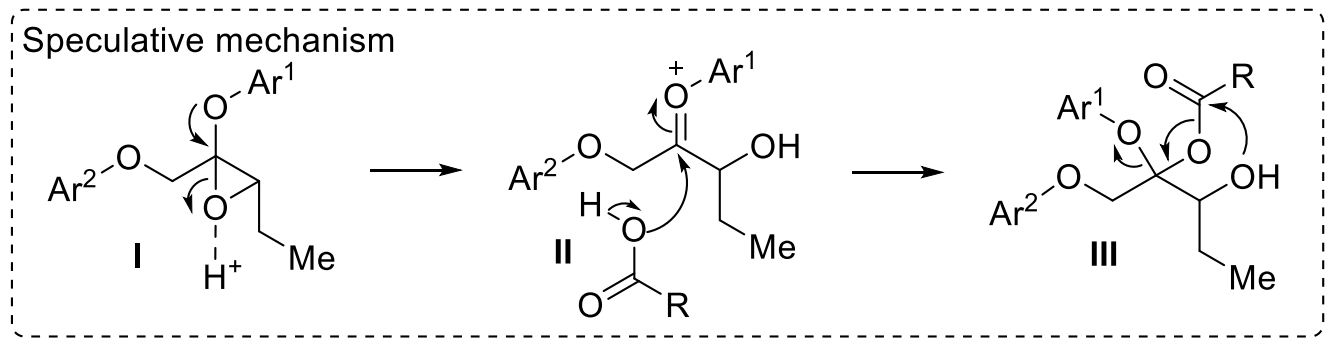

Scheme S8: Speculative mechanism for the epoxide opening with acid.

\section{Oxidation with Oxone ${ }^{\circledR}$ :}

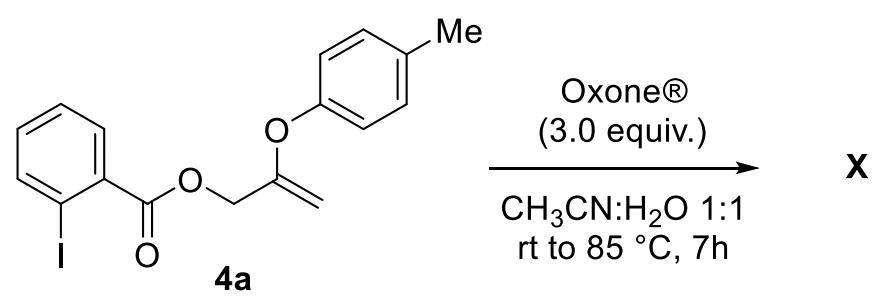

To a solution of $4 \mathrm{a}\left(20 \mathrm{mg}, 0.050 \mathrm{mmol}, 1.0\right.$ equiv.) in $\mathrm{CH}_{3} \mathrm{CN}(0.50 \mathrm{~mL})$ and $\mathrm{H}_{2} \mathrm{O}(0.50 \mathrm{~mL})$ was added Oxone ${ }^{\circledR}$ (92 mg, $0.15 \mathrm{mmol}, 3.0$ equiv.). The resulting mixture was stirred at room temperature for $5 \mathrm{~h}$

\footnotetext{
${ }^{23}$ a) C. L. Stevens, W. Malik, R. Pratt, J. Am. Chem. Soc. 1950, 72, 4758; b) C. L. Stevens, S. J. Dykstra, J. Am. Chem. Soc. 1953, 75, 5975; Review: c) A. Kirrmann, P. Duhamel, R. Nouri-Bimorghi, Justus Liebigs Ann. Chem. 1966, 691, 33.
} 
and at $85{ }^{\circ} \mathrm{C}$ for $2 \mathrm{~h}$. According to TLC, HRMS and NMR no reaction occurred and the starting $4 \mathrm{a}$ was recovered.

\section{Oxidation with Selectfluor ${ }^{\mathrm{TM}}$ :}

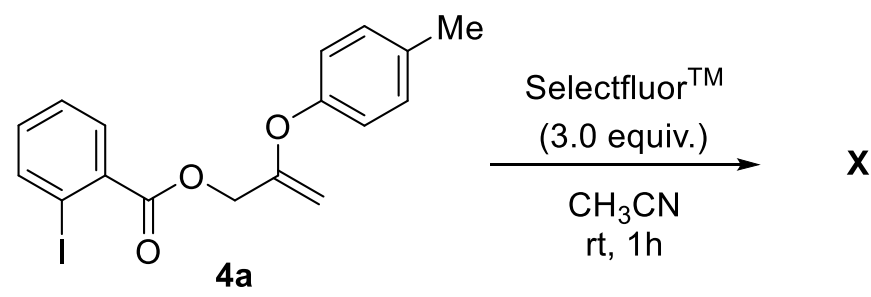

To a solution of $4 \mathrm{a}$ ( $20 \mathrm{mg}, 0.050 \mathrm{mmol}, 1.0$ equiv.) in $\mathrm{CH}_{3} \mathrm{CN}\left(0.50 \mathrm{~mL}\right.$ ) was added Selectfluor ${ }^{\mathrm{TM}}$ (54 $\mathrm{mg}, 0.15 \mathrm{mmol}, 3.0$ equiv.). The resulting mixture was stirred at room temperature. After $1 \mathrm{~h}$, full consumption of 4a was observed. According to NMR, decomposition occurred and a complex product mixture was recovered. 


\section{Mechanistic investigations}

\section{Nature of 4a:}

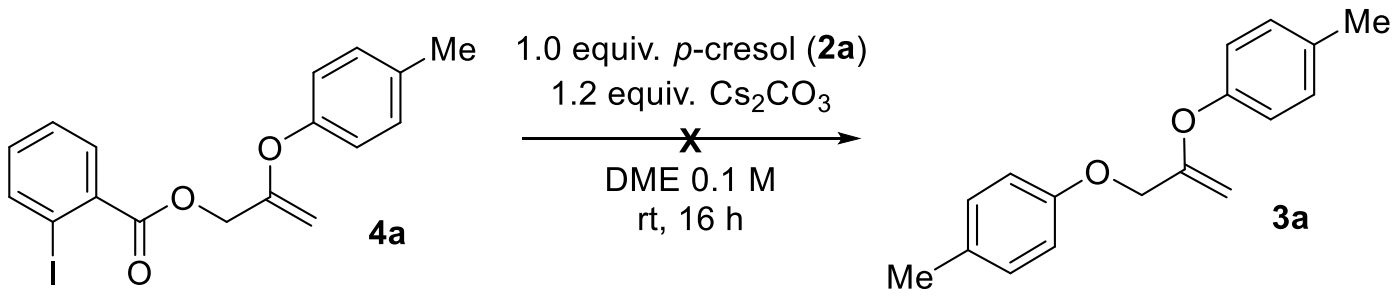

In a round-bottom flask were added $p$-cresol $(10.8 \mathrm{mg}, 0.100 \mathrm{mmol}, 1.00$ equiv.) and cesium carbonate ( $39.0 \mathrm{mg}, 0.120 \mathrm{mmol}, 1.20$ equiv.). Anhydrous DME ( $3.00 \mathrm{~mL}, 0.1 \mathrm{M}$ ) was and the solution was stirred at room temperature for $10 \mathrm{~min}$. Allyl-ester $4 \mathrm{a}(39.4 \mathrm{mg}, 0.100 \mathrm{mmol}, 1.00$ equiv.) was added to the reaction mixture under open air and the reaction mixture was stirred at room temperature for $16 \mathrm{~h}$.

\section{Cross-over experiment:}
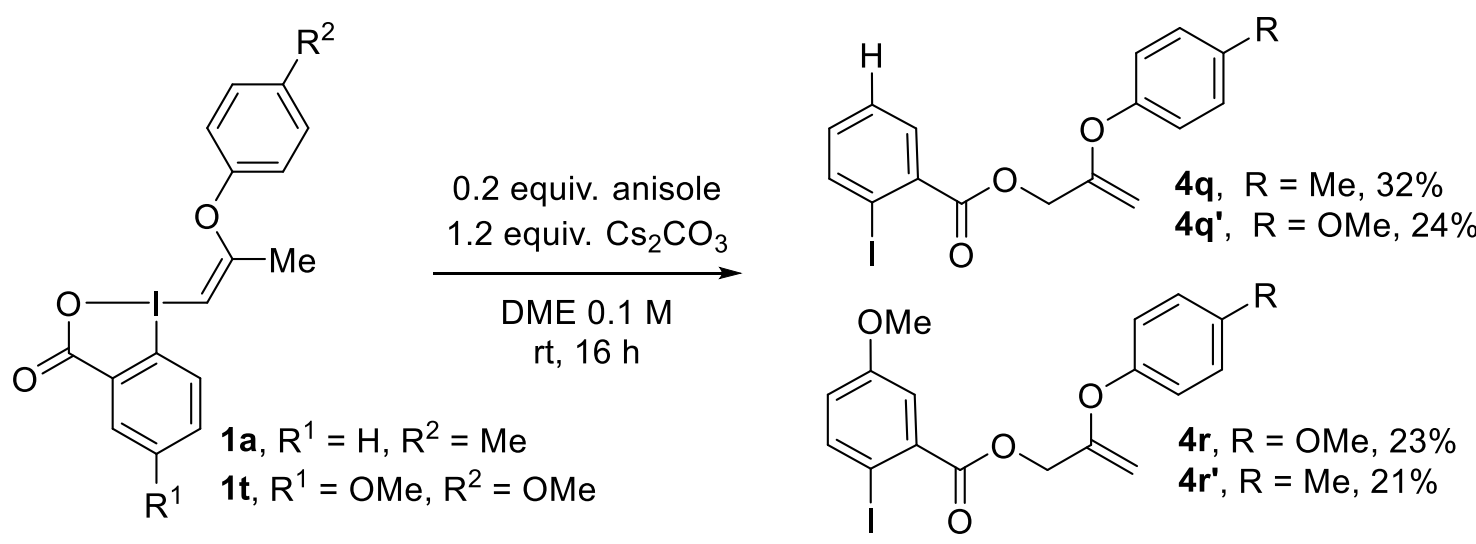

O-VBX 1a (0.020 g, $50.0 \mu \mathrm{mol}, 0.5$ equiv.), O-VBX 1t (0.022 g, $0.050 \mathrm{mmol}, 0.5$ equiv.) and cesium carbonate $(0.039 \mathrm{~g}, 0.12 \mathrm{mmol}, 1.2$ equiv.) were added to a $5 \mathrm{~mL}$ microwave vial. Anhydrous DME (1.0 $\mathrm{mL}$ ) was introduced to the vial by syringe. Then anisole $(2.17 \mu \mathrm{l}, 20.0 \mu \mathrm{mol}, 0.2$ equiv.) was added and the reaction mixture was stirred at room temperature, for $16 \mathrm{~h}$. The solution was then concentrated under reduced pressure. The crude material was purified by column chromatography (Pentane:Ethyl acetate 9:1) and 2-(p-tolyloxy)allyl 2-iodobenzoate $4 \mathrm{q}(0.013 \mathrm{~g}, 0.032 \mathrm{mmol}, 32 \%$ yield), 2-(4methoxyphenoxy)allyl 2-iodobenzoate $4 \mathbf{q q}^{\prime}(0.010 \mathrm{~g}, 0.024 \mathrm{mmol}, 24 \%$ yield), 2-(p-tolyloxy)allyl 2-iodo5-methoxybenzoate 4r' $(0.009 \mathrm{~g}, 0.021 \mathrm{mmol}, 21 \%$ yield) and 2-(4-methoxyphenoxy)allyl 2-iodo-5methoxybenzoate $4 \mathrm{r}(0.010 \mathrm{~g}, 0.023 \mathrm{mmol}, 23 \%$ yield) were obtained as a colorless oils.

\section{2-(p-Tolyloxy)allyl 2-iodobenzoate (4q)}<smiles>C=C(COC(=O)c1cc(I)ccc1I)Oc1ccc(C)cc1</smiles>

4q: ${ }^{1} \mathrm{H}$ NMR $(400 \mathrm{MHz}$, Chloroform-d) $\delta 8.01(\mathrm{dd}, J=8.0,1.2 \mathrm{~Hz}, 1 \mathrm{H}$, $\operatorname{ArH}), 7.87(\mathrm{dd}, J=7.8,1.7 \mathrm{~Hz}, 1 \mathrm{H}, \operatorname{ArH}), 7.41(\mathrm{td}, J=7.6,1.2 \mathrm{~Hz}, 1 \mathrm{H}$, $\operatorname{ArH}), 7.23-7.12(\mathrm{~m}, 3 \mathrm{H}, \mathrm{ArH}), 7.06-6.94(\mathrm{~m}, 2 \mathrm{H}, \mathrm{ArH}), 4.94(\mathrm{~s}, 2 \mathrm{H}$, $\left.\mathrm{CCH}_{2} \mathrm{O}\right), 4.55\left(\mathrm{~d}, \mathrm{~J}=2.4 \mathrm{~Hz}, 1 \mathrm{H}, \mathrm{CH}_{2} \mathrm{CO}\right), 4.23\left(\mathrm{~d}, \mathrm{~J}=2.3 \mathrm{~Hz}, 1 \mathrm{H}, \mathrm{CH}_{2} \mathrm{CO}\right)$, $2.33\left(\mathrm{~s}, 3 \mathrm{H}, \mathrm{CH}_{3}\right) .{ }^{13} \mathrm{C}$ NMR (101 MHz, Chloroform-d) $\delta 166.1,157.5$, 152.6, 141.6, 134.8, 134.2, 133.0, 131.4, 130.3, 128.1, 120.8, 94.5,

92.1, 65.0, 20.9. 


\section{2-(4-Methoxyphenoxy)allyl 2-iodobenzoate (4q')}<smiles>C=C(COC(=O)c1ccccc1I)Oc1ccc(OC)cc1</smiles>

4q': ${ }^{1}$ H NMR (400 MHz, Chloroform-d) $\delta 8.02$ (dd, $J=7.9,1.2 \mathrm{~Hz}$, $1 \mathrm{H}, \mathrm{ArH}), 7.88(\mathrm{dd}, J=7.8,1.7 \mathrm{~Hz}, 1 \mathrm{H}, \mathrm{ArH}), 7.42(\mathrm{td}, J=7.5,1.2 \mathrm{~Hz}$, $1 \mathrm{H}, \mathrm{ArH}), 7.17(\mathrm{td}, J=7.7,1.7 \mathrm{~Hz}, 1 \mathrm{H}, \mathrm{ArH}), 7.06-6.97(\mathrm{~m}, 2 \mathrm{H}, \mathrm{ArH})$, $6.92-6.84(\mathrm{~m}, 2 \mathrm{H}, \mathrm{ArH}), 4.94\left(\mathrm{~s}, 2 \mathrm{H}, \mathrm{CCH}_{2} \mathrm{O}\right), 4.51(\mathrm{~d}, J=2.3 \mathrm{~Hz}, 1 \mathrm{H}$, $\mathrm{CH}_{2} \mathrm{CO}$ ), 4.16 (d, $\left.J=2.4 \mathrm{~Hz}, 1 \mathrm{H}, \mathrm{CH}_{2} \mathrm{CO}\right), 3.80\left(\mathrm{~s}, 3 \mathrm{H}, \mathrm{OCH}_{3}\right) .{ }^{13} \mathrm{C} \mathrm{NMR}$ (101 MHz, Chloroform-d) $\delta 166.1,158.1,156.6,148.3,141.6,134.8$, 133.0, 131.4, 128.1, 122.2, 114.9, 94.5, 91.2, 65.0, 55.7.

\section{2-(4-Methoxyphenoxy)allyl 2-iodo-5-methoxybenzoate (4r)}<smiles>C=C(COC(=O)c1cc(OC)ccc1I)Oc1ccc(OC)cc1</smiles>

4r: ${ }^{1} \mathrm{H}$ NMR (400 MHz, Chloroform- $d$ ) $\delta 7.85(\mathrm{~d}, J=8.7 \mathrm{~Hz}, 1 \mathrm{H}, \mathrm{ArH})$, $7.42(\mathrm{~d}, J=3.1 \mathrm{~Hz}, 1 \mathrm{H}, \mathrm{ArH}), 7.08-6.98(\mathrm{~m}, 2 \mathrm{H}, \mathrm{ArH}), 6.92-6.83$ (m, 2H, ArH), $6.77(\mathrm{dd}, J=8.8,3.1 \mathrm{~Hz}, 1 \mathrm{H}, \mathrm{ArH}), 4.93\left(\mathrm{~s}, 2 \mathrm{H}, \mathrm{CCH}_{2} \mathrm{O}\right)$, $4.51\left(\mathrm{~d}, J=2.4 \mathrm{~Hz}, 1 \mathrm{H}, \mathrm{CH}_{2} \mathrm{CO}\right.$ ), $4.16\left(\mathrm{~d}, J=2.4 \mathrm{~Hz}, 1 \mathrm{H}, \mathrm{CH}_{2} \mathrm{CO}\right.$ ), 3.81 $\left(\mathrm{s}, 3 \mathrm{H}, \mathrm{OCH}_{3}\right), 3.80\left(\mathrm{~s}, 3 \mathrm{H}, \mathrm{OCH}_{3}\right) .{ }^{13} \mathrm{C}$ NMR $(101 \mathrm{MHz}$, Chloroformd) $\delta 165.9,159.7,158.0,156.6,148.3,142.1,135.6,122.2,119.6$, $116.9,114.9,91.3,82.8,65.0,55.7,55.7$.

2-(p-Tolyloxy)allyl 2-iodo-5-methoxybenzoate (4r')<smiles>C=C(COC(=O)c1cc(OC)ccc1I)Oc1ccc(C)cc1</smiles>

4r': ${ }^{1} \mathrm{H}$ NMR (400 MHz, Chloroform-d) $\delta 7.85(\mathrm{~d}, J=8.7 \mathrm{~Hz}, 1 \mathrm{H}, \operatorname{Ar} H)$, $7.42(\mathrm{~d}, J=3.1 \mathrm{~Hz}, 1 \mathrm{H}, \mathrm{ArH}), 7.20-7.11(\mathrm{~m}, 2 \mathrm{H}, \mathrm{ArH}), 7.05-6.94(\mathrm{~m}$, $2 \mathrm{H}, \mathrm{ArH}), 6.77(\mathrm{dd}, J=8.7,3.1 \mathrm{~Hz}, 1 \mathrm{H}, \mathrm{ArH}), 4.93\left(\mathrm{~s}, 2 \mathrm{H}, \mathrm{CCH}_{2} \mathrm{O}\right), 4.56$ (d, $J=2.3 \mathrm{~Hz}, 1 \mathrm{H}, \mathrm{CH}_{2} \mathrm{CO}$ ), 4.23 (d, J = $2.3 \mathrm{~Hz}, 1 \mathrm{H}, \mathrm{CH}_{2} \mathrm{CO}$ ), $3.80(\mathrm{~s}, 3 \mathrm{H}$, $\mathrm{OCH}_{3}$ ), $2.33\left(\mathrm{~s}, 3 \mathrm{H}, \mathrm{CH}_{3}\right) .{ }^{13} \mathrm{C}$ NMR (101 MHz, Chloroform-d) $\delta 165.9$, 159.6, 157.4, 152.7, 142.1, 135.6, 134.2, 130.3, 120.8, 119.6, 116.9,

$92.2,82.8,65.0,55.7,20.9$.

Tentative to trap intermediate:<smiles>COC(=O)c1ccccc1I(C)C=C(C)Oc1ccc(C)cc1</smiles>

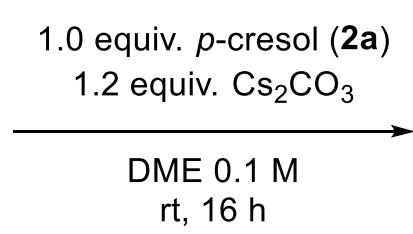<smiles>C=C(CO/C=C/C)Oc1ccc(C)cc1</smiles>

$3 a, 80 \%$ or 1 equiv. $p$-methoxystyrene

p-Cresol (10.8 mg, $0.100 \mathrm{mmol}, 1.0$ equiv.) and cesium carbonate $(39.0 \mathrm{mg}, 0.120 \mathrm{mmol}, 1.2$ equiv.) were added to a round-bottom flask. Anhydrous DME (0.1 M) was introduced at $0{ }^{\circ} \mathrm{C}$ and the solution was stirred at room temperature for $10 \mathrm{~min}$. O-VBX reagent $(39.0 \mathrm{mg}, 0.100 \mathrm{mmol}, 1.0$ equiv.) and TEMPO or $p$-methoxystyrene $(0.100 \mathrm{mmol}, 1.0$ equiv.) was added to the reaction mixture under open air and the reaction mixture was stirred at room temperature for $16 \mathrm{~h}$. The reaction mixture was filtrated, solvent was removed under reduced pressure and the crude material was purified by column chromatography (pentane: ethyl acetate 9:1). The 4,4'-(prop-2-ene-1,2- 
diylbis(oxy))bis(methylbenzene) 3a (20.0 mg, $80.0 \mu \mathrm{mol}, 80 \%$ yield) and the 2-( $p$-tolyloxy)allyl 2iodobenzoate $\mathbf{4 a}(7.80 \mathrm{mg}, 20.0 \mu \mathrm{mol}, 20 \%$ yield) were obtained as a colorless amorphous solids.<smiles>COC(=O)c1ccccc1I(C)/C=C(\C)Oc1ccc(C)cc1</smiles>

\section{Furan (co-solvent)}

0.2 equiv. anisole

1.2 equiv. $\mathrm{Cs}_{2} \mathrm{CO}_{3}$

DME $0.1 \mathrm{M}$

$\mathrm{rt}, 16 \mathrm{~h}$

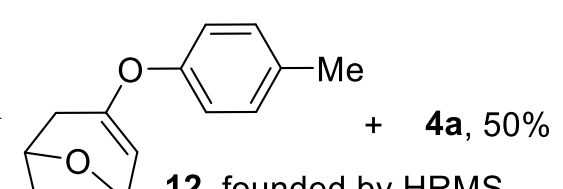

12, founded by HRMS

To a solution of O-VBX 1a (39.0 mg, $0.100 \mathrm{mmol}, 1.00$ equiv.) in DME and furan (0.1 M) were added cesium carbonate ( $39.0 \mathrm{mg}, 0.120 \mathrm{mmol}, 1.20$ equiv.) and anisole $(20.0 \mu \mathrm{L}, 20.0 \mu \mathrm{mol}, 0.20$ equiv.) and the solution was stirred at room temperature for 16 hours. The reaction mixture was filtrated, solvent was removed under reduced pressure and the crude material was purified by column chromatography (pentane:ethyl acetate 9:1). The 2-( $p$-tolyloxy)allyl 2-iodobenzoate 4 a $(19.6 \mathrm{mg}, 50.0 \mu \mathrm{mol}, 50 \%$ yield) was obtained as a colorless amorphous solid and the 3-(p-tolyloxy)-8-oxabicyclo[3.2.1]octa-2,6-diene 12 was observed by mass analysis. HRMS (APPI/QTOF) $\mathrm{m} / \mathrm{z}$ : $[\mathrm{M}+\mathrm{H}]^{+}$Calcd for $\mathrm{C}_{14} \mathrm{H}_{15} \mathrm{O}_{2}{ }^{+}$215.1067; Found 215.1063.<smiles>O=C1OI(C#CCC2CC2)c2ccccc21</smiles>

$5 q$

1.00 equiv. $p$-cresol (2a) $10 \mathrm{~mol} \% \mathrm{Cs}_{2} \mathrm{CO}_{3}$ DME $0.1 \mathrm{M}, \mathrm{rt}, 16 \mathrm{~h}$ then 0.20 equiv. anisole 1.20 equiv. $\mathrm{Cs}_{2} \mathrm{CO}_{3}$ DME $0.1 \mathrm{M}, \mathrm{rt}, 16 \mathrm{~h}$<smiles>Cc1ccc(O/C(=C/C2CC2)COC(=O)c2ccccc2I)cc1</smiles><smiles>O=C(COC(=O)c1ccccc1I)CC1CC1</smiles>

$14,4 \%$<smiles>C=C(/C=C/CCOC(=O)c1ccccc1I)Oc1ccc(C)cc1</smiles>

$13,53 \%$

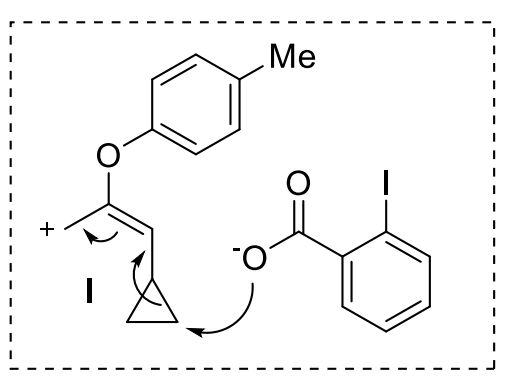

In a round-bottom flask, $p$-cresol $(0.108 \mathrm{~g}, 1.00 \mathrm{mmol}, 1.00$ equiv.) was dissolved in $10 \mathrm{~mL}$ of DME (0.1 M). Cesium carbonate ( $33.0 \mathrm{mg}, 0.100 \mathrm{mmol}, 0.10$ equiv.) was added and the mixture stirred vigorously for $5 \mathrm{~min}$. Then EBX $5 q$ was added in one portion $(0.326 \mathrm{~g}, 1.00 \mathrm{mmol}, 1.00$ equiv.) and the reaction was left stirring for 16 hours at room temperature. Then cesium carbonate $(0.391 \mathrm{~g}, 1.20 \mathrm{mmol}, 1.20$ equiv.) and anisole ( $22 \mu \mathrm{L}, 0.20 \mathrm{mmol}, 0.20$ equiv.) were added and the solution was stirred at room temperature for 16 hours. The reaction mixture was filtrated, solvent was removed under reduced pressure and the crude material was purified by column chromatography (pentane:ethyl acetate 9:1). The (E)-5-( $p$-tolyloxy)hexa-3,5-dien-1-yl 2-iodobenzoate (13) $(0.232 \mathrm{~g}, 0.534 \mathrm{mmol}, 53 \%$ yield), the (E)3-cyclopropyl-2-(p-tolyloxy)allyl 2-iodobenzoate $(4 \mathrm{~s})(78.0 \mathrm{mg}, 0.178 \mathrm{mmol}, 18 \%$ yield) and the 3- 
Cyclopropyl-2-oxopropyl 2-iodobenzoate (14) $(36.0 \mathrm{mg}, 36.0 \mu \mathrm{mol}, 4 \%$ yield) were obtained as sticky solids.

(E)-5-(p-tolyloxy)hexa-3,5-dien-1-yl 2-iodobenzoate (13)<smiles>C=C(/C=C/CCOC(=O)c1ccccc1I)Oc1ccc(C)cc1</smiles>

Rf: 0.29 (Pentane:EtOAc 9:1). ${ }^{1} \mathbf{H}$ NMR (400 MHz, Acetonitrile- $\left.d_{3}\right) \delta 8.03$ $-7.98(\mathrm{~m}, 1 \mathrm{H}, \operatorname{ArH}), 7.70$ (dd, J = 7.7, $1.7 \mathrm{~Hz}, 1 \mathrm{H}, \operatorname{ArH}), 7.47$ (td, J = 7.6, $1.2 \mathrm{~Hz}, 1 \mathrm{H}, \operatorname{ArH}), 7.23(\mathrm{td}, J=7.7,1.7 \mathrm{~Hz}, 1 \mathrm{H}, \operatorname{ArH}), 7.16-7.11(\mathrm{~m}, 2 \mathrm{H}$, $\operatorname{ArH}), 6.93-6.88(\mathrm{~m}, 2 \mathrm{H}, \mathrm{ArH}), 6.19(\mathrm{q}, J=1.9 \mathrm{~Hz}, 2 \mathrm{H}, \mathrm{OCCHCH}), 4.47$ $\left(\mathrm{d}, J=1.6 \mathrm{~Hz}, 1 \mathrm{H}, \mathrm{OCCH}_{2}\right), 4.38\left(\mathrm{t}, J=6.4 \mathrm{~Hz}, 2 \mathrm{H}, \mathrm{CHCH}_{2} \mathrm{CH}_{2} \mathrm{O}\right), 4.14(\mathrm{~d}$, $J=1.6 \mathrm{~Hz}, 1 \mathrm{H}, \mathrm{OCCH}_{2}$ ), 2.60 (tdd, $J=6.3,3.9,1.7 \mathrm{~Hz}, 2 \mathrm{H}, \mathrm{CHCH}_{2} \mathrm{CH}_{2} \mathrm{O}$ ), $2.29\left(\mathrm{~s}, 3 \mathrm{H}, \mathrm{CH}_{3}\right) .{ }^{13} \mathrm{C} \mathrm{NMR}\left(101 \mathrm{MHz}\right.$, Acetonitrile- $\left.d_{3}\right) \delta 167.6,159.2,154.7,141.9,137.2,134.2,133.6$, 131.3, 131.1, 129.2, 128.8, 128.5, 120.4, 95.1, 93.8, 65.4, 32.2, 20.7. IR 2987 (m), $2888(\mathrm{w}), 1729(\mathrm{~s})$, $1679(w), 1611(w), 1584(m), 1563(w), 1507(m), 1473(w), 1427(m), 1392(m), 1288(m), 1263(s)$, $1219(\mathrm{~m}), 1169(\mathrm{w}), 1135(\mathrm{~m}), 1103(\mathrm{w}), 1044(\mathrm{~m}), 1016(\mathrm{~m}), 740(\mathrm{~s}), 704(\mathrm{~m})$. HRMS (ESI/QTOF) m/z: $[\mathrm{M}+\mathrm{Na}]^{+}$Calcd for $\mathrm{C}_{20} \mathrm{H}_{19} \mathrm{INaO}_{3}{ }^{+}$457.0271; Found 457.0268.

\section{(E)-3-cyclopropyl-2-(p-tolyloxy)allyl 2-iodobenzoate (4s)}

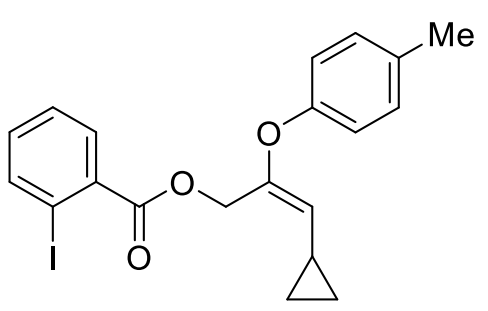

Rf: 0.50 (Pentane:EtOAc 9:1). ${ }^{1} \mathbf{H}$ NMR (400 MHz, Acetonitrile- $\left.d_{3}\right) \delta$ $8.02(\mathrm{dd}, J=7.9,1.2 \mathrm{~Hz}, 1 \mathrm{H}, \operatorname{ArH}), 7.74(\mathrm{dd}, J=7.8,1.7 \mathrm{~Hz}, 1 \mathrm{H}, \operatorname{Ar} H)$, $7.49(\mathrm{td}, J=7.6,1.2 \mathrm{~Hz}, 1 \mathrm{H}, \mathrm{ArH}), 7.24(\mathrm{td}, J=7.7,1.7 \mathrm{~Hz}, 1 \mathrm{H}, \mathrm{ArH})$, $7.19(\mathrm{~d}, J=8.2 \mathrm{~Hz}, 2 \mathrm{H}, \mathrm{ArH}), 7.00-6.94(\mathrm{~m}, 2 \mathrm{H}, \operatorname{ArH}), 4.96(\mathrm{~d}, J=9.1$ $\mathrm{Hz}, 1 \mathrm{H}, \mathrm{OCCHCH}), 4.62$ (d, J=2.3 Hz, 1H, OCH $2 \mathrm{CO}$ ), 4.11 (d, $J=2.3 \mathrm{~Hz}$, $\left.1 \mathrm{H}, \mathrm{OCH}_{2} \mathrm{CO}\right), 2.31\left(\mathrm{~s}, 3 \mathrm{H}, \mathrm{ArCH}_{3}\right), 1.50(\mathrm{dtt}, J=9.5,8.1,4.9 \mathrm{~Hz}, 1 \mathrm{H}$, $\mathrm{CH}), 0.76-0.64\left(\mathrm{~m}, 2 \mathrm{H}, \mathrm{CH}_{2}\right), 0.56$ (tddd, $\left.J=13.1,8.7,6.8,3.5 \mathrm{~Hz}, 2 \mathrm{H}, \mathrm{CH}_{2}\right) .{ }^{13} \mathrm{C} \mathrm{NMR}(101 \mathrm{MHz}$, Acetonitrile- $\left.d_{3}\right) \delta 167.0,161.4,153.8,142.0,137.1,135.1,133.7,131.3,131.2,129.3,121.3,93.8,91.5$, 80.0, 20.7, 14.3, 4.7, 3.6. IR v $3655(\mathrm{w}), 3546(\mathrm{w}), 3087(\mathrm{~m}), 3006(\mathrm{~m}), 2870(\mathrm{w}), 2602(\mathrm{w}), 1929(\mathrm{w})$, $1884(\mathrm{w}), 1728(\mathrm{~s}), 1646(\mathrm{~m}), 1609(\mathrm{~m}), 1584(\mathrm{~m}), 1506(\mathrm{~s}), 1464(\mathrm{~m}), 1430(\mathrm{~m}), 1281(\mathrm{~s}), 1247(\mathrm{~s}), 1223$ $(\mathrm{s}), 1167(\mathrm{w}), 1129(\mathrm{~m}), 1098(\mathrm{~s}), 1041(\mathrm{~s}), 1016(\mathrm{~s}), 959(\mathrm{~s}), 907(\mathrm{~m}), 833(\mathrm{~s}), 742(\mathrm{~s}), 687(\mathrm{~m}), 638(\mathrm{w})$. HRMS (ESI/QTOF) m/z: [M + Na] ${ }^{+}$Calcd for $\mathrm{C}_{20} \mathrm{H}_{19} \mathrm{INaO}_{3}{ }^{+}$457.0271; Found 457.0274.

\section{3-Cyclopropyl-2-oxopropyl 2-iodobenzoate (14)}<smiles>O=C(COC(=O)c1ccccc1I)CC1CC1</smiles>

Rf: 0.56 (Pentane:EtOAc 9:1). ${ }^{1} \mathbf{H}$ NMR $\left(400 \mathrm{MHz}\right.$, Acetonitrile- $\left.d_{3}\right) \delta 8.06$ (dd, $J$ $=8.0,1.2 \mathrm{~Hz}, 1 \mathrm{H}, \mathrm{ArH}), 7.91$ (dd, $J=7.8,1.7 \mathrm{~Hz}, 1 \mathrm{H}, \operatorname{ArH}), 7.52$ (td, $J=7.6,1.2$ $\mathrm{Hz}, 1 \mathrm{H}, \mathrm{ArH}), 7.27(\mathrm{td}, J=7.7,1.7 \mathrm{~Hz}, 1 \mathrm{H}, \mathrm{ArH}), 4.99\left(\mathrm{~s}, 2 \mathrm{H}, \mathrm{OCH}_{2} \mathrm{C}\right), 2.40(\mathrm{~d}, J=$ $\left.7.0 \mathrm{~Hz}, 2 \mathrm{H}, \mathrm{CCH}_{2} \mathrm{C}\right), 1.09-0.93(\mathrm{~m}, 1 \mathrm{H}, \mathrm{CH}), 0.62-0.47\left(\mathrm{~m}, 2 \mathrm{H}, \mathrm{CH}_{2}\right), 0.21-$ $0.13\left(\mathrm{~m}, 2 \mathrm{H}, \mathrm{CH}_{2}\right) .{ }^{13} \mathrm{C}$ NMR (101 MHz, Acetonitrile- $\left.d_{3}\right) \delta 204.3,166.6,142.3$, 135.6, 134.2, 132.0, 129.3, 94.3, 69.6, 44.6, 6.4, 4.8. IR $v 2990$ (m), 2941 (w), $2886(\mathrm{w}), 1729$ (s), 1672 (w), $1611(\mathrm{w}), 1584(\mathrm{~m}), 1564(\mathrm{~m}), 1508(\mathrm{~s}), 1473(\mathrm{w}), 1415(\mathrm{~m}), 1369(\mathrm{~m}), 1289(\mathrm{~m}), 1253(\mathrm{~s}), 1211(\mathrm{w})$, $1134(\mathrm{~m}), 1107(\mathrm{~m}), 1072(\mathrm{~m}), 1045(\mathrm{~m}), 1018(\mathrm{~m}), 952(\mathrm{w}), 826(\mathrm{w}), 740(\mathrm{~s}), 704(\mathrm{~m}), 639(\mathrm{w})$. HRMS (ESI/QTOF) $\mathrm{m} / \mathrm{z}:[\mathrm{M}+\mathrm{Na}]^{+}$Calcd for $\mathrm{C}_{13} \mathrm{H}_{13} \mathrm{INaO}_{3}{ }^{+}$366.9802; Found 366.9810 . 


\section{Spectra of new compounds}

\subsection{EBX}

2-(6-(3-Oxo-1,2-benziodoxol-3(1H)-yl)hex-5-yn-1-yl)isoindoline-1,3-dione (5f) ${ }^{1} \mathrm{H}$ NMR (400 MHz, Chloroform-d)

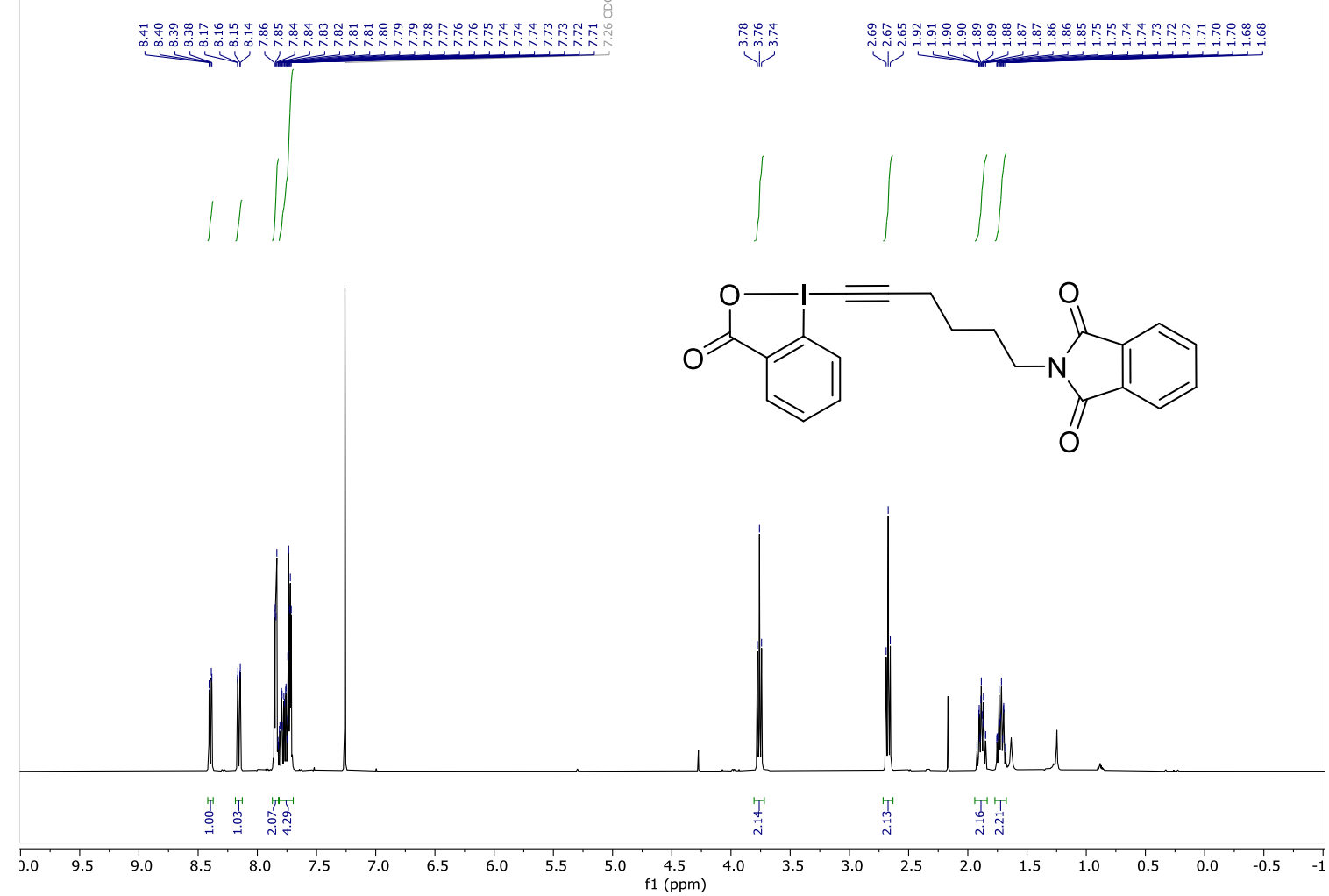

${ }^{13} \mathrm{C}$ NMR (101 MHz, Chloroform-d)

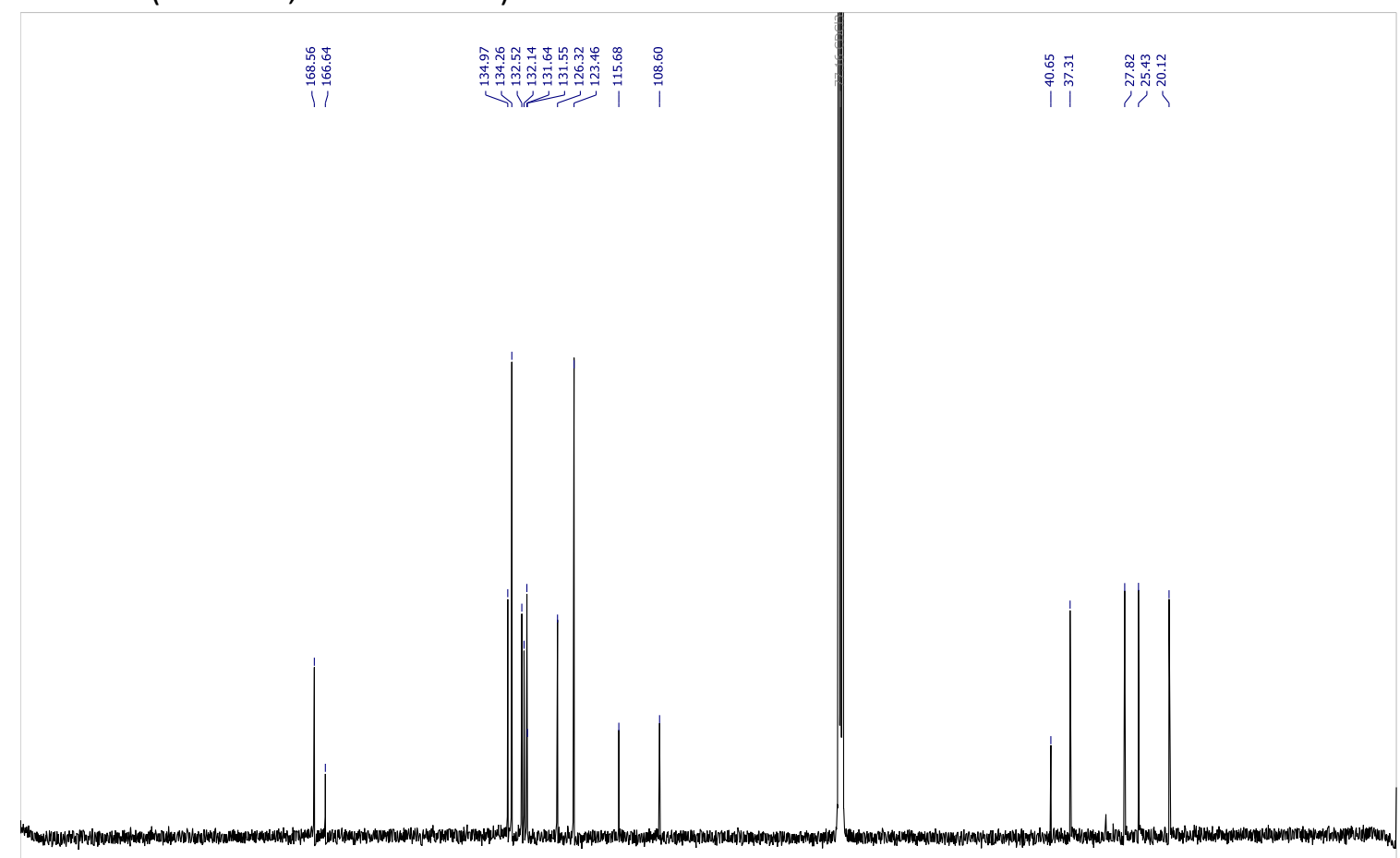

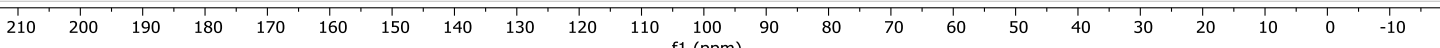


5-Methyl-propynyl-1,2-benziodoxol-3(1H)-one (5o)

${ }^{1}$ H NMR (400 MHz, Chloroform-d)

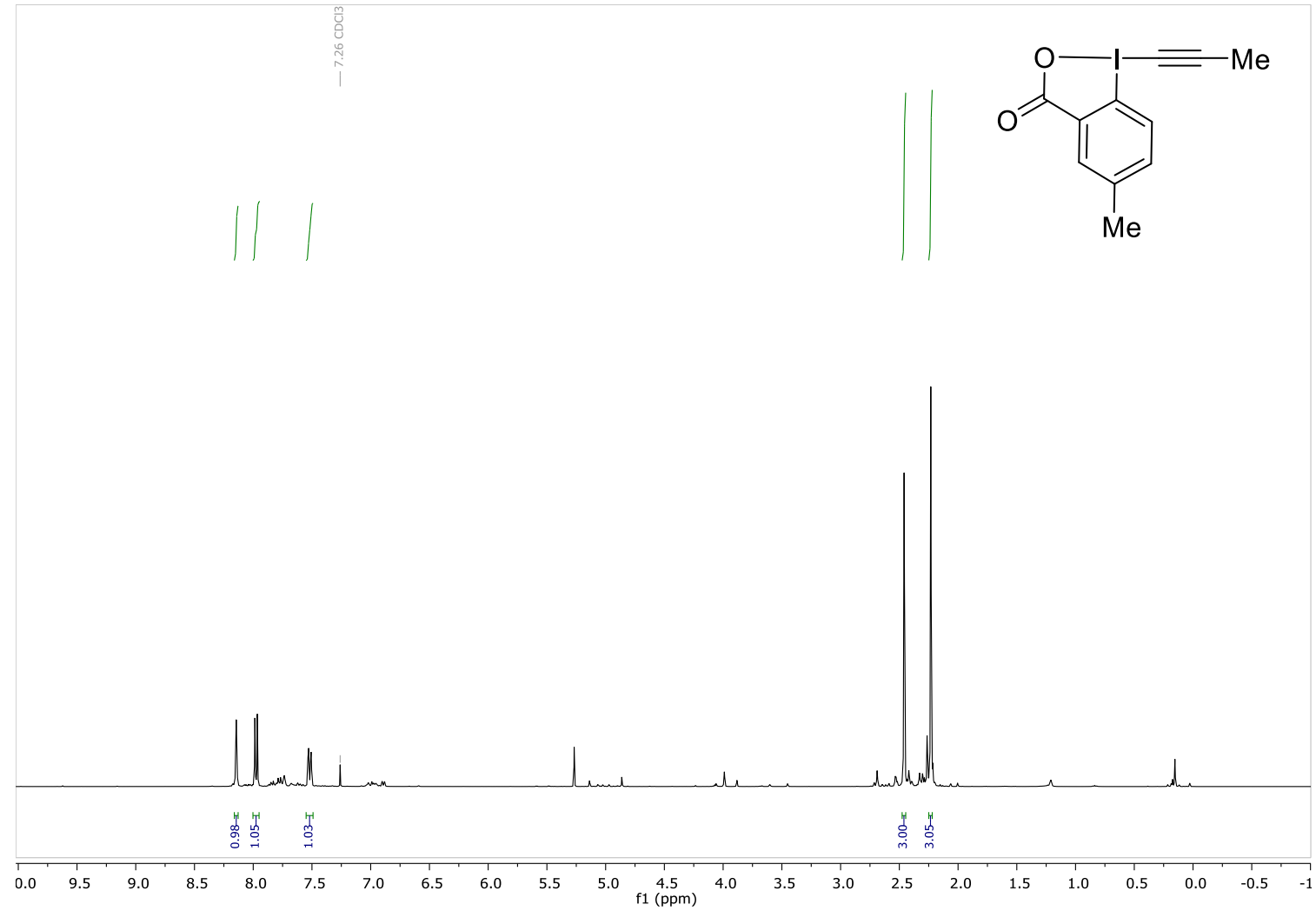

${ }^{13} \mathrm{C}$ NMR (101 MHz, Chloroform-d)

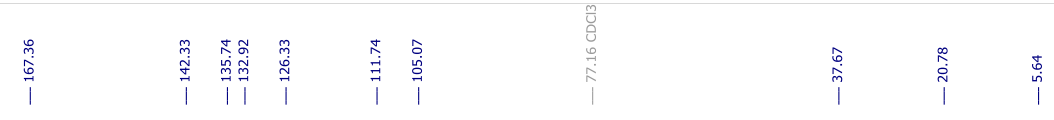

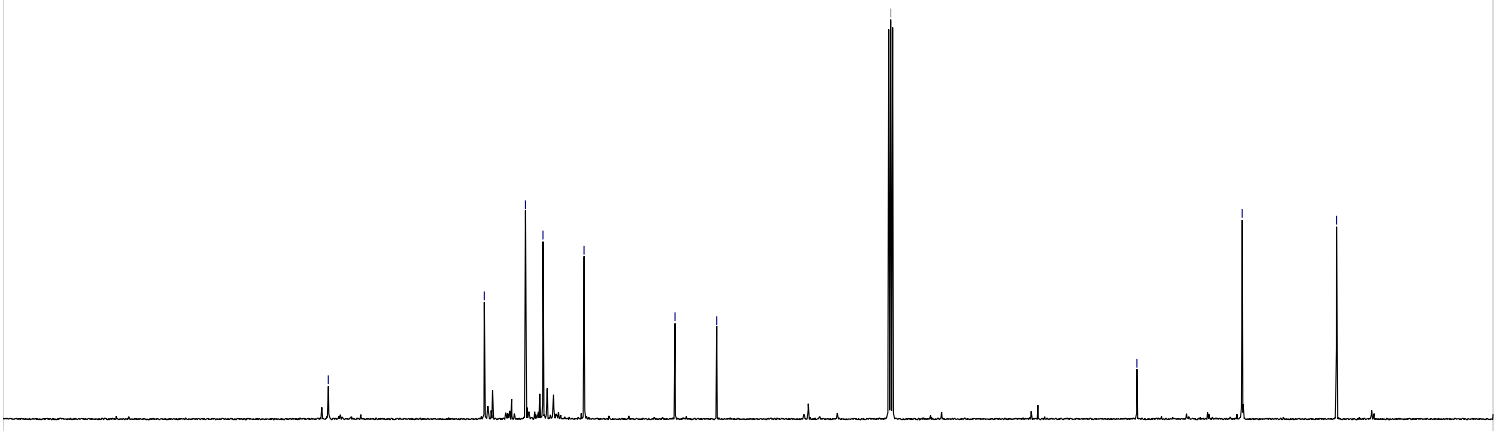

$\begin{array}{llllllllllllllllllllllllllllll}210 & 200 & 190 & 180 & 170 & 160 & 150 & 140 & 130 & 120 & 110 & 100 & 90 & 80 & 70 & 60 & 50 & 40 & 30 & 20 & 10 & 0 & -10\end{array}$ 
5-Methoxy-propynyl-1,2-benziodoxol-3(1H)-one (5p)

${ }^{1}$ H NMR (400 MHz, Chloroform-d)

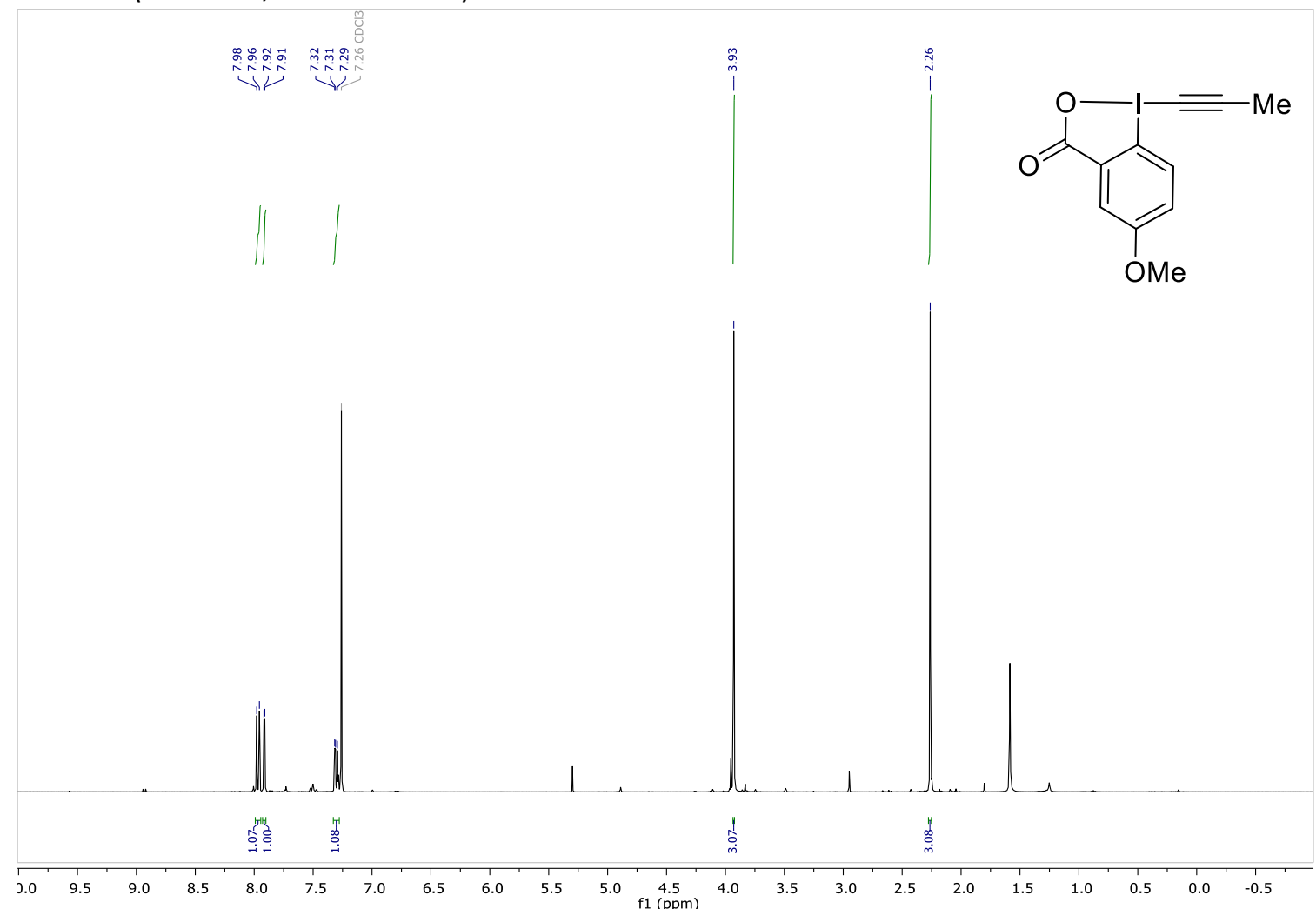

${ }^{13} \mathrm{C}$ NMR (101 MHz, Chloroform-d)

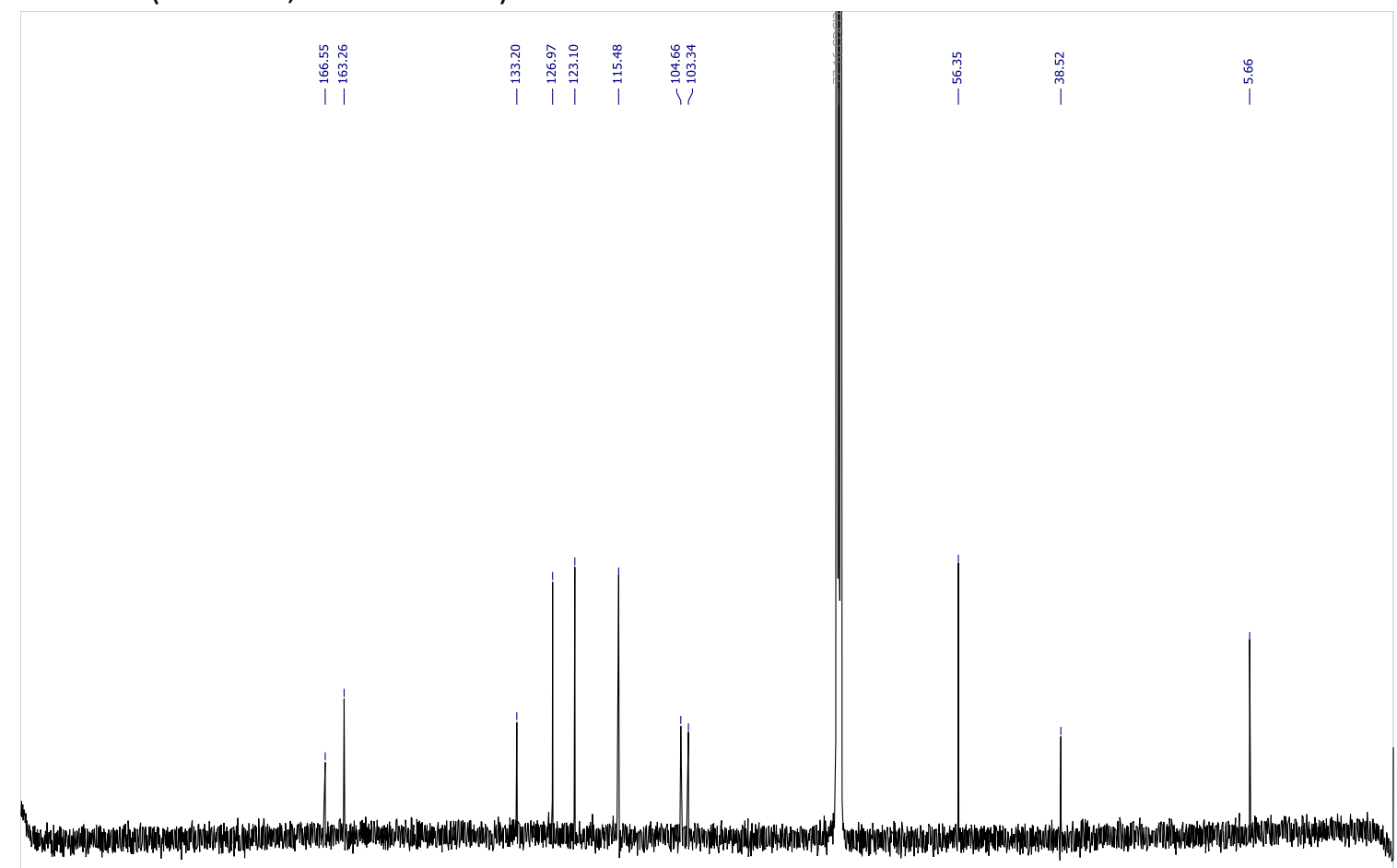

$\begin{array}{llllllllllllllllllllllllllllllll}210 & 200 & 190 & 180 & 170 & 160 & 150 & 140 & 130 & 120 & 110 & 100 & 90 & 80 & 70 & 60 & 50 & 40 & 30 & 20 & 10 & 0 & -10\end{array}$ 
1-(3-cyclopropylprop-1-yn-1-yl)-1,2-benziodoxol-3(1H)-one (5q)

${ }^{1} \mathrm{H}$ NMR (400 MHz, Methylene Chloride- $d_{2}$ )

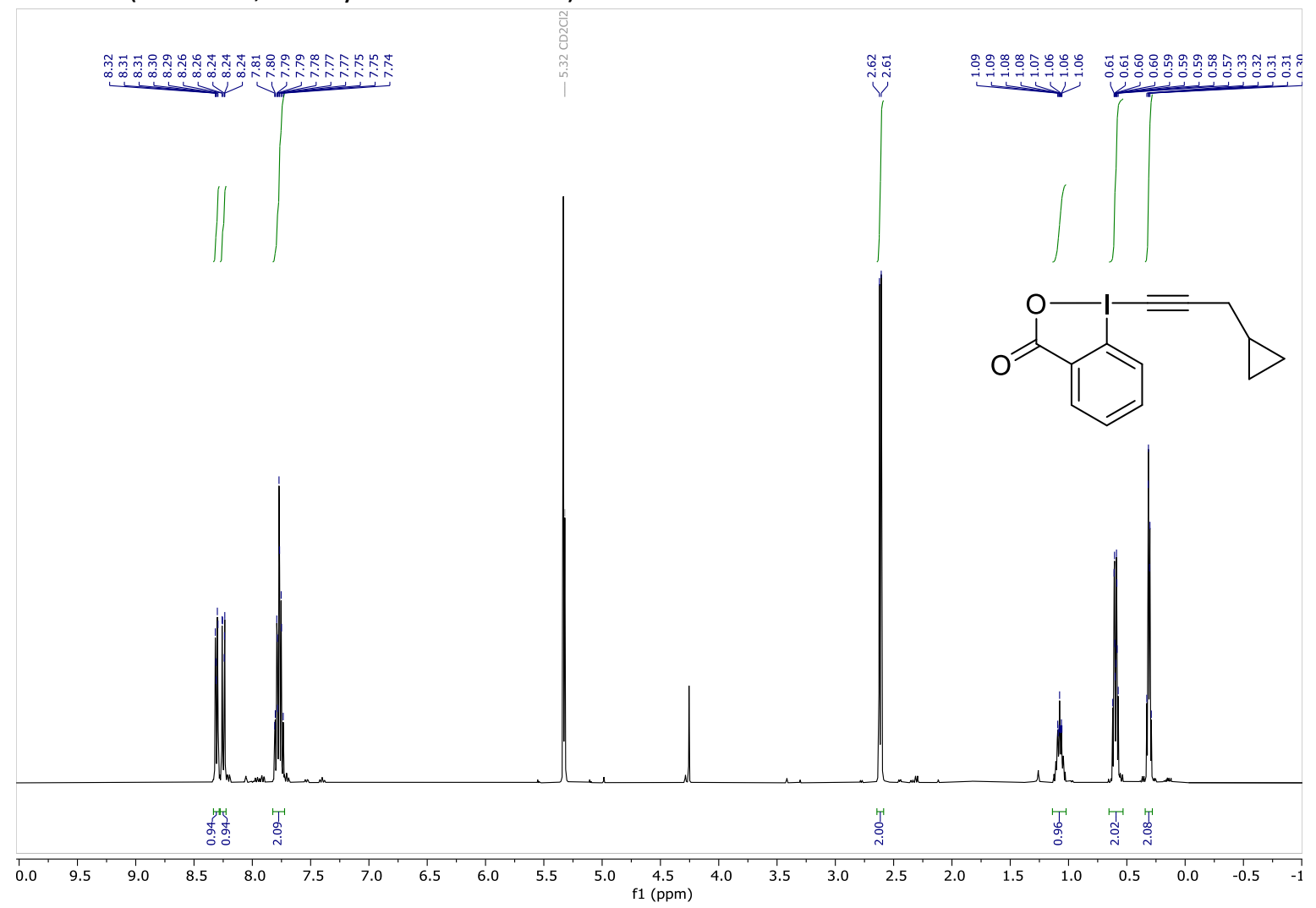

${ }^{13} \mathrm{C}$ NMR (101 MHz, Methylene Chloride- $d_{2}$ )

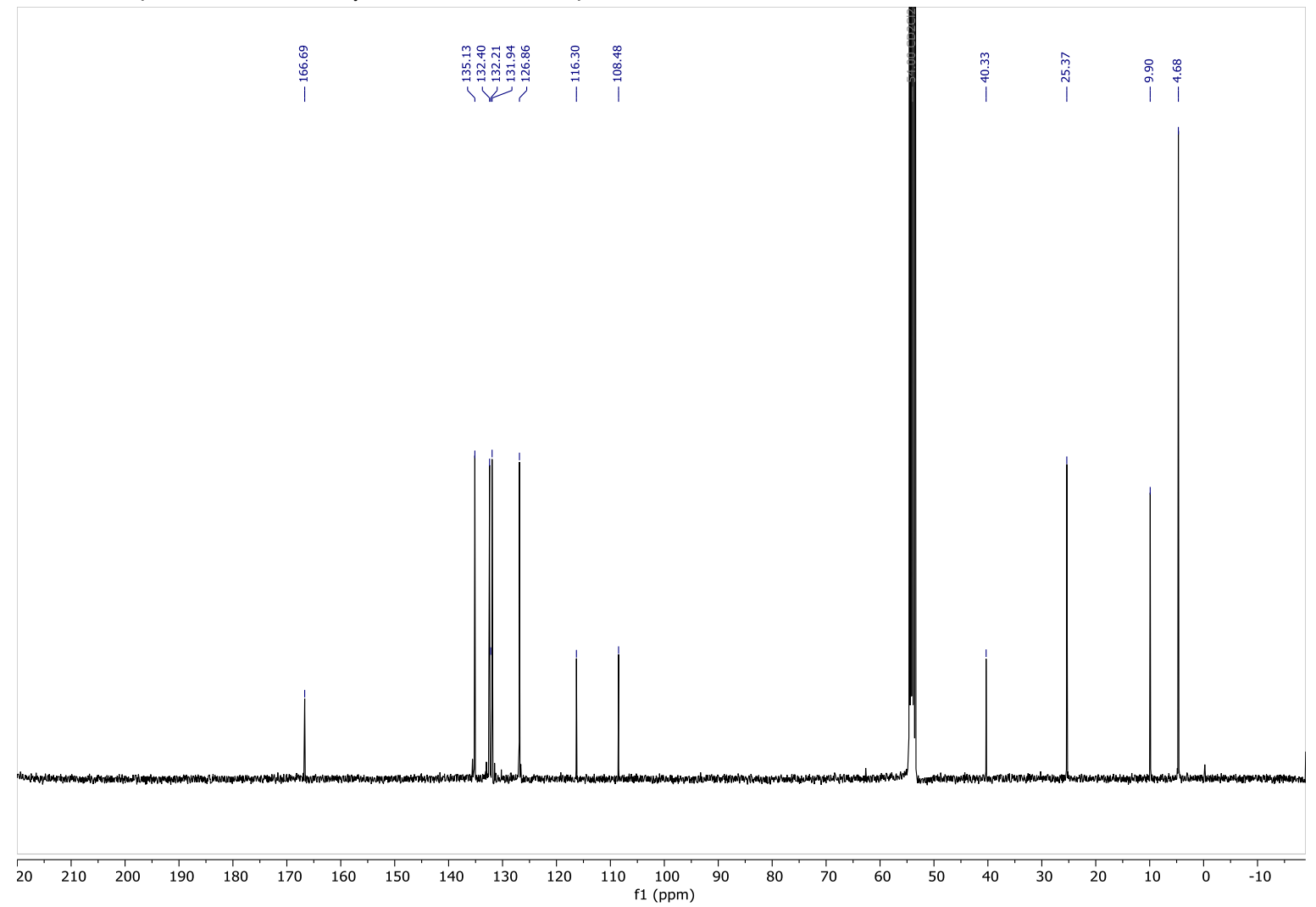




\subsection{O-VBX}

(Z)-1-(7-hydroxy-2-(p-tolyloxy)hept-1-en-1-yl)-1,3-benziodoxol-3-(1H)-one (1c)

${ }^{1} \mathrm{H}$ NMR (400 MHz, Chloroform-d)

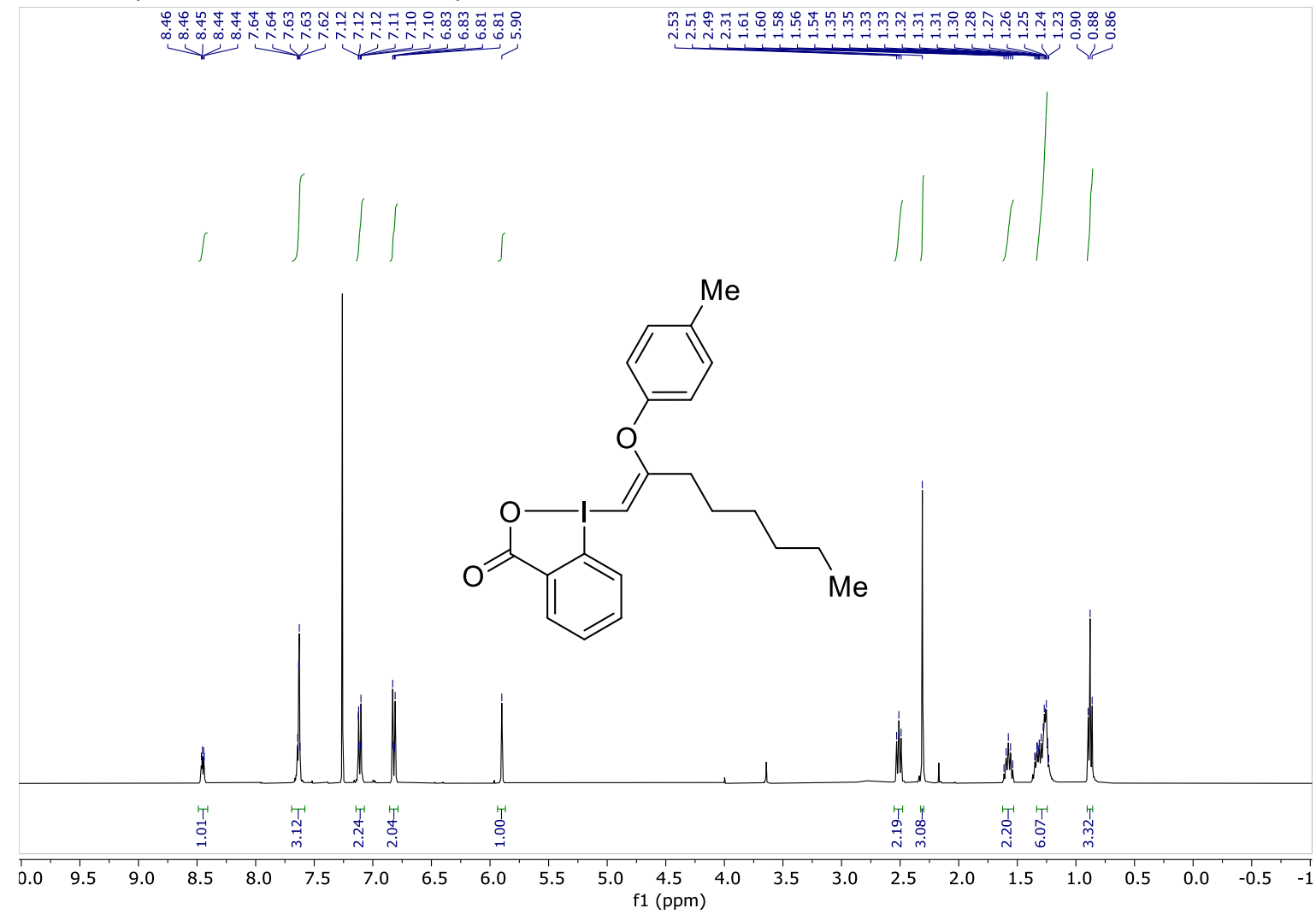

${ }^{13} \mathrm{C}$ NMR (101 MHz, Chloroform-d)

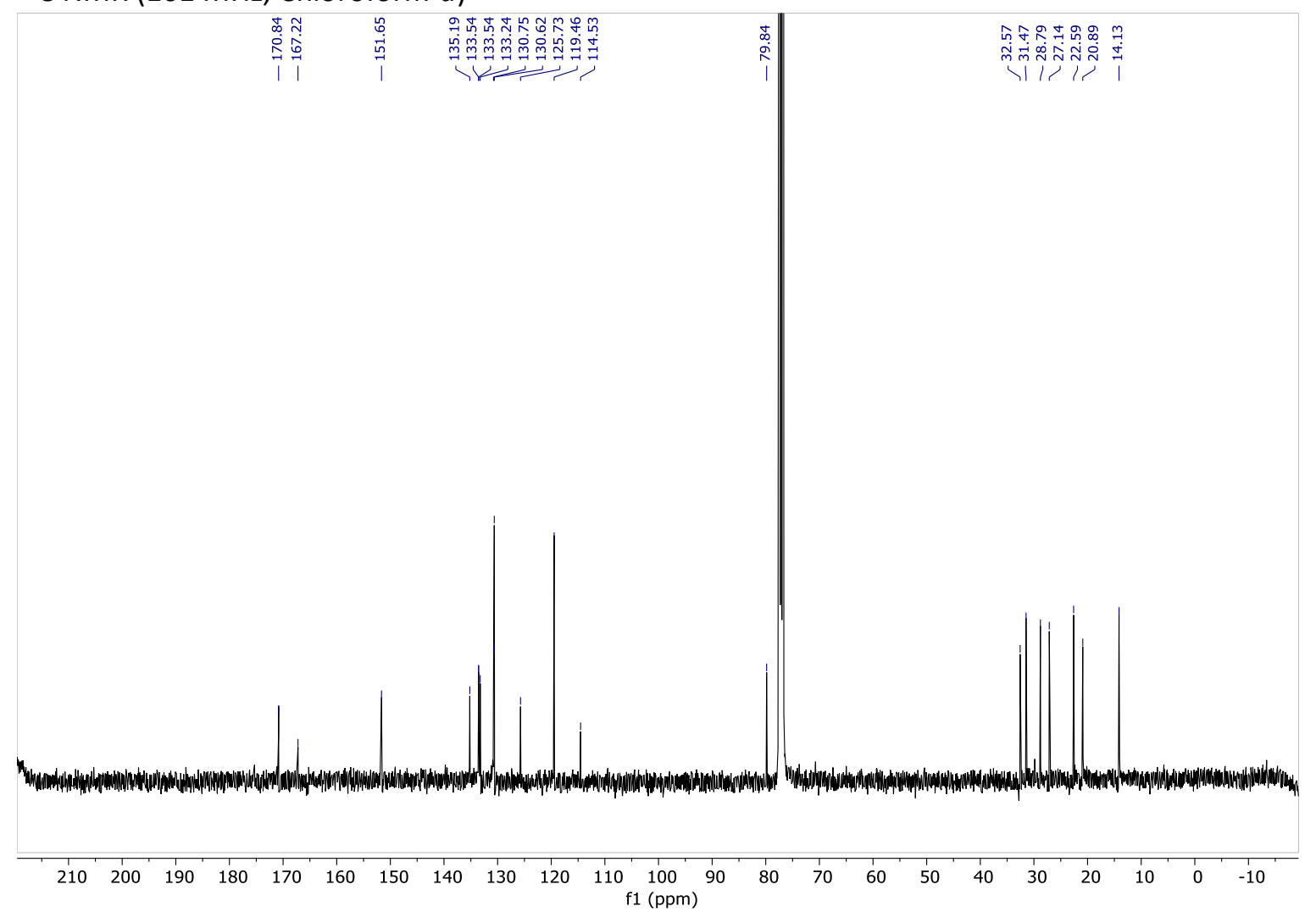


(Z)-1-(7-hydroxy-2-(p-tolyloxy)hept-1-en-1-yl)-1,3-benziodoxol-3-(1H)-one (1d)

${ }^{1} \mathrm{H}$ NMR (400 MHz, Methanol- $d_{4}$ )

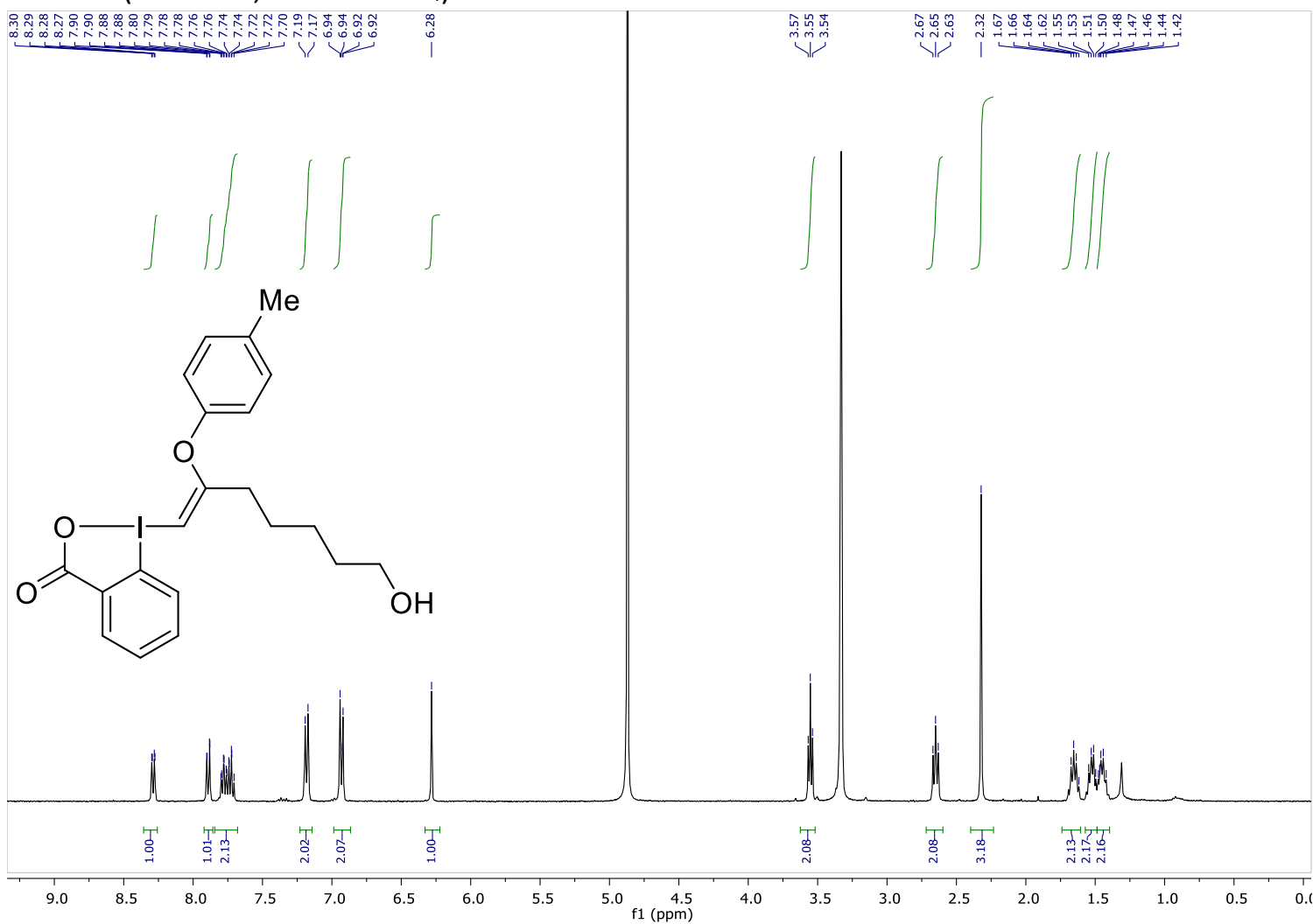

${ }^{13} \mathrm{C}$ NMR (101 MHz, Methanol- $\left.d_{4}\right)$

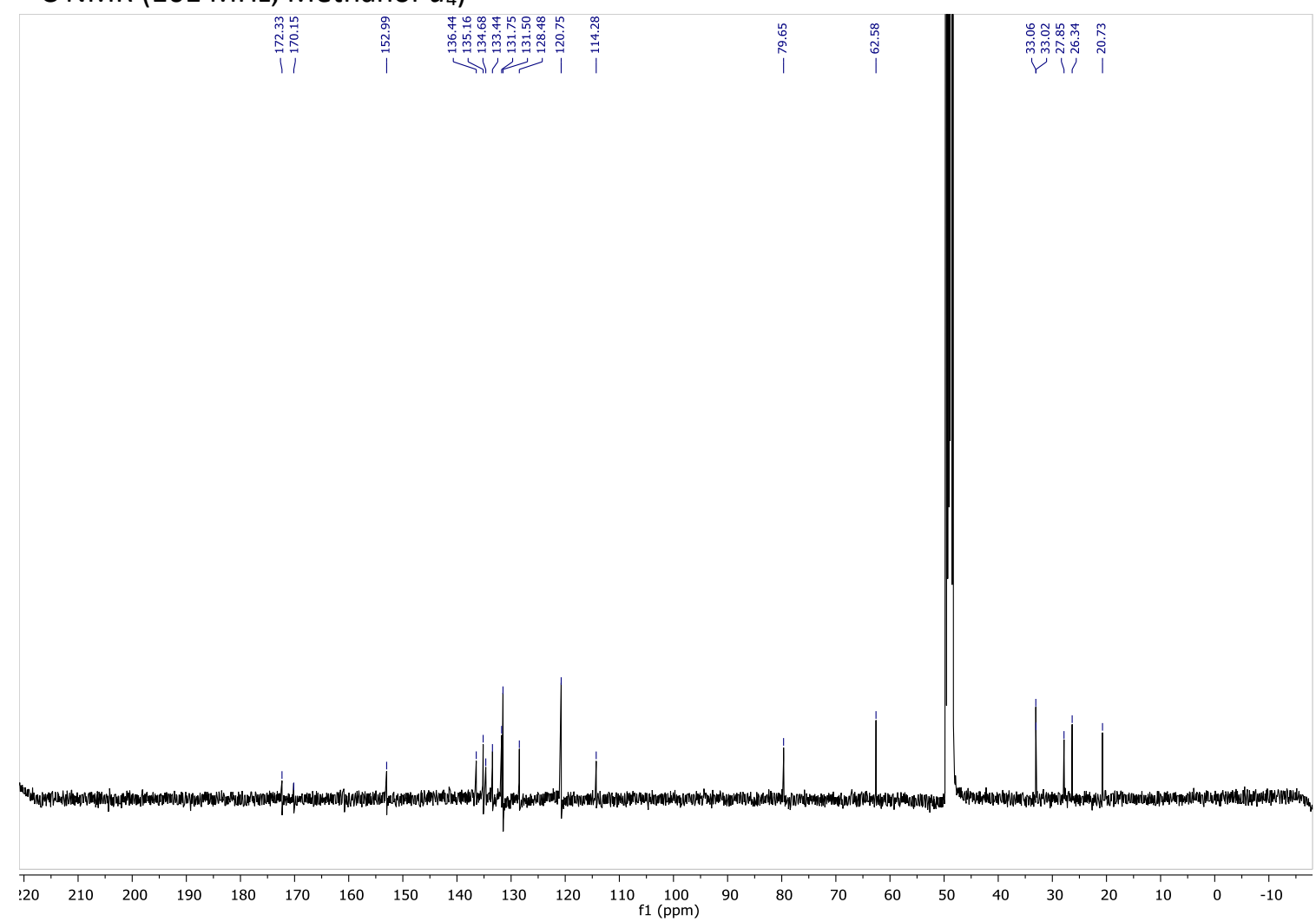


(Z)-1-(2-(p-tolyloxy)-8-(trimethylsilyl)oct-1-en-7-yn-1-yl)-1,3-benziodoxol-3-(1H)-one (1e) ${ }^{1} \mathrm{H}$ NMR (400 MHz, Methylene Chloride- $d_{2}$ )

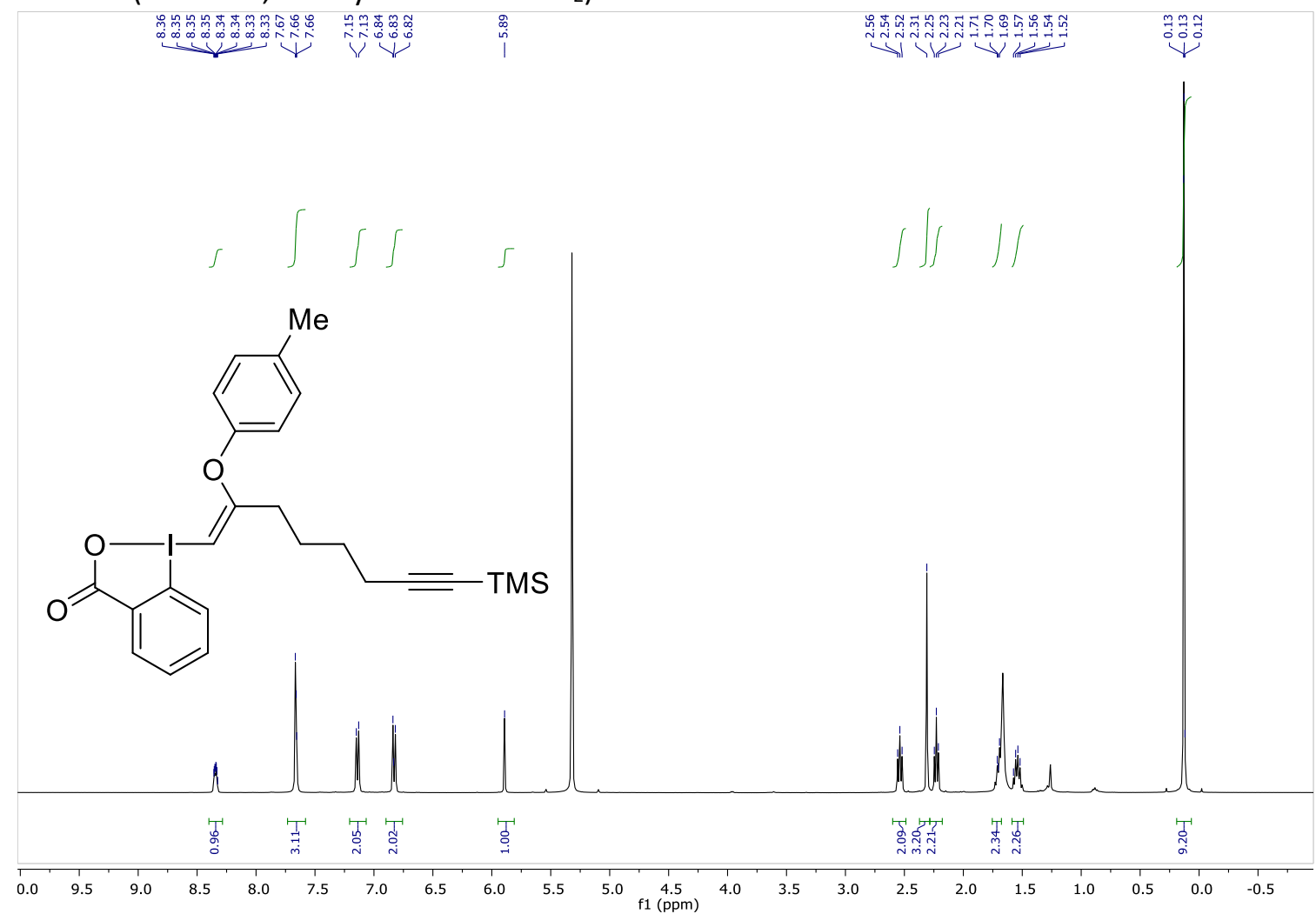

${ }^{13} \mathrm{C}$ NMR (101 MHz, Methylene Chloride- $d_{2}$ )

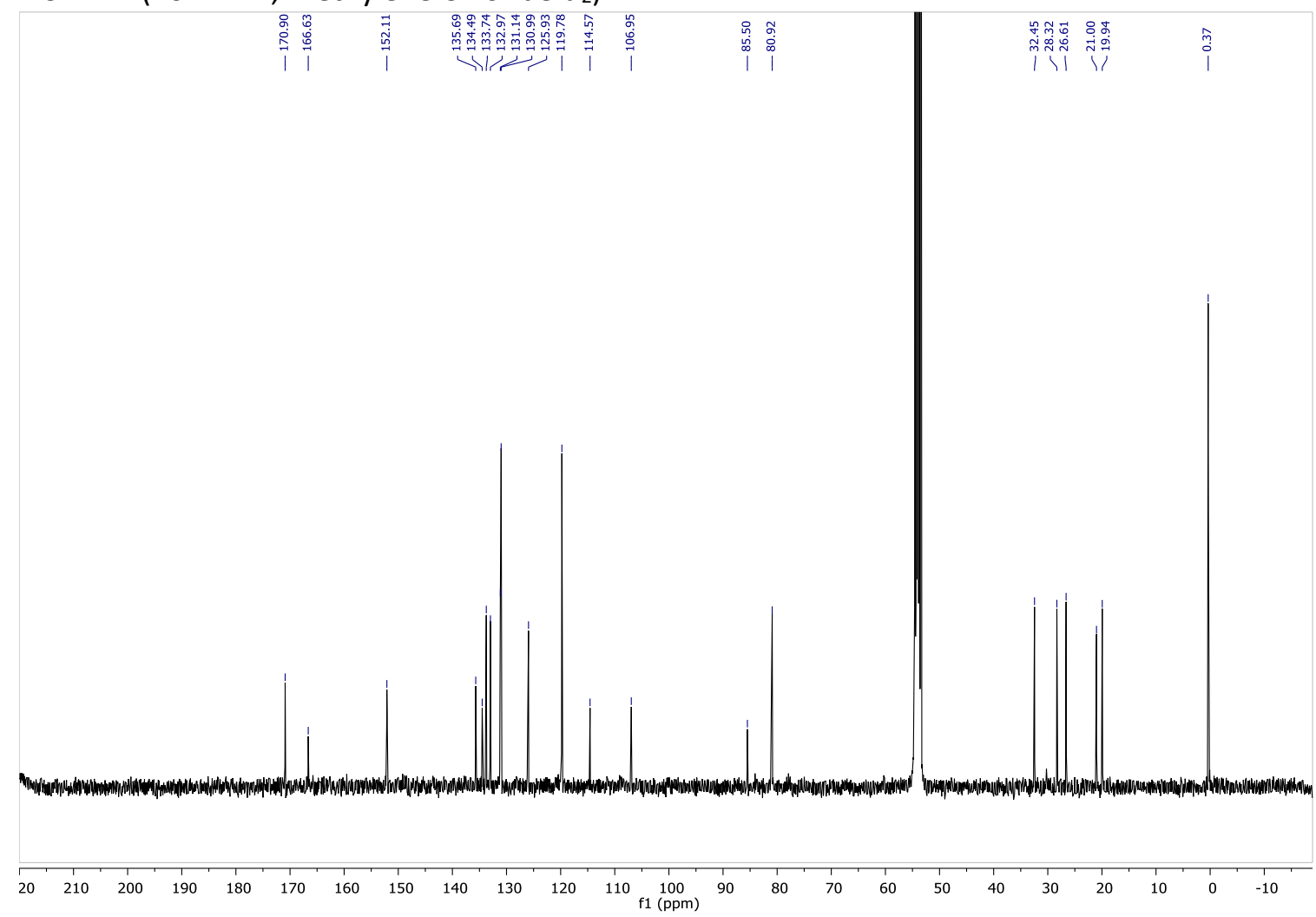


(Z)-2-(6-(3-Oxo-1,2-benziodoxol-3-(1H)-yl)-5-(p-tolyloxy)hex-5-en-1-yl)isoindoline-1,3-dione (1f) ${ }^{1} \mathrm{H}$ NMR (400 MHz, Chloroform-d)

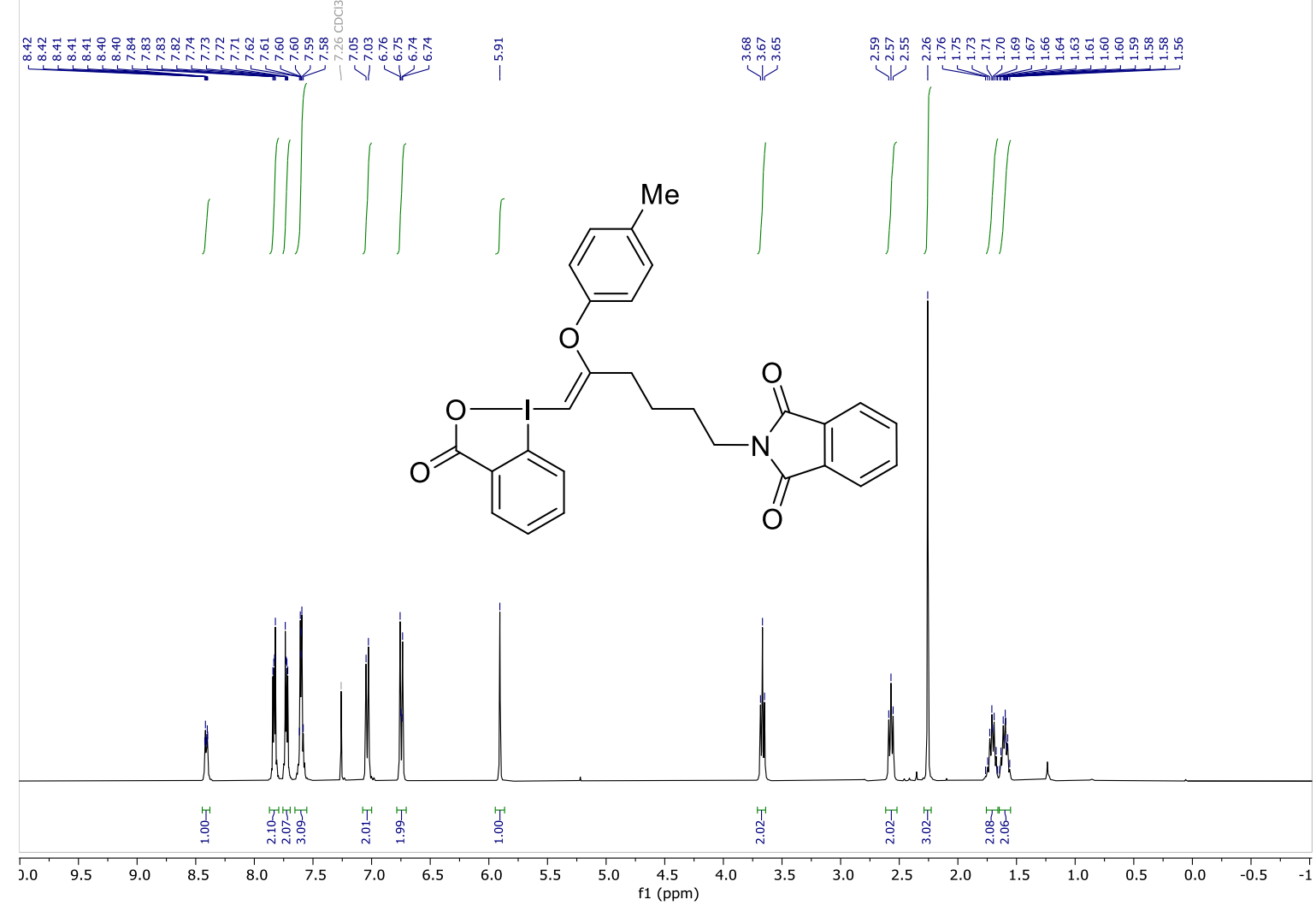

${ }^{13} \mathrm{C}$ NMR (101 MHz, Chloroform-d)

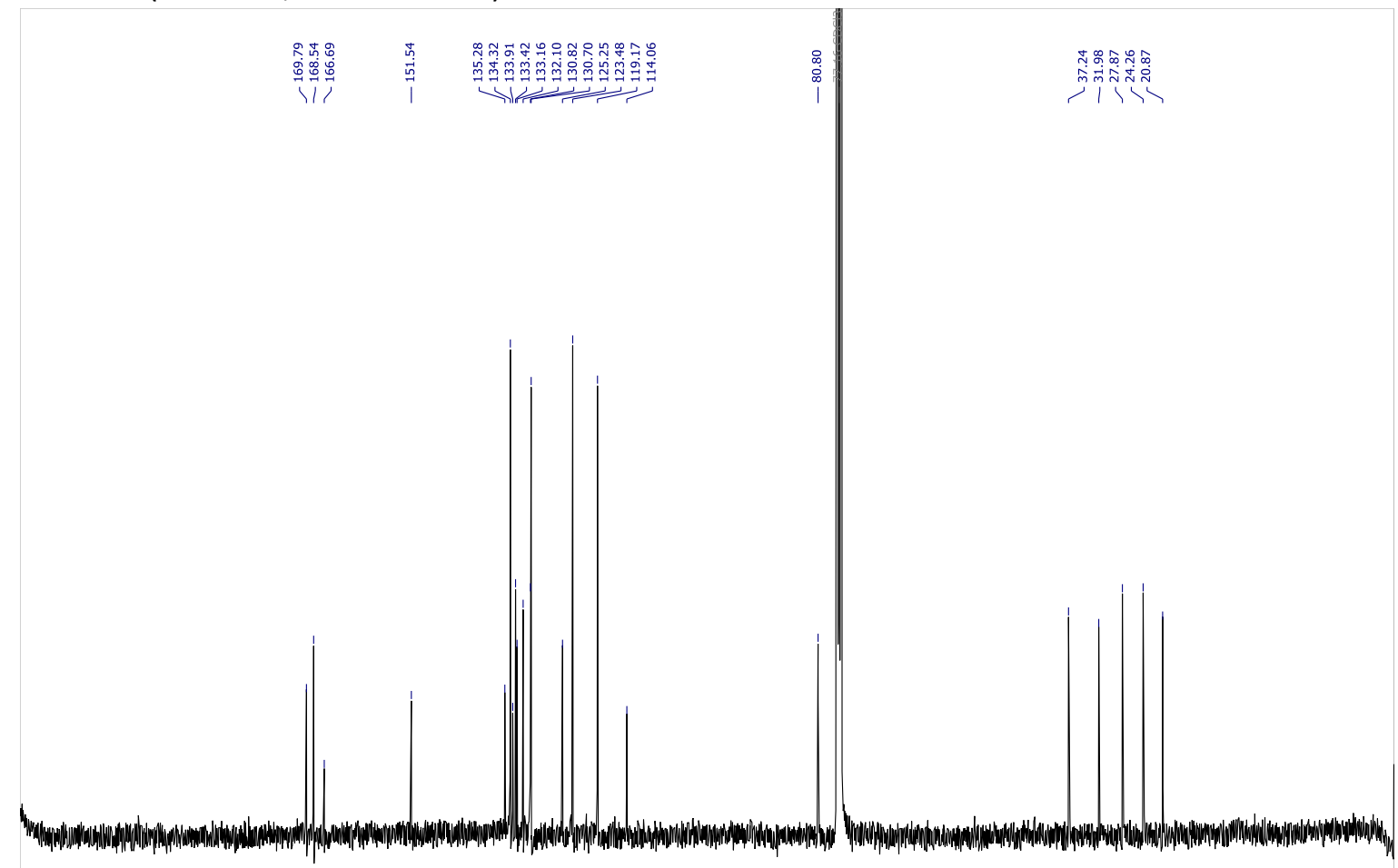

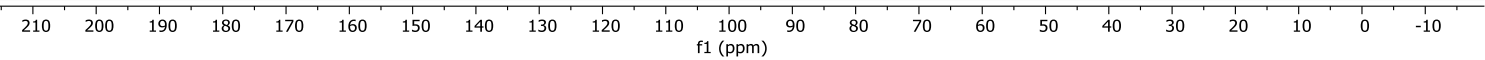


(Z)-1-(2-(2-iodophenoxy)prop-1-en-1-yl)-1,2-benziodoxol-3-(1H)-one (1g)

${ }^{1} \mathrm{H}$ NMR (400 MHz, Chloroform-d)

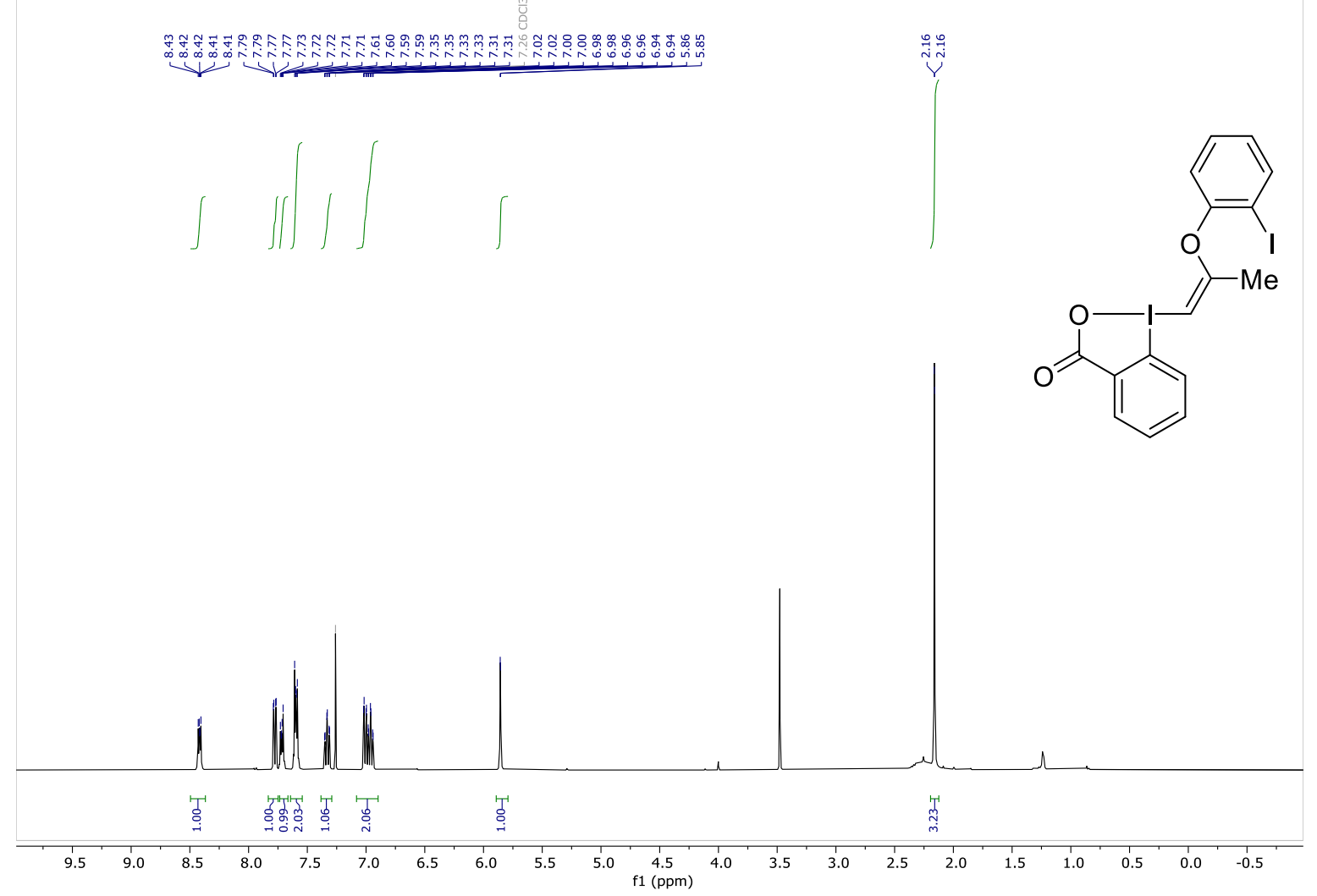

${ }^{13} \mathrm{C}$ NMR (101 MHz, Chloroform-d)

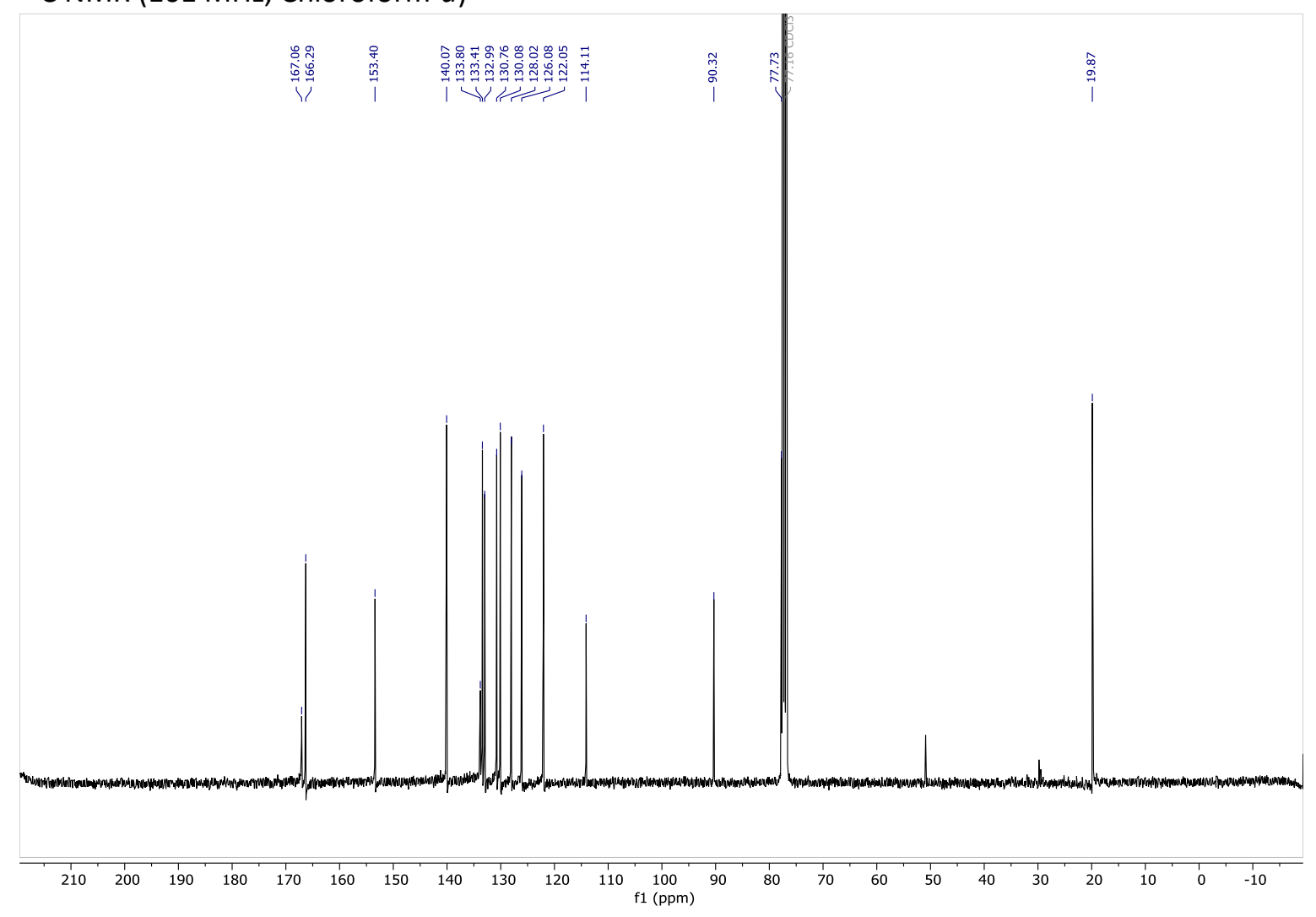


(Z)-1-(2-(2-iodophenoxy)pent-1-en-1-yl)-1,2-benziodoxol-3-(1H)-one (1h)

${ }^{1} \mathrm{H}$ NMR (400 MHz, Chloroform-d)

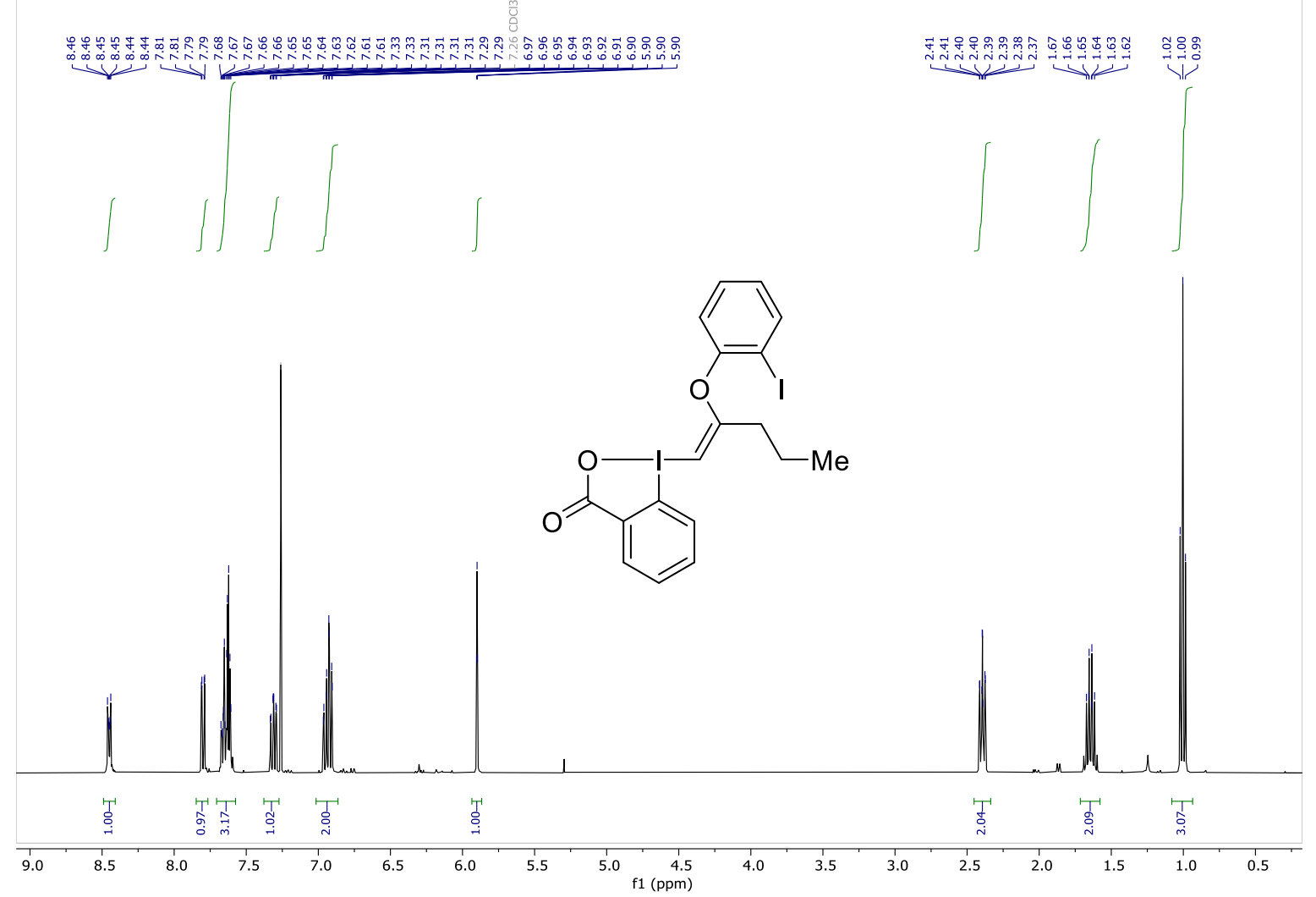

${ }^{13} \mathrm{C}$ NMR (101 MHz, Chloroform-d)

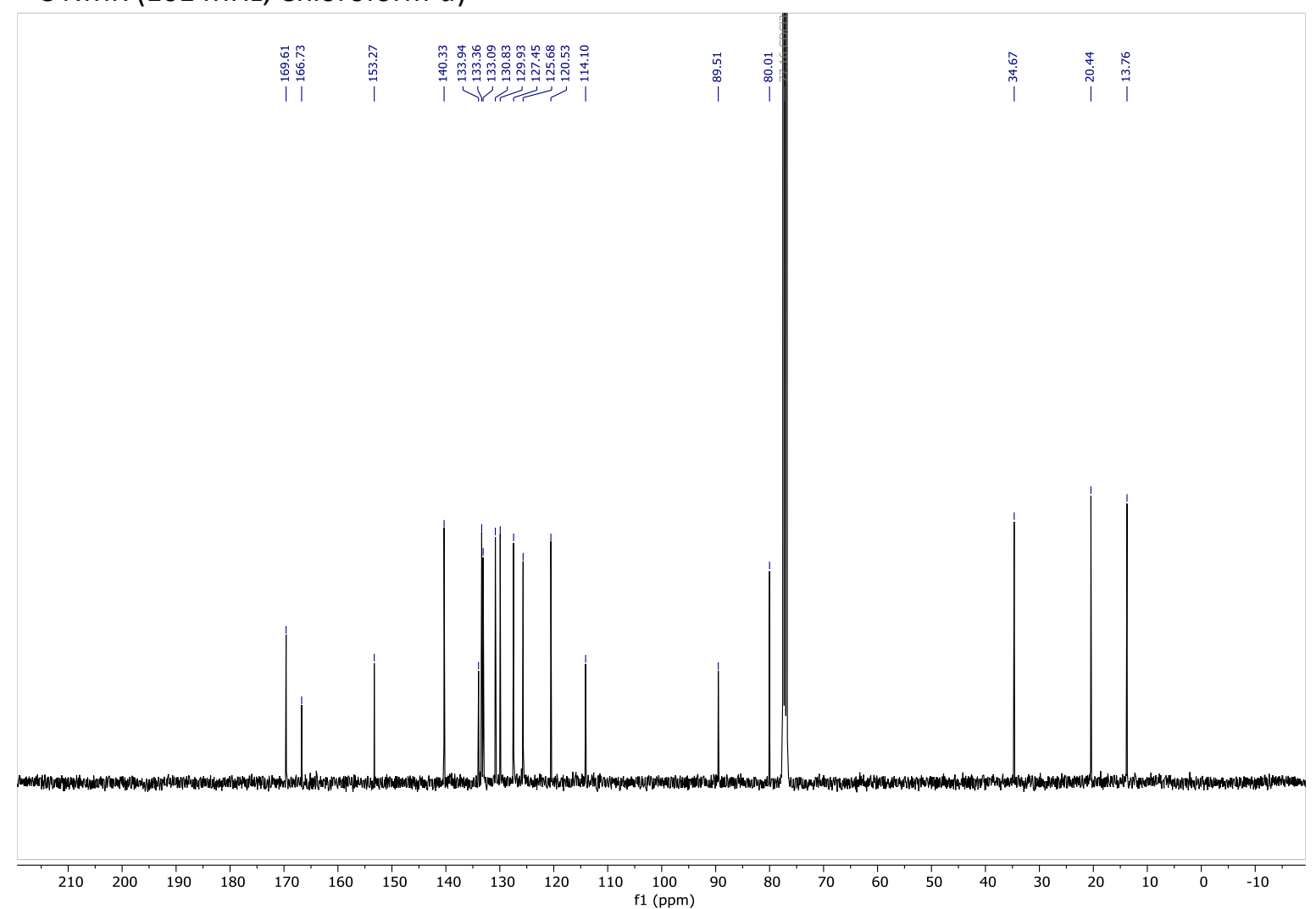


(Z)-1-(2-(2-lodophenoxy)oct-1-en-1-yl)-1,2-benziodoxol-3-(1H)-one (1i)

${ }^{1} \mathrm{H}$ NMR (400 MHz, Chloroform-d)

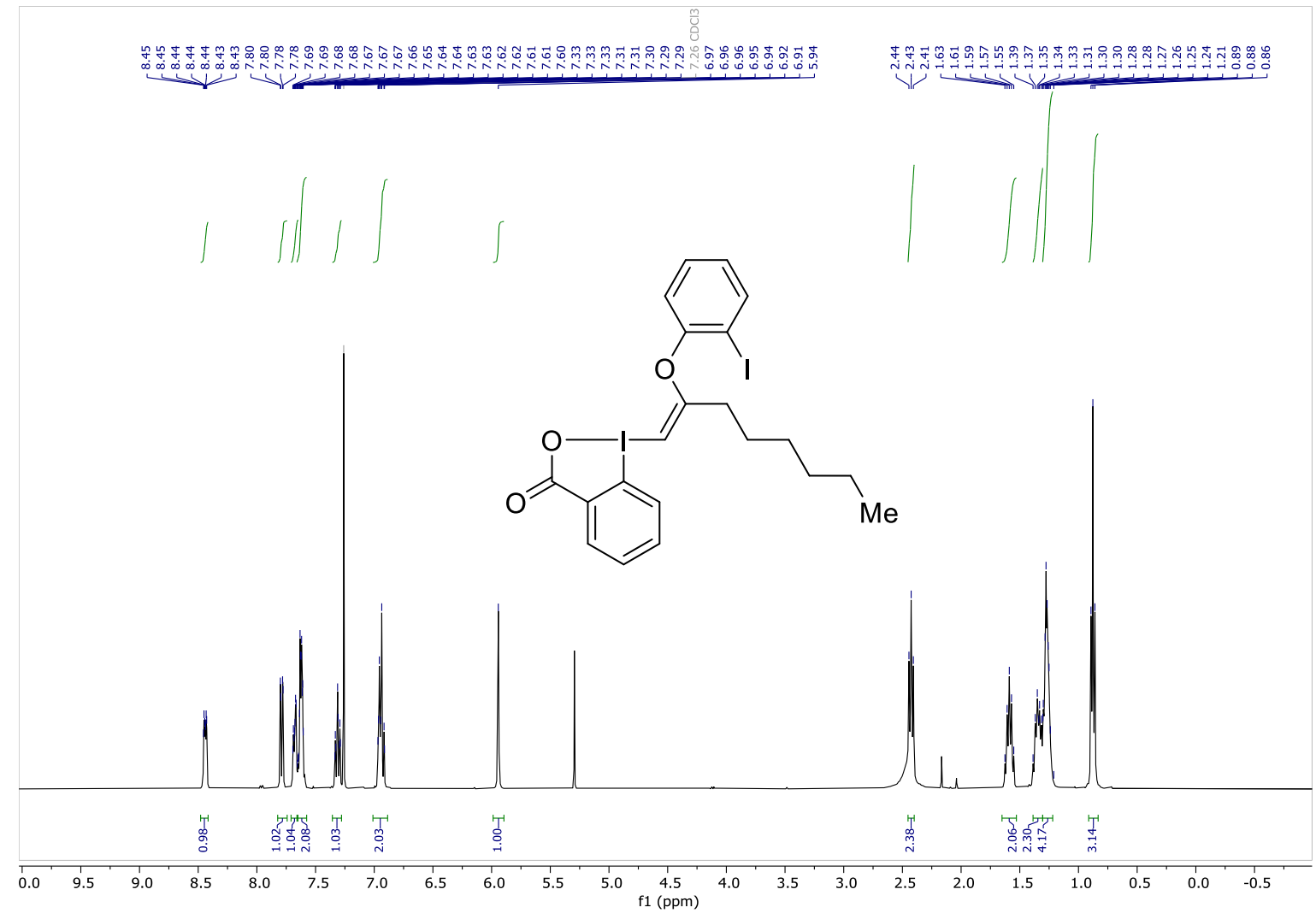

${ }^{13} \mathrm{C}$ NMR (101 MHz, Chloroform-d)

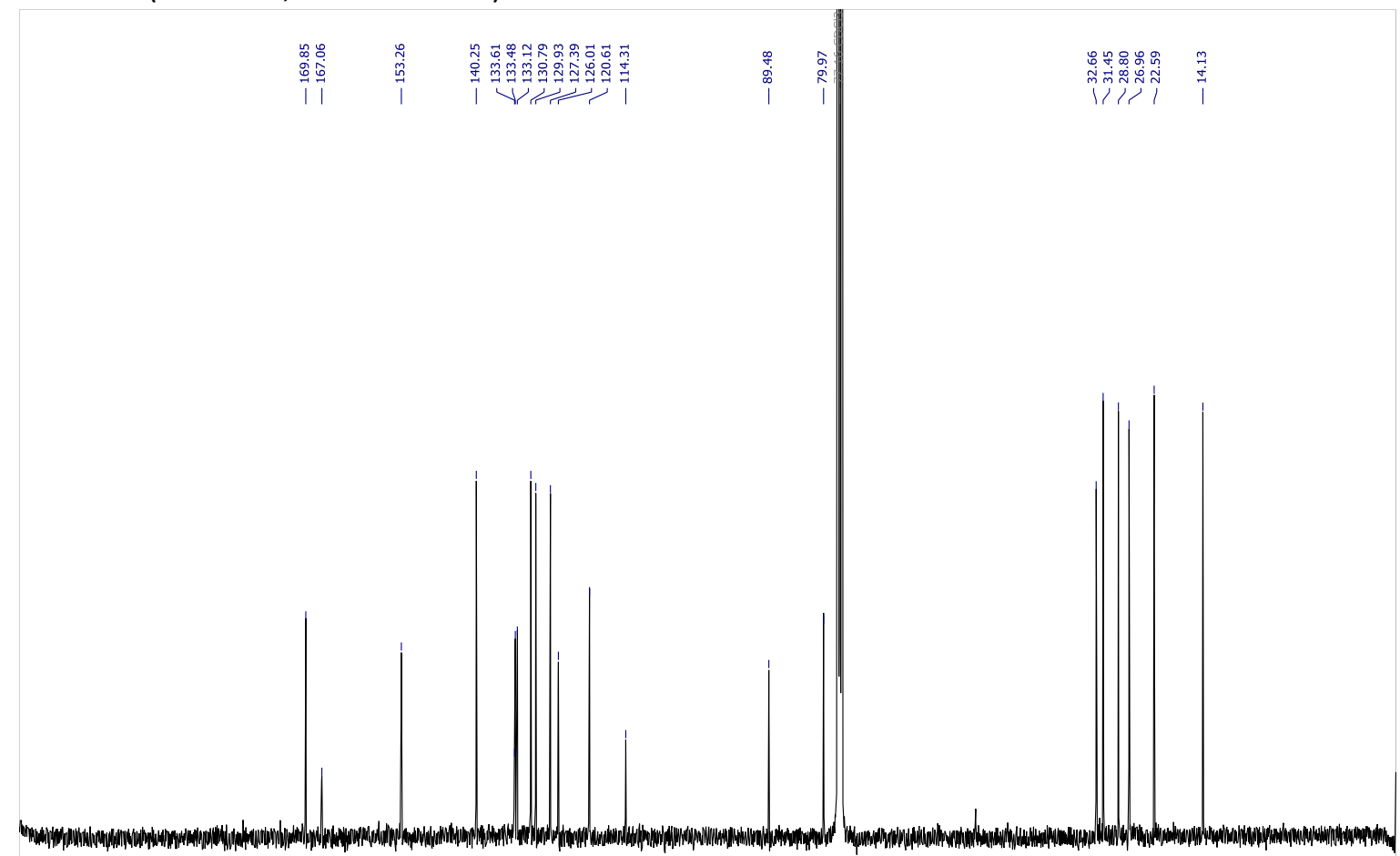

$\begin{array}{lllllllllllllllllllllll}\mid & 200 & 190 & 180 & 170 & 160 & 150 & 140 & 130 & 120 & 110 & \begin{array}{c}100 \\ \mathrm{f} 1(\mathrm{ppm})\end{array} & 90 & 80 & 70 & 60 & 50 & 40 & 30 & 20 & 10 & 0 & -10\end{array}$ 
(Z)-1-(2-(4-Methoxyphenoxy)pent-1-en-1-yl)-1,2-benziodoxol-3-(1H)-one (1j)

${ }^{1} \mathrm{H}$ NMR (400 MHz, Chloroform-d)

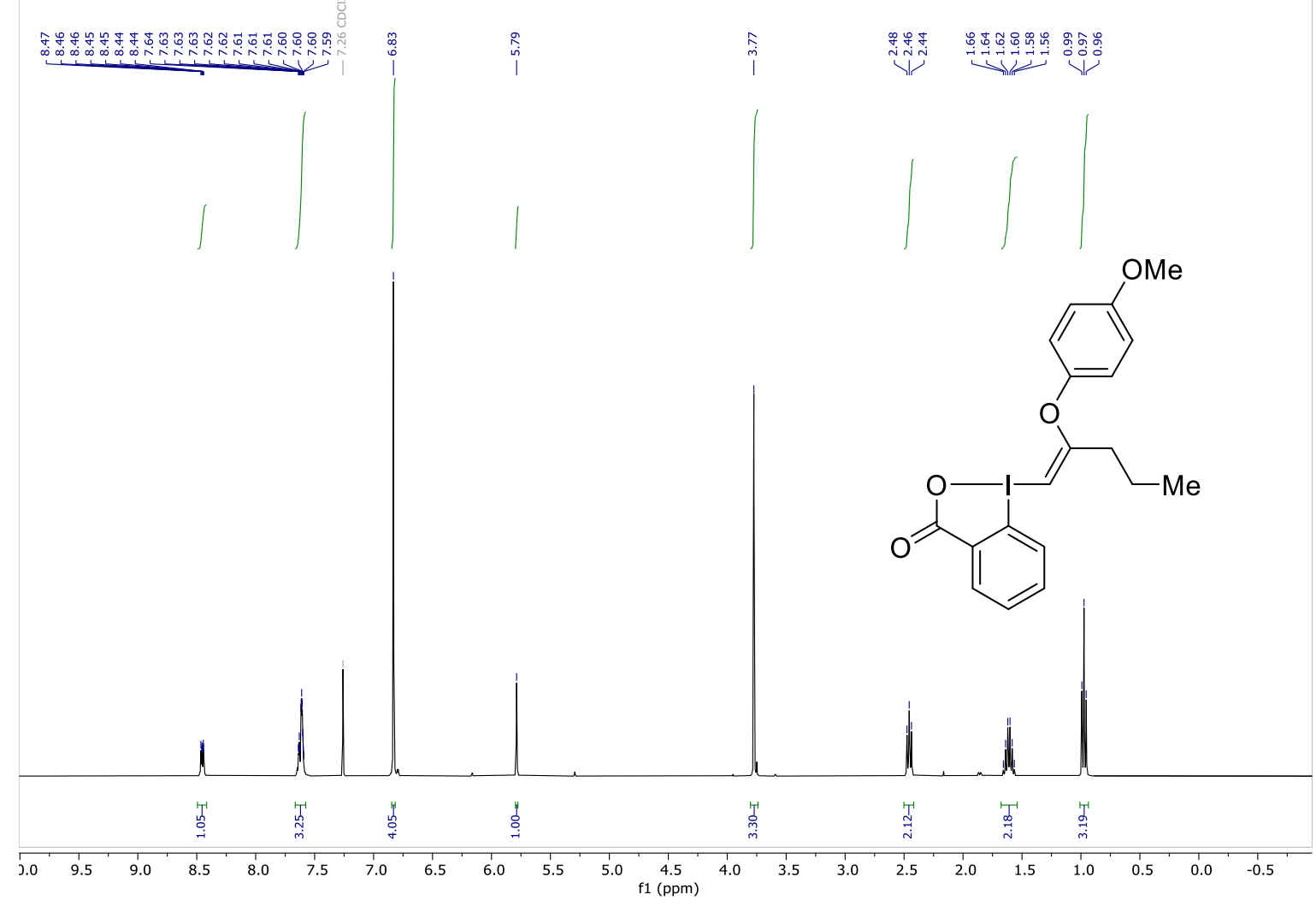

${ }^{13} \mathrm{C}$ NMR (101 MHz, Chloroform-d)

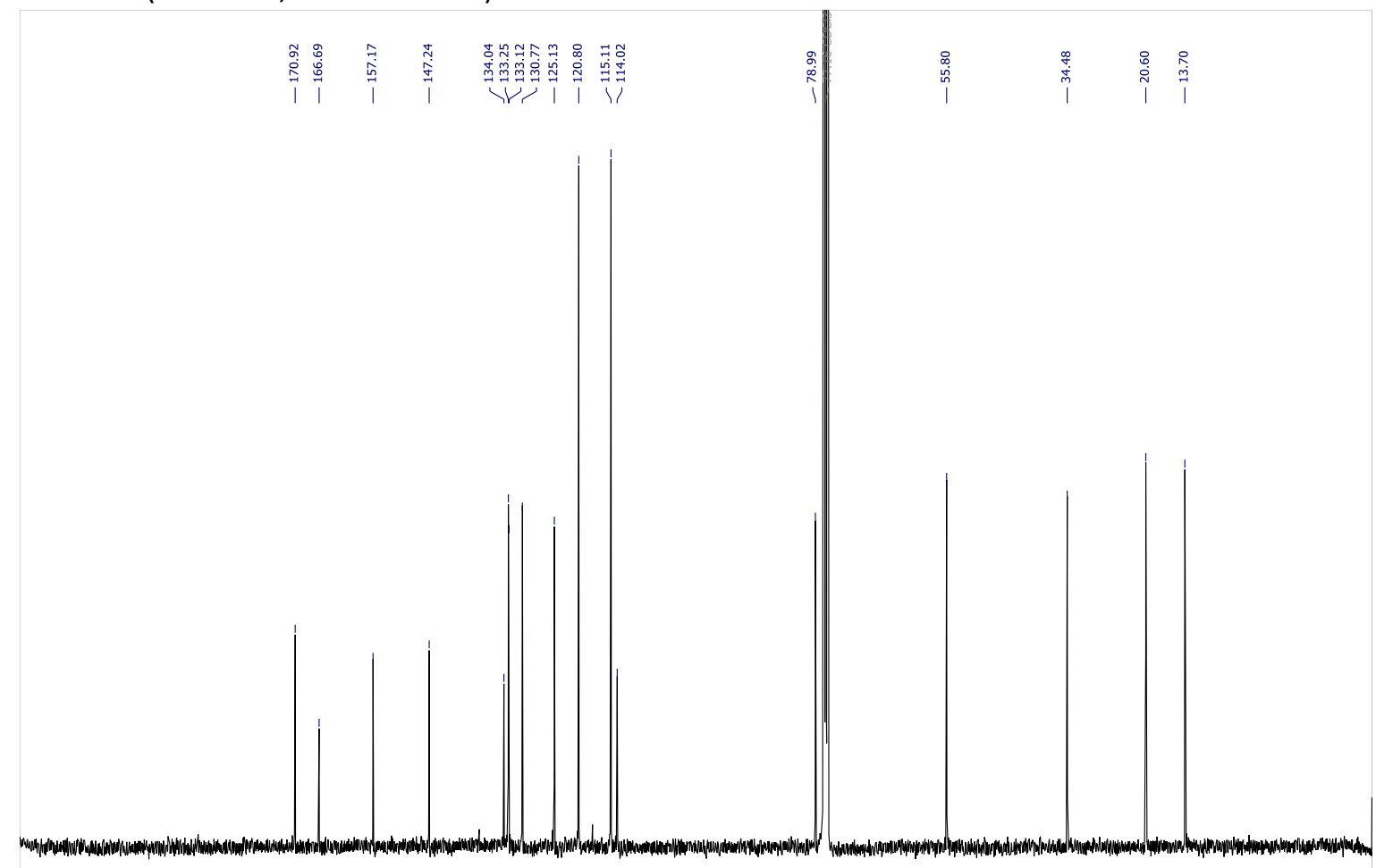

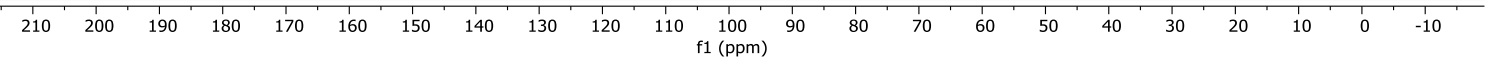


(Z)-1-(2-(4-methoxyphenoxy)prop-1-en-1-yl)-1,2-benziodoxol-3-(1H)-one (1k)

${ }^{1} \mathrm{H}$ NMR (400 MHz, Chloroform-d)

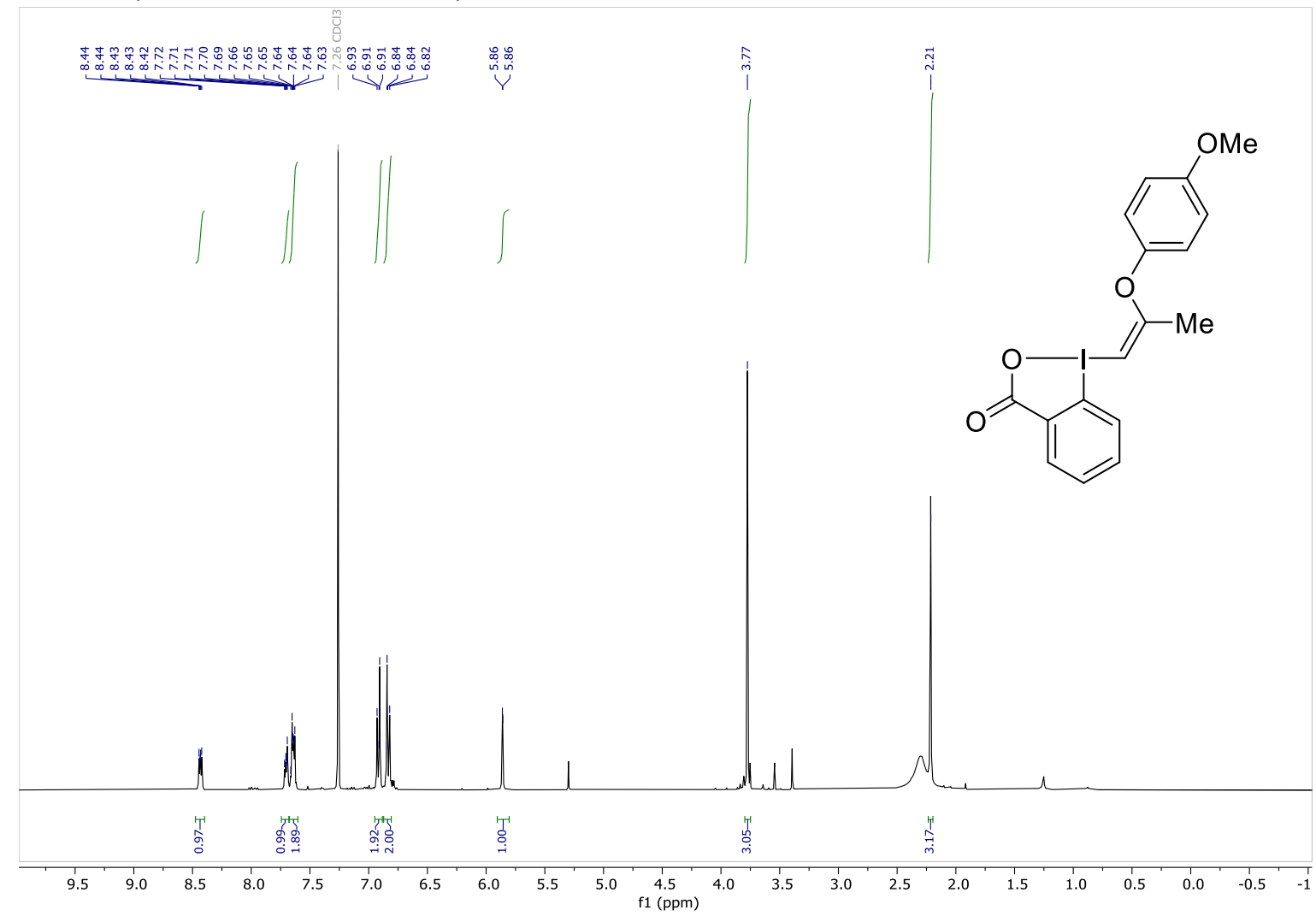

${ }^{13} \mathrm{C}$ NMR (101 MHz, Chloroform-d)

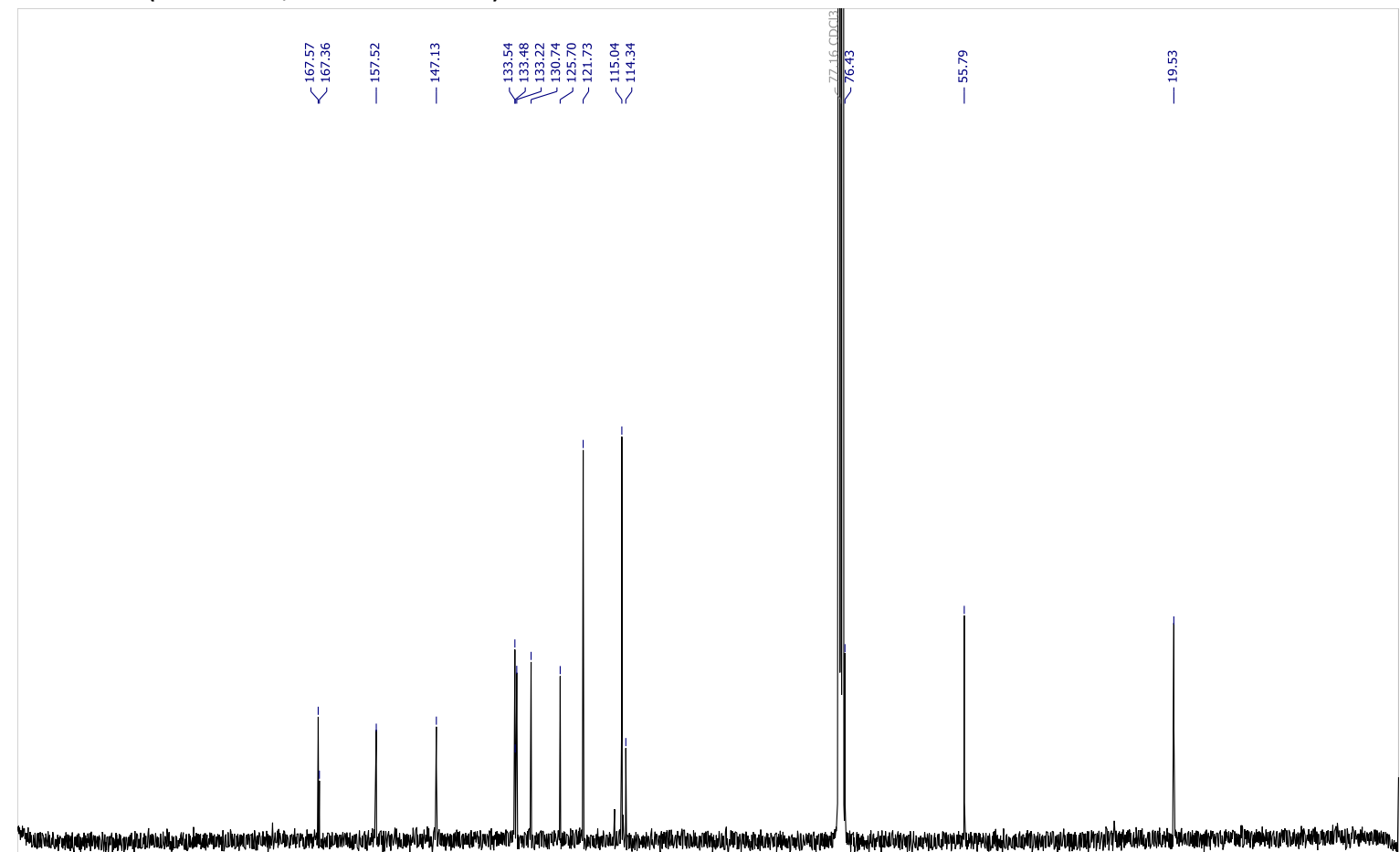

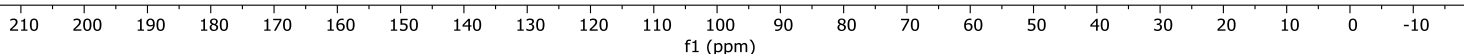


(Z)-1-(2-cyclopropyl-2-(p-tolyloxy)vinyl)-1|3-benziodoxol-3(1H)-one (1m)

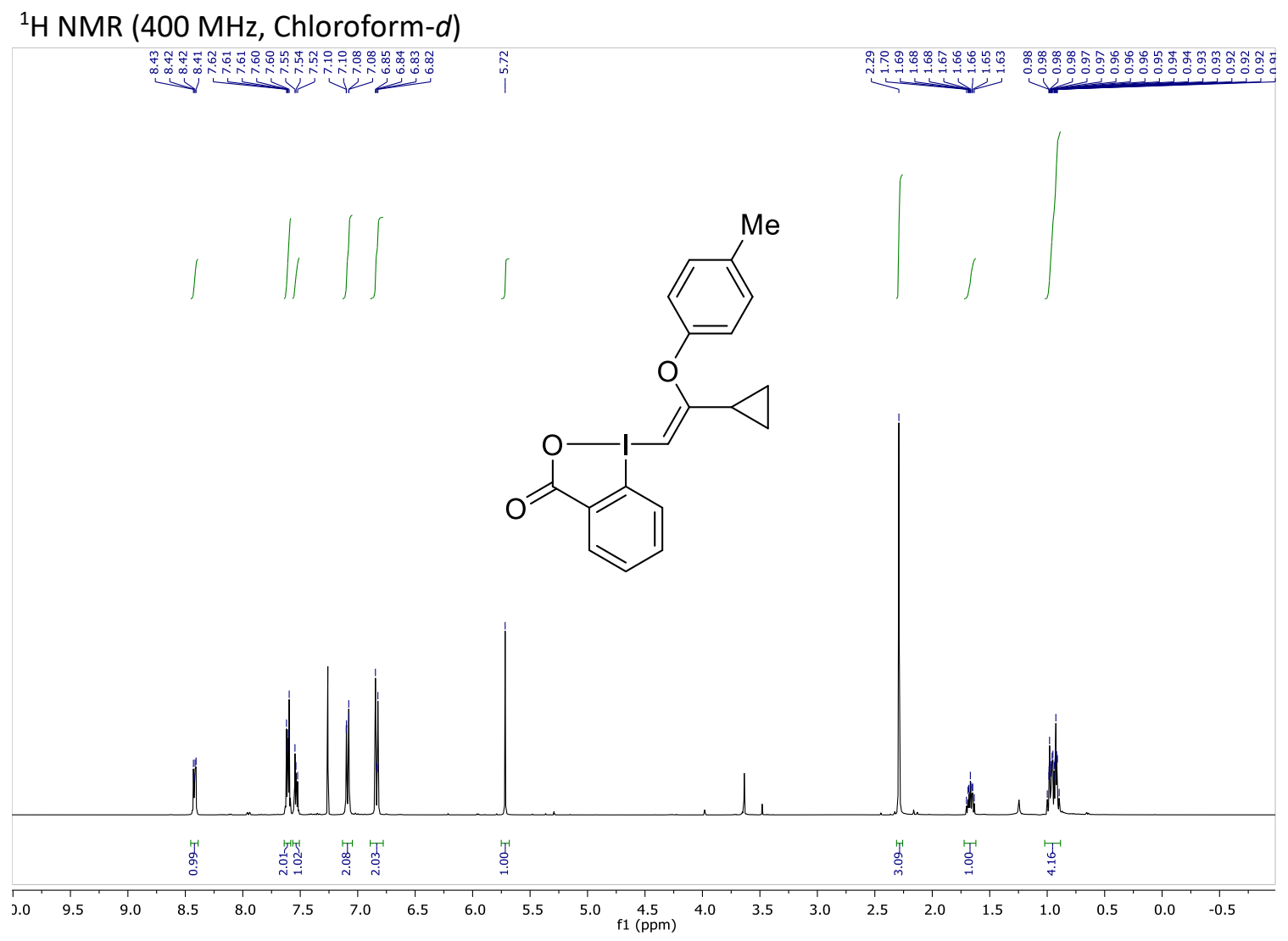

${ }^{13} \mathrm{C}$ NMR (101 MHz, Chloroform- $d$ )

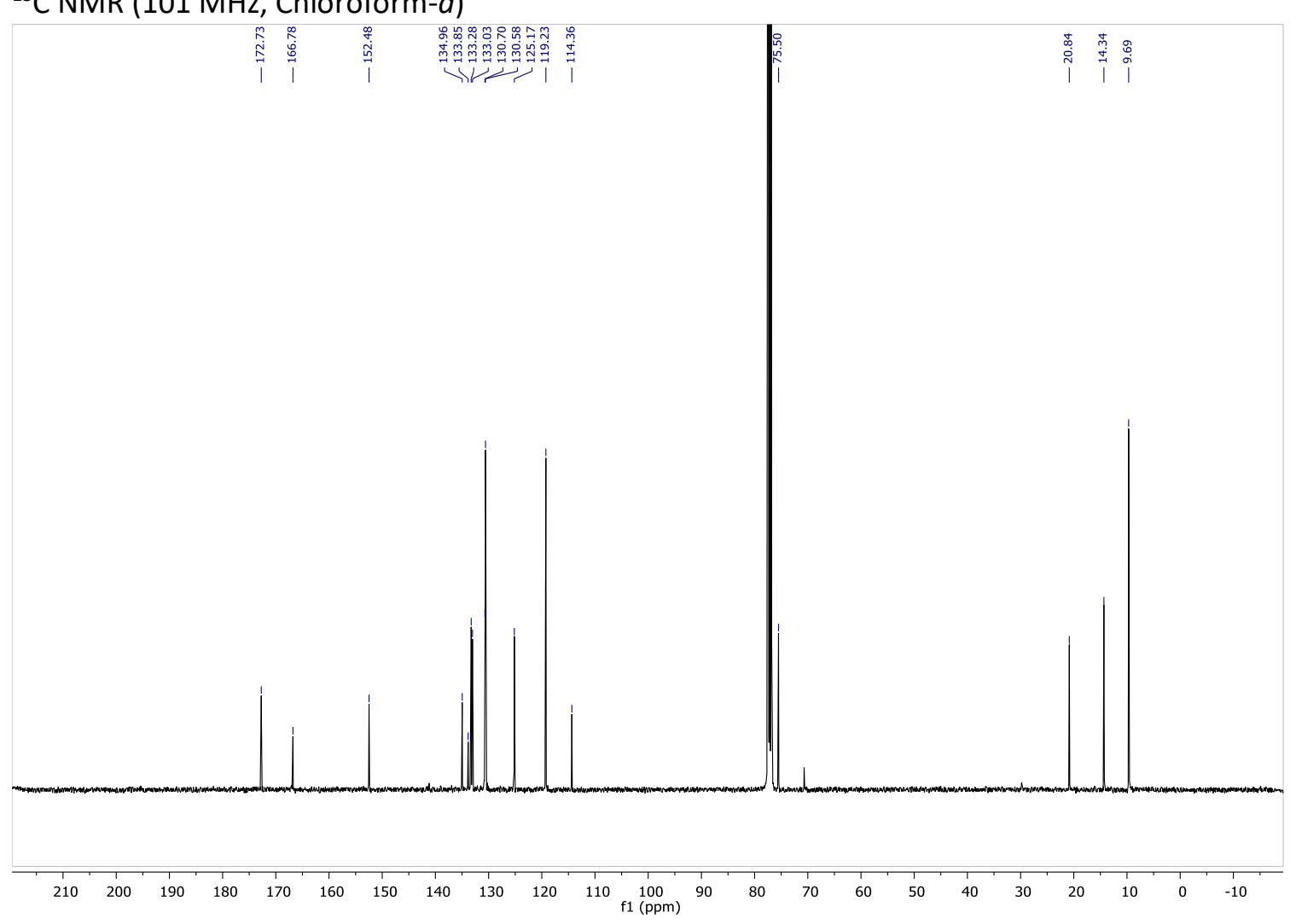

73 
(Z)-1-(2-cyclohexyl-2-(p-tolyloxy)vinyl)-1,2-benziodoxol-3-(1H)-one (1n)

${ }^{1} \mathrm{H}$ NMR (400 MHz, Chloroform-d)

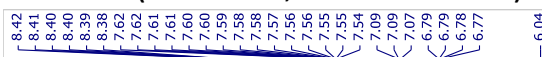

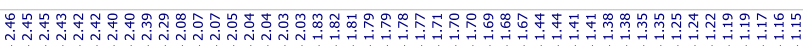

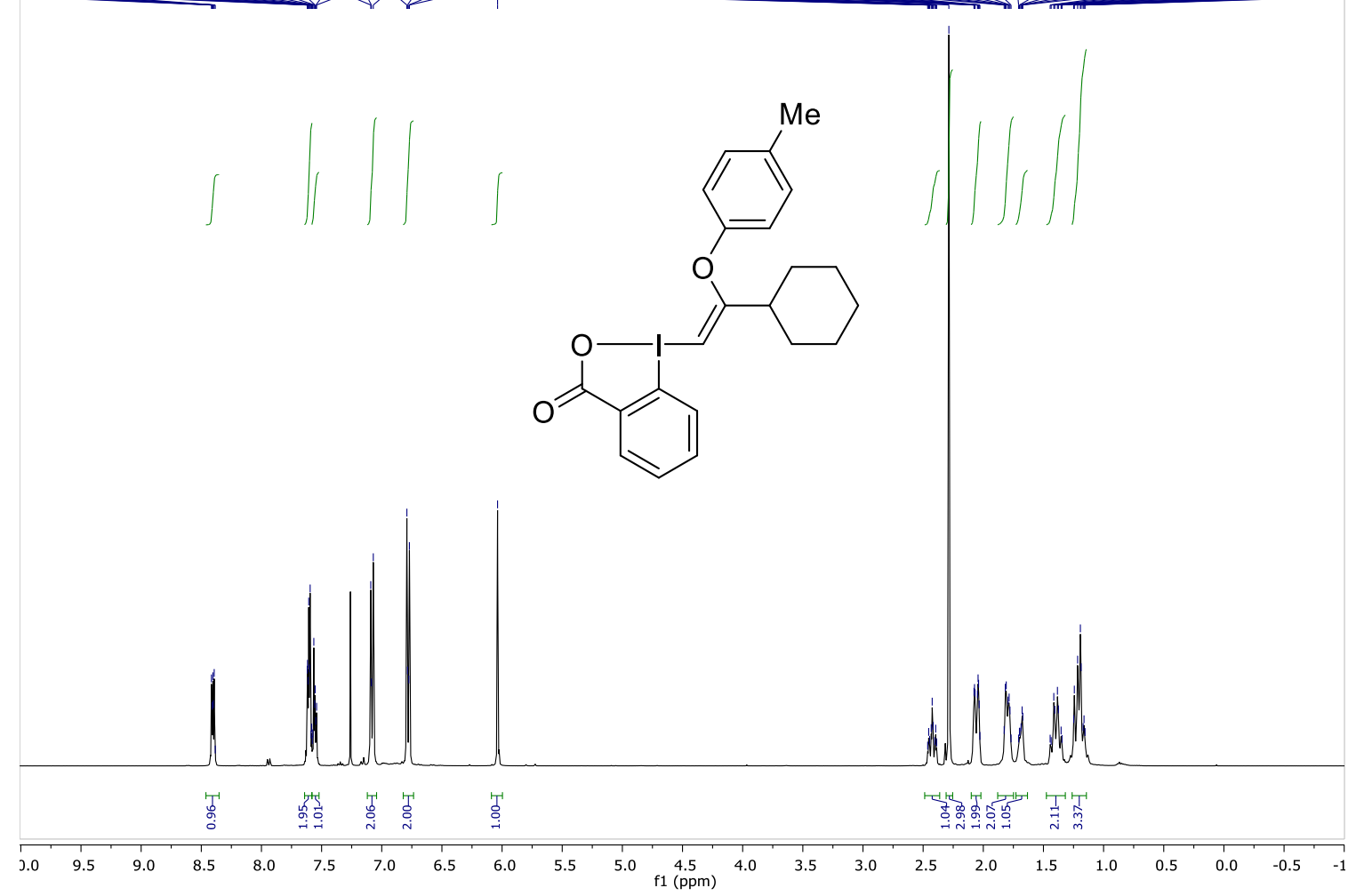

${ }^{13} \mathrm{C}$ NMR (101 MHz, Chloroform-d)

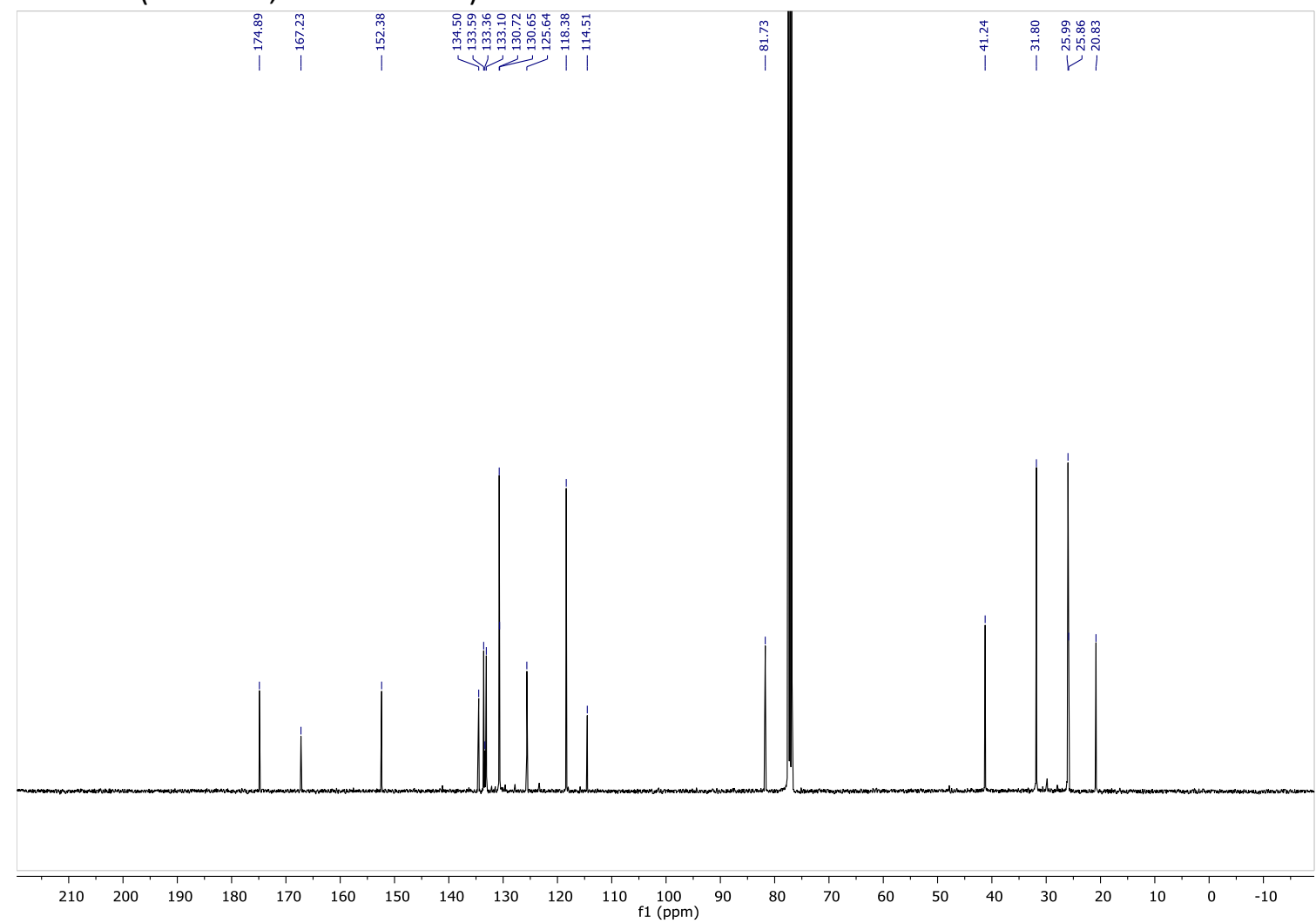


(Z)-1-(3,3-dimethyl-2-(p-tolyloxy)but-1-en-1-yl)-1,2-benziodoxol-3-(1H)-one (1p)

${ }^{1} \mathrm{H}$ NMR (400 MHz, Chloroform-d)

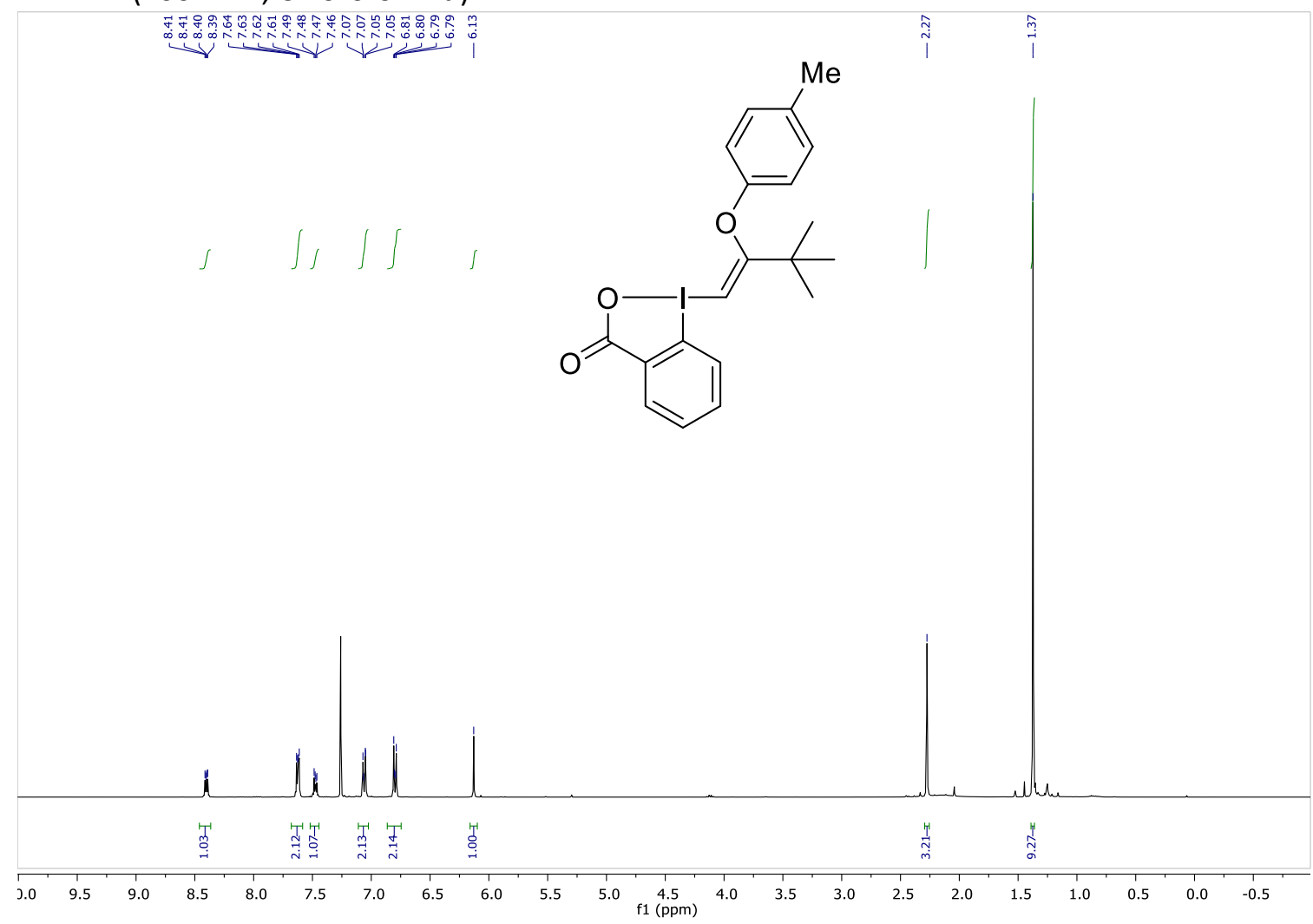

${ }^{13} \mathrm{C}$ NMR (101 MHz, Chloroform- $d$ )

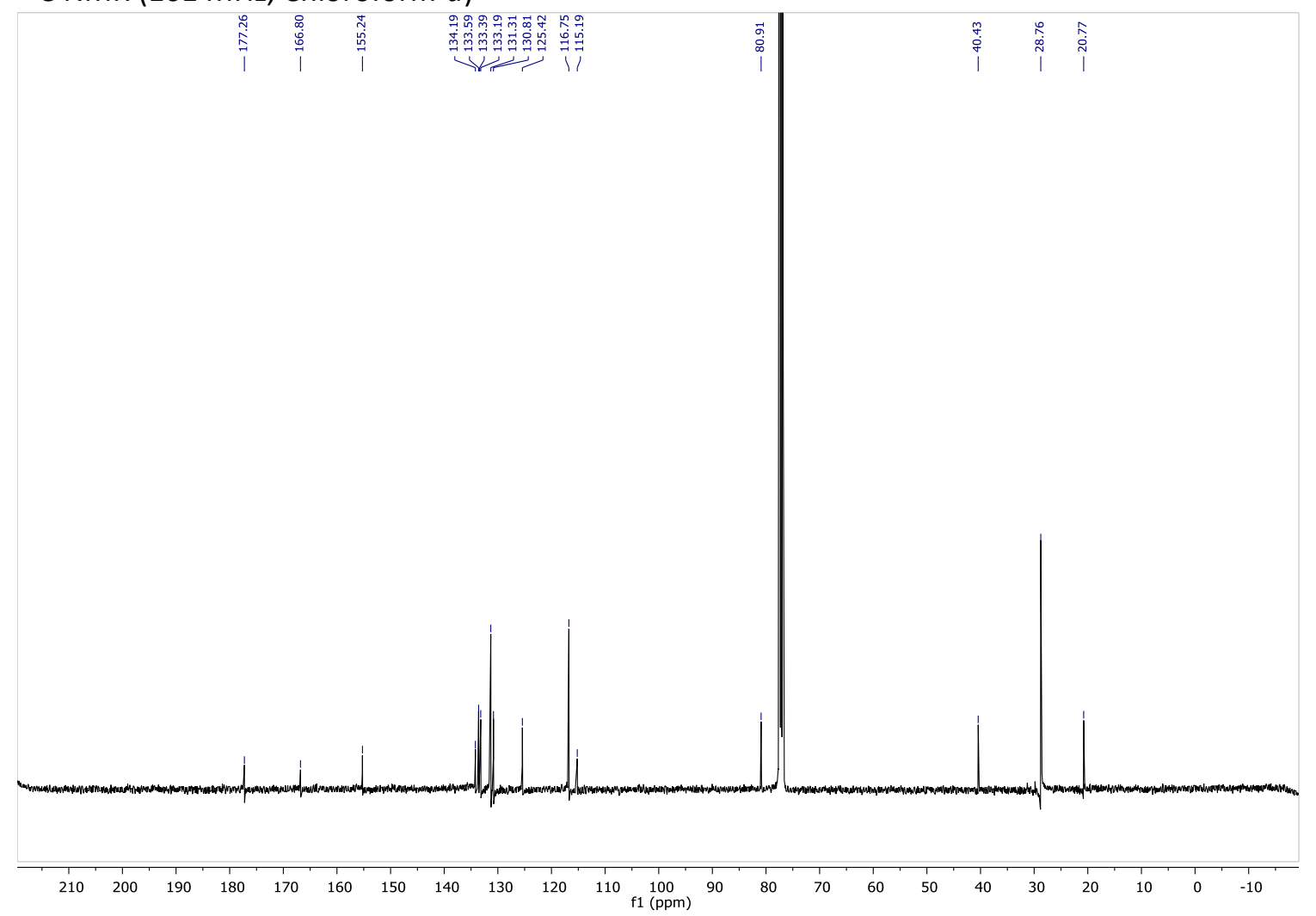


(Z)-1-(2-phenyl-2-(p-tolyloxy)vinyl)-1,2-benziodoxol-3-(1H)-one (1q)

${ }^{1} \mathrm{H}$ NMR (400 MHz, Chloroform-d)

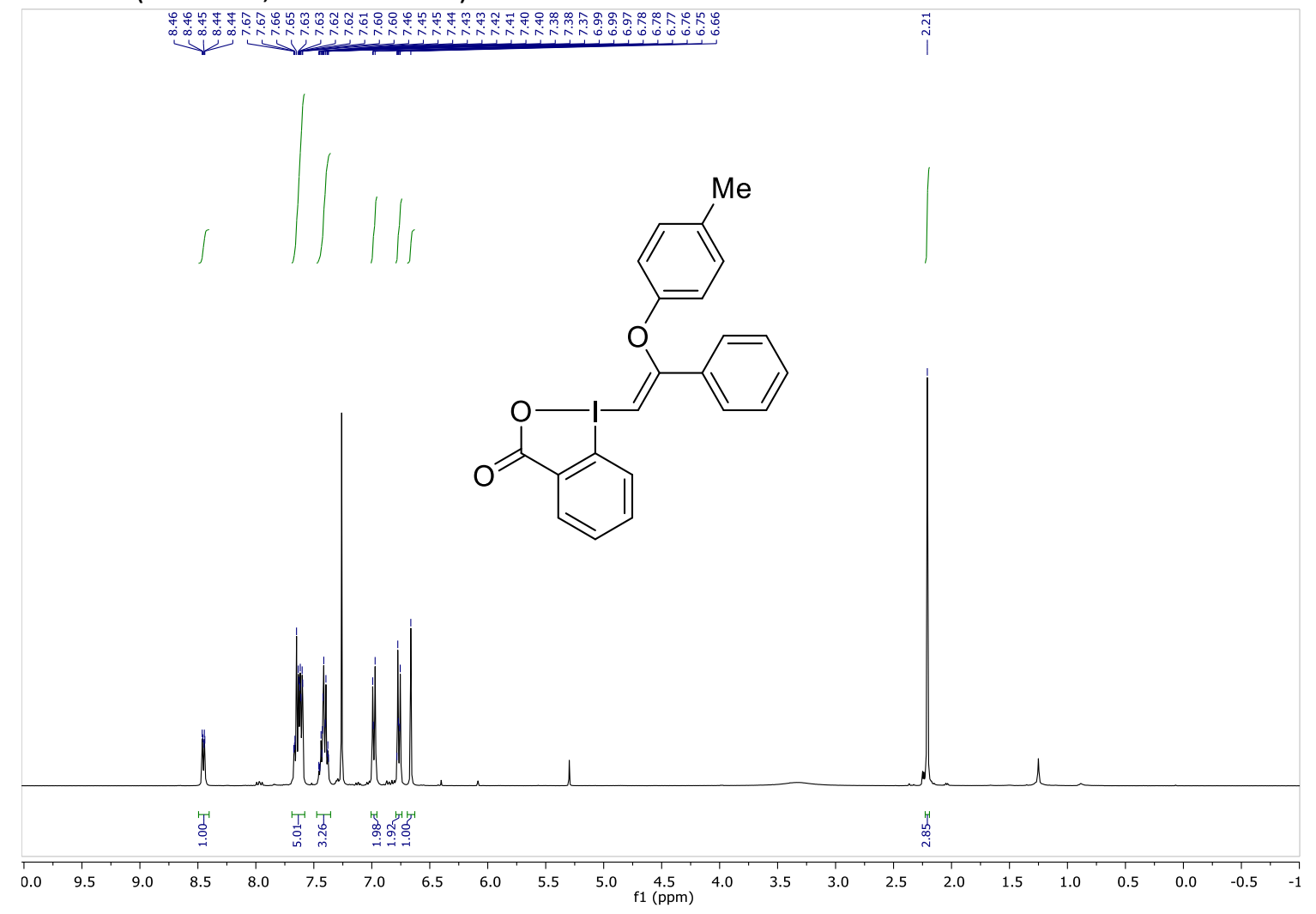

${ }^{13} \mathrm{C}$ NMR (101 MHz, Chloroform- $d$ )

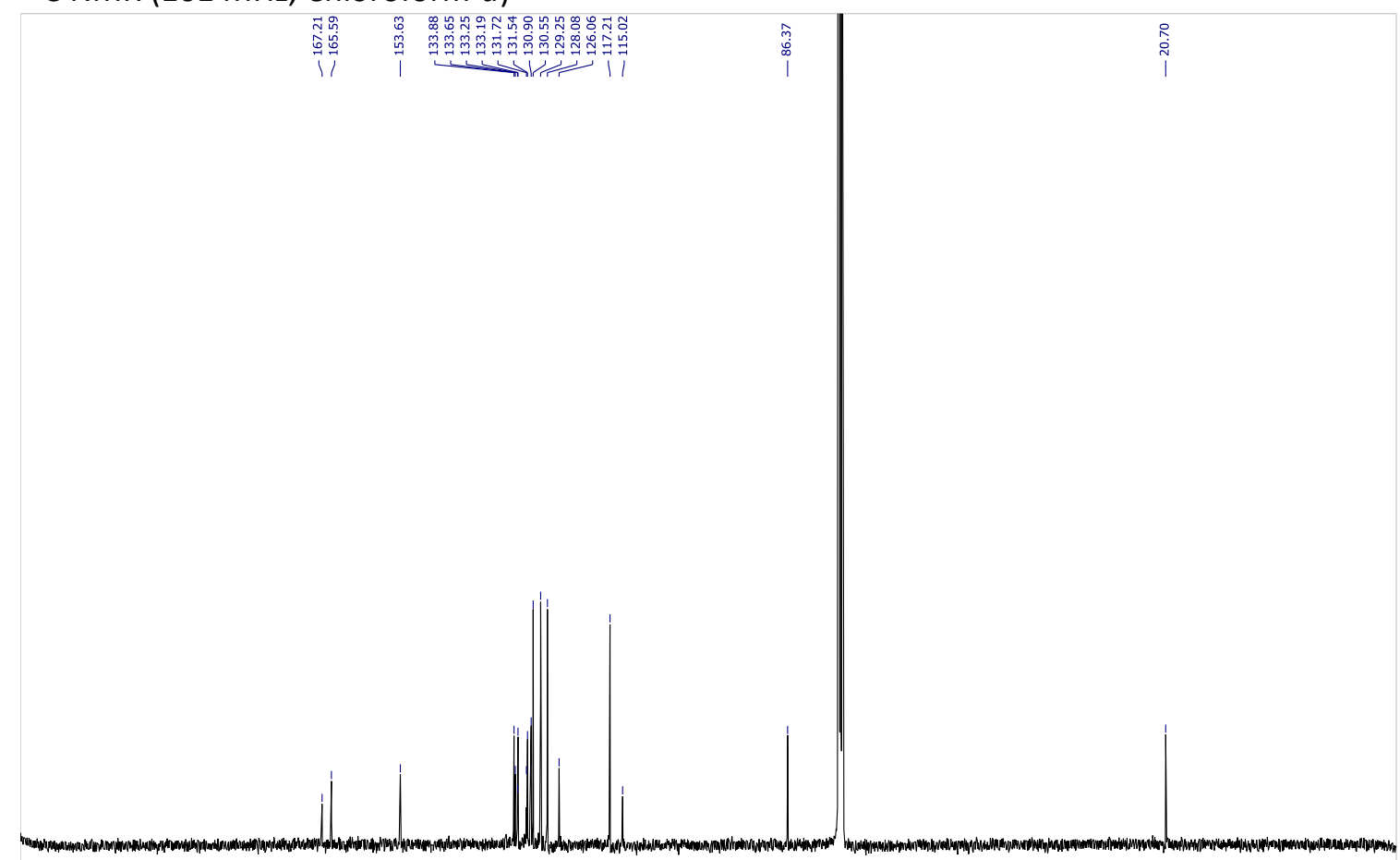

$\begin{array}{lllllllllllllllllllllllllllllllllll}210 & 200 & 190 & 180 & 170 & 160 & 150 & 140 & 130 & 120 & 110 & 100 & 90 & 80 & 70 & 60 & 50 & 40 & 30 & 20 & 10 & 0 & -10\end{array}$ 
(Z)-1-(2-(2,6-dichlorophenoxy)oct-1-en-1-yl)-1,3-benziodoxol-3(1H)-one (1r)

${ }^{1} \mathrm{H}$ NMR (400 MHz, Chloroform-d)

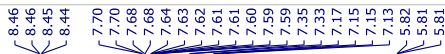

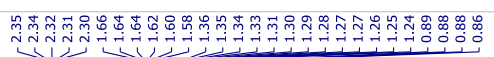

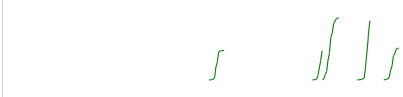

$\int$

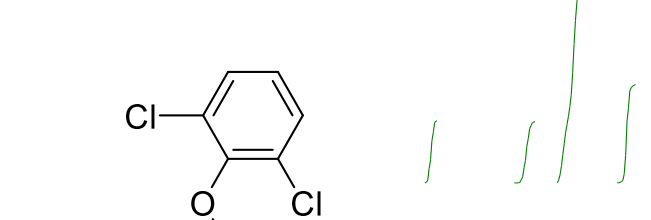<smiles>CCCCCC/C(C)=C/I1COC(=O)c2ccccc21</smiles>

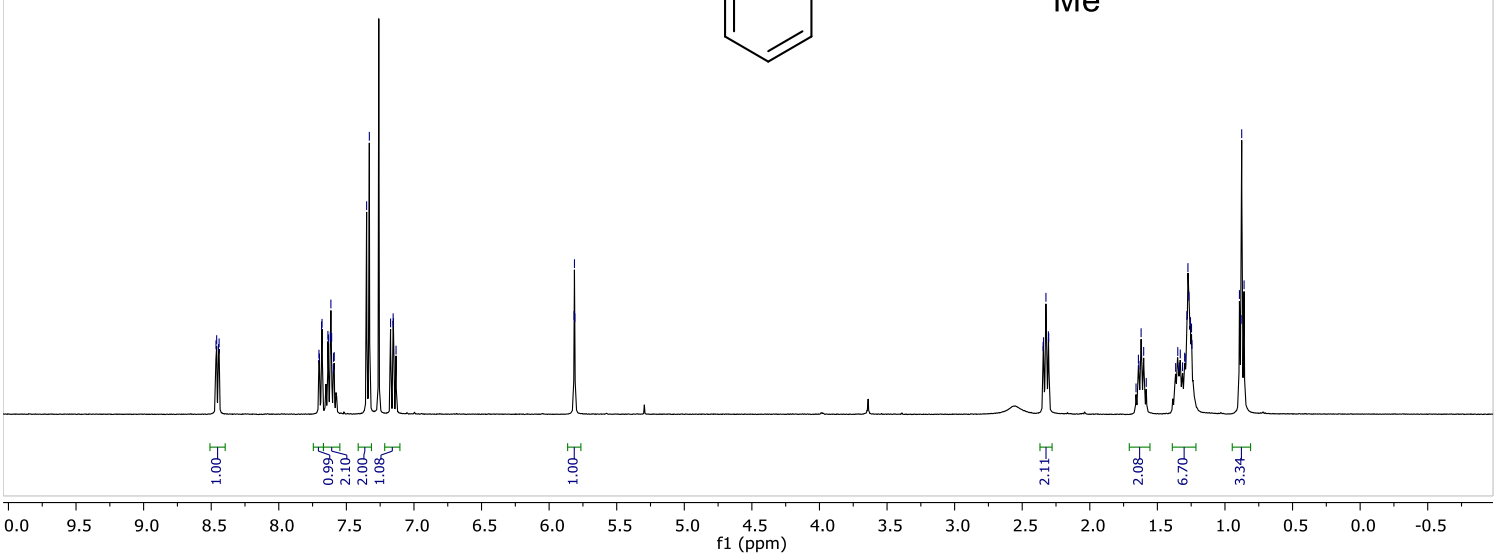

${ }^{13} \mathrm{C}$ NMR (101 MHz, Chloroform-d)

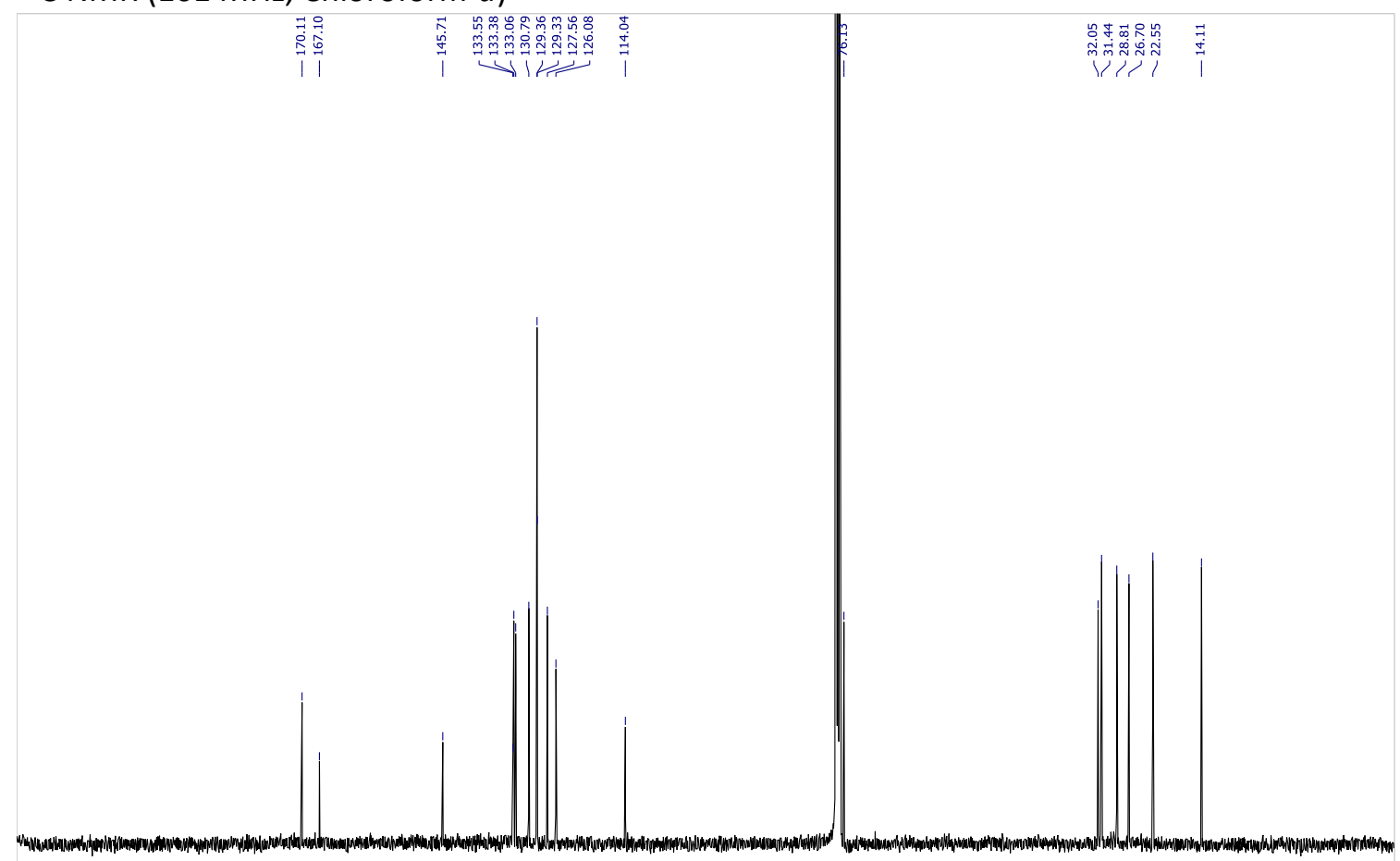

$\begin{array}{llllllllllllllllllllllllllllllll}210 & 200 & 190 & 180 & 170 & 160 & 150 & 140 & 130 & 120 & 110 & 100 & 90 & 80 & 70 & 60 & 50 & 40 & 30 & 20 & 10 & 0 & -10\end{array}$ 
(Z)-5-Methyl-1-(2-(4-methoxyphenoxy)prop-1-en-1-yl)-1,2-benziodoxol-3-(1H)-one (1s)

${ }^{1} \mathrm{H}$ NMR (400 MHz, Methylene chloride- $d_{2}$ )

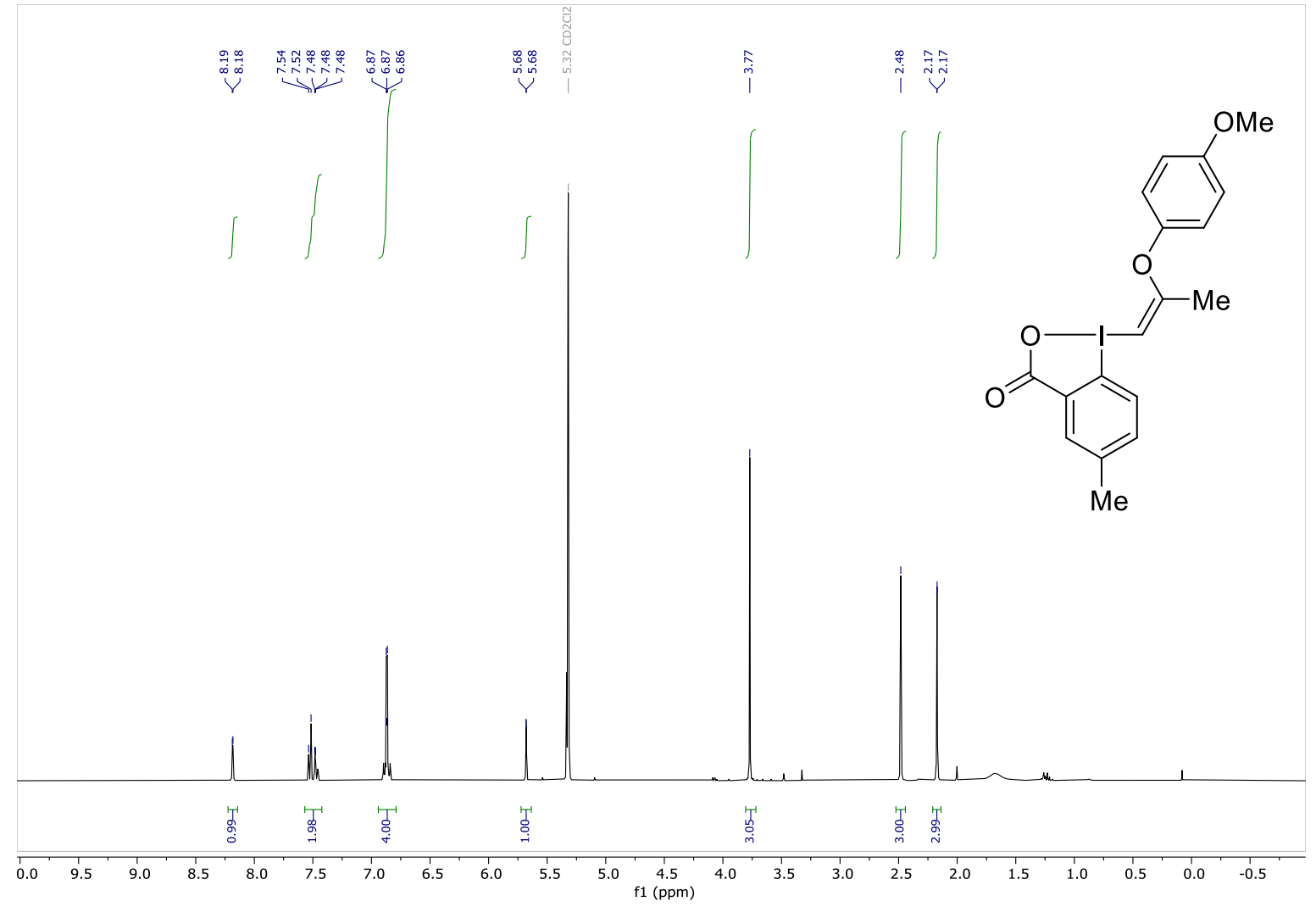

${ }^{13} \mathrm{C}$ NMR (101 MHz, Methylene chloride- $d_{2}$ )

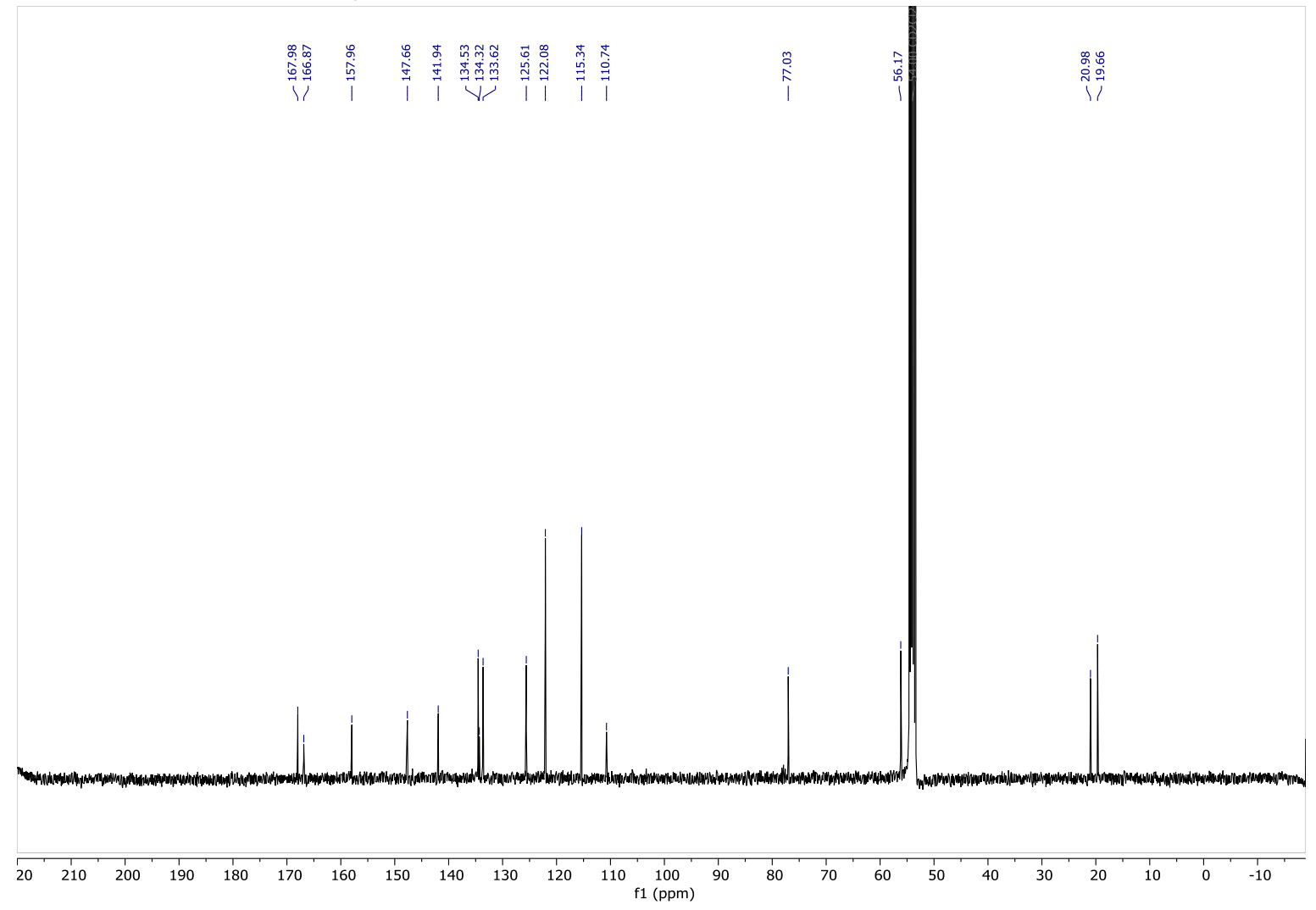


(Z)-5-Methoxy-1-(2-(4-methoxyphenoxy)prop-1-en-1-yl)-1,2-benziodoxol-3-(1H)-one (1t)

${ }^{1} \mathrm{H}$ NMR (400 MHz, Methylene chloride- $d_{2}$ )

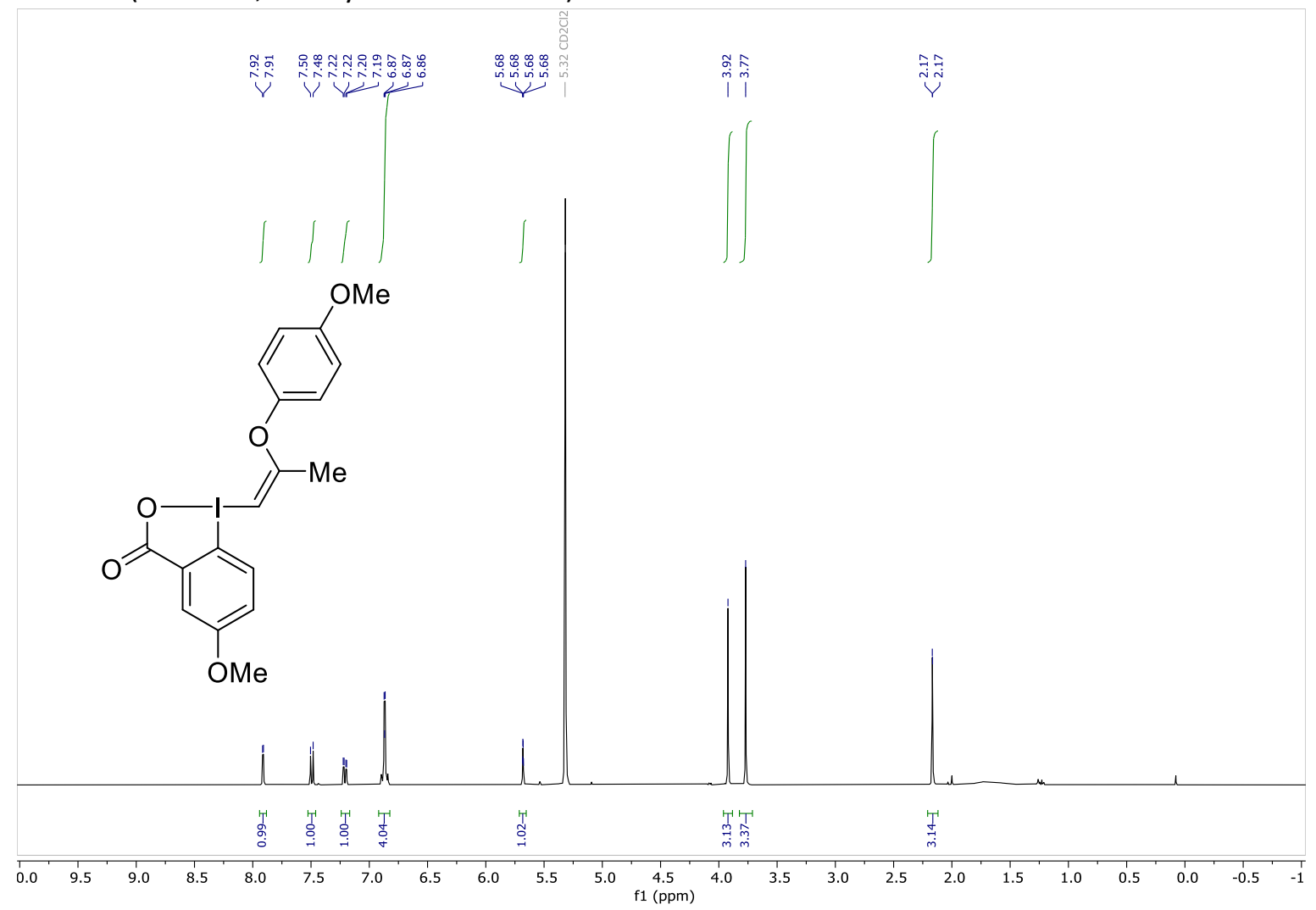

${ }^{13} \mathrm{C}$ NMR (101 MHz, Methylene chloride- $d_{2}$ )

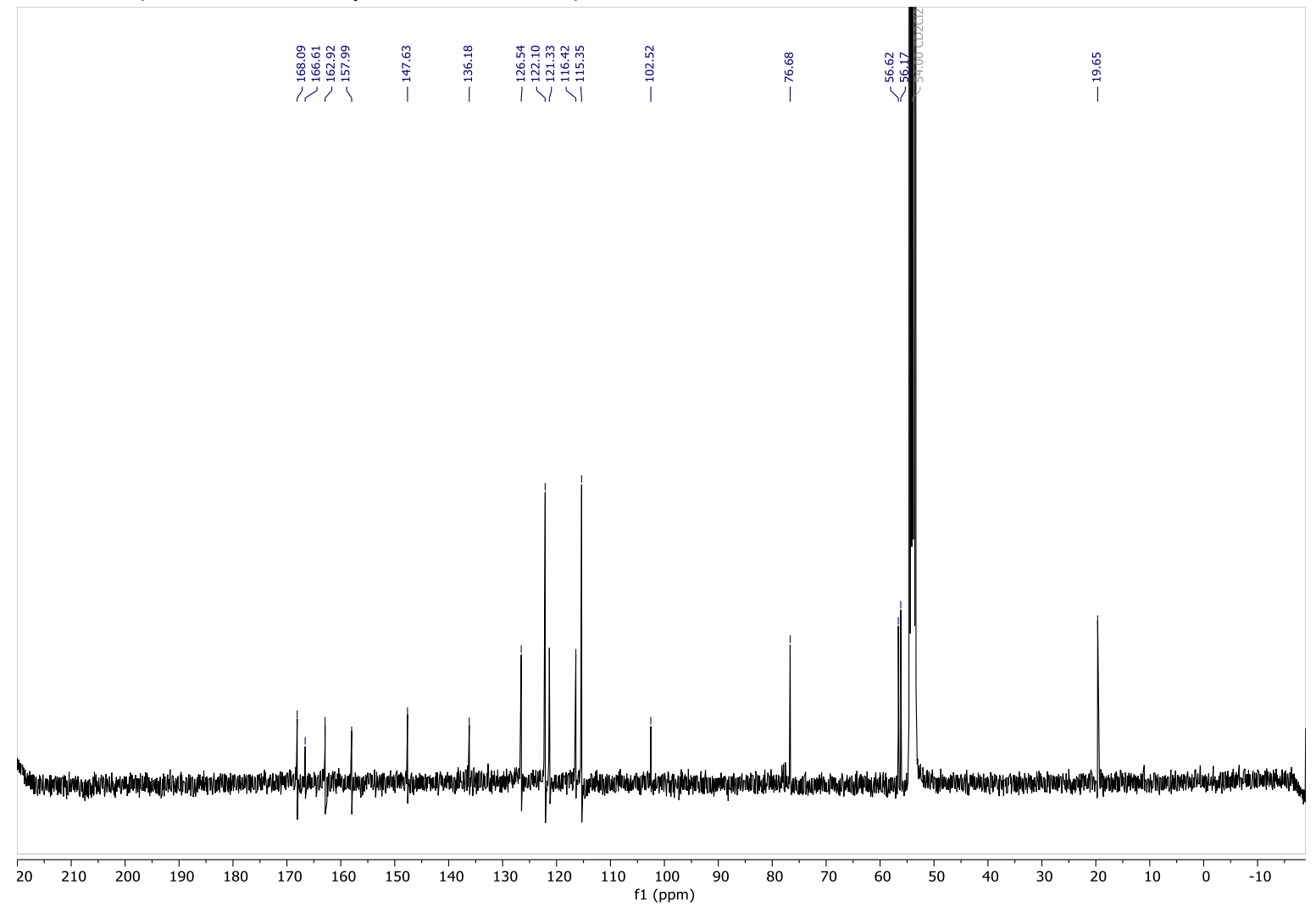




\subsection{Allylic alcohols}

4,4'-(prop-2-ene-1,2-diylbis(oxy))bis(methylbenzene) (3a)

${ }^{1} \mathrm{H}$ NMR (400 MHz, Chloroform-d)

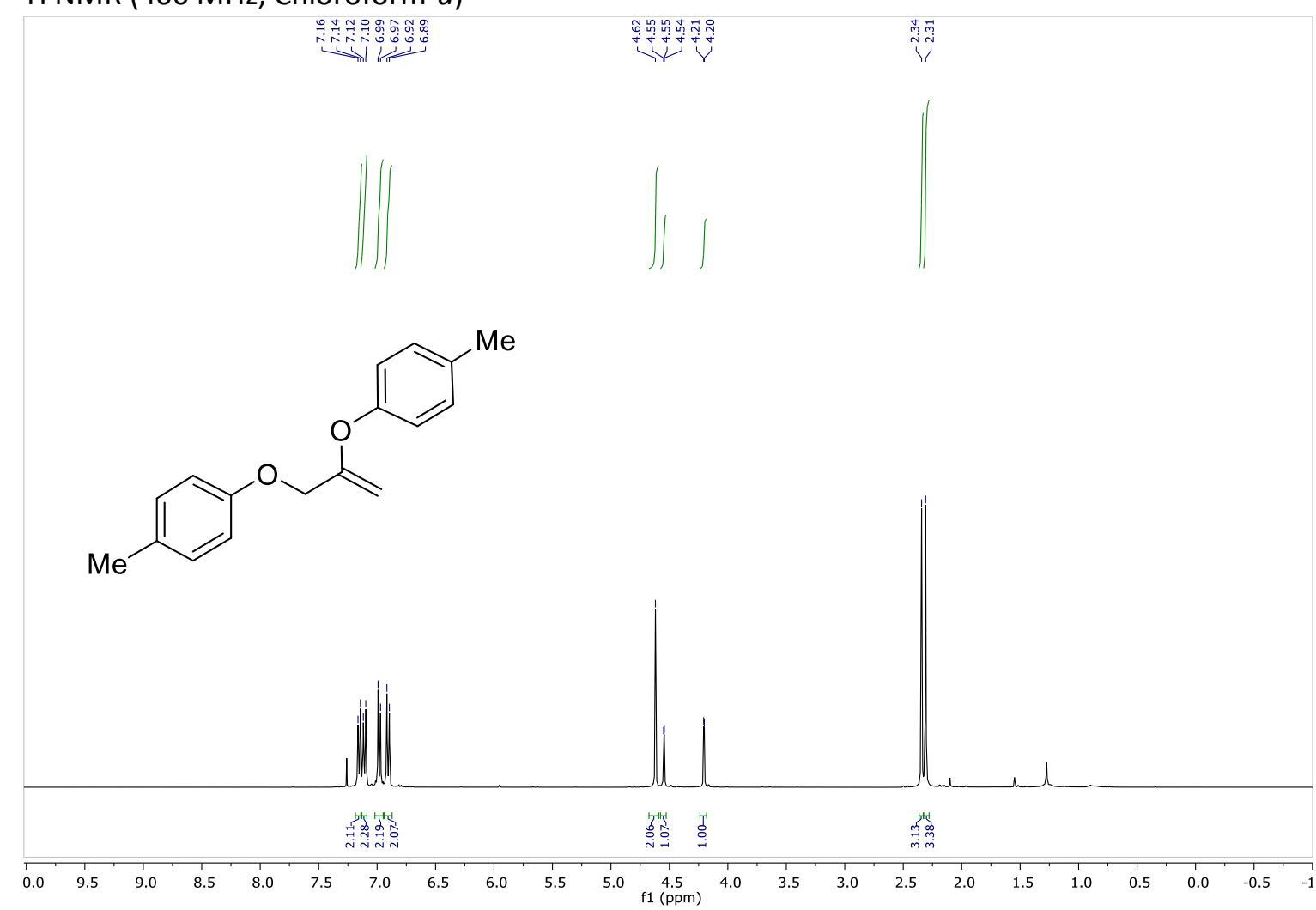

${ }^{13} \mathrm{C}$ NMR (101 MHz, Chloroform-d)

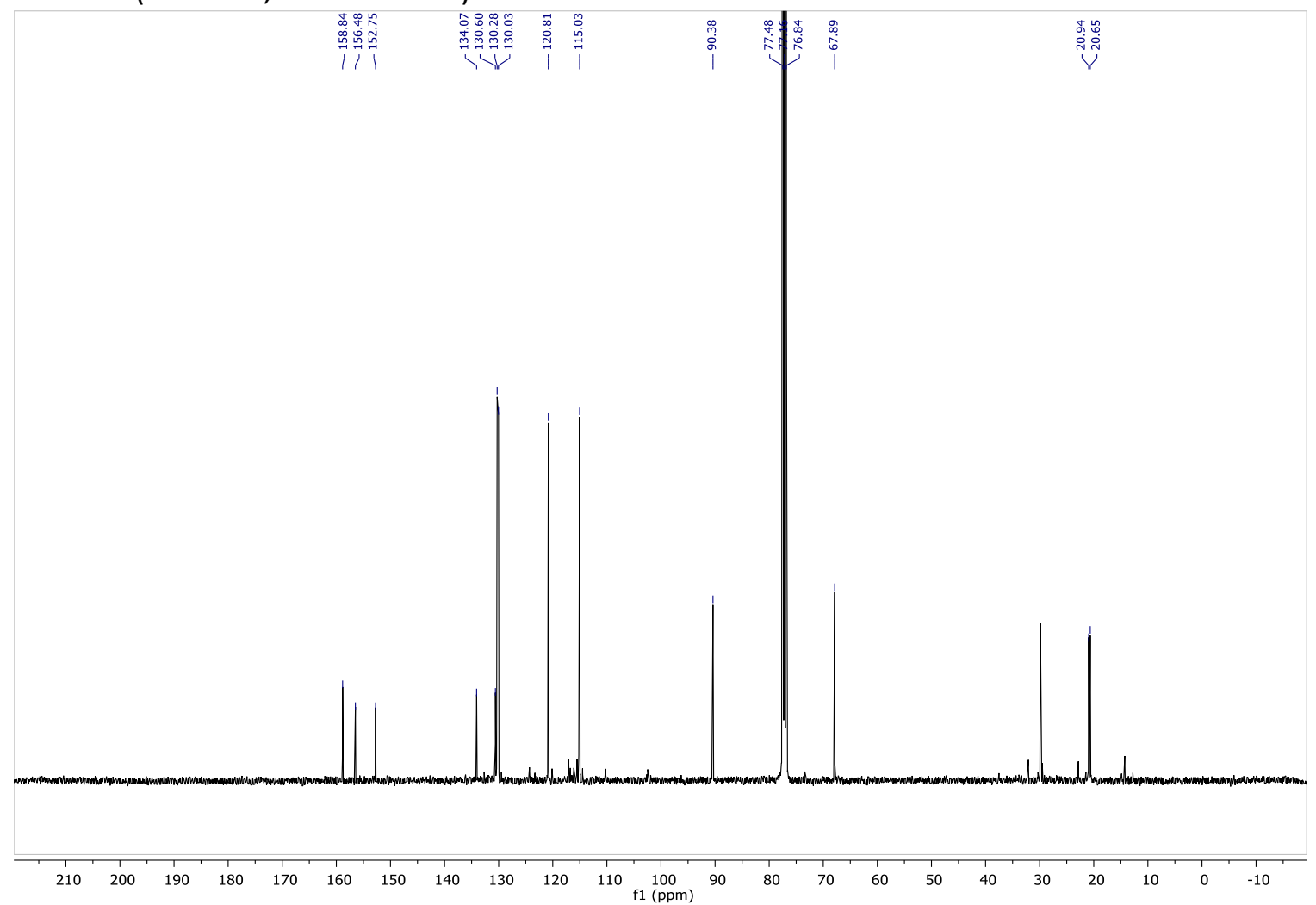


1-bromo-4-((2-(p-tolyloxy)allyl)oxy)benzene (3b)

${ }^{1} \mathrm{H}$ NMR (400 MHz, Chloroform-d)

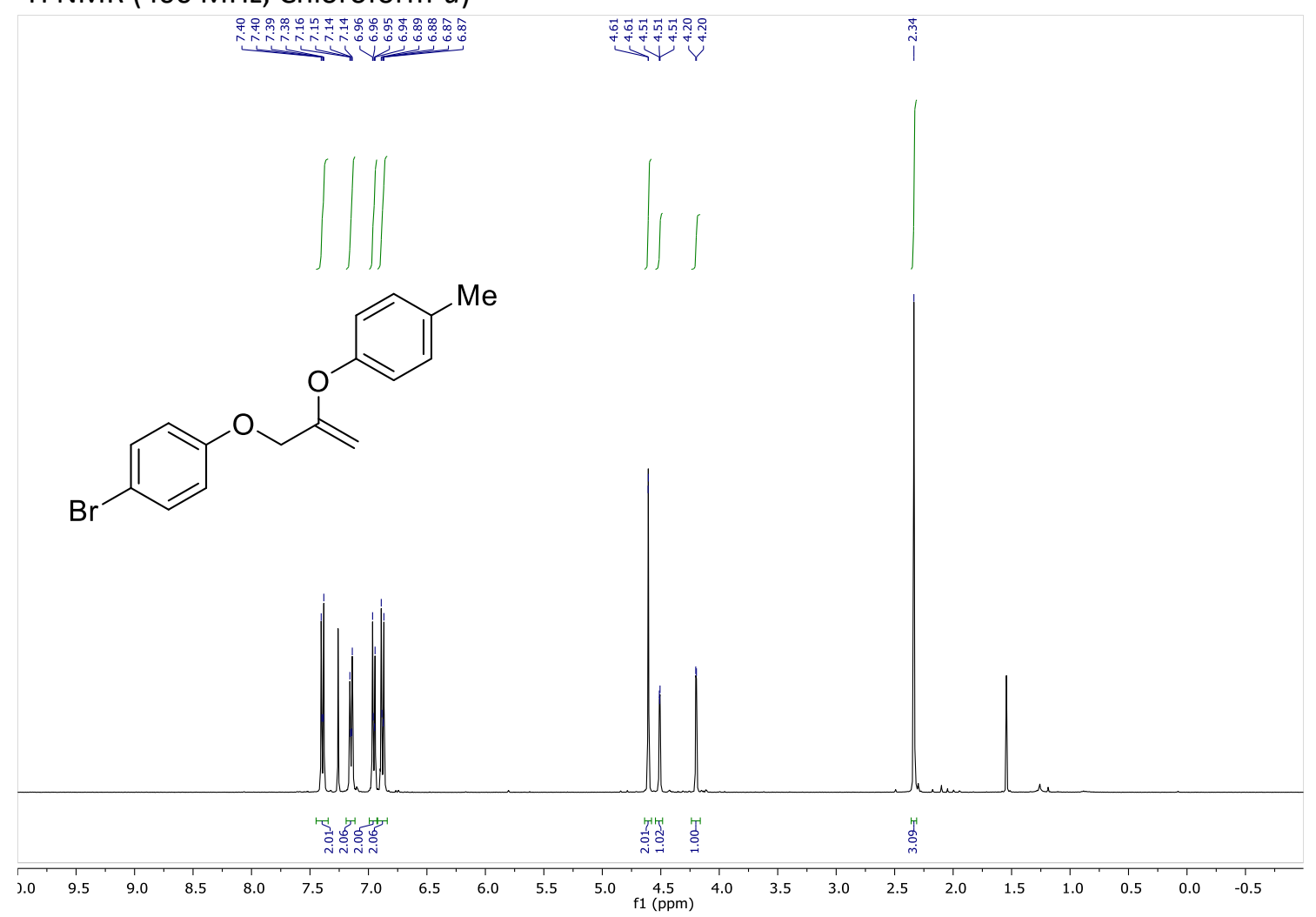

${ }^{13} \mathrm{C}$ NMR (101 MHz, Chloroform-d)

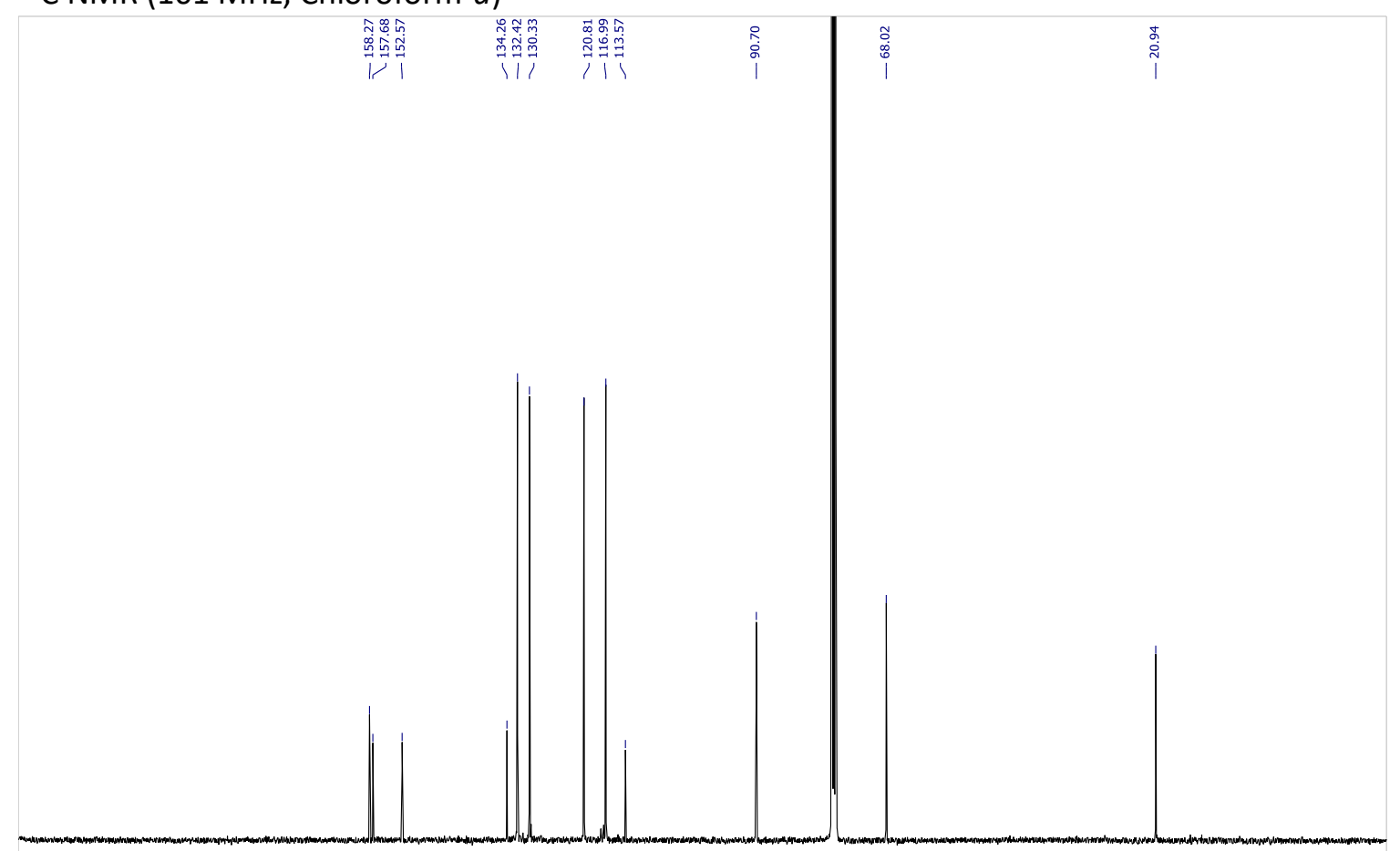

$\begin{array}{llllllllllllllllllllllllllllllll}210 & 200 & 190 & 180 & 170 & 160 & 150 & 140 & 130 & 120 & 110 & 100 & 90 & 80 & 70 & 60 & 50 & 40 & 30 & 20 & 10 & 0 & -10\end{array}$ 
1-iodo-4-((2-(p-tolyloxy)allyl)oxy)benzene (3c)

${ }^{1} \mathrm{H}$ NMR (400 MHz, Chloroform-d)

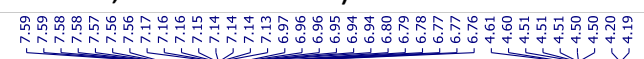

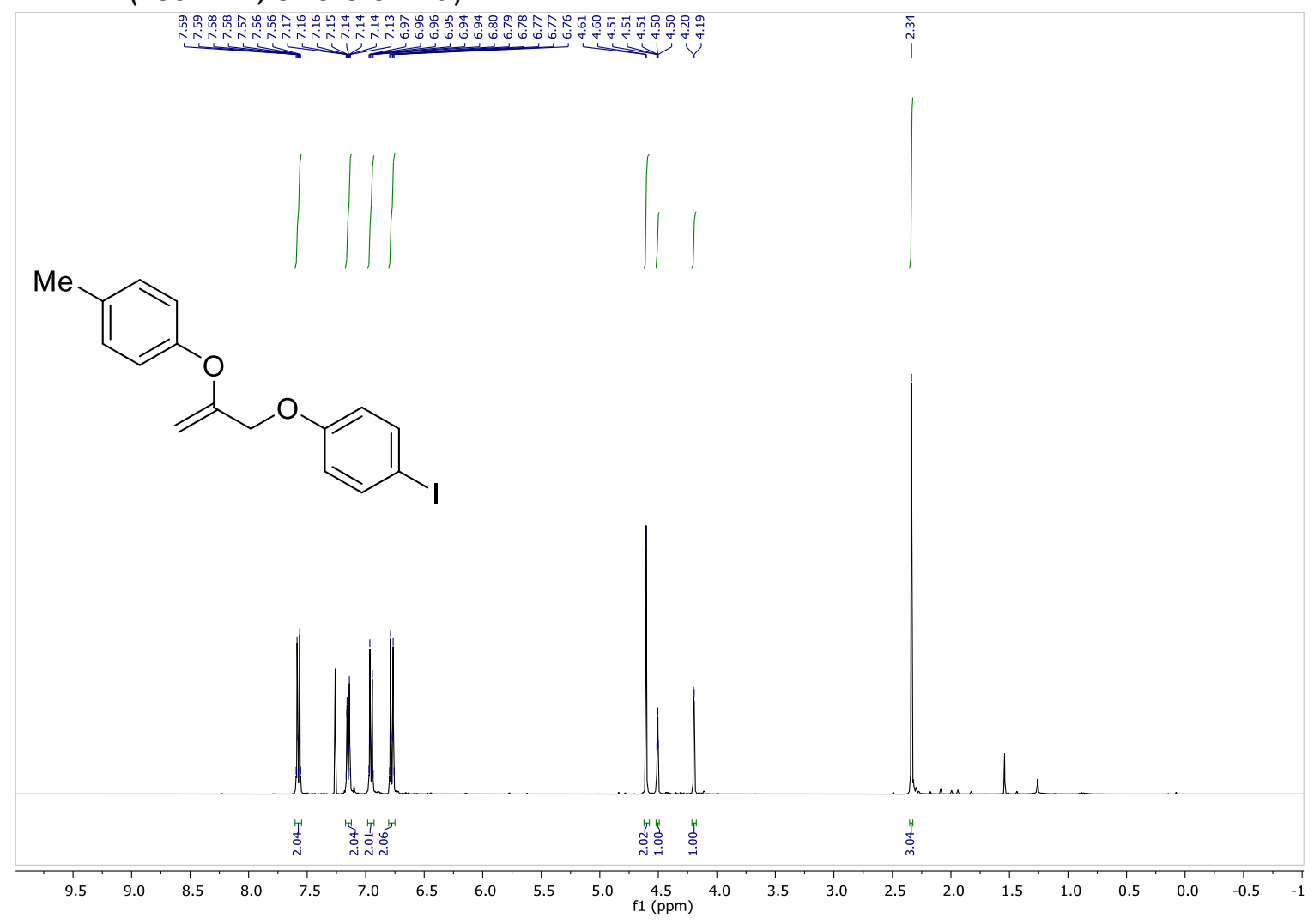

${ }^{13} \mathrm{C}$ NMR (101 MHz, Chloroform-d)

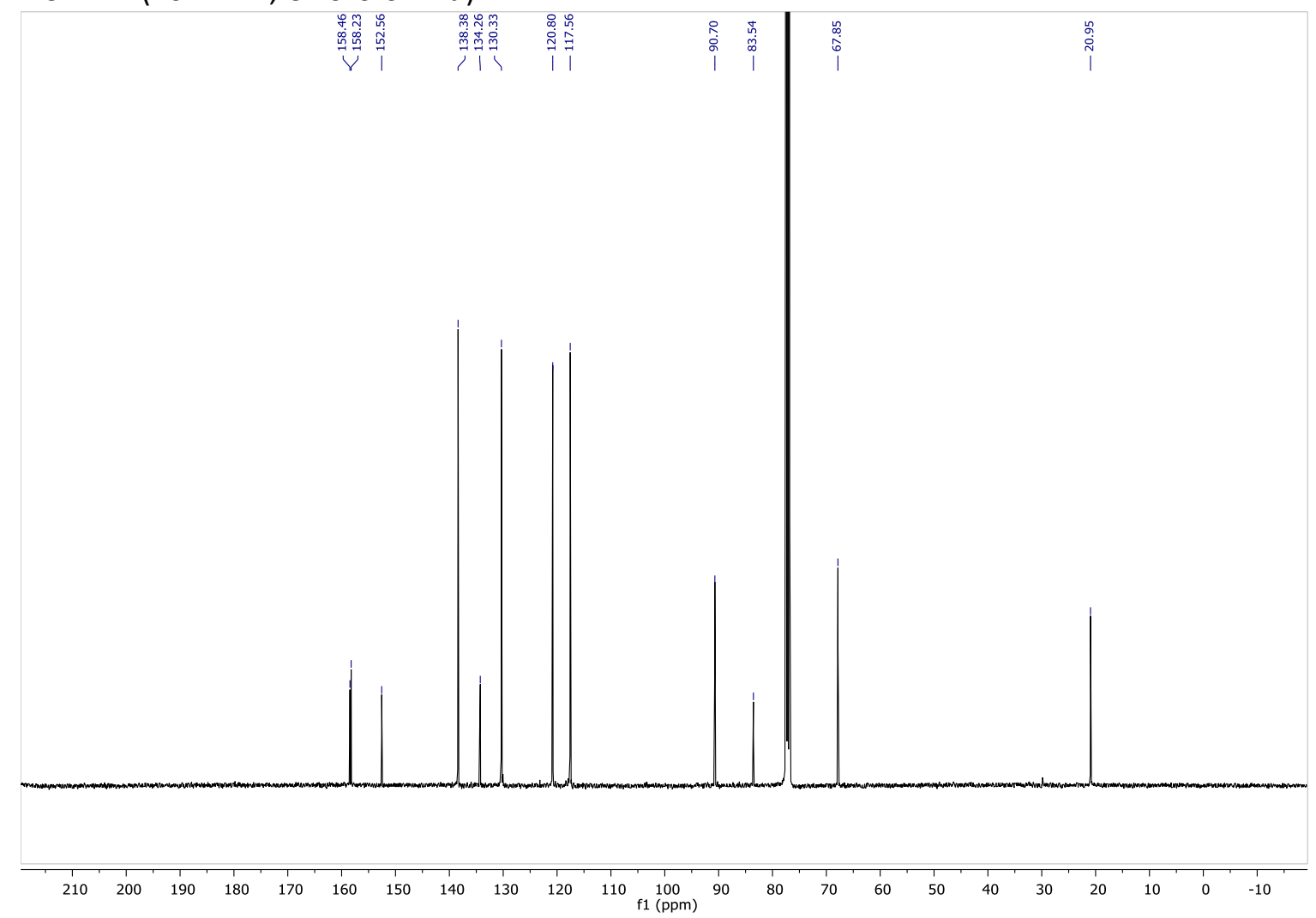

82 
1-Methoxy-4-((2-(p-tolyloxy)allyl)oxy)benzene (3d)

${ }^{1} \mathrm{H}$ NMR (400 MHz, Chloroform-d)

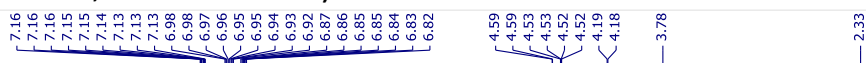

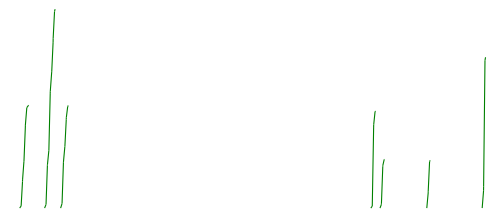

(c)

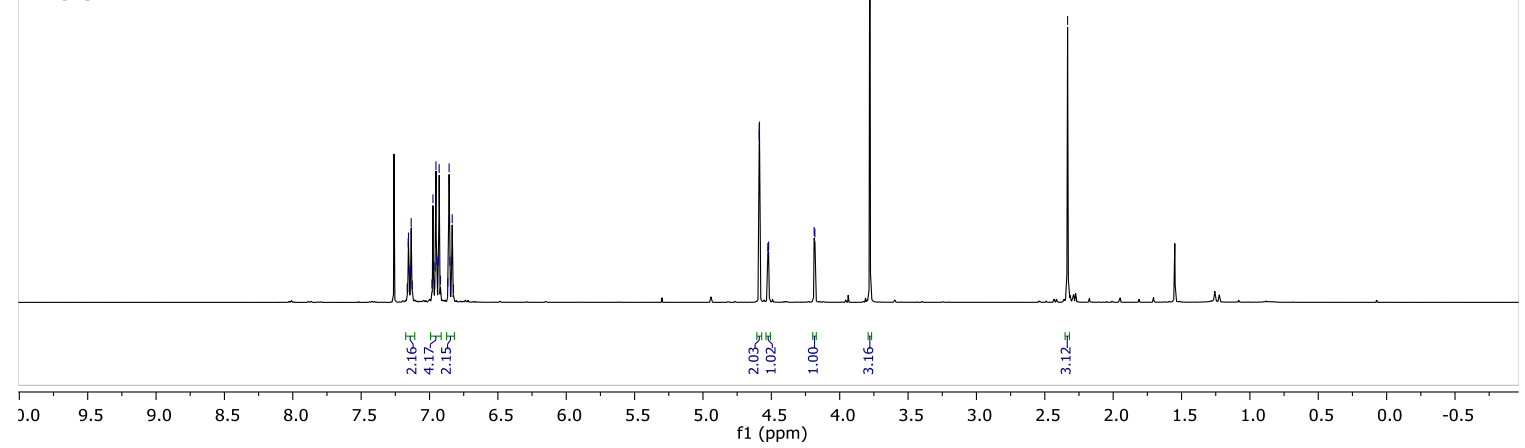

${ }^{13} \mathrm{C}$ NMR (101 MHz, Chloroform-d)

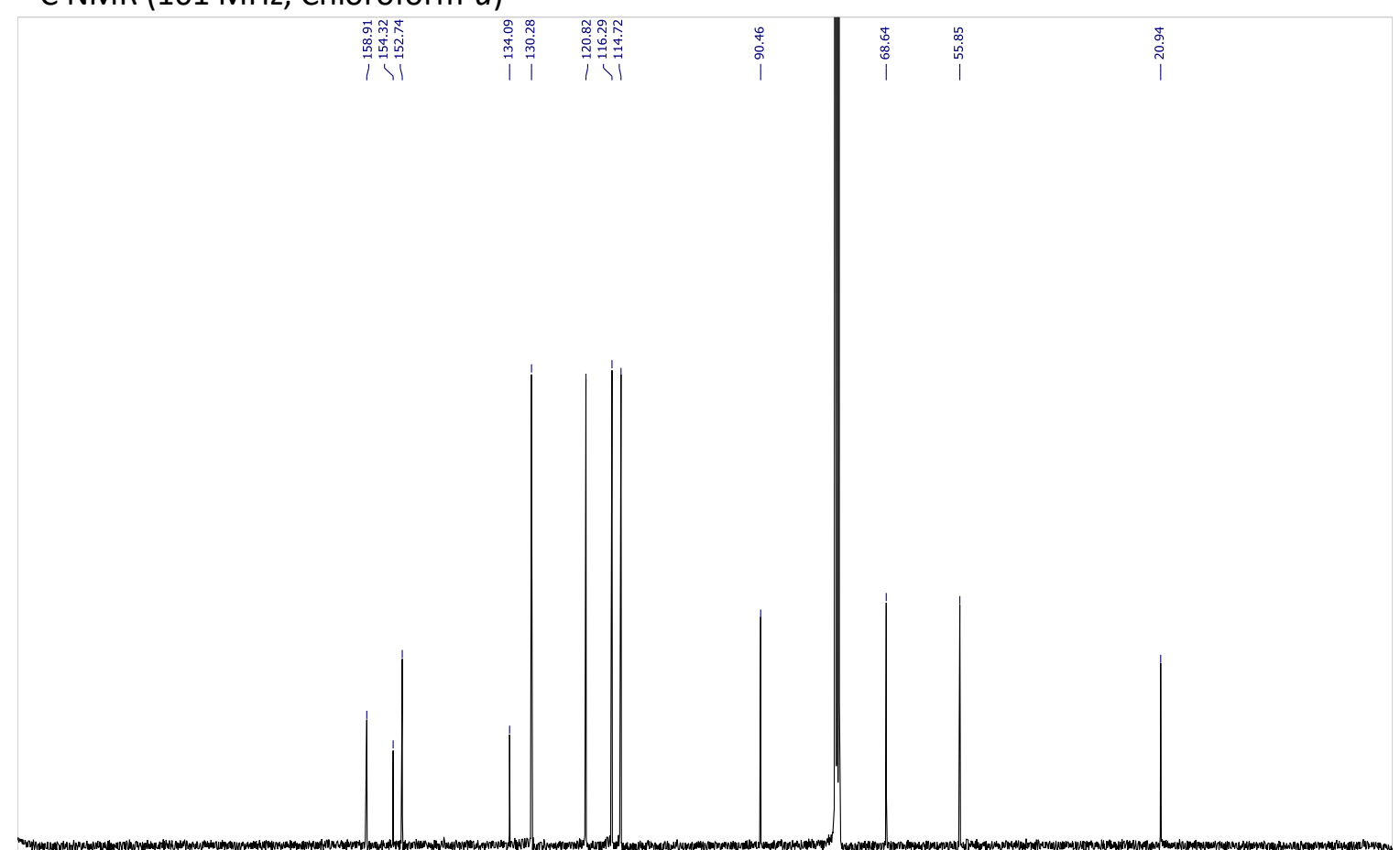

$\begin{array}{llllllllllllllllllllllllllllll}210 & 200 & 190 & 180 & 170 & 160 & 150 & 140 & 130 & 120 & 110 & 100 & 90 & 80 & 70 & 60 & 50 & 40 & 30 & 20 & 10 & 0 & -10\end{array}$ 
4,4,5,5-tetramethyl-2-(4-((2-(p-tolyloxy)allyl)oxy)phenyl)-1,3,2-dioxaborolane (3e)

${ }^{1} \mathrm{H}$ NMR (400 MHz, Methylene Chloride- $d_{2}$ )

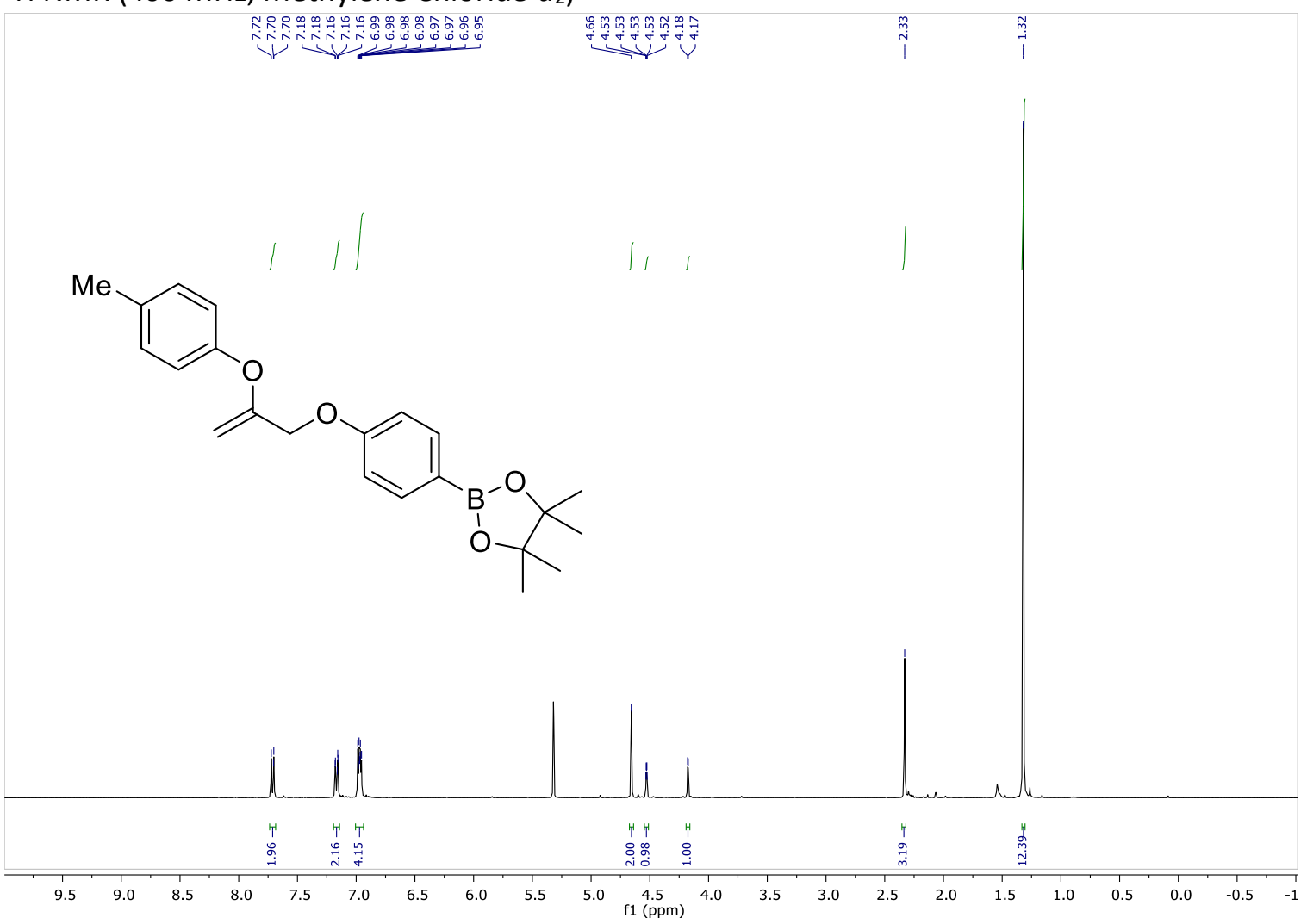

${ }^{13} \mathrm{C}$ NMR (101 MHz, Chloroform-d)

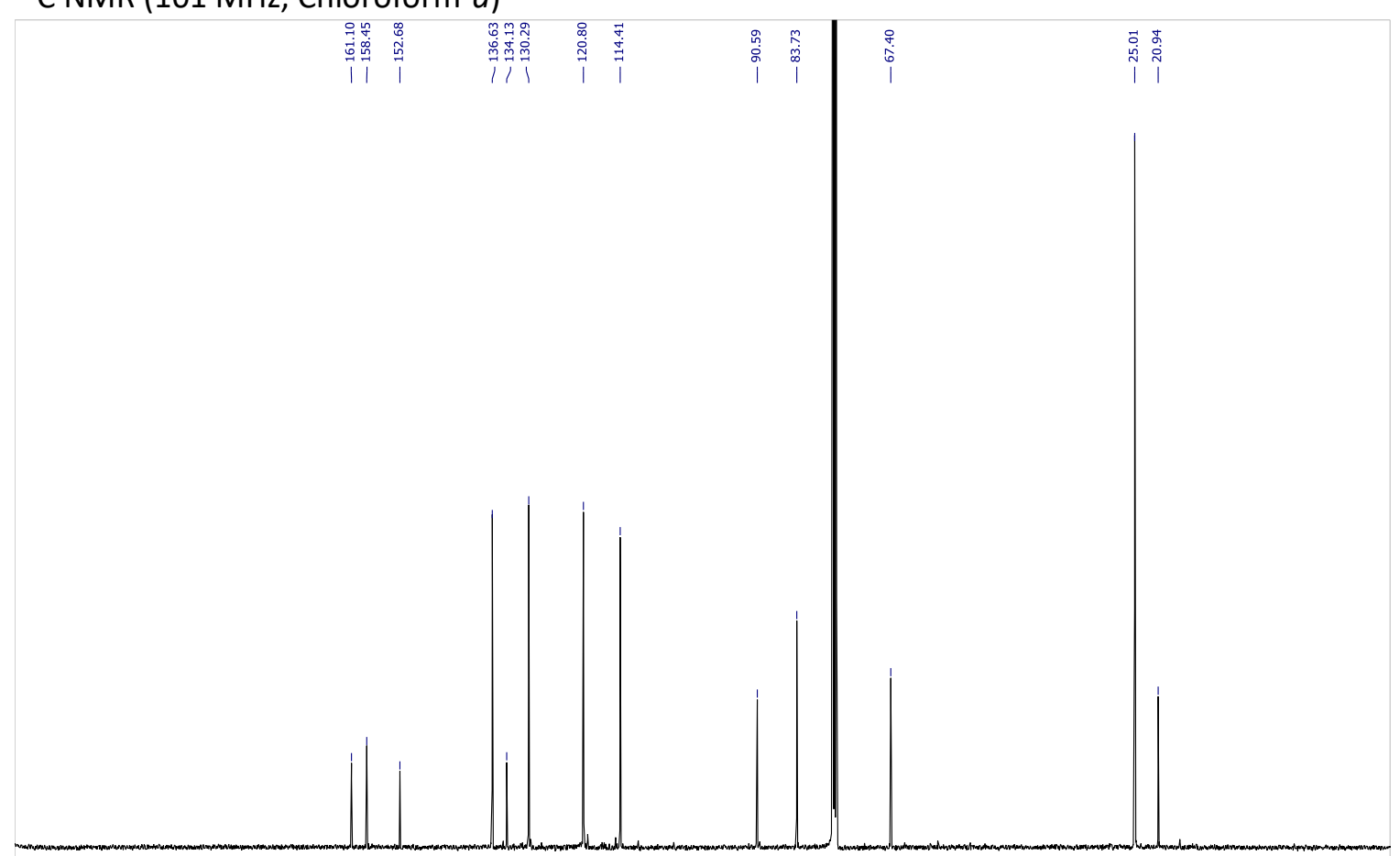

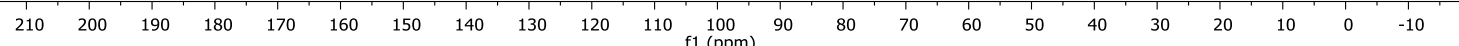


1-Methoxy-3-((2-(p-tolyloxy)allyl)oxy)benzene (3f)

${ }^{1} \mathrm{H}$ NMR $\left(400 \mathrm{MHz}\right.$, Acetonitrile- $\left.d_{3}\right)$

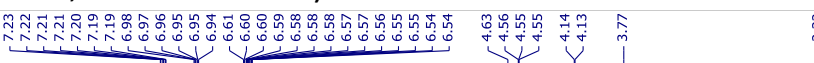

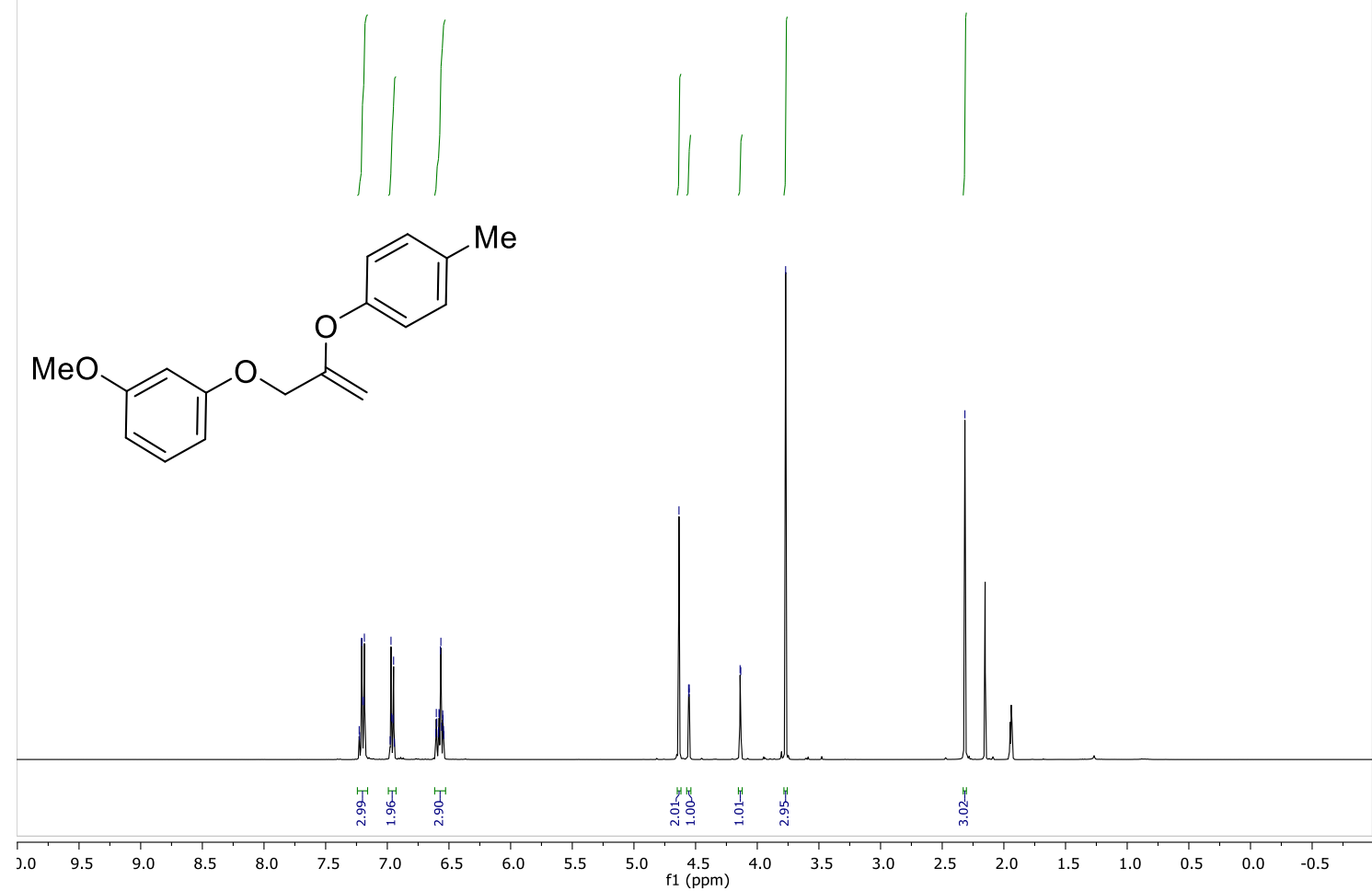

${ }^{13} \mathrm{C}$ NMR (101 MHz, Acetonitrile- $\left.d_{3}\right)$

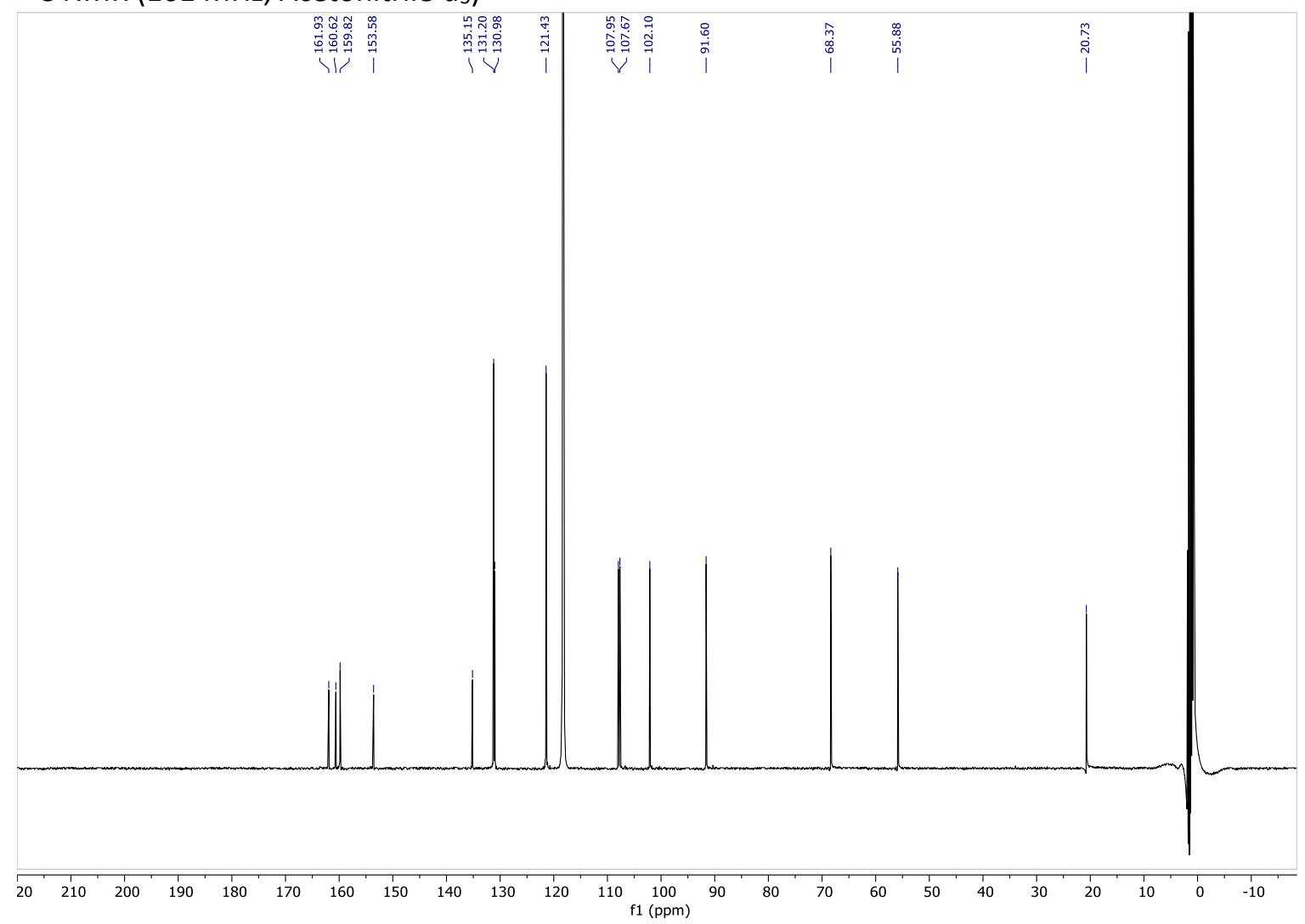


3-((2-(p-tolyloxy)allyl)oxy)aniline (3g)

${ }^{1} \mathrm{H}$ NMR (400 MHz, Chloroform-d)
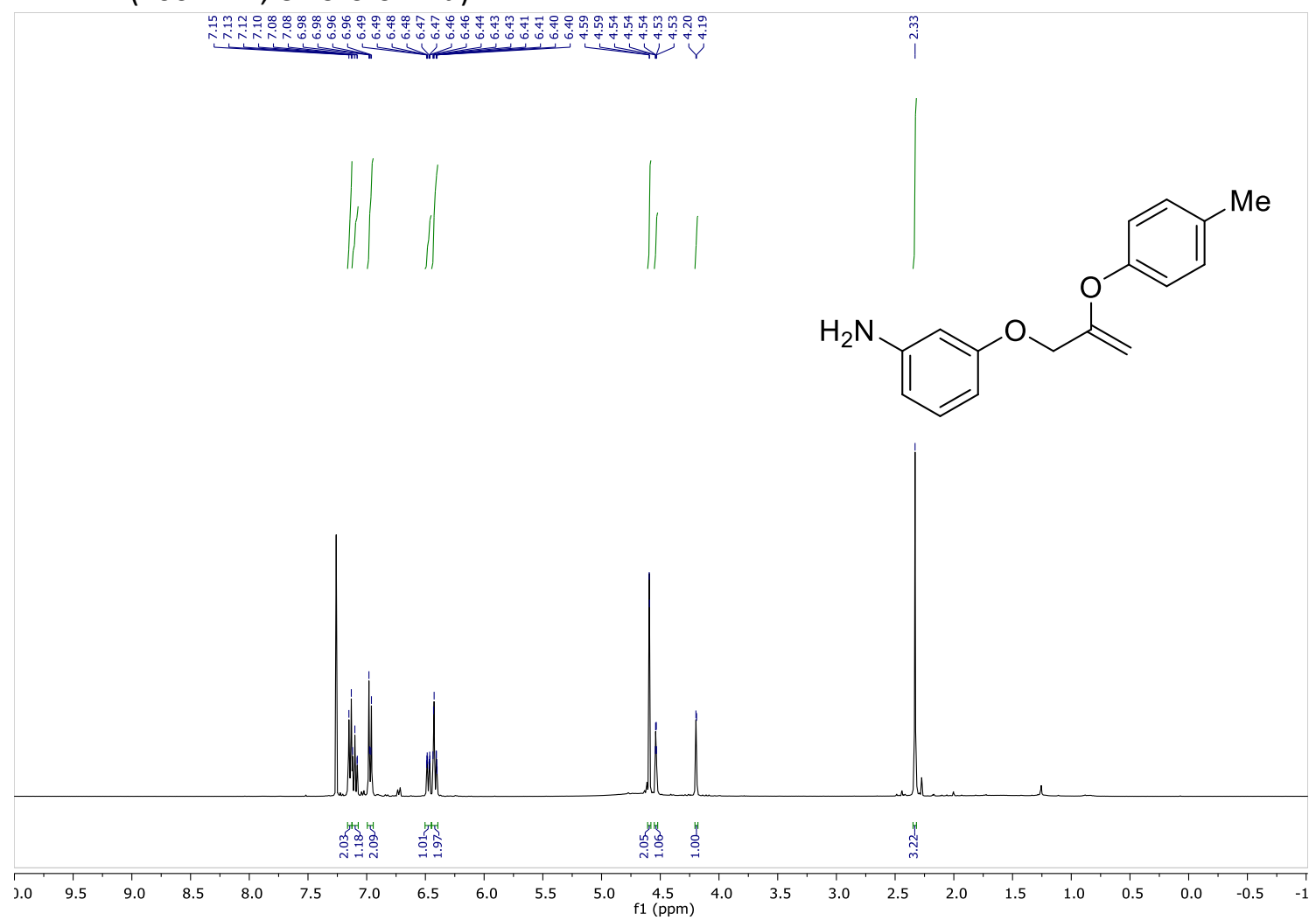

${ }^{13} \mathrm{C}$ NMR (101 MHz, Chloroform-d)

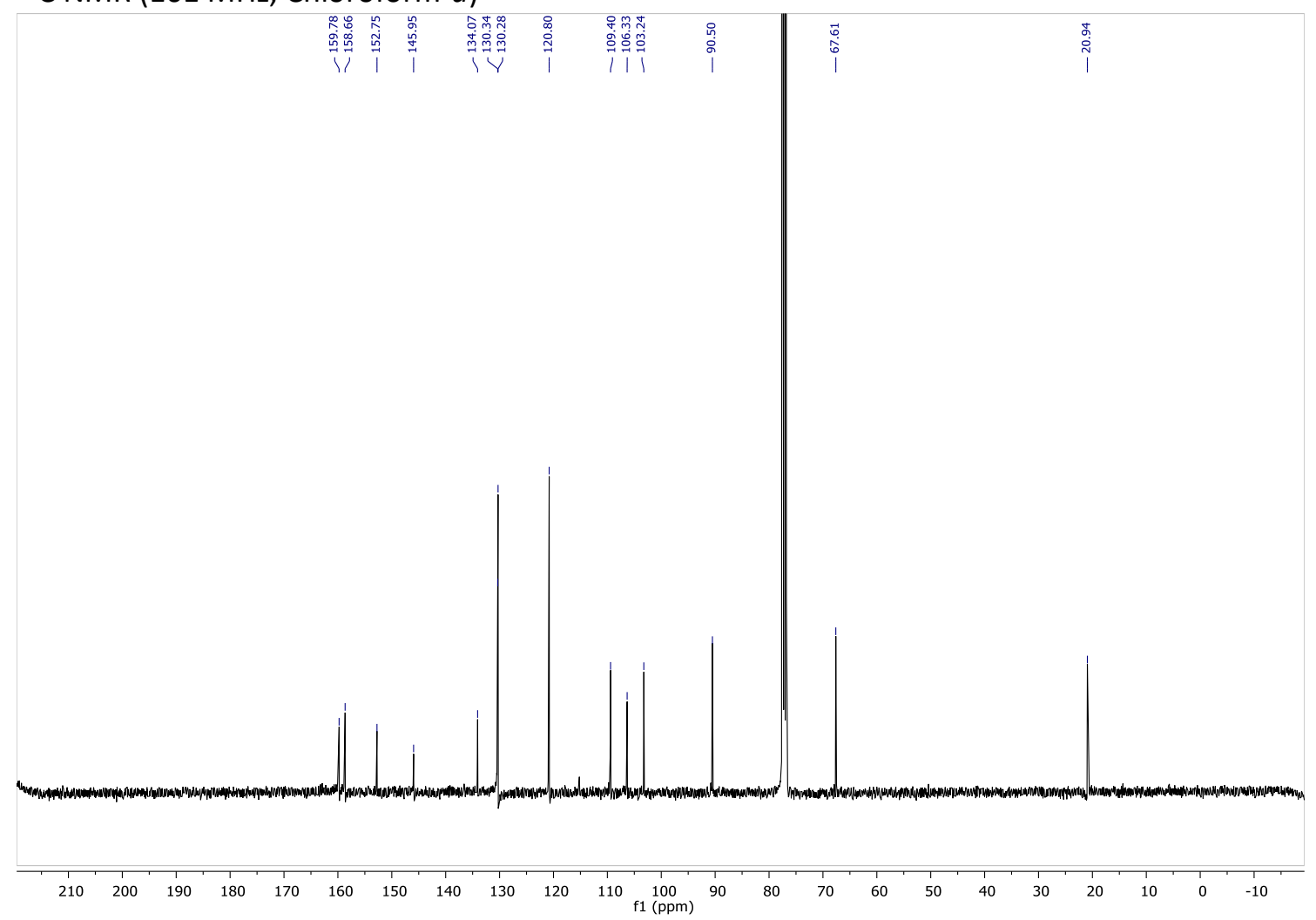


Methyl 2-((2-(p-tolyloxy)allyl)oxy)benzoate (3h)

${ }^{1} \mathrm{H}$ NMR (400 MHz, Chloroform-d)

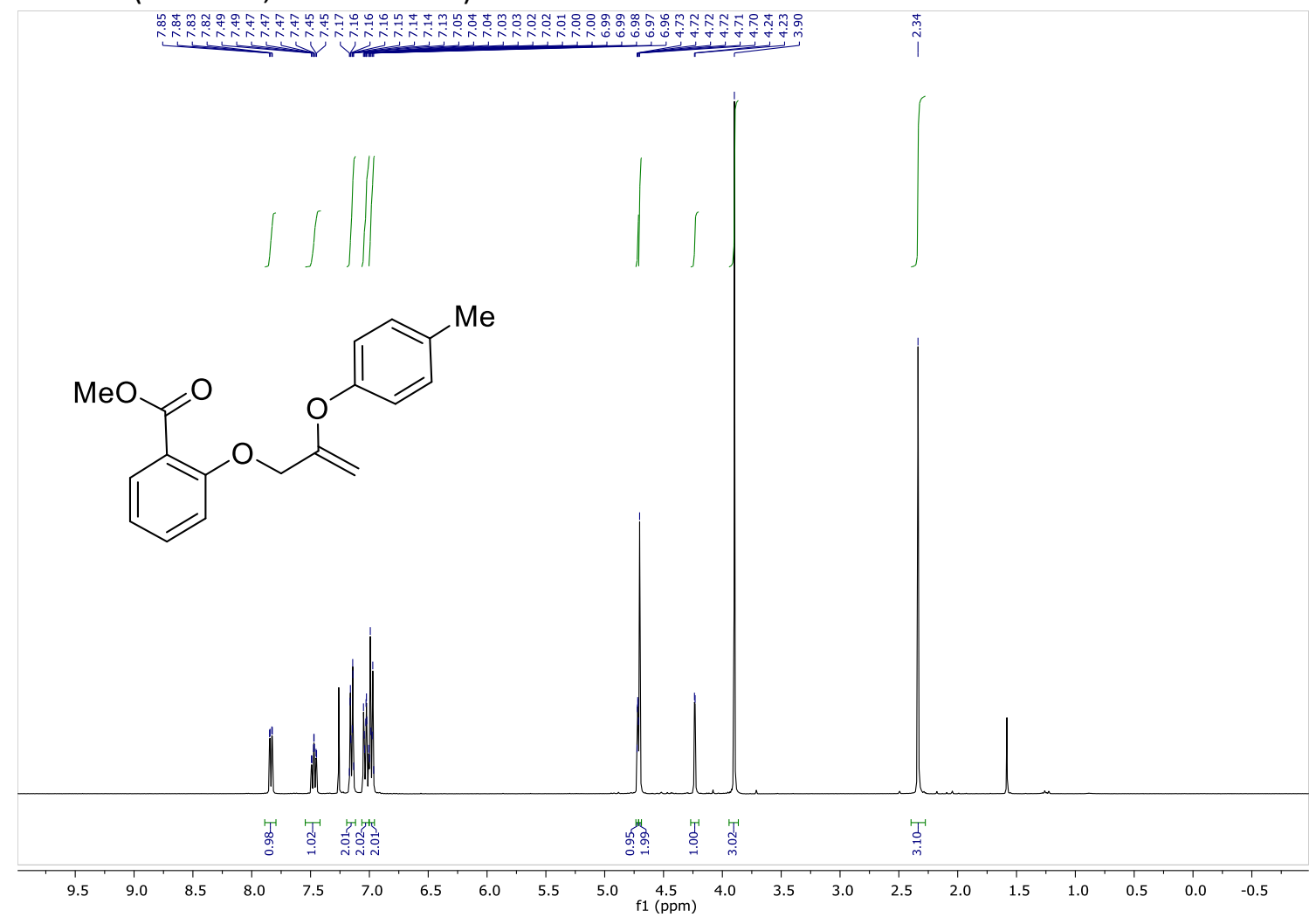

${ }^{13} \mathrm{C}$ NMR (101 MHz, Chloroform-d)

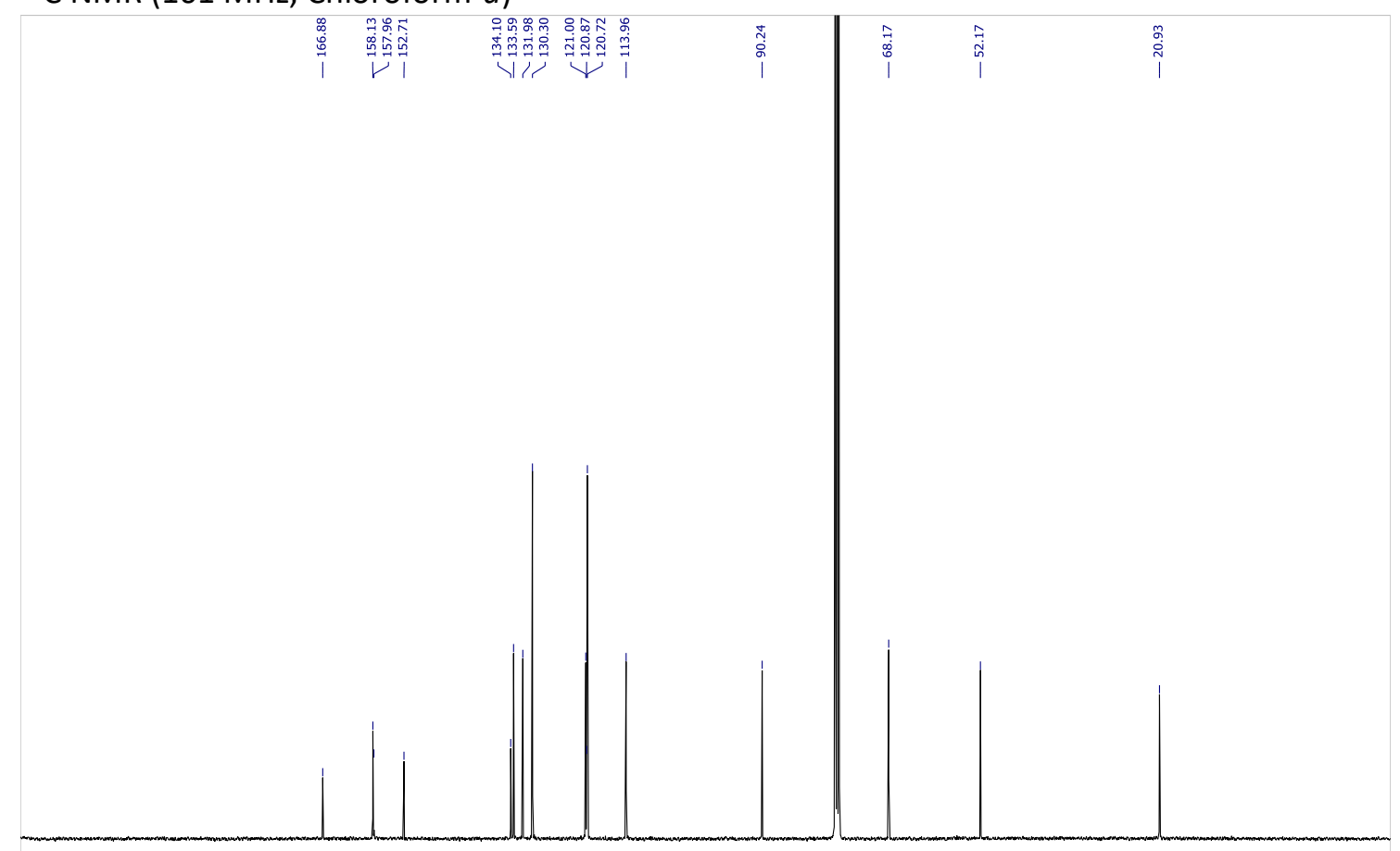

$\begin{array}{lllllllllllllllllllllll}210 & 200 & 190 & 180 & 170 & 160 & 150 & 140 & 130 & 120 & 110 & \begin{array}{c}100 \\ \mathrm{f} 1(\mathrm{ppm})\end{array} & 90 & 80 & 70 & 60 & 50 & 40 & 30 & 20 & 10 & 0 & -10\end{array}$ 
1-(3,5-di-tert-butyl-4-((2-(p-tolyloxy)allyl)oxy)phenyl)ethan-1-one (3i)

${ }^{1} \mathrm{H}$ NMR (400 MHz, Chloroform-d)

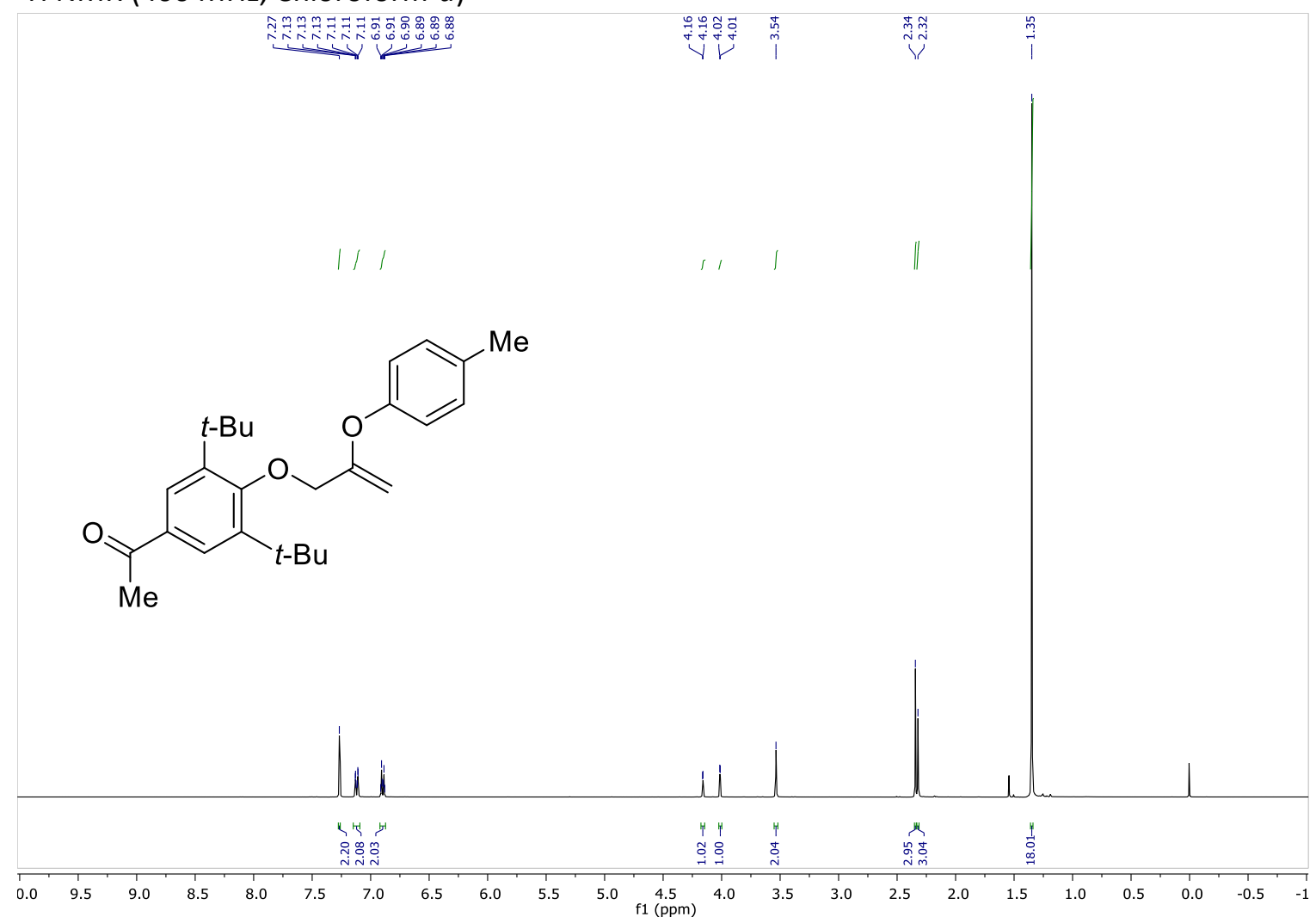

${ }^{13} \mathrm{C}$ NMR (101 MHz, Chloroform-d)

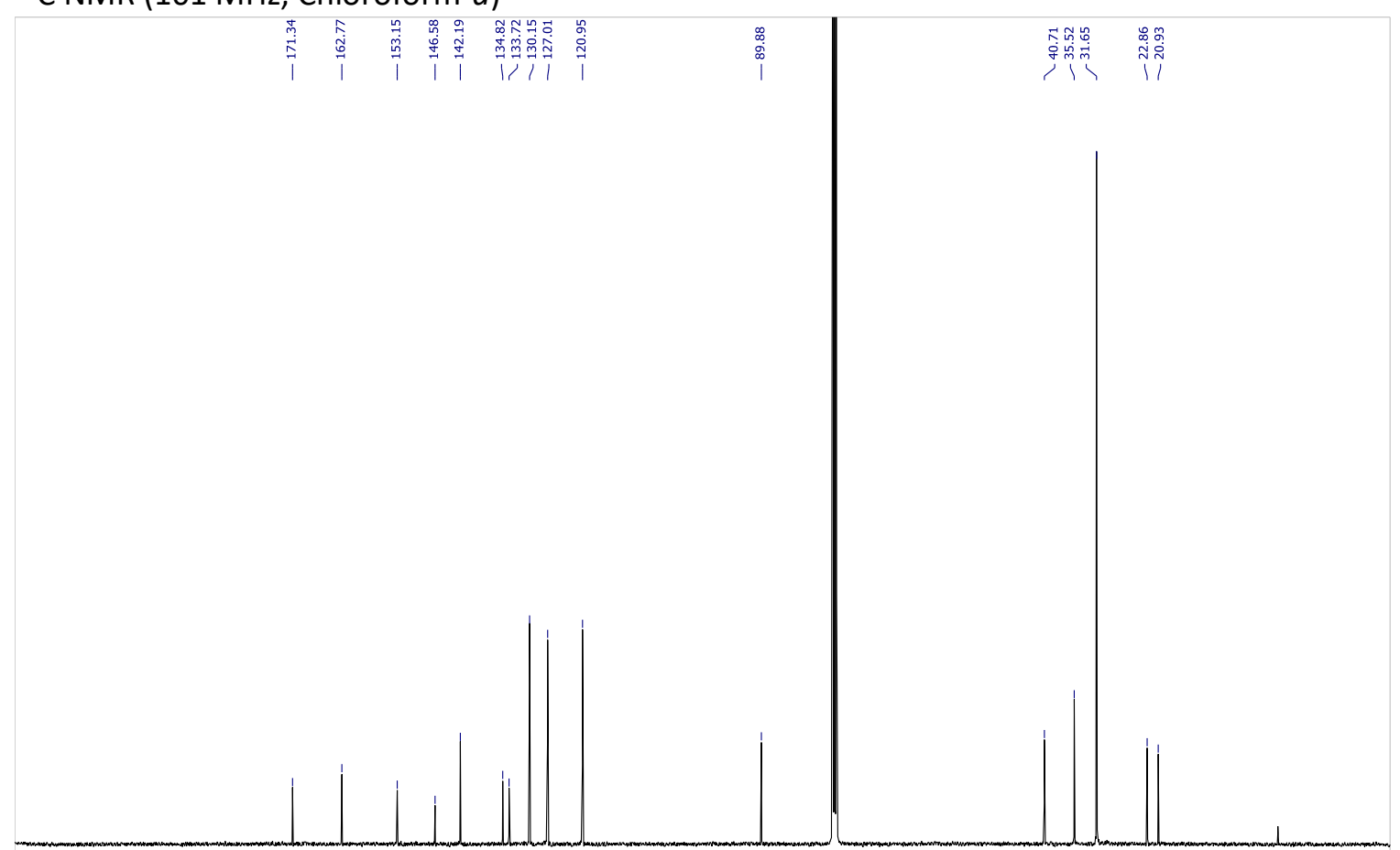

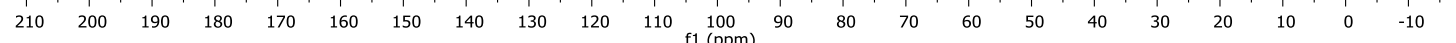


2-((2-(p-tolyloxy)allyl)oxy)naphthalene (3j)

${ }^{1}$ H NMR (400 MHz, Chloroform-d)

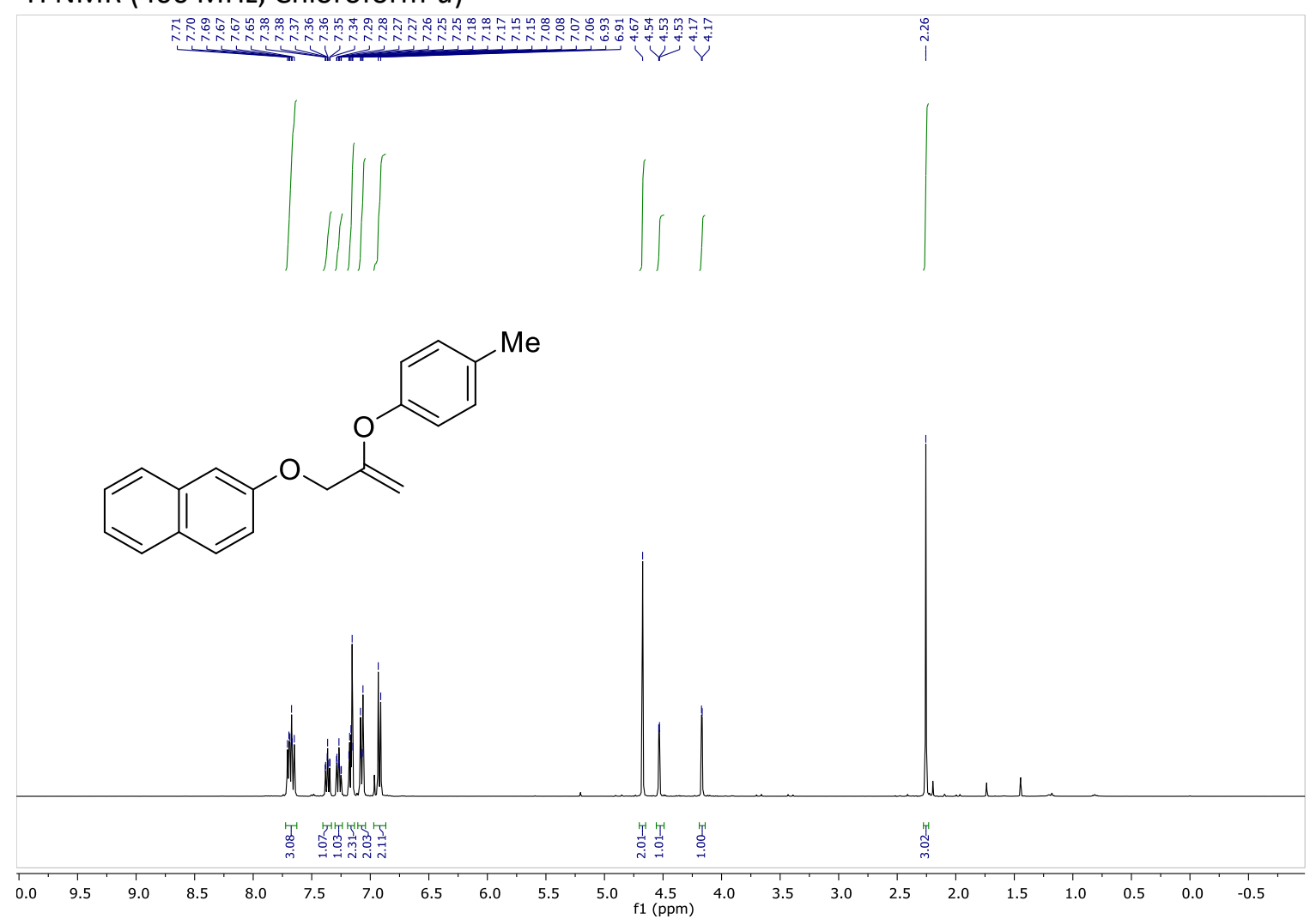

${ }^{13} \mathrm{C}$ NMR (101 MHz, Chloroform-d)

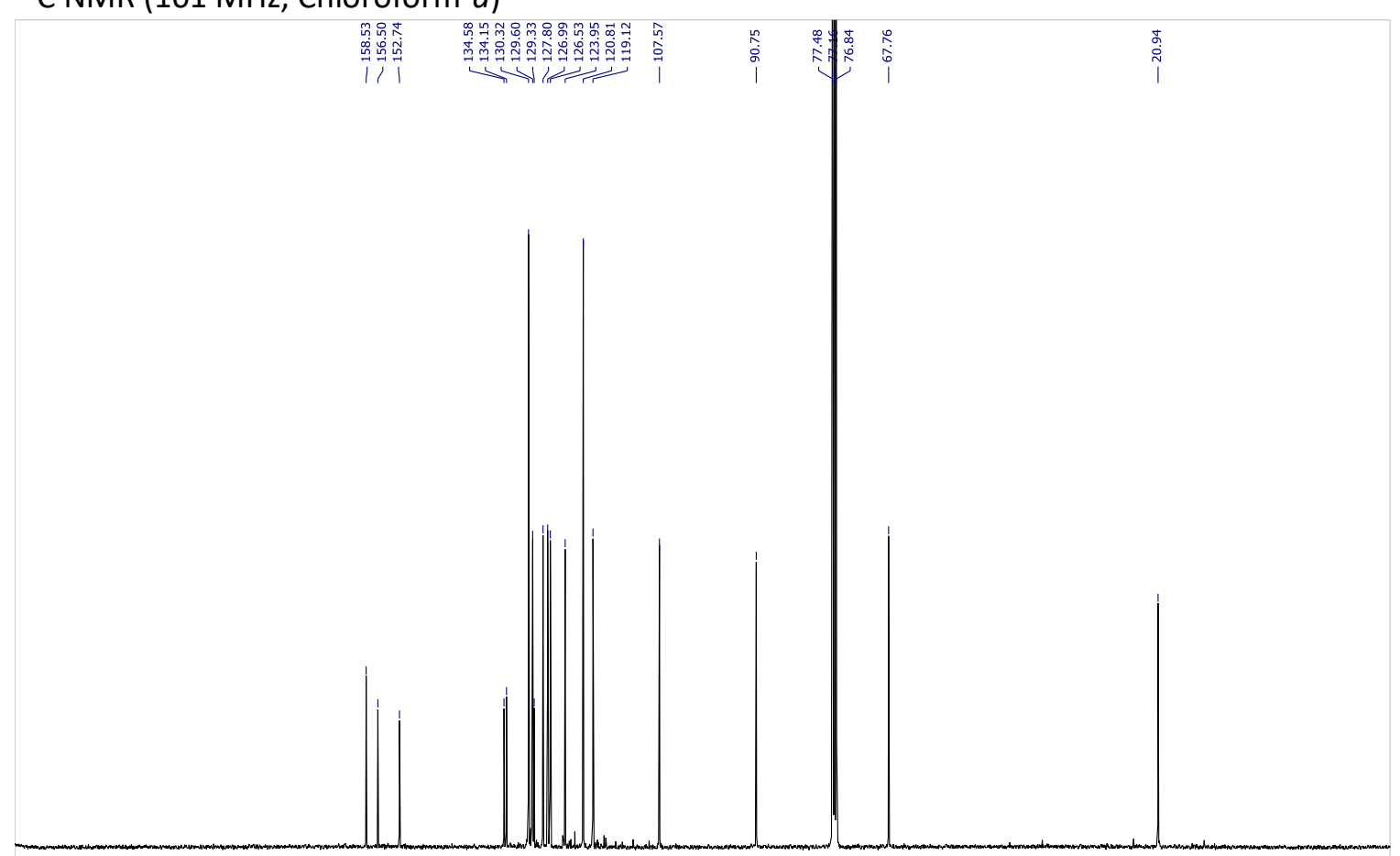

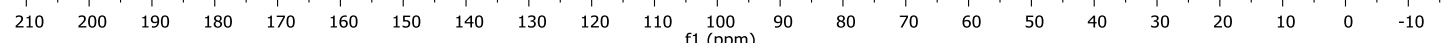


Ethyl (S)-2-acetamido-3-(4-((2-(p-tolyloxy)allyl)oxy)phenyl)propanoate (3k)

${ }^{1} \mathrm{H}$ NMR (400 MHz, Acetonitrile- $d_{3}$ )

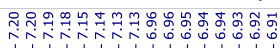

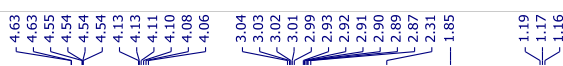
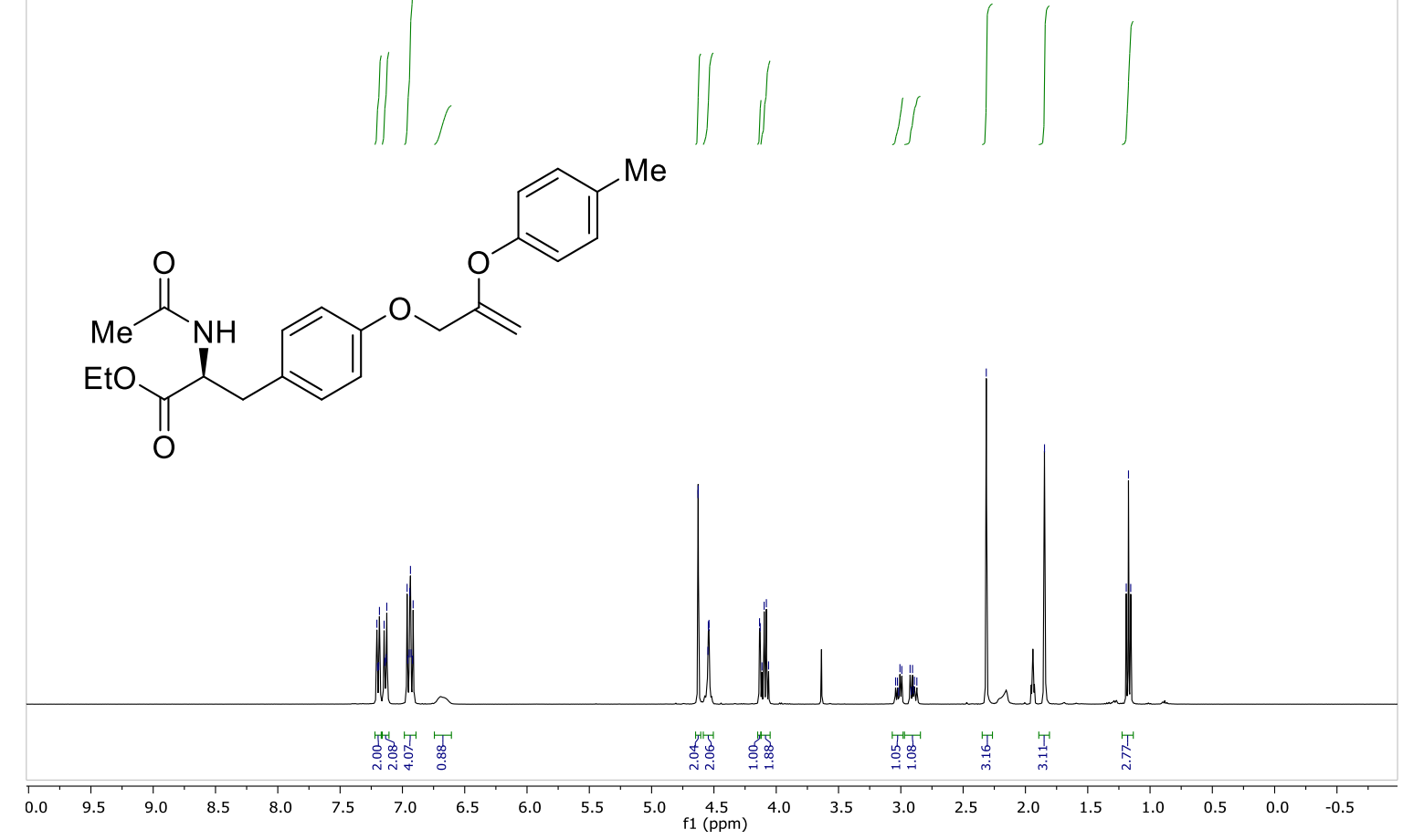

${ }^{13} \mathrm{C}$ NMR (101 MHz, Acetonitrile- $d_{3}$ )

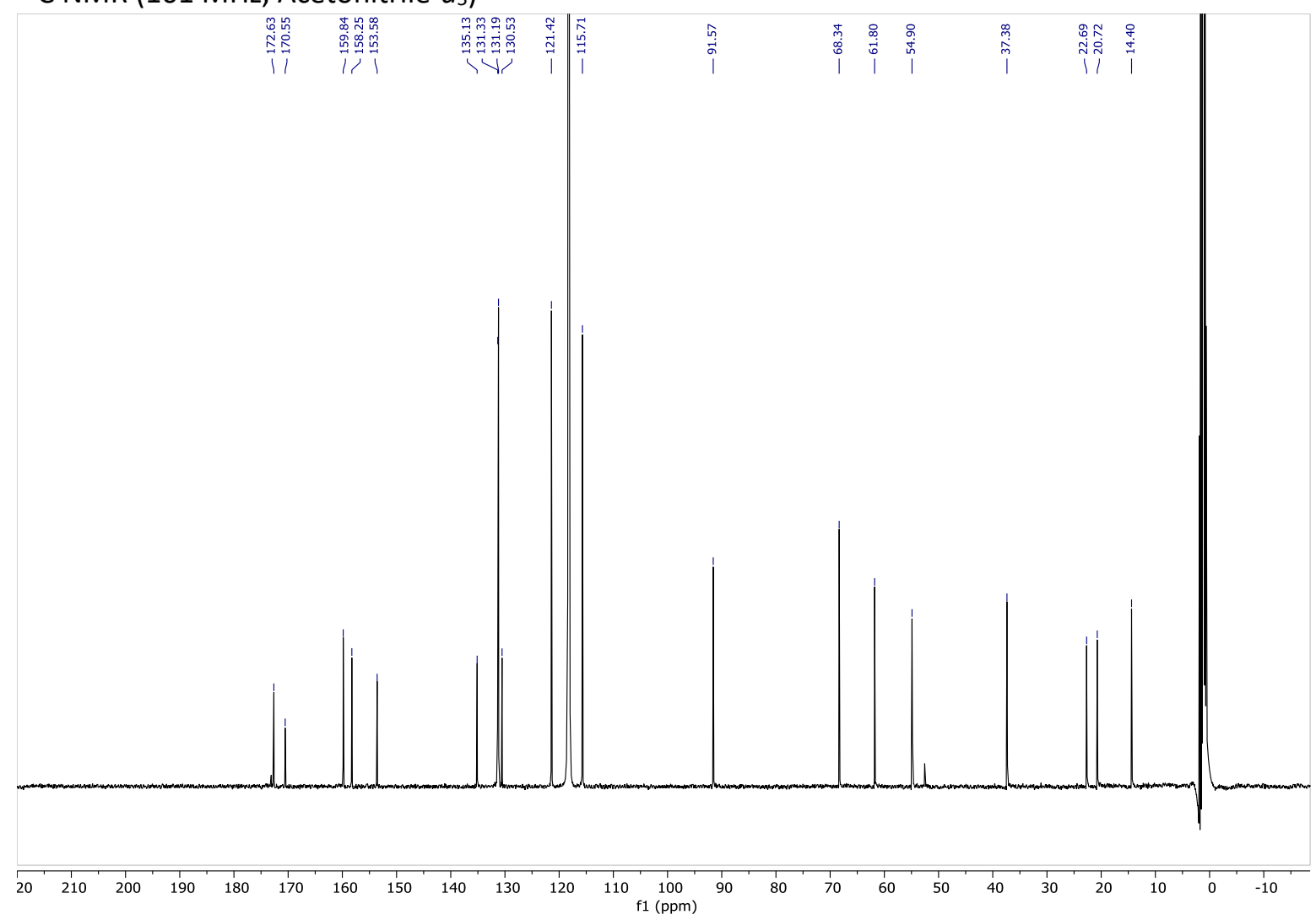


(E)-N-(4-)((3-)((8R,9S,13S,14S,17S)-17-Hydroxy-13-methyl-7,8,9,11,12,13,14,15,16,17-decahydro-6Hcyclopenta[a]phenanthren-3-yl)oxy)prop-1-en-2-yl)oxy)-3-methoxybenzyl)-8-methylnon-6enamide (3l)

${ }^{1} \mathrm{H}$ NMR $\left(400 \mathrm{MHz}\right.$, Acetonitrile- $\left.d_{3}\right)$

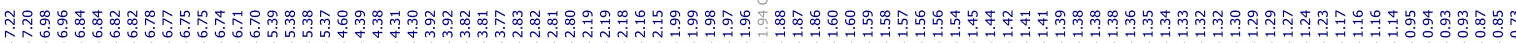

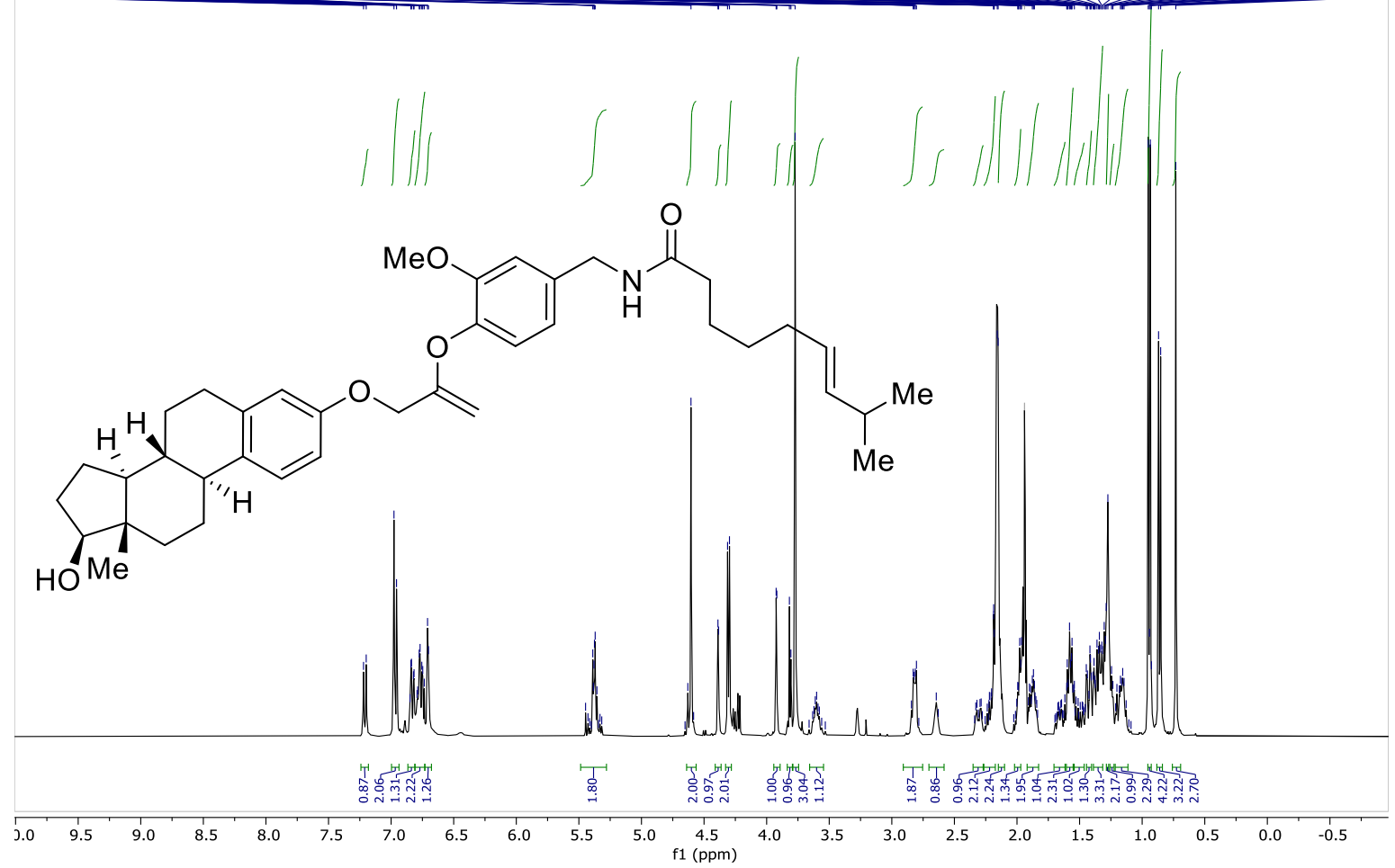

${ }^{13} \mathrm{C}$ NMR (101 MHz, Acetonitrile- $\left.d_{3}\right)$

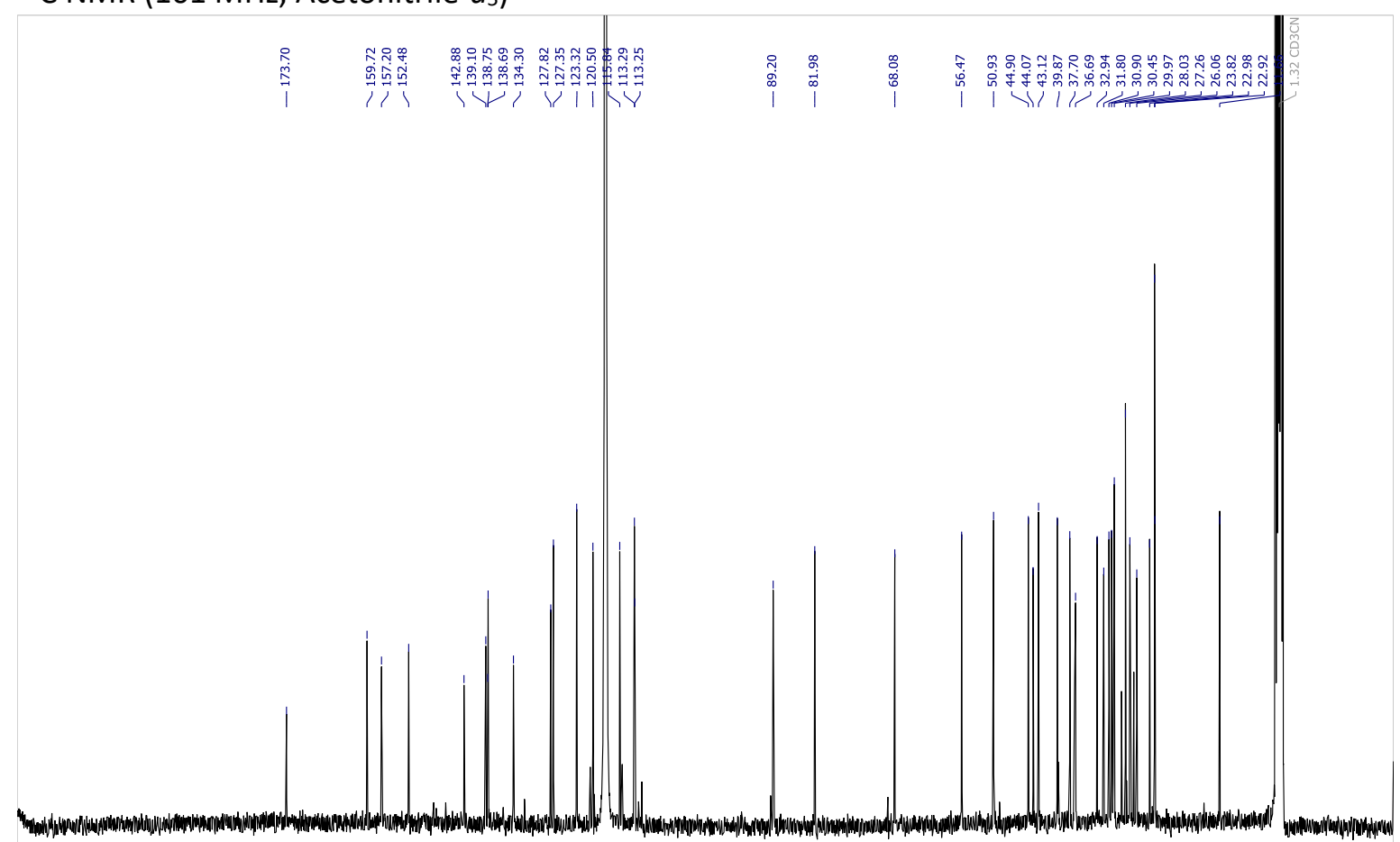

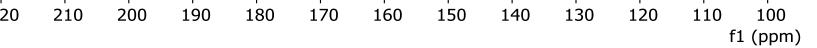


2-methyl-2-(2-(p-tolyloxy)allyl)cyclopentane-1,3-dione (3m)

${ }^{1} \mathrm{H}$ NMR (400 MHz, Chloroform-d)

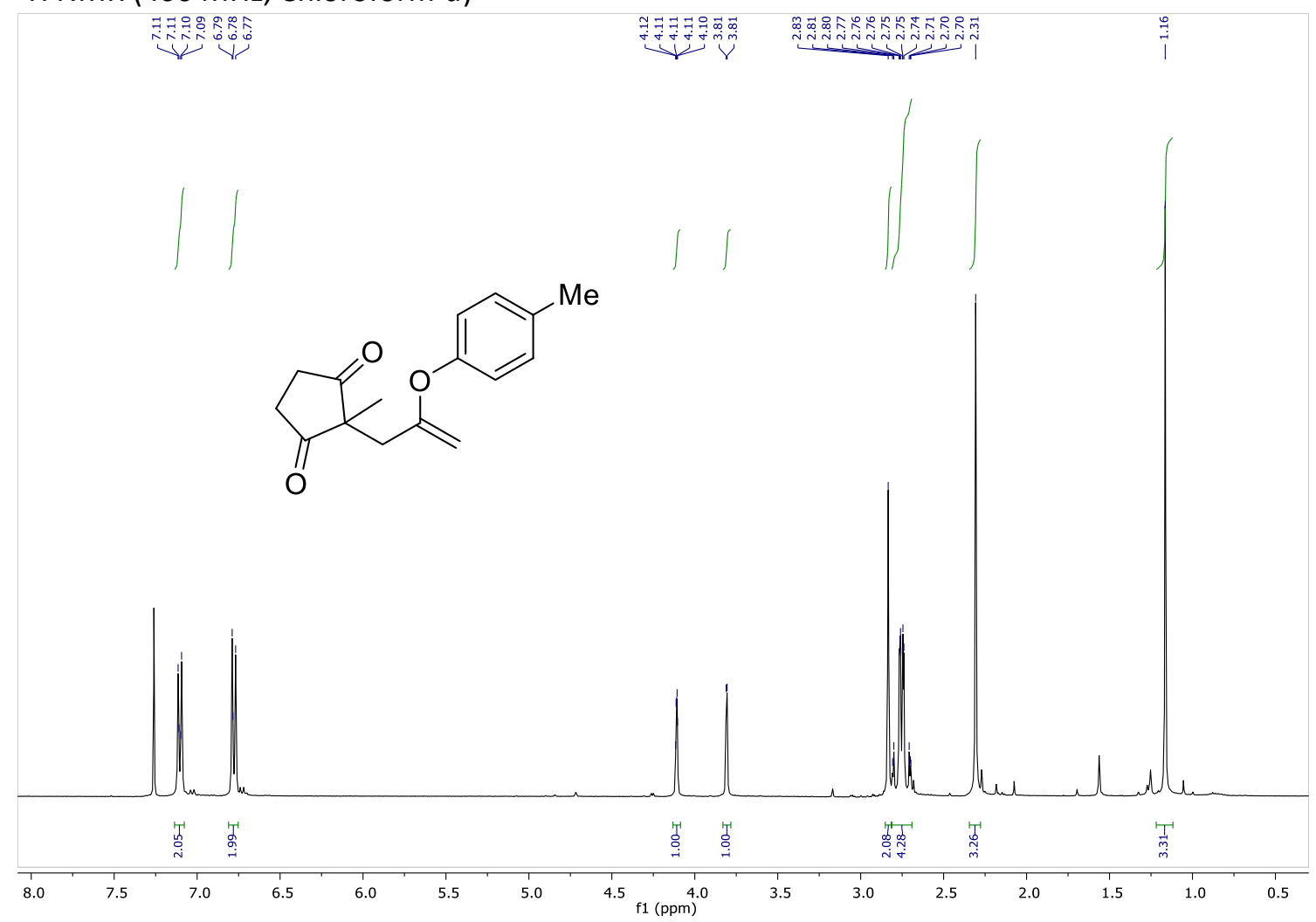

${ }^{13} \mathrm{C}$ NMR (101 MHz, Chloroform-d)

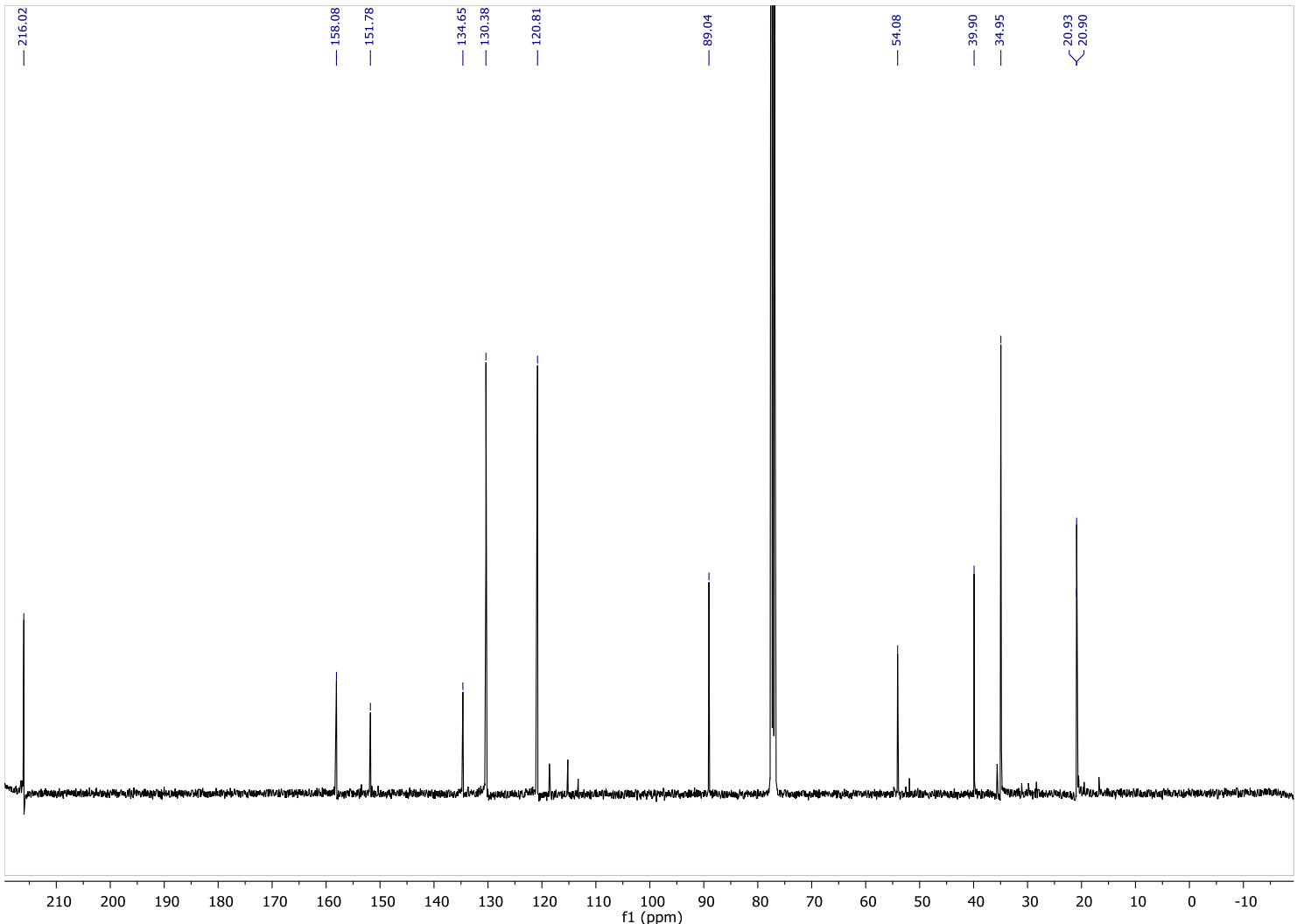


Ethyl 2-acetyl-2-methyl-4-(p-tolyloxy)pent-4-enoate (3n)

${ }^{1} \mathrm{H}$ NMR (400 MHz, Chloroform-d)

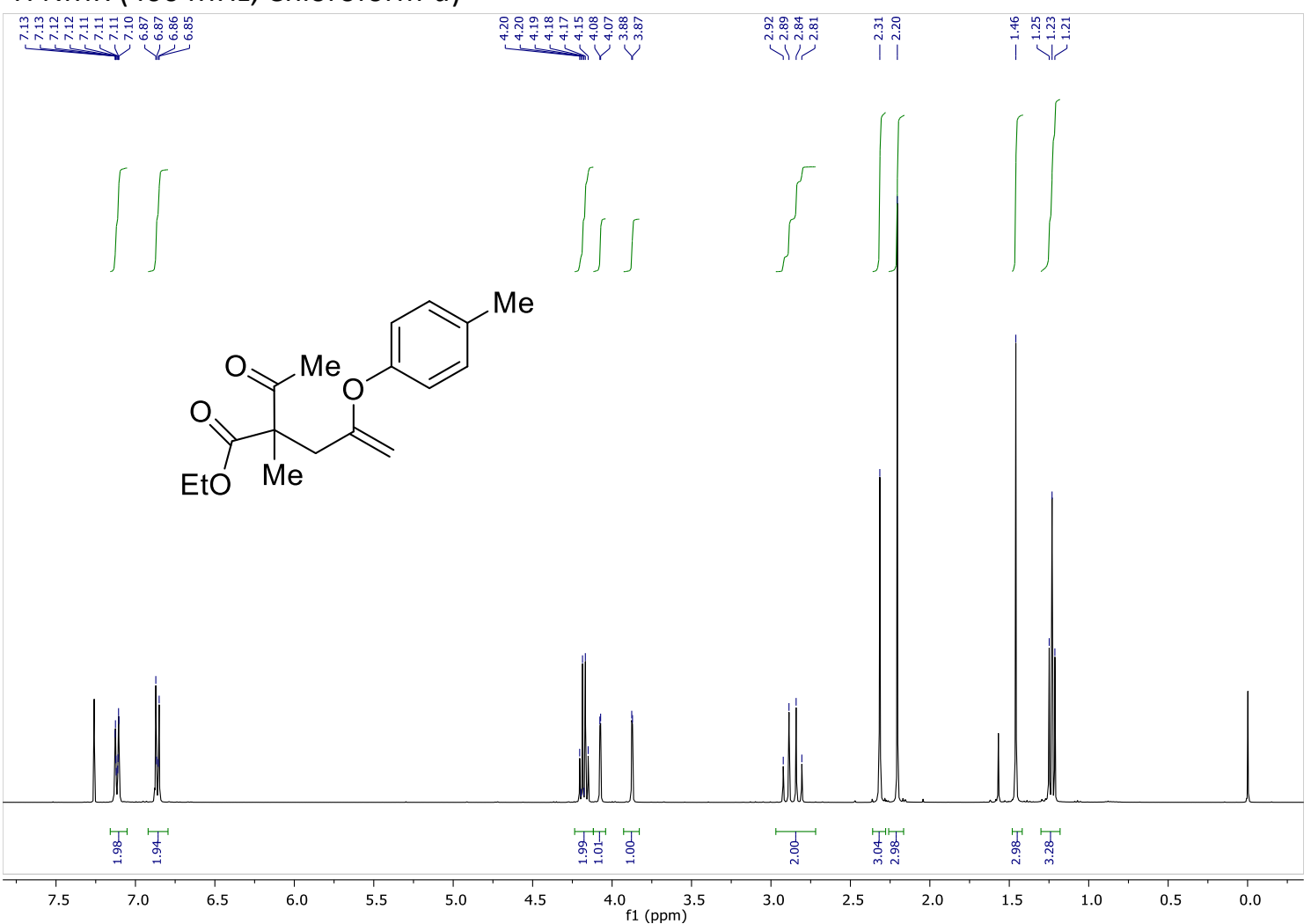

${ }^{13} \mathrm{C}$ NMR (101 MHz, Chloroform-d)

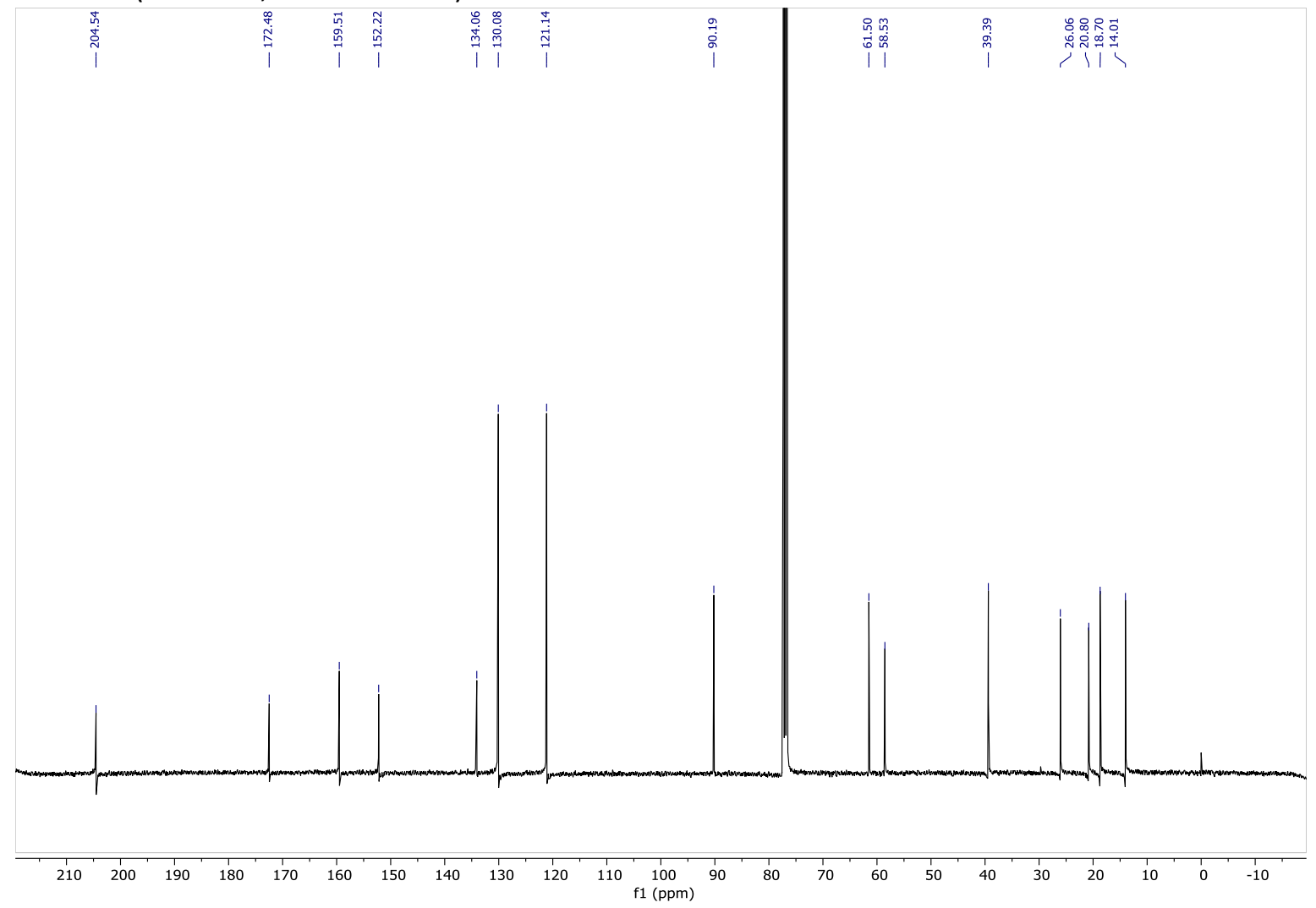


$N$-(4-methoxyphenyl)-4-nitro- $N$-(2-(p-tolyloxy)allyl)benzenesulfonamide (30)

${ }^{1} \mathrm{H}$ NMR (400 MHz, Methylene Chloride- $d_{2}$ )
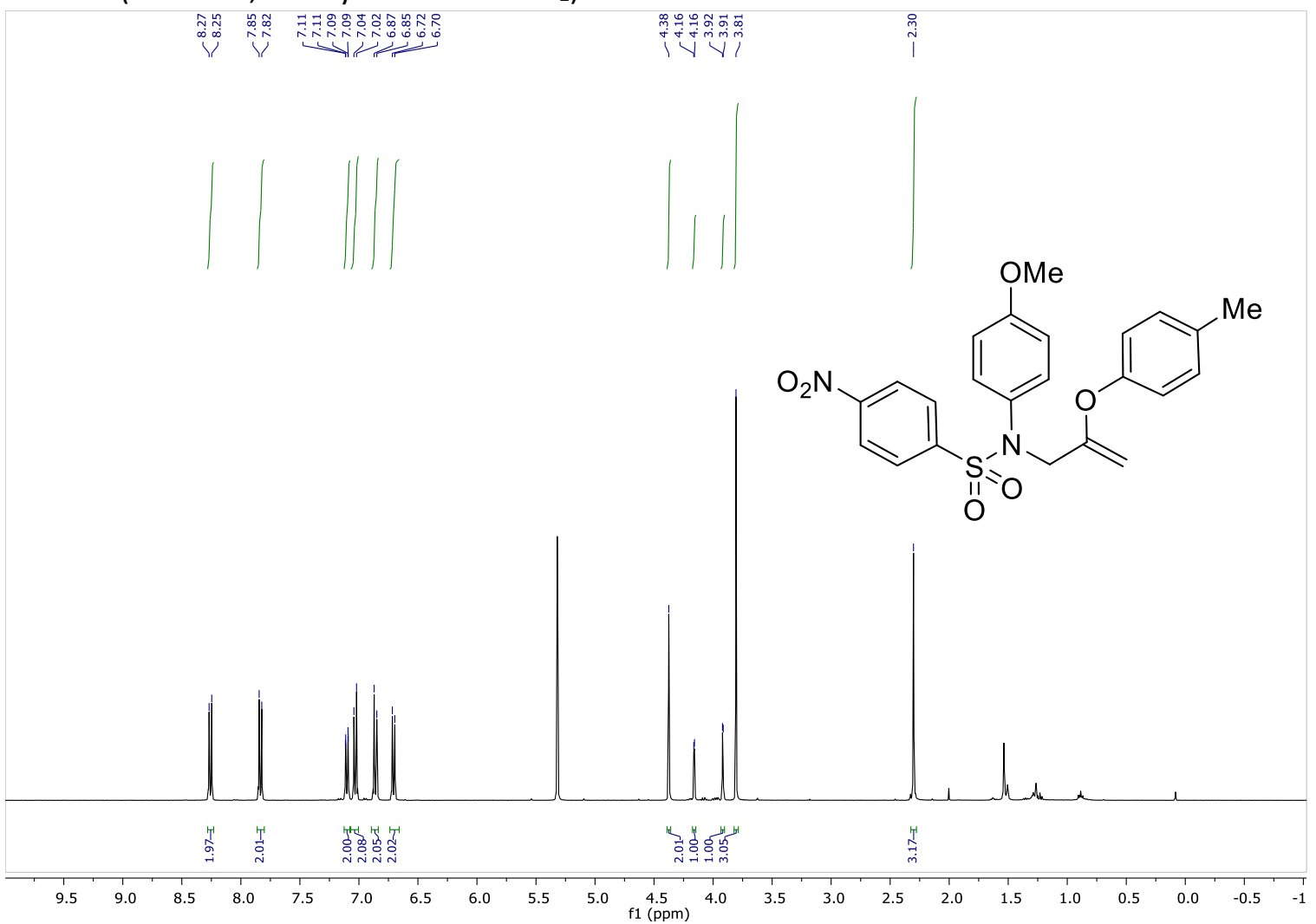

${ }^{13} \mathrm{C}$ NMR (101 MHz, Methylene Chloride- $d_{2}$ )

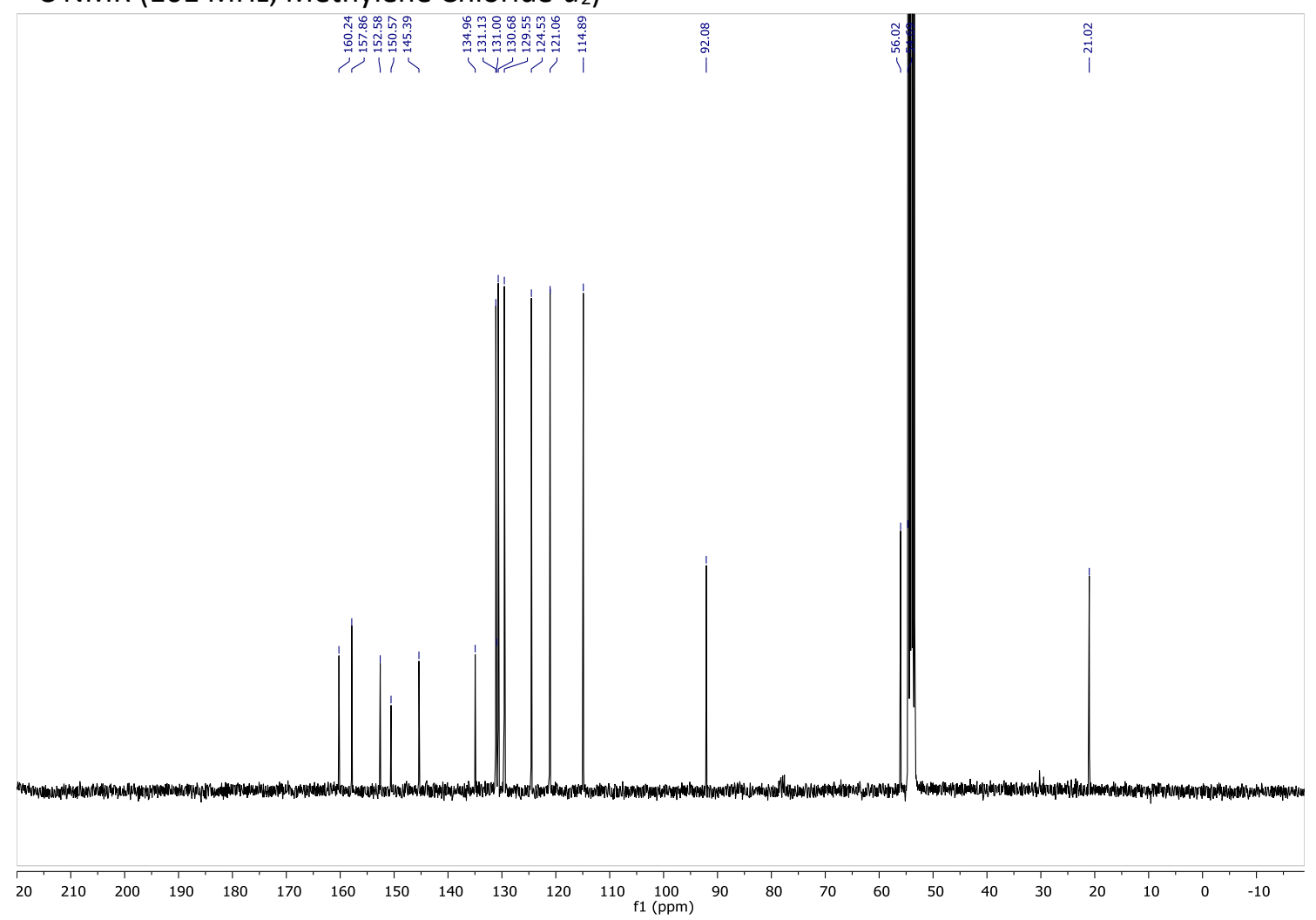


4,4'-(pent-1-ene-2,3-diylbis(oxy))bis(methylbenzene) (3p)

${ }^{1} \mathrm{H}$ NMR (400 MHz, Acetonitrile- $d_{3}$ )

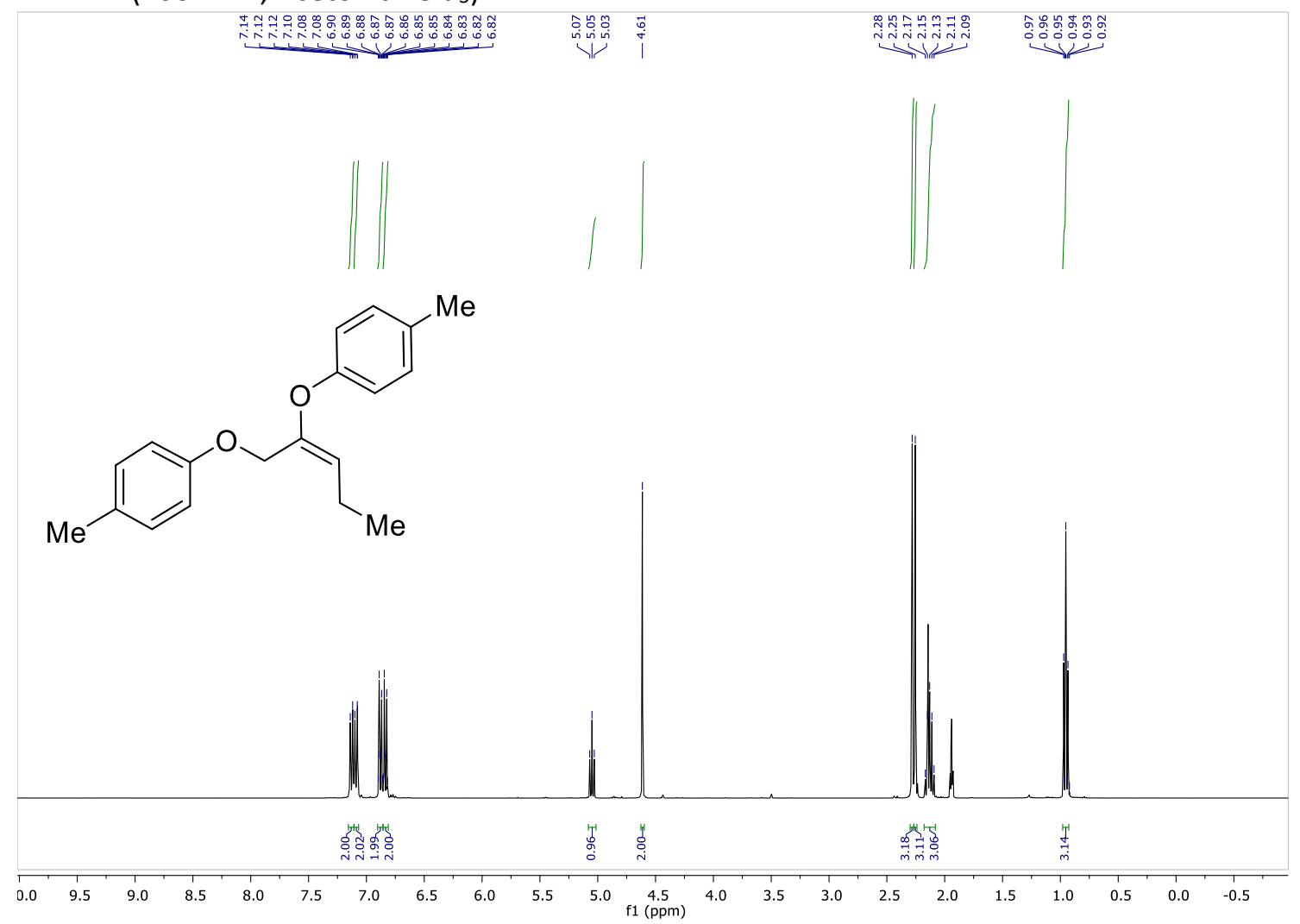

${ }^{13} \mathrm{C}$ NMR (101 MHz, Acetonitrile- $d_{3}$ )

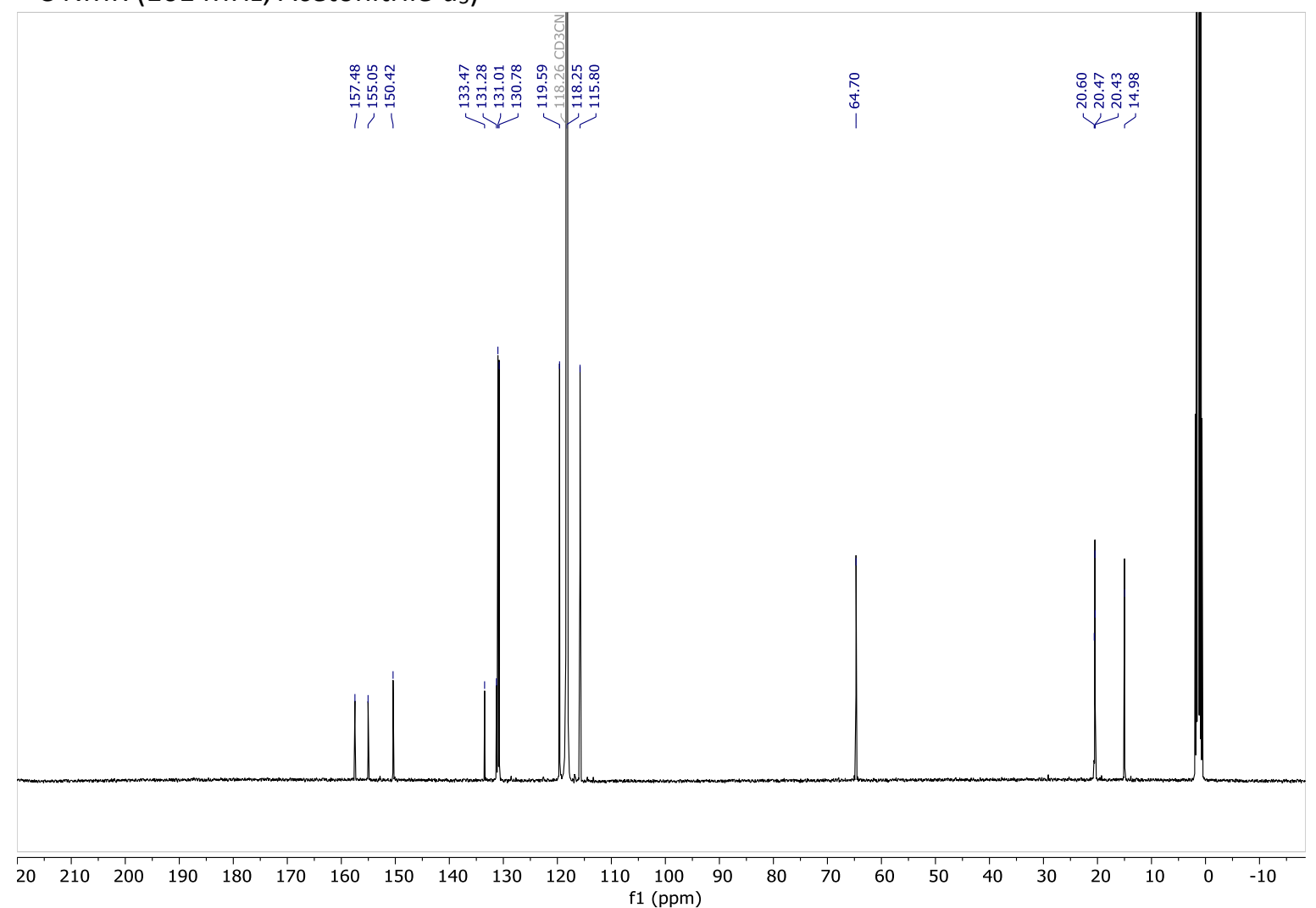


(E)-4,4'-(Oct-2-ene-1,2-diylbis(oxy))bis(methylbenzene) (3q)

${ }^{1} \mathrm{H}$ NMR $\left(400 \mathrm{MHz}\right.$, Acetonitrile- $\left.d_{3}\right)$

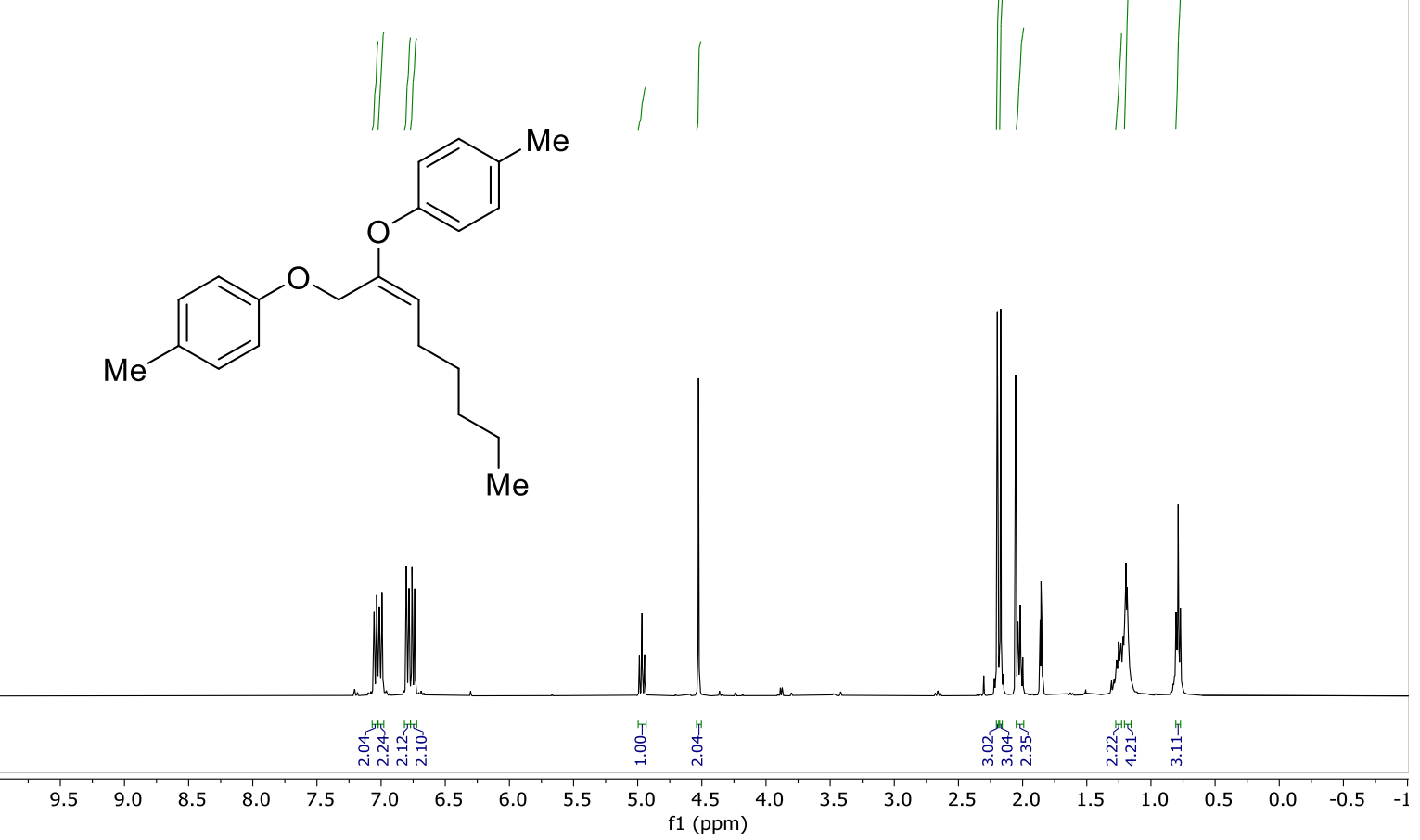

${ }^{13} \mathrm{C}$ NMR (101 MHz, Acetonitrile- $d_{3}$ )

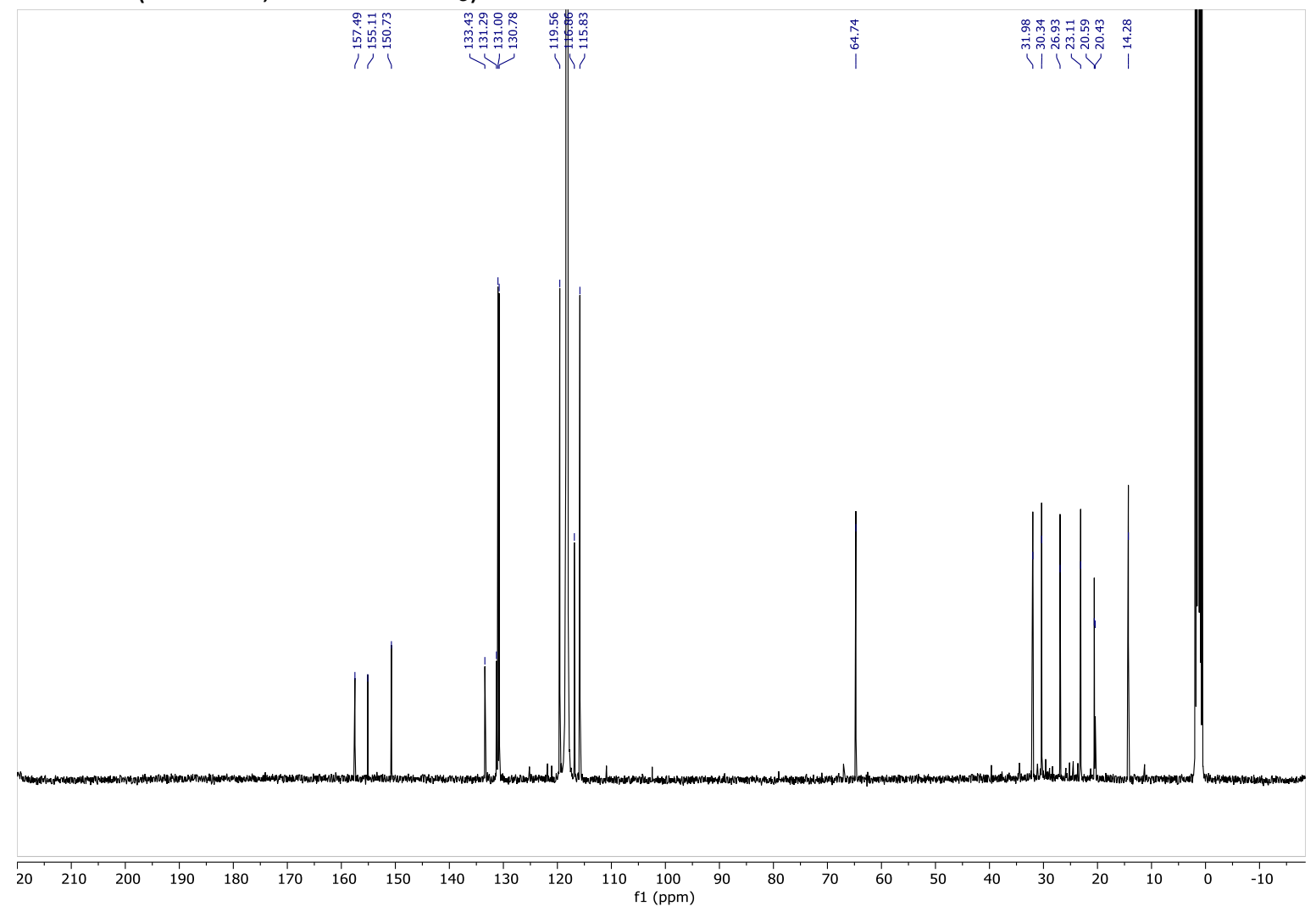


(E)-6,7-bis(p-tolyloxy)hept-5-en-1-ol (3r)

${ }^{1} \mathrm{H}$ NMR (400 MHz, Acetonitrile- $d_{3}$ )

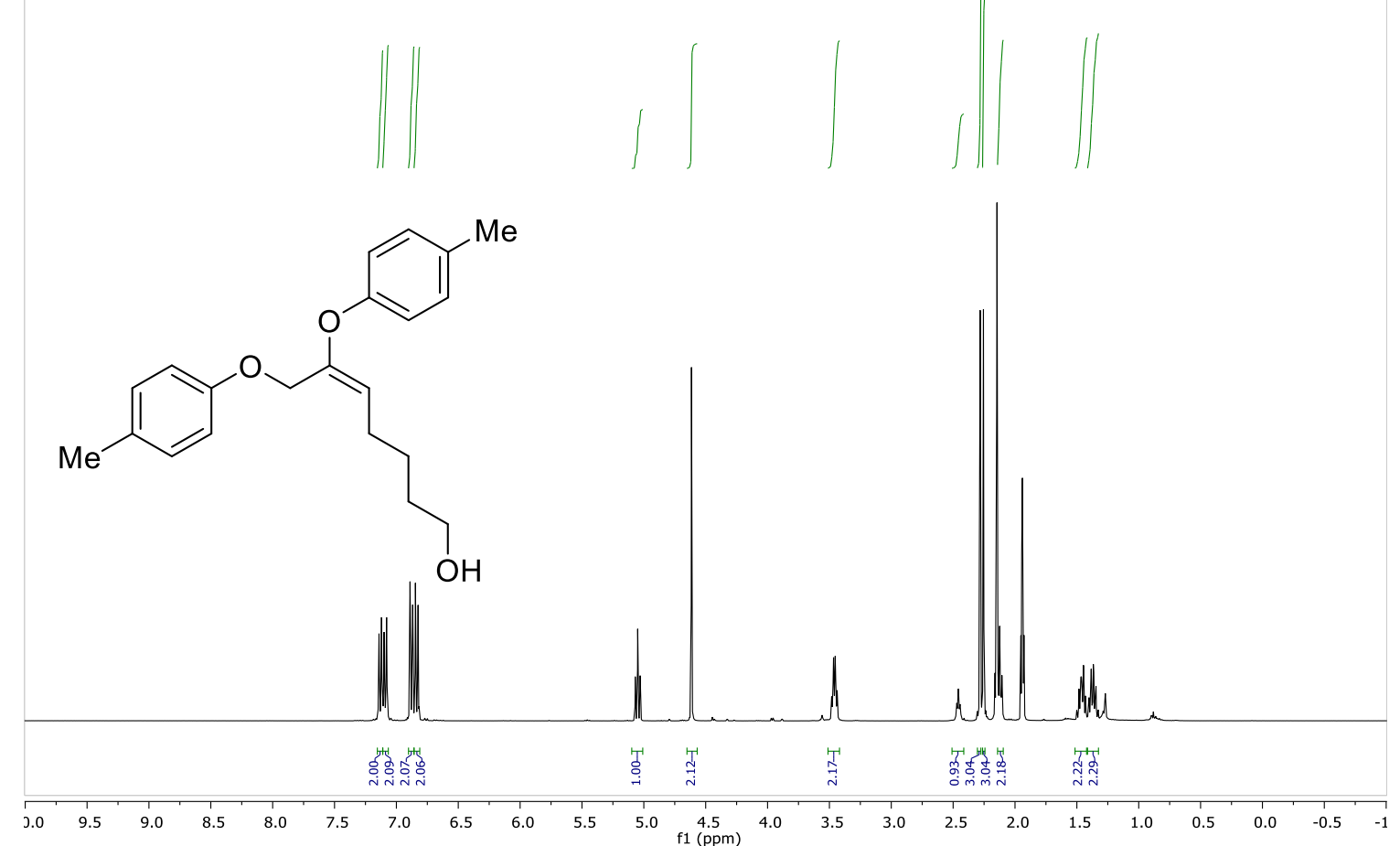

${ }^{13} \mathrm{C}$ NMR (101 MHz, Acetonitrile- $d_{3}$ )

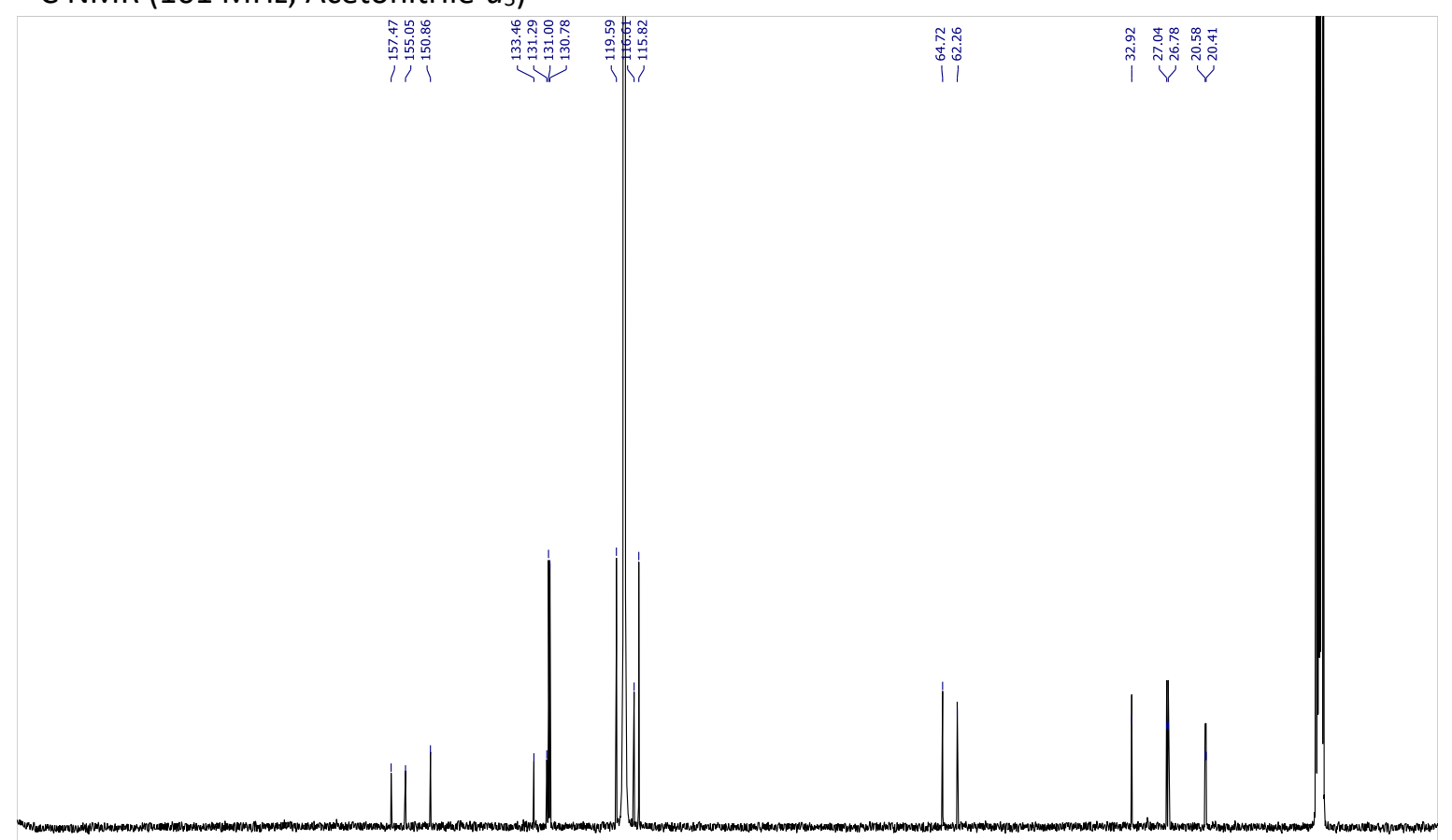

\begin{tabular}{lllllllllllllllllllllllllllll}
\hline 20 & 210 & 200 & 190 & 180 & 170 & 160 & 150 & 140 & 130 & 120 & 110 & 100 & 90 & 80 & 70 & 60 & 50 & 40 & 30 & 20 & 10 & 0 & -10
\end{tabular} 
(E)-(7,8-bis(p-tolyloxy)oct-6-en-1-yn-1-yl)trimethylsilane (3s)

${ }^{1} \mathrm{H}$ NMR (400 MHz, Acetonitrile- $d_{3}$ )

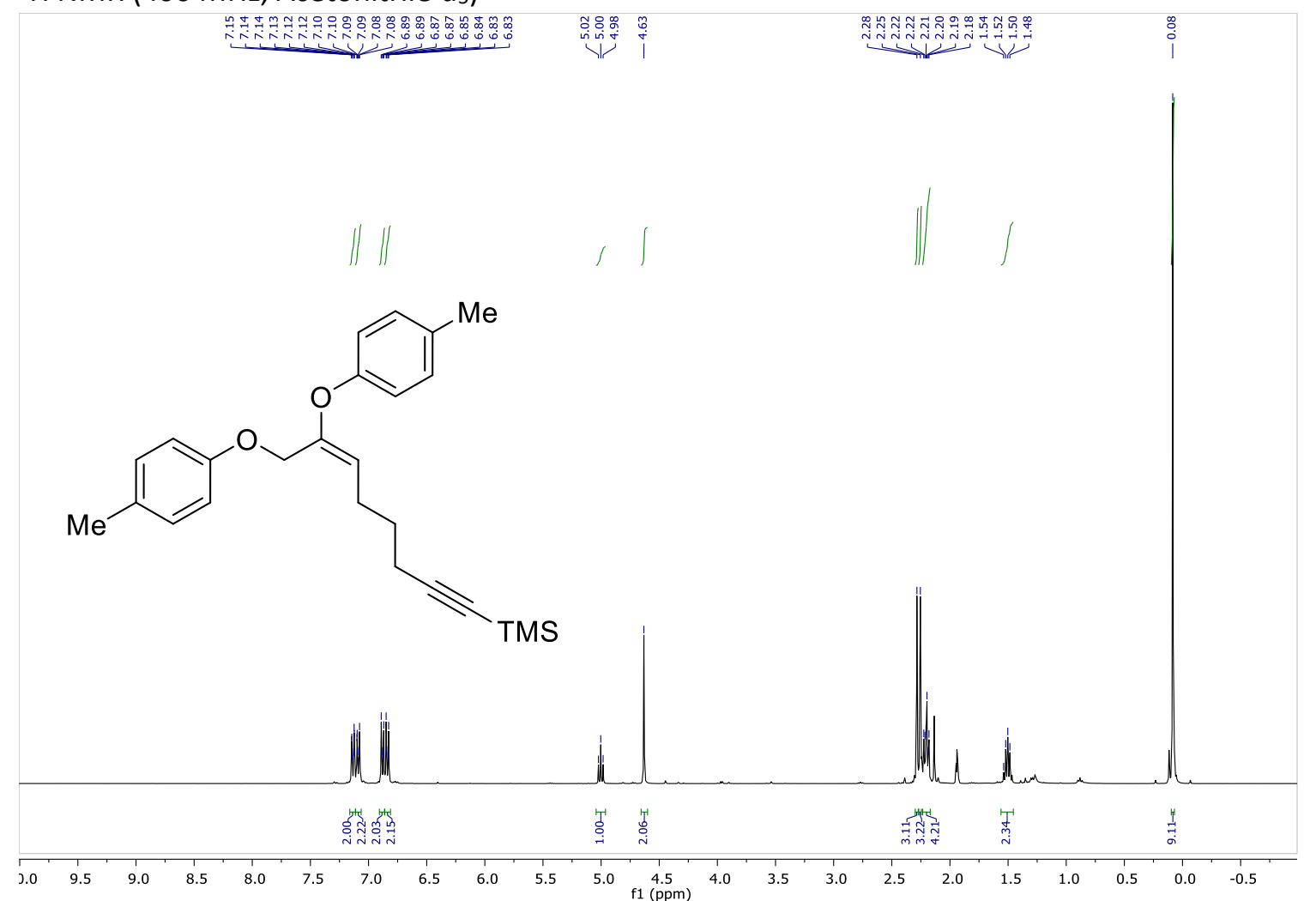

${ }^{13} \mathrm{C}$ NMR (101 MHz, Acetonitrile- $d_{3}$ )

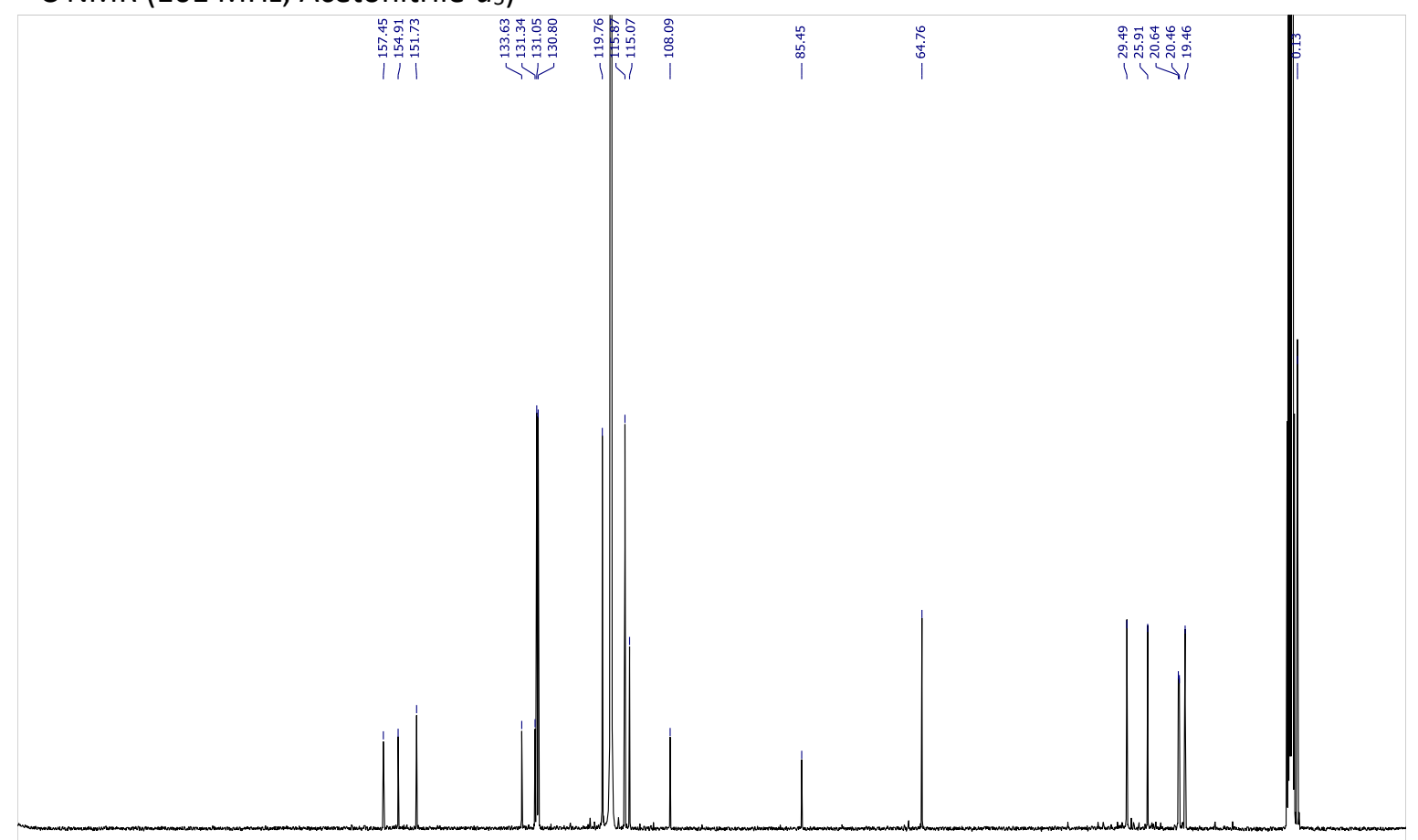

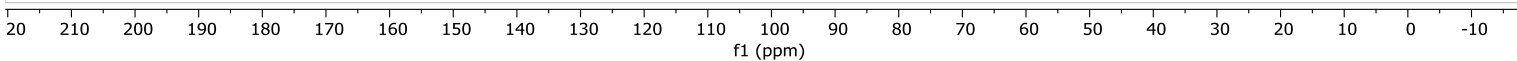


(E)-2-(5,6-bis(p-tolyloxy)hex-4-en-1-yl)isoindoline-1,3-dione (3t)

${ }^{1} \mathrm{H}$ NMR (400 MHz, Acetonitrile- $d_{3}$ )

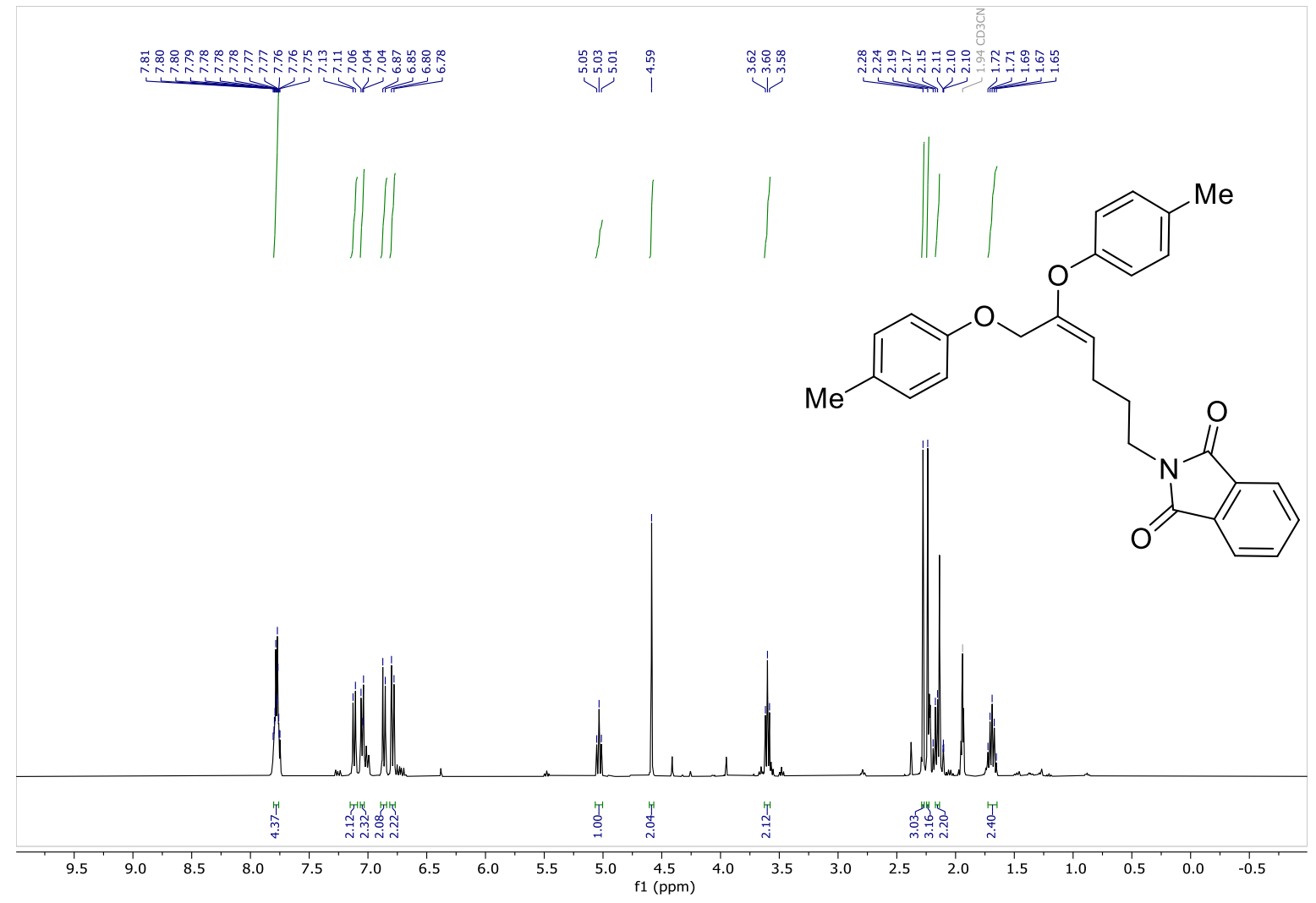

${ }^{13} \mathrm{C}$ NMR (101 MHz, Acetonitrile- $d_{3}$ )

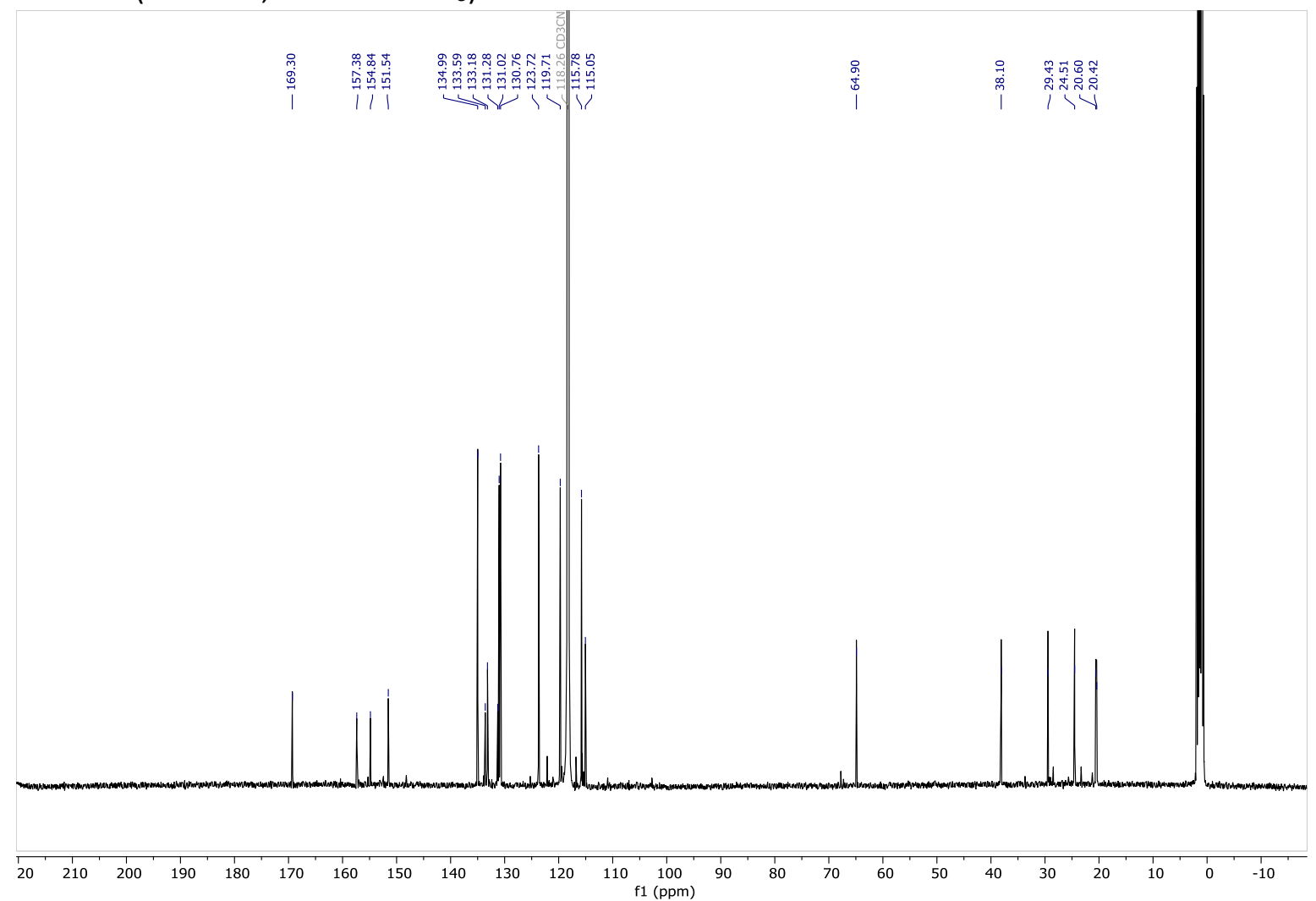


1-lodo-2-((3-(p-tolyloxy)prop-1-en-2-yl)oxy)benzene (3u)

${ }^{1} \mathrm{H}$ NMR $\left(400 \mathrm{MHz}\right.$, Acetonitrile- $\left.d_{3}\right)$

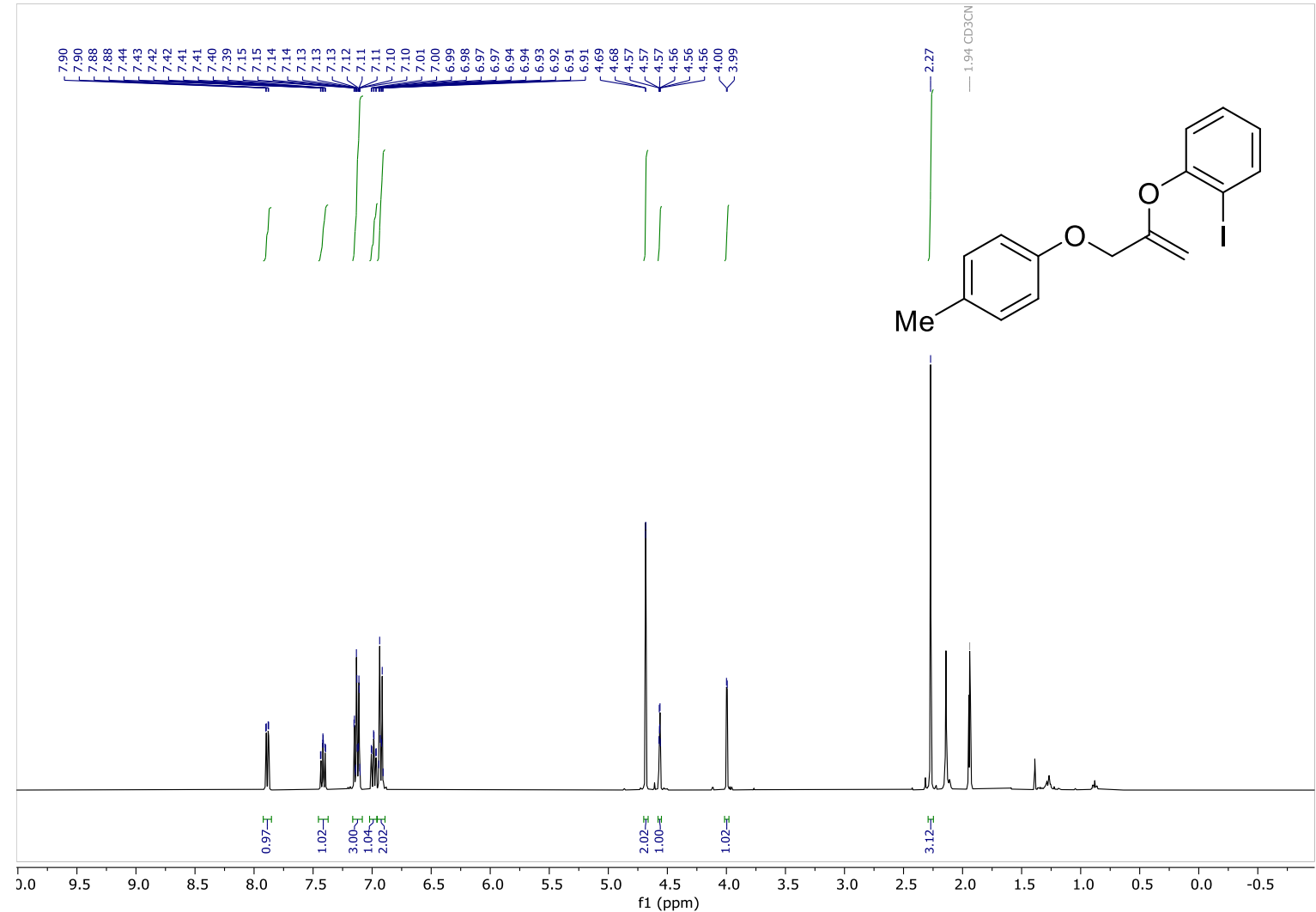

${ }^{13} \mathrm{C}$ NMR (101 MHz, Acetonitrile- $d_{3}$ )

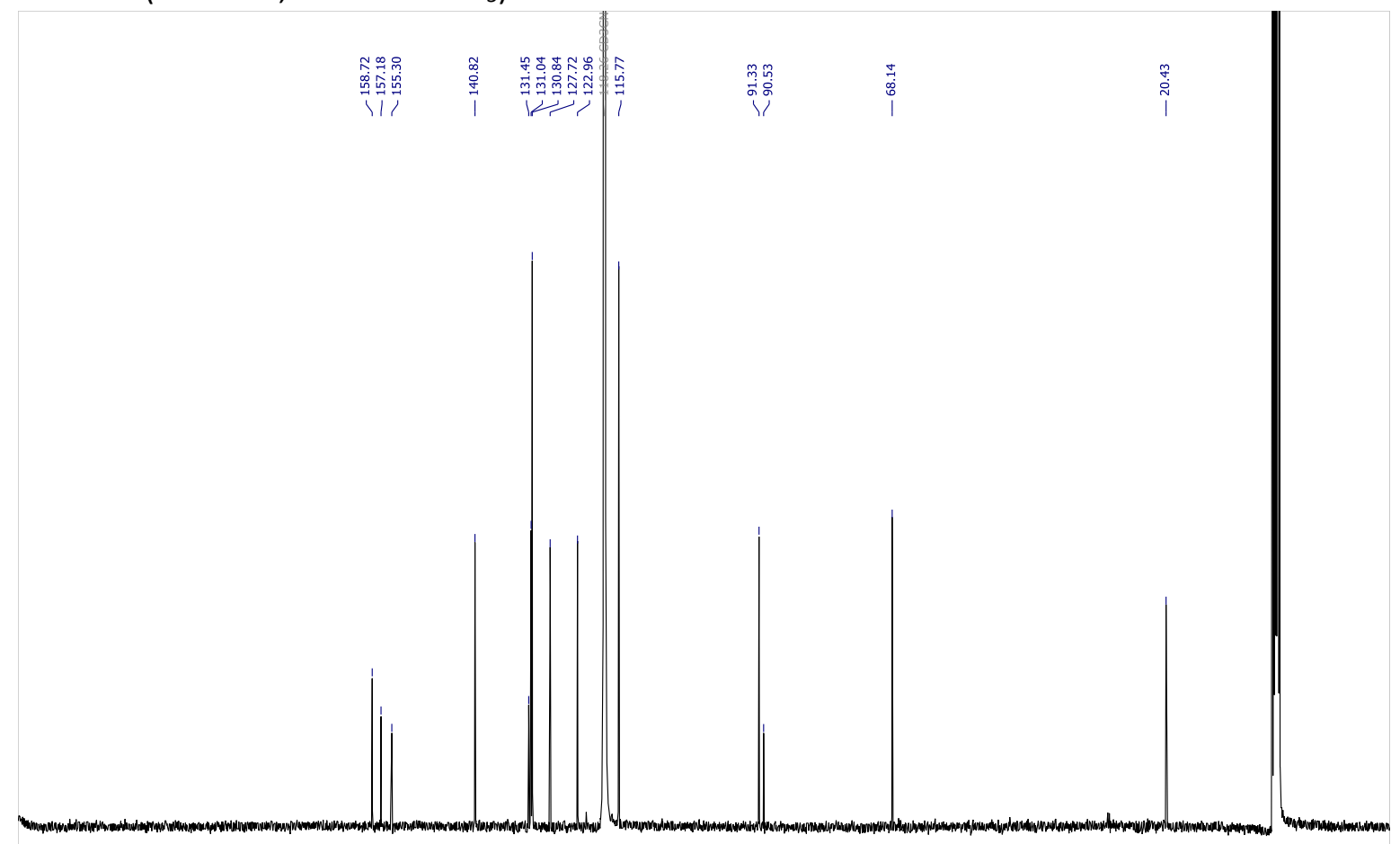

\begin{tabular}{lllllllllllllllllllllllll}
\hline & 210 & 200 & 190 & 180 & 170 & 160 & 150 & 140 & 130 & 120 & 110 & 100 & 90 & 80 & 70 & 60 & 50 & 40 & 30 & 20 & 10 & 0 & -10
\end{tabular} 
(E)-1-iodo-2-((1-(p-tolyloxy)pent-2-en-2-yl)oxy)benzene (3v)

${ }^{1} \mathrm{H}$ NMR $\left(400 \mathrm{MHz}\right.$, Acetonitrile- $\left.d_{3}\right)$

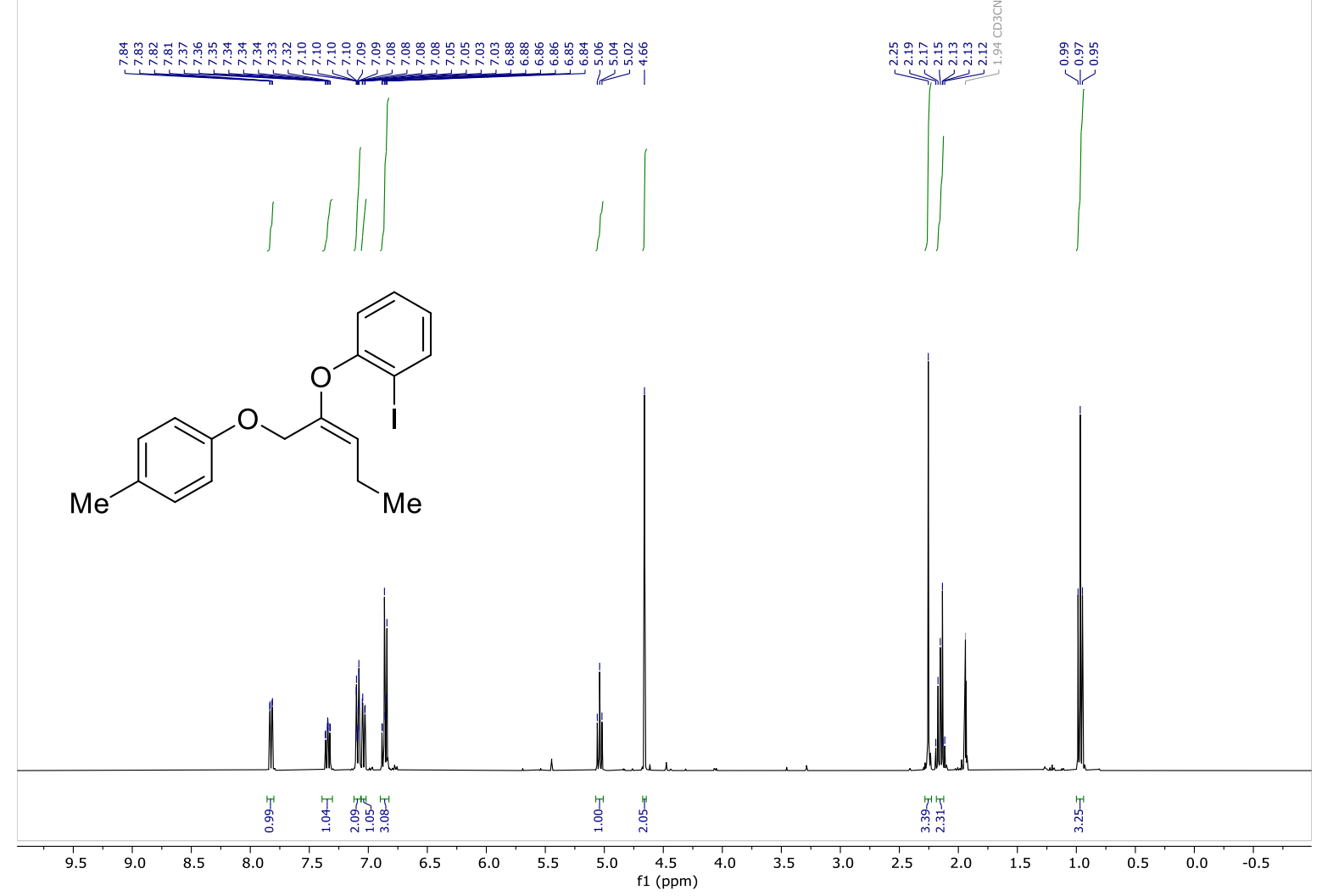

${ }^{13} \mathrm{C}$ NMR (101 MHz, Acetonitrile- $\left.d_{3}\right)$

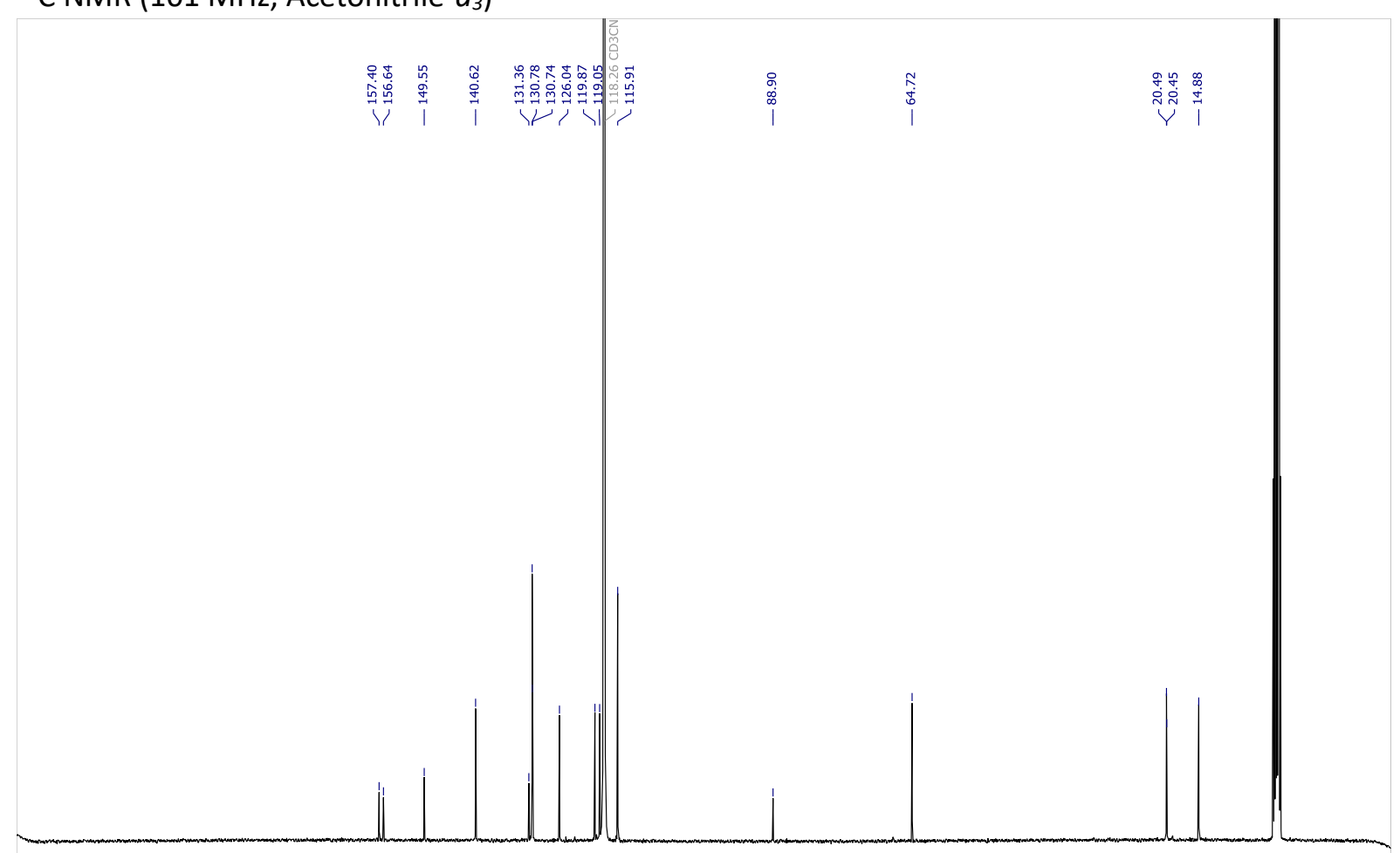

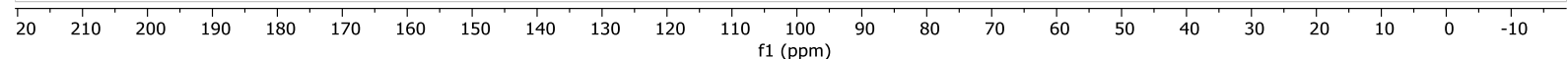


(E)-1-iodo-2-((1-(p-tolyloxy)oct-2-en-2-yl)oxy)benzene (3w)

${ }^{1} \mathrm{H}$ NMR (400 MHz, Acetonitrile- $d_{3}$ )

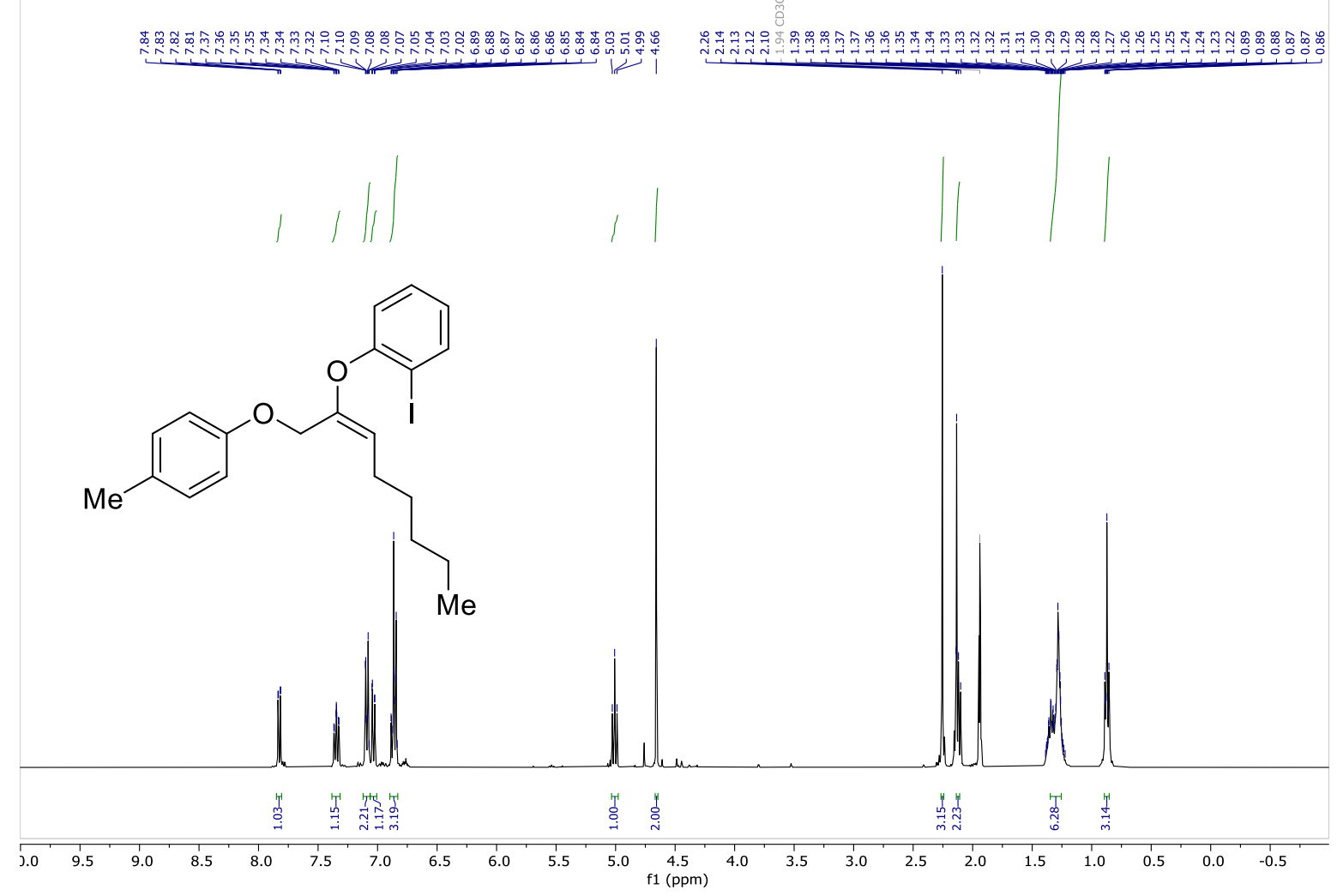

${ }^{13} \mathrm{C}$ NMR (101 MHz, Acetonitrile- $d_{3}$ )

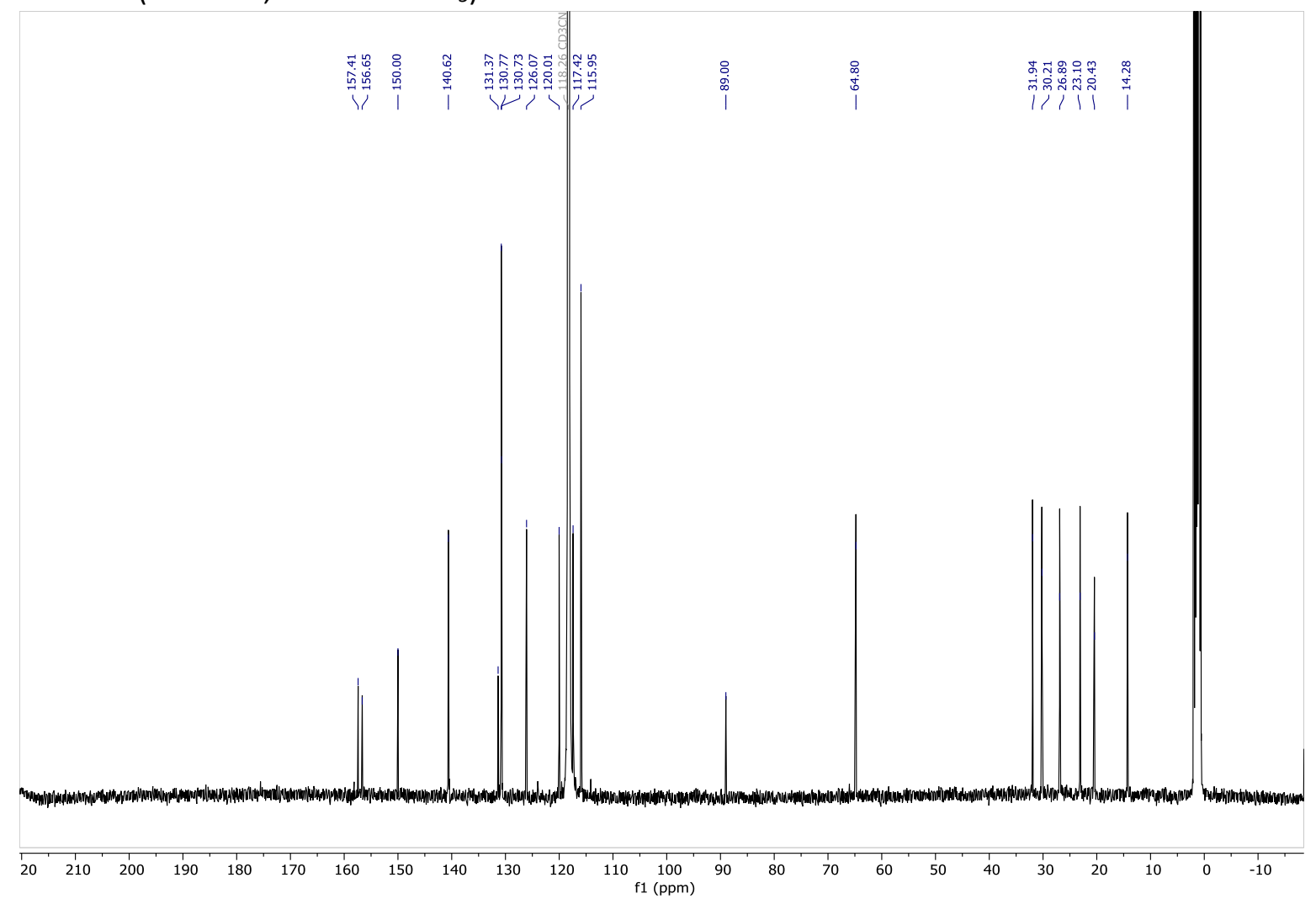


(E)-1-Methoxy-4-((1-(p-tolyloxy)pent-2-en-2-yl)oxy)benzene (3x)

${ }^{1} \mathrm{H}$ NMR (400 MHz, Acetonitrile- $d_{3}$ )

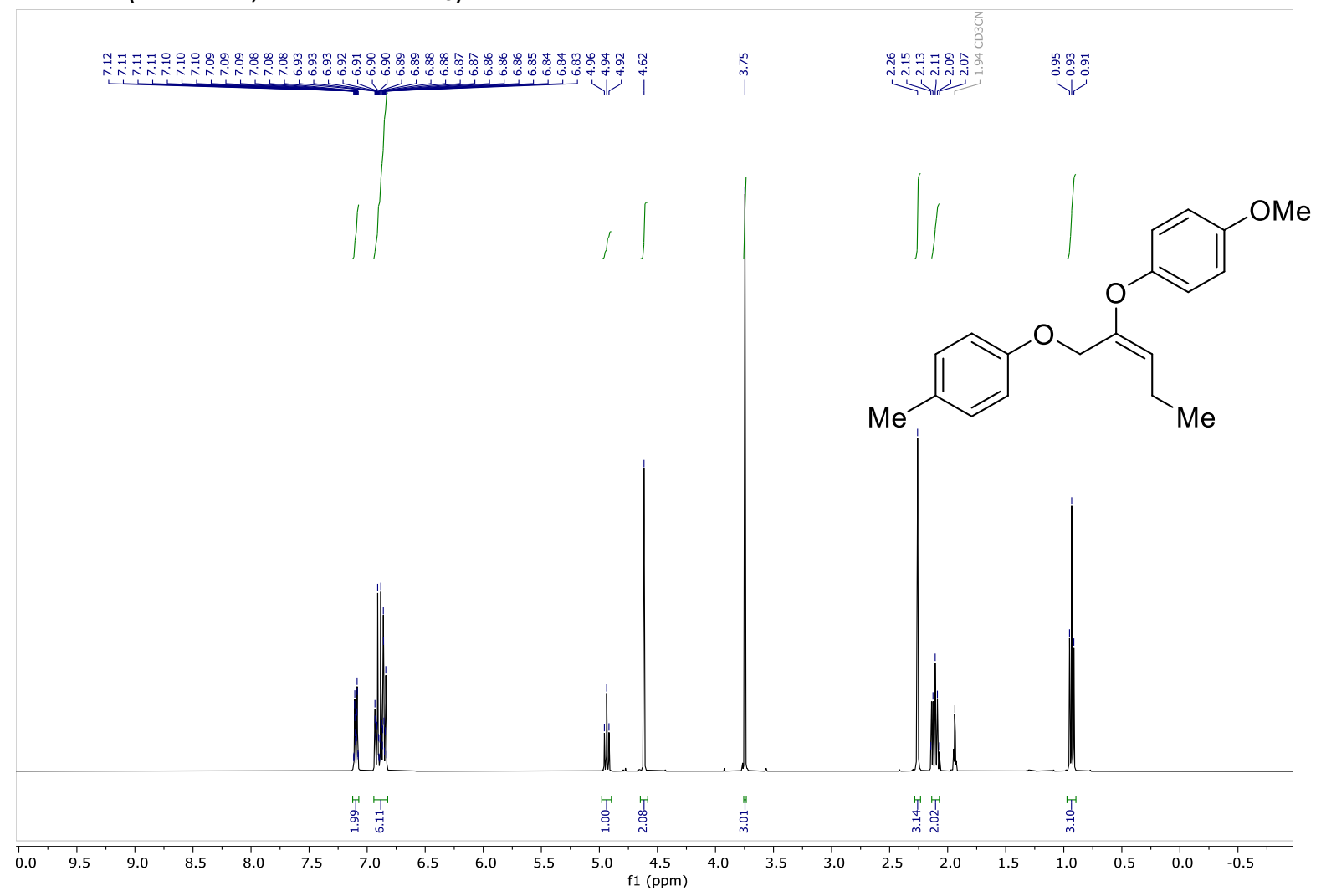

${ }^{13} \mathrm{C}$ NMR (101 MHz, Acetonitrile- $\left.d_{3}\right)$

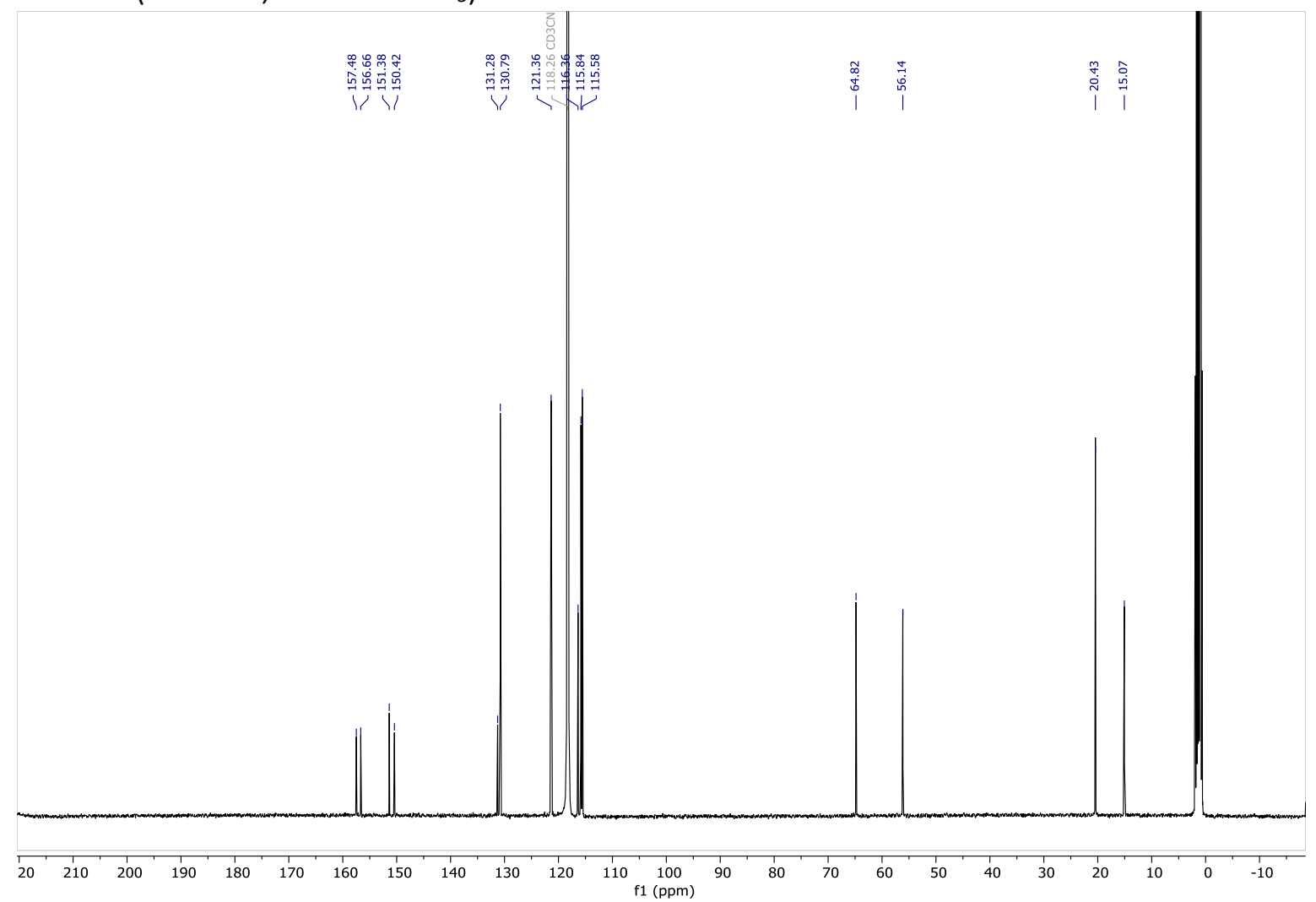


1-Methoxy-4-((3-(p-tolyloxy)prop-1-en-2-yl)oxy)benzene (3y)

${ }^{1} \mathrm{H}$ NMR $\left(400 \mathrm{MHz}\right.$, Acetonitrile- $\left.d_{3}\right)$

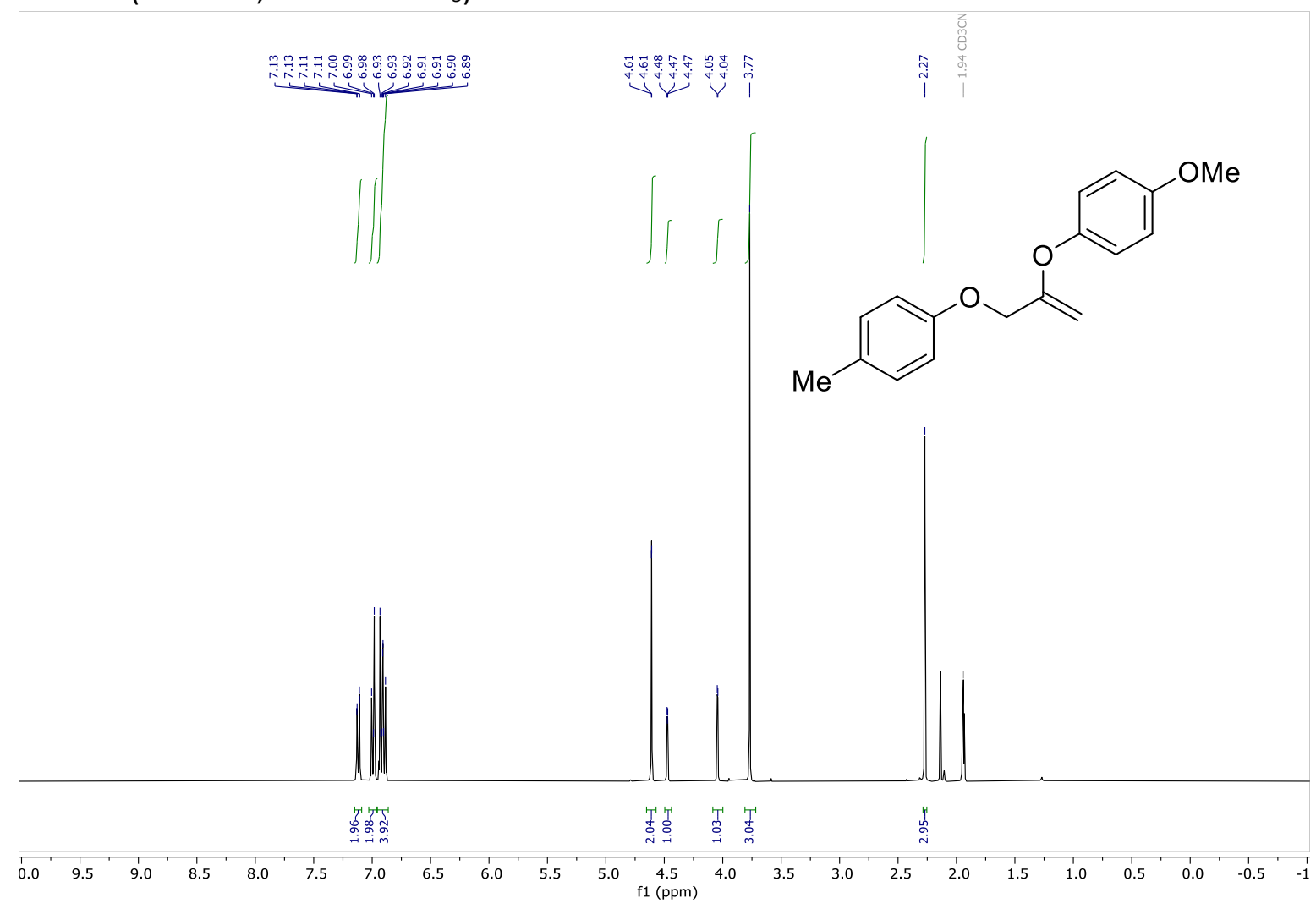

${ }^{13} \mathrm{C}$ NMR (101 MHz, Acetonitrile- $d_{3}$ )

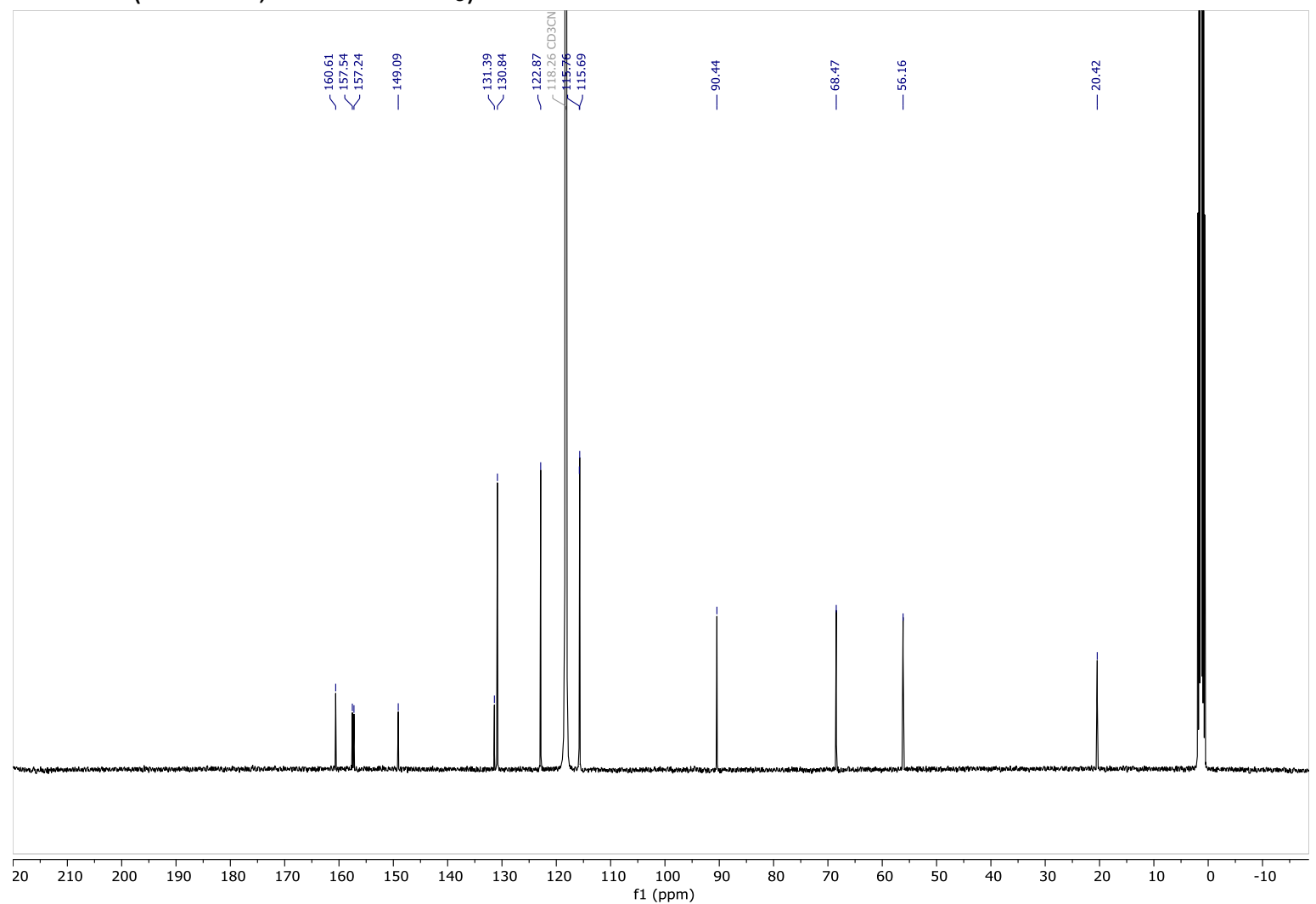




\subsection{Allylic esters}

2-(p-tolyloxy)allyl 2-iodobenzoate (4a)

${ }^{1} \mathrm{H}$ NMR (400 MHz, Chloroform-d)

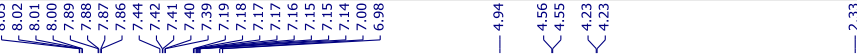
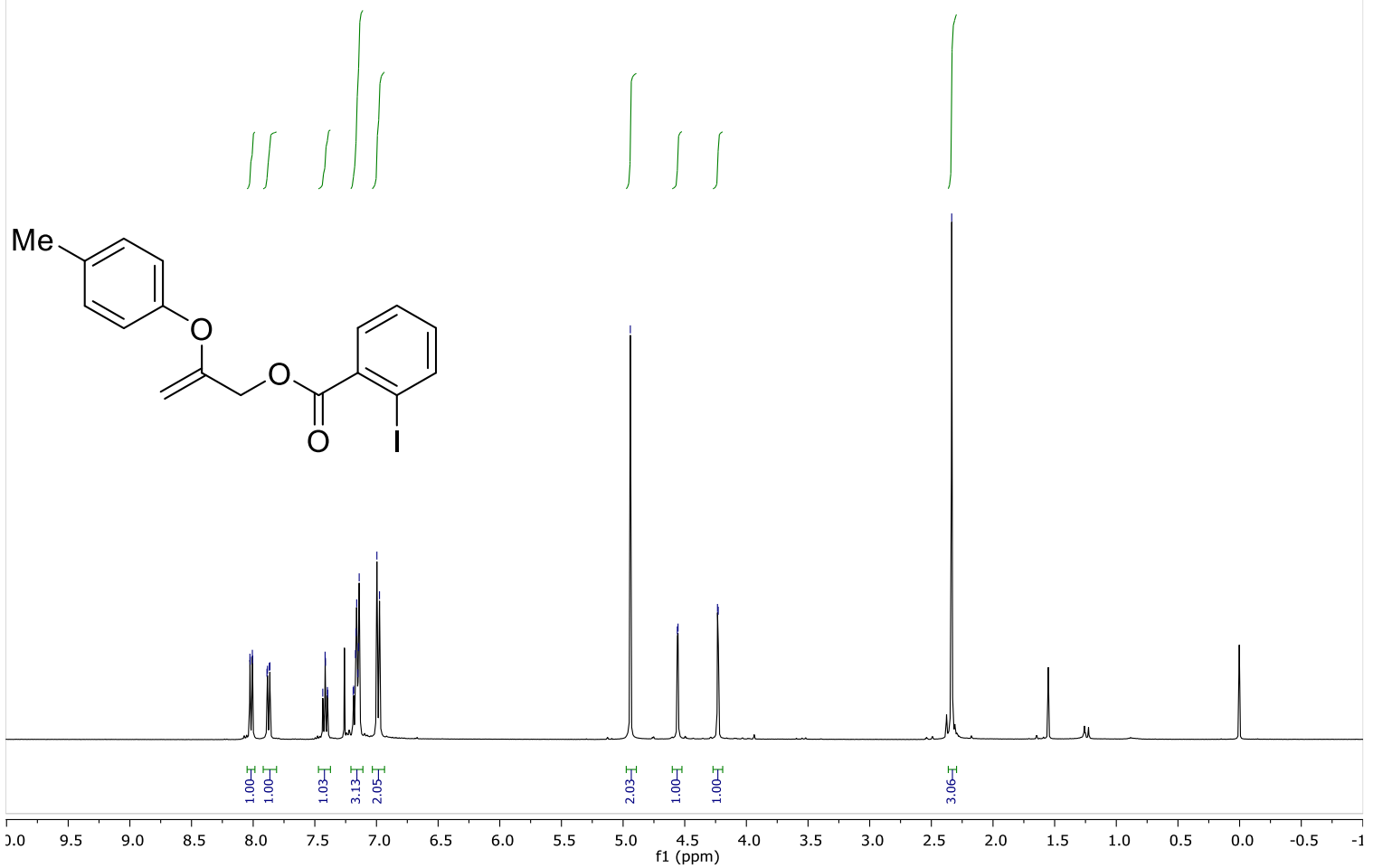

${ }^{13} \mathrm{C}$ NMR (101 MHz, Chloroform-d)

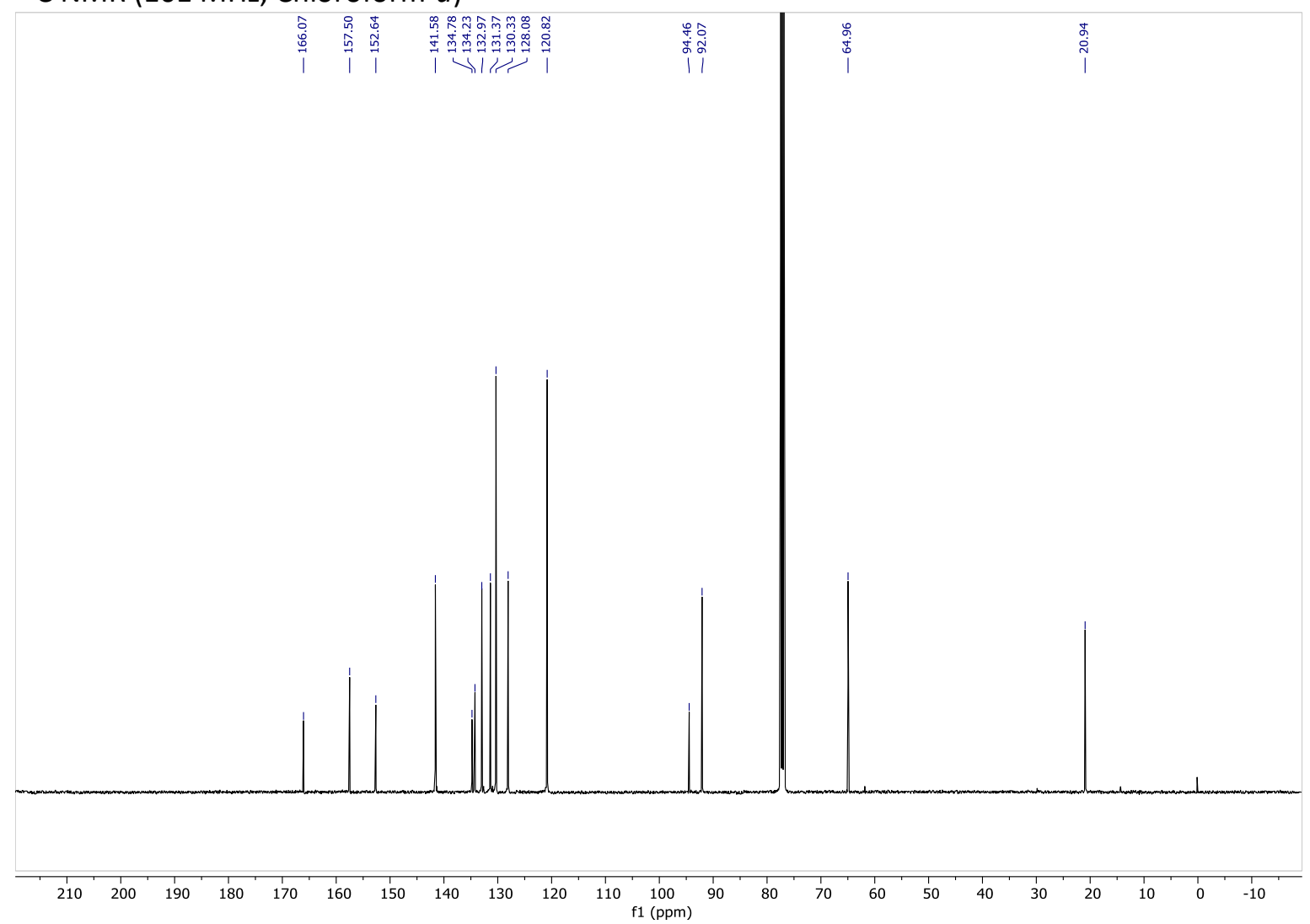


2-(p-tolyloxy)pent-1-en-3-yl 2-iodobenzoate (4b)

${ }^{1} \mathrm{H}$ NMR (400 MHz, Chloroform-d)

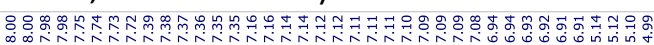

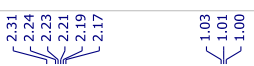

1111
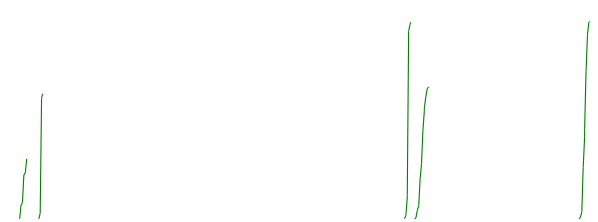<smiles>CC/C=C(/COC(=O)c1ccccc1I)Oc1ccc(C)cc1</smiles>
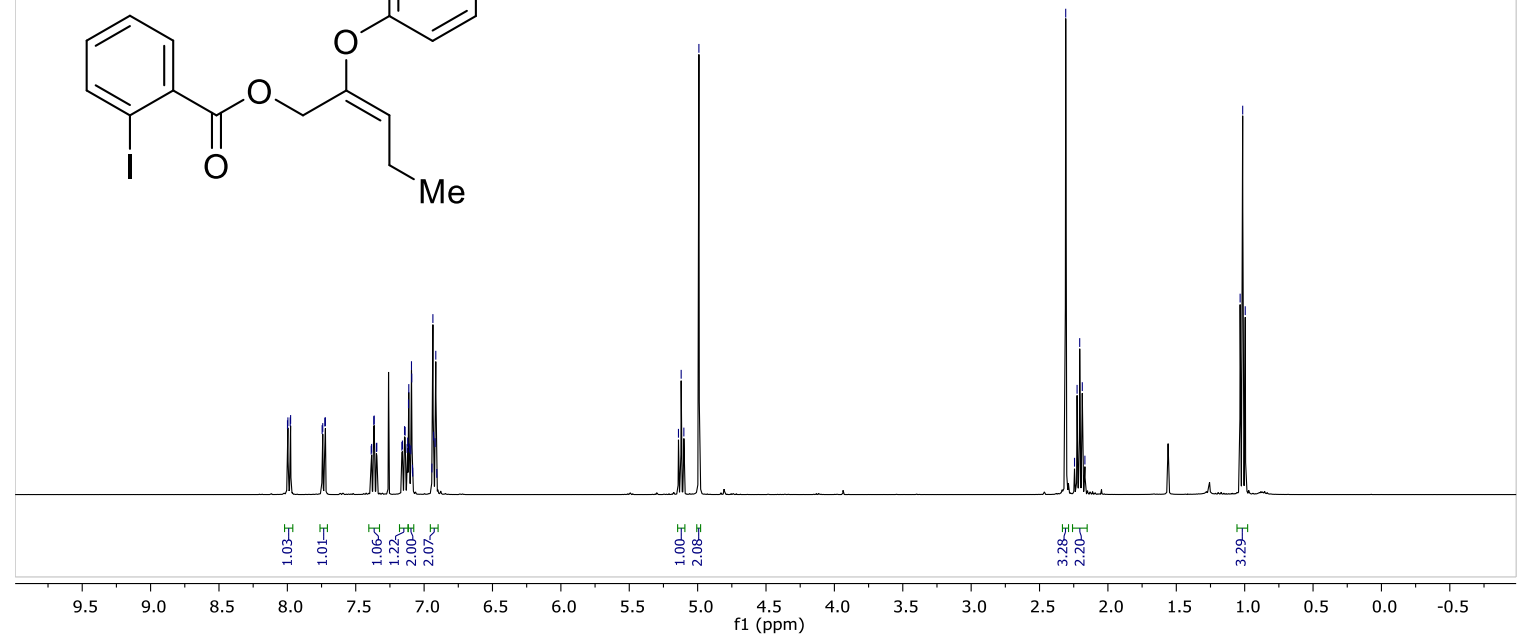

${ }^{13} \mathrm{C}$ NMR (101 MHz, Chloroform-d)

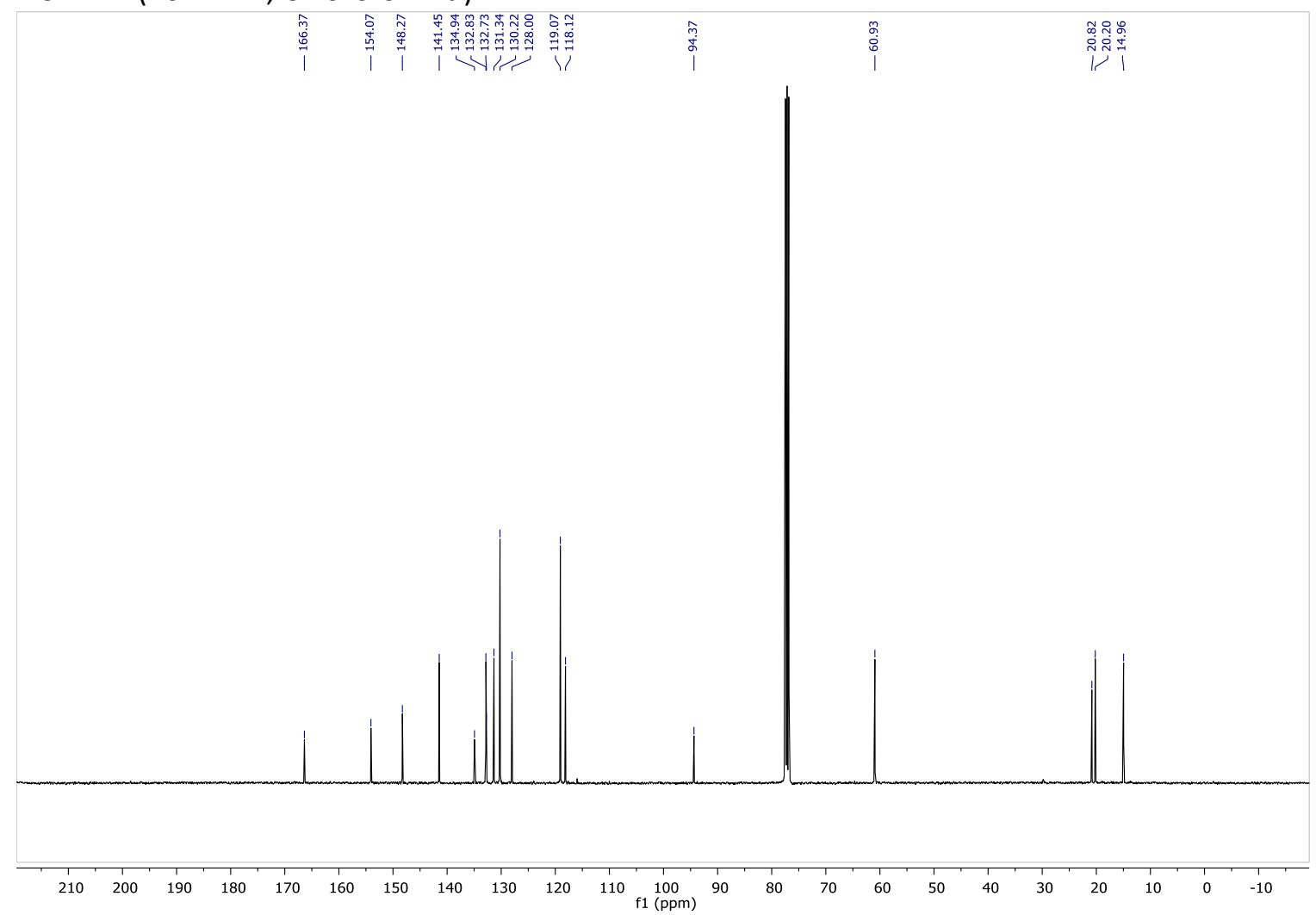


(E)-2-(p-Tolyloxy)oct-2-en-1-yl 2-iodobenzoate (4c)

${ }^{1} \mathrm{H}$ NMR (400 MHz, Chloroform-d)

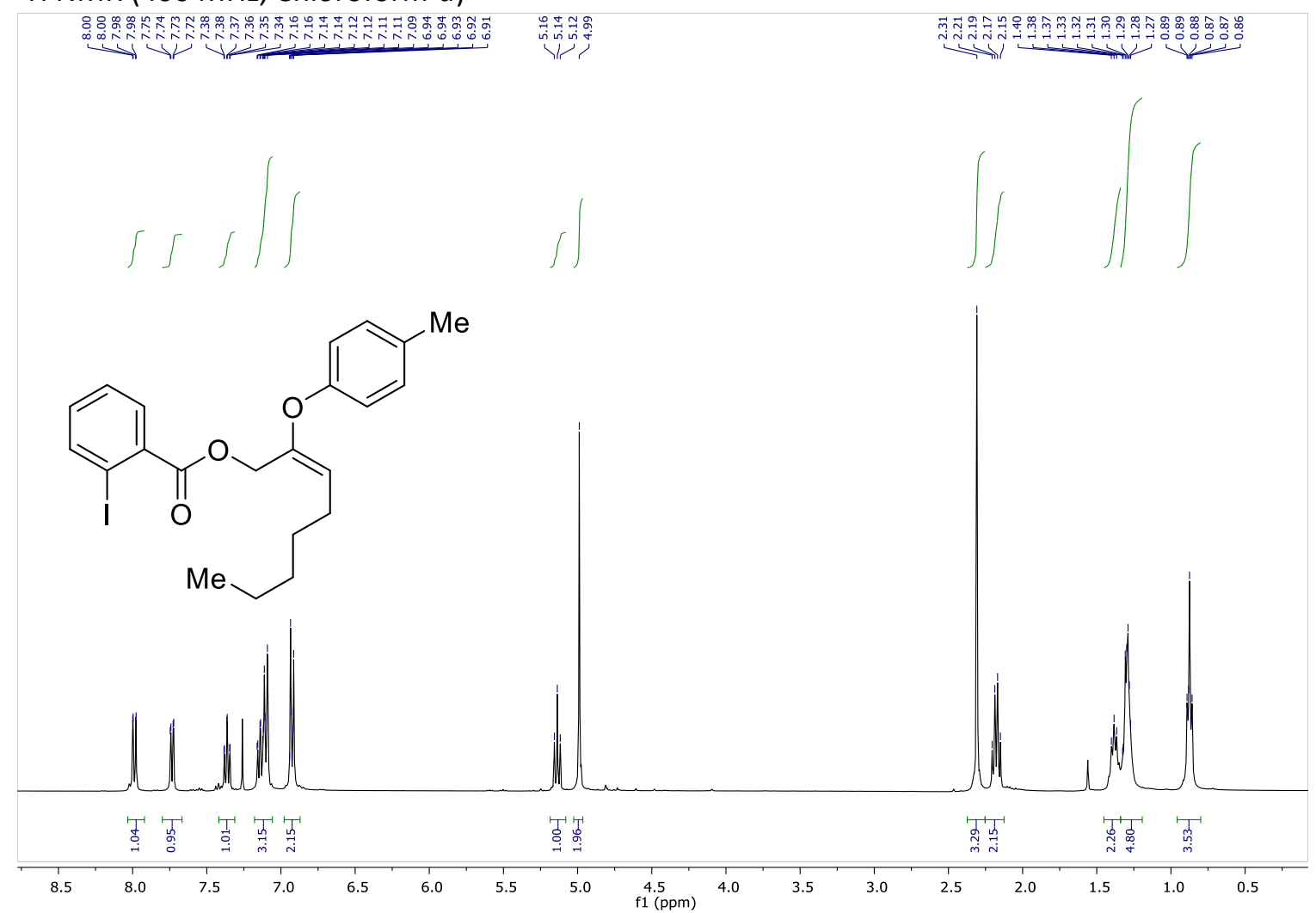

${ }^{13} \mathrm{C}$ NMR (101 MHz, Chloroform-d)

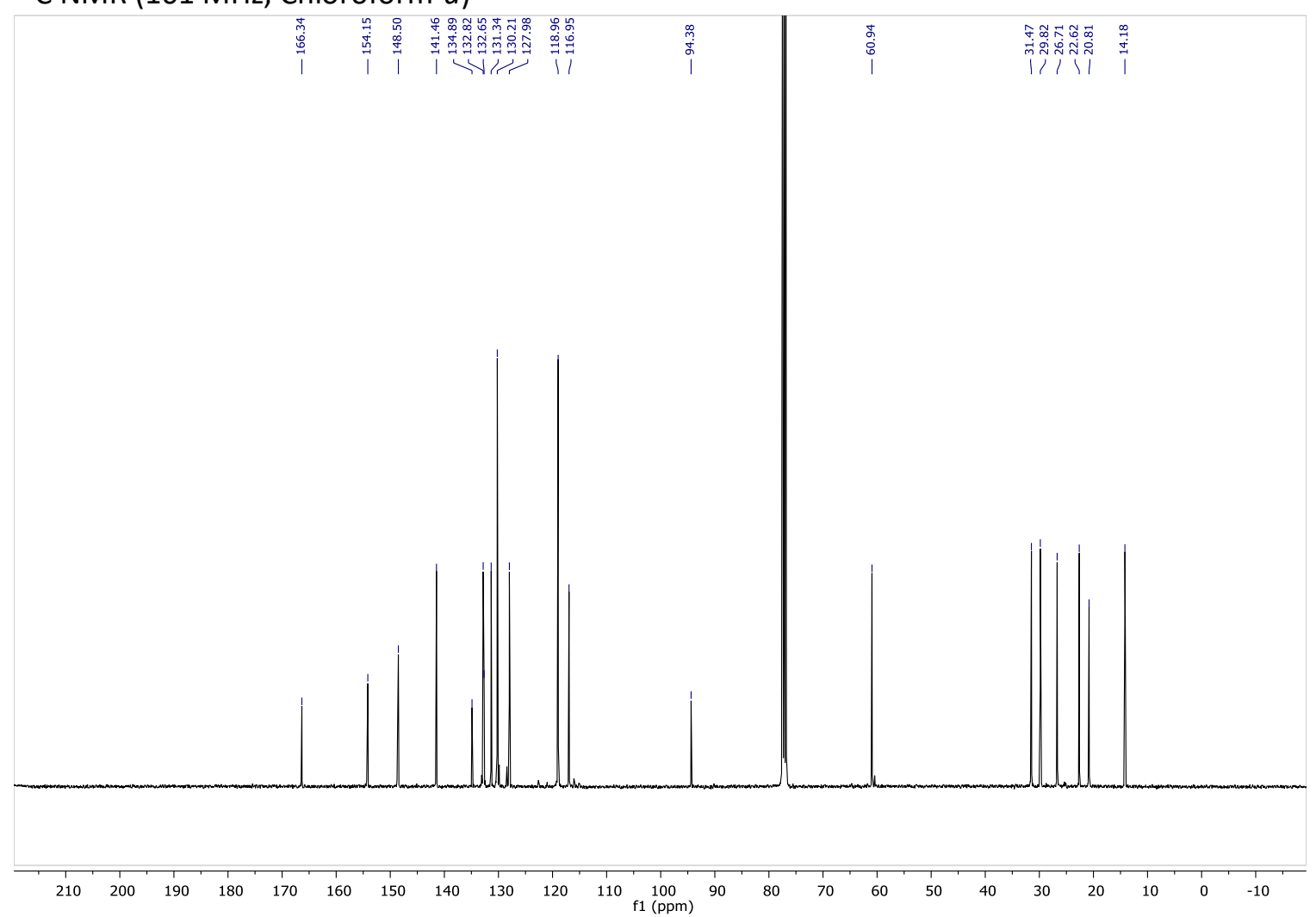


(E)-2-(p-tolyloxy)hexadec-2-en-1-yl 2-iodobenzoate (4d)

${ }^{1} \mathrm{H}$ NMR (400 MHz, Methylene Chloride- $d_{2}$ )

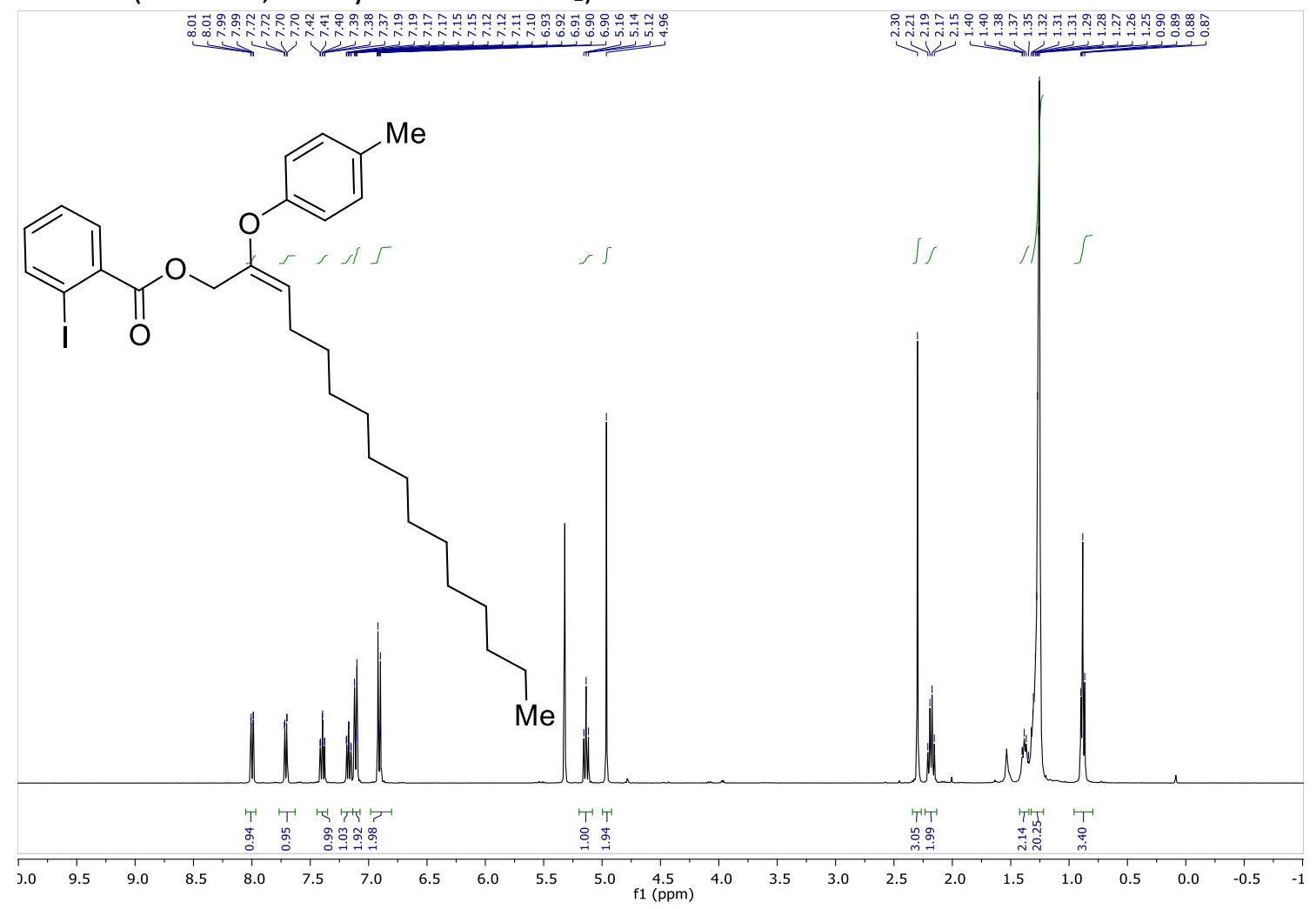

${ }^{13} \mathrm{C}$ NMR (101 MHz, Methylene Chloride- $\left.d_{2}\right)$

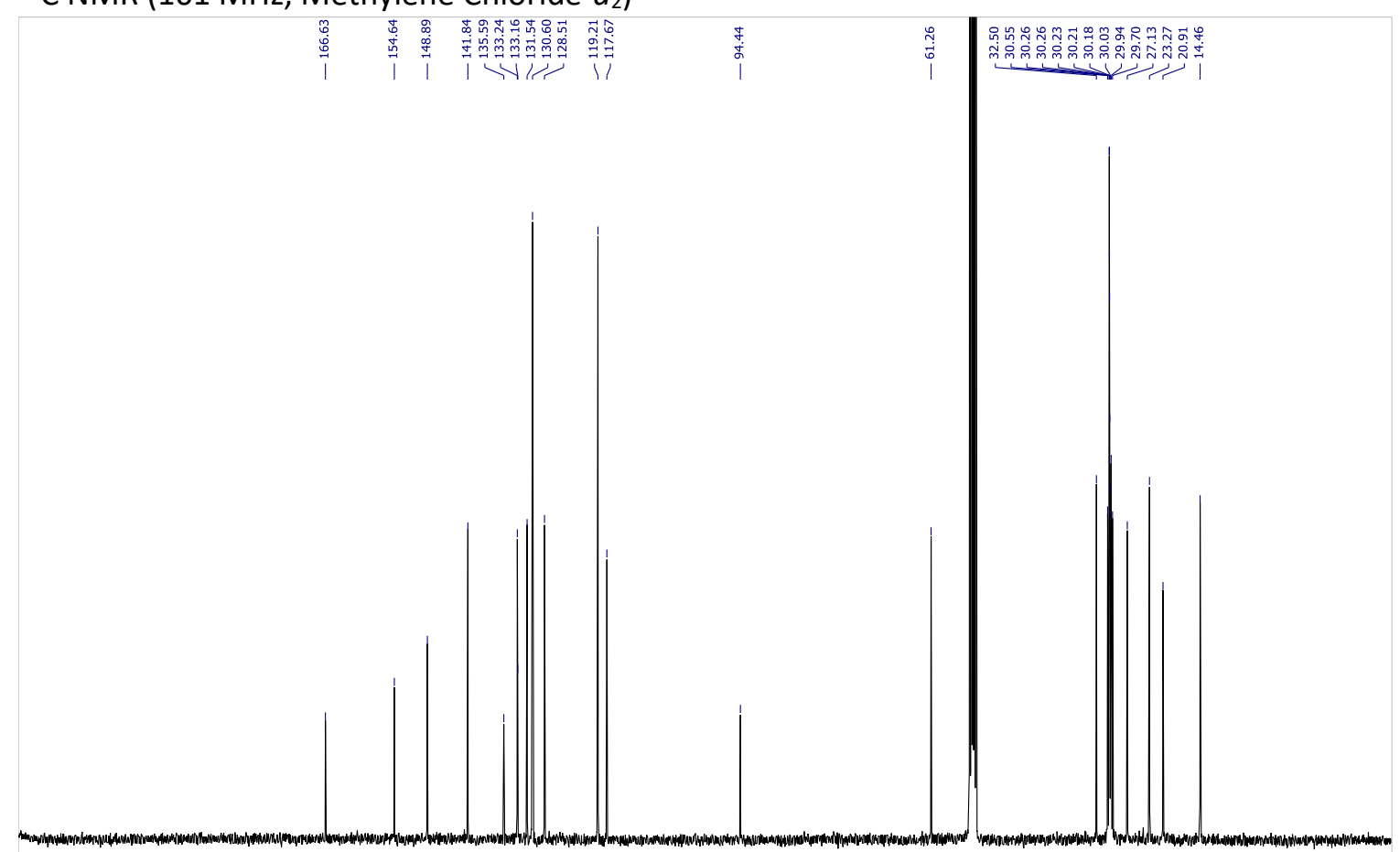

$\begin{array}{llllllllllllllllllllllllllllll}20 & 210 & 200 & 190 & 180 & 170 & 160 & 150 & 140 & 130 & 120 & 110 & 100 & 90 & 80 & 70 & 60 & 50 & 40 & 30 & 20 & 10 & 0 & -10\end{array}$ 
(E)-2-(p-tolyloxy)-8-(trimethylsilyl)oct-2-en-7-yn-1-yl 2-iodobenzoate (4e)

${ }^{1} \mathrm{H}$ NMR (400 MHz, Chloroform-d)

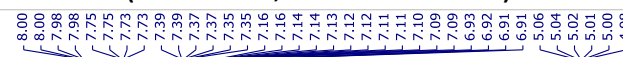

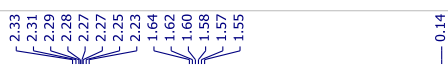

$1,1 / 1$<smiles>CC#CCCCC=C(COC(=O)c1ccccc1I)Oc1ccc(C)cc1</smiles>

TMS

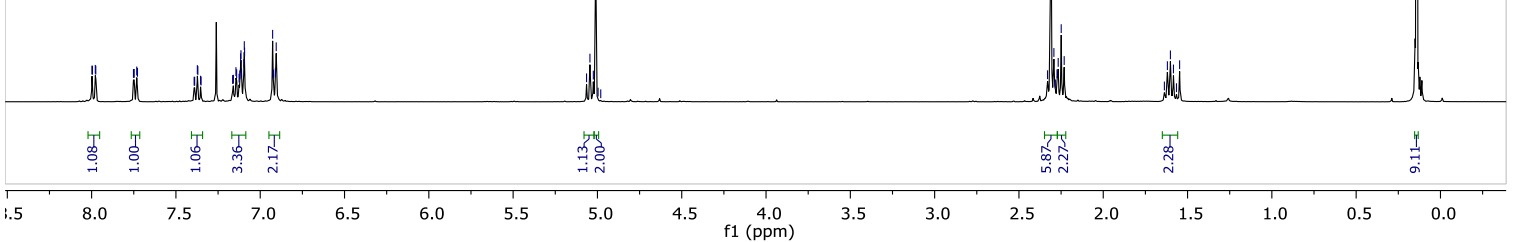

${ }^{13} \mathrm{C}$ NMR (101 MHz, Chloroform-d)

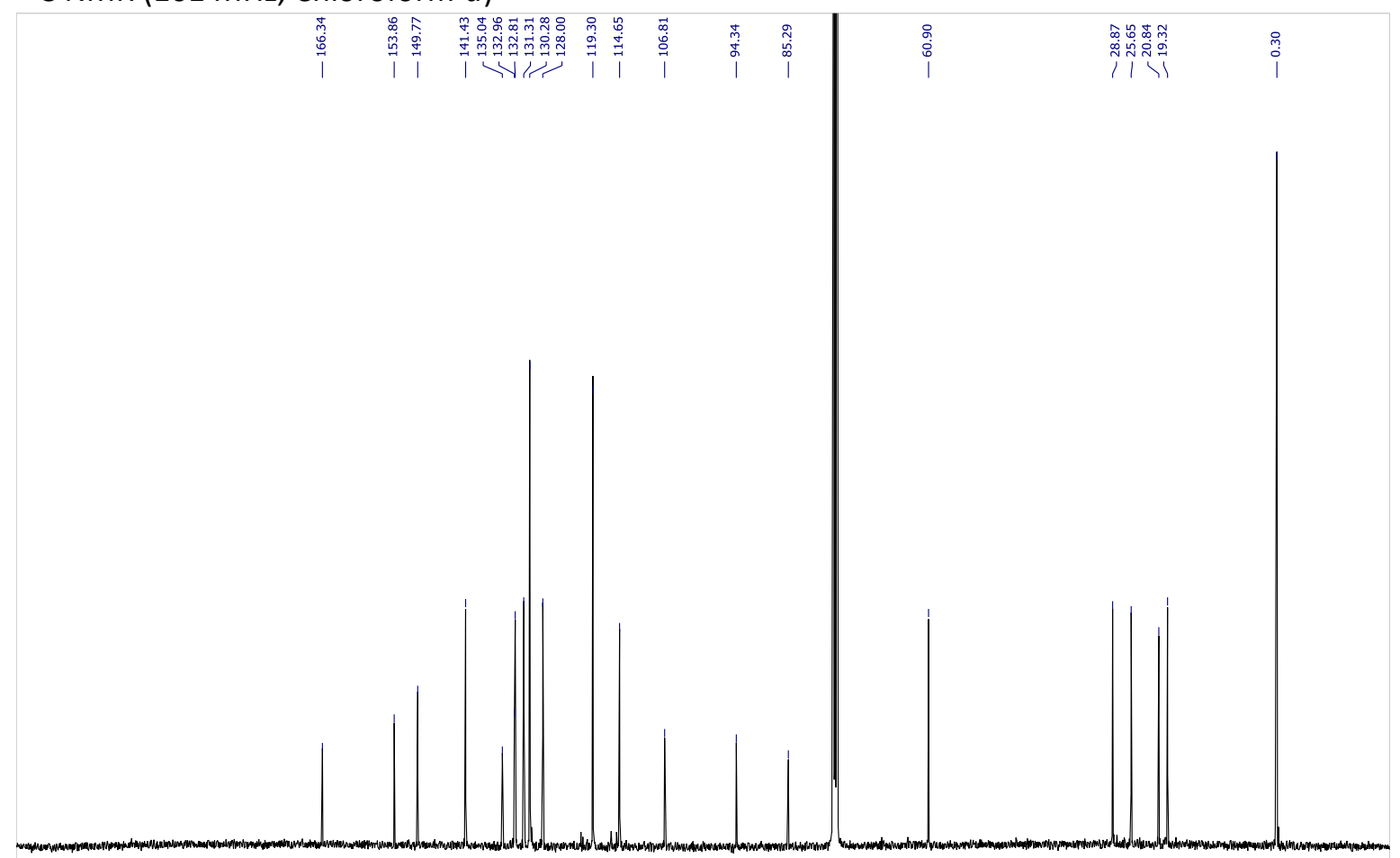

$\begin{array}{llllllllllll}210 & 200 & 190 & 180 & 170 & 160 & 150 & 140 & 130 & 120 & 110 & 100 \\ \mathrm{f} 1(\mathrm{ppm})\end{array}$ 
(E)-6-(1,3-Dioxoisoindolin-2-yl)-2-(p-tolyloxy)hex-2-en-1-yl 2-iodobenzoate (4f)

${ }^{1} \mathrm{H}$ NMR (400 MHz, Acetonitrile- $d_{3}$ )

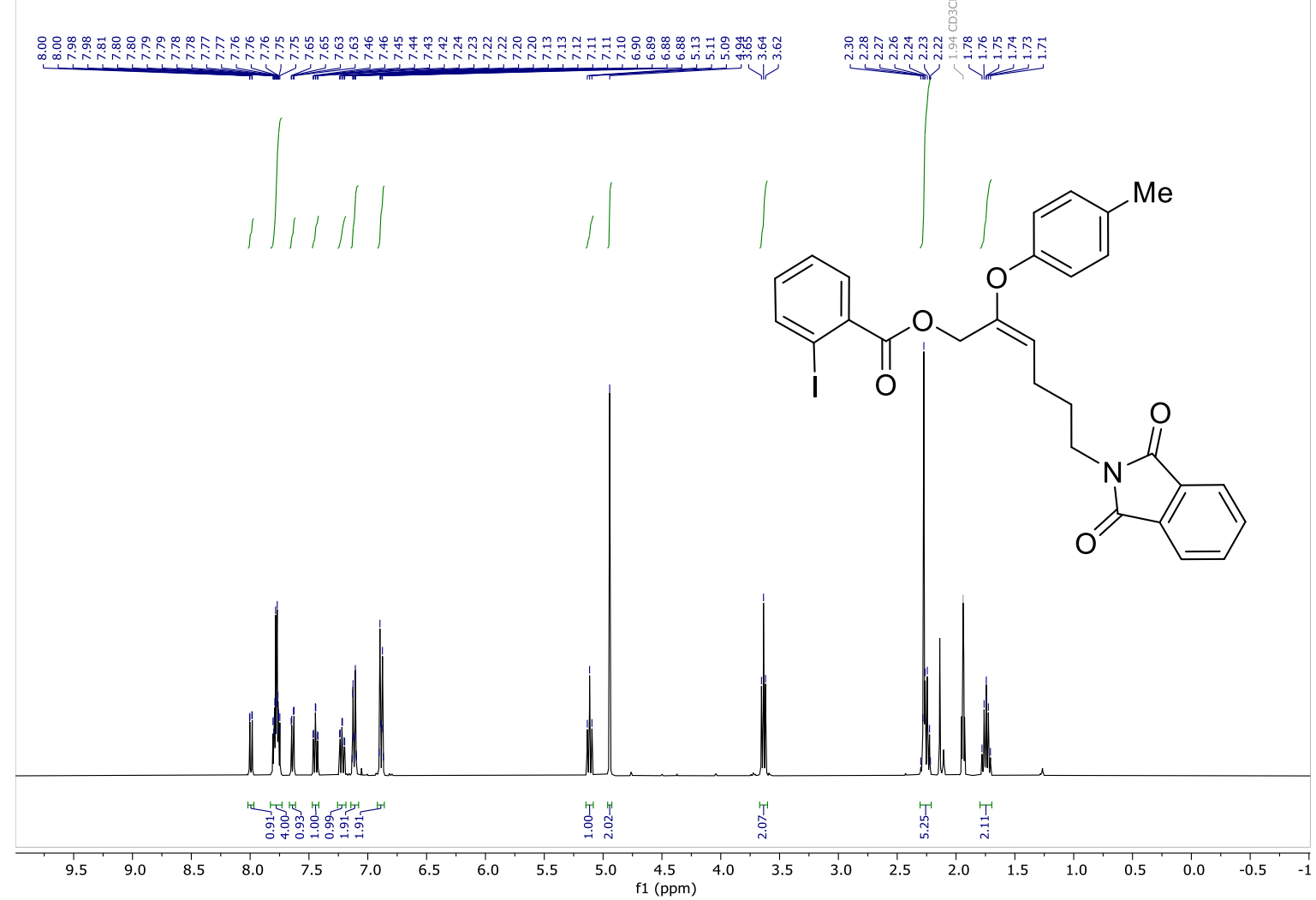

${ }^{13} \mathrm{C}$ NMR (101 MHz, Acetonitrile- $d_{3}$ )

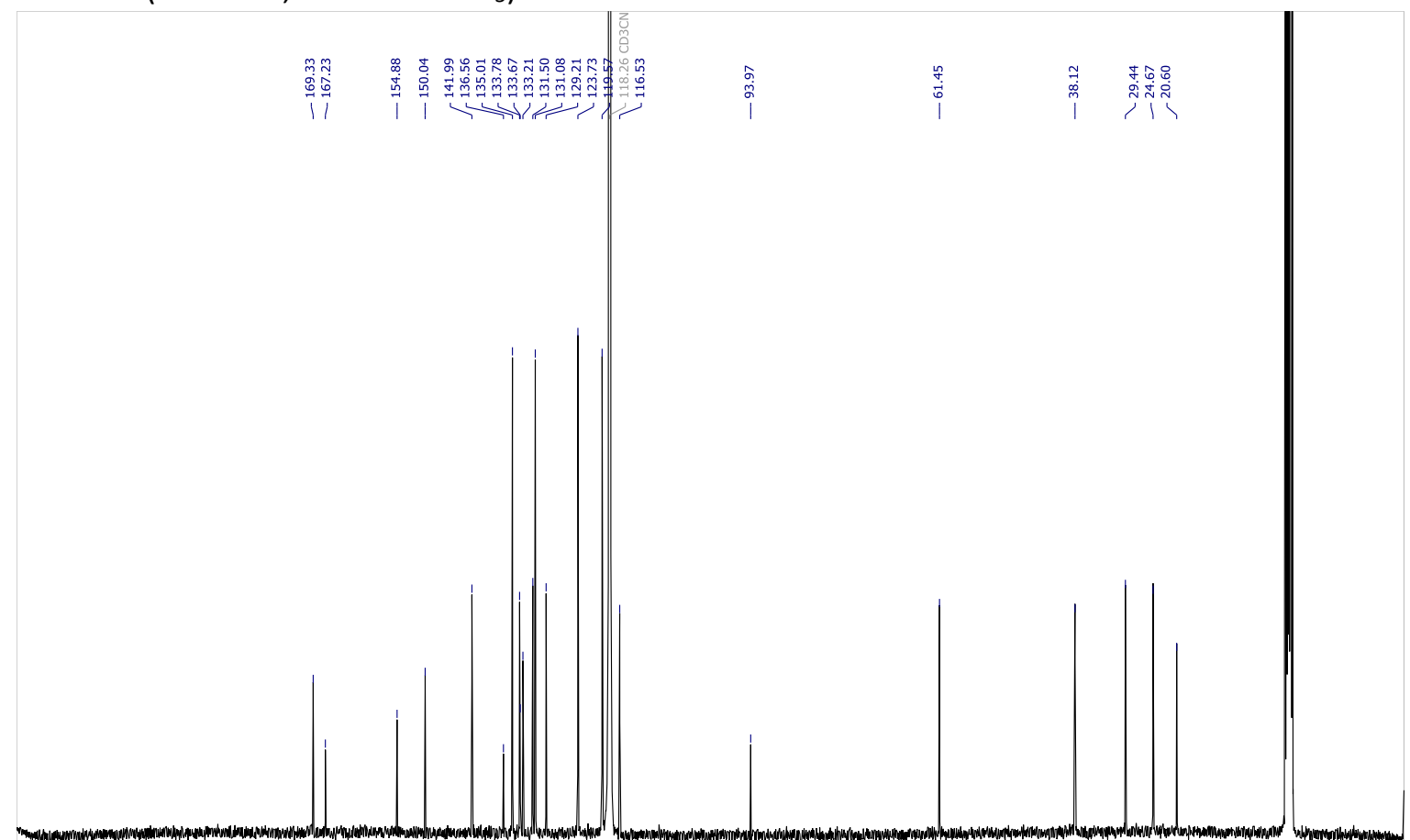

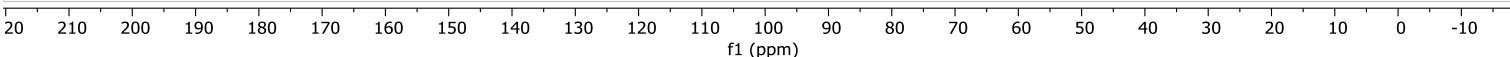


(E)-2-(p-tolyloxy)octa-2,7-dien-1-yl 2-iodobenzoate (4g)

${ }^{1} \mathrm{H}$ NMR (400 MHz, Chloroform-d)

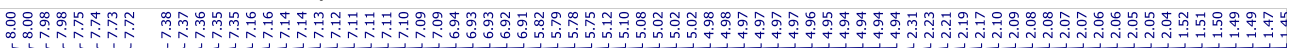

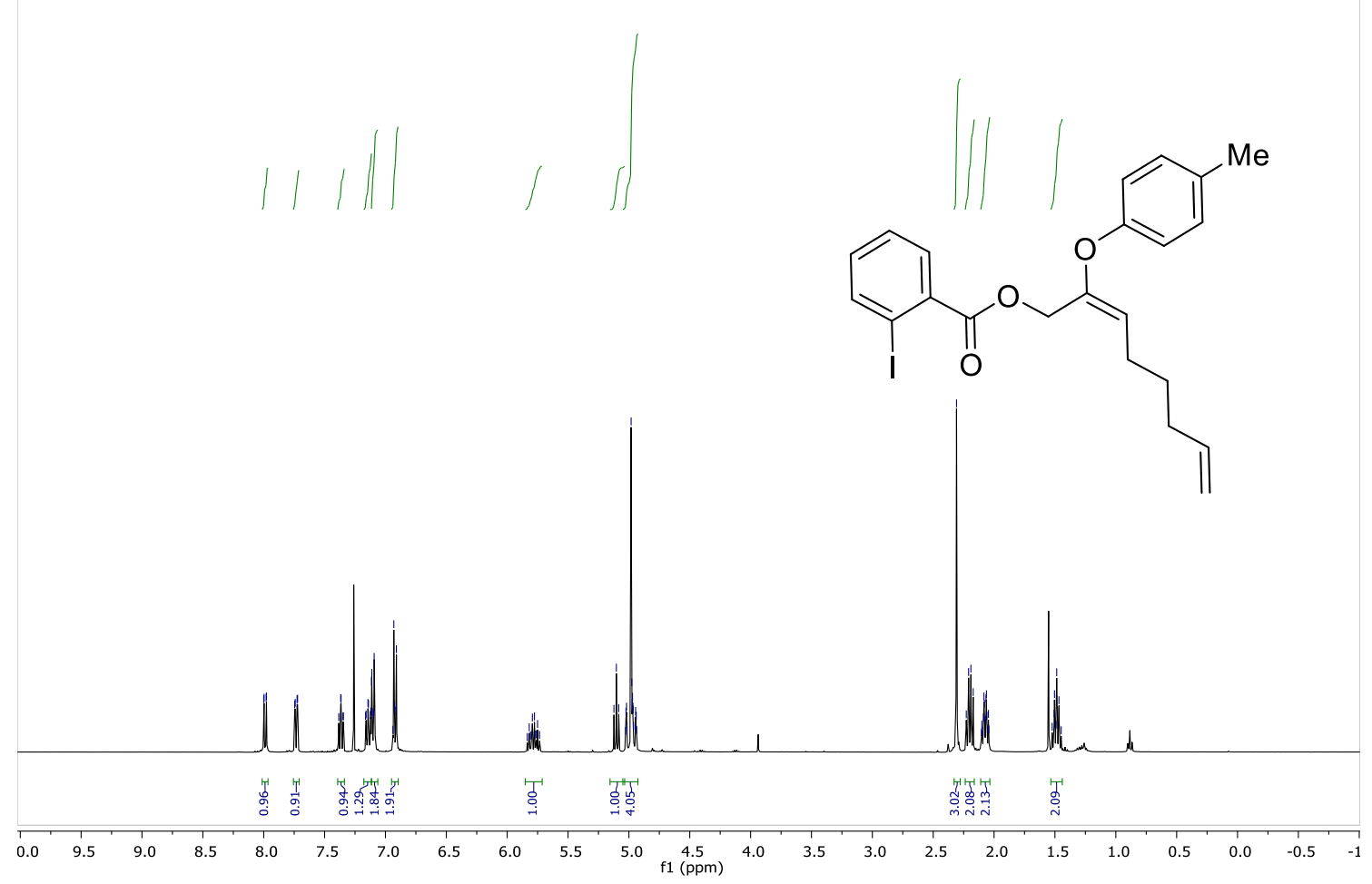

${ }^{13} \mathrm{C}$ NMR $(101 \mathrm{MHz}$, Chloroform- $d$ )

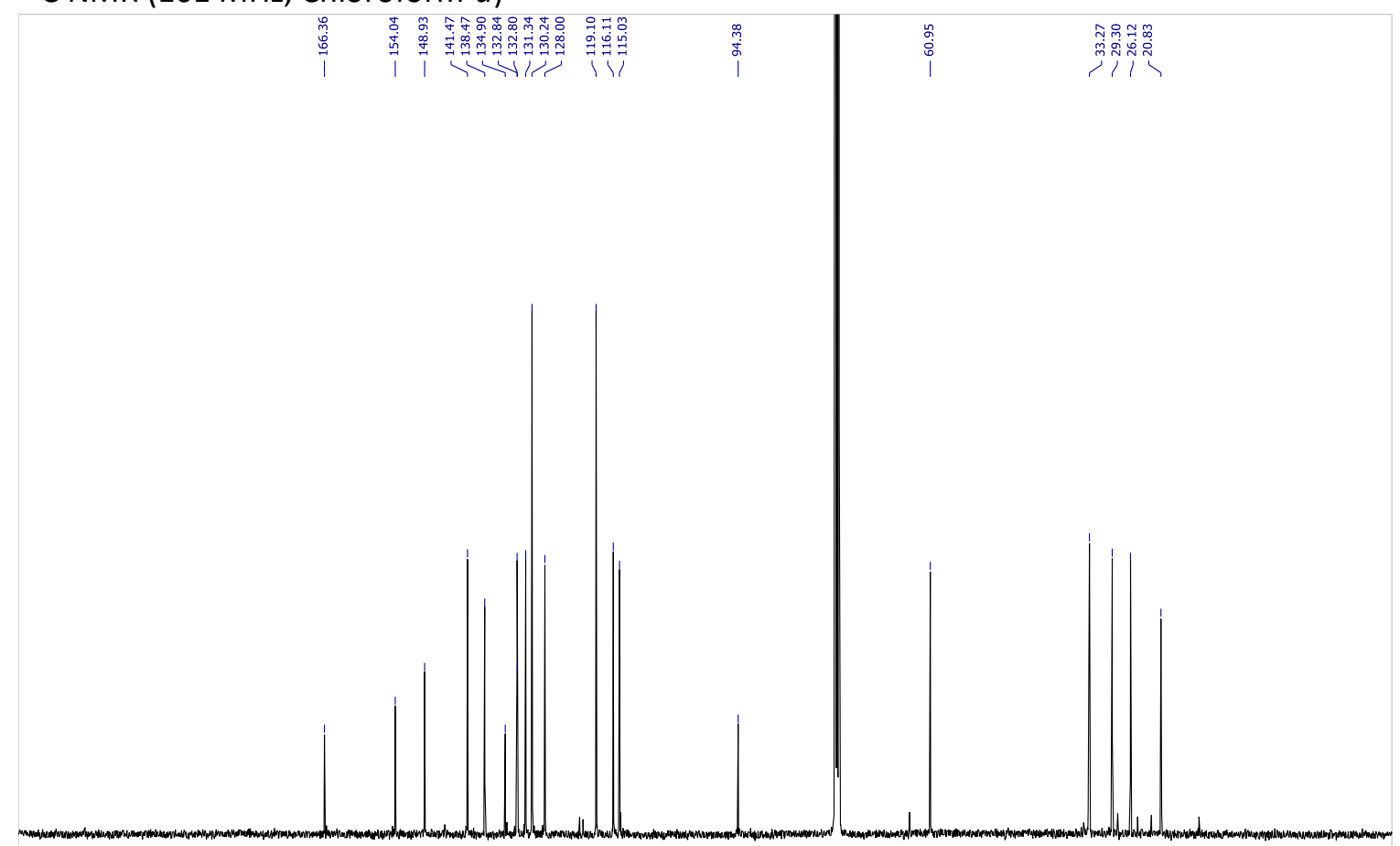

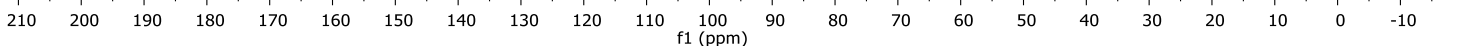


(E)-5-chloro-2-( $p$-tolyloxy)pent-2-en-1-yl 2-iodobenzoate (4h)

${ }^{1} \mathrm{H}$ NMR (400 MHz, Chloroform-d)

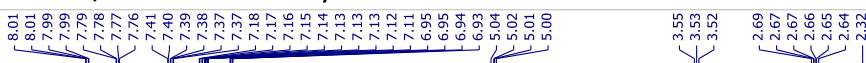

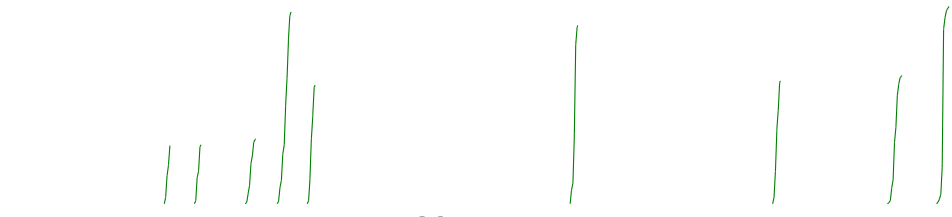<smiles>Cc1ccc(O/C(=C/CCCl)COC(=O)c2ccccc2I)cc1</smiles>

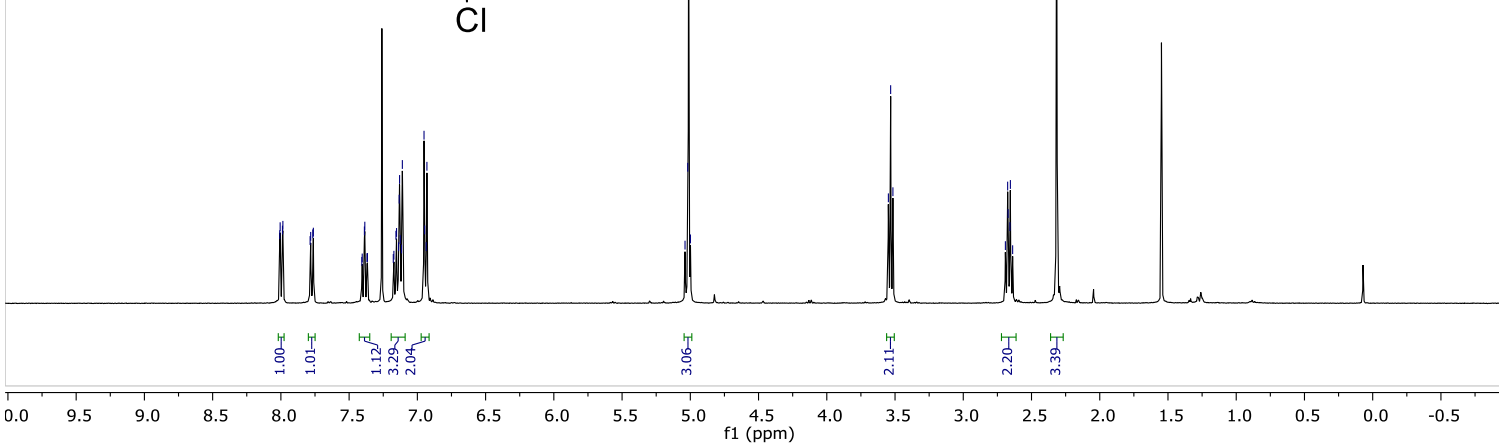

${ }^{13} \mathrm{C}$ NMR (101 MHz, Chloroform-d)

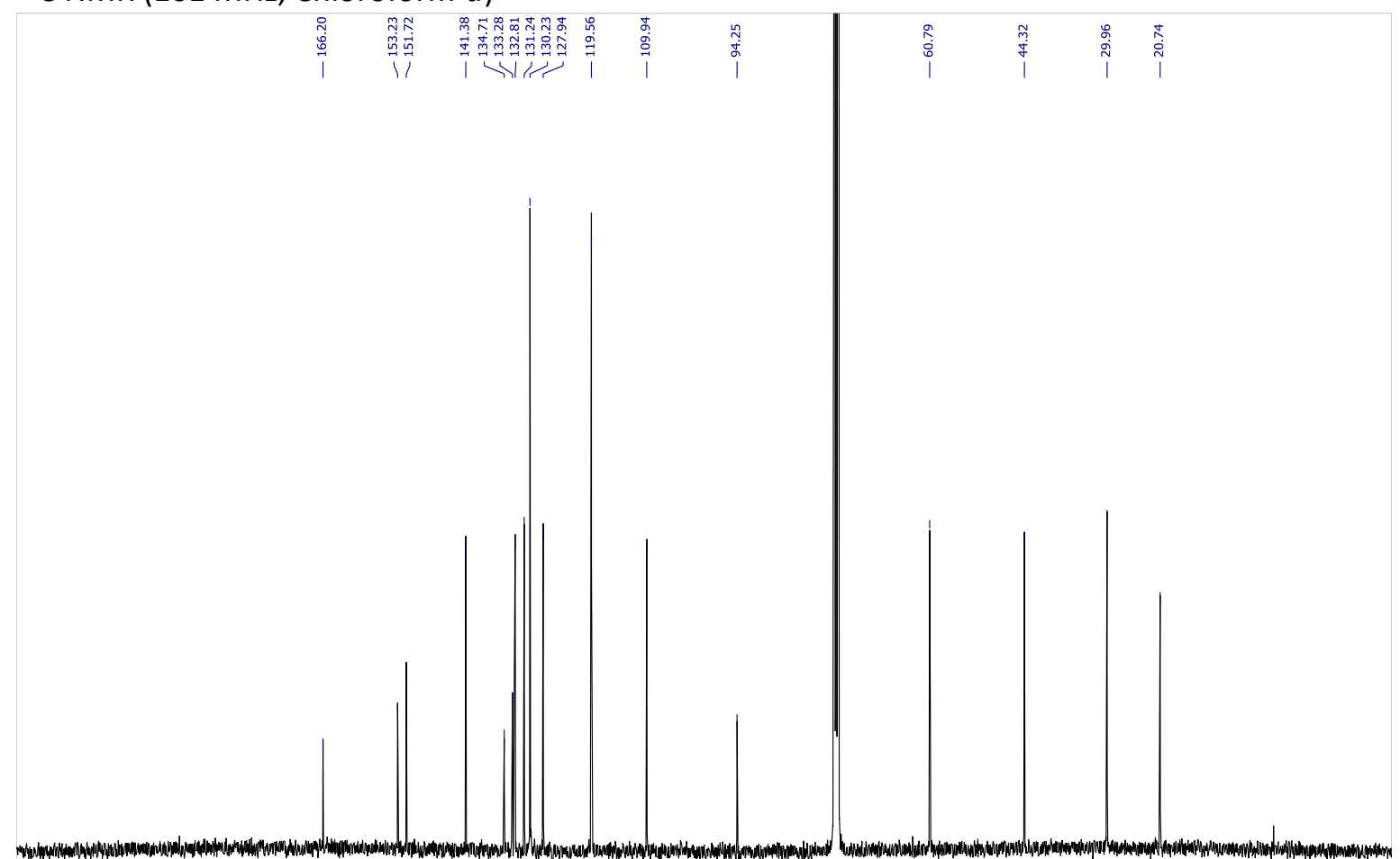

$\begin{array}{llllllllllll}210 & 200 & 190 & 180 & 170 & 160 & 150 & 140 & 130 & 120 & 110 \underset{\mathrm{f} 1(\mathrm{ppm})}{100} & 90\end{array}$ 
2-(2-lodophenoxy)allyl 2-iodobenzoate (4i)

${ }^{1} \mathrm{H}$ NMR $\left(400 \mathrm{MHz}\right.$, Acetonitrile- $d_{3}$ )

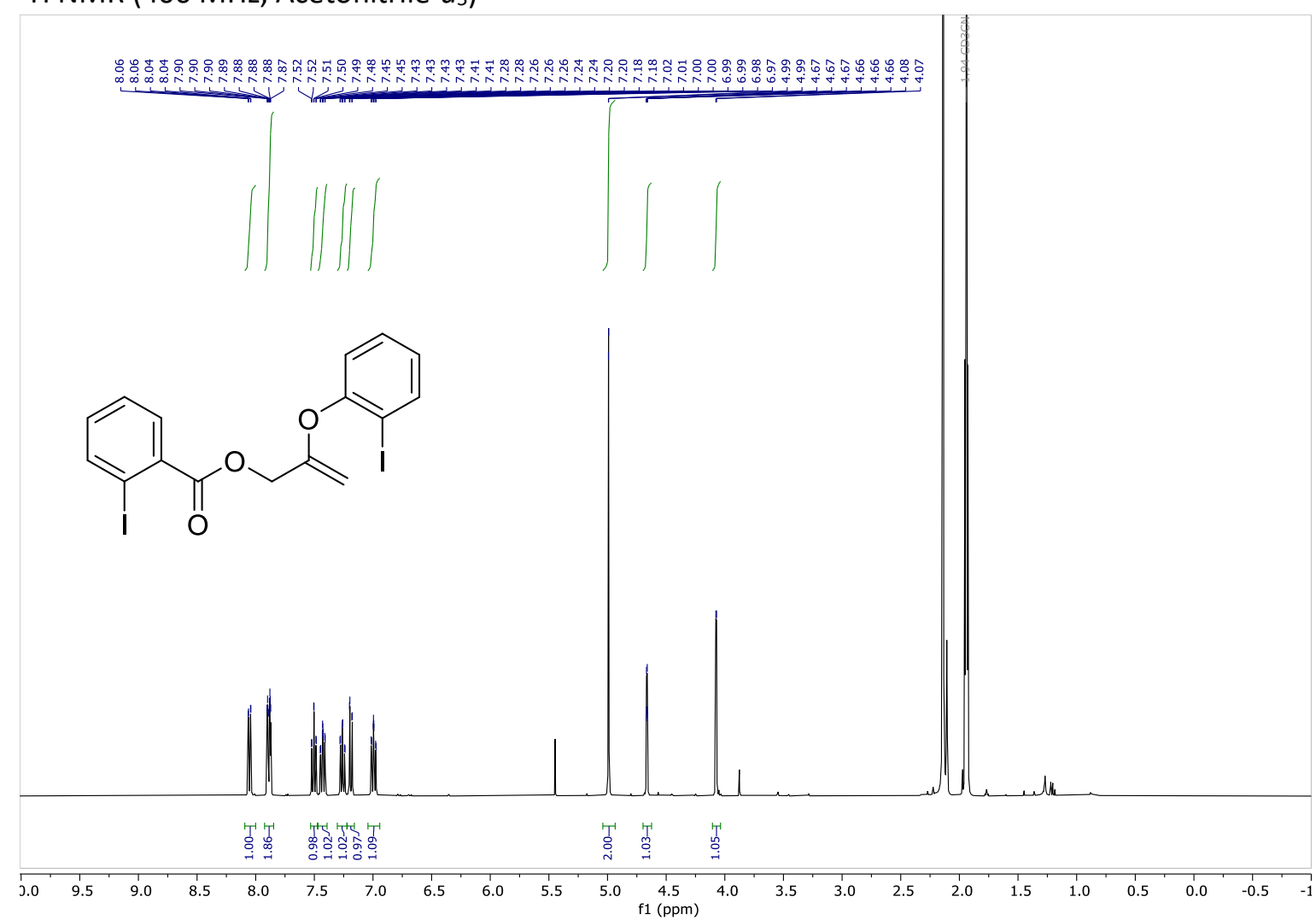

${ }^{13} \mathrm{C}$ NMR (101 MHz, Acetonitrile- $d_{3}$ )

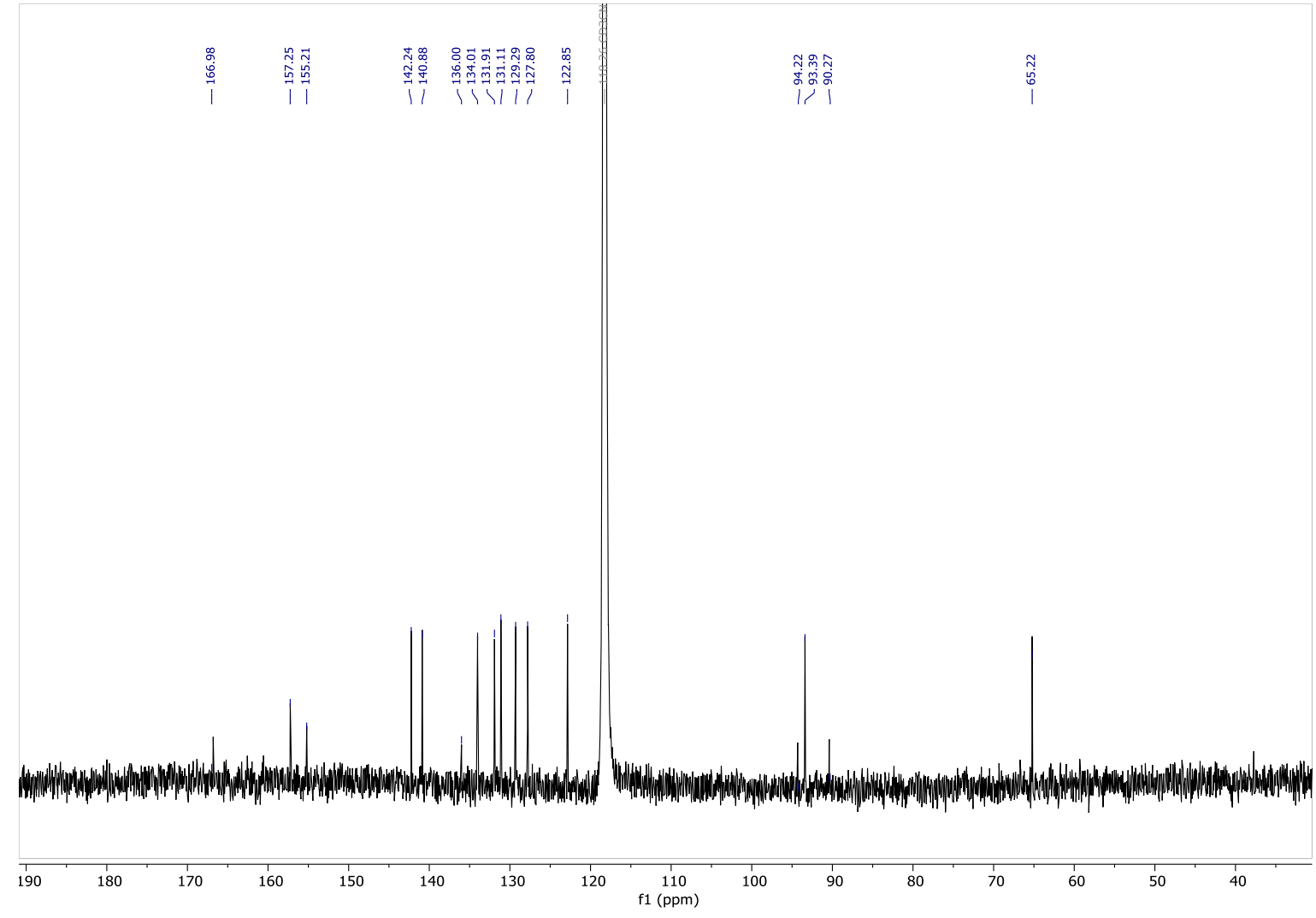


(E)-2-(2-iodophenoxy)pent-2-en-1-yl 2-iodobenzoate (4j)

${ }^{1} \mathrm{H}$ NMR $\left(400 \mathrm{MHz}\right.$, Acetonitrile- $\left.d_{3}\right)$

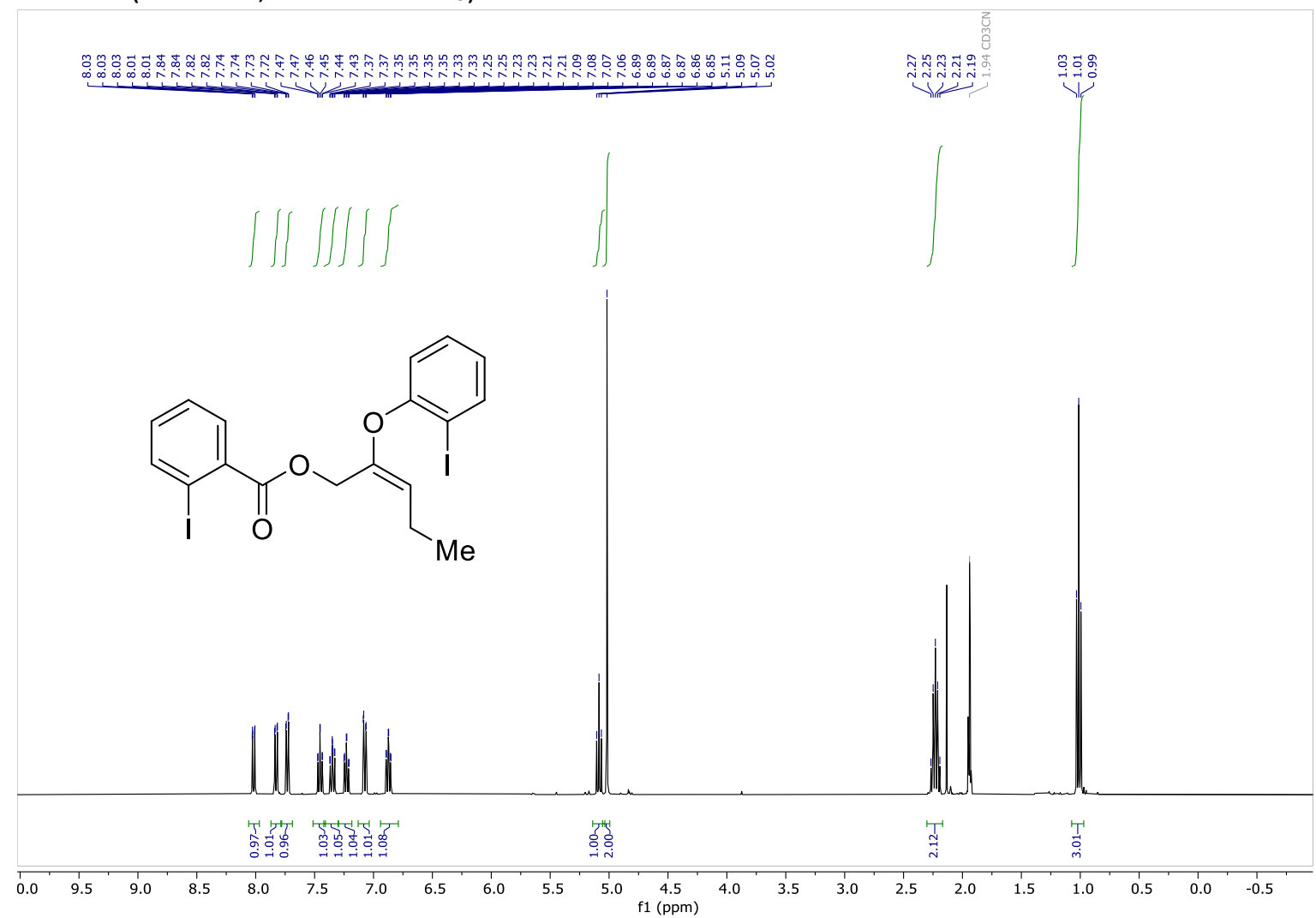

${ }^{13} \mathrm{C}$ NMR (101 MHz, Acetonitrile- $d_{3}$ )

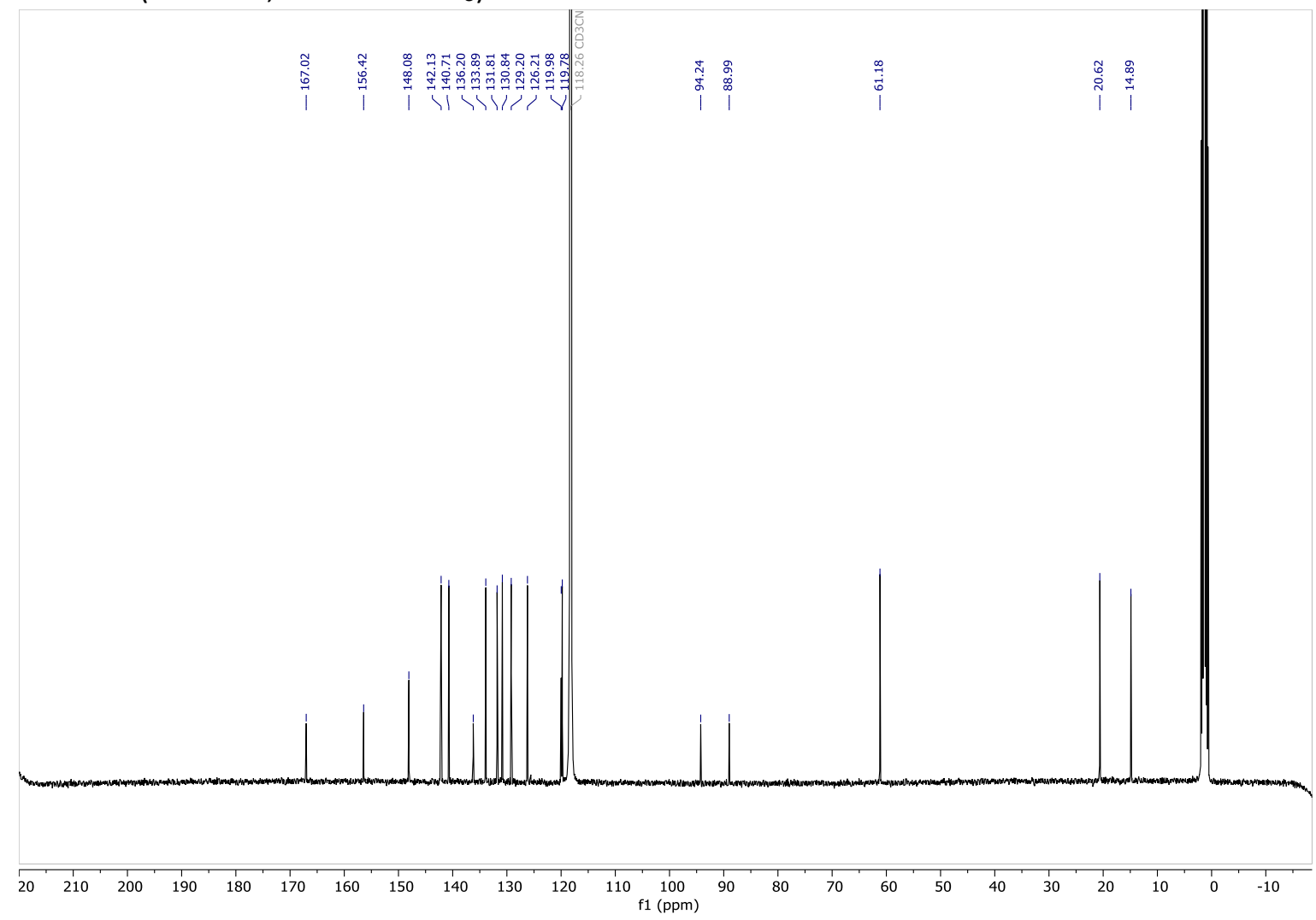


(E)-2-(2-iodophenoxy)oct-2-en-1-yl 2-iodobenzoate (4k)

${ }^{1} \mathrm{H}$ NMR (400 MHz, Acetonitrile- $d_{3}$ )

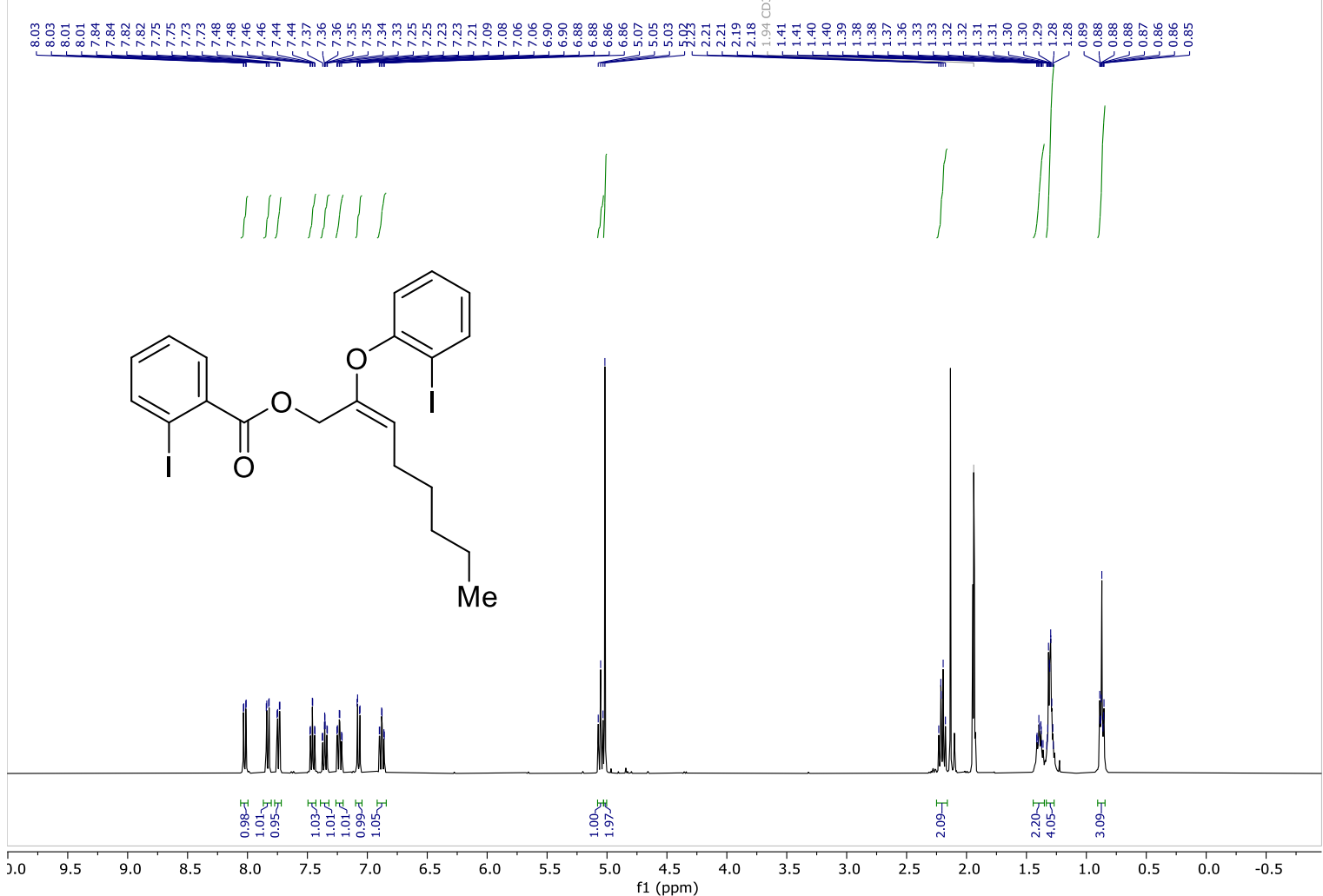

${ }^{13} \mathrm{C}$ NMR (101 MHz, Acetonitrile- $\left.d_{3}\right)$

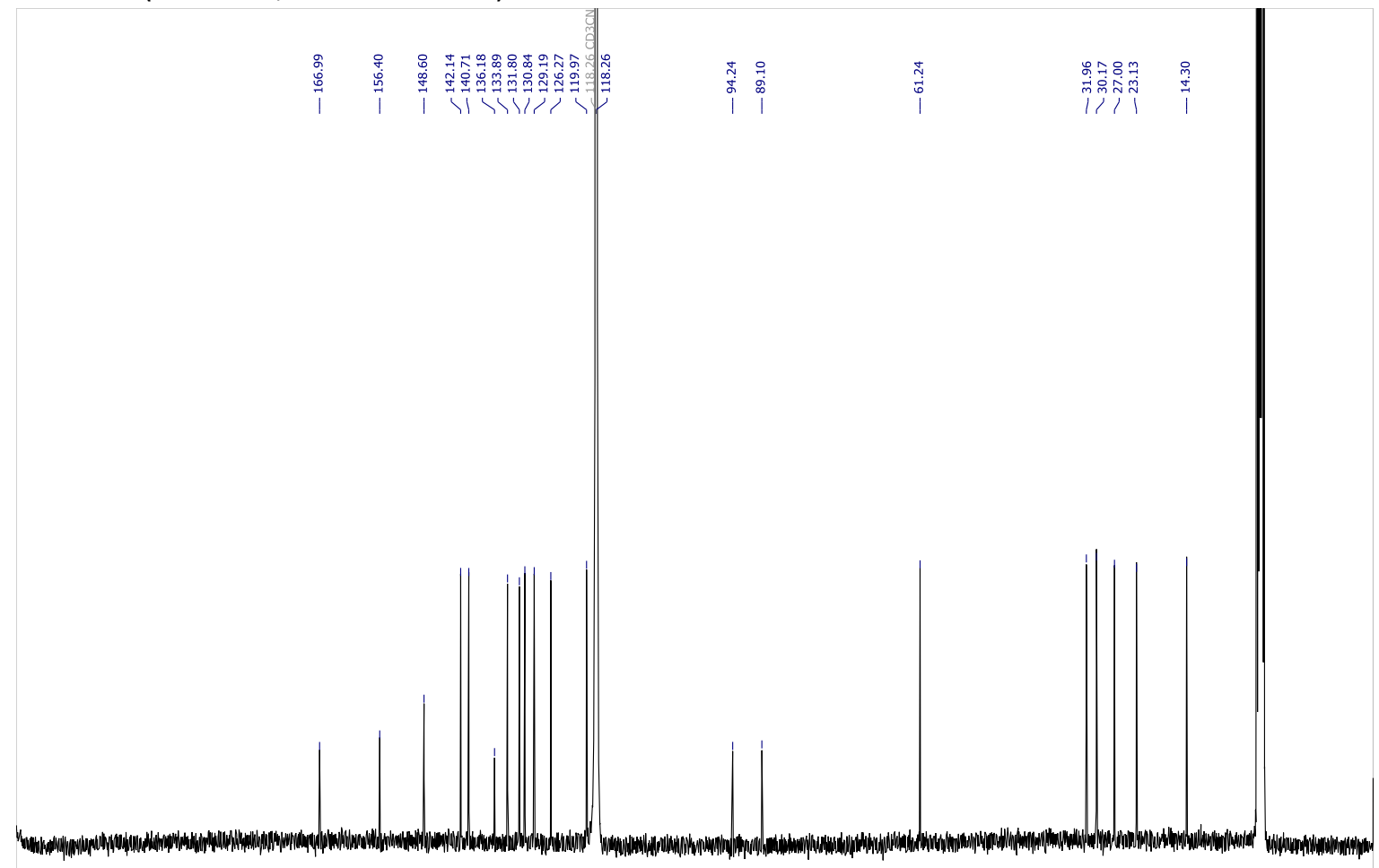

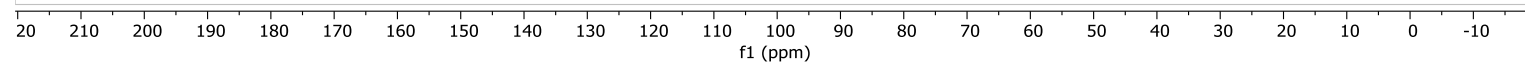


(E)-2-(3-bromophenoxy)-5-chloropent-2-en-1-yl 2-iodobenzoate (4I)

${ }^{1} \mathrm{H}$ NMR (400 MHz, Chloroform-d)

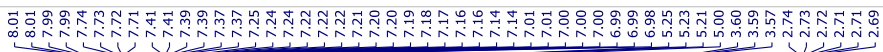

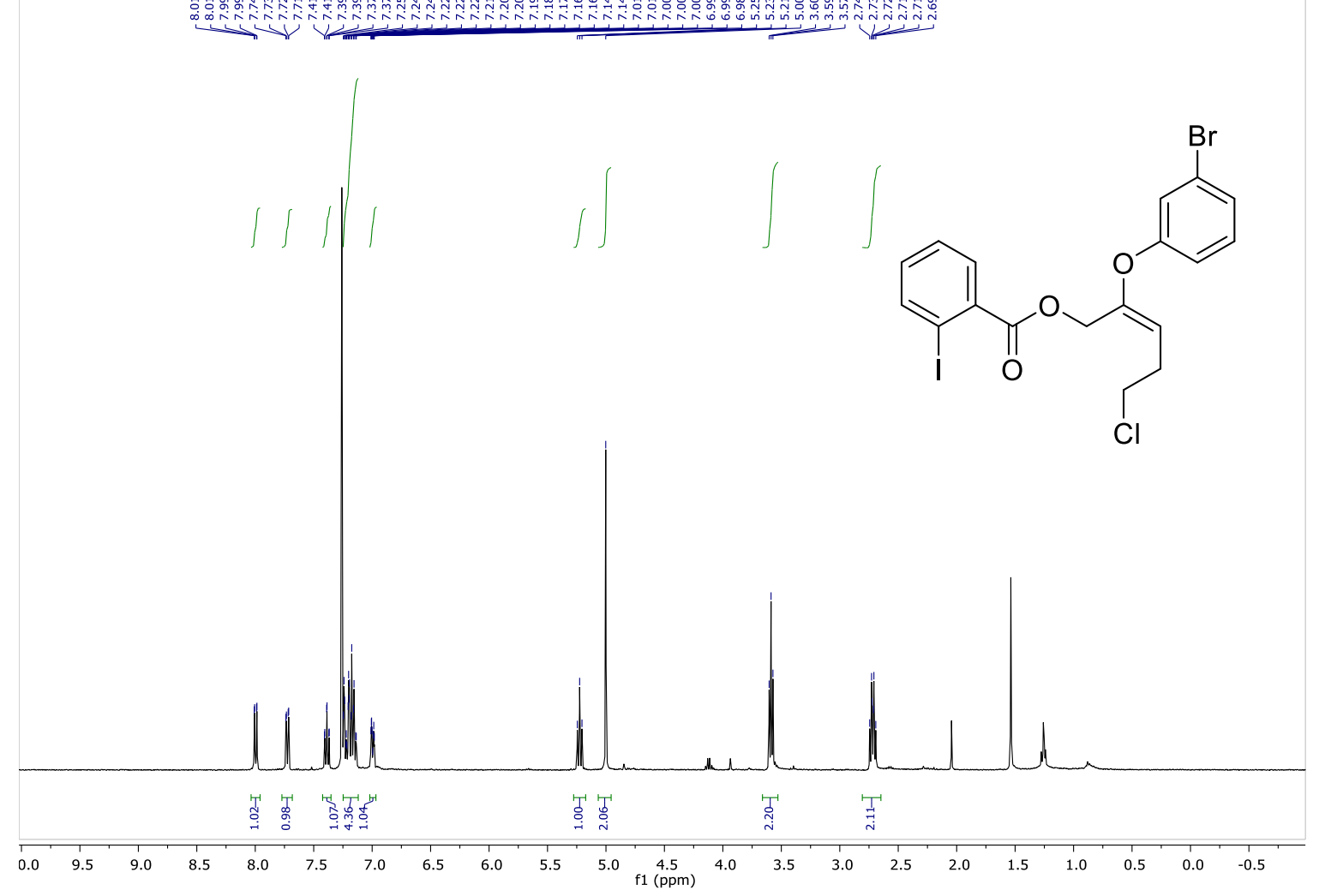

${ }^{13} \mathrm{C}$ NMR (101 MHz, Chloroform-d)

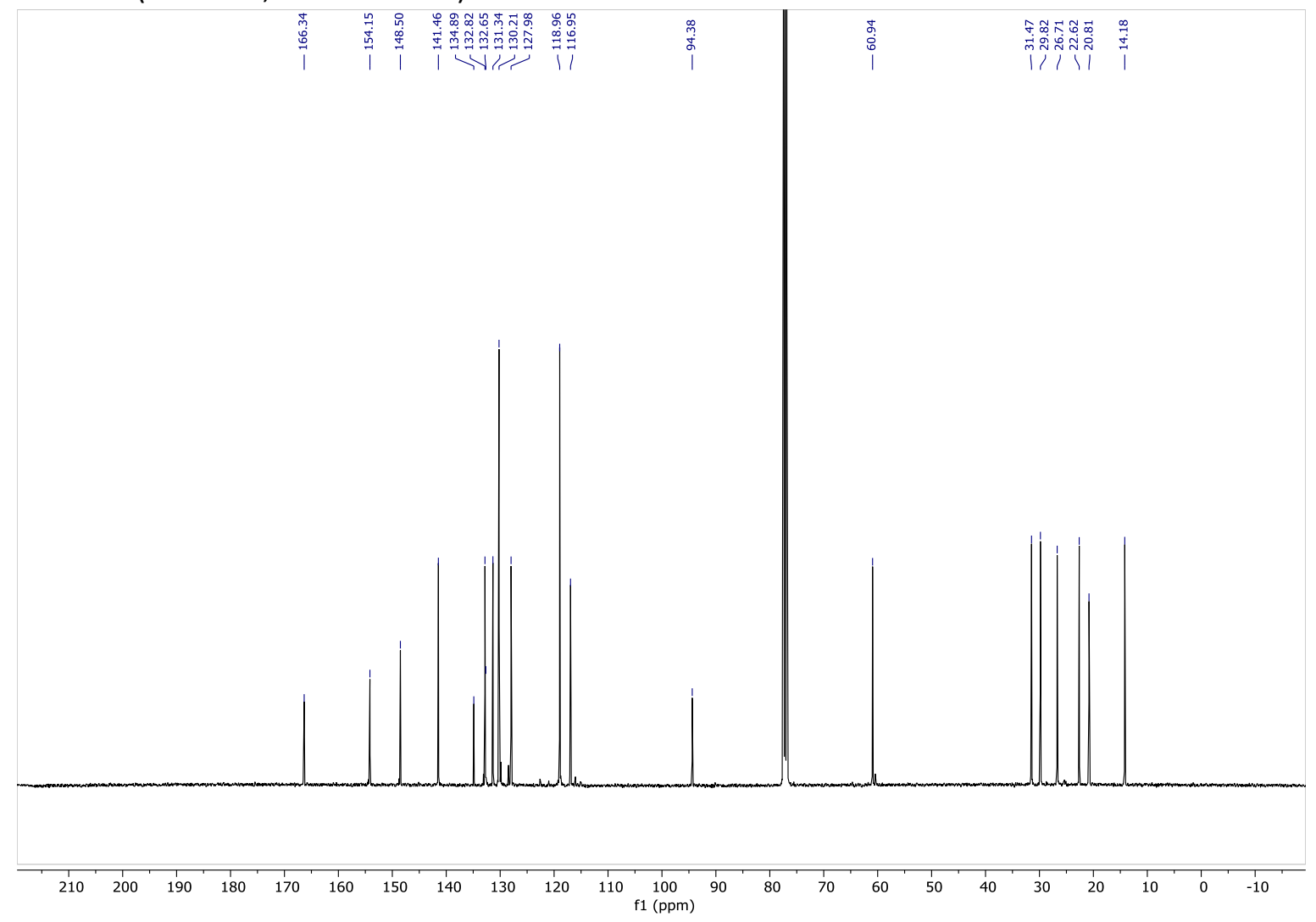


2-(perfluorophenoxy)pent-1-en-3-yl 2-iodobenzoate (4m)

${ }^{1} \mathrm{H}$ NMR (400 MHz, Chloroform-d)

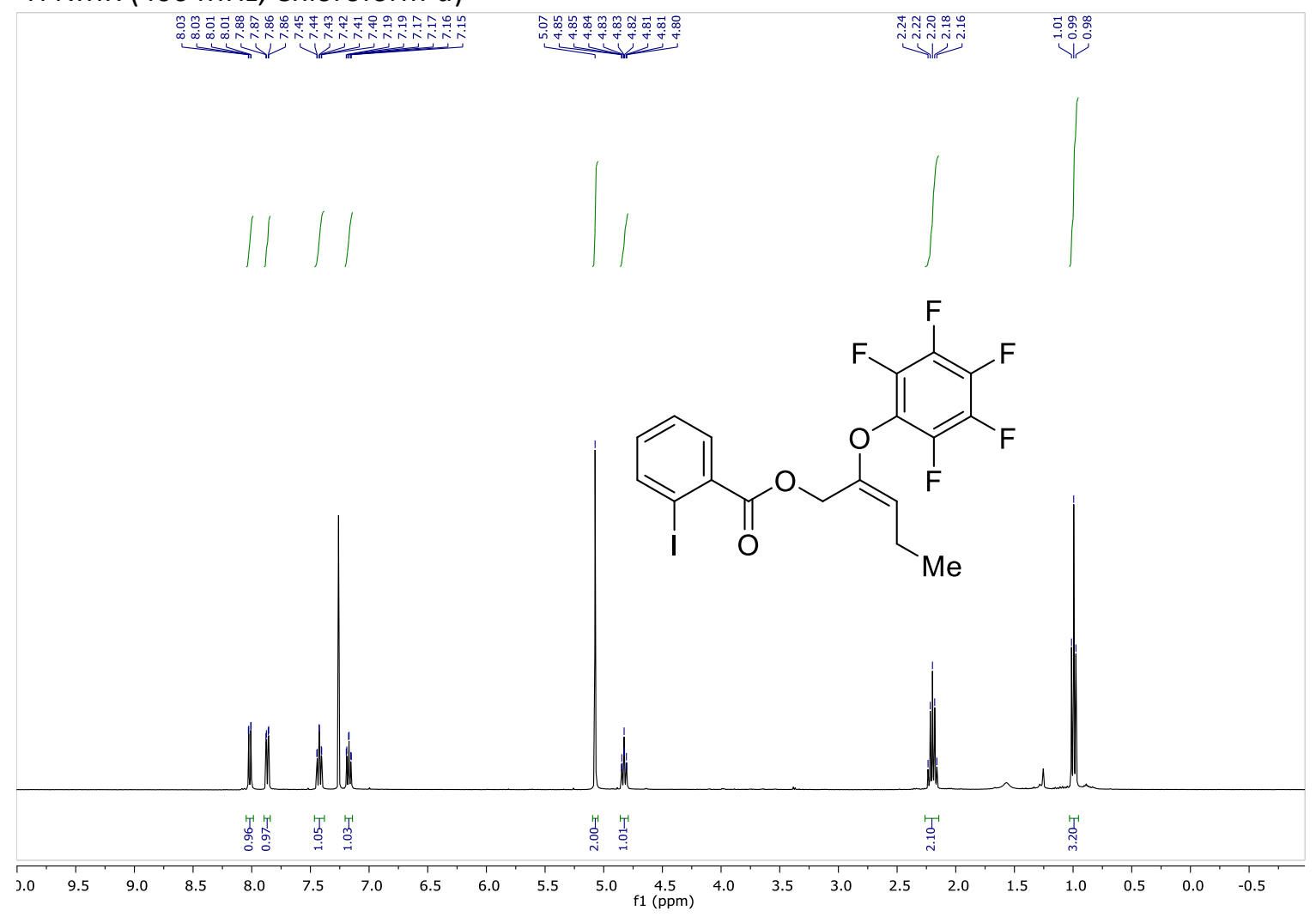

${ }^{13} \mathrm{C}$ NMR (101 MHz, Chloroform-d)

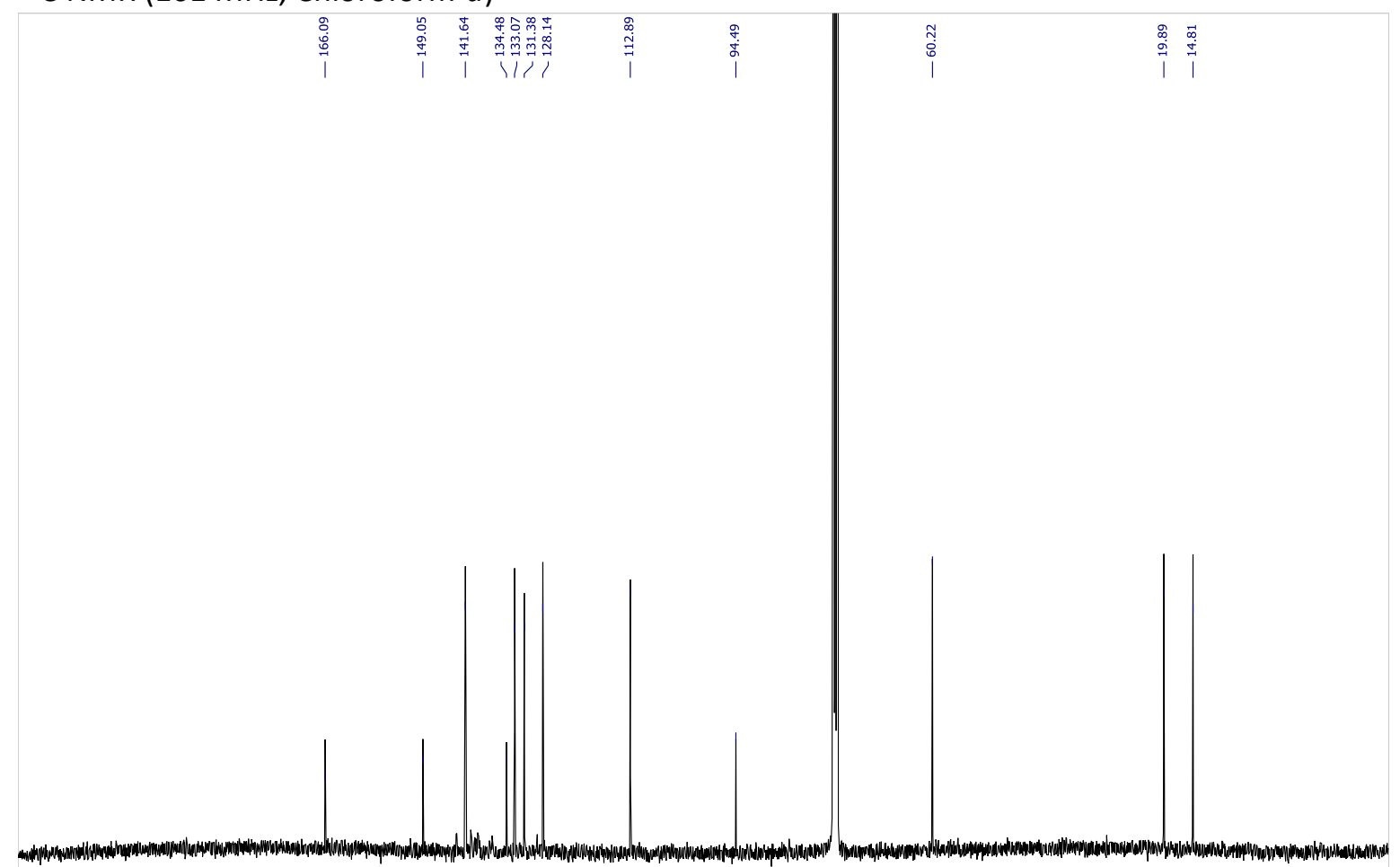

$\begin{array}{lllllllllllllllllllllllllllll} & 210 & 200 & 190 & 180 & 170 & 160 & 150 & 140 & 130 & 120 & 110 & 100 & 90 & 80 & 70 & 60 & 50 & 40 & 30 & 20 & 10 & 0 & -10\end{array}$ 
${ }^{19}$ F NMR (376 MHz, Chloroform-d)

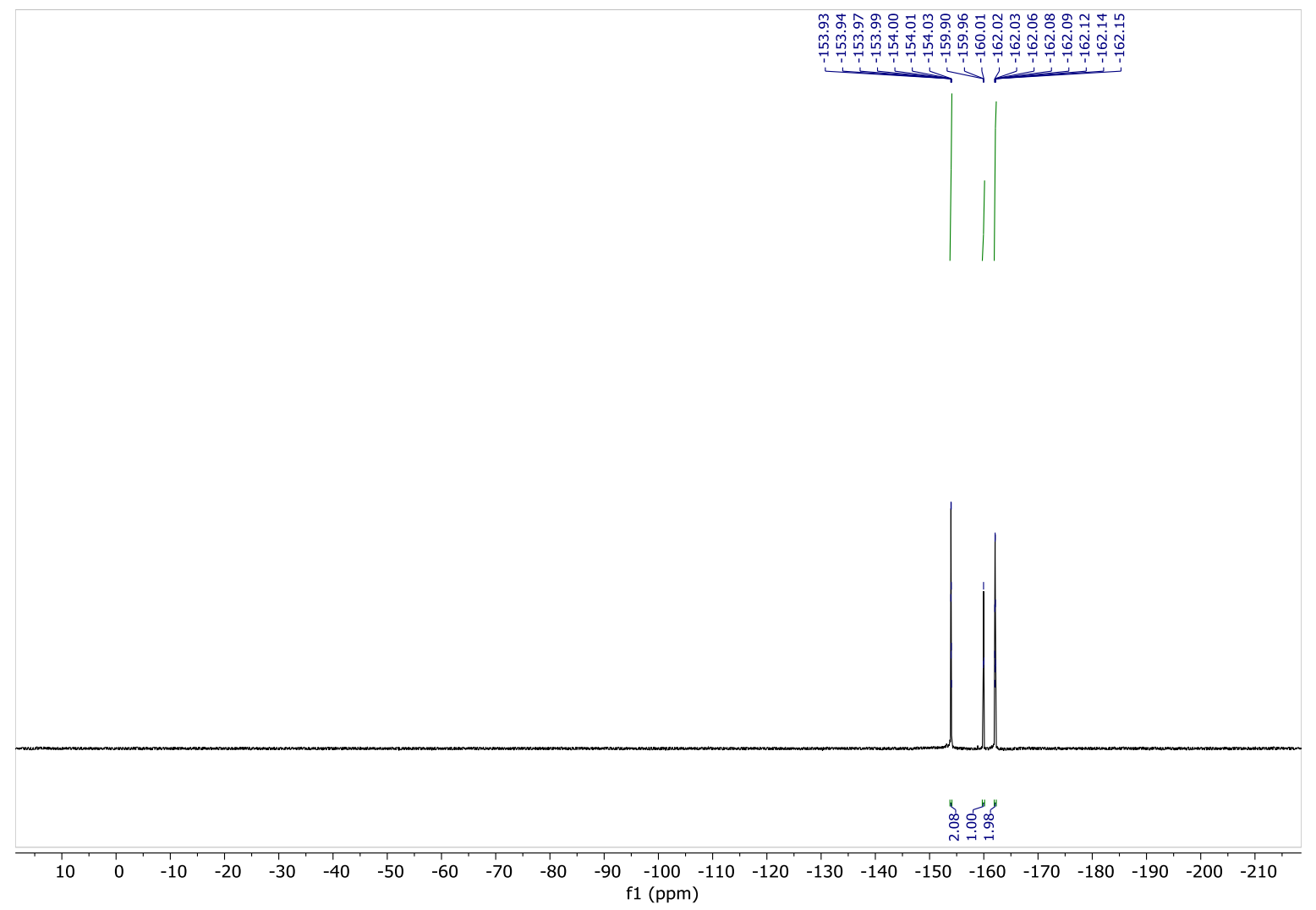


(E)-2-(4-Methoxyphenoxy)pent-2-en-1-yl 2-iodobenzoateiodobenzoate (4n)

${ }^{1} \mathrm{H}$ NMR (400 MHz, Acetonitrile- $d_{3}$ )

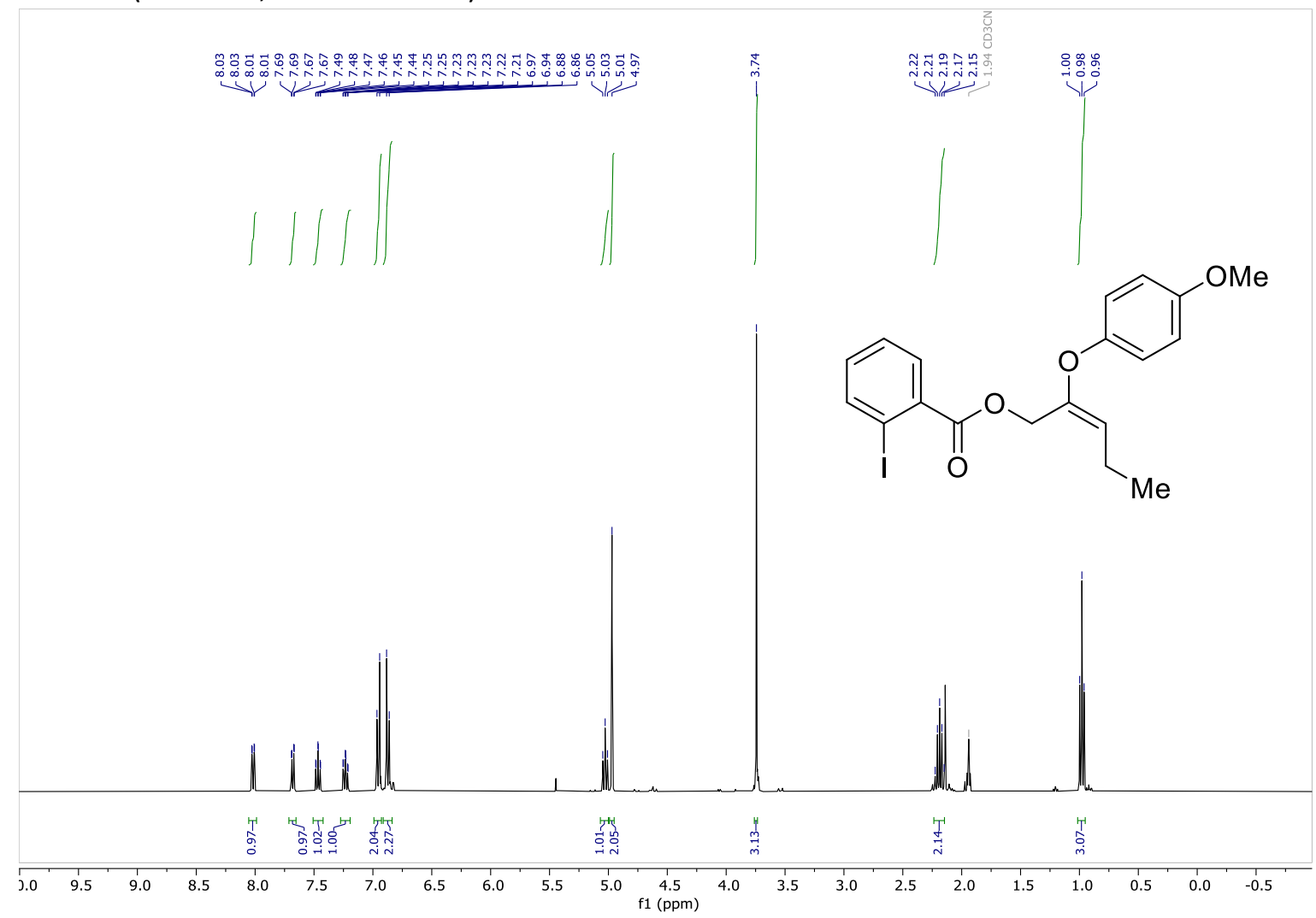

${ }^{13} \mathrm{C}$ NMR (101 MHz, Acetonitrile- $d_{3}$ )

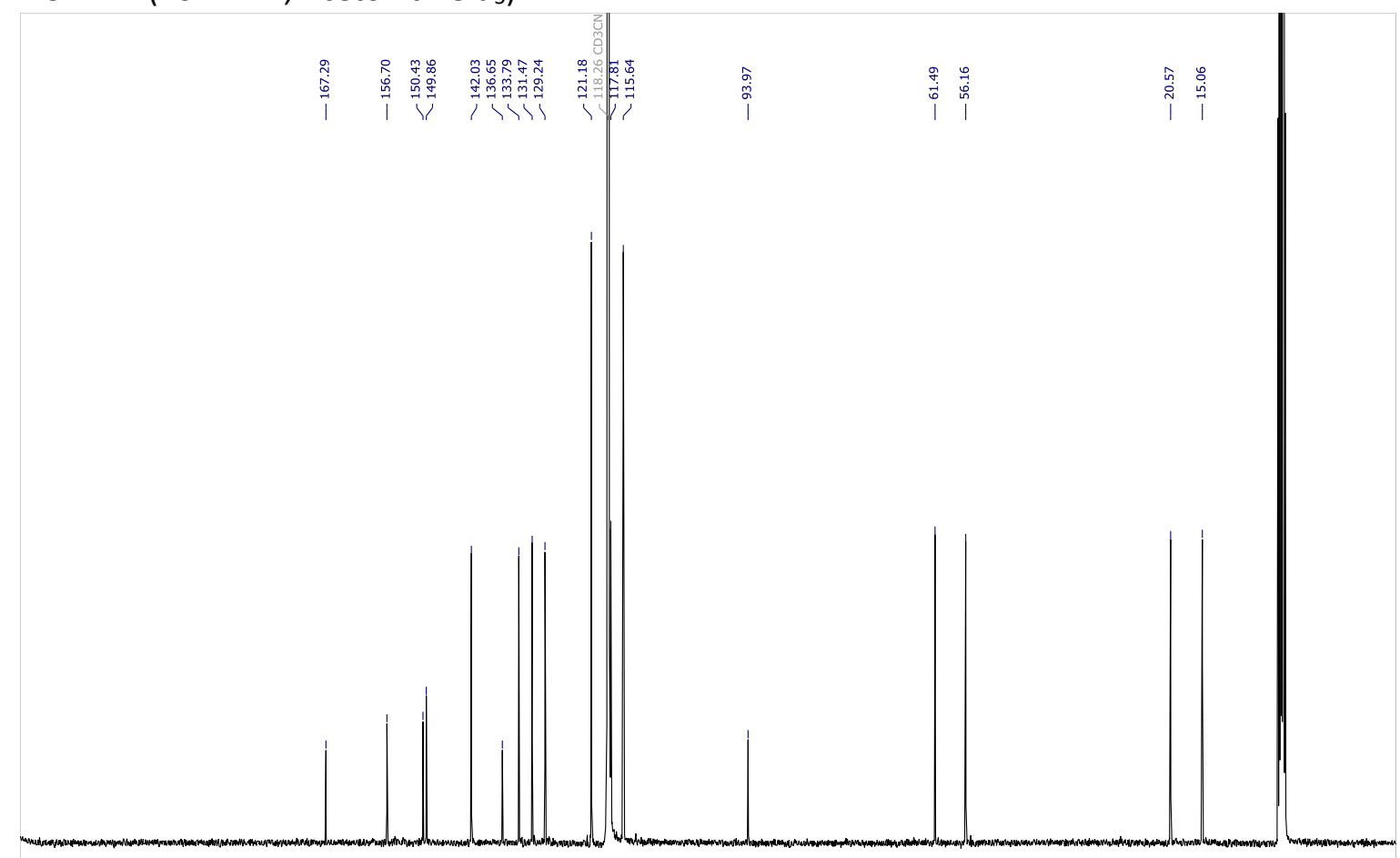

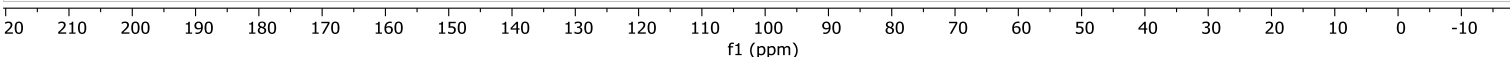


2-(4-Methoxyphenoxy)allyl 2-iodobenzoate (40)

${ }^{1}$ H NMR (400 MHz, Chloroform-d)

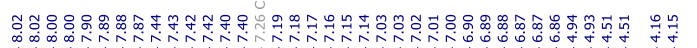<smiles>C=CC#CC(C)C=C</smiles>

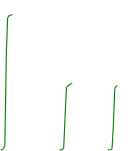<smiles>C=C(COC(=O)c1ccccc1I)Oc1ccc(OC)cc1</smiles>
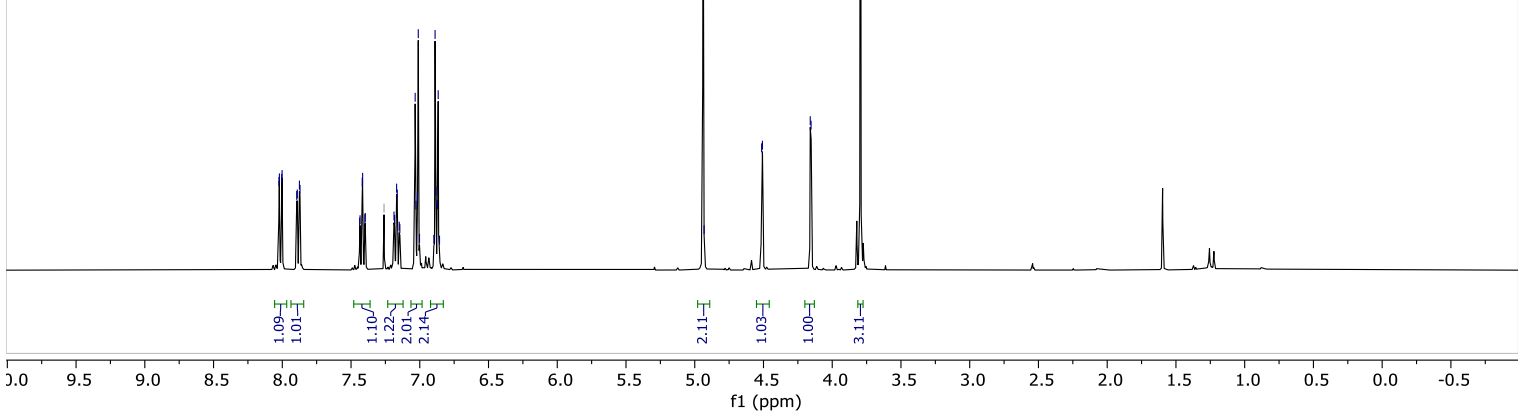

${ }^{13} \mathrm{C}$ NMR (101 MHz, Chloroform-d)

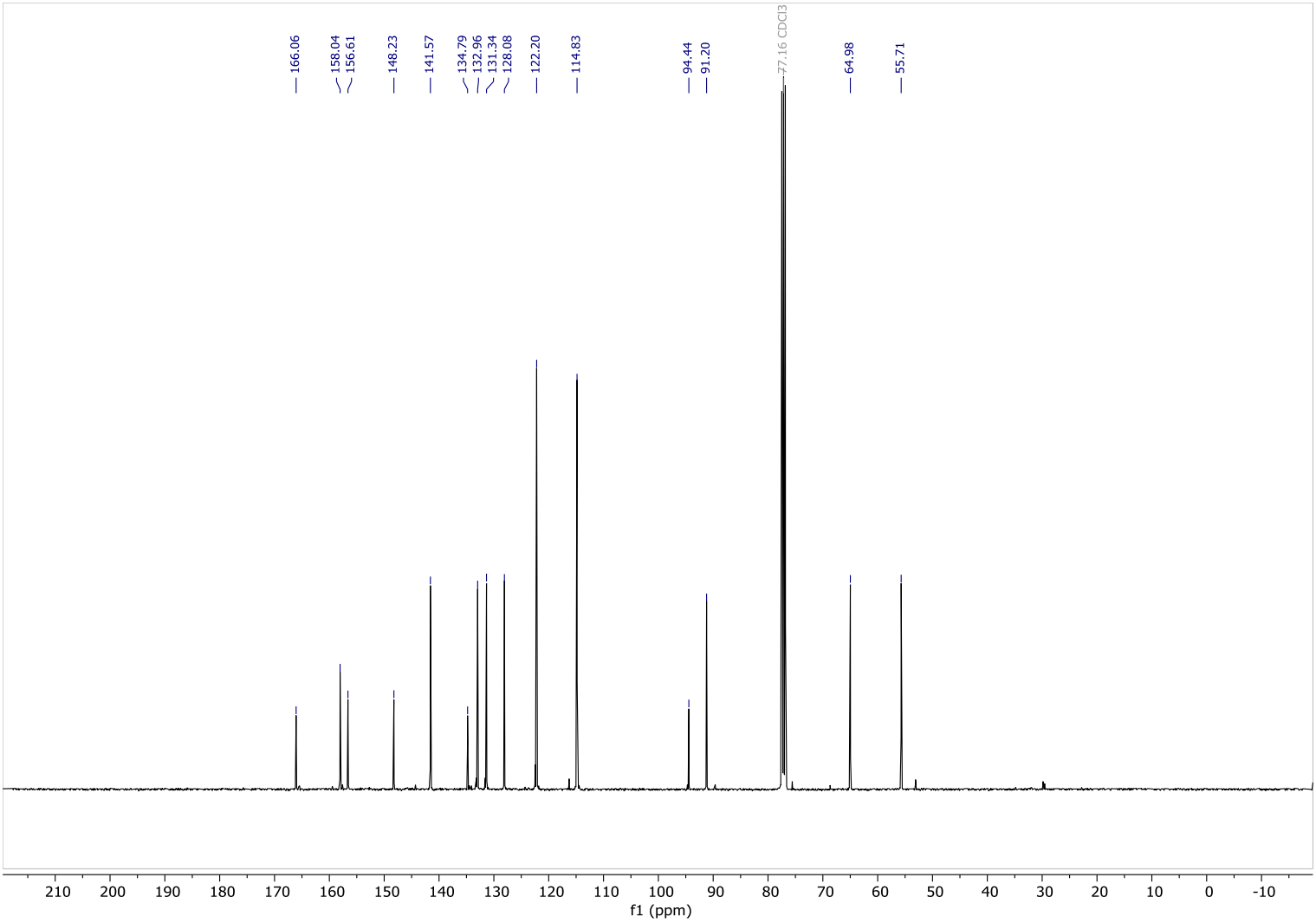




\section{(E)-2-(2-formylphenoxy)oct-2-en-1-yl 2-iodobenzoate (4p)}

${ }^{1} \mathrm{H}$ NMR (400 MHz, Acetonitrile- $d_{3}$ )

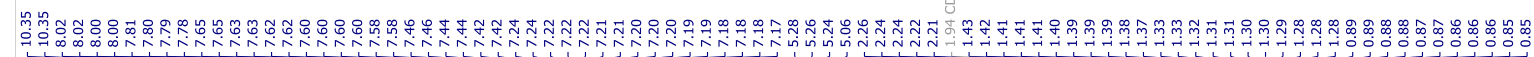

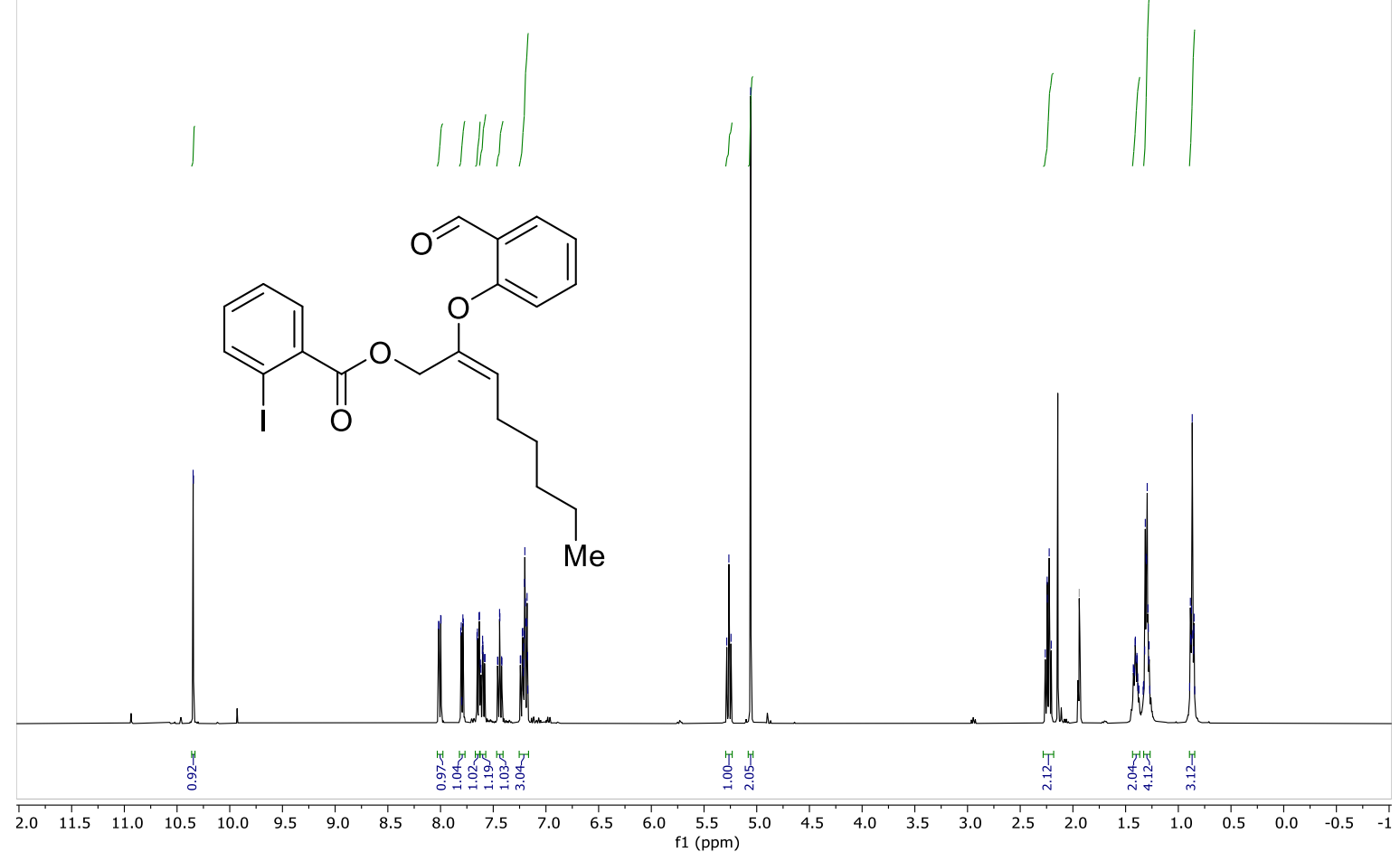

${ }^{13} \mathrm{C}$ NMR (101 MHz, Acetonitrile- $d_{3}$ )

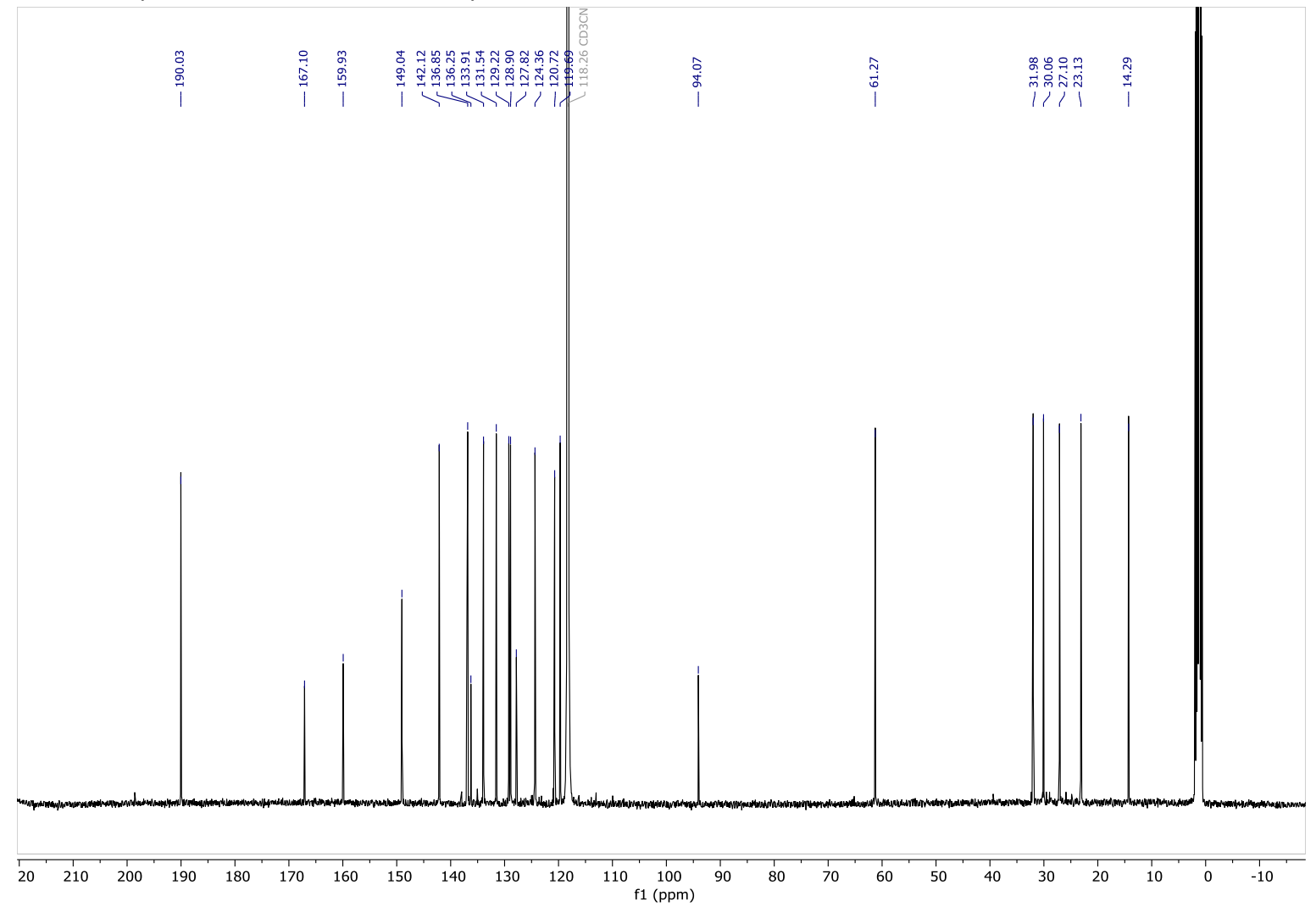




\subsection{Product modifications}

\section{2-((p-Tolyloxy)methyl)benzofuran (6)}

${ }^{1} \mathrm{H}$ NMR (400 MHz, Methylene Chloride- $d_{2}$ )

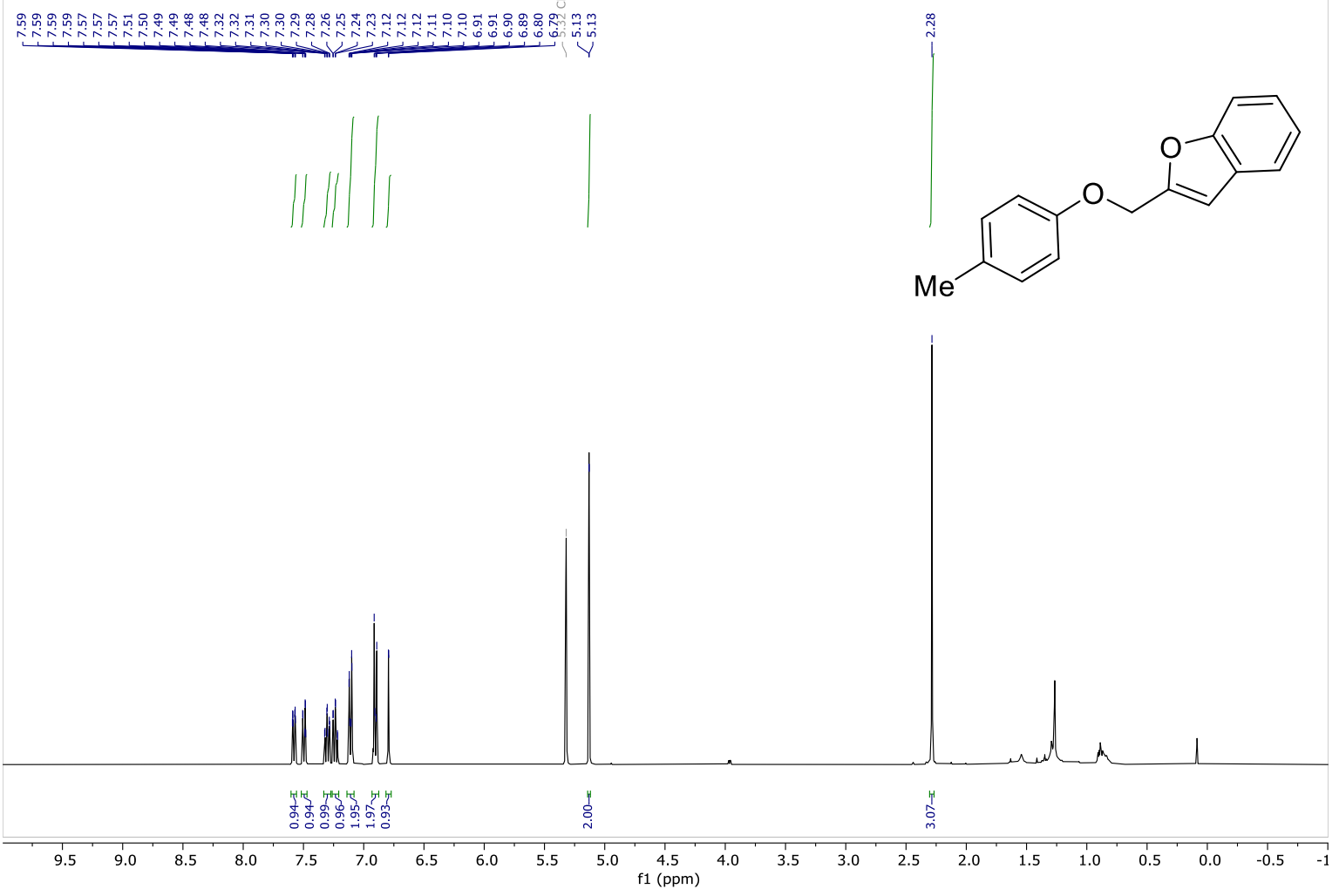

${ }^{13} \mathrm{C}$ NMR (101 MHz, Methylene Chloride- $d_{2}$ )

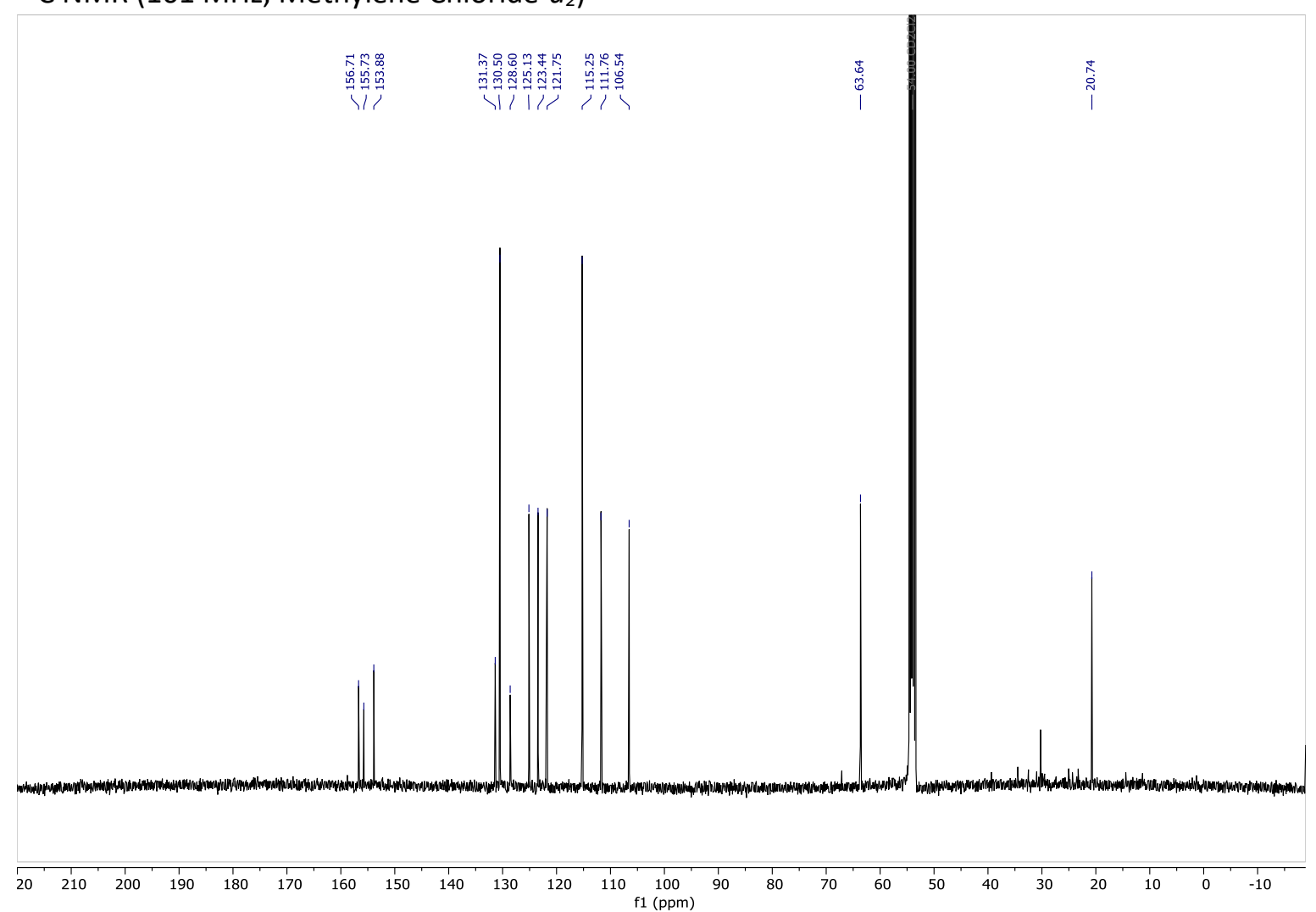


2-oxopentyl benzoate (7)

${ }^{1} \mathrm{H}$ NMR $\left(400 \mathrm{MHz}\right.$, Acetonitrile- $\left.d_{3}\right)$

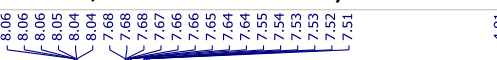

14.

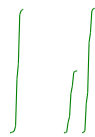<smiles>CCCC(=O)COC(=O)c1ccccc1</smiles>

$\mathrm{Me}$

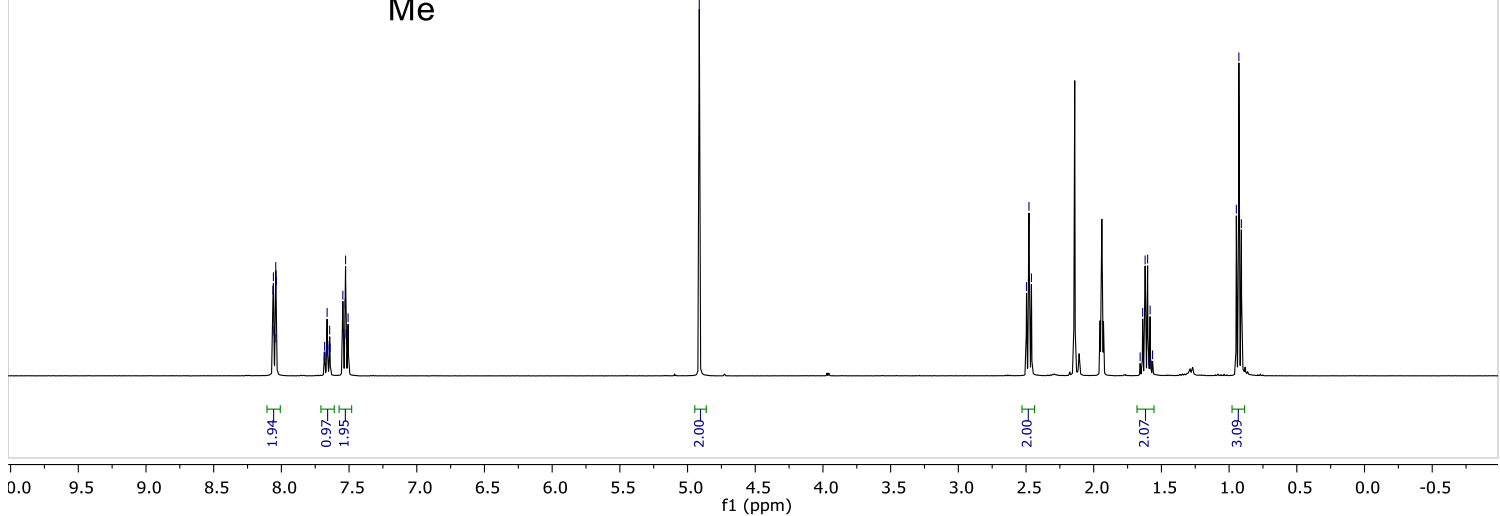

${ }^{13} \mathrm{C}$ NMR (101 MHz, Acetonitrile- $d_{3}$ )

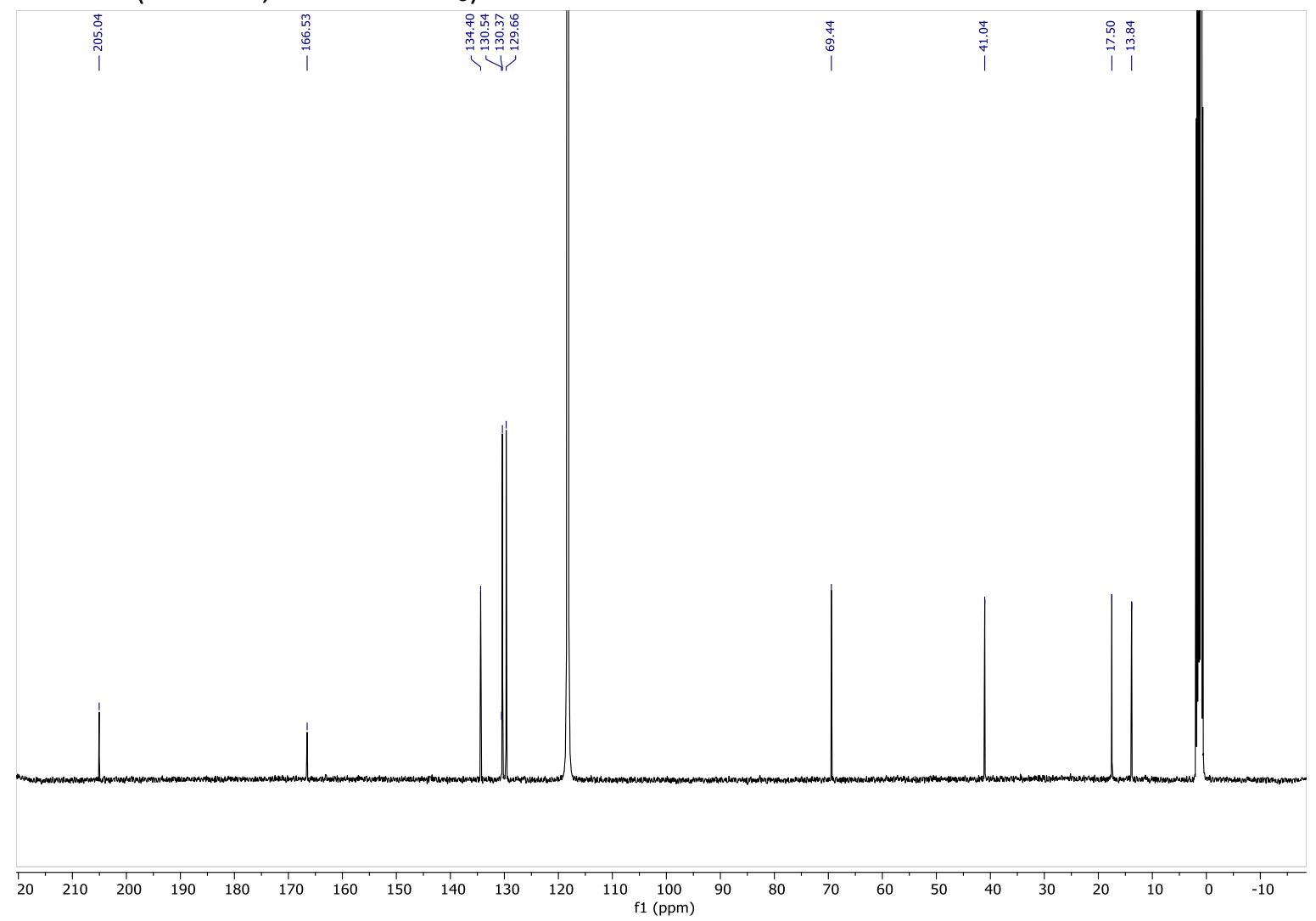


3-ethyl-2-(p-tolyloxy)-2-((p-tolyloxy)methyl)oxirane (8a)

${ }^{1} \mathrm{H}$ NMR $\left(400 \mathrm{MHz}\right.$, Acetonitrile- $\left.d_{3}\right)$

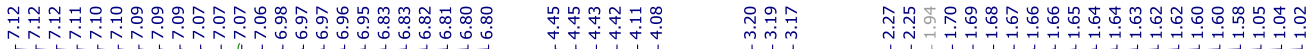

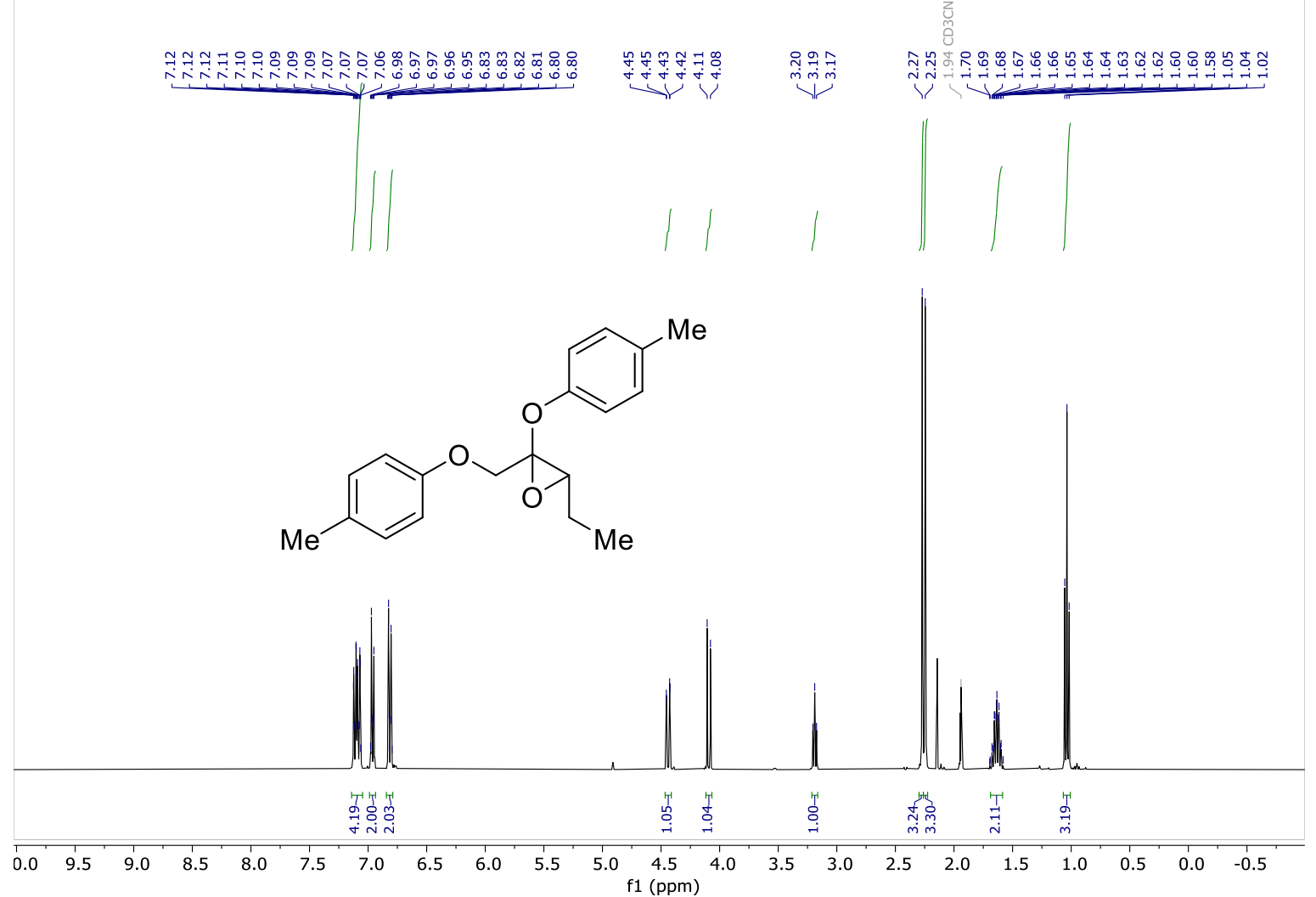

${ }^{13} \mathrm{C}$ NMR (101 MHz, Acetonitrile- $\left.d_{3}\right)$

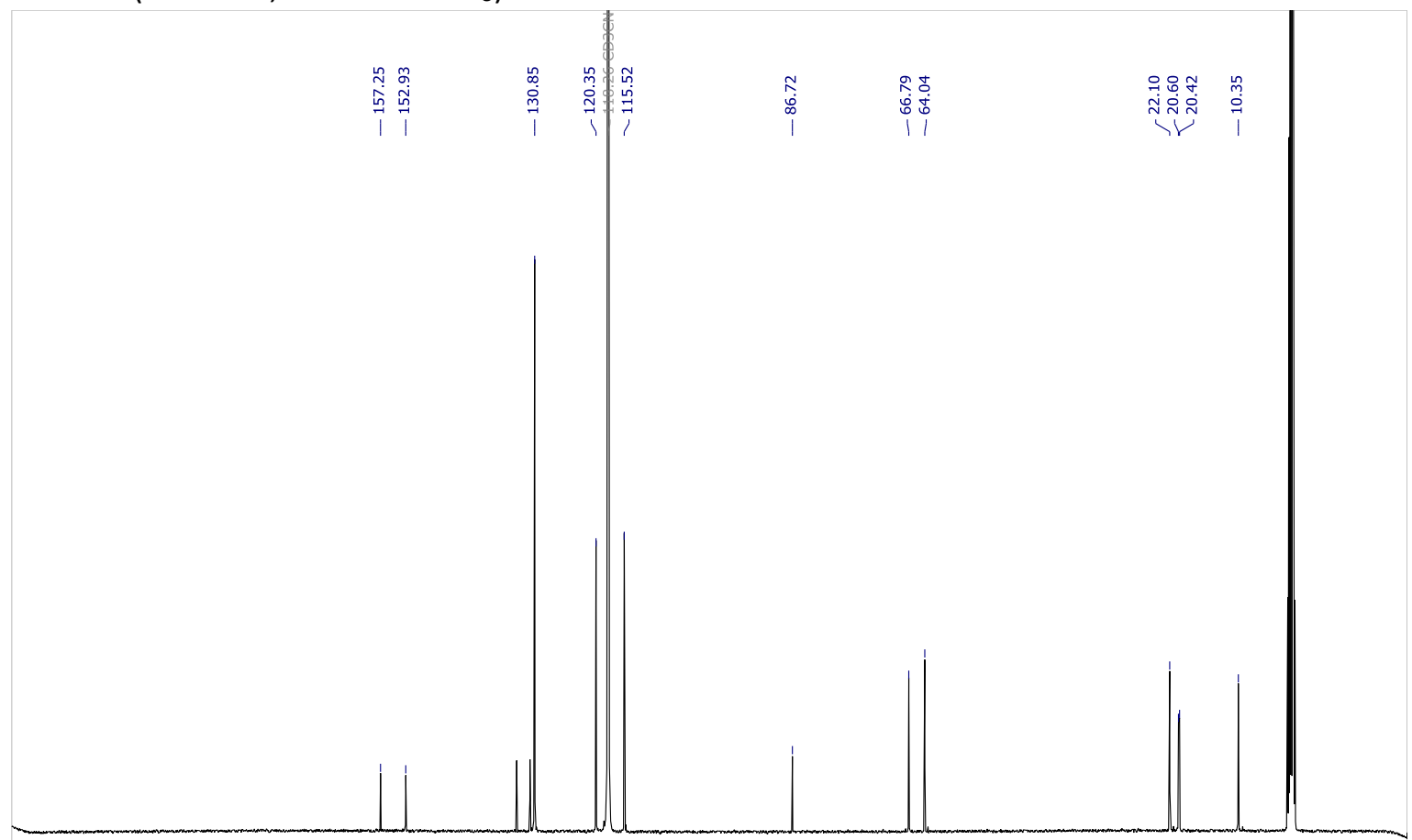

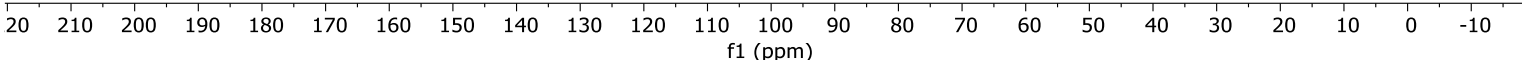


3-ethyl-2-(4-methoxyphenoxy)-2-((p-tolyloxy)methyl)oxirane (8b)

${ }^{1} \mathrm{H}$ NMR (400 MHz, Acetonitrile- $d_{3}$ )

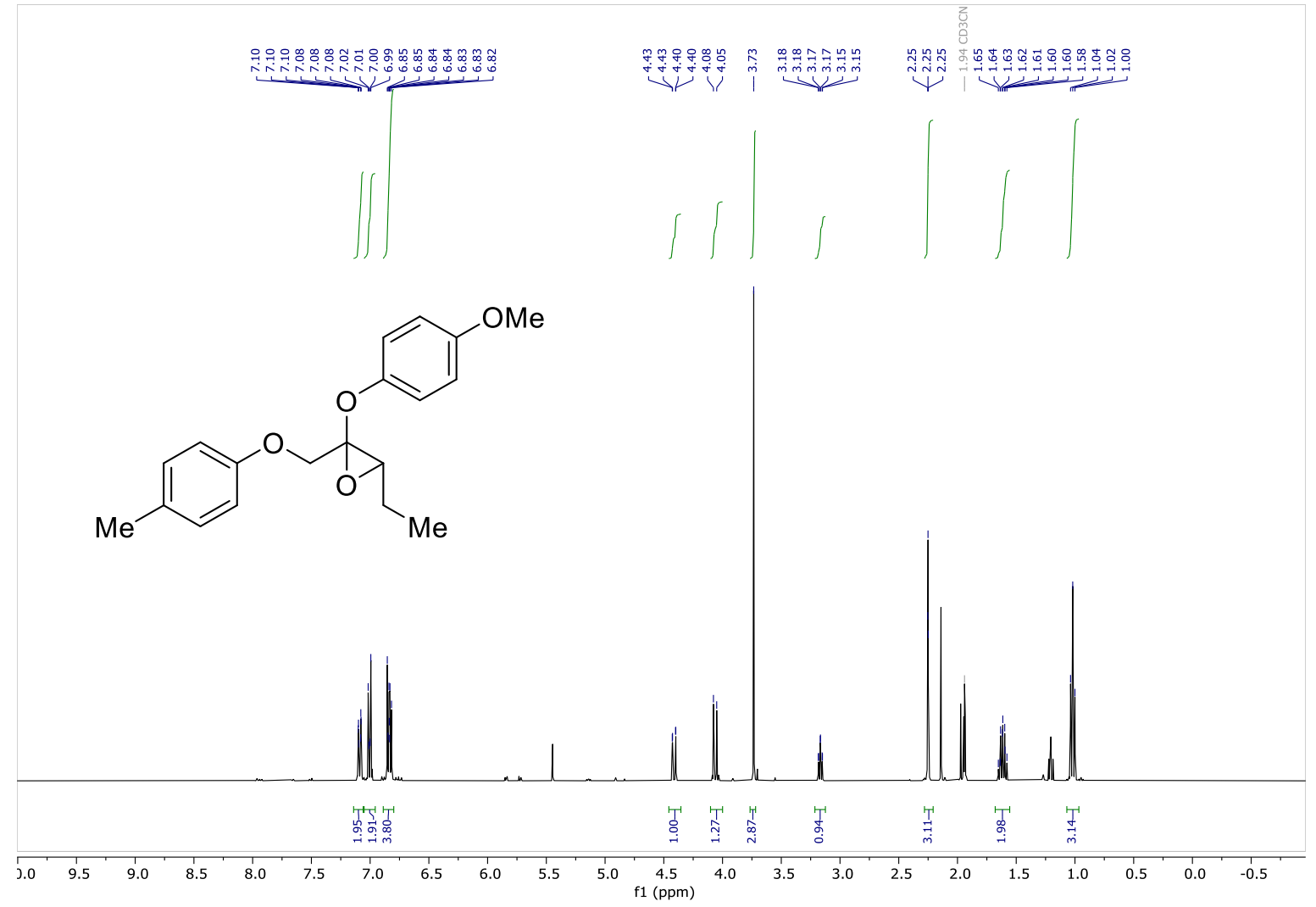

${ }^{13} \mathrm{C}$ NMR (101 MHz, Acetonitrile- $d_{3}$ )

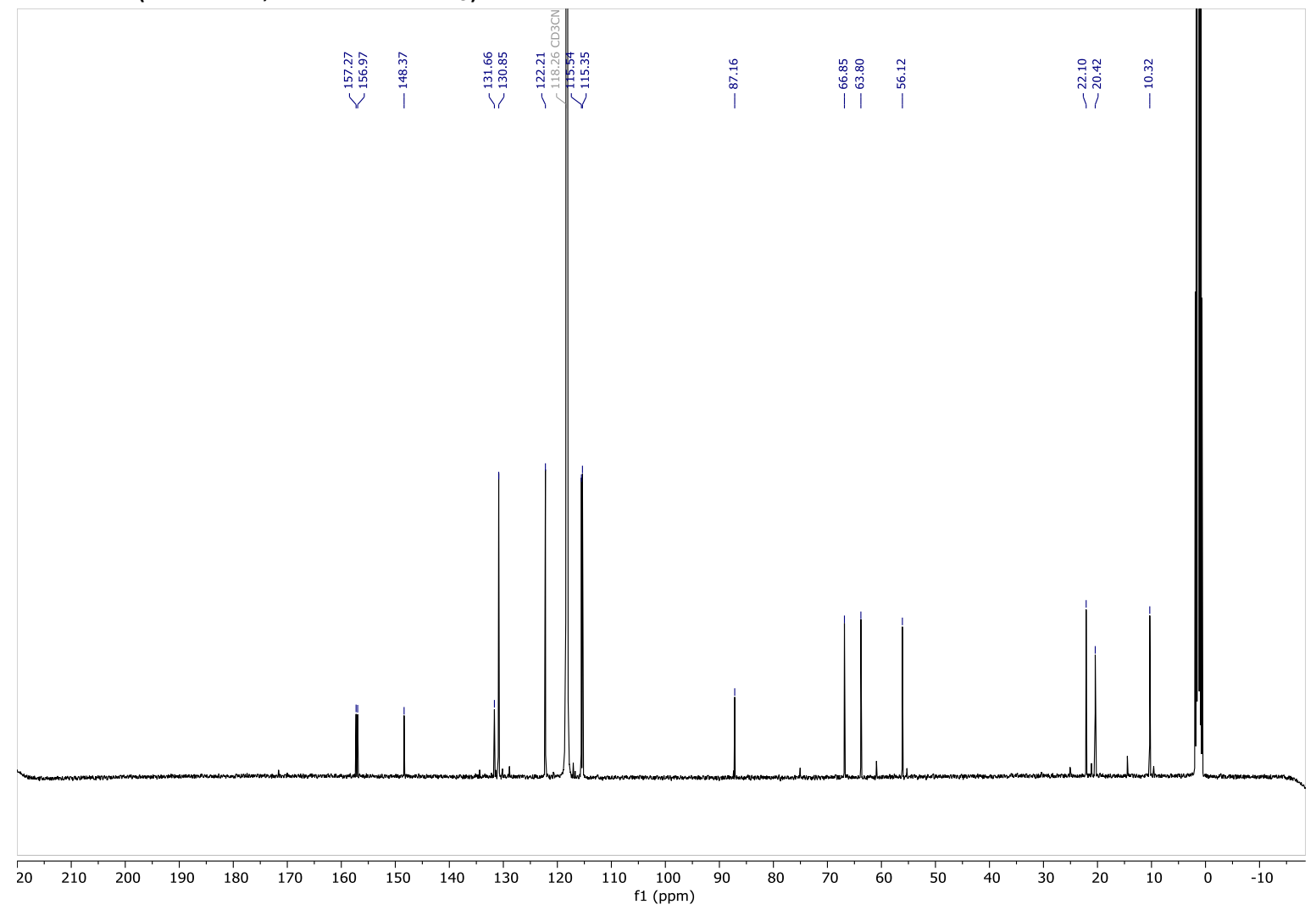


2-oxo-1-(p-tolyloxy)pentan-3-yl 3-chlorobenzoate (9a)

${ }^{1} \mathrm{H}$ NMR (400 MHz, Acetonitrile- $d_{3}$ )

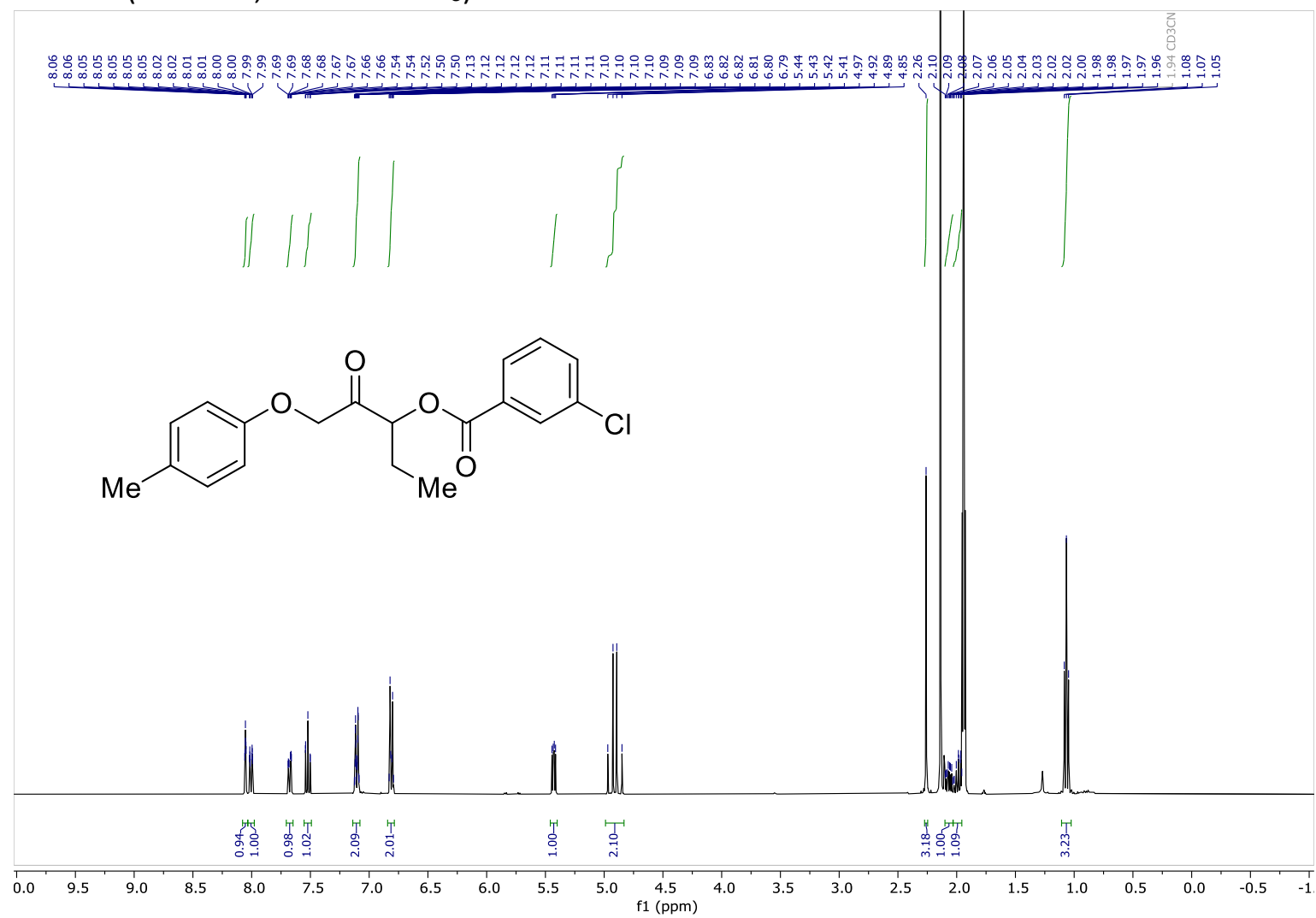

${ }^{13} \mathrm{C}$ NMR (101 MHz, Acetonitrile- $d_{3}$ )

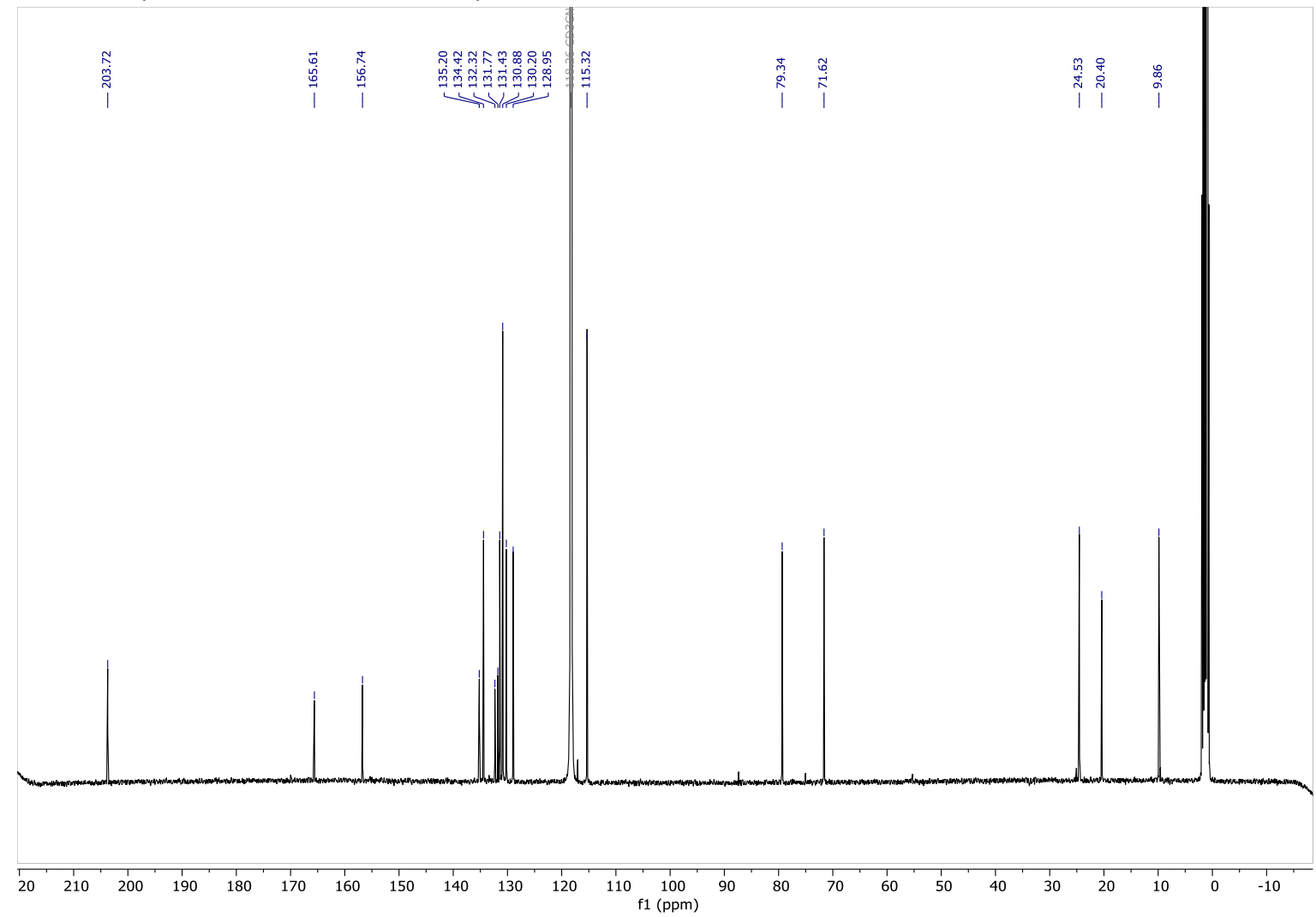


2-oxo-3-(p-tolyloxy)propyl 3-chlorobenzoate (9b)

${ }^{1} \mathrm{H}$ NMR $\left(400 \mathrm{MHz}\right.$, Acetonitrile- $\left.d_{3}\right)$

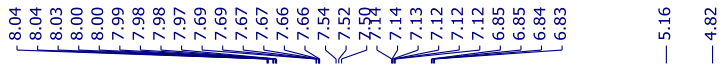
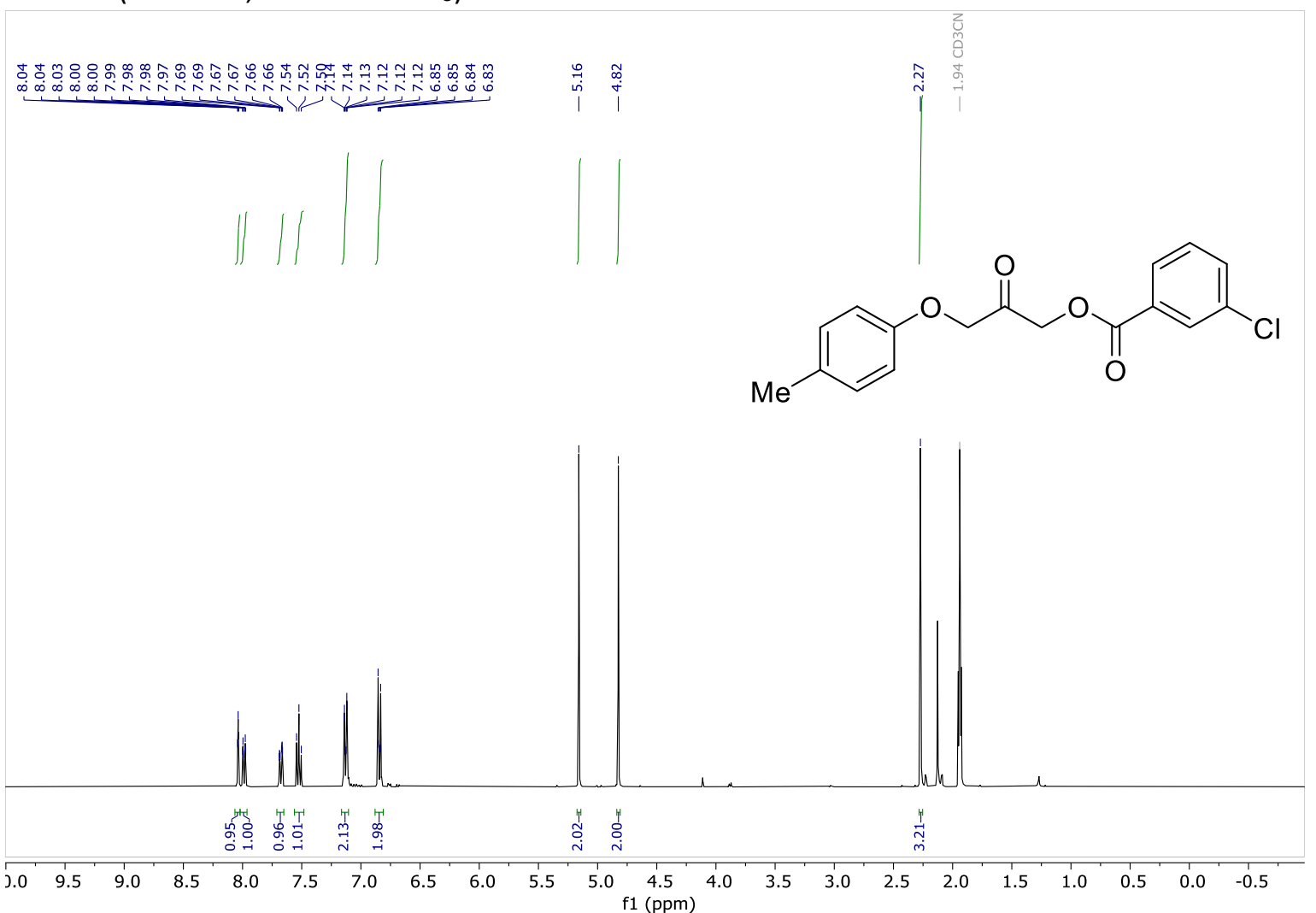

${ }^{13} \mathrm{C}$ NMR (101 MHz, Acetonitrile- $d_{3}$ )

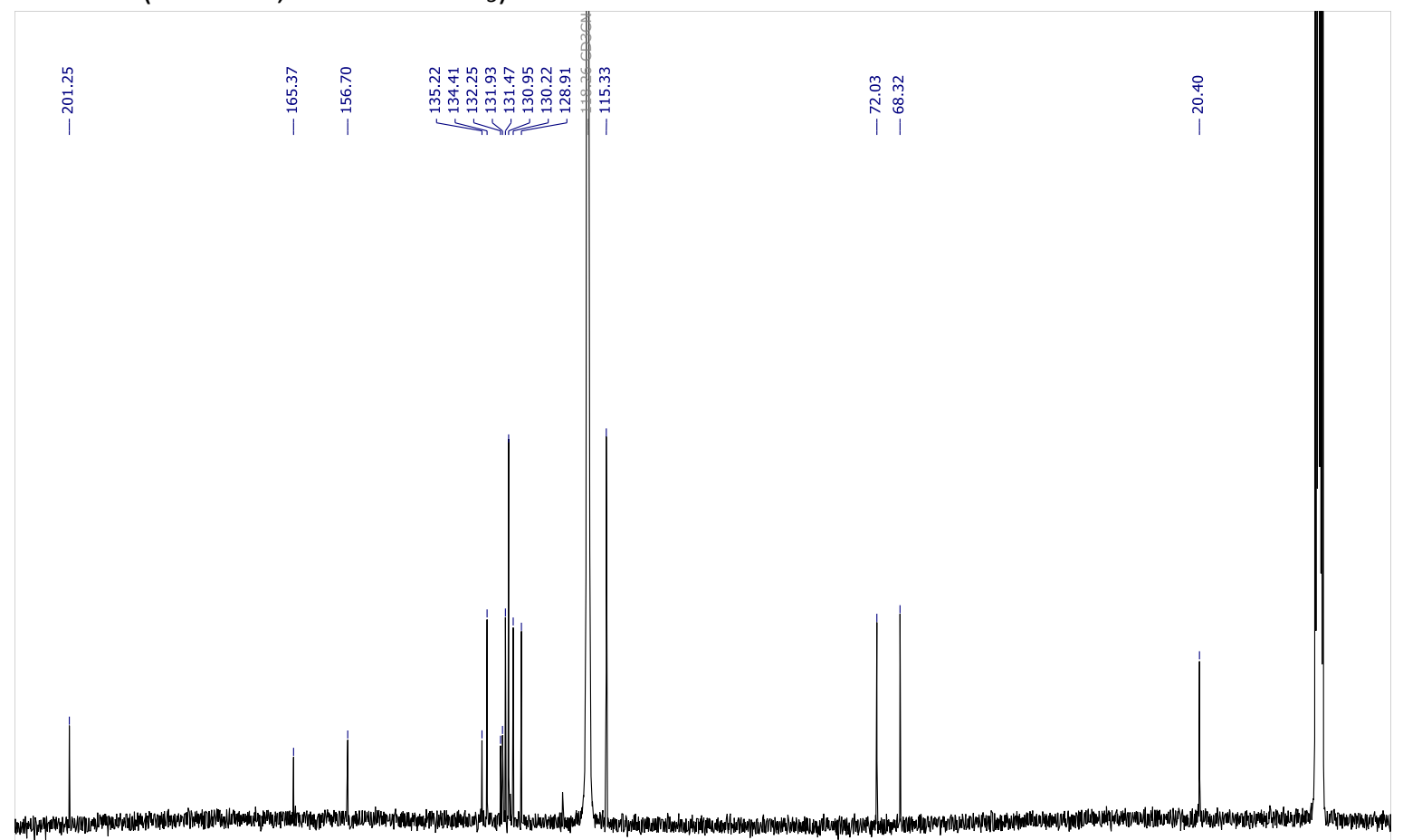

$\begin{array}{lllllllllllllllllllllllll}10 & 200 & 190 & 180 & 170 & 160 & 150 & 140 & 130 & 120 & 110 & \begin{array}{c}100 \\ \mathrm{f} 1(\mathrm{ppm})\end{array} & 90 & 80 & 70 & 60 & 50 & 40 & 30 & 20 & 10 & 0 & -1\end{array}$ 
2-oxo-1-(p-tolyloxy)pentan-3-yl acetate (10)

${ }^{1} \mathrm{H}$ NMR (400 MHz, Methylene Chloride- $d_{2}$ )

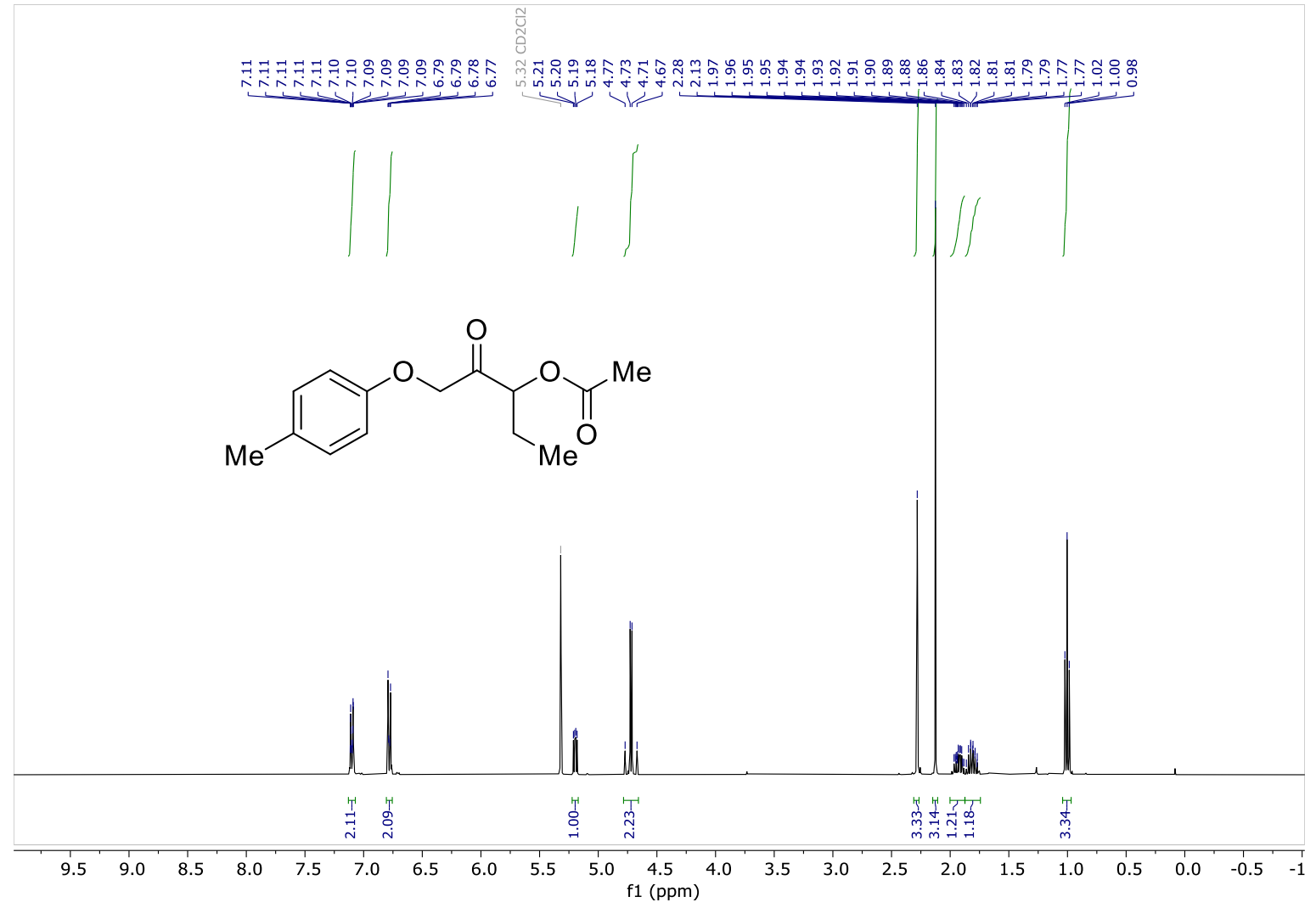

${ }^{13}$ C NMR (101 MHz, Methylene Chloride- $d_{2}$ )

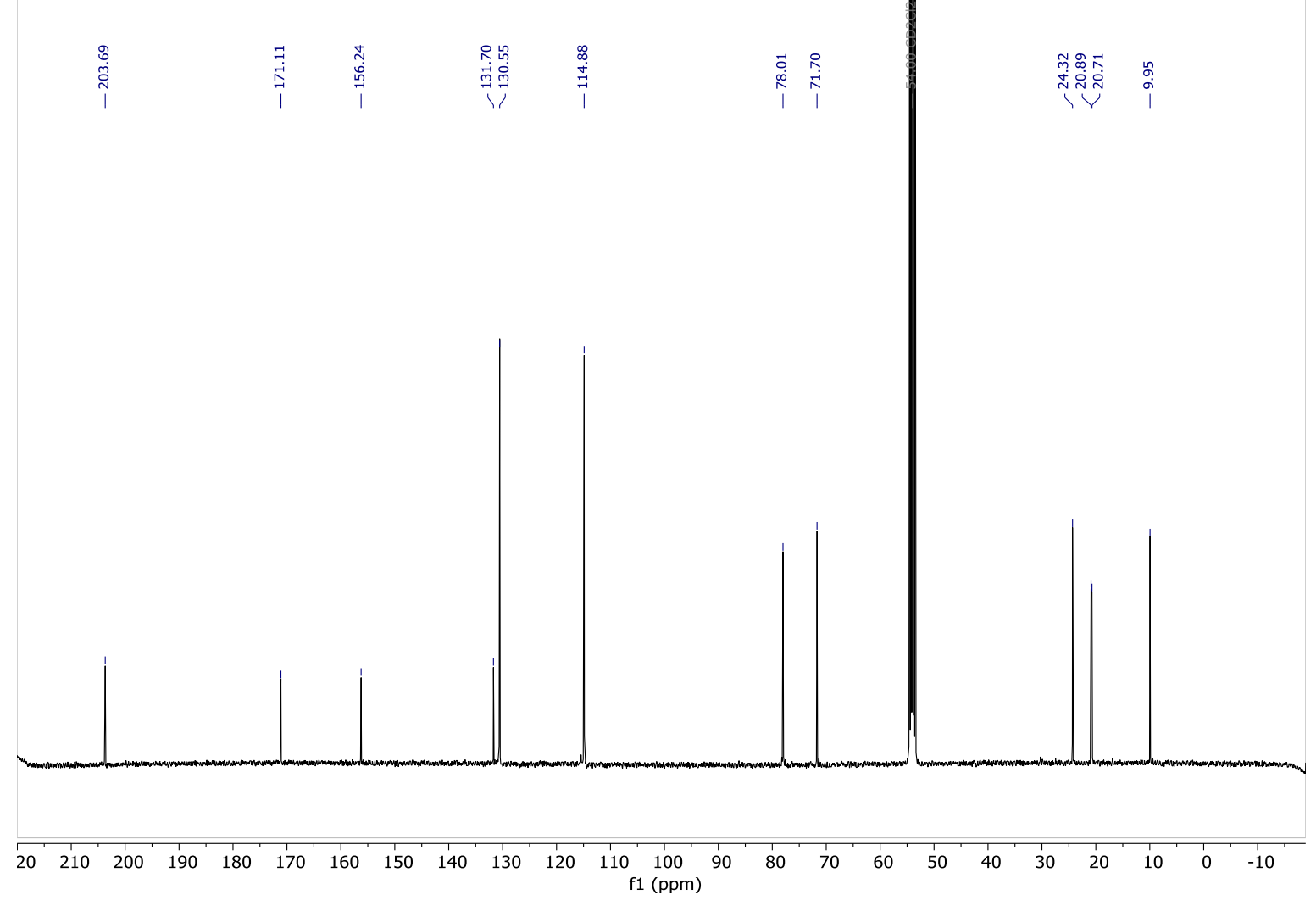




\subsection{Mechanistic investigations}

2-(p-Tolyloxy)allyl 2-iodobenzoate (4q)

${ }^{1} \mathrm{H}$ NMR (400 MHz, Chloroform-d)

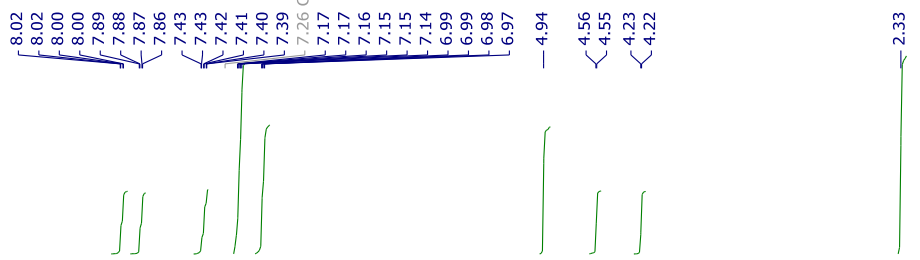<smiles>C=C(COC(=O)c1ccccc1I)Oc1ccc(C)cc1</smiles>

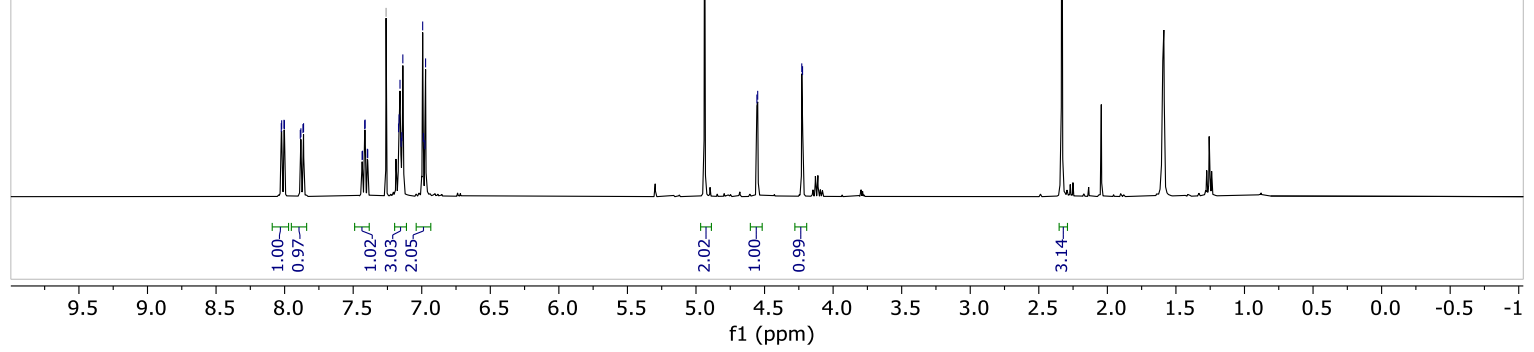

${ }^{13} \mathrm{C}$ NMR (101 MHz, Chloroform-d)

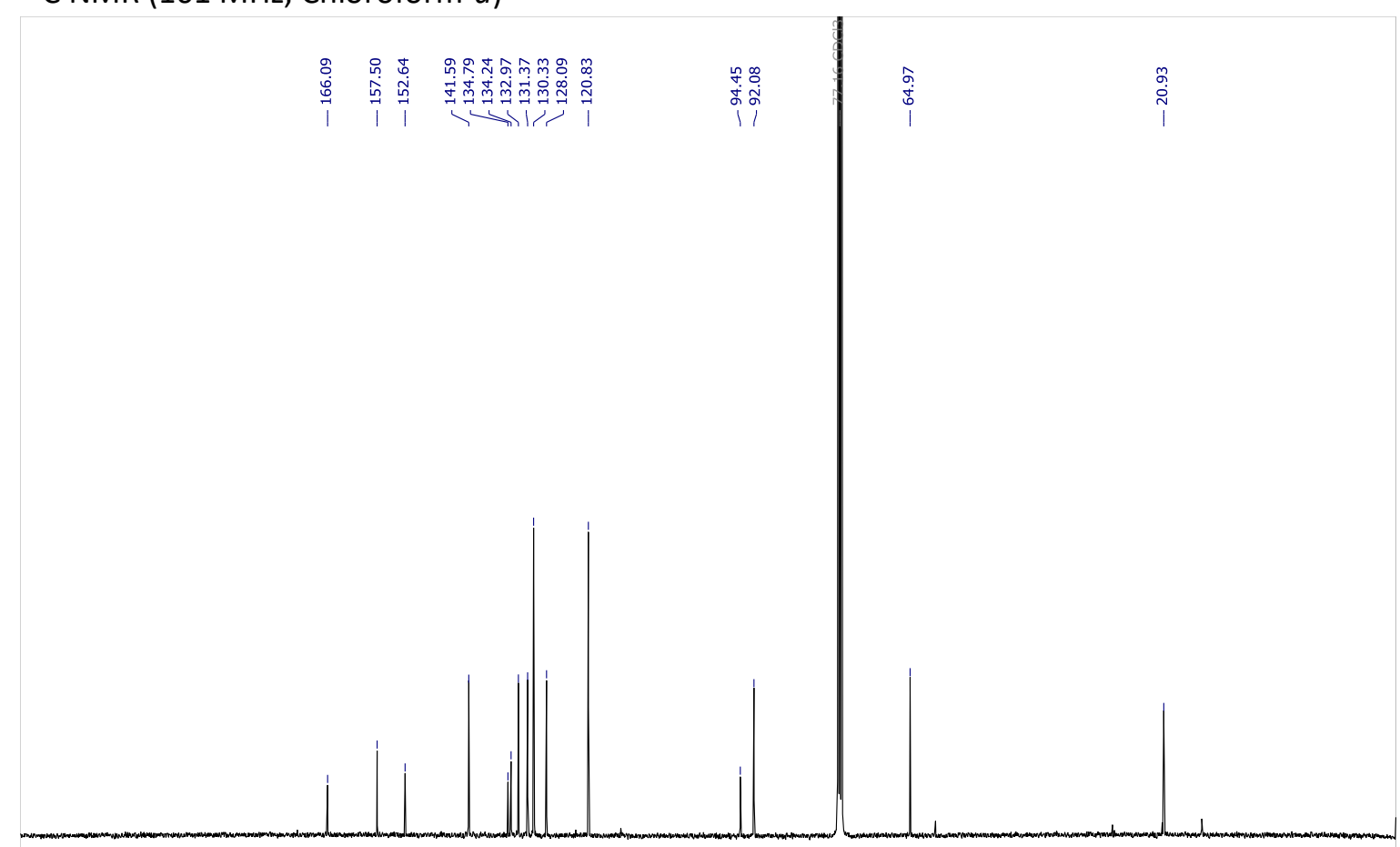

$\begin{array}{lllllllllllll}210 & 200 & 190 & 180 & 170 & 160 & 150 & 140 & 130 & 120 & 110 & 100 & 90\end{array}$ 
2-(4-Methoxyphenoxy)allyl 2-iodobenzoate (4q')

${ }^{1} \mathrm{H}$ NMR (400 MHz, Chloroform-d)

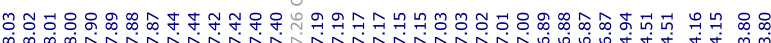<smiles>C=CC1CCCCC1C</smiles><smiles>C=C(COC(=O)c1cc(I)ccc1I)Oc1ccc(OC)cc1</smiles>

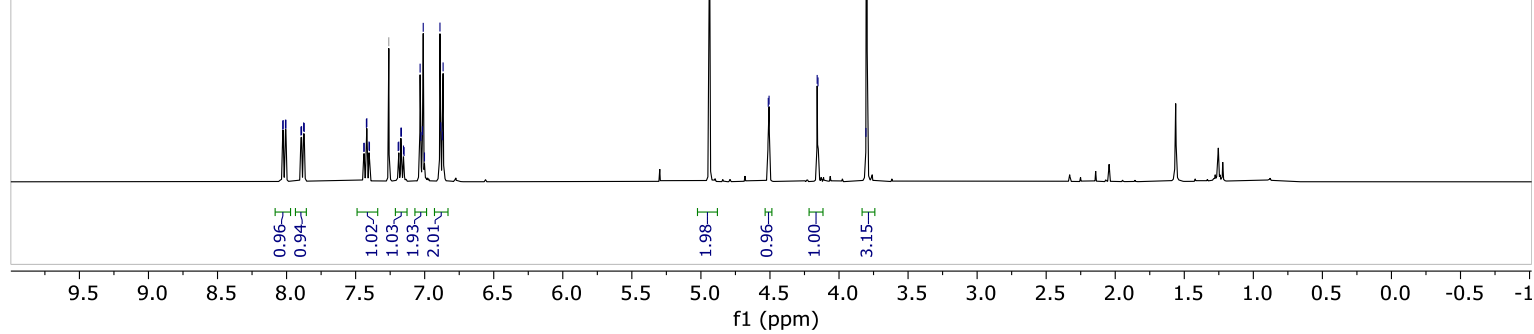

${ }^{13} \mathrm{C}$ NMR (101 MHz, Chloroform-d)

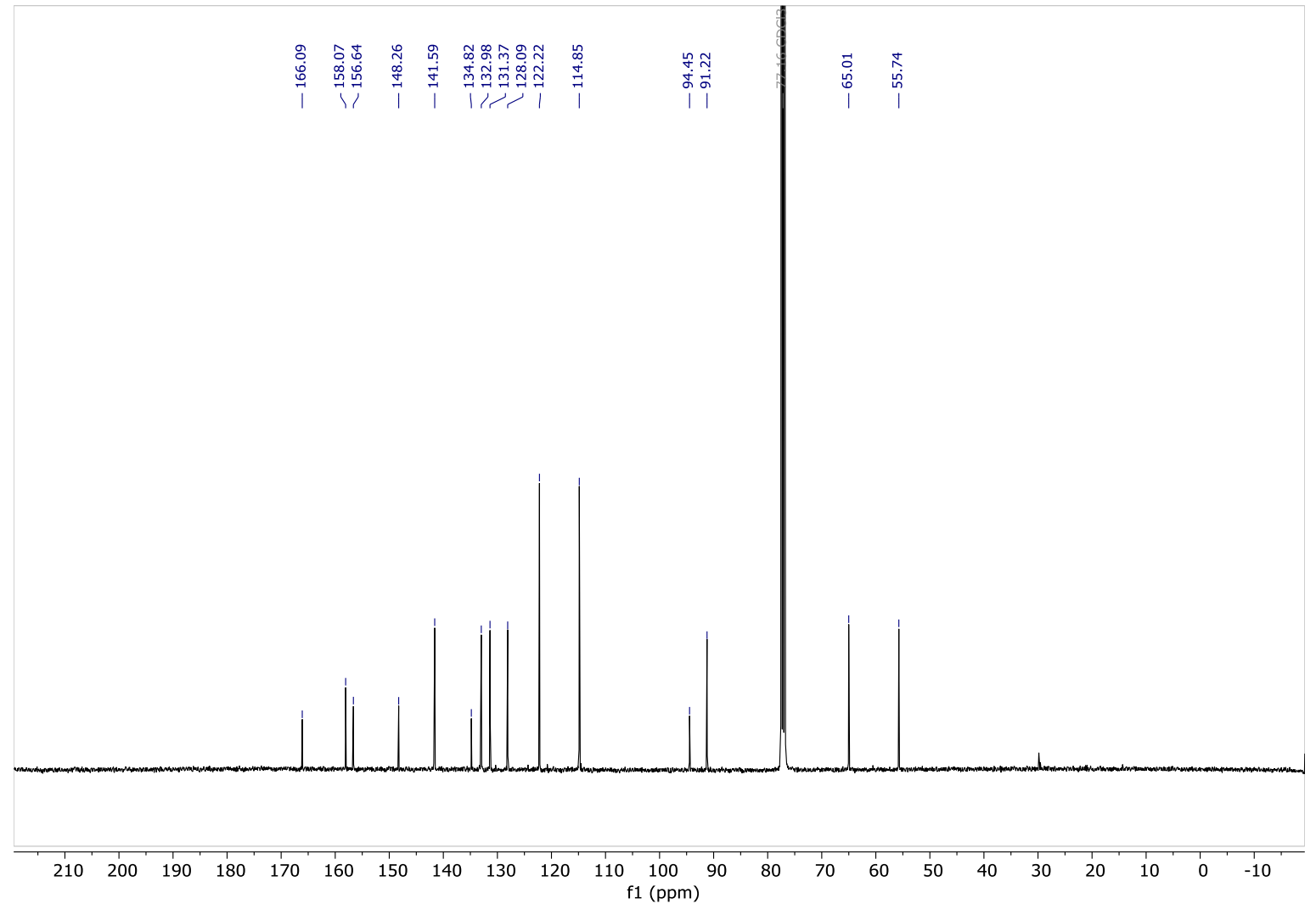


2-(4-Methoxyphenoxy)allyl 2-iodo-5-methoxybenzoate (4r)

${ }^{1} \mathrm{H}$ NMR (400 MHz, Chloroform-d)

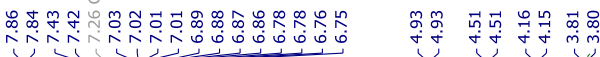<smiles>C1#CC2C[CH]C1C2</smiles><smiles>C=C(COC(=O)c1cc(OC)ccc1I)Oc1ccc(OC)cc1</smiles>
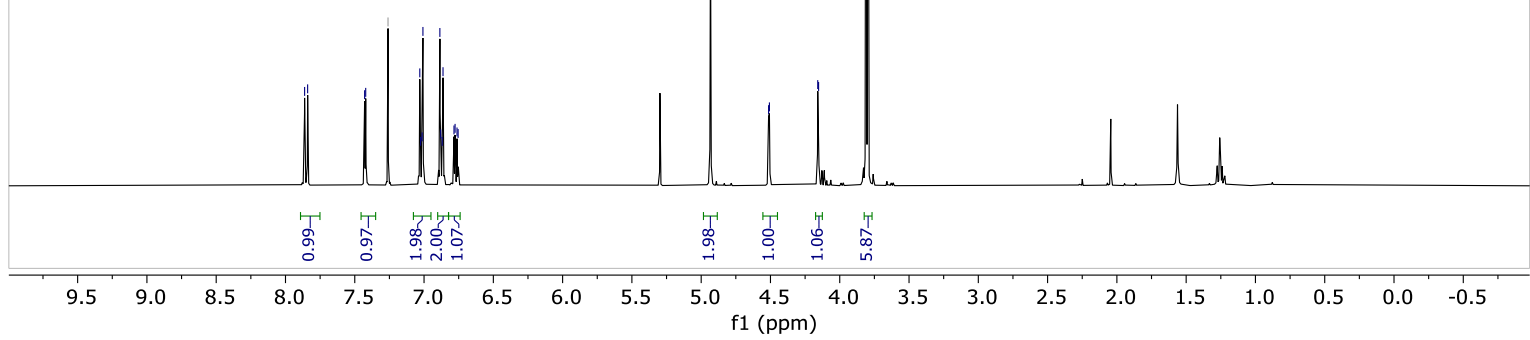

${ }^{13} \mathrm{C}$ NMR (101 MHz, Chloroform-d)

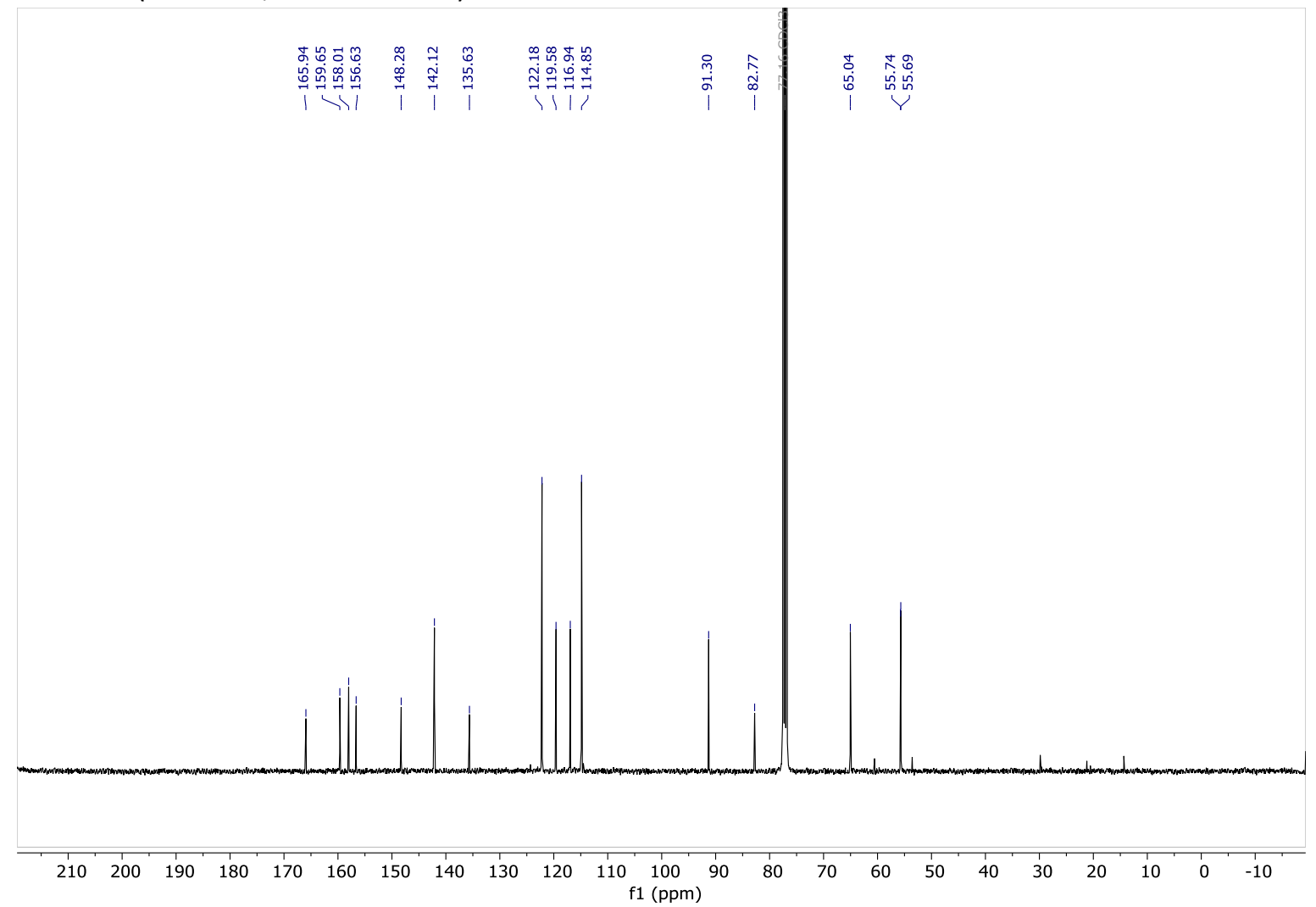


2-(p-Tolyloxy)allyl 2-iodo-5-methoxybenzoate (4r')

${ }^{1} \mathrm{H}$ NMR (400 MHz, Chloroform-d)

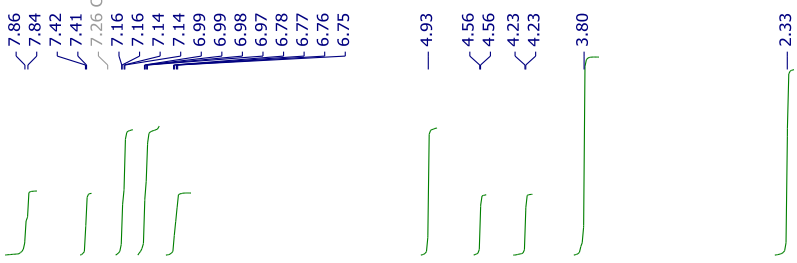<smiles>C=C(COC(=O)c1cc(OC)ccc1I)Oc1ccc(C)cc1</smiles>

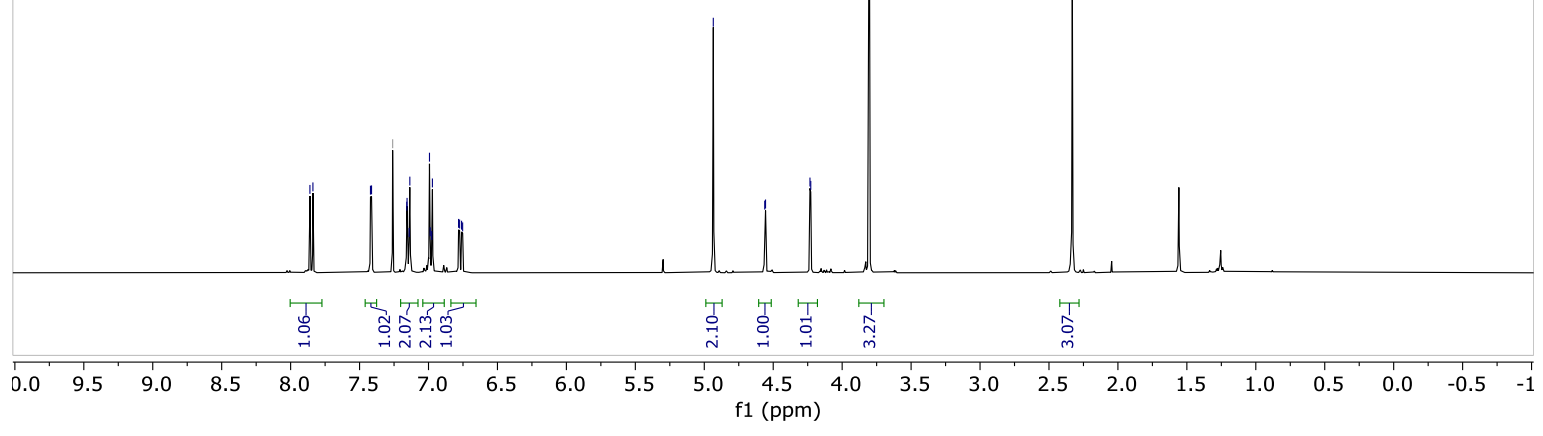

${ }^{13} \mathrm{C}$ NMR (101 MHz, Chloroform-d)

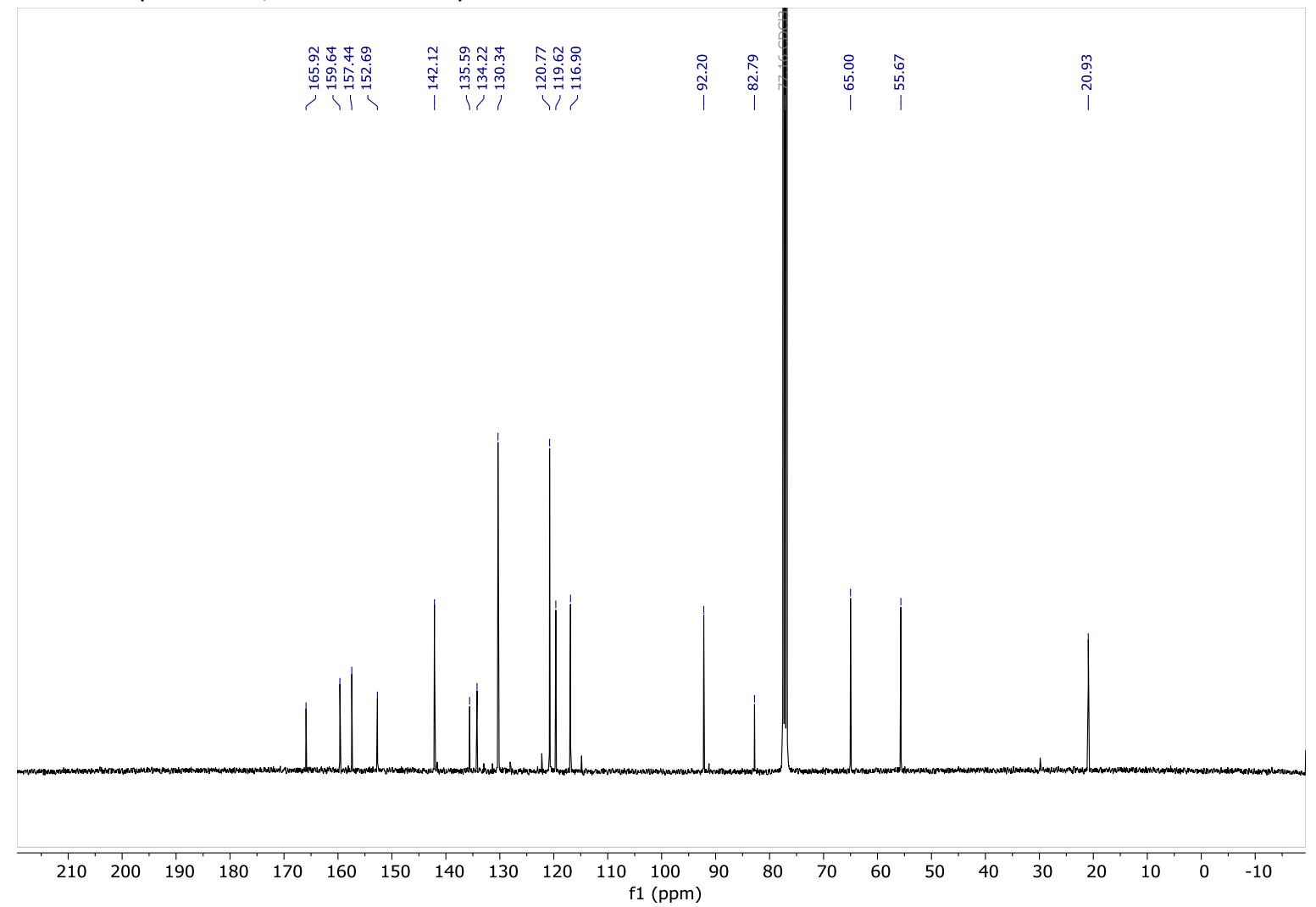


(E)-5-(p-tolyloxy)hexa-3,5-dien-1-yl 2-iodobenzoate (13)

${ }^{1} \mathrm{H}$ NMR (400 MHz, Acetonitrile- $d_{3}$ )

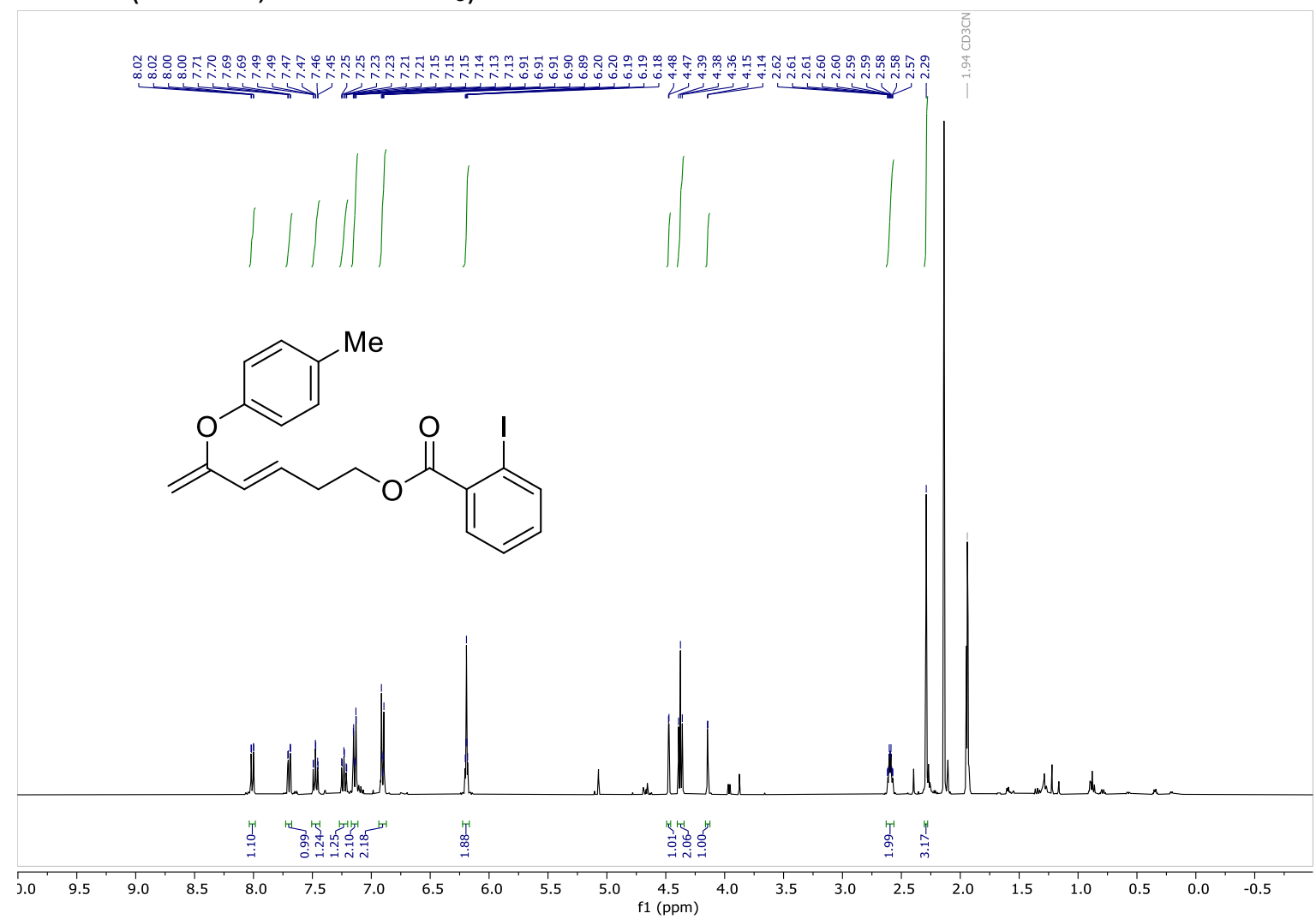

${ }^{13} \mathrm{C}$ NMR (101 MHz, Acetonitrile- $d_{3}$ )

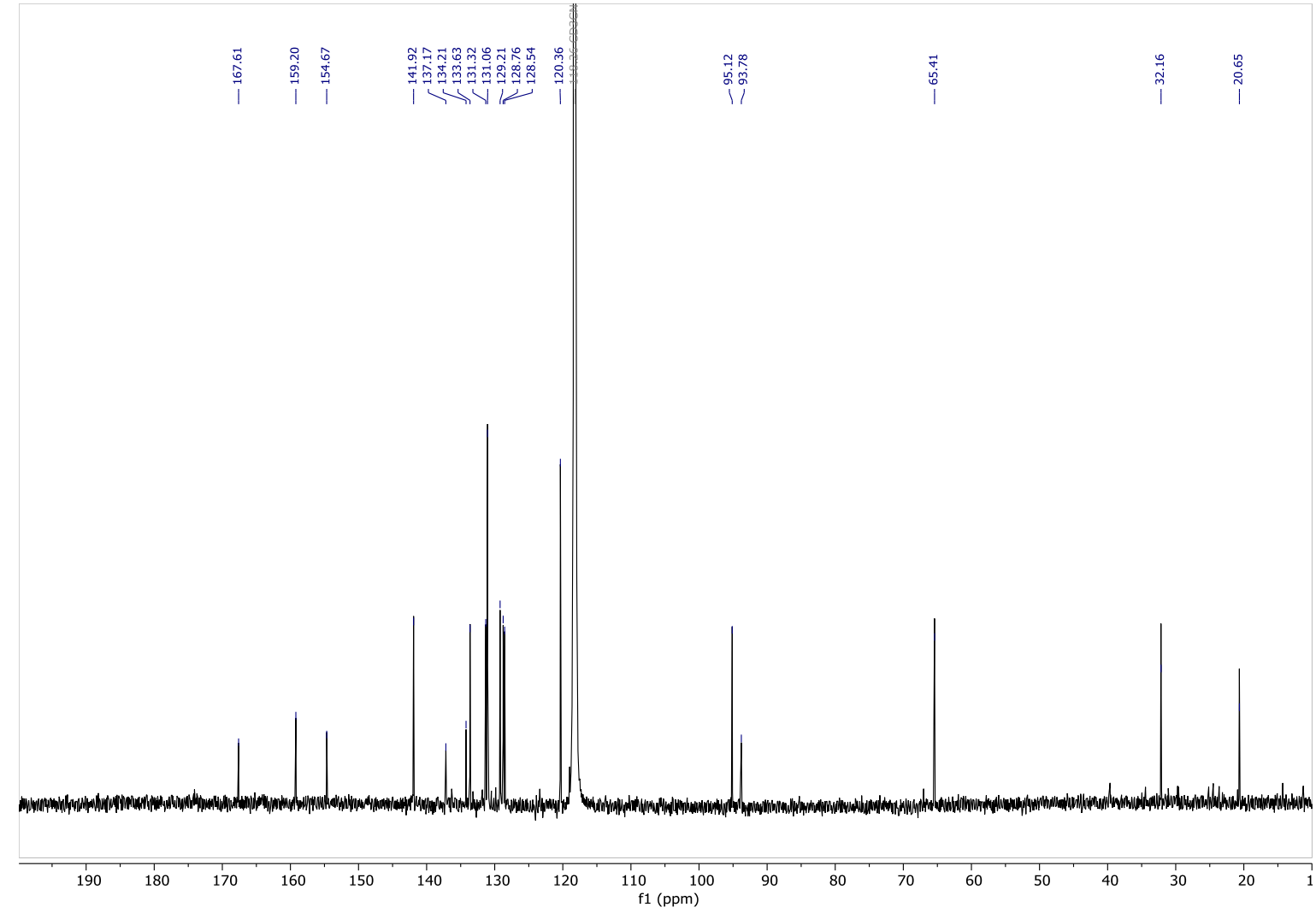


(E)-3-cyclopropyl-2-(p-tolyloxy)allyl 2-iodobenzoate (4s)

${ }^{1} \mathrm{H}$ NMR (400 MHz, Acetonitrile- $d_{3}$ )

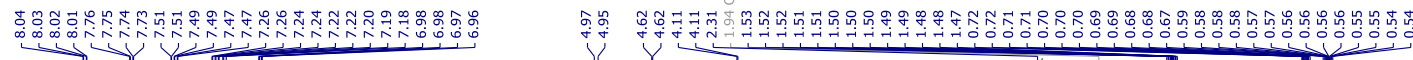

$\int 1 \int 1$<smiles>Cc1ccc(O/C(=C/C2CC2)COC(=O)c2ccccc2I)cc1</smiles>

H.t.
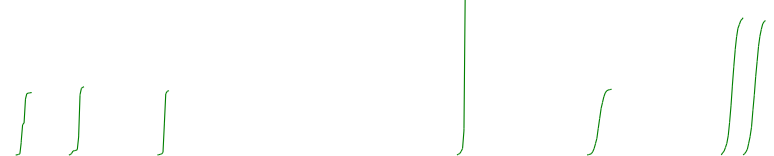

${ }_{1}^{M e}$

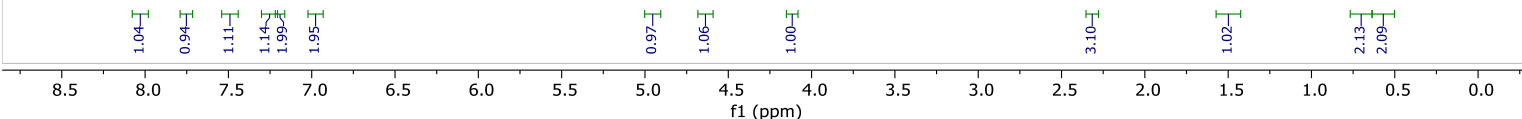

${ }^{13} \mathrm{C}$ NMR (101 MHz, Acetonitrile- $d_{3}$ )

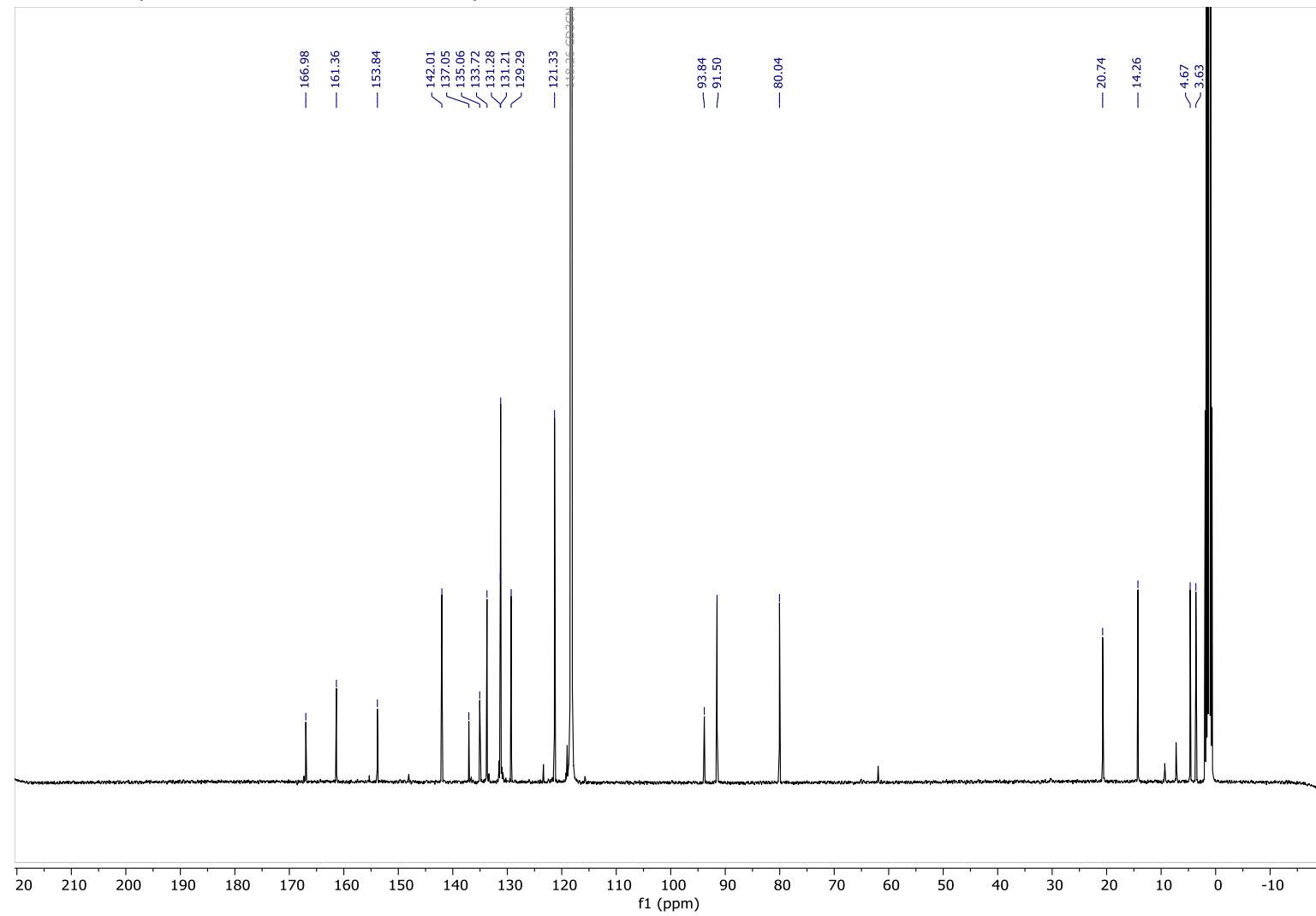


3-cyclopropyl-2-oxopropyl 2-iodobenzoate (14)

${ }^{1} \mathrm{H}$ NMR (400 MHz, Acetonitrile- $d_{3}$ )

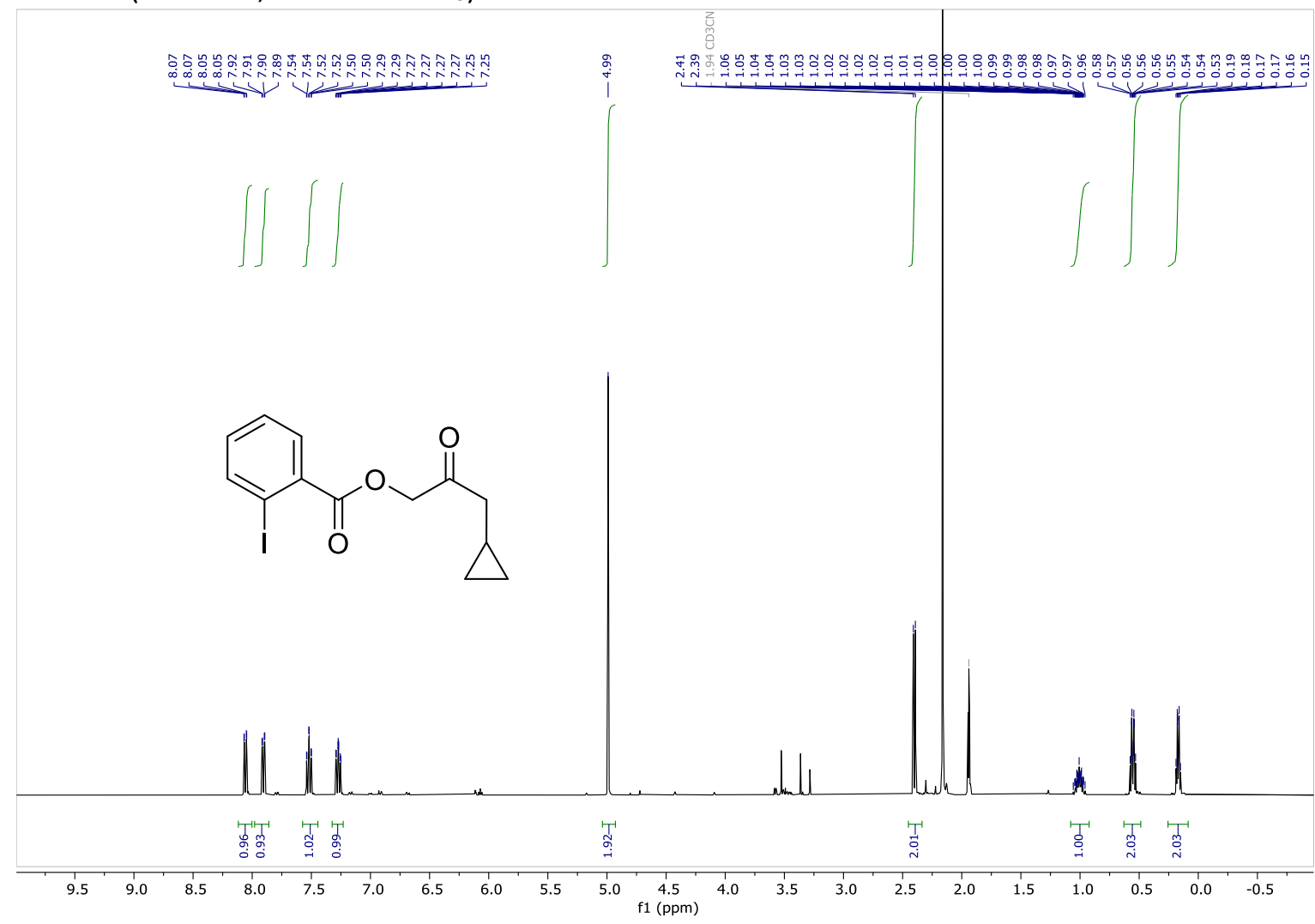

${ }^{13} \mathrm{C}$ NMR (101 MHz, Acetonitrile- $d_{3}$ )

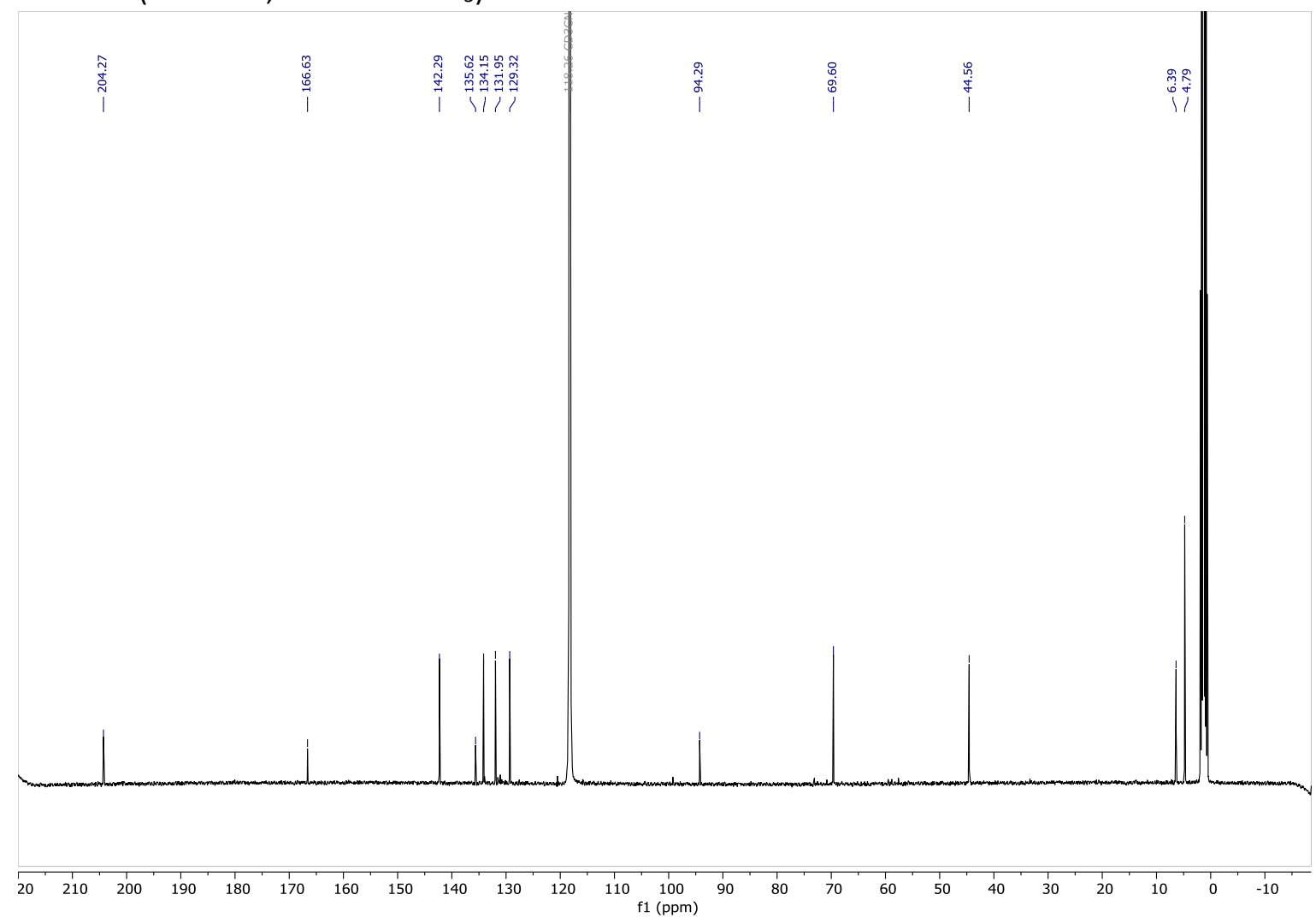

